

\section{- 20 a}

、 mâ

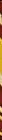
1ลลลลลลล जด

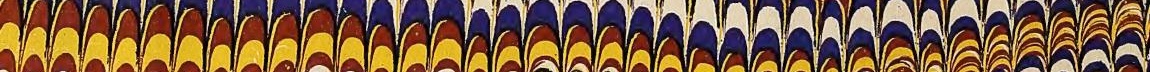

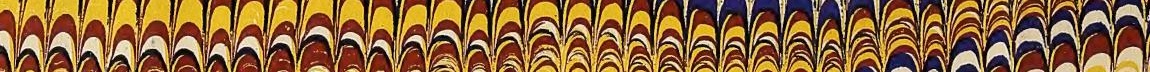

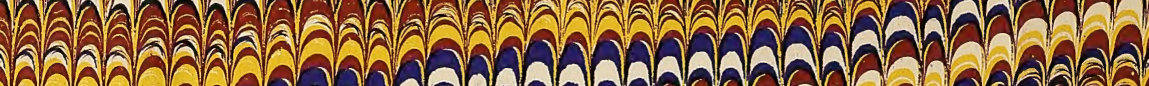
ล

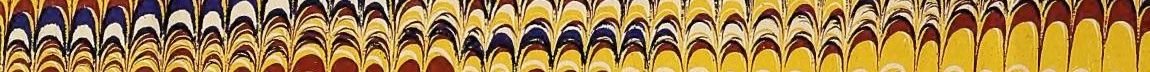

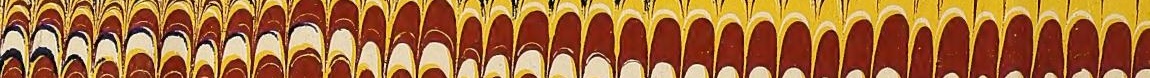
1 I

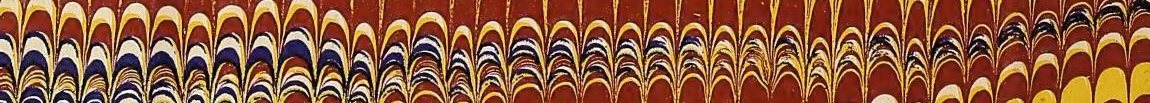

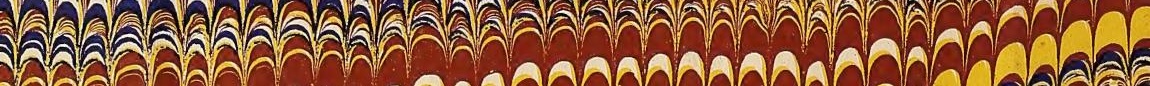

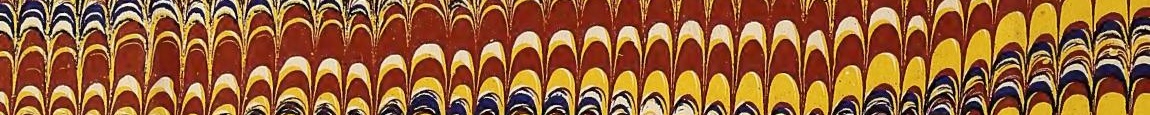

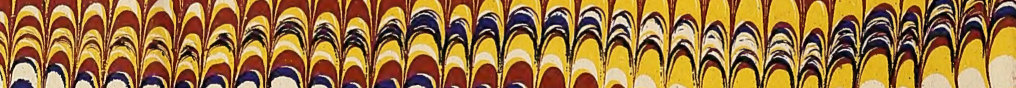

Thดลดนhดh

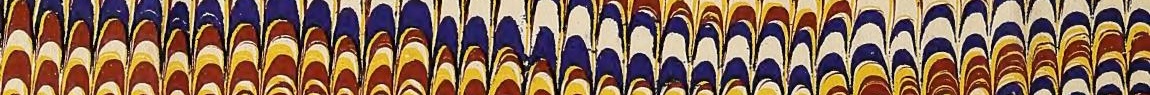

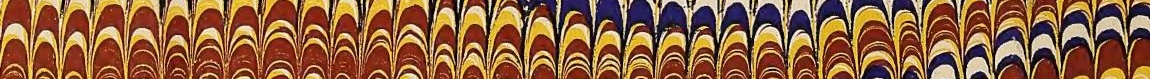

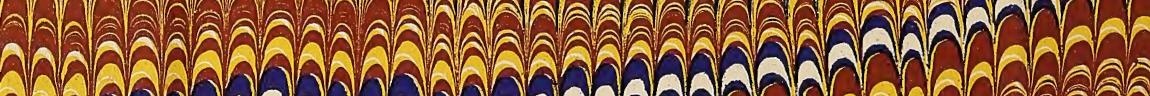

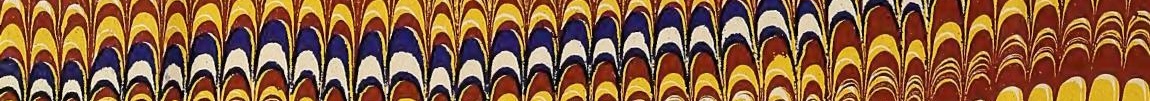

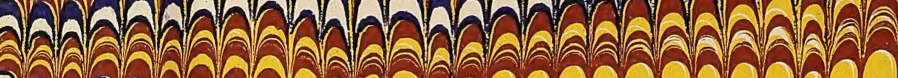

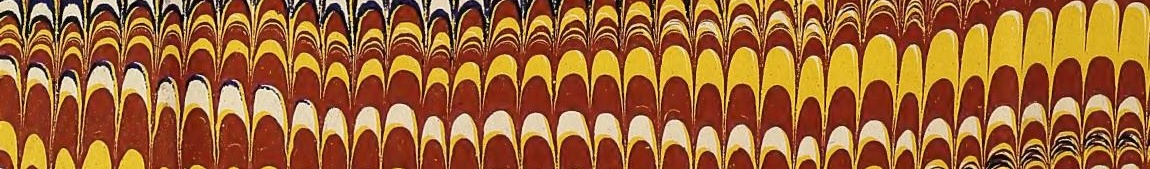

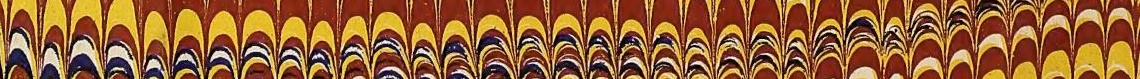

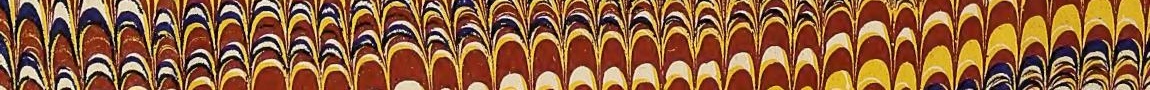

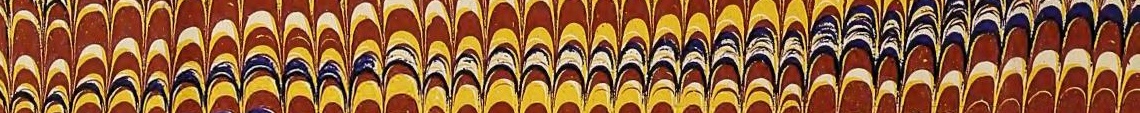

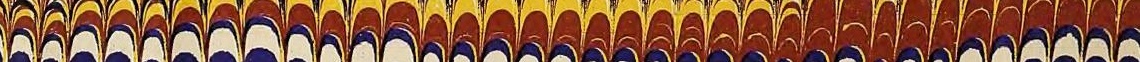

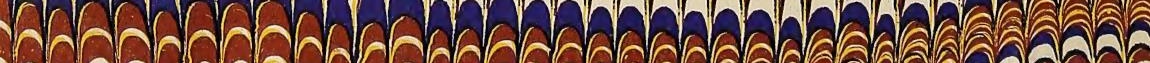

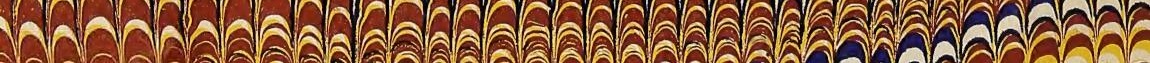

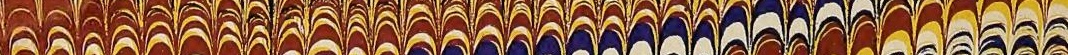









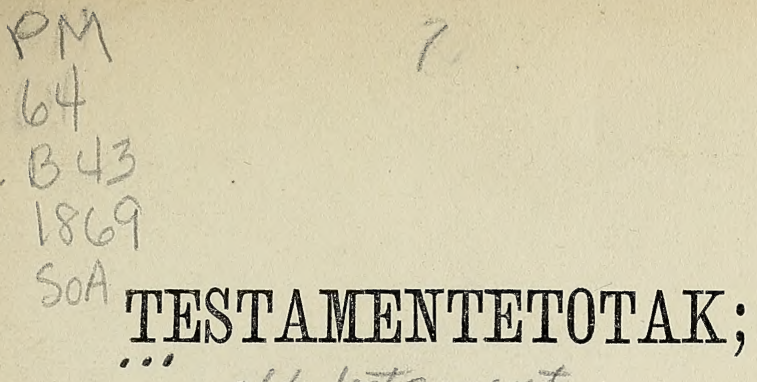

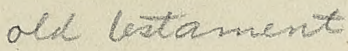

\section{JOSUAB AGLANGINIT， ESTERIB}

\section{AGLANGIT TIKKILUGIT.}

her beara $x$ conving to.
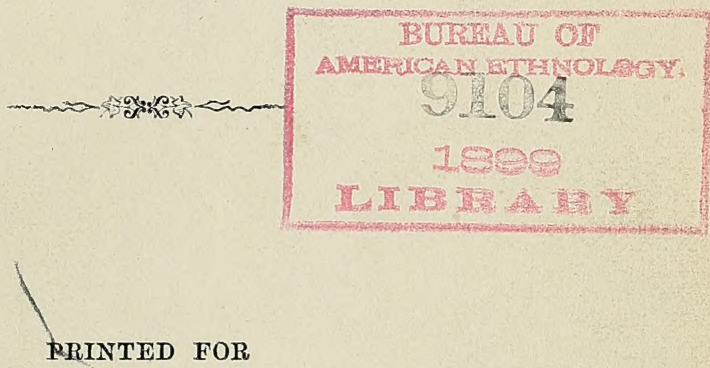

CThe Sititish and forcign Bible Society in fonton, FOR THE USE OF THE MORAVIAN MISSION IN LABRADOR.

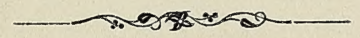

\section{STOLPEN :}

GUSTAV WINTERIB NÊNERLAUKTANGIT.

1869. 


$$
\begin{aligned}
& P M 64 \\
& B 43 \\
& 1869
\end{aligned}
$$




\title{
AGLAIT INGMIGOLINGAJUT:
}

\author{
axtom
}

Josua • • • • • • • . Pag. 1

Idluarsaijut : . . . . . . . 48

Ruth . . . . . . . . . . 95

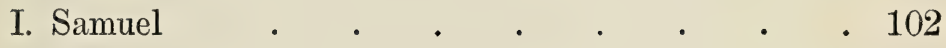

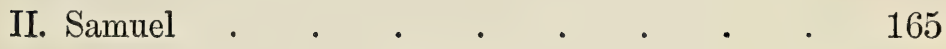

I. Attannit . . . . . . . 218

II. Attannit . . . . . . 280

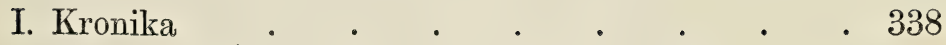

II. Kronika • • . • • • • • • 395

Esra . . . . . . . . . . . . 462

Nehemia . . . . . . . . . 482

Ester . . . . . . . . . 511 



\section{JOSUAB AGLANGIT.}

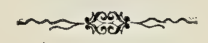

\section{KAPITEL $\mathrm{I}$.}

\section{Josuab aulatsïjokojauninganitk.}

MosESIB Nalekab kivgangata tokkungata kingorngane, $\mathrm{Na}=$ legak Josuamut Nunib erninganut, Mosesib kivganganut, okarpok:

2 Kivgara Mosese tokkovok; tagva prrngnailerit manna, Jordanelo tamna ikkarlugo pit, igvit innugiartoksuillo ukkox illunatik, nunamut tunnilauktamnut tapkonunga, Jsraelib kittornganginnut.

3 Allupse tullerarviginiartangit tamaita tunnilaukpakka illipsingnut, sorlo Mosese okautilaurapko.

4 Sunakajuitomit, Libanonemillo mattomiangat, koksoarmut Fratemik attelingmut tikkilugo; Hethiterit nunangat illunât, imarbiksoarmut kangianetomut kiglilervise illingajuksauvut.

5 Kialonêt akkerartoraksaringilâtit inôsingne tamât. Sorlo Mosese aiparilaurapko taimak aiparijomavagittauk. Kemagomangilagit aularviginaglonêt.

6 Maksoalerit mungungajuilutillo; nunamik aviksijuksaugavit innugiartoksoarnut ukkununga, angerutigilauktamnik atâtagijanginnut, tunnijomagapko tapkonunga.

7 Maksoatuinarit kuviasudlarlutillo, mallitsiarkovlutit, pinniarmarikovlutillo perkojet 'Mosesib kivgama perkolauktangit illingnut malliklugit. Senniagorniarnagit tallerpingmullonêt saumingmullonêt, sillatutsiartunnik illusekarungnarkovlutit pinniaraksarne tamaine.

8 Aglaillo makkoa perkojaktaggit kannernit nûlerkoniarnagit, nautsertorkille uvlorme unnuarmelo, mallitsiarkovlutit pinniarmarikovlutillo aglaksimajut tamakkonane malliklugit. Tagva sullitsiarniarpotit sillatutsiarlutillo piniarungnarniarpotit.

9 Ahak perkolaukpagit maksoalutillo kuviasuktuksaugavit. Keungominak, kakkilarnaglo; Nalekab Gudivit illagingmatit pinniarnerne pinniarneriniartarne tamaine. 


\section{KAPITEL IV.}

\section{Ujarket erkaumajqujukset Jordanib seniane iminganelo.}

NaLEKABLo Josua okautiva :

2 Angutinnik 12 nik tigusilerit, kinguvarêt nelliênit attautsemik,

3 Perkolugillo okaritselo: Jordanib illuanit, ajochertuijut ittiggangita nangervinganit, ujarkenik 12 nik kiviksileritse, neksarluselo tapkoninga tamaunga, kemaktaukovlugit sinniktarvipsingnut unutarme tamattomane.

4 Tagva Josuab angutit 12 it kaikovait, Israelib kittornganginnut annerijaumalauktut, kinguvarêt nelliennit attausek,

5 Okautilugillo: Aileritse Nalekab Gudipse iklervingata sânganut, Jordanib kerkânut; attunnillo ujarak attausek kivik siuk, illilugillo tuipsingnut, Israelib kittorngangita kinguvarêngita unnurningit malliklugit;

6 Nellunaikutaukovlugit akkunapsingne. Kittorngarijapse kingorngagut atâtatik apperijomâjarangagit okarlutiglo: Kannok ujarket ukkua tagvane sullidlarkât?

7. Okarkovluse tagva tapkonunga, kannok Jordanib iminga avilaungmat Nalekab iklervingata sivorngagut, Jordanekut akkigartautillugo, ujarket ukkua erkaumajaksaukovlugit soraijuitomik Israclib kittornganginut.

8 Tagva Israelib kittorngangit taimailiorput, sorlo Josuamut perkojaulauramik, ujarkello 12 it tiguvait Jordanib kerkanit, sorlo Nalekab Josua okautilaungmago, Israelib kittorngangita kinguvarêngita unnurningit malliklugit, neksarpaillo sinniktarvingmut; tagvanekolugillo.

9 Josuablo ujarket 12 it nappartipait Jordanib kerkâne, ajochertuijut, angersimanerub iklervinganik akkigartut ittiggangita nangervigilauktangane, tagvanepullo sulle uvlok tamanna tikkilugo.

10. Ajochertuijut iklervingmik akkigartut nangermatta Jordanib kerkâne, tamaita sorærtitaukârtinnagit Nalekab perkolauktangit Josuamut, innungnut okauserilugit; sorlo Mosesib Josua perkolaungmago. Innuillo tuavilukarput ailutiglo akkianut.

11 Innugiartoksuillo illuêngarlutik akkianut pilaukârtillugit, Nalekab iklervinga akkigartaugivok akkianut, innugiartoksuillo ajochertuijut sivorlerivait.

12 Rubenikullo Gadikullo, kinguvarêllo Manassekut ketterkangit, sakkolijarlutik Israelib kittorngangita sivorlerilaukpait, sorlo Mosesib okautilaungmagit;

1340,000 diokassait oppalungersimavlutik unnatadlarnermut, ailaukput Nalekab sângane sorsungnermut Jerichub naternangane. 
14 Uvlorme taipsomane Nalekab Josua angijorortipa Israelitit illunamassiatik sângane; sivoragivâllo sorlo Mosese sivoragilaungmatsuk innosinga nâlugo.

15 Nalekablo Josua okautiva:

16 Ajochertuijut kigligiudsinerit iklerviktanganik akkigartut perkokit, kakkikovlugit Jordanemit.

17 Taimak Josuab ajochertuijut perkovait okarlunelo: Jordanemit kakkileritse.

18 Ajochertuijullo Nalekab angersimaningata iklervinganik akkigartut kakkitillugit Jordanemit, tuttitillugillo allumingnut pannertomut, Jordanib iminga pilaurivok innigivaktaminut, kolunelo sivornganetut siksangangne illunane.

19 Takkib sivorlerpangata uvlungita 10 ninganne innugiartoksuit kakkilaukput Jordanemit, tangmârlutiglo Gilgaleme, iglugaseksuit Jerichub kittâne.

20 Ujarkello 12 it Jordanemit tigulauktangit Josuab nappartipait Gilgaleme,

21 Okarlunelo Israelib kittornganginnut: Kittorngarijapse atâtatik mattoma kingorngagut apperijomâjarangagit, okarlutiglo: Ujarket ukkua suksauvat?

22 Tagva kaujititaksarivasse okautilugillo: Israelitit ipterilingajokut pissulaukput Jordanekut;

23 Nalekab Gudipse Jordanib iminga ipteriliktilaungmago sivunapsigut ikârkârtinase; sorlo Nalegak Gudise pinniarlaungmat imarbingme iviksoatalingme suvlolingnik, ipteriliktilauktanga sivunaptigut ikkârkârtinnata.

24 Nunakattigêksuit nunamêtut illunatik illitarksilerkovlugit Nalekab agganginnik, kannok pitsartunekarninginnik; sivurakovluse Nalekamik Gudipsingnik soraijuitomik.

\section{KAPITEL V.}

Kanaaniterit mungungalerninganit. Israelitit Tippijauninginik, nervininginiglo saugarsungmik aularviksiorutiksamik.

AMORITERIT attanningit illunatik Jordanib akkiane kangiane nunakalauktut, Kanaaniterillo attanningit illunatik tussaramik, kannok Nalekab Jordanib iminga ipterilingatilaungmage, Israelib kittorngangita sivorngatigut, akkianut tikkikârtinagit, tagva omattingit mungungalerput maksoanekarungnailunelo tapkonane, Israelib kittorngangit pitjutigællugit,

2 Nelliutome taipsomane Nalegak Josuamut okarpok: Savingnik ujarkenik sennalerit, kippilugillo ama Israelib kittorngangit.

3 Tagva Josuab savit ujarkenik sennavait, Israeliblo kittorngangit kippivait kakkarulângme Aralothemik attelingme.

4 Tamannalo pitjutaulaukpok suna pivlugo Josuab innugi- 
lugit, utterkârtinagit udlatitik, kingorngane apkotigijariakartaptingnik agvertaritik.

17 Angutigle okarpuk tapsomunga: Angुermaringnermit tigulauktarnit uvaptingnit kellarudsartaujomavoguk,

18 Kainiarupta nunamut, aklunaglo aupaluktak una ningitijutigijet uvaptingnik kellaksorlungikungne igalangmut, kattitilungikungnelo iglungnut, atâtet anânello, kattangutitillo, atâtavillo iglomiokottingit illunaita.

19 Iglovillo upkoanganit annijub tapsoma aunga niakominut pile, uvaguglo passijaksaungilaguk; illunaitale iglungnetut kialo aggangimnut aktortaukpatta, aunget niakuptingnut pijuksauvok.

20 Kimullo sunamik pinniarkojauniptingnit tamattomangat okarniaruvit, angermaringnermit, tigulauktarnit uvaptingnit kellarudsartaujomavoguk.

21 Tamna okarpok: Taimailerle sorlo okaraptik; aularkolugiglo. Tapkoalo aularpuk. Tapsomalo aklunak aupaluktak kellaksorpa igalangmut.

22 Tapkoale aivuk tikkilutiglo kakkamut, tagvanêlutiglo uvlunik pingasunik, taipkoa utterkârtinagit udlatitingit. Kennerlaungmatta tapkungninga apkosinerne tamaine, nachvaratiglo.

23 Taimak angutik tapkoa utterivuk, attersarlutiglo kakkamit, ikârlutiglo tikkilarmiglo Josuamut, Nunib erninganut, unipkarlutiglo tamainik, kannok tukkisilauktamingnik,

24 Okarpuglo Josuamut: Nalekab nuna :illunât tunnilaukpa aggaptingnut, taimaktauk nunab innungita illunatik maksoajuijutigivâtigut.

KAPITEL. III.

\section{Isractitit pissungninginit Jordanelut.}

JoSUALO uvlarârsukut parngnailerpok, aularlutiglo Sittimemit, tikkilarmiglo Jordanemut, tamna Israeliblo kittorngangit illunatik, tagvanelerlutiglo unnuarme ikârkâratik.

2 Uvlulle pingasut nângmatta angajokajet tangmarvik pissugvigivât,

3 Perkolugillo inugiartoksuit, okarpullo: Tækkoniarupsiuk Nalekab Gudipse angersimaningata iklervinga akkigartautillugo ajochertuijunuut Levitinnit pijunnut, tagva aularitse innigijapsingnit malliklugolo:

4 Imagle, akkumapsingne, tapsoma illipselo inniksakarkovlugo tallitut 2000 ditukassait takkitigijomik. Kaglijaksaringilasse, kaujimakovluse apkutit nelliene agvertartuksaumangapse; apkut tamanna sivorngagut agvertarvigilaunginapsiuk. 
5 Josualo okarpok innugiartoksoarnut: Ivsornaitititse Nalegak kaupat tattamnartulliniarmat akkunapsingne.

6 Ajochertuijunnullo okarpok: Angersimanerub iklervinga akkigarsiuk ailerluselo innugiartoksuit sivorngænne. Tagva tapkna angersimanerub iklervinga akkigarlugolo aivut innugiartoksuit sivorngænne.

7 Nalekablo Josua okautiva: Uvlome pigiaromavunga angijorortilutit Israelitit illunamassiatik sanginne, kaujimakovlugit sorlo Mosese illagilaurapko illagingmigapkit.

8 Igvillo ajochertuijut angersimanerub iklervinganik akkigartut, perkokit, okalutillo: Tikkikupse Jordanib imingeta sinâta illuanut, tagva nokkaritse.

9 Josuablo Israelib kittorngangit okautivait: Kaititse tussarluselo, Nalekab Gudipse okausinginnik;

10 Okarlunelo: Tamattomangat tukkisilertuksauvose omartomik Gudekarmat akkonapsingne; aulartitsiniarmallo sirunapsigut Kanaaniterinnik, Fethiterinnik, Heviterinnik, Feresiterinnik, Girgasiterinnik, Amoriterinnik, Jebusiterinniglo.

11 Ahâk, attaniotitub sillaksoarmut tamainut angersimaningata iklerlervinga sivorleriniarpasse Jordanib illuanut.

12 Tagva manna angutinnik 12 nik tigusileritse Israelib kinguvarêngninginnit, kinguvarêt nellienit attautsemik.

13 Mannalo ajochertuijut akkigartut attanniotitub sillaksoarmut tamainut angersimaningata klervinganik, allutik pitipattigik Jordanib imminganut, tagva immek timânit kôktok avingniarpok, kallerêktilunelo nokkarniarpok.

14 Tagva manna innugiartoksuit annitillugit tuppermingnit, Jordan ikâromavlugo ajochortuijullo angersimanerub iklervinganik akkigartillugit innugaseksuit sivorngænne,

15 Jordanemullo pitillugit, ittiggamingniglo missuktillugit imermut, (Jordanele ullingalaukpok inninginne tamaine, kattersiviub nelliuninga navlugo.)

16 Tagva imek timânit kôktok nokkarpok kallerêktilunelo, ungasidlartomik, iglugaseksoarnit Adamemik attelingnit Zarthanib senianetomit; immerle attânut pijok imânut, inânut tarriolingmut kêtaulerpok nungulunelo. Taimak innugiartoksuit ikârput Jericho sâlugo.

17 Ajochertuijullo Nalekab angersimaningata iklervinganik akkigartut, nangerput taimak ipterilingajome Jordanib kerkane. Isráelitillo illuêngarlutik ikârput erkagut ipterilingajokut, innugiartoksuit illumamassiatik Jordanib akkianut tikkikârtinagit. 
10 Tagva Josuab innugiartoksuit angajokângit perkovait, okarlunelo:

11 Tangmarvik pissugvigissiuk innugiartoksuillo perkogissigik, okarluselo: Takkoaksamik kattersoileritse uvlut pingasut nâkpatta Jordan una ikkarniarapsiuk, tikkilerkovluse tigusilerluselo nunamik Nalekab Gudipse tunniniartanganik illipsingnut.

12 Rubenekunnullo Gadekunnullo kinguvarênullo Manassib kinguvangita ketterkanginut Josua okarpok:

13 Erkarsigik okautsit taipkoa, Mosesib Nalekab kivgangata okauserilauktangit illipsingnut, okarlunelo: Nalekab Gudipse merngoêrservingmut âtsarêrpâse tunnitsivigiluselo nunamik ominga.

14 Nulliakotisse sorrusekotisselo nergjutekotisselo kemmaksigik nunamut Mosesib tunnilauktanganut illipsingnut Jordanib miksâne; illipsele, angutit sorsugungnartut sakkulijarluse kattangutisse sivorlervigijaksarivase ikkajoraksarilugillo,

15 Kattangutisse merngoêrservingmut âtaukârtinnagittauk Nalekamut illipsetut, aksarnikârtillugillotauk nunamik Nakab Gudipse tunniniartanganik tapkonunga. Tagva uttertuksaugivose nunapsingnut, Mosesib Nalekab kivgangata tunnilauktanganut illipsingnut perkutaukovlugo illipsingnut, Jordanib miksâne, sekkerngub nuilervingata nellane.

16 Tapkoalo Josua kiolugolo okarput: Hllunaita perkolauktatit uvaptingnut pinniarutigijomavavut, namullo tilliklerviginiartarnut uvaptingnik, tagvunga aijomavogut.

17 Sorlo Mosese nalelauraptigo, taimak igvittauk nalegomavaptigit; kissiane Nalekab Gudivit illagituinarpattit, sorlo Mosese illagilaungmago.

18 Kannernik nalengitok, nalengnanelo okausernik, tamaine perkojarne uvaptingnut, tamna tokkojuksauvok. Maksoatuinarit mungungajuilutillo.

\section{KAPITEL II.}

\section{Nautsertortuksâk Rahabemut piulïauvuk.}

JoSUABLE Nunib erningata angigutsivlune nautsertortuksâk magguk tillilaukpâk Sittimemit okautilaukpâglo: Aileritik nuna nautsertoritikko Jericholo. Tapkoa aivuk, tikkilutiglo anguniarpaktub Rahabemik attiggub iglunganut, sinniktarlutiglo tapsomane.

2 Tagva attannek Jerichome okautsauvok: Ahâk, unuarme tamattomane tikkitokarpok ovunga angutinik Israelitinnit nuna nautsertoromavlugo. 
3 Tagva Jerichub attanertanga tilliklerpok Rahabemut okantsaukolugolo: Angutik tikkituk illingnut iglungnut kaikik; tikkisimangmannik nuna nautsertoromavlugo.

4 Arnable tapsoma angutik tapkoa ijertorpâk, okarlunelo imâk: Tikkitokalauralloarpok angutingnik uvamnut, kaujimalungitungale nakit pingmangangnik.

5 Upkoaksuillo pallangertaujuksautillugit tâktillugo annilaukpuk, kaujikonanga namut aivingangnik. Erinesukluse udlassigik anguniarapsigik.

6 Tapsomale tapkoa majoarkovak iglub kânganut, ijertorpaglo ivaluksaijakset ivingita issivimalauktame iglub kângane attânut.

7 Angutille udlatorpakik apkosinerme Jordanemut, ikkarvik tikkilugo. Upkoaksoarlo pallangertaulaukpok, taipkoa udlaniktut taipkungninga annilaukârtillugit.

8 Angutiglo sinnikârtinagik, majoarlune iglub kânganut aivigivak,

9 Okautivlugiglo: Kaujimavunga Nalekab nunamik mattominga tunnitsivigingmasse; kvaksarnerub tupvigingmattigut sivunapsigut, nunablo innungita illunatik tikkitsomâlnise maksoajuijutigivât.

10 Tussalaurapta kannok Nalekab immak immarbingme iviksoatalingme suvlolingnik ipteriliktilaungmago sirunapsigut Egiptenemit aulartilluse, pinniarnerilauktapsingniglo Amoriterit attannigingnut, Sihonemut Ogemullo Jordanib ungatanêtungnut, nungulaurapsigik.

11 Taimangallo tamattominga tussarlamapta, omattivut mungungavut, maksoarnekarunginailunelo nelliane tikkiniarnise pitjutigæellugo; Nalegak Gudise Gudeôngmat, tamangne pâno killangme, attânelo nuname.

12 Tagva Nalekame angervigitinga mamna, napkiningnermik sakkertitsivigigaptik illipsetauk atâtama iglomiokattinginnut napkiningnermik sakkertitsikovluse, nellonaikutamiglo nellonangitomik tunnitsivigitinga,

13 Inôkogapsigik atâtaga, anânagalo, kattanğntikkalo anğutit arnello, tamaitalo illangit, piulilugillo tarnivut tokkom?

14 Angutik tapkoa okarpuk tapsomunga: napkiningnermik nellagornermiglo sakkertitsiniangikupta illingnut, Nalekab nunamik ominga tunnitsivigikpattigut, tagva tarnivił inangêrluse tokkojuksauvuk, kissiane pinniarkojauniptingnik sakkertitsikonak.

15 Tagva igalakut aklumamut ningitipak, iglunga iglugaseksuit avalungita karmangita tungânelaungmat, kânganelaungmallo.

16 Tapsomalo okautivak: Kakkanut aileritik parsikonagiilliptingnik udlatitik, ijertorlutiglo tagvano urlut pingasat nât- 
artoksuit illunaita Egiptenemit aularlauktut, angutit, kippilaungmagit; sorsuktukset illunatik tokkolaungmatta apkomelutik sunakajuitome Egiptenemit aularlutik,

5 Inuille illunaita aularlauktut kippimmalaukput, innuille illunaita sunakajuitome erniangolauktut apkome, Egipternemit aularlutik, kippijaulaungilet.

6 Israelib kittorngangit agvertarlaungmatta, Jahrit 40 git nâlugit sunakajuitome, innuit illunaita angutit sorsuktukset, Egiptenemit aularlauktut nungukârtinagit, tamana pivlugo Nalekab nippinga nalelaungimatsuk; Nalekab okautimarilaungmagit, nuna tækkojaksaringimatsuk, Nalekab angerutigilauktanga atâtagijanginnut, tunnijomavlugo uvaptinguut, nuna imungmik igupsaujartullo orksuanik kôlik.

7 Tapkoa kittorngangit inangêrlugit innojut, Josuab kippivait; kippimalaungimatta, apkome kippijaulaungimatta.

8 Inugiartoksuillo illunaita kippisimagamik tangmarvingme najortamingnetuarlaukput mamikârtinagit.

9 Nalegarlo Josuamut okarpok: Uvlome Egiptenib kangunarninga aksakartilaukpara illipsingnit. Tangmarviglo tamanna Gilgalemik taijaulaukpok, uvlok tamanna tikkilugo,

10 Israeliblo kittorngangit taimak tangmarsimalutik Gilgaleme, Passamik attorput, takkib taipsoma uvlungita 14 ingata unuuangane, Jerichub naternangane,

11 Nerrivlutiglo nunab kakkojaksanginnit, Passab uvlungita aipangane, kakkojamik sêrnakangitomik, kakkojaksaniglo jarrasimajunnik, uvlorme taipsomane.

12 Manalo sorailaukpok, nerrivingæta nunab kakkojaksangjunik uvlungata aipangane, Israelib kittorngangit Mannakarungnimatta, nerrilaukpulle Kanaanib nunangata kakkojaksanginik Jahremit taipsomangàt,

13 Pijokalaukporlo Josua Jerichub kannitanganêdlune, ijingminut tækkosarlaurame, mallugosulerporlo nangertokangmat sakkamine angumik, tigumiartomik aggangminut saviksoarmik poksimangitomik. Josuablo aivigivlugo okautiva; Uvaptingnut illingavît, omigijiptingnullonêt?

14 Tamna okarpok: Aukak, angajokaksoangovungale Nalekab kattimgajokotingimut, tikkipungalo manna. Tagva Josua kênaminik pûlune nunamut pamakterpok, tuksiarlunelo okautiva: Kannoêtunik Nalegara okausiksakarka kivgaminut?

15 Angajokaksoarlo Nalekab kattimgajokotinginnut Josuamut okarpok: Ittigagutit pêrkit ittiggangait; nuna nangervigijat ivsornaimat. Josualo taimailiorpok. 


\section{KAPITEL VI.}

\section{Jerichub asserortauninganik.}

JERICHOLE pallangersimalaukpok, udsertortaulunelo Israelib kittorngangit pitjutigallugit, annijokarungnarkonnago, ittertokarungnarkonagolonêt.

2 Nalekable Josua okautiva: Tagga ahâk, Jericho, attanertangallo sorsuksoakotingillo illaliudlugit, tunnimavakka aggangnut.

3 Angutit sorsuktukset pissukokit iglugaseksuit avatâtigut attausiarlutik taimailiorlutillo uvlunne 6 inne.

4 Uvlulle 7 ingane ajochertuijut Jahrib nertordlernartub tiptulautingit 7 it tigulerligit iklerviub sivorngane; uvlullo 7 ingane tamattomane 7 êrtorluse aileritse iglugaseksuit avatâtigut, ajochertuijullo tiptulautit tiptulausijautigiligit.

5 . Jahriblo nertordlernartub tiptulautinga tiptulausijartaukpat, imiatitaakpallo, tiptulautit tussarupsigik, tagva innugiartoksuit illunamassiatik unatadlarnerub nippevaksoanganik pijuksauvut, tagva iglugaseksuit avalungita karmangit ochoniarput innugiartoksuillo ittertuksauvut illunatik attunit sivumut.

6 Tagva Josuab Nunib erningata ajochertuijut kaikovait okautivlugillo: Angersimanerub iklervinga akkigarsiuk, ajochertuijullo 7 it neksarlit Jahrib nertordlernartub tiptulautinginnik 7 nik Nalekab iklervingata sivorngane.

7 Inugiartoksoarnulle okarpok: Aileritse pissukluselo iglugaseksuit avatâtigut; oppalungersimajullo ailerlit Nalekab iklervingata sivorngane.

8 Josuab innugiartoksuit tamattominga okautijarêrlaungmagit, ajochertuijut 7 it Jahrib nertordlernartub tiptulautinginik 7 nik akjarsivut Nalekab iklervingata sivorngane tiptulausijarlutiglo; Nalekablo angersimaningata iklervinga mallikpok tapkoninga.

9 Oppalungersimajullo ajochertuijut tiptulausijartut sivorngane pisulaukput; innugiartoksuillo mallikput iklervingmik, ajochertuijullo sivumuarlutik tiptulausijarput.

10 Josuable innugiartoksuit perkovait okarlunelo: Unatadlarnerub nippivaksoanganik pijuksaungilase, nippiselonêt tussartaukojaksaringilasse, annititsinaselonêt okautsemik attautsemik kannipsingnit, uvlok tamna tikkilugo okarviga illipsingnut: Unatadlarnerub nippivaksoanganik pileritse; tagva unatadlarnerub nippivaksoanganik pileritse.

11 Taimak Nalekab iklervinga ingergavok attausiarlune iglugaseksuit avatâtigut, kailutiglo tangmarvingmut, nokkarlutiglo tagvane.

12 Josuale uvlarme uvlarârsukut makkijongolaukpok, ajochertuijullo Nalekab iklervinga akkigarpât.

13 Taimak ajochertuijut 7 it Jahrib nertordlernartub tip- 
8 Iglugaseksuille aksarnigarijarêrupsigik ikkilersigik ikkomamut, pinniarluselo Nalekab okausingit malliklugit. Ahak perkovapse tamattominga.

9 Taimak Josua tilliklerpok tagvunga, aivullo terliarsiviksamingnut, nokkangavullo Beth-Elib Aiblo akkorngangne, Aib kangiane. Josuale unnuarme innugiartoksuit akkornganetualerpok,

10 Uvlarmelo uvlarârsukut makkijajovok, parngnaitilugillo innugiartoksuit, majorarlunelo Israelib angajokangit illagilugit, inugiartoksuit sivorlerilugo takpaunga Aimut.

11 Inuillo sorsuktuksat illagijangit majorarlaukput, kaglilutiglo tikkilutiglo iglugaseksuit sakkânut, nokkangalutiglo Aib tachane, naksakartuinarmat tapkoa Aiblo akkorngane.

12 .Tiguvaillo angutit 5000 dikassait pitilugillo terliarsiviksamut Beth-Elib Aiblo akkorngangnut iglugaseksuit kangianut.

13 Pitipaillo innugiartoksuit kattimgajut illunaita; iglugaseksuit tachanelauktut; kingorlingit pingmatta iglugaseksuit kangia tikkidlugo. Taimak Josua unnuame taipsomane ailaukpok nâksab kerkanut.

14 Aiemiulle attaningat tamattominga takkolaurame, tuavidlalaukput, tavalekullo parngnailerput, iglugaseksuillo angutingit anisarput Iraelitit paromavlugit unatadlarnermut inniksame nellonarungnaititaumajome naternaub miksane, innukotine illunaita illagallugit. Kaujimalaunginame uttakkijokarmat tapsominga terliarsivingme iglugaseksuit ungatane.

15 Josuale Israelitillo illunatik pissertorlutik salauniarsertorput tapkonunga kemâlutiglo apkome sunakajuitomut sangmijome.

16 Tagva iglugaseksoarmiut illunamasiatik niplialerput unangmiklertokarkovlugo tapkoninga.

17 Unangmivâllo Josua, annilutiglo iglugaseksoarnit, angutekarungnailaungmat attautsemiglonêt Aiemê Beth-Elemêlo annilaungitomik Israelitit unangmilugit, iglugaseksuillo pallangersimanagit kemakpait Israelitit unangmilugit.

18 Tagva Nalegak Josuamut okarpok: Kallugiak aggangnetok issautiuk Aiemut tunnijomagapkit aggangnut. Josuablo kallugiak issautingmago iglugaseksoarnut.

19 Tagva terliarsivingmetut aularsarput innigijamingnit akpangerlutiglo, aggangit issalaukârlugit, tikkilutiglo iglugaseksoarnut sâlagilugillo, tuavilaukpullo, ikkilaitalo ikkomamut.

20 Angutillo Aiemiut keviarput, tækkosarlutiglo uttimut, tækkolutiglo iglugaseksuit isserlunganik kôngmuartomik killangmut, kemâviksakangilello ikkungalonêt tagvungalonêt. Innuillo sunnakajuitomut kemâjut tunnulerput udlatorlugit.

21 Josuale, Israelitillo illunatik tækkogamik, terliarsivingmelauktut iglugaseksuit aksarnigaringmattigik, iglugaseksuit 


\section{isserlunga kongmuarmat, utterivut ullimarlugillo angutit} Aiemiut.

22 Tapkoalo iglugaseksoarnetut anilaurivut pârlugit, taimak Israelitit akkornganemarilaungmatta, ikkangat ovangallo ullimarpaillo pitakarungnaikârtinago, tapkonangat nellienik, kemâjokarungnarkonagolonêt.

23 Aiblo attannertanga tiguvât innotillugo âlâlo Josuamut.

24 Israelitillo Aiemiut illunaita naternamelauktut, sunnakajuitomelauktullo udlalauktatik tokkojarêrmattigik, ochojarêrmattalo saviksub kênanganut, illunatik nungukârtinagit, tagva Israelitit illuêngarlutik salerput Aiemut, ullimârlugillo saviksub kênanganut.

25 Illunatiglo ocholauktut uvlorme taipsomane tamarmik angutit arnello 12000 diolaukput, illunatik Aiemiut.

26 Josuablo aggane kallugiarmik issaktijutigijane erkitilaungilait nungukârtinnagit Aiemiut illunaita.

27 Iglugaseksuille nergiutingit aksartaujutigijangillo Israelitit avitipait ingminut, Nalekab okausingit, Josuamut perkolauktangit malliklugit.

28 Josuablo Ai nungutimarikpait ikkomamut, katsiktungortilaitalo soraijuitomik, tagvanetomik sulle uvlome.

29. Aiemiullo attanningat nivingataukova nappartomut unnuk tikkilugo. Sekkinerle nippijarêrmat, perkojivok timinga anernengêrtok akkartitaukovlugo nappartomik, egipâllo iglugaseksuit itterviksoanganut, ujarkello unuktut perorliutivait kânganut, uvlok tamanna tikkilugo tagvanetut.

30 Tagva Josuab Altare sennava Nalekamut Israelib Gudinganut, kakkame Ebalemik attelingme;

31 Sorlo Mosesib Nalekab kivgangata Israelib kittorngangit perkolaungmagit, sorlo aglaksimangmat Mosesib aglanginne perkojaktalingne, Altare yjarkenit illuitunnit, kikkiengmut ullimartaulungitunnit; tunnergutiksaniglo oteksanik kujalijutikseniglo tunnijivok Nalekamut.

32 Aglaklunelo tagvane ujarkenut, perkojet adsinginik, Mosesib Israelitinut perkolauktanginik.

33 Israelitillo illuêngarlutik, angajokajatik, aulatsijitiglo idluarsaijitiglo illagilugit nangerput iklerviub senneragingne tamangne, ajochertuijut Levitillo Nalekab angersimaningata iklervinganik akkigartut sanginne, tujormijat illagijangillo, ketterkangit aipaingit kakkab Grisimemik attigub senniane, aipaingillo kakkab Ebalemik attiggub senniane, sorlo Mosese Nalekab kivganga sivorngane perkojilaungmat, innugiartoksuit Israelitit saimartilugit.

34 Kingorngane perkojet okausertangit illunaita, okartut saimanermik ominarnermiglo kaigarsuktaukoveit, sorlo aglangne perkojaktalingne aglaksimangmat. 
runik, illuniarpâtigut, attivullo pejarniarpait nunamit. Sunamiglo pinniaromavît atterne angijome?

10 Tagva Nalegak Josuamut okarpok: Makkitit! sôg taimak pamangavit kenêt pûlugo?

11 Israelitit idluitulilaukput, angersimaneralo perkolauktara tapkonunga tappagilaukpât; tigusilauramik nungutaksamik, tiglilaukpullo taimak, missiarlutiglo, illijilutiglo perkutigijamik akkornganut.

12 Israelib kittorngangit nangerungnangilet omigijimik sanginne, tunnuleriakarpaille omigijitik; ominautiksakaramik. Mannamit illaginiangilapse, ominautiksak pêjarniangikupsiuk illipsingnit.

13 Makkitit innugiartoksuillo ivsornaitikit, okarlutillo: Ivsornaititse kaupemut. Nalegak Israelib Gudinga imak okarmat: Ominautiksakarpok akkonapsingne Israelitiojose; taimaimat nangerungnangilase omigijipse sanginne ominautiksak pejartaukârtinago illipsingnit.

14 Uvlakullo kaijuksauvose, kinguvarêt nellekârlutik; kinguvarêllo Nalekab nellauniartangita abvakungit nellekarlutik kaijuksauvut; abvakullo Nalekab nellauniartangita iglomiukattingit nellekârlutik kaijuksauvut; iglomiukattillo Nalekab nellauniartangita angajokangit nellekârlutik kaijuksauvut.

15 Nachvartauniartorlo ominautiksakarlune tamna ikkitaujuksaurok ikkomamut, pigijane tamaita illaliudlugit, tamanna pivlugo, Nalekab angersimaninga tappagilaungmago sillainermiglo pinniarlaungmat Israeleme.

16 Tagva Josua uvlarme uvlarâsukut makkijajovok, âtsilunelo Israelitinnik, kinguvarêt nellekârlutik; Judablo kinguvarêngit nellautaulaukput.

17 Judablo abvakungit âlaungmagit, Serab abvakungit nellautaulaukput. Serablo abvakungit âlaungmagit ig̣̣t - angajokangit nellekârlutik, Sabdi nellautaulaukpok.

18 Iglomiukattingillo âlaungmagit, angutit nellekârlutik, Achan nellautaulaukpok, Karmib erninga, Sabdib erningata Serab erningata Judab kinguvarênginit.

19 Josualo Achanemut okarpok: Ernîk nertordlernermik tumnijilerit Nalekamut Israelib Gudinganut, nakorilugolo pît, okautilungalo, sunamik pinialaukit? missiarviginangalo sunamik.

20 Tàgva Achanib Josua kiova, okarlunelo: Ahamarik Nalegak Israelib Gudinga idluitullivigilaukpara; imâkk imâglo pinniarlaukpunga.

21 Aksarnigaujat akkorngane tækkolaukpunga ullingmik pivianartomik Babelemiomik, Silberemiglo Sekelenik 200 dinnik, okangoamiglo Goldemik Sekeletut 50 gitut okumaitigijomik, tapkoninga ikligulaukpunga tigusilungalo. Ahaglo saumavlutik ijersimavut nuname tuppimme, Silberelo akkornganepok. 
22 Tagva Josua nautsertortuksanik tilliklerpok, tapkoa akpangerput tuppermut; ahâglo, ijersimalaukput tuppingane, Silberelo akkornganêdlune.

23 Tiguvaillo tuppermit âlugillo Josuamut, Israeliblo kittornganginut illunainut, navilaitalo Nalekab sânganut.

24 Tagva Josuab Israelitillo illunatik Achan Serab eirninga tiguvât, Silberelo, ulliglo, okangoarlo Golde, erningillo panningillo, tuktuvakotingillo, siutekoktojokotingillo, saugakotingillo tuppingalo, tamaitalo pigijangit illaliudlugit, tessiorpaillo paunga naksamut Achoremik attelingmut.

25 Josualo okarpok: Kiksartilauraptigut Nalekab kiksartilitit uvlorme tamattomane. Israelitillo illunatik millorpât, ikkilugillo ikkomanut. Millorêramigillo,

26. Ujarket unnuktut perorliutivait kanginnut, tapkoa tagvaneput sulle uvlok tamanna tikkilugo. Tagva Nalegak ningautsime illungertorninganit allarpok. Tamanna pivlugo nuna tamanna taijauvok, Achorib naksanganik uvlok tamannatikkilugo.

\section{KAPITEL VIII.}

Iglugaselssuit Ai salagïjauvit. Saimanarnek ominarnerlo okautigïauvuk kriklkangne Garizimeme Ebalemelo.

Nalegarlo Josuamut okarpok: Sivuraniarnak mungunganaglo. Inuit sorsuktuksat illunaita illasearikit, parngnaîlutillo majorarlutillo takpaunga Aiemut. Tagga ahâk, Aib attannertangat, innukotingit illaliudlugit tunnijarêrpakka aggangnut.

2 Aielo, attanertangallo pinniarvigijaksarivattit, sorlo Jericho attanertangallo pinniarvigilaurangne, kissiane aksartaujutigijangit omajokotingillo avigeksarigapsigik illipsingnut. Uttakkijokarkojilerille terliarsivingmêtunik iglugaseksuit ungatane.

3 Tagva Josua parngnailerpok sorsuktuksallo illunatik majoraromavlutik Aiemut. Josuablo angutit sorsuktuksat 30,000 dit annerivait tillilaitalo unnuarme;

4 Perkolugillo okarpok: Udsertoritse, illipse uttakkijuksauvose terliarsivingme, iglugaseksuit ungatane ; kanningiluartomulle iglugaseksoarnit ainiarase, oppallungersimaluselo illunase.

5 Uvangale innugiartoksuillo illagijakka kaglileromavogut iglugaseksoarnut. Opaklutalo pachreartorpattigut sivurnganetut kemâjomavavut.

6 Udlatuikovlugit uvaptingnik iglugaseksuit sillatânut, âtsikârtinata tapkoninga iglugaseksuit sillatânut. Issumakarniarpulle kemâgaptigik sivurnganetut. Kemâtillutalo tapkoninga,

7 Illipse nulertnksauvose terliarsivipsingnit iglugaseksuillo aksarnigarilugit; Nalekab Gudipse tunniniarmagit aggapsingnut. 
tulautinginik 7 nik akjarsivut Nalekab iklervingata sivorngane, sivumuarlutiglo tiptulausijarput; oppalungersimajullo sivorlerivait, innugiartoksuillo mallikput Nalekab iklervinganik, ajochertuijullo sivumuarlutik tiptulausijarput.

14 Uvlub aipanganetauk attausiarlutik ailaukput iglugaseksuit avatâtigut uttermilutiglo tangmarvingmut. Taimailiorlaukput uvlunne pingasujortunne.

15 Uvlulle 7 ingane kaub aupallungninga nuilertillugo, uvlarârsukut aularlaukput, ailutiglo illusek tamanna malliklugo 7 êrtorlutik iglugaseksuit avatâtigut; taimak uvlorme attautseme taipsomane 7 êrtorlutik iglugaseksuit avatâtigut pivut.

167 inganelo ajochertuijut tiptulausijartillugit, Josuab innugiartoksuit okautivait: Unatadlarnerub nippivaksoanganik pileritse, Nalekab tunnitsivigingmasse iglugaseksoarnik.

17 Iglugaseksuille tamakkoa illuanêtullo tamaita nungutuksauvut Nalegak pivlugo. Anguniarpaktok Rahabe kissime innojuksaurok, iglomiokattingillo illunatik; nautsertortuksâk tillilauktâkput ijertorlaungmagik.

18 Kissiane illimasugitse nungutæksanik, nungutaksautikonnasse, nungutakset illangit tigugupsigik, nungutaksautilugolo Israelib tangmarvinga, pitilugolo ogguarnartomut.

19 Silberele, Goldelo tamaita, illulerkutit kannujet kikkiello illaliudlugit ivsornaititaksauvut Nalekamut, pilerkovlugit Nalekab ivlernartokotinginnut.

20 Tagva inugiartoksuit unatadlarnerub nippivaksoanganik pilerput, ajochertuijut tiptulausijartillugit. Inugiartoksuille tiptulautib nippinganik tussaleramik unnatadlarnerub nippivaksoanganik pivut; avalullo karmait ochovut, innugiartoksuillo ittarput iglugaseksoarnut attunnit sivumuarlutik. Taimak iglugaseksuit salagivait.

21 Asserorlugillo iglugaseksoarnêtut tamaita saviksub kênanganut, tamarmik angutit arnello, innuosuktut innutokaujullo, tuktuvaillo saukello siutekoktojullo.

22 Josuable angutik nautsertoriartorlauktuk nunamik okautivak: Itteritik anguniarpaktub iglunganut, arnarlo tamna annitikko tagvangat pigijangit tamaita illaliudlugit, sorlo angervigimarilauraptikko.

23 Tagva innuosuktuk nautsertoriarlauktuk itterpuk annitilugolo Rahabe, atâtanga, anânangalo, kattangutingillo illaliudlugit, pigijangillo tamaita, illagijangillo illunaita, kemaklugillo Israelib tangmarvingata sillatanut.

24 Iglugaseksuille ikkipait ikkomamut, illuanetullo tamaita illaliudlugit. Silberele Goldelo, illulerkutillo kannujat kikkiello pitipait ivlernartunnut Nalekab iglunganut.

25 Rahabele anguniarpaktok, atâtangata iglomiokattingit illaliudlugit, pigijangillo tamaita, innokojaulaukput Josuamut. 
Nunakarporlo Israeleme uvlok tamanna tikkilugo, tamanna pivlugo, tillijaujuk Josuab tillilauktagik Jerichomut, nautsertoriarkovlugik ijertorlaungmagik.

26 Nelliutome taipsomane Josua kigligiudsimarilaukpok okarlunelo: Ominarsijaule angut taimna Nalekab sângane, nappartitsijok igloliortorlo iglugaseksoarnik Jerichomik ukkuninga. Illiguniuk tungavingat erninga angajuklek pijaule, upkoaksoangillo illigunigit erninga nukkârdlek pijaule.

27 Taimak Nalekab Josua illagiva, okautigijaulaungmat nunane illunaine.

\section{KAPITEL VII.}

\section{Achanib tiglingnek pivlugo pitlartauninganik.}

ISRAELITILLE tammarlaukput tigusinermut nungutaksamik; Achan Karmib erninga, Sabdib erningata, Serab erningata, Judab kinguvânginnit, tigusilaungmat nungutakset illanginnik. Tagva Nalekab ningausingata Israelib kittorngangit illungertorutigivait.

2 Tagva mana Josuab, angutit tillingmagit Jerichomit Aimut, Beth-Avenemetomut Bethelib kittanetomut okautilugillo: Takpaunga majorarluselo nuna nautsertorsiuk; majorarlauramiglo Ailo nautsertorlaukârlugit,

3 Utterput Josuamut okautivlugolo: Inugiartoksuit illuêngarlutik majorarkoniarnagit, angutille 2000 dikasait 3000 dillonêt majorarlit, ullimarnikovlugit Aimik, innugiartoksuit illuêngarlutik sullijaksakartitaukonagit tapkonunga, ikkitotuinaungmatta:

4 Taimak innugiartoksoarnit angutit 3000 dikasait majorarput, kemâvullo angutinik Aimiunik.

5 Aimiullo ullimarpait tapkonangat 36 it kemâtilaitalo upkoaksoarmit Sebarim tikkilugo, ullimarlugillo attersartillugit. Tagva innugiartoksuit omætingit mungungalerput, imektut îlerdlutik.

6 Josuab annorâne alliktorpait pamakterlunelo kênaminik pûlune nunamut Nalekab iklervingata sânganut, unnuk tikkidlugo Israelitit angajokangit illagilugit, sannillo egipait niakomingnut.

7 Josualo okarpok: Nalegak Nalegak sôg innugiartoksuit ukkua tessiorlaukpigît Jordanib akkianut, unnertutigiluta Amoriterit agganginnut, tokkoluta? Kanoktôk Jordanib akkianetsainarlaurupta, sorlo pigialaurapta.

8 Nalegara kanoêtunik okausekartuksauvik, Israelitit tunnungmatta omigijimingnit?

9 Kanaaniterillo nunamiullo illunatik tamattominga tussa- 
35 Okausekalaungilak attautsemiglonêt perkojaulauktomik Mosesemut, Josuab kaigarsuktaukolaungitanganik illagêkiut Israelitit illunatik tækkoæne arnallo sorrutsillo tujormijallo, agvertartut tapkoa akkorngane tækkoane.

\section{KAPITEL IX.}

\section{Gibeonemiut pingitsertorninginits.}

MANNA attannit, Jordanib ungatane kakkane naksanello, imârbiksublo siksangane nunakalauktut, tapkoatauk kakkab Libanonib senianelauktut, imaitut, Hethiterit Amoriterillo, Kanaaniterillo, Feresiterillo, Heviterillo, Jebusiterillo, tamattominga tussaramik;

2 Kattimalerput illajêklutik unatadlaromavlutik Josuamut Israelitinnullo.

3 Iglugaseksuille Gibeonemik attiggit innungit tussaramik sunamik Josuab pinniarnerilauktanganik Jerichomut Aiemullo issumasivut pingitsertornermik.

4 Aivut tilliklerlutiglo okausijartortuksanik, tigusilutiglo pôngnik nutaungitunik siutekoktojokotimingnut,

5 Weinekautiniglo nutaungitunik alliktorsimalauktunik illarsimajuniglo, ittiggagutiniglo nutaungitunik. illarsimajuniglo ittikkamingnut, attilugillo anorângnik nutaungitunik, niakojallo neksartangit illunatik sittijolaukput sapkaulutiglo.

6 Aivullo Josuamut tangmarvingmut Gilgalemut, okarlutiglo tapsomunga Israelitinullo illumainut: Kaivogut nummanit kanningitunnit, tagva manna angerkattigêngnermik pileritse uvaptingnut.

7 Tagva Israelitit illunatik Hethiterinut okarput: Imakka akkonaptingne nunakarungnarajarpose, kannok tagva angerkattigêngnermik pijungnarkita illipsingnut?

\& Tapkoale okarput Josuamut: Kivgaksaurogut illingnut. Josuable okautiveit: Kikuvise, nakillo pivise?

9 Tapkoa okarput: Kivgattit numnanit kanningitoksoarnit tikkiput Nalekab Gudivit attinga pivlugo; tussaumajatuninga tussarlauraptigo, tamaitalo pinniarnerilauktangit Egipteneme.

10 Tamaitalo pinniarnerilauktangit Amoriterit attunningangnut maggungnut Jordanib ungatanetungnut, Sihonemut Hesbonib attanertanganut, Ogemullo Basanib attannertanganut, Astharotheme nunakalauktomut.

11 Tamanna pivlugo angajokavut nunagijaptale innungit illunatik okarput: Tokkoaksamik neksaritse ingerganermut, ailuselo pachrerarlugit, okautilugillo: Kivgauvogut illipsingnut. Taimak mana angerkattigêngnermik pileritse uvaptinginut.

12 Niakojavut ukkua, nerkiksaptingnut igluptingnit neksarlanktarut, nutaumarilaukput aulartilluta illipsingnut, mande ahâk, sittijovut sapkakalutiglo; 
13 Weinekautillo ukkua nutangotillugit immerlaukpavut, ahâglo alliktorsimavut; annoravullo ukkua ittiggagutivullo nutaungitolerput ingerganermut sivitojomut.

14 Tagva angajokajet tapkoa nerkingit illelliorpait, appertsoratiglo Nalekab kanninganik.

15 Josuablo ullapirsautekarkattigilerpait angerkattigêngnermiglo pilune tapkonunga, innojuksaungmatta. Illagêktullo angajokangita angervigimarikpait.

16 Uvlulle pingasut nâlermatta, angerkattigêngnermik pilaukârlutik, tussarput taipkoa kanidlarmatta akkonermingnelo nunakarniarmatta.

17 Israelible kittorngangit aularamik uvluk pingajuangne tikkiput taipkoa iglugaseksoakotinginut, attekartunut Gibeonemik, Kafiramiglo, Beerotemiglo, Kiriat-Jearimemiglo.

18 Ullimarlaungilaillo, tamanna pivlugo Israelitit angajokangita angervigimarilaungmattigik Nalekame Israelib Gudingane. Illagêktulle illunamassiatik okalokingmatta angajokajanut,

19 Illagêktut illunamassiatik angajokangit okarput: Angervigimarilaukpavut Nalekame Israelib Gudingane; tamanna pivlugo aktorungnangilavut.

20 Imaitomigle pinniarvigijomavavut: Innôkosigik ningautsemik pijokarkonago uvaptingnut, angermaringnek angerutigilauktavut tapkonunga pivlugo.

21 Angajokajello okautivait: Innôkosigik kitsiorteolerkovlugit immertarteolerkovlugillo illagêktunut illunainut, sorlo angajokajet angervigilaungmattigik.

22 Tagva Josuab kaikoveit okarkattigilugillo okarpok: Sôg terliarlaukpittigut, okarluselo kaningidlarapse uvaptingnit nunakaralloartilluse akkonaptingne?

23 Tamanna pivlugo ominarnerartaujuksauvose, akkonapsingne sorailerkonagit kivget, kitsiortut imertartullo Gudima iglunganut.

24 Tapkoa Josua kiovât okarlutiglo: Kivgattit okautsaulaukput, Nalekab Gudivit kivgane Mosese perkolaungmago nuna illuêngarlune tunnijomangmago illipsingnut, nunablo innungit illunamassiatik nungutsomangmagit sivunapsingne. Tagva innôtsivut pivlugit sivoragidlalaukpapse, taimailiorlutalo.

25 Mannale tækkoit aggangnêpogut; namanasugijarnik idluarasugijarniglo pinniarvigiluta, tamattominga piniarlaurit.

26. Tapsomalo taimak piniarvigivait, piulilugillo Israelib kittorngangita agganginit tokkotsikonagit tapkoninga.

27 Taimak Josuab uvlorme taipsomane kitsiortungortilaukpait, imertartungortilugillo illagêktunut, Nalekablo Altaringanut uvlok tamanna tikkilugo, iniksame anneriniartamine (Altaremut.) 


\section{KAPITEL. $\mathrm{X}$.}

Josuab salakarninganik tattamnadlartomik Amoriterinik.

A ADONI Zedek Jerusalemib attanertanga tussarame Josuab Ai sâlagilaungmagit nungutilaungmagillo, Ailo, attanertanga illaliudlugo pinniarvigilaungmagit, sorlo Jericho attanertangalo piniarvigilaungmagit, Gibeonemiullo ullapirsautekarkattigêngnermik pilaungmatta Israelitinut, tapkoalo akkornganut pilaungmatta,

2 Sivoradlalerput (Gibeon iglugaseksoangolaungmatta angijunik, iglugaseksoatitut attaneliktutitut, anginersaulaukporlo Aiemit angutingillo illunatik sorsugungnarlaukput.)

3 Tilliklerporlo Hohamemut, Hebronib attanertanganut, Pireamemullo Jarmutib attanertanganut, Jafiamullo Lachisib attanertanganut, Debiremullo Eglonib attanertanganut, okautsaukolugillo:

4 Majorarluse kaititse takpaunga uvamnut ikkajorsingalo Gibeonemik ullimarnikovluta; Josuamut Israeliblo kittornganginut ullapirsautekarkattigêngnermik pilaungmatta.

5 Tagva kattimalerput majorarlutiglo Amoriterit attanningit tellimat, tagva Jerusalemib attanertanga, Hebronib attanertanga, Jarmutib attanertanga, Lachisib attanertanga, Egloniblo attanertanga, sorsuktuksaksoakotitik illagilugit Gibeonelo illuvat sorsugvigilugillo.

6 Gibeonemiullo tilliklerput Josuamut tangmarvingmut Gilgalemut, okautsaukovallo: Aggaktit erkitiniarnagit kivgarnit, majorarlutillo kaisarit uvaptingnut, piulilutalo ikkajortigut; Amoriterit attaningit kakkane nunakartut illunatik illagêlermatta akkerartorvigiluta.

7 Josua Gilgalemit takpaungarpok, sorsuktuksello illunatik illagivat, angutillo illunatik sorsugungnartut.

8 Nalegarlo Josuamut okarpok: Sivoraginiarnagit aggangnut tunimagapkit; nelliellonêt tapkonangat nangerungnarniangilak sângne.

9 Taimak Josuab suerganit tikkipait, unuak nâlugo majorarmat Gilgalemit.

10 Nalekable erksitipait Israelitit sangane, ullimarnikovlugit unnatadlarnermik angijomik Gibeoneme, udlapaillo apkome Bet-Horonemut torartome, ullimarpaillo Aseka Makedalo tikkilugik.

11 Israelitillo kemângmagit attersarlutik apkome BetHoronemut, Nalekab kattagviokovait killangmit nettarkonâlungnik Aseka tikkilugo, tokkolerkovlugit. Unuktunniglo tokko- 
taujokarnersaulaukpok nettarkonâlungnut Israelib kittorngangita tokkolauktanginit saviksoarmut.

12 Tagva Josuab Nalegak okarvigiva uvlorme taipsomane Nalekab unertuiviane Amoriterinik Israelib kittorngangita sangane, okarlunelo Israelitit tækkoane: Sekkinek nokkarit Gibeonib nellâne, takkerlo Ajalonib naksangata nellâne!

13 Tagva sekkinerlo takkerlo nokkarpuk, innugiartoksuit omigijitik kiglugvigikârnagit. Tamanna aglaksimangilâk idluartut aglangine? Taimak sekkinek nokkarpok killaub kerkane mulludlarlunelo nippinerminut uvlormik illuitokasangmik.

14 Uvlokalaungilarlo adsekartomik ominga, sivorngagullonêt kingorngagullonêt, Nalekab nalegvianik angutib nippinganik; Nalegak sorsungmat Israelitit pivlugit.

15 Josuale utterivok tangmarvingmutGilgalemut, Israelitillo illunatik illagivât.

16 Attanille tellimat taipkoa kemâlaukput ijerlutiglo kairosungmut Makedametomut.

17 Tagva Josua okautsauvok: Attannit tellimat taipkoa nennilaukpavut ijersimatillugit kairosungme Makedametome.

18 Josua okarpok: Tagva ujaraksuit aksakartisigik kairosub pânganut, angutekarkojileritselo udsertortunik taipkoninga.

19 Illipsele nokkarniarase omigijisele udlasigik, kingorlingillo ullimarsigik, ittertailitisigiglo iglugaseksoarmingnut; Nalekab Gudipse tunnimangmagit aggapsingnut.

20 Josuablo Israeliblo kittorngangita unatadlarnek angijoksoak una soraitilaungmatsuk tapkonane, nungulaungmattigiglo; amiakungit iglugaseksoarnut avalulingnut pirut.

21 Tagva innugiartoksuit illunatik ullapirsautekarlutik utterivut Josuamut, tangmarvingmut Makedamut, nelliatalo okane aulatijaksarilaungila Israelib kittorngangita sangine.

22 Josuale okarpok: Kairosub pânga upkoarsiuk, attannillo tellimat taipkoa kailersigik uvamnut.

23 Taimailiorput, attannillo tellimat tapkoa âpait kairosungmit tapsomunga, Jerusalemib attanertanga, Hebronib attannertanga, Jarmutib attanertangat, Lachisib attanertanga, Eglonib attanertanga.

24 Attannille tellimat tapkoa âtaumangmatta tapsomunga, Josuab Israelitit illuêngarlutik kaikovait, okarlunelo sorsuktukset illagijame angajokanginut: Kaititse ovunga, attannillo ukkoa tuttisigik ittikkapsingnut kongesingitigut! Kaivullo tagvunga, tuttiluiiglo ittikkamingnut tapkoa kongesinginut.

25 Josuablo okautivait: Sivoraniarase kvaksarniaraselo, maksoanekaluselo mungungajuititse; taimak Nalekab omigijise illunaita sorsugvigijase piniarviginiarmagit.

26 Kingornganelo Josuab ullimarpait tokkolugillo nivingati- 
lugillo nappartunut tellimanut; nivingalaukpullo nappartune unnuk tikkilugo.

27 Sekkinerle nippijarêrmat perkojivok akkartitaukovlugit nappartunit egitaukovlugillo kairosungmut ijervigilauktanganut, ujarkaniglo angijunik illijivut kairosub pângata sanganut. Tapkoa tagvaneput sulle uvlok tamana tikkilugo.

28 Uvlorme taipsomane Josuab Makedatauk salagilaurivait ullimârlugillo saviksub kênanganut, attaningat illaliudlugo nungutipaillo, tarnillo tamaita tapkonanelauktut, amiakokarkojilaungilarlo nellianik; Makedablo attannertanga pinniarvigiva sorlo Jerichub attannertanga pinniarvigilaungmago.

29 Tagva Josua Israelitillo illuêngarlutik illagilugo aularput Makedamit Libnamut, sorsugvigivaillo.

30 Nalekablo tapkoatauk tunnigivait Israelitit agganginut, attanertangat illaliudlugo; ullimarpaillo saviksub kênanganut, tarnillo illunaita tapkonanelauktut; amiakokarkojilaungilarlo nellianik tapkonane; attanningallo pinniarvigiva sorlo Jerichub attanertanga pinniarvigilaungmago.

31 Kingorngane Josua Israelitit illuêngarlatik illagilugit aularpok Libnamit Lachisemut, illulugillo sorsugvigivait.

32 Nalekablo Lachisetauk tunnivait Israelitit agganginnut salakarkovlugit tapkoninga uvlub aipangane, ullimarpaillo saviksub kênanganut, tarnillo illunaita tapkonanelauktut, taimamarik sorlo Libna pinniarvigilaungmagit.

33 Nelliutome taipsomane Horame Geserib attannertanga takpaungarpok Lachis ikkajoromavlugit; Josuable ullimarpa innukotingit tamaita illaliudlugit, amiakokarungnaikârtinago tapkonangat.

34 Josualo Israelitit illuêngarlutik illagilugit aularpok Eglonemut illulugillo sorsugvigivait;

35 Salagilaitalo uvlorme taipsomane, ullimarlugillo saviksub kênanganut, tarnillo illunaita tapkonanelauktut nungutipait uvlorme taipsomane, taimamarik sorlo Lachis pinniarvigilaungmagit.

36 Kingorngane Josua Israelitit illuêngarlutik illagilugit takpaungarpok Eglonemit Hebronemut, sorsugvigivaillo,

37 Salagivaillo ullimarlugillo saviksub kênanganut, attaningallo iglugaseksoakotingillo illaliudlugit, tarnillo illunaita tapkonanelauktut, amiakokarkojingilarlo nellianik, taimamarik sorlo Eglon pinniarvigilaungmagit, nungutipaillo tarnillo illunaita tapkonanelauktut.

38 Tagva Josua Israelitillo illuêngarlutik illagilugit utterivok Debiremut, sorsugvigivaillo.

39 Salagilaitalo attaningat iglugaseksoakotingillo illunaita illaliudlugit, ullimarpaillo tarnit illunaita tapkonanelauktut, 
amiakokarkojingilarlo nellianik. Sorlo Hebron pinniarvigilaungmagit, taimak Debire attanningallo pinniarvigivaittauk, sorlo Libna attaningallo pinniarvigilaungmagit.

40 Taimak Josuab nunnat illunaita, kakkane sekkinerdlillo, nâksanelo kôngnelo attaningit illaliudlugit ullimartautipait, amiakokarkojilaungilak nellianik, tamaitalo annerneggit nungutipait, sorlo Nalegak Israelib Gudinga perkojilaungmat.

41 Ullimartautipaillo Kades Bar̀neamit Gasa tikkilugo, nunalo Gosen illunât, Gibeon tikkilugo.

42 Attanillo tapkoa, nunangit illaliudlugit salagivait attautsekut; Nalegak Israelib Gudinga sorsungmat Israelitit pivlugit.

43 Josualo utterivok tangmarvingmut Gilgalemut, Israelitit. illuêngarlutik illagilugit.

\section{KAPITEL XI.}

Attanit nunab Kanaanib avane nunaliartut illagêlerput Isroelitit akkerartoromavlugit, salagïaulutiglo. Josuab salakarningit. J ABINIB Hazorib attanertangata tamanna tussaramiuk tilliklerpok Jobabemut, Madonib attannertanganut, Simroniblo attannertanganut, Achsafiblo attannertanganut,

2 Attannernullo avane kakkane nunakalauktunut, naternamelo Cinerotib sekkerngane, naksanelo, Nafot Doremelo imarbiub senniane nunakalauktunut.

3 Kanaaniterit kittanetut kangianetullo Amoriterit, Hetiterillo, Feresiterillo, Jebusiterillo kakkane, taimaktauk Heviterit kakkab Hermonemik attiggub umangane nuname Mizpamik attelingme.

4 Tapkoa illunatik aularput sorsuktuksaksoakotitik kattimgajut illunaita illagilugit, innugaseksuit, siorkatitut imarbiub siksanganêtutitut unnuktigijut, horsillo aksalloaggillo unnuktovaksuit.

5 Attannit tapkoa illunatik kattimalerput kailutiglo tangmarput kattimgavlutik tetserme Meromemik attelingme, Israelitit. sorsugvigijomavlugit.

6 Nalegarlo Josuamut okarpòk: Sivorajutiginiarnagit, kaupalle nelliutome tamattomane illunaita ullimarsimalutik unnertutigijomavakka Israelib kittornganginut; horsekotingit sibvêtitaksarivattit, kamutekotingillo aksalloaggit ikkomamut ikkitaksarivattit.

7 Josuablo suerganit tikkipait, innugiartoksuillo sorsuktukset illagilugit tetserme Meroneme, opingarpaillo.

8 Nalekablo tunnivait Israelitit agganginut, ullimarpaillo kemâtilaitalo Zidon angijok tikkidlugo, imiksuillo onartut tikkidlugit, Mizpiblo senia tikkilugo kittâne ullimarpaillo amiakokarungnaikârtinago tapkonangat. 
9 Tagva Josuab pinniarvigivait, sorlo Nalekab okautilaungmago, horsekotingillo sibvêtipait aksalloalikotingillo ikkipait.

10 Utterlunelo nelliutome taipsomane, Hazorelo salagivait attannertangallo ullimarpa saviksoarmut (Hazorle sivorngagut attaniovît tamakkoa iglugaseksoanginit anginerpaulaukpok.)

11 Tarnillo illunaita tapkonanêlauktut ullimarpait saviksub kênanganut nungupaillo, amiakokarkojingilarlo nellianik anernelingmik, ikkipaillo Hazor ikkomamut.

12 Tappilugillo attannit tapkoa iglugaseksoangit illunaita attannertangit illaliudlugit Josuab salagivait, ullimarlaitalo saviksub kênanganut, nungutipaillo, sorlo Mosese Nalekab kivganga perkojilaungmat.

13 Israelible kittorngangita iglugaseksuit kakkarulangnelauktut ikkilaungilait, Hazorele kisseta Josuab ikkipait.

14 Iglugaseksuillo tamakkoa aksartaujutigijangit, nergjutillo Israelib kittorngangita avikpait ingmingnut; innuille illunaita ullimarpait saviksub kênanganut nungutsikârtinagit tapkoninga, amiakokarkojilaungilallo nellianik anernelingmik.

15 Sorlo Nalekab Mosese kivgane, Mosesiblo Josua perkolaungmago, taimak Josua pinniarlaukpok, pinniartaililaungilarlo sunamik Nalekab perkolauktanganik Mosesemut.

16 Taimak Josuab nuna tamanna illunat kakkanetok aksarnigariva, illunâllo aunga illingajok, nunalo Gosen illunat, naksello, naternello, Israeliblo kakkamaringit, naksangit illaliudlugit.

17 Kakkamit avititsijomit nunamik takpaunga Seiremit, Baal Gade tikkilugo kakkab Libanonib senianetok kakkabHermonib attâne. Attaningit illunaita salagivait ullimarlaitalo, tokkolugillo.

18 Akkunêrtomigle attanit tapkoa unatadlarvigivait.

19 Iglugaseksoakalaungilarle attautsemiglonêt, ullapirsantekaromavlutik tunnijunik ingminik Israelib kittornganginut, (Heviterit Gibeoneme nunakalauktut assinginik) illunaitale sorsungnermut salagivait.

20 Tamannalo taimak Nalekamit pilaukpok omattingit mangaititaukovlugit, sorsuklutik Israelib kittorngangit pârlugit, nungutaukovlugit, saimanermiglo pilerkonagit, pêjartaukovlugillo, sorlo Nalekab Mosese perkolaungmago.

21 Nelliutome taipsomane Josuab tikkilunelo Enakiterit (innukpait) pêjarpait kakkamit, Hebronemit, Debiremit, Anabemit, Judab kakkanginit tamainit, Israeliblo kakkamaringinit, nungutipaillo iglugaseksoangit illaliudlugit.

22 Amiakokarkojilaungilarlo Enakiterinik Israelib kittorngangita nunangane, kissiane Gasame, Gathemelo, Asdodemelo amiakokalaukpok sulle.

23 Taimak Josuab nuna illunât aksarnigariva, taimamarik sorlo Nalekab Mosese okautilaungmago, tunnivalo Israelitinut 
kingormgutijaksauvlune tapkonunga, kinguvarêt illunatik attunit ikkupianganik. Nunamelo unnatadlarnekarungnailaukpok.

\section{KAPITEL XII. \\ Attanit salagijaumajut.}

TAM EDSA nunab attanertangit Israelitit ullimarlauktangit aksarnivigijangillo nunanginik Jordanib akkiane kittane, kongmit Arnonemetomit kakkak Hermon tikkilugo, naternarlo illunât kittâne.

2 Sihon, Amoriterit attaningat, Hesboneme nunakalauktok, attaniotilauktorlo Aroeremit kôb Arnonemetub siksangane kôblo kerkanetomit, Gileadiblo ketterkanganut, kôk Jabok tikkilugo, kiglilerviojok Amonib kittornganginut.

3 Naternamullo Cinnerotib imanga tikkilugo kittane, imarbiglo naternametok tikkilugo, tagva immak tarriok kittane, apkosinek Bet-Jesimotemut senniagorlugo, ângallo kakkab Pisgab kôngine.

4 Taimaktauk Ogib Basanemiut attaningata kiglilervia, amiakolauktub sulle innukpangnit, nunakalauktublo Astaroteme Edreimelo,

5 Attanniotilauktublo kakkamut Hermonemut Salchamullo Basanemullo illunânut, Gesurib Maachaliblo kiglilervigik tikkidlugik, Gileadiblo ketterkanganut, kiglilerviolauktomut Sihonemut Hesbonib attanertanganut.

6 Mosesib Nalekab kivgangata Israeliblo kittorngangita ullimarlaukpait. Mosesiblo Nalekab kivgangata tunnilaukpait perkutautilugit Rubenekunut Gadekunullo, kinguvarrêllo Manassekut ketterkanginut.

7 Tamadsa nunab attannertangit, Josuab Israeliblo kittorngangita ullimarlauktangit Jordanib miksane, kangiane, Baal-Gam demit Libanonib senianetomit, kakkak tikkilugo avititsijok nunamik Seiremit, Josuablo tunilauktangata Israelib kinguvarênginut perkutautilugo, kinguvarêt attunit ikkupianganik;

8 Kakkanelauktut, nâksanelauktullo, naternanelauktullo, kôngnelauktullo sunakangitomelauktullo ânelauktullo; Hetiterit, Amoriterit, Kanaaniterit Feresiterit, Heviterit Jebusiterillo.

9 Jerichub attanertanga, Aib Betelib senianetub attanertanga,

10 Jerusalemib attanertanga, Hebronib attanertanga,

11 Jarmutib attanertanga, Lachisib attanertanga,

12 Eglonib attanertanga, Geserib attanertanga,

13 Debirib attanertanga, Gederib attanertanga,

14 Harmab attanertanga, Aradib attanertanga,

15 Libnab attanertanga, Adullamib attanertanga, 
16 Makedab attanertanga, Betelib attanertanga,

17 Tapuahib attanertanga, Heferib attanertanga,

18 Afekib attanertanga, Lasaronib attanertanga,

19 Madonib attanertanga, Hazorib attanertanga,

20 Simron-Meronib attannertanga, Achsafib attanertanga,

21 Taenachib attanertanga, Megidob attanertanga,

22 Kedesib attanertanga, Jakneab Karmelemetub attanertanga,

23 Nafot-Dorib attanertanga, nellojut Gilgaleme attaningat, 24 Tirzab attanertanga. Tagva attanit 31 git.

\section{KAPITEL XIII.}

\section{Josuab perkojauninganik nuna aviklugo.}

JoSUA mana îtodlartillugo uvlokartillugolo unnuktunik, Nalekab okautiva: Innutokauvotit uvlokarlutillo unnuktunik, amiakokarporlo sulle nunnanik unuktunik aksarnigaujariakarıunik.

2 Tagva Filisterit Galilæakotingat illunât, Gesurillo illunât,

3 Sihoremit kôktomit Egiptenib sângagut, Ekronib kiglilervinga tikkilugo, avane, kittitaumajok Kanaaniterinut, Filisterit angajokangit tellimat, Gasamiut, Asdodemiut, Askalonemiut, Gethemiut, Ekronemiut, Avimiullo.

4 Anget Kanaaniterit nunangat illunât, Mearalo Zidonemiunut illingajok Afek tikkilugo Amoriterit kiglilervingat tikkilugo;

5 Taimaktauk Gibliterit nunangat illunât, Libanonelo illunât, kittane Baal-Gademit kakkab Hermonib attânetomit Hamatib itterviat tikkilugo;

6 Tapkoa illunaita kakkane nunakartut Libanonemit puailavit onartut tikkilugit, Zidonerillo illunaita. Pejaromavakka Israelib kittorngangita sivorngane; imerautigituinarkit, aviklugit Israelitinut, sorlo perkolaurapkit.

7 Tagva manna nuna tamanna aviguk kingormgutijaksauvlune kinguvarênut 9 enut, kinguvarênullo Manassekut ketterkanginut.

8 Rubenekut Gadekullo kingormgutijamingnik pijarêrlaungmatta kinguvarêt Manassekut ketterkangit, aipaingit illagilugit Mosesib tunnilauktanganik tapkonunga Jordanib akkiane kittanê; sorlo Mosesib Nalekab kivgangata tamanna tunnilaungmago tapkonunga.

9 Aroeremit kôb Arnoneme kôktub siksanganetomit, iglugaseksoarnillo kôb kerkanetunit, Medbadiblo erkâ illunat Dibone tikkilugo;

10 Sihoniblo Amoriterit attaningata Hesbonemelauktub iglugaseksoakotingit tamaita, Amonib kittorngangita kiglilervingat tikkilugo. 
11 Tappilugillo Gilead, Gesuriblo Maachatiblo kiglilervigik, kakkarlo Hermon illunât, Basanelo illunât Salcha tikkilugo;

12 Ogiblo Basanib attanertanga, Astaroteme Edreimelo nunakalauktub, amiakolauktub sulle innukpanit attaniovinga illunât. Mosesible ullimârlugillo pingujarlaukpait.

13 Israelible kittorngangita Gesuremiut Maachatemiullo aulartilaungilait; tamarmigle Geșuremiut Maachatemiullo nunakarput Israelitit akkorngane uvlok tamanna tikkilugo.

14 Kinguvarêlle Levikut kingormgutijaksamik tunnitsivigilaungilait; Nalekab Israelib Gudingata tunnergusiarijangit kingormgutigingmattigik, sorlo okautilaungmagit.

15 Taimak Mosesib kinguvarêt Rubenib kittorngangit tunnitsivigivait abvakungit malliklugit;

16 Kiglilervingat pilaungmat Aroeremit kôb Arnonemetub siksanganetomit, iglugaseksuillo kôb kerkanêtut, naternangat illunât, illaliudlugo Medba tikkilugo;

17 Hesbon iglugaseksoangillo tamaita naternametut; Dibon, Bamoth-Baal, Beth-Baal- Meonelo,

18 Jahza, Kedemot, Mefathelo,

19 Kiriataim, Sibma, Zeret-Sahar naksarme kakkametome,

20 Beth Peor, kôt Pisgamit pijut Beth-Jesimotelo,

21 Iglugaseksuillo tamaita naternamêtut Sihoniblo Amoriterit attaningata, Hesbonemelauktub, Mosesib ullimarlauktangata attaniovinga illunât Midianib, Eviblo, Rekemiblo, Zuriblo, Huriblo, Rebablo angajokangit, Sihonib pitsartunelikotingit, nuname nunakalauktut illalindlugit.

22 Bileamelo Beorib erninga nellautaijok Israelib kittorngangita tokkokassiutilaurivat saviksoarmut ullimarsimajut illaliudlugit.

23 Rubeniblo kittorngangita kiglilervingat Jordaneôlaukpok. Tamana Rubenib kittorngangita kingormgutijangat abrakungit malliklugit iglugaseksuit iglugasaillo.

24 Mosesib kinguvarêt Gadib kittorngangit, abvakungita akkorngane tunnitsivivait,

25 Kiglilervingat Jaesereôlaungmat, iglugaseksuillo illunaita Gileademetut, Amoniblo kittorngangita nunangata ketterkanga Aroer Rabbab siroranetok tikkilugo.

26 Hesbonemit Ramat - Mizpe Betonimelo tikkilugik, Mahanaimillo Debirib kiglilervinga tikkilugo.

27 Naksarmele Beth-Haram, Beth-Nimra, Suchot Zafonelo, amiakolauktut Sihonib Hesbonib attanertangata attaniovinganit, Jordan kiglilerviolaungmat; Cineretib imanga tikkidlugo Jordanib milksane kittane.

28 Tamanna Gadib kittorngangita kingormgutijangat abvakungine, iglugaseksoangit iglugasaingillo. 
29 Mosesiblo kinguvarêt Manassib kittorngangita ketterkangit, tunitsivigivait abvakungit malliklugit,

30 Kiglilervingit pilaungmatta Mahanaimemit Basan illunât, Ogib Basanib attanertangata attaniovinga illunât, Jairiblo iglugasaingit Basanemetut illunaita, iglugaseksuit 60 git.

31 Gileadiblo ketterkanga, Astarot, Edrei, Ogib Basanib attanertangata iglugaseksoagilauktangit, tunnijauvut Machirib kittornganginut, Manassib erningata, tagva Machirib kittorngangita ketterkanginut abvakungit malliklugit.

32 Tamædsa Mosesib avilauktanga, Moabiterit naternangane, Jordanib akkiane Jerichub sângane kittane.

33 Kinguvarêlle Levikut Mosesib kingormgutijaksamik tunnitsivigilaungilait; Nalegak Israelib Gudingât kingormgutigingmatsuk sorlo okautilaungmagit.

\section{KAPITEL XIV.}

\section{Nunab Kanaanib immerautigïjauninga, Kaleb Fingormguti- jaksanga.}

Tamannauvorle Israelib kittorngangita aksarnigarilauktangat nuname Kanaanene, ajochertuijub Eleaserib, Josuablo Nunib erningata, atatello angajokaunerojut Israelib kittorngangita kinguvârengningita akkorngane, avilauktangat tapkonunga,

2 Avilaukpalle tapkonunga imerautigivlugo, sorlo Nalegak Mosesekut perkojilaungmat, kinguvarêt 9 it koppangalo tunnitsivigilugit.

3 Kinguvarêlle maggoit kinguvarêllo attautsit ketterkangit Mosesib kingormgutijaksamik tunnitsivigilaukpait Jordanib akkiane. Levitille kingormgutijaksamik tunnitsivigilaungilait tapkoa akkorngane.

4 Josefib kittorngangit kinguvarêngolermatta maggoit, Manasse Efraimelo: tamanna pivlugo Levitit illangiutivikartilaungilait nunamik, iglugaseksoarnigle iglogijaksanginik, aiparuserniglo iglugaseksuit avatanê nergjutekotinginut perkutinginullo.

5 Sorlo Nalekab Mosese perkolaungmago, taimailiorlaukput Israelib kittorngangit, aviklugolo nuna.

6 Tagva Judab kittorngangit kaivut Josuamut Gilgaleme, Kaleblo Jefunib erningata Kenesikunit pijub okautiva: Kaujimavotit kanoêtunik Nalekab okauserilauktanginik, Mosesemut Gudib angutekotinganut Kades-Barneame pivlunga, pivlutillo.

7 Jahrekalaukpunga 40 ginik Mosesib Nalekab kivgangata 
tillilaungmanga Kades Barneamit, nuna nautsertoriartorlugo, okautilaungmigapkolo issumagilauktakka malliklugit.

8 Kattangutimale takpaunga majorarkattigilauktama innugiartoksuit omattingit mungungatilaukpait; uvangale Nalegak Gudiga mallilaukpara illuêngarlunga.

9 Tagva Mosese uvlorme taipsomane angermarilaukpok okarlunelo: Nuna tullerarvigilauktat ittiggangnut, kingormgutijaksauvok illingnut kittorngangnullo soraijuitomik; tamana pivlugo Nalegak Gudiga mallilaurangne nertornartomik.

10 Mannalo takkoit, Nalekab innokolaukpânga, sorlo okalaurame. Jahreôvut manna 45 git Nalekab okarvianit taimaitomik Mosesemut, Israelitit arvertarvigilauktangit sunakajuitome. Mannalo takkoit, uvlome Jahrekarpunga 85 ginik,

11 Uvlomelo sulle, uvlorme tamattomane taimak sangitigijovunga uvlutut taipsomanetut Mosesib tilliklervianetut uvamnik; sorlo nukkekarnera îlaungmat taipsomane, taimainivok sulle, sorsuklunga, aularlungalo, itterlungalo.

12 Tagva tunnitsivigilaunga manna kakkanik ukkuninga, Nalekab okautigilauktanginik uvlorme taipsomane; igvit tussarlaurangne uvlorme taipsomane. Enakimit nunakarmatta takpâne, iglugaseksoakarlutiglo angijunik songojuniglo; Nalekab illaginiarmangangma, aulartitsikovlunga tapkoninga, sorlo Nalegak okalaungmat.

13 Tagva Josuab saimartipa tunitsivigivalo Kale Jefunib Erninga Hebronemik kingormgutijaksamut.

14 Tamanna pivlugo Hebron kingormgutijaulaukpok uvlok tamanna tikkilugo Kalemut Jefunib erninganut, Kenesikunnut illingajomut, Nalegak Israelib Gudinga mallilauramiuk nertornartomik.

15 Hebronele sivorngane attekalaukpok Kiriath-Arbamik, tamna innukpangolaukpok Enakimit akkorngane. Nunalo unatadlarnekarungnailaukpok.

\section{KAPITEL XV.}

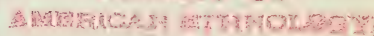

\section{Judab kinguvangita kiglilervingat iglugaseksoangillo.}

\section{KINGUVA
ita akkorn
sekkerngan
rekungane}

2 Kiglilervingat âne pilaungmat imarbiub tarriub terrekunganit, tagva okaujartomit aunga sangmijomit;

3 Pilunelo tagvangat takpaunga Akrabbimemut, ailunelo 
Zinekut, sangmilunelo ama angat Kades Barneamut, pilunelo Hezronekut, ailunelo takpaunga Adaremut, sangulunelo Karkab avatâtigut;

4 Ailunelo Azmonekut, tikkilunelo Egiptenib konganut, kiglilerviub nâninga imarbiksoangokovlugo. Tamæda kiglilervise âne.

5 Kiglervigle kittâne illingavok imarbingmit tarriomit Jordanib nâninga tikkilugo. Kiglilervigle avane pivok imarbiub okaujartunganit Jordanib sennianetomit;

6 Ailunelo takpaunga Beth-Haglamut, sangmilunelo avangat Beth-Arbamut, tikkilunelo takpaunga Bohenib Rubenib erningata ujaranganut;

7 Ailunelo Debiremut, Achorib naksanganit, iglugasengnillo avane Gilgalemut salertunit takpane Adumimib akkianetunit âne kôngmetub. Tagvangat aivok kongmut En-Semesemik attelingmut, tikkilunelo Rogelib puailavinganut.

8 Tagvangat aivok takpaunga Hinomib erningata naksanganut, Jebusiterit sekkerngane, tagva Jerusalem; tikkilunelo takpaunga kakkab Himonib naksangata sânganetub avangat, kanganut, aktuajub avane Refaimib naksangata terekunganut.

9 Tagvangat pivok kakkab tamattoma kânganit Neftoab puailavinganut, ailunelo kakkat Efronemik attiggit iglugaseksoanginut, sangulunelo Baala, tagva Kireat-Jearime tikkilugo.

10 Sangulunelo Baalamit avunga Seirib kakkanginut, ailunelo Jearimib kakkangita seniagut avangat, tagva Chessalonemit, tikkilunelo kannunga Beth-Semesemut, pilunelo Timnakut.

11 Pilunelo Ekrone senniagorlugo avunga, sangmilunelo Sichronemut, pilunelo kakkab Baalamik attiggub kangagut, pilunelo Jabneelemut nâninga imarbiksoangongmat.

12 Kiglilervigle kangiane imarbiksoangovok. Tamædsa Judab kittorngangita kiglilervingat avatane illunane, abvakungine.

13 Kalele Jefunib erninga tunnitsiviolaukpok ikkupianganik Judab kittorngangita akkorngane, sorlo Nalegak perkojilaungmat, imaitomigle, Kiriat-Arbamik, Enakib atâtangata, tagva Hebronemik.

14 Kaleblo aulartipait tagrangat Enakib erningit pingasut: Sesai, Ahiman, Talmailo, Enakib ernertarilauktangit.

15 Ailaukporlo tagvangat takpaunga Debiremiunut. Debirele mattoma sivorngagut attekalaukpok Kiriat-Seferemik.

16 Kalelo okarpok:- Kina Kiriat-Seferemik ullimarniktok aksarniktorlo, tamna panimnik Achsamik tunnitsivigijomavara nulliaksanganik.

17 Tagva Atnielib Kenasib Kaleb nukangata erningata 
aksarnigarivait; tapsomalo paniminik Achsamik tunnitsivigiva nulliaksanganik.

18 Pijokalaukporlo, tamna itterlaungmat tilliortaulaukpok kennukovlugo perorsêvingmik atâtaminit; akkarporlo siutekòktojomit. Tagva Kaleb okautiva: Konoêkit?

19 Tamna okarpok: Saimanermik tunnitsivigilaunga; tunnitsivigilauramga perorsêvingmik sekkernganetomik, tunnitsivigilaungatauk puailavilingmik immermik. Tagva puailavilingmik kollâne attânelo tunnitsivigiva.

20 Tamædsa kinguvarêt Judab kittorngangita kingormgutijangat abvakungita akkorngane.

21 Kinguvarêllo Judab kittorngangita iglugaseksoangit, issungmit aipanganit aipanga tikkidlugo Edomiut kiglilerviane âne, imailaukput: Kabzeel, Eder, Jagur,

22 Kina, Dimona, Ad-Ada.

23 Kedes, Hazor, Itnan,

24 Sif, Telem, Bealot,

25 Hazor-Hadata, Kiriot, Hezron tagva Hazor.

26 Amam, Sema, Molada,

27 Hazar-Gadda, Hesmon Beth-Palet,

28 Hazar-Sual, Beer-Seba, Bisjot-Ja,

29 Baela, Ijim, Azem,

30 El-Tolad, Chesil, Harma,

31 Ziklag, Madmanna, San-Sanna,

32 Lebaoth, Silhim, Ain, Rimon, Tagva iglugaseksuit 29 it iglugasaingillo.

33 Naksarnelo, Estaol, Zarea, Alna,

34 Sanoah, En-Ganim, Tapuah, Enam,

35 Jarmut, Adullam, Socho, Aseka,

36 Saarim, Adithaim, Gedera, Gederothaim. Tagva iglugaseksuit 14 it iglugasaingillo,

37 Zenan, Hadasa, Migdal-Gad,

38 Dilean, Mizpe, Jaktiel,

39 Lachis, Bazekat, Eglon,

40 Chabon, Lahmom, Kitlis,

41 Gederot, Beth-Dagon, Naema, Makeda. Tagva iglugaseksuit 16 it, iglugasaingillo.

42 Libna, Ether, Asan,

43 Jeftah, Asna, Nezibe,

44 Kegila, Achsibe, Maresa. Tagva iglugaseksuit 9 it iglugasaingillo.

45 Ekron, iglugaseksoakattingillo, iglugasengillo illaliudlugit.

46 Ekronemit imarbiksoak tikkilugo, tamaita Asdonib kanitanganetut, iglugasengillo.

47 Asdod iglugaseksoakattingillo, iglugasengillo illaliudlugit; Ga- 
sa iglugaseksoakattingillo, iglugasengillo illaliudlugit Egiptenib kônga tikkidlugo; imarbiksoarlo kiglilerviovok.

48 Kakkanello, Samir, Jathir, Socho,

49 Dana, Kiriat-Sana, tagva Debir,

50 Anabe, Estemo, Anime,

51 Gosen, Holon, Gilo. Tagva iglugaseksuit 11 it iglugasengillo.

52 Arabe, Duma, Esean,

53 Janum, Beth-Tabuah, Afeka

54 Humta, Kiriat-Arba, tagva Hebron, Zior. Tagva iglugaseksuit 9 it iglugasengillo.

55 Maon, Karmel, Sif, Juta,

56 Jesreel, Jakdeam, Sanoah,

57 Kain, Gibea, Thimna. Tagva iglugaseksuit 10 it iglugasengillo.

58 Halhul, Betzur, Gedor.

59 Maarat, Beth-Anoth, Eltekon. Tagva iglugaseksuit 6 it iglugasengillo.

60 Kiriath-Baal, tagva Kiriath-Jearim, Harabba; iglugaseksuit magguit iglugasengillo.

61 Sunakangitome, Beth-Araba, Middin, Sechacha.

62 Nibsan, iglugaseksuillo sioraujaggit, Engedilo. Tagva iglugaseksuit 6 it iglugasengillo.

63 Jebusiterille Jerusaleme nunakalaukput, Judablo kittorngangita aulartitsungnarlaungilait. Taimak Jebusiterit Judab kittorngangit illagilugit Jerusalemetuarlaukput uvlok tamanna tikkilugo.

\section{KAPITEL XVI.}

\section{Josefib kitiorngangita ikkupiangat, piluartomik Efraime- kut kiglilevingat.}

IMERAULLO nellaulaukpok Josefib kittornganginut Jordanemit Jerichub sivuranetomit, kôk Jerichometok tikkilugo kittânit, sunakangitorlo sangmijok takpaunga Jerichomit Bethelib kakkangitigut;

2 Bethelemillo tikkipok Lusemut, ailunelo Archi-Atharotib kiglilervingagut;

3 Sangmilunelo kannunga paunga Jafletib kiglilervinganut, Beth-Horonib atsiktub kiglilervia tikkidlugo, Gaserelo tikkilugo, nâningalo imarbingmepok.

4 Tamattominga Josefib kittorngangit Manassekullo Efraimekullo pilaukput kingormgutijaksamingnut.

5 Efraimib kittorngangita kingormgutijangata kiglilervingat abvakungita akkorngane imailaukpok: kittâne AtharotAdaremit, Beth-Horon kôgvasinerpak tikkilugo; 
6 Ailunelo Michmeteme avanetome avunga; tagvane sangulerpok iglugaseksuit Thaenat-Silomik attiggit kittânut tapkutigunalo pilune unungat aivok Janohamut;

7 Attersarlunelo Janohamit Atharotemut Naaratemullo aktoalunelo Jerichomut nâlunelo Jordaneme.

8 Thapuamit aivok paunga Nahal-Kanamut; nâningalo imarbingmepok.

Tamædsa kinguvarêt Efraim kittorngangita kingormgutijangit abvakungita akkorngane.

9 Efraimiblo kittorngangita kiglilervingita iglugaseksoangit, iglugasengit illagilugit akkusimalaukput Manassib kittorngangita kingormgutijangata akkorngane.

10 Aulartilaungilaillo Kanaaniterit Gaserme nunakatauktut. Taimak Kanaaniterit Efraimekut akkornganetualaukput uvlok tamanna tikkilugo, akkilejuksangortitaulutiglo.

\section{KAPITEL XVII.}

\section{Manasekut ikkupiangat kiglilervingallo.}

IMERAULLO nellaulaukpok kinguvarênut Mannassekunut, Josefib erniangokârtokotigilaungmago; nellaulunelo Machiremut Manassib erninganut angajuklermut Gileadib atâtanganut, anguteolaungmat sorsugungnartomik, tamanna pivlugo Gilead Basanelo pilaukpak.

2 Mannassible kittorngangita assingit nellautaulaurivut abvakungita akkorngane, tagva Abieserib kittorngangit, Helekib kittorngangit, Asrielib kittorngangit, Sechemib kittorngangit, Heferib kittorngangit, Semidablo kittorngangit. Tamædsa Manassib Josefib erningata kittorngangit, angutit abrakungita akkorngane.

3 Zelafehadele Heferib erninga, Gileadib erningata Machirib erningata, Mannassib erningata ernekalaungilak, pantekartuinarlaukporle, attingillo tapkoangorut: Mahela, Noa, Hagla, Milka, Thirza;

4 Kailaukpullo ajochertuijub Eleaserib Josuablo Nunib erningata, angajokaunerojullo sanganut okarlutiglo: Nalekab Mosese perkolaukpa kingormgutijaksamik tunnitsivigijaksaringmattigut kattangutipta akkorngane. Tunnitsiviolaukpullo kingormgutijaksamik atâtangata kattangutingita akkorngane, Nalekab perkojininga malliklugo.

5 Manassekunulle pivut ikkupianik 10 nik, nunab Gileadib Basaniblo, Jordanib ungatânetub assinginik.

6 Manassib paningit kingormgutijaksamik illangiutivikara- 
mik erningita akkorngane; nunalo Gileade Manassib kittorngangita assinginut illingalaukpok.

7 Manassekullo kiglilervingat pilaukpok Asseremit Michmethatemut, Sechemib sivoranetomut, tallerpingmullo tokpok En-Tapuamut.

8 Tapuab nunanga Manassekut pigilaungmatsuk; kiglilerviolunelo Manassekunut Efraimib kittorngangine.

9 Tagvangat attersarpok Nahal-Kanamut iglugaseksuit kôngnetut Efraimekut pigijangita. Manassekut iglugaseksoangita akkorngane sekkernganut; avangalle Manassekut kiglilervingat kongmepok, nâlunelo imarbiksoarme,

10 Ane Efraimekunut illingavok avanelo Manassekunut, imarbiksoarlo kiglilerviovok; aktuajuksauvorlo avane Asserkunnut unnanelo Isascharekunut.

11 Taimak Manassekuit pekalaukput Isascharekut Asserekullo akkorngane Beth-Seanemik iglugasenginiglo, Iblaamik iglugasenginiglo, Doremetuniglo iglugasenginiglo, Endoremetuniglo iglugasenginiglo, Taanachemetuniglo iglugasenginiglo, Megiddometuniglo iglugasenginiglo, Nafetiblo pingajuanik.

12 Manassiblo kittorngangita iglugaseksuit tamakkoa aksarnigarijungnarlaungilait, Kanaaniterille nunakalerlaukput nuname tamattomane.

13 Israelible kittorngangit songolerlauramik Kanaaniterit akkilêjuksangortipait aulartinagillo.

14 Tagva Josefib kittorngangita Josua okarvigivat okarlutiglo: Sôg uvagut immeraumik attausênarmik kingormgutijaksablo ikkupiaktanganik attauseînarmik tunnitsivigilaukpittigut? Inugaseksoangogalloartilluta, sorlo , Nalekab saimauserlaungmattigut.

15 Tagva Josuab okautivait: Innugaseksoangogapse takpaungaleritse nappartunut, kippitsiluselo tagvane Feresiterit innukpaillo nunangane, Efraimekut kakkangit nerrokigigapsigik.

16 Tagva Josefib kittorngangit okarput: Kakkakattigêt aksarnigarijungnarniængilavut, Kanaaniterit illunatik nunab naksangine nunakartut kamutekangmatta kikkiengnik, tapkoa Beth-Seanemetut iglugasenginetullo, Jesereelemetullo naksangme.

17 Josua Josefib iglunganut, Efraimekunnut Manassekunullo okarpok: Inugaseksoangôvose, unnuktogapselo immeraumik attausênarmik pekartuksaungilase;

18 Kakkalle nappartuggit perkutigijaksarivasse, tapkoa kippisigik, tagva tapkoa imeraukotipse nâninganut illinganiarput, Kanaaniterit kikkiengnik kamutekartut songojullo aulartigupsigik. 


\section{KAPITEL XVIII.}

\section{Benjaminekut kiglilervingat.}

ILLAGÊLLO illuêngarlutik Israelib kittorngangit kattimalerput Silome, nappartitsilutiglo tagvane kattimaviub tuppinganik, nunablo nalegarivait.

2 Amiakokalaukporlo sulle Israelib kittornganginit, kinguvarênik 7 nik, kingormgutijaksamik pilaungitunik sulle.

3 Josuablo okarpok Israelib kittornganginut: Kannok akkunêrtigijomik taimak erkeasukise, ainase nuna pigilerlugo Nalekab atatagijapse Gudingata tunnilauktanga illipsingnut?

4 Kinguvarênit illuainit attunit angutekarle pingasunik, tilliklerkovlunga tapkoninga, aularkovlugit nuna arvertarvigilugo nellonaijaijutigilugolo kingormgutijaksatik malliklugit, kailutiglo uvamnut.

5 Nuna aviksiuk ikkupianut 7 nut, Juda kiglilervingminetsainartuksauvok ângat, Josefekullo kiglilervingminetsainartuksauvut avangat.

6 Illipsele ikkupiat 7 it nunangat aglagutigisiuk, kaisigiglo tamaunga uvamnut; imeraromavunga tagva pivluse, Nalekab Gudipta sângane.

7 Levitille ikkupiakangilat akkunapsingne, Nalekable ajochertuiviksanga kingormgutigivât. Gadekulle Rubenekullo, kinguvarêllo Manassekut ketterkangit ikkupiangmingnik pijarêrput Jordanib akkiane kittane, Mosesib Nalekab kivgangata tunnilauktanganik tapkonunga.

8 Tagva angutit tapkoa parngnailerput aularlutiglo; Josuablo perkovait aulartillugit nuna aglagutigijomavlugo okarlunelo: Aileritse nunalo arvertarvigisiuk, nellonaijaijutigilugolo, kaimiluselo tamaunga uvamnut, mane imerarkovlunga pivluse Nalekab sângane Silome.

9 Taimak angutit tapkoa aivut, nunalo arvertarvigivât, aglagutigilâlo allakapsamut, iglugaseksuit avitisimajut ikkupianut 7 nut malliklugit, tikkilutiglo Josuamut tangmarvingmut Silometomut.

10 Tagva Josuab imerautigivait Silome Nalekab sângane, nunalo avikpa tagvane Israelib kittornganginut, illunatik attunit ikkupianganik.

11 Kinguvarêllo Benjaminib kittorngangita imerautingat nellaulaukpok abvakungit malliklugit, imerautingatalo kiglilervinga pivok Judab kittorngangita Josefiblo kittorngangita alkkorngangnit.

12 Kiglilervingallo nuvungmeląukpok avane Jordanemit, 
sangmilunelo takpaunga Jerichub seniane avangat, pivorlo kakkanut kangiane sâlunelo Beth-Avenib sunakajuitunganut;

13 Annilunelo tagvangat Lusemut, Luse senniagorlugo sekkerngagut, tagva Beth-Elemut, attersarlunelo unnunga AtarothAdaremut, kakkak Beth-Horonib atsiktub sekkernganetok tikkilugo.

14 Tagvangallo salerpok sangulunelo nuvungmut kangianêtomut kakkab Bethhoronib sekkernganetub sekkernganut, nâlunelo Kiriath-Baaleme, tagva Kiriath-Jearime, Judab kittorngangita iglugaseksoakotingine; tamadsa nuvua kangiane.

15 Nuvuale sekkerngane pigiarpok Kiriath-Jearimemit sangmilunelo paunga, pilunelo Nefthoab puailavinganut.

16 Attersarporlo tagvangat kakkab, Himonib erningata naksangata, Refaimib naksanganetub tachane, sânganetub naninganut, attersarporlo Himonib naksangagut, Jebusiterit sekkerngane, pilunelo unnunga Rogelib puailavinganut.

17 Sivumuarporlo avangat, pilunelo, En-Semesemut, sivumuartuarporlo Gililotemut Adumimib sivuranetomut, attersarlunelo Bohenib Rubenib erningata ujaranganut;

18 Sangmilunelo senianut naternak tachanetok senniagorlugo, pivorlo unnunga naternamut.

19 Beth-Hagalalo seniagorpa tachane, naningalo immarbiub tarrioggub okaujartunganepok tachane, Jordanib pângata sekkerngane. Tamædsa kiglilervik âne.

20 Jordanelo kiglilerviojuksauvok kittane. Tamædsa Benjaminib kittorngangita kingormgutijangat, kiglilervingmingne avatâtigut abvakungita akkorngane.

21 Benjaminiblo kittorngangita kinguvangita iglugaseksoangit abvakungita akkorngane imaiput: Jericho, Beth-Hagla, Emek-Keziz,

22 Beth-Araba, Zemaraim, Beth-El;

23 Avim, Hapara, Ofra,

24 Kafar-Amonai, Afni, Gaba. Tagva iglugaseksuit 12 it, iglugasengillo.

25 Gibeon, Rama, Beeroth,

26 Mizpe, Kafira, Moza.

27 Rekem, Jerpeel, Thareala,

28 Zela, Elef, Jebuselo, tagva Jerusalem, Gibeath, Kiriath; iglugaseksuit 14 it iglugasengillo. Tamædsa Benjaminib kittorngangita kingormgutijangat abvakungine. 


\section{KAPITEL XIX.}

\section{Kinguvarêt assingita 6 it, Josuablo kingormgutijangit.}

$K_{\text {IN }}$

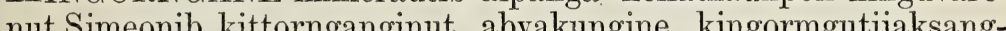
allo Judab kittorngangita kingormgutijangata akkornganelaukpok.

2 Kingormgutigilerpaillo Bersaba, Seba, Molada,

3 Hazar-Sual, Bala, Azem,

4 El-Tholad, Bethul, Harma,

5 Ziklag, Beth-Markabot, Hazar-Sussa,

6 Beth-Lebaot, Saruhen, tagva iglugaseksuit 13 it, iglugasengillo.

7 Ain, Rimon, Ether, Asan. Tagva iglugaseksuit sittamat iglugasengillo.

8 Illaliudlugillo iglugasait illunaita iglugaseksuit tapkoa avatinginetut Balat-Beer-Ramoth ânetok tikkilugo. Tamædsa kinguvarêt Simeonib kittorngangita kingormgutijangat abvakungine.

9 Simeonib kittorngangata kingormgutijangat Judab kittorngangita ikkupiangata akkornganemat. Judab kittorngangita kingormgutijangat angiluarlaungmat tapkonunga, tamanna pivlugo Simeonib kittorngangit kingormgujilaukput tapkoa kingormgutijangata akkorngane.

10 Imerautik pingajuak nellaulaukpok Sebulonib kittornganginut abvakungine; kingormgutijangatalo kiglilervinga pilaukpok Sarid tikkilugo,

11 Ailunelo paunga Marealamut, aktoalunelo Dabasetemut, aktoalunelo kôngmut Jakneamib sivorane koktomut.

12 Sangulunelo Saridemit ununga Cislot-Thaborib kiglilervinga tikkilugo; pilunelo Daberatemut illingalunelo takpaunga Jafiamut,

13 Tagvangallo aivok unnunga Gita - Heferekut, Ithakullo, Kazinekullo, pilunelo Rimonemut Mitoaremullo, Neamullo.

14 Salunelo avangat Nathonemut nâlunelo Jeftah-Elib naksangane,

15 Katath, Nahalal, Simron, Jedeala Bethlehemelo. Tagva iglugaseksuit 12 it iglugasengillo.

16 Tamædsa Sebulonib kittorngangita kingormgutijangat abvakungine; tamædsa iglugaseksoangit iglugasengillo.

17 Imerautit sittamangat nellaulaukpok Isascharib kittorngang inut abvakungit malliklugit.

18 Kiglilervingallo imailaukpok, Jesreel, Chesullot, Sunem,

19 Hafaraim, Sion, Anaharat,

20 Rabit, Kiseon, Abez, 
21 Remeth, Enganim, Enhada, Beth-Pazez,

22 Aktoalunelo Thaboremut, Sahazimamullo, Beth-Semesemullo, nâningalo Jordanemepok. Iglugaseksuit 16 it iglugasengillo.

23 Tamædsa kinguvarêt Isascharib kittorngangita kingormgutijangat abvakungine, iglugaseksoangit iglugasengillo.

24 Imerautit tellimangat kinguvarênut Asserib kittornganginut nellaulaukpok, abvakungit malliklugit. felo,

25 Kiglilervingallo Helkateolaukpok, Hali, Beten, Achsa-

26 Ala-Melech, Amead, Miseal; aktoalunelo Karmelemut imarbingme, Sihor-Libnathemullo;

27 Sangulunelo sekkerngub nuijavianut Beth-Dagonemut, aktoalunelo Sebulonemut, naksarmullo Jeftah-Elemik attelingmut, Beth-Emekib, Nigieliblo tachangangnut annilunelo Kabulib saumiane.

28 Ebron, Rehob, Hamon, Kana, Zidon angijok tikkilugo.

29 Sâlunelo Ramamut, iglugaseksuit avaluliksuit Zoremik attiggit tikkilugit, sâlunelo Hossamut, nâlunelo imarbingme, torarmariklune Achsibemut. illo.

30 Uma, Afek, Rehobelo. Iglugaseksuit 22 it iglugaseng-

31 Tamædsa kinguvarêt Asserib kittorngangita kingormgutijangat abvakungine, iglugaseksoangit iglugasengillo

32 Imerautit pingasojoat nellaulaukpok Naftalib kittornganginut abvakungine,

33 Kiglilervingillo Helefemit pilaukput Elonemut, Zaenanimut, Adami-Nekebemut Jabneelemullo, Lakumemut tikkilugo, nâlunelo Jordaneme;

34 Sâlunelo kangianut Asnoth-Thaboremut, annilunelo tagvangat Hukokemut, aktoalunelo Sebulonemut sekkerngane, Asseremullo kangiane, Judamullo Jordaneme, sekkerngub nuijarviane;

35 Iglugaseksoakarporlo songojunik, Zidim; Zer, Hamath, Rakath, Cinareth,

36 Adama, Rama, Hazor,

37 Kedes, Edrei, Enhazor,

38 Jereon, Migdal-El, Harem, Beth-Anath, Beth-Semes. Iglugaseksoarnik 19 nik iglugasenginiglo.

39 Tamadsa kinguvarêt Nafthalib kittorngangita kingormgutijangat abvakungine, iglugaseksoangit iglugasengillo.

40 Imerautit 7 ingat nellaulaukpok kinguvarênut Danib kittornganginut, abvakungit malliklugit.

41 Kingormgutijangetalo kiglilervingit imailaukput: Zarea, Esthaol, Irsames,

42 Saelabin, Ajalon, Jethla, 
43 Elon, Timnatha, Ekron,

44 Eltheke, Gibethon, Baalath,

45 Jehud, Bene-Barak, Gath-Rimon,

46 Me-Jarkon, Rakon, kiglilervingit illaliudlugit Jafomut illingajut.

47 Tagvanelo Danib kittorngangita kiglilervingat nâlaukpok. Daniblo kittorngangit takpaungarlaukput sorsugíarlutiglo Lesememut, salagivaillo ullimarlugillo saviksub kênanganut, aksarnigarivaillo innigilugillo, atserarpaillo Danemik, atatagijamik attinga malliklugo.

48 Tamædsa kinguvarêt Danib kittorngangita kingormgutijangat, abvakungine, iglugaseksoangit iglugasengillo.

49 Nunalo illunât kiglilervingit illaliudlugit avitsarêrlaungmatsuk, Israelib kittorngangita Josua Nunib erninga tunnitsivigivât kingormgutijaksamik akkonamingne,

50 Tunnitsivigivâllo Nalekab perkojininga malliklugo iglugaseksoarnik tussugijanginik, Timnath-Seramik, Efraimib kakkanginetunik. Tagva iglugaseksuit tapkoa sennavait innigilugillo.

51 Tamædsa kingormgutijat Eleaserib ajochertuijoksub, Josuablo Nunib erningata atatello angajokaunerojut kinguvarêt akkorngane, imeraumut avilauktangit Israelib kittornganginut Silome Nalekab sângane kattimaviub tuppingata upkuangata sângane; sorailerlutiglo taimak nunab aviktauninganik.

\section{KAPITEL XX.}

\section{Iglugaseksuit pitlartaujungnaivit unertutigijauningat.}

$\mathrm{N}$

NaleKablo Josua okarvigiva okarlunelo:

2 Israelib kittorngangit okautikit: Akkunapsingne iglugaseksoarnik pitlartaujungnairvingnik tunnijititse, Mosesekut okautigilauktamnik illipsingnut;

3 Tokkotsijut kemâviksanginik, kinamik pijarinane kaujimananelo tokkotsijok, akkonapsingne pijaukonagit aungmik akkiniartemut.

4 Kemâjorlo iglugaseksuit tapkoa illanganut nangertuksauvok sillatâne iglugaseksuit upkoaksoangata sângane, okartuksaulunelo illinganerminik iglugaseksuit angajokaunerôjungita sângane; tagvalo illallioraksarivât ingmingnut iglugaseksoarnut, inniksakartilugolo, tagvane nunakarkovlugo.

5 Aungmiglo akkiniartib unangmikpago, tagva unnertutigijaksaringilât tapsoma agganginut, pijarinane innukattine anaulaungmago omiginagolo sivorngagut.

6 Taimak iglugaseksoarne tamakkonane nunakartuksauvok, 
nangerkârtinago illagêt sângane erkartuivingme, ajochertuijoksoak tokkokârtinnago ajochertuijoksoangojok nelliutome taipsomane; tagva tokkotsijok kaijuksaugivok iglugaseksoaminut iglominullo, iglugaseksoarnut kemâlauktaminut.

7 Tagva unertutigivait, Kedes Galilæame Naftalib kakkangine, Sechemelo Efraimib kakkangine, Kiriat-Arba tagva Hebron, Judab kakkangine.

8 Jordaniblo akkiane Jerichomit nuijarviub nellâne, tunnilaukpait Bezer sunakangitome natername, kinguvarênit Rubenekunit, Ramothelo Gileademe kinguvarênit Gadekunit, Golanelo Basaneme, kinguvarênit Manassekunit.

9. Tamædsa, iglugaseksuit unertutigijaumajut Israelib kittornganginut illunainut, tujormitsanullo illunainut tapkoa akkorngane nunakartunut, kemâvigilugit tokkotsijub kimik pijarinane, tokkotaukonnago aungmik akkiniartemut, nangerkầrtinago illagêt sângine.

\section{KAPITEL XXI.}

\section{Levitit issumagïjauningat.}

TAGVA atâtet angajokaunerôjut Levitit akkorngane kaivut ajochertuijomut Eleaseremut, Josuamullo Nunib erninganut, angajokaunerôjunullo Israelib kittorngangita kinguvarêngine.

2 Okarkattigilugillo Silome nuname Kanaaneme, okarlutiglo: Nalegak Mosesekut perkojilaukpok, iglugaseksoarnik tunnitsivigiluta igluksaptingnut aiparutsiniglo iglugaseksuit avatâne nergutekotiptingnut.

3 Tagva Israelib kittorngangita Levitit tunnitsivigivait kingormgutijamingnit Nalekab perkojininga malliklugo, iglugaseksoarnik makkoninga aiparusinginiglo avatâne.

4 Imeraullo nellaulaukpok Kahatib abvakunginut, Aroniblo ajochertuijub kittorngangit Levitinit pijut, pilaukput imeraumut iglugaseksoarnik 13 nik, kinguvarênit Judakunit, kinguvarênit Simeonekunit, kinguvarênillo Benjaminekunit.

5 Kahatib kittorngangita assingit abvakungine immeraumut pilaukput iglugaseksoarnik 10 nik, kinguvarênit Efraimekunit, kinguvarênit Danekunit, kinguvarênillo Manassekut ketterkanginit.

6 Gersonible kittorngangit tapsomatsainaub kinguvangit pilaukput imeraumut iglugaseksoarnik 13 nik, kinguvarênit Isascharekunit, kinguvarênillo Asserkunit, kinguvarênillo Naftalikunit, kinguvarênillo Manassekut ketterkanginit Basaneme,

7 Merarib kittorngangita abvakungit pilaukput iglugasek- 
soarnik 12 nik, kinguvarênit Rubenekunit, kinguvarênit Gadekunit, kinguvarênillo Sebulonekunit.

8 Taimak Israelib kittorngangita Levitit tunnitsivigivait imeraumut iglugaseksoarnik makkoninga aiparusinginiglo iglugaseksuit avatane, sorlo Nalekab Mosese perkolaungmago.

9 Kinguvarênit Judab kittornganginnit kinguvarênillo Simeonib kittornganginit tunnijilaukput iglugaseksoarnik makkoninga tailauktamingnik attinginut.

10 Aronib kittornganginut Kahatib abvakunginut Levib kittornganginit; imeraut sivorlek tapkonunga illingalaungmat.

11 Taimak tunnitsivigilaukpait Kiriath Arbamik, Enakib atâtanganut illingalauktomik, tagva Hebronemik, Judab kakkangine, aiparusinginiglo avatâne.

12 Iglugaseksuille perorsêvingit, iglugasengillo tunnivait Kalemut Jefunib erninganut kingormgutijanganut.

13 Taimak Aronib kittorngangit tunnitsivigivait tokkotsijut kimik pitlartaujungnaivinganik Hebronemik aiparusinginiglo avatâne, Libnamiglo aiparusinginiglo avatâne,

14 Jatiremiglo aiparusinginiglo avatâne, Esthemoamiglo, aiparusinginiglo avatâne,

15 Holonemiglo aiparusinginiglo avatâne, Debiremiglo aiparusinginiglo avatâne,

16. Ainemiglo aiparusinginiglo avatâne, Jutamik aiparusinginiglo avatâne, Beth-Semesemik aiparusinginiglo avatâne; iglugaseksoarnik 9 nik kinguvarênit maggoinit tapkonangat.

17 Kinguvarênillo Benjaminekunit tunnijilaukput iglugaseksoarnik sittamainik: Gibeonemik aiparusinginiglo avatâne,

18 Gebamiglo aiparusinginiglo avatâne, Anatotemiglo aiparusinginiglo avatâne, Almonemiglo aiparusinginiglo avatâne.

19 Aronib ajochertuijub kittorngangita iglugaseksoangit kattilugit 13 ôlaungmatta aiparusingit avatâne illaliudlugit.

20 Kahathible kittorngangita assingita abvakungit, Levitit, imerautimikut pilaukput iglugaseksoarnik sittamanik kinguv arênit Efraimekunit.

21 Tunnitsivigivaillo tokkotsijut pitlartaujungnaivinganik Sechememik aiparusinginiglo avatâne Efraimib kakkangine, Geseremik aiparusinginiglo,

22 Kibzaimik aiparusinginiglo, Beth-Horonemik aiparusinginiglo.

23 Kinguvarênit Danekunit iglugaseksoarnik sittamainik: Eltekemik aiparusinginiglo avatâne, Gibtonemik aiparusinginiglo avatâne,

24 Aijalonemik aiparusinginiglo avatâne, Gath-Rimonemik aiparusinginiglo avatâne.

25 Kinguvarênit Manassekut ketterkanginit iglugaseksoar- 
nik magguinik: Thaenachemik aiparusinginiglo avatâne, GathRimonemik aiparusinginiglo avatane.

26 Kahatib kinguvangita assingita kittorngangita iglugaseksoangit 10 ôlaungmatta, aiparusingit avatâne illaliudlugit.

27 Gersonible kittorngangit Levitit abvakunginit tunnitsiviovut kinguvarênit Manassekut ketterkanginit iglugaseksoarnik magguinik: Tokkotsijut pitlartaujungnaivinganik, Golanemik Basaneme aiparusinginiglo avatâne, Beestharamik aiparusinginiglo avatâne.

28 Kinguvarênit Isascharekunit, iglugaseksoarnik sittamainik: Kis-Jonmik aiparusinginiglo avatâne, Dabrathemik aiparusinginiglo avatâne,

29 Jarmuthemik aiparusinginiglo avatâne, Enganimik aiparusinginiglo avatâne.

30 Kinguvarênit Asserkunit iglugaseksoarnik sittamainik: Misealemik aiparusinginiglo avatâne, Abdonemik aiparusinginiglo avatâne,

31 Helkathemik aiparusinginiglo avatâne, Rehobemik aiparusinginiglo avatâne,

32 Kinguvarênit Nafthalikunit iglugaseksoarnik pingasuinik: pitlartaujungnairvingmik tokkotsijunut Kedesemik Galilæame, aiparusinginiglo avatâne, Hamoth-Doremik aiparusinginiglo avatâne, Karthanemiglo aiparusinginiglo avatâne.

33 Gersonib kinguvangita iglugaseksoangit kattilugit 13 ôlaungmatta aiparusingit illaliudlugit.

34 Merarible kittorngangita abvakungit Levitit assingit, tunnitsiviolaukput kinguvarênit Sebulonekunit iglugaseksoarnik sittamainik: Jakneamemik aiparusinginiglo avatâne, Karthamik aiparusinginiglo avatâne.

35 Dimnamik aiparusinginiglo avatâne, Nahalemik aiparusinginiglo avatâne.

36 Kinguvarênit Rubenkunit iglugaseksoarnik sittamainik: Bezeremik aiparusinginiglo avatâne, Jahzamik aiparusinginiglo avatâne,

37 Kedemothemik aiparusinginiglo avatâne, Mefaathemik aiparusinginiglo avatâne,

38 Kinguvarênit Gadekunit iglugaseksoarnik sittamainik: tokkotsijut pitlartaujungnairvinganik Ramothemik Gileademe aiparusinginiglo avatâne,

39 Mahanaimemik aiparusinginiglo avatâne, Hesbonemik aiparusinginiglo avatâne, Jaeseremik aiparusinginiglo avatâne.

40 Taimak Merarib kittorngangita iglugaseksoangit abvakungita Levitit assingita akkorngane, imerautingat malliklugo 12 ôlaukput.

41 Levitit iglugaseksoangit Israelib kittorngangita kingorm- 
gutijangata akkorngane kattilugit 48 ôlaukput, aiparusingit avatâne illaliudlugit.

42 Iglugaseksuillo tapkoa attunit aiparusekalaukput avatimigut, aipaingit aipaingititut.

43 Taimak Nalekab Israelitit tunnitsivigilaukpait nunamik illunânik, angermarijutigilauktaminik atatagijanginut tunnijijomavlune, aksarnigarilaukpâllo innigilugolo.

44 Nalekablo ullapitaujuinermik tunnitsivigivait avatinginêtut tamaita pivlugit, sorlo atatagilauktangit angermarilaungmagit; omigijingitalo illangatalonêt akkerartorlaungilait, omigijingille illunaita tunnilaukpait tapkoa agganginut.

45 Kingomajaujokalaungilak ajungitut illanganiglonêt, Nalekab okauserilauktangita Israelib iglomiokottinginut; kailaukput illunatik.

\section{KAPITEL XXII.}

\section{Kinguvarêt maggoit koppaillo angerarkojauningit, sennaning- allo Altaremik.}

TAGVA Josuab Rubenekut Gadekullo kinguvarêllo Manassekut ketterkangit kaikovait,

2 Okautilugillo: Tamainik pinniarlaukpose Mosesib Nalekab kivgangata perkolauktanginik illipsingnut, nippimniglo naletsiarlaukpose tamaine perkolauktamne illipsingnut.

3 Kattangutise kemmalaungilasse akkunit, uvlok tamanna tikkilugo, ungalaukposelo Nalekab Gudipse perkojanganut.

4 Nalekab Gudipse manna kattangutise merngoêrservingmut âtsarêrmagit sorlo okarvigilaungmagit, tagva sanguleritse manna, ailuselo iglupsingnut, kingormgutijapse nunanganut, Mosesib Nalekab kivgangata tunnilauktanganut illipsingnut Jordanib akkiane.

5 Manale katsungailuse attaruilititse pinniarkovluse Nalekab perkojanga illusiokojangalo, Mosesib Nalekab kivgangata perkolauktanga illipsingnut malliklugo, naglikovluse Nalegapsingnik Gudipsingnik, arvertarluselo apkosiningine tamaine, mallikovluselo perkojanginik, ungaluselo tapsomunga, kivgartorkovluselo tapsominga, omamut tamanut tarnemullo tamanut.

6 Taimak Josuab saimarpait aularkolugillo, ailaukpullo iglomingnut.

7 Mosesib kinguvarêt Manassekut ketterkangit tunnitsivigilaukpait Basaneme, ketterkangillo aipaingit Josuab tunitsivigivait kattangutingita akkorngane Jordanib miksâne kangiane: Aularkolaungmagillo iglunginut, saimarlaukârlugillo,

8 Okarpok tapkonunga: Utterpose iglupsingnut perkuteli- 
jarluse unnuktunik, nergjutinik unnuktunik, Silberemik, Goldemik, kanujarmik, kikkiengmik, anorangniglo; tagva manna omigijipse aksartaujutigijangit aviksigik kattangutipsingnut.

9 Taimak Rubenekut Gadekullo, kinguvarêllo Manassekut ketterkangit utterput, aularlutiglo Israêlib kittornganginit Silomit, Kanaanib nunanganetomit, aikovlugit nunamut Gileademik attelingmut, nunamut kingormgutijamingnut, kenuarijamingnut Nalekab perkojininga malliklugo Mosesekut.

10 Tikkinamiglo kiglilervingmut Jordaneme Kanaanib nunanganetomut, Rubenekut Gadekullo, kinguvarêllo Manassekut ketterkangit sennavut tagvane Jordaneme Altaremik angijomik ânanamik.

11 Israelible kittorngangit tussaramik okartokarmat: Ahâk Rubenib kittorngangit, Gadiblo kittorngangit, kinguvarêllo Manassekut ketterkangit Altaremik sennalaukput, nuna Kanaane akkerartorlugo, Jordaneme Israelib kittorngangita miksane,

12 Tagva kattilerput illagêt illunamassiatik illagilugit $\mathrm{Si}$ lome unatadlarnermut, aivigijomavlugit.

13 Tilliklerpullo tapkonunga nunamut Gileademut, Pinehasemik Eleaserib ajochertuijub erninganik,

14 Illakartilugolo angajokaunerojunik 10 nik atatamik iglomiokattingita akkornganetunik, kinguvarênit illunainit attunit attausemik.

15 Tikkigamigillo nuname Gileademe, okarkattigivait okarlutiglo:

16 Nalekab illagêktokotingita illunatik imak okautsaukovâse : Kanok idluitullivise taimak Israelib Gudinganut, tunnulerapse uvlome Nalekamit, senanipsingnut Altaremik, kattakapse Nalekamit?

17 Peorib idluitullijutigijanga mikkigivittigo? sujungaititaujutigingitavut sulle, uvlok tamanna tikkilugo, kappianartorlo tikkilaukpok Nalekab illagêktokotingita akkornganut.

18 Illipselo uvlome sangulerpose Nalekamit, uvlomelo Nalegak kemakpasse, uvlome kaupallonêt ningaumajalerkovlugo Israelib illagêngningit illunaita pivlugit.

19 Kingormgutijapse nunangat ivsornarasugigupsiuk, tagva kaileritse akkianut, nunamut Nalekab pigijanganut, Nalekab iglungata nangervigijanganut, kingormgujileritselo akkonaptingne, Nalegarlo kemangniarassiuk, kemangniaratigullo, sennanipsingnut Altaremik illipsingnut, Nalekab Gudipta Altarekotingata assianik.

20 Achan Serab erninga idluitullilaungilâk nungutaksat pitjutigallugit, ningaumajarnerlo pirok illagêktunut illunainut? kissiovlunelo pejartaulaungilak idluitullinine pivlugo.

21 Tagva Rubenib kittorngangit, Gadiblo kittorngangit, 
kinguvarêllo Manassekut ketterkangit kiovut, okarlutiglo, Israelib angajokanginut anginerojunginullo:

22 Gude pitsartujok, Nalegak, Gude pitsartujok, Nalegak tamna kaujimavok, Israelitillo kaujimagivut kattakupta, idluitulliguptalonêt Nalekamut; tagva ikkajorniaranittigut uvlome!

23 Tamana pivlugo Altaremik senalaurupta sangujomagapta Nalekamit, tunergutinik otaksanik, tunergutiniglonêt nerrijaksanik tunijijomavluta kângane, tunnerguteniglonêt kujalijutiksanik illijiluta kângane Nalekab sângane, tagva tamna nangminek akkiortaujomalerle!

24 Piniangârlaungikuptalo illimasungnermut, imaitomik okarapta: Uvlojomârtune kittorngase okarajarmatta kittorngaptingnut: Sunamik illangiutivikarkise Nalekamik Israelib Gudinganik?

25 Nalekab Jordane kiglilervingortilaukpa uvagut illipselo Rubenib Gadiblo kittorngangita akkonaptingnut; illangiutivikangilase Nalekamik. Taimailiornermut kittorngapse kittorngavut nûtinajarpait sivoranermit Nalekamik.

26 Tamana pivlugo okalaukpogut: Altaremik senalerta, tunergutiksamuungitok, tunnergutiksanullonêt otaksanut:

27 Kigligiudsijokovlugole akkonaptingne akkonapsingnelo, kinguvârijomârtaptalo akkorngane, Nalegak kivgartorungnarlugo sângane, tunnergutiksaptingnut otaksanut, tunnergutiksaptingnullo kujalijutiksanut, tunnergutiptalo assinginut; kittorngase, nelliutsomârtome okarungnarkonagit: Illangiutivikangilase Nalekamik.

28 Taimagle okarajarpatta, kinguvaptingnullonêt nelliutsomârtome, tagva okarungnarajarput: Tækkoleritse Nalekab Altaringata adsinganik, atatagijapta senalauktanganik, tunnergutiksamuungitok, tunnergutingnullonêt otaksanik, kigligiudsijomulle akkonaptingne pivluse pivlutalo.

29 Tamana ungasigle uvaptingnit kernainivut Nalekamik, uvlome sangujomagapta tapsomangat, Altaremiglo senajomagapta tunnergutiksanut otaksanik, tunnergutiksanullo nerrijaksanik, tunergutiksanullonêt assinginut, Nalekab Gudipta Altaringata iglungata sânganetub assianik.

30 Pinehasible ajochertuijub, illagêktullo angajokangita, Israelitit attaniotijungita, tapsoma illagijangita, okautsit makkoa tussaramigit, Rubenib, Gadiblo, Manassiblo kittorngangita okauserijangit, namagivait.

31 Pinehaselo Eleaserib ajochertuijub erninga okarpok Rubenib Gadiblo Manassiblo kittornganginut: Uvlome tukkisivogut Nalekab akkonaptingneninganik, idluitullilaunginipsingnik Nalekamut piniarnermut mattomunga. Manna Israelib kittorngangit piulilaukpasse Nalekab agganginit.

32 Tagva Pinehas Eleaserib ajochertuijub erninga angajo- 
kajallo aularput nunamit Gileademit Rubenib Gadiblo kittornganginit, nunamut Kanaanemut Israelib kittornganginut, okarlutiglo tamattominga tapkonunga.

33. Israeliblo kittorngangita tamana idluarivât, Israeliblo kittorngangita Gudingat nakorivât, okarungnailutiglo takpaungaromagamik kattimgajunut unatadlarvigijomavlugit, nuna asseroromavlugo Rubenib Gadiblo kittorngangita innigijangat.

34 Rubeniblo Gadiblo kittorngangita Altare tamna taivât: kigligiudsijokovlugo akkonaptingne pivluta, pivluselo, Nalegarlo Gudeôngmat.

\section{KAPITEL XXIII.}

\section{Josuab Israelitit katterkovait kaiblarlugillo.}

AKKUNILLO Nalekab âtsilaungningata Israelitinik merngoêrsernermut, omigijingit avatinginetut tamaita pivlugit kingorngane, Josualo mana îtolaurame uvlokaramelo unuktunik;

2 Israelitit illuêngarlutik, angajokangillo, angajokaunerôjungillo idluarsaijungillo, aulatsijungillo kaikovait okautilugillo: Innutokauvunga manna, uvlokarlunga unnuktunik;

3 Tækkolaukposelo tamainik Nalekab Gudipse piniarnerilauktanginik innukattigêksoarne tamakkonane tamaine sivunapsigut: Nalekab Gudipse nangminek sorsugutigilaungmasse,

4 Ahâk innukattigêt amiakojut sulle imerautigilugit avilaukpakka illipsingnut, kinguvarêt illunatik attunit kingormgutijaksanginik, Jordanemit, innukattigêllo illunaita nungutilauktakka, imarbiksoak angijok sekkerngub nippijarviata nellânetok tikkilugo.

5 Nalekablo Gudipse pingujarniarpait sivunipsigut, aulartiniarlugillo illipsingnit, aksarnikovluse nunamik; sorlo Nalekab Gudipse okautilaungmasse.

6 Tagva manna maksoadlaritse, sapkutsitailikovluse, pinniarkovluselo tamainik aglaksimajunik Mosesib aglakotingine perkojaktalingne senniagorkonnase tamakkonangat tallerpingmullonêt, saumingmullonêt.

7 Inukattigêt amiakojut makkoa illipsingnetut akkornganut pikonase; issumaksarsiorkonaselo angerkonaselo Gudingoata attingine, kivgartorkonaselonêt tapkoninga, tuksiarviginagillonêt.

8 Ungakovlusele Nalekamut Gudipsingnut, sorlo uvlok tamanna tikkilugo pilaurapse.

9 Tagva Nalekab Gudipse nunakattigêksuit unuktôjut songojullo pingujarniarpait sivunipsigut; uvlorlo tamanna tikkilugo nangertokarungnarlaungilak sâpsingne. 
10 Illapse illangata 1000 dit kemâtiniárpait Nalekab Gudipse sorsugutigingmasse, sorlo okautilaungmasse.

11 Taimaimat attaruilinerpangôjomik tarnise sapputisigik, naglikovluse Nalekamik Gudipsingnik.

12 Sangulerupse, innukattigêllo tapkoa amiakojut ungagigupsigik, aipaniktigêgupse akkornganut pikovluse, tapkoalo akkonapsingnut pilutik;

13 Tagva kaujimaleritse Nalekab Gudipse innukattigêt ukkua pingujarungnainiarmagit sivunipsigut; tapkoale kellerutingorniarlutiglo nulluangorniarput illipsingnut, issakautingorniarpullo sennerapsingne, kapputingorniarlutiglo ijipsingne, pêjartaukârtinase tapsomunga nunamit ajungitomit tamattomangat, Nalekab Gudipse tunnilauktanganit illipsingnut.

14 Ahâk, uvlome aivunga sillaksoarmiut illunatik aivigivaktanganut, illipselo kaujijuksauvose omamut tamânut, tarnemullo tamânut, okautsemik attautsemiglonêt kingomajaujokalaungimat ajungitunit Nalekab Gudipse okauserilauktanginit illipsingnut; tamaita tikkisimavut, tikkingitokangilarlo attautsemiglonêt.

15 Sorlo manna ajungitut tamaita tikkisimangmatta Nalekab Gudipse okautigilauktangit: taimak Nalekab idluitut tamaita tikkerkojomârivait illipsingnut, pejaikârtinago illipsingnik nunamit ajungitomit mattomangat Nalekab Gudipse tunilauktanganit illipsingnut;

16 Tappagigupsiuk Nalekab Gudipse angerutigijanga, perkolauktanga illipsingnut, aigupselo kivgartorlusèlo Gudib assinginik (Gudingoanik) tuksiarvigigupsigiglo, tagva Nalekab ningausinga illungertorniarpok pivluse, pêjarniarpâselo nunamit ajungitomit tunnilauktaminit illipsingnut.

\section{KAPITEL XXIV.}

\section{Josuab kingorlerpâmits Israelitit katterkovait Sicheme. Tap-} somalo Eleaseriblo tokkonigik.

JOSUAB Israelib kinguvarêngit illunaita katterkovait, kaikolugillo Israelitit angajokangit, angajokaunerôjungillo, idluarsaijungillo, aulatsijungillo. Gudiblo sanganut ablorêrmatta,

2 Okarpok inugiartoksoarnut illunainut: Imâk Nalegak Israelib Gudinga okarpok: Atatagilauktase nunakalaukput itsaksoarme kôksuit akkiane, Tharah, Abrahamib Nahoriblo atâtangak, kivgartorlutiglo Gudib assinginik (Gudingoanik).

3 T'agva Abraha atatagijase tigulaukpara koksuit akkiane, arvertarkolugolo nuname Kanaaneme illunane, unnuksitilugillo kingurangit, tunitsivigilaukparalo Isaangmik. 
4 Isaaglo tunnitsivigilaukpara Jakomik Esaumiglo, Esaulo tunnitsivigilaukpara nunaksanganik kakkanik, Seiremik attelingnik. Jakole kittorngangillo attersarlaukput Egiptenemut.

5 Tagva Mosese Aronelo tillilaukpâka, Egiptenemiullo kappiasuktilaukpakka, sorlo akkorngane pinnialaurama. .

6 Kingornganelo illipse atâtagijaselo aulartilaukpapse. Imarbingmullo pilaurapse, Egiptenemiullo atâtagijasse udlalaungmattigik aksalloalikutigut horsekutigullo imarbingmut iviksoatalingmut suvlolingnik,

7 Tagva Nalekamut nipliadlalaukput, tapsomalo tâksoak pitilaukpa, illipse Egiptenemiullo akkorngænut, imarbiksoarlo âlugo kânginut, ullilaukpait. Ijisselo tækkolaukput sunamik piniarnerilauktamnik Egipteneme. Illipse nunakalaukpose sunakangitome akkunit.

8 Uvangalo âlaukpapse Amoriterit, Jordanib akkiane nunakalauktut, nunanganut; unatadlarvigilaungmasselo tunnilaukpakka aggapsingnut, nunanganik perkutisiniarluse; nungutilaukpakkalo sivunipsigut.

9 Tagva Balak, Ziporib erninga Moabiterit attaningat opalungailerlaukpok, sorsugvigilugillo Israelitit, tilliklerlunelo kaikojaukojilunelo Bileamik Beorib erninganik, ominarsijaukojikovlugo illipsingnik;

10 Uvangale tussaromalaungilara. Saimartilaukpâselo, uvangalo piulilaukpapse taipsoma agganginit;

11 Jordanemiglo ikkartilluse Jerichomullo piluse, Jerichomiut sorsugvigilaukpâse, Amoriterit Feresiterit, Kanaaniterit, Hethiterit, Girgositerit, Heviterit, Jebusiterit, tunnilaukpakka aggapsingnut.

12 Igupsaksuillo tillilaukpakka sivunipsigut, tapkoa aulartilaukpait sivunipsigut, Amoriterit attannigik, saviksoapsingnuungitok pitiksipsingnuungitorlonêt.

13 Nunamiglo tunnitsivigilaukpapse sennavigilaungitapsingnik, iglugaseksoarniglo iglogaselliorlaungitapsingnik, iglokarkovluse tapkoninga, nerriluselo perorsêvingnit Weineliksatalingnit, perorsêvingnillo orksuksatalingnit, illilaungitapsingnit.

14 Tagva manna Nalegak sivoragisiuk kivgartorlugolo nertornartomik idluartomiglo, Gudingoallo atâtagijapse kivgartorlauktangit kôksuit akkiane, Egiptenemelo ipperarsigik, kivgartorluselo Nalekamik.

15 Kuviagijaungipalle illipsingnut, Nalegak kivgartorlugo, tagva uvlome annerosugitse kinamik kivgartoromangmangapse, Gudemik tapsominga, atâtagilauktapse kivgartorlauktanganik kôksuit akkiane, Amoriterillônet nunagijapse innungita Gudingoanginik. Uvangale iglomiokattimalo Nalegak kivgartoromavavut.

16 Tagva innugiartoksuit kiovlutiglo okarput: Tamanna 
ungasigle uraptingnit, kemailuta Nalekamik, kivgartorlutalo Gudib assinginik Gudingoanik.

17 Nalekab Gudipta uvagut atatagijavullo tessiorlaungmattigut Egiptenemit kippaloviub iglunganit, ijiptalo sângine nellonaikutakartitsilaukpok angijunik, sapputilaukpâtigullo apkome ingergarvigilauktaptingne illunane, innukattigêksuillo illunatik akkorngane tapkutiguna apkosarutigilauktapta;

18. Sivunipsigullo innukattigêksuit Amoriterit illunaita nunakalauktut nuname pingujarlaukpait: Tamanna pivlugo Nalegak kivgartoromavavuttauk; Gudigigaptigo.

19 Josua inugiartoksoarnut okarpok: Nalegak kivgartorungnangilasse; Gudeôngmat heiligeojok, Gudeôngmallo illungertortok, tappanipsingnik ajornipsingniglo pitlartautitsitailiniangitok.

20 Nalegarle kemmagupsiuk, Gude tækkorngartak kivgartorlugo pigupse, tagva allarniarpok, kappiasuktiniarluselo nungutiniarpâselo ajungitullivigilaukârluse.

21 Innugiartoksuillo Josuamut okarput: Taimangolungitok, Nalegarle kivgartoromavavut.

22 Tagva Josua innugiartoksoarnut okarpok: Illipse kigligiudsijôvose pivluse, Nalegak annerigapsiuk kivgartoromavlugo. Tapkoalo okarput: Ahaila.

23 Tagva manna Gudingoat tækkorngartat akkonapsingnêtut pêjarsigik illipsingnit, omattiselo mannisigik Nalekamut Israelib Gudinganut.

24 Innugiartoksuillo Josuamut okarput: Nalegak Gudivut kivgartoromavavut nippingalo nalegomavavut.

25 Taimak Josuab uvlorme taipsomane inugiartoksuit angerkattigivait, mannijivigilugillo Sichememe perkojanik idluarkutaunerniglo.

26 Josuablo tamakkoa tamaita aglalaukpait Gudib aglakotinginut perkojaktalingnut; tigusilunelo ujarkamik angijomik, nappartilugolo tagvane, nappartub Eichemik attiggub Nalekab ivsornaitovikotingata kanitanganêtub attâne.

27 Okarlunelo innugiartoksoarnut illunainut: Ahâk ujarak una kigligiudsijojuksauvok akkonaptingne; tussarlaurame Nalekab okalaninginik tamainik, okauserilauktanginik uvaptingnut; kigligiudsijojuksauvorlo pivluse, missiarkonnase Gudipsingnik.

28 Taimak Josuab innugiartoksuit aularkovait, illunaita attunit kingormgutijanginut.

29 Pijokartullo tamakkoa kingorngane pijokalaukpok Josua, Nunib erninga tokkolaungmat Jahrekarlune 110 nik.

30 Illijartortaulaukporlo kingormgutijame kiglilervingane, Thimnath Serahme, Efraimib kakkanginetome, kakkab Gaasemik attiggub tachâne. 
31 Israelitillo Nalegak taimak akkunêrtigijomik kivgartorlaukpât Josua innotillugo, angajokajallo akkunit Josuab kingorngagut innôlauktut, kaujimalauktullo Nalekab pinniarninginik tamainik, pinniarnerilauktanginik Israelitinut, innôtillugit.

32 Josefib timinga Israelib kittorngangita neksarlauktangat Egiptenemit illijartorpât Sicheme, natername Jakob pissiarilauktangane Hemorib, atâtaulauktub Sichememut kittornganginit, kênaujanut 100 dinut, kingormgutijaulaukporlo Josefib kittornganginut.

33 Eleaserelo Aronib erninga tokkolaurivok, illijartorpâllo Gibeame, erningata Pinehasib pigijangane tunergusearilauktangane Efraimib kakkangine.

\section{Idluarsaijut aglangit.}

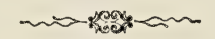

\section{KAPITEL I.}

Kinguvarêt Judałeut aulatsivut unatadlarnermik Josua innangêrlugo.

J OSUAB tokkungata kingorngane Israelib kittorngangita $\mathrm{Na-}$ legak appertsorpât, okarlutiglo: Kina akkunaptingne unatadlarnermik Kanaaniterinut aulatsijuksauva?

2 Nalegak okarpok: Judakut aulatsijuksauvut tamattominga. Ahâk, nuna tunnimavara aggenginut.

3 Tagva Judakut kattangutimingnut Simeonekunut okarput: Takpaungarkattigitigut immerautiptingnut, sorsugiarluta Kanaaniterinut, tagva aularkattaujomagivogut imerautipsingnut. Taimak Simeonekut aularkattigivait.

4 Judakut manna takpaungarmatta, Nalekab Kanaaniterit, Feresiterillo tunnivait tapkoa agganginut, ullimarpaillo Besekeme angutit 10,000 dit, 
5 Naipipâllo Adoni-Besek Besekeme, sorsugvigivâllo; ullimarpaillo Kanaaniterillo Feresiterillo.

6 Adoni-Besekele kemâvok, unangmivâllo, angugamitsuglo, kublugik aggangine, puttokogiglo ittiggangine nakkarpait.

7 Tagva Adoni Besek okarpok: Attannit 70 git kippiviomajut kublomingnik aggangine, puttogomingniglo ittiggangine, kattersoilaukput nerrimârvingma attâne. Sorlo manna uvanga pinnialaurama, taimak Gudib akkinniarvigivânga. Atauvorlo Jerusalemut, tokkolunelo tagvane.

8 Judable kittorngangita Jerusalem sorsugvigilerpait, salagivaillo, ullimarlugillo saviksub kênanganut, iglugaseksuillo ikkipait.

9 Kingorngane Judab kittorngangit attersarput, Kanaaniterit, nunakalauktut kakkane sekkernganelo, nâksanelo sorsugvigilugit.

10 Judakullo aularput Kanaaniterinut Hebroneme nunakartunut (Hebronele mattoma sivorngagut Kiriath-Arbamik attekalaukpok) Sesailo, Ahimanelo Thalmailo ullimarpait.

11 Tagvangallo aivut Debiremut (Debirele mattoma sivorngane attekalaukpok Kiriath Seferemik)

12 Kalelo okarpok: Kina Kiriath-Seferemik ullimarniktok sâlakartorlo, tamna pannimnik Achsamik tunnitsivigijomavara nulliaksamut.

13 Tagva Athnielib, Kenasib, Kaleb nukkangata erningata salagivait. Tapsomalo tunnitsivigiva panniminik Achsamik nulliaksamut.

14 Pijokalaukporlo, tamna ittermat okautsaulaukpok, kennukovlugo atâtaminit perorsêvingmik; akkarporlo siutekoktojomit. Tagva Kaleb okautiva: Kannoêkit?

15 Tamna okarpok: Saimanermik tunnitsivigilaunga. Perorsêvingmik sekkernganêtomik tunnitsivigilauramga, tunnitsivigilaungatauk immektalingmik, Tagva tunnitsivigiva immẹtar lingmik, kollâne attânelo.

16 Keniteriblo Mosesib sakkingata kittorngangit, takpaungarput iglugaseksoarnit Palmitalingnit, Judab kittorngangit illagilugit Judab sunakajuininganut, iglugaseksuit Arademik attiggit sekkernganetomut, ailutiglo, nunakarlutiglo innugiartoksuit akkorngane.

17 Judakullo aularput kattangutitik Simeonekut illagilugit, ullimarpaillo Kanaaniterit Zefateme, nungupaillo, iglugaseksuillo atserarpait Harmamik.

18 Tamakkoa tappilugit Judakut Gaza illagijangit illaliudlugit salagivait, Askalonelo illagijangit illaliudlugit, Ekronelo illagijangit illaliudlugit.

19 Nalekablo Judakut illagivait, sâlakarkovlugit kakkane- 
tunik, naksarmiulle salagijungnarlaungilait, kikkiengnik aksalloalikutekalaungmatta.

20 Kalelo tunnitsivigivât Hebronemik, sorlo Mosese okalaungmat; tapsomalo aulartipait tagvangat Enakib erningit pingasut.

21 Benjaminible kittorngangita Jebusiterit Jerusaleme nunakalauktut aulartilaungilait, Jebusiterille Benjaminib kittorngangine Jerusaleme nunakalaukput uvlok tamanna tikkilugo.

22 Taimaktauk Josefib kittorngangit takpaungarlaukput Bethelemut, Nalekablo illagivait.

23 Josefekullo Bethele, sivorngane Lusemik attekalauktut, nautsertoriartorpait.

24 Nautsertortullo angut iglugaseksoarnit annijok tækkovât, okautilugolo: Unertotitigut, naukut tikkitsungnarniptingnik iglugaseksoarnut, tagva napkiningnermik pinniarvigijomavaptigit.

25 Unertotimagillo naukut iglugaseksoarnut tikkininganik, ullimarpait iglugaseksoarmiut saviksub kênanganut; angulle tamna, illangillo illunaita aularkovait.

26 Tagva angut tamna ailaukpok Hetitherit nunanganut, sennalunelo iglugaseksoarnik, taisilunelo tapkoninga Lusemik; sullelo uvlome, uvlorme tamattomane taimak taijauvut.

27. Manassekullo Beth-Seanemiut iglugaseksoakattingitalo innungit aulartilaungilait, Taenahemiullonêt iglugaseksoakattingitalo innungit, Toremiullonêt iglugaseksoakattingitalo innungit, Meggidomiullonêt iglugaseksoakattingitalo innungit; Kanaaniterillo nunakalerlaukput nuname tamattomane.

28 Israelitille songolerlauramik Kanaaniterit akkilêjuksangortipait, aulartilaungilaillo,

29 Taimaktauk Efraimekut Kanaaniterit Gaseremelauktut aulartilaungilait: Kanaaniterille tapkoa akkorngane nunakalaukput Gasereme.

30 Sebulonekullo Kitronemiut Nahalolemiullo aulartilaungilait; Kanaaniterillo tapkoa akkorngane nunakalaukput akkilêjuksangortitaumalutiglo.

31 Asserekut Akomiut, Zidonemiullonêt, Ahelabemiullonêt, Achsibemiullonêt, Helbamiullonet, Afikemiullonet, Rehobemiullonêt aulartilaungilait;

32 Asserekulle nunakalaukput Kanaaniterit nunamelauktut akkorngane; aulartilaungimattigik.

33 Naftalikut Bethsemesemiut, Beth-Anatemiullonet aulartilaungilait; nunakalaukpulle Kanaaniterit nuname nunakalauktut akkorngane; Bethsemesemiulle Beth-Anatemiullo akkilêjuksangolerput.

34 Amoriterillo Danib kittorngangit singipait kakkanut, naksarnullo tikkitailitipait.

35 Amoriterillo nunakalerput kakkane Heresemik attilingne, 
Ajaloneme, Saalbimemelo. Josefekulle aggangit okkumailualilaukput tapkonunga, akkilêjuksangolerpullo.

36 Amoriterillo kiglilervingat takpaungarvingmelaukpok apkome Akrabimut, kairtomillo, kotsiktomillo.

\section{KAPITEL II.}

\section{Israelib kittorngangito nippunganingat, kattangningallo pitlartauningallo.}

NALEKABLE Engelinga kailaukpok takpaunga Gilgalemit Bochimemut, okarlunelo: Tessiorlaukpapse takpaunga Egiptenemit, tikkiutiluselo nunamut atâtagijapsingnut angerutigilauktamnut, okalaukpungalo: angernera illipsingnut sokkosertitsomanginapko issokangitomut:

2 Illipse angerkattigêngnermik pijuksaunginapse nunab tamattoma innunginut; Altarekotingillo serkomitaksarigapsigik. Illipsele nippiga nalelaungilasse. Sôg taimailiorlaukise?

3 Tagva okalaurivunga: Pêjaromangilakka sivunapsingne, kellerutiksangolerkovlugit illipsingnut, Gudingoangillo nulluangolerkovlugit.

4 Nalekablo Engelinga taimaitunik okautsinik okarêrlaungmat Israelib kittornganginut illunainut, inugiartoksuit nippitik kagvarpait kêalutiglo.

5 Innelo tamana atserarpât Bochimemik, tunnijilutiglo tunnergutinik Nalekamut,

6 Josuable innugiartoksuit aularkolaungmagit, Israeliblo kittorngangit aularlaungmatta, attunit kingormgutijamingnut, nuna aksarnigarilugo,

7 - Innugiartoksuit Nalegak kivgartorlaukpât Josua innotillugo, angajokajallo, akkunit Josuab kingorngane innôlauktut, tækkolauktullo Nalekab pinniarnersoanginik tamainik, piniarnerilauktanginik Israelitinut, innotillugit.

8 Josualo nunib erninga, Nalekab kivganga tokkolaungmat Jahrekarlune 110 nik,

9 Illijartorpât kingormgutijangata kiglilervingane, Thimnath-Hereseme, Efraimib kakkanginetome, kakkab Gaasemik attiggub tachâne.

10 Illunatiglo, nelliutome taipsomane innôlauktut, kattersortaujarêrlaungmatta atâtagijamingnut, tagva tapkoa kingorngane kinguvarêkalerpok assianik, Nalekamik illitarksilungitunnik, pinniarnerniglonêt pinniarnerilauktanginik Israelitinut.

11 Tagva Israelib kittorngangit idluitullixut Nalekab sângane, Baalillo kivgartorpait,

12 Kemakpâllo Nalegak atâtagijangita Gudingat, tessior- 
nilauktok tapkoninga nunamit Egiptenemit, malliklutiglo Gudib assinginik, innukattigêksuillo avatinginelauktut Gudinginiktauk, tuksiarvigivaillo, Nalegarlo ningarosuktipât.

13 Nalegak kemakattarmatsuk, kivgartorlutiglo Baalemik, Astharotemiglo.

14 Taimak tagva Nalekab ningarninga illungertudlalaukpok Israelitit pivlugit, tunnivaillo arktainiktut tapkoninga agganginut, aksarnikovlugit tapkoninga, niorvgutigivaillo omigijingita avatânetut agganginut. Akkerartorungnarungnailaukpaillo omigijitik;

15 Naukulle annijomavingine, Nalekab aggangita akkerartorpait pillorijuijutiksanginut, sorlo Nalekab okautilaungmagit angervigimarilaungmagillo; angijomiglo okumaiksartitaulaukput.

16 Nalegak tagva idluarsaijokartitsilaurangat piuliklertunik tapkoninga, arktaijut agganginit,

17 Tagva idluarsaijut nalelaunginivait; ajortullilutigle Gudib assinginut, tuksiarvigivaillo, sangusarailutiglo apkomit atâtagijamik arvertarvigilauktanganit Nalegak naleklugo, pinniarlaungilello taipkotitunak.

18 Nalekable idluarsaijokartilaurangagit, tagva Nalekab idluarsaijok aiparilaukpa, ikkajorlugillo omigijingita agganginit idluarsaijok innotillugo. Nalekab tapkoa ogguarningit, taipkoa okkumaiksartitsijut tapkoninga pivlugit, illapsugosujutigingmagit.

19 Idluarsaijorle tokkolaurangat, tagva sangulerput, asseroinersaulutiglo atâtagijamingnit, mallingnermut Gudib assinginik, kivgartorlugit, tuksiarvigilugillo; allailaungilet kajusimanermingnik, illinganermingniglonêt tappatonelingmik.

20 Tamanna pivlugo Nalekab ningarninga illungertulerlaukpok Israelitit pivlugit, okarkovlugo: Inugiartoksuit ukkoa angernera tappagilaungmatsuk atâtagijanginut perkolauktara, nippigalo nalelungimatsuk,

21 Tagva mattoma kingorngagut nellojut Josuab amiakotilauktangit tokkotillugo pêjaromanginivakka;

22 Oktorkovlunga Israelitinik tapkonane, Nalekab apkosininganênarmangatta, agvertarlutiglo tamattomane, sorlo atâtagijangit tamattomanênarlaungmatta, pingimangattalonêt.

23 Taimak Nalekab nellojut taipkoa tunnilaungitane Josuab agganginut, tagvanekovait pêjarsârnagit. 


\section{KAPITEL III.}

\section{Athniel, Ehud, Samgar, pinniarningillo.}

\section{TAMæDSA nellojut Nalekab tagvanekojangit, oktorkovlugo Is-} raelitinik tapkonane, kaujimalaungitunik, Kanaanib unatadlarninginik.

2 Israeliblo kittorngangita abvakungit kaujilerkovlugit, illinnialerkovlugillo sorsungnermik, sivorngagut kaujilaungitut tamattominga.

3 Imagle: Filisterit attanningit tellimat, illunaitalo Kanaaniterit, Zidonemiullo, Heviterillo, nunakalauktut kakkame Libanoneme, kakkamit Baal Hermonemik attalingnut Hemathe tikkilugo.

4 Tapkoa amiakolaukput, Israelitit oktorlugit tapkonane, sakkijarkovlugo Nalekab perkojangit nalengmangagit, atâtagijanginut perkolauktangit Mosesekut.

5 Israelitit tagva manna taimak nunakalauramik Kanaaniterit, Hetiterillo, Amoriterillo, Feresiterillo, Heviterillo, Jebusiterillo akkorngane,

6 Tapkoa panninginik nullianikput, pannitiglo tunnilaukpait taipkoa erninginut, kivgartorlutiglo taipkoa Gudingoanginik;

7 Idluitullilutiglo Nalekab sângane, Nalegarla Guditik puigorlaukpât, kivgartorlutiglo Baalemik, perorsêvingniglo nappartolingnik.

8 Tagva Nalekab ningarninga illungertulerlaukpok Israelitit pivlugit, niorvgutigivaillo Kusanib Risataimib Mesopotamiab attanertangata agganginut; taimaglo Israelitit Kusan-Risataime kivgartorpât Jahrinik sittamajortunik.

9 Tagva Israelib kittorngangit niplialerput Nalekamut, Nalekablo piuliklertekartilaukpait, piuliklerniartomik tapkoninga, Athnielemik, Kenasib Kaleb nukkangata erninganik.

10 Nalekablo Annerninga illumigiva, idluarsaijolaukporlo Israelitinik, aularlunelo unatadlarnermut; Nalekablo Siriab attanertanga Kusan - Risathaime tunniva tapsoma agganginut, aggangit tapsomunga songelualilaungmatta.

11 Tagva nuna innilangalaukpok Jahrinik 40 ginik. Athnielelo Kenasib erninga tokkovok.

12 Israelible kittorgangit kingorngane idluitullilaurivut Nalekab sângane. Tagva Nalekab Eglon Moabiterit attaningat ajugautipa Israelitinut, tamanna pivlugo idluitullilaungmatta Nalekab sângane.

13 Tapsomalo illasearivait ingminut Amonib kittorngangit 
Amalekiterillo; aularlaukporlo sâlagilugillo Israelitit, iglugaseksuillo Palmitaggit aksarnigarivait.

14 Israelitillo Eglon Moabiterit attaninga kivgartorlaukpât Jahrinik 18 nik.

15 Tagva niplialerput Nalekamut, Nalekabło piuliklertekartipait, Ehudemik Gerab Jeminib erningata erninganik, tamna saumiolaukpok. Israeliblo kittorngangit tapsomuna pilliutiksanik tillikliudjilaungmatta Eglonemut, Moabiterit attaninganut,

16 Ehudib saviksoak igluktut kênalik sennava piksane, tallertut takkitigijok, tapsilugolo annorâme illuane makkitimigut tallerpingmigut,

17 Pilliutiksarlo âpa Eglonemut, Moabiterit attaninganut. Eglonele anguteôlaukpok kuinidlartok.

18 Pilliutiksarlo unertutigijarêrlauramiuk, innuit pilliutiksamik akjarsilauktut aularkovait;

19 Utterlunelo Gudingoanit Gilgalemelauktunit, okautsaukojilunelo: Attanek! angiarijaujariakartunik okausiksakarpunga illingnut. Tamnale nippangerkojivok; annivullo illunatik avatingane nangertut.

20 Ehudiblo ittervigiva; tamnale iksivavok iglomine aujaksiume, tapsomungaênak illingalanktome. Ehudelo okarpok: Okausekarpunga Gudemit pijunik illingnut. Tagva nikkovipok iksivautamit.

21 Ehudible aggane saumine issakpait saviksoarlo makkitiminit tallerpingminit tiguva, kappivâlo nângagut,

22 Ipubtauk saviktane mallingmago, kiksautillo ipo ullingmatsuk, (tapsoma saviksoak amulungimago nânganît) anaksak anningmat tapsomangat.

23 Ehudelo iglomit annivok, upkoarlo upkoarpa pallangairlugolo.

24 Annijarêrmat mana, kivgangit itterput tækkolutiglo iglub aujaksiub upkoanga pallangairsimangmat, okarlutiglo: Anâriatorpallaivok iglub aujaksiub aiparusinganut.

25 Taimagle akkunêrtigijomik uttakkilauramik kangusulerkârtinagit (upkoak anjaksiume upkoêrtaungimat kinamut) pêrut tiguvât pallangairudjarlugolo; ahâk tagva angajokangat tokkungavlune nellavok netterme.

26 Ehudele kemmâlaukpok uttakkitillugit, Gudingoallo sennerkopait, kemâlunelo Seirath tikkilugo.

27 Tagvungalo pigame, tiptulaut tiptulausijautigivâ Efraimib kakkangine. Israeliblo kittorngangita aularkattigivât Efraimib kakkanginit, tamnalo sivorlerivât.

28 Okautilugillo: Malliksinga, Nalekab Moabiterit omigijise tunningmagit aggapsingnut. Malliklugolo akpalliutivut, ikkârviglo Jordaneme, Moamut illingajok aksarnigarivât, ikkarkojilungilello kinamik. 
29 Ullimarpaillo taipsomane Moabiterit angutit 10,000 dit, illunatik angutiôjut sorsugungnärnerpângôjut, attauserlonêt kemmânane.

30 Taimak Moabiterit taipsomane ajugaujuêrtitauvut Israelib kittorngangita agganginut. Nunalo innilangalaukpok Jahrinik 80 ginik.

31 Kingornganelo Samgar, Anathib erninga pivok; tapsoma Filisterit 600 dit annaulerlugit tokkopait ajaupiamut, tuktuvângnut attortaumajomut, Israelitillo piulilaurivait.

\section{KAPITEL IV.}

\section{Deborab, Barakib, Jaeliblo maksoaninginik.}

ISRAELIBLE kittorngangit ama idluitullilaurivut Nalekab sângane, Ehud tokkolaungmat.

2 Nalekablo niorvgotigivait Jabinib Kanaaniterit attaningata Hazoremelauktub agganginut; sorsuktuksoakotingitale angajokangat Sisseraôlaukpok, nunakalaukporlo Harosetheme nellojunut illingajome.

3 Israeliblo kittorngangit niplialerput Nalekamut; aksalloalikutekalaungmat kikkiangnik 900 dinik, Israeliblo kittorngangit aksorornermut okumaiksartipait Jahrinik 20 ginik.

4 Nelliutomelo taipsomane idluarsaijolaukpok Israelitinik nellautaijok arnak Debora, Lapidotib nullianga.

5 Nunakalaukporlo Deborab Palmikotingita attâne, Ramab Betheliblo akkonigingne, Efraimib kakkangine. Israeliblo kittorngangit takpaungarlaukput tapsomunga idluarsaivingmut.

6 Tamna tilliklerpok Barakemut Abinoamib erninganut, Kedesemetomut Nafthalime, okautsaukolugolo: Nalekab Israelib Gudingata perkolaungilatit: Aularit ailutillo kakkamut Thaboremut, illasearikillo angutit 10,000 dit, Nafthalib Sebuloniblo kittornganginit?

7 Sissera Jabinib sorsuktuksaksoakotingita angajokangat aulartitsomagapko illingnut kôngmut Kisonemut, aksalloalikotingit unnuktokotingillo illaliudlugit, tunnijomavaralo aggangnut.

8 Barak okarpok tapsomunga: Aikattiginiarumga, aijomavunga; aikattigingikumgale, aijomangilanga.

9 Tamna okarpok: aikattigijomavagit, nertortaunerle, nangmineriniangilet aularnerne tamattomane; Nalekable Sissera tunniniarpa arnab agganginut. Taimak Debora parngnailerpok, aularlunelo Barak aiparilugo Kedesemut.

10 Tagva Barakib Sebulonekut, Nafthalikullo kaikovait Kedesemut, pissuklunelo aularpok angutit 10,000 dit illagællugit. Deborablo aularkattigiva. 
11 Heberele Kenitereôjok, Keniterinnit, Hobab, Mosesib sakkingata kittornganginit aularlaukpok, tangmarlunelo Zaanaimib Eichingine, Kedesib senniane.

12 Tagva Sissera okautsaulerpok, Barak Abinoamib erninga aularsimangmat kakkamut Thaboremut.

13 Tapsomalo aksalloalikotine illunaita katterkovait, aksalloaggit kikkiengôjut 900 dit, innugiartoksuillo illagijine illunaita, nellojut Harosetinganit, kôngmut Kisonemut.

14 Deborale Barakemut okarpok: Atte! tamanna uvloôvok Nalekab tunnijivinga Sisseramik aggangnut, Nalegak sivorleriniarangne. Taimak Barak kakkamit Thaboremit attersarpok, 10,000 dillo tapkoa mallikpât.

15 Nalekable Sissera kvaksartipa, aksalloalikotingit illunaita, sorsuktuksoakotingillo illunaita illaliudlugit, Barakib saviksoangata kênanganut, Sissera niungmat aksalloalikotiminit kemâlunelo pissuklune.

16 Barakible aksalloaggit, sorsuktuksoakattigêllo udlapait, nellojut Harosetingat tikkilugo. Sisserablo sorsuktuksoakotingit illunaita ochovut saviksub kênanganut amiakokaratik attautsemiglonêt.

17 Sisserale pissuklune kemâvok Jaelib Heberib, Kenitereôjub nulliangata tuppinganut; Attannek Jabin Hazoreme, Heberiblo Kenmitereôjub iglomiokotingit ullapirsautekarkattigêlaungmatta.

18 Jaele annivok Sissera pachrearlugo okautivâlo: Itterit, Nalegâk, itterviginga, sivoranaglo. Ittervigivâlo tuppinganut; ullikpâlo ulliksoarmut.

19 Tapsomale okautiva: Nagliktara immilaunga immermik immerudlarama. Tagva immukautit illangat mattoêrpa immilâlo, ulliklugolo.

20 Tapsomalo okautiva: Tuppiub pânganut nangerit, tikkitokarpallo appertsortomik, mânêtokangimangat kimik, tagva okarit: Ahaila.

21 Tagva Jaelib Heberib nulliangata kikkiek tuppermit tiguva ujaratsiarlo aggangminut, terrêdlunelo ittervigiva, kikkierlo katsukpa niakungata tigliktângagut nunamut pilune. Tamnale sinnisilerpok issumairotilunelo tokkolerpok.

22 Barakible Sissera udlangmago Jaelieb pachreartorpâ annilune, okautilugolo: Tamaungarit, angut kennertat tækkojautitsomavara illingnut. Ittervigingmagolo Sissera tokkungavlune nellavok netterme, kikkierlo niakungata tiglertanganelaukpok.

23 Taimak Nalekab nelliutome taipsomane Jabin Kanaaniterit attaningat ajugaujuêrtipa Israelib kittorngangita sângine.

24 Israeliblo kittorngangita aggangit piganerlaukput song- 
olutiglo Jabinemut Kanaaniterit attaninganut nungutsikârtinagit tapsominga.

\section{KAPITEL V.}

\section{Deborab Barakiblo imgerutingit salakarnermik opigotiksat.}

T

TAGVA Debora Baraklo Abinoamib erninga imgerpuk nelliutome taipsomane okarlutiglo:

2 Nalegak nertorsiuk, Israelitit kippalojungnaimingmatta, innugiartoksuillo kunnutuilaungmatta tamattomunga.

3 Nalagitse attanniojose, kamatsiarluselo angajokaksoangôjose! Uvanga, illa uvanga Nalegak imgervigijomavara, Nalegak Israelib Gudingat imgervigijomavara nertordlerutiksanik.

4 Nalegak Seiremit annilauktillutit, pitillutillo Edomib naternanganit, tagva nuna sajukpiludlalaukpok, killak kussidlalaukpok, nuvujallo kussidlalaukput immermik.

5 Kakkat auksilaukput Nalekab sângane, illa Sinai Nalekab Israelib Gudingata sângane.

6 Samgarib Anatib erningata nelliuningane, Jaeliblo nelliuningane, apkosinekarungnailaukpok; ingergartuksaulauktullo, agvertarlaukput apkutitigut niungapattekeatigut.

7 Iglugasengne Israeleme innuit ikkinersaupallialaukput. Uvanga Debora pilerkârtinanga, pilerkârtinanga anânaujunga Israeleme.

8 Gudib nutâk amerimava, upkoaksuit sorsugvigilaukpait. Sakkijartokalaungilak Israeleme sappulutamiglonêt kallugiarmiglonêt, 40,000 dit akkorngane.

9 Omattima Israelib aulautsijingit ungagivait, kunnutuitut innugiartoksuit akkorngane. Nalegak nertorsiuk,

10 Ablangajose siutekoktojune kakkortane, idluarsaivingme iksivajose, imgeritselo apkosinerme agvertartose.

11 Pittiksertut nipliarvigilauktangine immertarvit akkorngane, tagvane Nalekab idluarninga okautigijaulaukpok, iglugaseillo Israeleme innungita idluarningat; tagva Nalekab innukotingit attersarput upkuaksoarmut.

12 Atte, atte Debora, atte, atte, imgerutiksamik imgerit: Parngnailerit Barak, tigulugillo tigujomajitit, igvit erniôjotit Abinoamut!

13 Tagva kemaktaumajut attanniotilaukput innungnut sangijunut; Nalegak uvapkut attanniotilaukpok songudlartunut.

14 Efrainememit amângat pivok Amalek akkerartorlugo, kingunakut Benjamin innukotingne. Machiremit attannernik pijokarpok, Sebulonemillo pijokarpok aulatsijunik, aglaktub ajaupiangagut.

15 Angajokaksuillo Isascharemit Debora illagilaukpât; Isa- 
scharekullo Barak illagilaukpât naksarme, tillijauvlutik innukotingit pissuktut illagivlugit. Rubenekut piojorilaukput, avitilutiglo uvaptingnit.

16 Sôg nergjutit avalulingita akkornganetuarkise, kattimgajut nipliadlarningit tussarlugit, piôjoriluselo, avitiluselo uvaptingnit?

17 Gileadekut Jordanib akkianetuarlaukput; sunalo pivlugo Danekut umiaksoarnekât? Asserekut immarbiksub siksangane iksivalaukput, iglugasenginetuarlaukput asserorsimajune.

18 Sebulonekulle Nafthalikullo innolaukput tarnemingnik tunijomalauktut tokkomut naternaub timâne.

19 Attanit kailaukput sorsuglutiglo, tagva Kanaaniterit attaningit sorsulaukput Thaanacheme Meggidob kôngane; sullilaungilalle.

20 Killangmit sorsugvigijaulaukput; uvloritset ingergarnermingne sorsulaukput Sisseramut.

21 Kôb Kisonib aksakatilaukpait, kôb Kaduminib, kôb Kisonib. Tarnik, songojut tuttidlarkit.

22 Tagvane Horsit ittiggangit kikkergalijarlaukput, ablangajingita songojut mungunganingit pivlugit.

23 Iglugaseksuit Meros ominarsijaukosigik, Nalekab Engelinga okalaukpok; inungit ominarsijaukosigik, tikkilaungimatta Nalegak ikkajorlugo, Nalegak ikkajorlugo songojunut.

24 Saimartaule arnat akkorngane Jaele, Heberib Kenitereôjub nullianga, saimartaule tupperme arnat akkorngane.

25 Imungmik tunijilaukpok imermik kaitsikojitillugo, imublo orksuanik kaitsivok pogutame ânanâme.

26 Aggangminut kikkiek tigulaukpa, tallerpingminullo sabviortib ujaratsianga, katjuklunelo Sisseramik niakungagut, serkomitsilunelo, puttojilunelo niakungata tiglertanganik.

27 Ittiggangine nimalaukpok, ochovok nellalunelo, nimavok ocholunelo ittigganginut; nimavingmine nellavok asserorsimavlune.

28 Sisserab anânanga ijainilaukpok igalakut kaggudlarlunelo killapatakut: Sog aksalloalikotinga mulludlarka tikkingimat? Kanok aksalloakotingita aksalloajungit kingoraidlarkât?

29 Sillatunersat nulliangita akkorngane kiovut, tapsoma okautsine ogguarutiksane attupsatsainarmagit:

30 Nachvartuksaungilelle, avititsilutiglo aksarnigaujunik? angutinut illunainut attunit uigarsungmik attautsemik maggungniglonêt perkutiksanginut, Sisseramullo annorângnik minguarutelingnik aglaksimajuniglo perkutiksanganut; annorângnik mingoarutelingnik sunatuinarnik aglaksimajuniglo kongesinganut, piksanganut?

31 Taimak nungutuksauvut, Nalegak omigijitit illunaita! 
Tapsomingale naglingniktut îtuksauvut sekkerngub nuiningatut songonermine. Nunalo inilangalaukpok Jahrenik 40 ginik.

\section{KAPITEL VI.}

\section{Gideon idluarsaïungortitauvok.}

ISRAELIBLO kittorngangit idluitullilaungmatta Nalekab sấngane, Nalekab tunnilaukpait Midianiterit agganginut Jahrit 7 it nâlugit.

2 Midianiterillo aggangit songoluarlaungmatta Israelitinut, Israelib kittorngangit senavut ingmingnut ittersanik kakkane, kairosungniglo, ineniglo avaluksoalingnik.

3 Israelitillo sunamik karngasuilaurangatta, tagva Midianiterit Amalekiterillo, tapkoalo unanemiut takpaungarput tapkonunga;

4 Tangmarlutiglo akkerartorlugit, perortullo nuname illunaita asserorpait, Gaza tikkilugo, amiakokartitsilaungilello nerkiksamik sunamiglonêt Israeleme, saukamiglonêt, tuktuvangmiglonêt, siutekoktojomiglonêt.

5 Takpaungarlauramik nergutekotitik tuppitiglo neksarlugit piglertaksoatitut unuktovaksoatitut, tapkoalonêt Kamelekotingillonêt kittiksaulaungimatta; ittarlaukpullo nunamut, asseroromavlugo.

6 Taimak Israelitit angijomik aklutitaulaukput Midianiterinut. Tagva Israelib kittorngangit niplialerput Nalekamut.

7 Nalekamulle nipliarmatta Midianiterit pivlugit,

8 Nalekab nellautaijok tilliva tapkonunga, tapsoma okautivait: Imâk Nalegak Israelib Gudingat okarpok: Egiptenemit tessiorlaukpapse, aulartiluselo iglomit kippalovingmit ;

9 Piulilaukpapselo Egiptenemiut agganginit illunatiglo agganginit okumaiksartitsilauktut illipsingnik, pingudlalaukpakkalo sivunapsigut, nunangallo tunilaukpara illipsingnut.

10 Okautilaukpapselo: Nalegauvunga Gudise, Amorterit nunangine nunakarvigijapse Gudingit sivoraginiarasigik. Illipselo nippiga nalelaungilase.

11 Nalekablo Engelingita illangat kaivok ingilunelo Eichit (nappartut) illangata Oframelauktut attânut, perkutaulauktub Joasemut, Esriterit atâtanganut, erningalo Gideon Weizinik katjulaukpok iptêjaivingme, kemâkovlugo Midianiterinik.

12 Tagva Nalekab Engelingata sakkervigiva, okautilugolo: Nalekab aiparilitit, songojotit sorsungnerme!

13 Gideonible okautiva: Nalegâk, Nalekab illagikpattigut, sôgle tagva tamakkoninga tamainik pilaukita? Nanekâllo tattamnartulliningit tamaita atâtagijapta unipkautigilauktangit 
uvaptingnut, okarlauklutiglo: Nalekab Egiptenemit tessiorlaukpâtigut? Mannale Nalekab kemmalaukpâtigut, tunilutalo Midianiterit agganginut.

14 Nalekable savigiva okarlunelo: Ailerit pitsartunerne tamattomane, igvit Israelitit piulijaksarivattit Midianiterit agganginit. Ahak tillilaukpagit.

15 Tamnale okarpok tapsomunga. Nalegâk, sunamut Israelitit piulijaksarivakka? Ahak illakka mingnerôvut Manassekunit, uvangalo mingnerôvunga Atatama iglomiokotinginit.

16 Nalekable okautiva: Aiparijomavagit Midianiterit ullimaraksarigangne, angutetut attautsetut sorlo.

17 Tapsomale okautiva: Nagliktara saimaneksimaguma sângne, tagva nellunaikutekartilaunga igviôgavit okarkattigijara.

18 Nûniarnak tikkitsearilunga, neksarlungalo tunnergutiksamik nerkiksamik, tunniniartamnik sângne. Tamna okarpok: Ovanetuaromavunga tikkitsêaringmilutit.

19 Gideonelo kaivok, tokkotsilunelo tuktungajomik angusalloârsungmik, tigusilunelo senaukamik sernakangitomik Efa trettælugo, illijilunelo nerkemik amargaujamut, kajorlo pitipa ukkusingmut, atsilunelo tamakkoninga sillamut Eichib attânut, najutilunelo.

20 Gudible Engelingata okautiva: Nerke tiguleruk, sernakangitorlo, illilugillo kairtomut manêtomut, kajorlo koviuk. Taimailiorlaukporlo.

21 Tagva Nalekab Engelingata ajaupiak tigumiartane aggangminut, issautiva aktorlugiglo nuvuanut nerke sennaugarlo sernakangitok. Ikkomalo annivok kairtomit, nerrilunelo nerkemik sennaukamiglo sernakangitomik. Nalekablo Engelinga nippakpok ijigingnit.

22 Gideon mana tukkisigame Nalegab Engeligingmago okarpok: Nalegak, Nalegak, taimak illa Nalekab Engelingata kênanga tækkolaukpigo?

23 Nalekable okautiva: Ullapirsautekarit! sivoraniarnak, tokkoniangilatit.

24 Tagva Gideon tagvane Altaremik sennavok Nalekamut; tailugolo Nalekamik ullapirsautelingmik. Tamna tagvanêtsainarpok sulle uvlok tamana tikkilugo Oframe, atâtaulauktome Eseriterinut.

25 Unnuarmelo taipsomane Nalekab okautiva: Atâtavit tuktuvakotinginit angusallormik tigusilerit, angusallormiglo assianik Jahrelingmik 7 inik; serkomitsilutillo Baalib Altaringanik, perkutaumajomik atâtarnut, nakkatsilutillo nappartunik Gudingoartalingnik sennianêtunik;

26 Sennalutillo Altaremik kairtub kângane Nalekamut Gudingnut, akiksorlugolo, tigusilutillo angusallub aipanganik, tunnijilutillo tunnergutiksamik ôtaksamik kejungmut nappartunit Gudingoatalingnit nakkarlauktarnit. 
27 Tagva Gideon tigusivok kivgaminit angutinik tellimaujortunik piniarlunelo sorlo Nalekab okautilaungmago. Sivoralaukporle tamattominga piniarlune uvlôningane, atâtame iglomiokotingit pivlugit; piniarlunelo unuarme.

28 Tagva mana innuit iglugaseksoarne uvlarme makkimatta, ahakBaalib Altaringa serkomisimalaukpok, nappartullo Gudingoataggit senianelauktut nakkarsimavlutik; angusallublo aipanga tunergutiksauvlune Altarib senajaumajub kângane.

29 Okautiutivullo ingminut: Kina tamattominga pinialauka? Kennerlaungmatta apperkutekalaungmattalo okartokarpok: Gideon Joasib erninga tamattominga pinialaukpok.

30 Tagva iglugaseksuit innungit Joasemut okarput: Ernît kaitsuk; tokkotaksauvok, Baalib Altaringa serkomilaungmago, nappartullo Gudingoataggit tapsomanelauktut nakkalaungmagit.

31 Joasele illunainut nangerkattigijaminut okarpok: Baal aivajutigijomavissiuk? Ikkajoromavissiuk? Aivajok tamna pivlugo, tamna uvlarme tamattomane tokkojuksauvok. Gudeôgune pivlune akkiniarle Altaringa serkomisimangmat.

32 Uvlormit tamattomangat taijaulaukpok Jerub Baalemik, okartokarporlo: Baal nangminek pivlune akkiniarle Altaringa serkomisimangmat.

33 Tagva manna Midianiterit illunatik Amalekiterillo, tapkoalo unanemiut attautsemut kattimangmatta nunakorlutiglo, tangmarlaungmatta Jesreelib naksangane;

34 . Nalekab Anerningata Gideon kajungersarpa; tiptulautiniglo tiptulausijautaukojilaukpok, kaikolugillo Abieserekut mallikovlugit tapsominga.

35 Tilliklerporlo akpartitsijunik Manassekunut illunainut, kaikolugillo mallikovlugittauk tapsominga. Tilliklerpoktauk akpartitsijunik Asserekunut Sebulonekunullo Nafthalikunullo, tapkoa takpaungarput pachrearlugo.

36 Gideonelo Gudemut okarpok: Israelitit aggapkut piulijomagungne sorlo okalauravit;

37 Tagva ammek merkolik katjugvingmut (kakkojaksanik) illijomavara. Missuksoak ammermetuinarniarpat, nunalo illunane pannerpat, tagva mallugosugomavunga, Israelitit aggapkut piulijomagangne sorlo okalauravit.

38 Taimaglo pilaukpok. Kaulermallo uvlakut makkijajolaurame amek silvotorpa, illulerkullo tættæetipa immermik tamattominga.

39 Gideonelo okarpok Gudemut: Ningarnivit illungertorviginiaraninga, ama okarmigama. Ama oktoromavunga amek attorlugo. Panertuinarle amerme, missuksoakartillugo nuname illunane.

40 Gudelo taimailiorlaukpok unuarme taipsomane, paner- 
tuinarlaungmat amerme, missuksoakartillugolo nuname illunane.

\section{KAPITEL VII.}

\section{Gideonib Midianiterit salagivait.}

TAGVA Jerub-Baal, tagva Gideon, uvlararsukut aularjajovok innugaseksuillo illagijangit illunatik, tangmarlutiglo imertarvingme Harodemik attelingme, taimak Midianiterit kattimgajokattigêningit tachanelaungmatta udsertorviub kakkarulangita ungatane, naksarme.

2 Nalekable Gideon okautiva: Innuit illagijattit unuluadlarput, Midianiderit tunnijaksarigapkit agganginut; Israelitit ingminik nertortinajarput akkerartorlunga, okarlutiglo: Aggapta piulilaukpâtigut:

3 Tagva mana kaggudlarkojilerit inugaseksuit siutingita sângine, okarkojilutillo: Illerasuktokarpat, mungungolunelo pijok, utterle nûsarlunelo Gileadib kakkanginit. Tagva innuit 22000 dit utterput, amiakokartuinarlaungmat, 10000 dinik.

4 Nalegarlo Gideonemut okarpok: Inuit sulle unuluadlarput. Tessiorkit angmut imermut, tagvane oktoromavakka sângne, nelliatalo okautiginiartama illingnut aiparijaksaringmattit, tapsoma aiparijaksarivâtit, nelliatale okautiginiartama aiparijaksaringimattit, tamna illaujuksaungilak.

5 Tessiorlaukpaillo inugaseksuit angmut imermut. Nalegarlo Gideonemut okarpok: Nelliat okaminut alluktok imermik, sorlo kingmek allungmat, tamna ingmigolitiuk; taimaktauk serkorsorlune immertok.

6 Tagva tapkoa allulauktut aggamingnit kanermut, 300 deôlaukput; inuit assingit illunatik serkortorlutik imerlaukput.

7 Nalegarlo Gideonemut okarpok: Angutitigut 300 ditigut allulauktutigut piulijomavapse, tunijijomalungalo Midianiterinik aggangnut; inugaseksuille assingit aikokit iniksanginut.

8 Inuillo tapkoa nerkiksamik takkoaksamik neksarput, tiptulautekotimingniglo. Israelitille assingit aikovait attunit iglogijanginut; tamnale pitsartutivok angutinut 300 dinut. Midianiterillo kattimgajokattigêningit tangmarsimalaukput sakkangane atsiktome naksarme.

9 Nalekablo unuarme taipsomane okautiva: Makkitit attersarlutillo tangmarvingmut: tunimagapkit aggangnut.

10 Attersariarle erksigigungne, tagva kivgat Pura attersarkattigiuk tangmarvingmut,

11 Tussarkovlutit okauserijanginik; kingorngane pitsartunelijarlutit attersartuksauvotit tangmarvingmut. Tagva Gideon kivgane Pura aiparilugo attersarpok pigârtut tangmarvingmelauktut ininganut. 
12 Midianiterillo Amalekiterillo unanêmiullo illunatik nellalaukput naksarme piglertatitut unuktovaksoatitut; Kamelekotingillo kittiksaulaungilet unumut, siorkatitut imarbiub siksanganetutitut.

13 Gideonelo tikkitillugo, ahâk tagva tapkoa illangata illagijane sinektomanermik unipkautiva, okarlunelo : Ahâk sinektomalaukpunga, tautukorlaukpunga niakojak Gersteovinek ôsimajok aksakâvok Midianiterit kattimgajokotinginut; tikkimallo tuppernut annaunikpok tapkoning a,ochotilugillo pussiktilugillo, kângit alliotilugit, tuppit imiksimangmatta.

14. Tagva aipanga akkiorpok okarlunelo: Tamana sunaungilak assianik Gideonib Joasib Israeliteôjub erningata saviksoanganit. Gudib Midianiterit kattimgajoksoangit illagilugit tunimavait tapsoma agganginut.

15 Gideonib tamna tussaramiuk sinnektomanermik taimaitomik unipkartok, tukkekartitauningalo, pamangavlune tuksiarpok, uttermilunelo Israelitit kattimgajunginut, okarlunelo: Parngnaileritse Nalekab Midianiterit kattimgajungit, tunningmagit aggapsingnut.

16 Angutillo 300 dit tapkoa avikpait pingasuilivlugit attunîllo tunnitsivigivait tiptulautemik agganginut, macharniglo illulekangitunik, nennergoaniglo takkamane.

17 Okautivaillo: Tækkonârsinga taimailiorluselotauk; ahâglo kattimgajut ininganut piguma, piniarniptut taimak piniarivose.

18 Tiptulaumik tiptulausijautekaruma, illunatiglo illagijakka, tagva illipsetauk tiptulausijartuksauvose, kattimgajoksuit illunatik avatâtigut okarluselo: Mâne Nalegak Gideonelo!

19 Taimak Gideon, angutillo 100 dit illagilugit tikkipok kettimgajoksuit inninganut, udsertortut sivorlinginut, udsertorkojaujunut tuppaktilugillo tiptulausijarlutiglo, machaillo aggamingnetut serkomipait.

20 Taimak kattimgajut pingasuit illunatik tiptulausijarput machaillo serkomilugit pivut. Nennergoalle tigumiarpait aggangmingnut saumingminut, tiptulautillo aggangmingnut tallerpingmingnut, tiptulausijarlutik kaggudlarlutiglo: Mâne Nalekab saviksoanga Gideoniblo!

21 Attunîllo inimingne nangerput kattimgajoksuit avatâne. Tagva katimgajoksuit illunamassiatik akpangertitaulaukput, kaggudlarlutiglo kemâvut.

22 Angutillo 300 dit tiptulausijartillugit, Nalegak pititsivok kattimgajune illunaine attunît illangata saviksoanga torartitaungmat illaminut. Kattimgajoksuillo kemâvut Beth-Sitta-Zeratamut tikkilutik, Abel-Meholablo Tabathemetub kiglilervia tikkilugo.

23 Angutillo Israelitit Nafthalikunit, Asserekunillo, Ma- 
nassekunillo illunainit, kaggudlarlutik Midianiterit udlapait.

24 Gideonelo akpartitsijunik tilliklerpok Efraimib kakkanginut illunainut, okarkojilunelo: Attersaritse Midianiterit pachrearlugit, imiksuillo Beth Bara Jordanelo tikkilugo tikkerkârsigik tapkonangat. Tagva illunatik Efraimekunit pijut kaggudlarput akpangerlutiglo, tikkerkârlutiglo imiksoarnut BethBaramut Jordanemullo.

25 Angusilutiglo Midianiterit angajokaksoanginit maggungnik, Orebemik Sebemiglo, Orebelo tokkopât Orebib kairtungane, Sebelo, Sebib iptêjaivingane (Weinemik); Midianiterillo kemâtipait. Orebiblo Sebiblo niakogik âpakit Gideonemut Jordanib akkianut.

\section{KAPITEL. VIII.}

\section{Midianiterit amiakojut sulle salagïjavut, Sebalo Zalmunalo}

\section{pïjauvuk.}

ANGUTILLO Efraimemit okautivât: Sôg taimailiorvigilaukpittigut, kaikolaunginaptigut aularlutit unatadlarnermut Midianiterinik? Aivadlarpâllo.

2 Tapsomale okautivait: Sunamik pinialaukik mana piniarnipsetut nelligêktomik? Efraimib nappartungita Weineliksat akkerungat attausek namanersaungilâk Abieserib kattersininginit Weineliksanik illunainit?

3 Gudib Midianiterit angajokaksoagik Orebe Sebelo tunilaukpak aggapsingnut. Kannok uvanga taimailiorungnarajalaukik? Taimaitomik okautingmagit ningausingata allarpa.

4 Gideonelo Jordanemut pigame ikkârpok, angutit 300 dit illagijangit illagilugit, merngortorlaukpullo, udlanilutiglo sulle.

5 Innungnullo Suchotemetunut okarpok: Nagliktakka innuit nalektikka tunnitsivigissigik kakkojat illanginik; merngortormatta, udlanikovlunga Midianiterit attannigingnik Sebamik Zalmunamiglo.

6 Angajokajallo Suchoteme okautivât: Sebab Zalmunablo aggangit aggangnejarêrkât, kattimgajokotitit kakkojanik tunnitsivigijaksarigaptigik?

7 Gideon okarpok: Attauk, Nalekab Seba Zalmunalo tunniniarpagik aggamnut, tagva uvinise orpinut kakkilautilingnut sunakajuitomit pijunut kessertutinullo nerkersudlartiniarpakka.

8 Tagvangallo aularpok Pnuelemut, taimaglotauk okarvigivait. Innuillo Pnueleme akkiorpât Suchotemetutitut.

9 Innungnullo Pnueleme okarpok: Ullapirsautekarlunga utterniaruma, nuvugoseksoak una serkomitsomavara. 
10 Sebale Zalmunalo Karkoremelaukpuk, kattimgajokotingillo illagilugit, 15000 diokassait, amiakolauktut kattimgajoksoarnit illunainit unanemiunit. 120000 dit saviksoarmik amusijungnarlauktut ochomalaukput.

11 Gideonelo majorarpok apkosinekut tuppernik iglokarvikut ununga, Nobamut Jagbehamullo, ullimarpaillo kattimgajut; terlilaungmatta kattimgajut.

12 Sebalo Zalmunalo kemâvuk; Gideonible udlapait, angulugiglo Midianiterit attannigik Seba Zalmunalo, kattimgajullo illunaita siamartipait.

13 Gideonelo, Joasib erninga, uttermat unatadlarnermit, sekkinek nuikârtinago,

14 Nukapiak anguva Suchotemiunit, apperilugolo; tapsomalo aglagvigiva angajokajanik Suchoteme, angajokaunerojuniglo, angutinik 77 ginik.

15 Tikkilaukporlo innungnut Suchotemetunut okarlunelo: Ahâk mânêpuk Seba Zalmunalo mittautekarutigilauktâkse uvamnik, okarluselo: Sebablo Zalmunablo aggangit, aggangnêjarêrkat, innutitatit merngortortut kakkojanik tunnitsivigijaksarigaptigik?

16 Tiguvaillo iglugaseksuit angajokangit, orpillo kalkkilantiggit, kessertutillo, ikpigijautipaillo Suchotemiunut.

17 Pnueliblo nuvugosektanga serkomipa, iglugaseksoarmiullo tokkopait.

18 Sebamullo, Zalmunamullo okarpok: Angutit Taboreme tokkolauktasse kannoêtomik tautukalaukât? Tapkoa okarpuk: Illiktut ilaukput, illunatiglo attunit ânanaulaukput, attanniub kittorngangititut.

19 Tamnale okarpok: Kattangutaulaukput uvamnut, anânama ernerilaukpait. Sorlo miksekârtomik Nalegak inôngmat, tokkonajangilaptik, taipkoa innokolaurupsigik.

20 Ernerminullo erniangokârtomut Jetheremut okarpok: Nikovilerit tokkolugiglo. Nukapiable saviksoane amulaungila, sivoralaurame, nukapiangolaurame sulle.

21 Sebale Zalmunalo okarpuk: Igvit nikkovitit opaklunug10; angut illinganermitut pitsartunekarametauk. Taimak Gideon nikkovipok, tokkopaglo Seba Zalmunalo; tigusilunelo mattarêkutinik Kamelingita kongesinginêlauktunik,

22 Tagva Israelitit illangit okarput Gideonemut: Nalegaulerit uvaptingnut, igvit, ernillo ernerpillo erninga, piulilauraptigut Midianiterit agganginit.

23 Gideonele okarpok tapkonunga: Nalegaujomalungitunga illipsingnut, erneralo nalegaujuksaunginivok illipsingnut, Nalegarle nalegaujuksauvok illipsingnut.

24 Gideonele okarpok tapkonunga: Attausek tussugivara illipsingnit; illunase attunit kaurutit aksarnigarijase tunnissigik 
uvamnut. Ismaeliteôlaungmatta kaurutekalaukput Goldenik.

25 Tapkoa okarput: Tapkoninga tunnitsivigijomavaptigit. Annorâglo issivipât, attunillo kaurutitik aksarnigarilauktatik illivait kânganut.

26 Kaurutillo Goldit, kaikojangita okumainingit, Sekeliolaukput Goldit 1700 dit, kittikassiutinagit mattarêkutit, kellaumgujallo, annorâllo aupalluktat, Midianiterit attaningita attortangit, Kamelillo kongesermiutingit kittikassiutinagit.

27 Gideonelo ikkiamik sennavok tapkonangat, pitipalo iglugaseksoarminut Oframe. Israelitillo illuêngarlutik ajortullijutigivât tagvane, sinaungajutaulerlunelo Gideonemut iglomiukattinginullo.

28 Taimak Midianiterit mannigotititaulaukput Israelib kittornganginut, niakotiglo kagvarungnailaukpait. Nunalo innilangalaukpok Jahrinik 40 ginik Gideon innôtillugo.

29 Jerub-Baalo Joasib erninga, aivok iglokarlunelo iglomine.

30 Gideonelo ernekalaukpok 70 ginik, makkitiminit pilauktunik; unnuktunik nuliakalaurame.

31 Panervgakpângalo pigilauktanga Sicheme ernitarilaurivok tapsomunga, tamna Abi Melechemik atserarpa.

32 Gideonelo Joasib erninga kiglimorlune tokkolerpok, illijartortaulunelo atâtame Joasib illuvingane Oframe, atâtaulauktub Eseriterinut.

33 Gideonele tokkojarêrmat, Israelib kittorngangit sangulerput, ajortullilutiglo Baalim malliklugit, Gudiotipâllo ingminut Baal Berite.

34 Israeliblo kittorngangita Nalegak Guditik issumagilaungilât, piulijitik omigijimik illumajimik illunatik agganginit.

35. Napkiningnermiglo pinniarlaungilet Jerub Baalib, tagva Gideonib iglomiokattinginut, sorlo tamna ajungitunik tamainik pinniarlaungmat Israelitinut.

KAPITEL IX.

Abimeleche nangminênarasuarlune attanningortipok Israelitinut, naggatekarporle kappianariomik.

ABIMELECHELE Jerub-Baalib erninga aivok Sichemut, anâname anninginut, tapkoalo anânamelo atâtangata iglomiokotingita abvakungit illunaita okarkattigivait, okarlunelo:

2 Nagliktakka, angutit Sichemiut illunatik siutingita sângine okaritse: Suna nekkornersauva illipsingnut, angutit 70 git, illunatik kittorngaujut Jerub-Baalemut, nalegaupatta illipsing- 
nut, ubvalo angut attausek nalegaukpat illipsingnut? Erkaikassiudjileritsetauk saunekattigilungalo uvinekattigigapsinga.

3 Tagva anânangata anningita okautsit tamakkoa tamaita tapsomangat okauserivait, angutit Sichemetut siutingita sângine; omattingillo mannilerput Abimelechemut, issumagamik: Kattangutigivavut.

4 Tunnitsivigivâllo Silberlinginik 70 ginik, Baal-Beritib iglunganit, Abimelechiblo tamakkonunga angutit uivêtut kuksalaitullo pissiarivait malliktut tapsominga.

5 Tikkilunelo atâtame iglunganut Oframut, tokkotsilunelo kattangutiminik Jerub-Baalib kittornganginik, angutinik 70 ginik ujarkame (attautseme). Amiakovorle Jerub-Baalib erningita nukardlerpângat, ijersimalaungmat.

6 Sichemiullo angutingit illunatik kattimalerput, Milloblo iglomiokotingit illunatik, ailutiglo attanningortipâllo Abimeleche Eicheme pôktojome Sicheme nappajome.

7 Tamanna okautigijaulaungmat Jotamemut, aivok nangeriartorlunelo kakkab Grisimib kôtsiktoninganut, nippinelo kagvarpa, kaigarsuklunelo okarpok tapkonunga: Tussarsinga angutiojose Sicheme, Gude tussarkovlugotauk illipsingnik:

8 Nappartut ailaukput attannekartitsijomavlutik mingoarlugo ingmingnut, okarlutiglo nappartomut orksulingmut: Attaniolerit uvaptingnut.

9 Nappartuble orksuliub kiolune okautivait: Orksukarnera Gudinut innungnullo nertortaujok uvamne, sapkotaksarivigo, ailungalo, kollangiarkovlunga nappartut kângine?

10 Tagva nappartut nappartomut Feigelingmut okarput: Igvit kailerit attaniolutillo uvaptingnut.

11 Nappartuble Feigeliub okautivait: Mamarnera paungakkalo ânanât sapkotaksarivakka, ailungalo kollangiarkovlunga nappartut kângine?

12 Tagva nappartut nappartomut Weineliksamut okarput: Igvit kailutillo attanniolerit uvaptingnut.

13 Nappartable Weineliksab okautivait: Iptera sapkotaksarivigo, Gudinik innungniglo kuviasuktitsijok, ailungalo kollangiarkovlunga nappartut kângine?

14 Tagva nappartut illunatik orpingmut kakkilautilingmut okarput: Igvit kailutillo attanniotit uvaptingnut.

15 Orpiglo kakkilautelik okarpok nappartunut: Taimaimarikpat attanermut mingoarapsinga illipsingnut, tagva kaileritse unertutigiluselo tachamnut; taimaitsomangikupsele tagva ikkoma annilerle orpimit kakkilautelingmit, nungutsilunelo Cederinik Libanonemêtunik.

16 Idluartomik nellagortomiglo pinnialaukise manna, Abimeleche attanningortilaurapsiuk? Namaktomiglo pinnialaukise 
Jerub-Baaleme, iglomiokotinginelo; pinniarvigilaukpissiuglo sorlo pivluse pijaksaulaungmat,

17 Atâtaga pivluse sorsulaungmat, tarninelo egilaungmago, piuliluse Midianiterit agganginit?

18 Illipselo manna kippalidlarpose atâtama iglunganut, innuarluselo kittornganginik, angutinik 70 ginik ujarkame attautseme; attaningortitsiluselo Abimelechemik, kivgangata erninganik, angutinut Sichemetunut, kattangutigigapsiuk.

19 Manna idluartomik nellagortomiglo pinnialaurupse JerubBaaleme iglunganelo uvlorme tamattomane, tagva Abimeleche kuviasutigisiuk, tapsomalo kuviasutigilise.

20 Taimaingipalle ikkoma annilerle Abimelechemit, nungutsilunelo angutinik Sichemiunik, Milloblo iglomiokotinginik, ikkomalo annilerletauk angutinit Sichemiunit, Milloblo iglomiokotinginit nungutsilunelo Abimelechemik.

21 Jothamelo kemâvok nûlunelo, ailunelo Beremut, nunakalunelo tagvane, erksinermut angajuminik Abimelechemik.

22 Abimelechelo manna Jahrinik pingasunik attanniotijarêrlaungmat Israelitinut,

23 Gude tilliklerpok pijomanermik idluitomik Abimelechiblo angutillo Sichemiut akkornganut. Angutille Sichemiut Abimeleche nalegungnailerpât;

24 Issumagilugolo idluitullinek pilauktok Jerub-Baalib erninginut 70 ginut, tapkoalo aungat illivât Abimelechemut kattangatinganut, tokkotsilauktomut tapkoninga, angutinullo Sichemiunut agganginik pitsartutitsilauktunut tamattomunga, kattangutiminik tokkotsikovlugo.

25 Angutillo Sicheme terliarsijunik tilliklerlaukput kakkat kânginut, arktaivigivaillo illunaita apkosinerme ingergarasuarlauktut tapkonunga. Abimelechelo okautsaulaukpok tamattominga.

26 Gaalele Ebedib erninga kattangutingillo tikkiput, itterlutiglo Sichemut. Angutillo Sichemiut sungertutigivât.

27 Annilaukpullo perôrsêvingmat, pejailutiglo paungakotimingnik Weineliksanik, iptêjailutiglo, okigêserlutiglo, ailarmiglo Gudimik iglunganut, nerilutiglo immerlarmiglo, okarnerlutigivâllo Abimeleche,

28 Gaalelo Ebedib erninga okarpok: Kinauva Abimeleche? Sunauvâlo Sicheme, kivgartoraksarigaptigo? Jerub-Baalib erneringikaûk? Sebulelo kivgane pitilaukpa avunga angajokangortilugo Hemorib Sichemib atâtangata innukotinginut. Suna pivlugo kivgartoraksarivittigo?

29 Gudelo pijomale innugiartoksuit aggamnênajarninginik, singitsikovlunga Abimelechemik. Abimelechelo okautsaurok: Sorsuktuksakotitit unnurnersautikit aularlutillo.

30 Sebulele iglugaseksuit angajokangat tussarame Gaalib 
Ebedib erningata okausinginik, illungertudlalerpok ningarnermut.

31 Tilliklerlunelo angigutsivlune akpartuksanik Ábimelechemut, okautsaukovâlo: Ahak, Gaal Ebedib erninga kattangutingillo tikkisimavut Sichemut, akkerartortipaillo iglugaseksoarmiut illingnut.

32 Tagva parngnailerit manna unnuarme, igvit innukotitillo illingnêtut, terliarsivingmiglo âkiksoilerit perorsêvingme,

33 Uvlarmelo sekkinek nuilerpat annijajolerit opingârlugillo iglugaseksuit. Tamnalo tagva, innugaseksuillo tapsomanêtut anniniarpatta illingnut, tagva pinniarvigiuk aggakpît pijungnarningititut.

34 Abimeleche makkipok unnuarme, innugaseksuillo tapsomanêlauktut illunatik, torarlunelo Sichemut, kattimgajunut sittamainut.

35 Gaalelo Ebedib erninga annivok, nangeriartorlunelo upkoab iglugaseksuit itterviksoangata sânganut. Abimelechele annilerpok terliarsivingmit, innugaseksuit illagilugit.

36 Gaaliblo manna innugaseksuit tækkogamigit, okarpok Sebulemut: Ahâk innugaseksuit attersarput kakkab kôtsiktominganit. Sebulible okautiva: kakkat tachangit innunasugivattit.

37 Gaalelo unnurnersanik okausekalaukpok sulle, okarlunelo: Ahâk innugaseksuit, nunab kerkanit attersarput, kattimgajulle attautsit aggerput apkosinerme Eicheme- angakoktorvingme.

38 Tagva Sebulib okautiva: Nanêka manna kanît, okalauktok: Kinauva Abimeleche, kivgartoraksarigaptigo? Innugaseksoangongilât tapkoa nachogilauktatit? Aularit manna sorsugvigilugolo.

39 Gaalelo aularpok angutinik Sichemiunik sivorlerlune, sorsugiarlunelo Abimelechemut.

40 Abimelechible aijalikpa, kemâtilâlo, unnuktullo ullimarsimajut ochovut itterviksub upkoanga tikkilugo:

41 Abimelechelo Arumame nokkangavok. Sebulible Gaale kattangutingillo kemâtipait Sichemetsungnarkonagit.

42 Kaulermalle innugaseksuit annilerput perorsêvingmut. Abimeleche okautsaulermat tamattominga;

43 Innugaseksuit tigurait, avitilugillo pingasuilivlugit terliarsijokartitsilunelo tapkoninga perorsêvingme. Tækkogamelo manna innuit iglugaseksoarnit anningmatta makkijutigivait, ullimârlugillo.

44 Abimelechible, kattimgajullo tapsomanêlauktut opingârpait, tikkilutiglo iglugaseksuit upkoaksoanganut; kattimgajullo maggoit opingârrpait illunaita perorsêvingmelauktut, ullimarpaillo.

45 Tagva Abimelechib iglugaseksuit sorsugvigivait uvlok 
tamna nâvlugo, salagivaillo, innuillo illuanelauktut tokkopait, serkomilugillo iglugaseksuit, sioraujamiglo kattaraivigivait.

46 Angutit Sichemib nuvugosektanganelauktut tamattominga tussaramik, tagva itterput Gudingoab Beritemik attiggub iglungata avaluksoanginut.

47 Abimelechelo tussarame angutit illunatik Sicheme nuvugosermelauktut kattimangmatta;

48 Aivok kakkamut Zalmonemut, innukotine tapsomanelauktut illunaita illagilugit, tigusilunelo ullimaumik aggangminut, nakkatsilunelo akkerkomik nappartunit, kiviklugolo illiva tuiminut, okarlunelo inugaseksoarnut tapsomanêlauktunut: Tækkojase pinniarnerijara, tamanna illipsetauk pinniarsârsiuk uvaptut.

49 Tagva innugaseksuit illunatik attunnit akkerkomik nakkatsilerput malliklutiglo Abimelechemik, illijilutiglo tapkoninga avaluksoarnut, ikkitsilarmiglo ikkomamut, tokkolaungmattatauk angutit illunatik Sichemib nuvugosektanganelauktut, angutit arnallo kattilugit 1000 diôkassait.

50 Abimelechele aivok Thebezemut, illulugillo salagilaitalo.

51 Nuvugosekalaukporle songojomik iglugaseksuit kerkâne, tagvunga kemâvut angutit arnallo illunatik, iglugaseksuillo innungit illunatik, tunnumikullo pallangairsivut majoarlutiglo nuvogutsib kânganut.

52 Tagva Abimeleche kaivok nuvogusermut sorsugvigivâlo, najutilunelo nuvogutsib upkoanganut, ikkitsomavlugo ikkomamut.

53 Arnalle illangata serkallitsiviub ujaraktangata illanga kattaktipa Abimelechib niakunganut, makkanganiglo serkopjêvigiva.

54 Tagva errinesuklune kivgane, sakkunginik akjarsijok, kaikova okautilugolo: Saviksuit amuleruk tokkolungalo, okautaukonanga: Arnab tokkolaukpa. Tagva kivgangata kappiva, tokkokovlugo.

55 Israelitille illagijangit tækkogamik Abimeleche tokkungmat, illunatik attunit aivut nunagijamingnut.

56 Taimak Gudib Abimeleche akkiniarvigiva idluitullinek pivlugo, atâtaminut pinniarnerilauktanga, kattangutiminik 70 ginik tokkotsilaurame.

57 Taimaktauk angutit Sichemiut idluitulliningit taimaita Gudib akkiniarutigivait niakunginut, tikkitaulutiglo Jothamib Jerub-Baalib erningata ominautekarkojininganut. 


\section{KAPITEL X.}

\section{Israelitit kivgartornek Gudingoanit pivlugo Filisterinut kappiasuletitaulerput.}

ABIMELECHIB kingorngane Thola makkilerpok Israelitit ikkajorlugit, angut Isascharekunit, Puab Dodob erningata erninga. Nunakalaukporlo Samireme, Efraimib kakkangine.

2 Idluarsailunelo Israelitinik Jahrenik 23 ginik, tokkolerlunelo, illuvertaularmelo Samireme.

3 Tapsoma kingorngane makkilerpok Jair, Gileademiok, idluarsailunelo Israelitinik Jahrinik 22 ginik;

4 Ernekalaukpok 30 ginik ablangalauktunik siutekoktojut nochangine 30 gine; iglogaseksoakalaukporlo 30 ginik, attekartunik Jairib iglugasenginik, uvlok tamanna tikkilugo, Gileademêpullo.

$\check{5}$ Jairelo tokkolerpok, illuvertaulaukporlo Kamoneme.

6 Israelible kittorngangit idluitullilaurivut Nalekab sângane, kivgartorlutiglo Baalinik, Astarotemiglo, Siriamiullo Gudinginik, Zidonemiullo Gudinginik, Moabiterillo Gudinginik, Amoniblo kittorngangita Gudinginik, Filisterillo Gudinginik, Nalegarlo kemmakpât kivgartornagolo.

7. Tagva Nalekab ningarningata Israelitit illungertorutigivait, niorvgotigilugillo Filisterit Amoniblo kittorngangita agganginut.

8 Tapkoalo Israelib kittorngangit tullerarpait ullimarlugillo, Jahremit taipsomanemit, Jahrit 18 kassait nâlugit, illa Israelib kittorngangit illunaita Jordanib akkianelauktut Amoriterit nunagilauktangine, tagva Gileademe.

9 Tamannalo tappilugo Amonib kittorngangita Jordane ikkârlaukpât, sorsugiarlutiglo Judakunik, Benjaminekuniglo, Eframiblo iglomiokotinginik, taimak, Israelitit kappiasuktitaulaungmatta angijomik.

10 Tagva Israelib kittorngangit nipliarput Nalekamut, okarlutiglo: Idluitullilaukpogut illingnut: Gudivut kemmalauraptigo, Baalelo kivgartorlaukpavut.

11 Nalekable Israelib kittorngangit okautivait; Egiptenemiut, Amoriterillo, Amoniblo kittorngangita, Filisterillo,

12 Zidonemiullo, Amalekiterillo, Moaniterillo, aksorortilaunginivasse, uvangalo ikkajorlaukpapse agganginit, nipliarvigilaurapsinga?

13 Sulle kemalaukpapsinga, Gudillo assingit kivgartorlugit pilaukpose; tamanna pivlugo ikkajoromairpapse.

14 Aileritse Gudillo anerijasse nipliarvigisigik; tapkoa ikkajorlise kappiasugvipsingne. 
15 Israelible kittorngangit Nalekamut okarput: Idluitullilaukpogut, igvit piniarvigituinartigut namagijarnut; kissiane piulitigut nelliutome tamattomane.

16 Ipperarpaillo Gudit tækkorngartat, Nalegarlo kivgartorpât. Illapsugosutigilaukpalo, Israelitit taimak kappiasuktitaulaungmatta.

17 Amoniblo kittorngangit nipliadlarput, tangmarlutiglo Gileademe; Israelible kittorngangit kattimalerput, tangmarlutiglo Mizpame.

18 Innugiartoksuillo angajokajello Gileademe ingminut okalerput: Pigialertok sorsugiarlune Amonib kittornganginut, tamna niakôjuksauvok, illunainut Gileademêtunut.

\section{KAPITEL XI.}

\section{Jeftab annerïauninga, salakarningalo, angerningalo.}

\section{$\mathrm{J}_{1}$} ainâgaulaukporle. Gileadible Jefta ernetarilaukpa.

2 Gileadible nullianga kittorngilaungmat tapsomunga, nulliablo tapsoma kittorngangit innumarioleramik, pingujarpât Jafta, okautilugolo: kingormgujijuksaungilatit atâtapta iglungane, arnab assiata erneringmattit.

3 Tagva kemâvok kattangutiminit, nunakalunelo nuname Tobemik attelingme. Kattimavullo tapsomunga innuit uivêtut aularkattigilugolo.

4 Ovaitsiarolo kingorngane Amonib kittorngangita Israelitit sorsugvigivait.

5 Amoniblo kittorngangit taimak sorsugialaungmatta Israelitinut, angajokajat Gileademit aivut Jefta aijomavlugo Tobib nunanganit,

6 Okautilugolo: Kailaurit angajokaulatillo uvaptingnut, sorsukovluta Amonib kittornganginut.

7 Jeftable Gileademiut angajokangit okautivait; Illipseungilase omisulauktut uvamnik, pingunilauktullo uvamnik atâtama iglunganit? Mannalo tikkipapsinga kappianartomênapse.

8 Angajokajat Gileademit Jefta okautivât: Tamanna pivlugo manna tikkipogut ama illingnut, illaukovlutit uvaptingnut, ikkajorkovlutillo uvaptingnik, sorsukluta Amonib kittornganginut, niakungôkovlutillo uvaptingnut, illunainut Gileademe nunakartunut.

9 Jeftab angajokajat Gileademit okautivait: Aimigupsinga sorsugiarlunga Amonib kittornganginut, Nalekablo tunniniarpagit uvamnut, tagva niakôjuksauvîk illipșingnut? 
10 Angajokajat Gileademit Jefta okautivât: Nalegak tussarteôle akkonaptingne pinniarniangikupta okauserijaktut.

11 Taimak Jeftab angajokajat Gileademit illagivait, innugiartoksuillo niakungortipât angajokangortilugolo ingmingnut. Jeftablo tamanna tamât okauseriva Nalekab sângane Mizpame.

12 Tagva Jefta tilliklerpok akpartuksanik Amonib kittorngangita attaninganut okautsaukolâlo: Susiorvigijaksarivinga tikkinavit nunaga sursugvigilugo?

13 Amonib kittorngangita attaningata Jeftab tillijangit kiovait: Tamanna pivlugo, Israelitit Egiptenemit aularlutik nunaga aksarnigarilaungmatsuk Arnonemit Jabokemut, Jordanemullo tikkilutik; tagva manna sattortautiuk uvamnut ullapirsautekarnerme.

14 Jeftable ama akpartuksat tillivait Ammonib kittorngangita attaninganut,

15 Tapkoa okautivât: Jefta okarpok imâk: Israelitit nunamik aksarnilaungilat Moabiterinillonêt, Ammonib kittornganginillonêt.

16 Egiptenemille aularlauramik Israelitit agvertarlaukput sunakajuitokut imarbik ivilik suvlulingnik tikkilugo, tikkilutiglo Kadesemut.

17 Tilliklerlutiglo akpartuksanik Edomiterit attaninganut, okarlutiglo: Nunarkut ingergarkotigut. Edomiterille attaningata tussarlaungilait. Tilliklerlaurivuttauk Moabiterit attanninganut, tamna kunnulaurivok. Taimak Israelitit Kadesemetuarlaukput.

18 Agvertarlaukpullo sunakajuitome, Edomiterillo Moabiterillo nunagik kaivalerpakit, tikkilutiglo sekkerngub nuijarviata nellânit Moabiterit nunanganut, tangmarlutiglo Arnonib akkiane, tikkinatiglo Moabiterit kiglilervinganut, Arnone kiglilerviôngmat Moabiterinut.

19 Israelitillo tilliklerput akpartuksanik Sihonemut Amoriterit attaninganut, Hesbonemelauktomut, okautsaukolugolo: Nunarkut ingergarkotigut inniksaptingnut.

20 Sihonible Israelitit tettigilaungilait ingergarkonagit kiglilervikotimigut; inukotinele illunaita kattertilaukpait, tang* marlunelo Jazame, Israelitillo sorsugvigilugit pivut.

21 Nalekable Israelib Gudingata Sihone innukotingit illunaita illaliudlugit tunnilaukpait Israelitit agganginut, ullimarnikovlugit tapkoninga. Taimak Israelitit Amoriterit, nuname tamattomane nunakalauktut nunangat tamât aksarnigarilaukpât.

22 Amoriterillo kigilervingit illunaita aksarnigarilaukpait, Arnonemit Jabok tikkilugo, sunakajuitomillo Jordane tikkilugo.

23 Taimak manna Nalekab Israelib Gudingata Amoriterit pejârlaukpait innukotime Israelitit sivorngane; igvillo aksaromavigît? 
24 Tapkoa aksaraksarivattit, Gudivit Kamosib pêjartitangit, uvagullo tapkoninga illunainik aksarnikoluta Nalekab Gudipta pêjartilauktanginik sivunaptingne.

25 Issumavît idluarnekarnersaugavit Balakemit Ziporib erninganit, Moabiterit attaninganit? Tamna akkerartorlauralloariva unatadlalaukâlonêt Israelitinut?

26 Israelitit manna Jahrit 300 dit nâlugit nunakajarêralloarmatta Hesboneme iglugasenginelo, Aroeremelo iglugasenginelo, iglugaseksoarnelo tamaine Arnonemetune? Sôg piulilaungipissigik nelliutome taipsomane?

27 Idluitullivigilaungilagit sunamik, igvillo taimak idluitullivigivarma sorsugiaravit uvamnut. Nalegak uvlome erkartuijôle Israelitit Amoniblo kittorngangita akkorngane.

28 Amoniterille attaningata Jeftab okalaningit tillikliutilauktangit tapsomunga, tussarlungilait.

29 Tagva Nalekab Annerninga Jeftamut pivok, aularporlo Gileadekut, Mannasekullo, Mizpakullo Gileademetokut, Mizpamillo Gileademetomit Amonib kittornganginut.

30 Jeftablo Nalegak angernermik angervigimarikpa, okarlunelo: Ammonib kittorngangit aggamnut tunniniarungne;

31 Igluma upkoanganit annijok pachrearlunga, Ammonib kittornganginit ullapirsautekarlunga utteruma, tamanna Nalekamut illingajuksauvok, tunnergutautitsomavara ikkoalajaksamut.

32 Taimak Jefta ailaukpok Amonib kittornganginut, sorsugvigilugit. Nalekablo tunnivait tapsoma agganginut.

33 Ullimarpaillo Aroeremit, Minnite tikkilugo, iglugaseksuit 20 git, perorsêvit nappartolingnik Weineliksanik naternangat tikkilugo, unatadlarnermik angijomik. Taimaglo Amonib kittorngangit mannigotititaulaukput Israelib kittornganginut.

34 Jeftalo tikkilermat Mizpamut iglominut, tagva panninga annivok pachrearlugo, killautersoalijarlune imgerlunelo, kittorngatualaukporlo, assianiglo ernekalaungilak pannekalaungilarlonêt.

35 Tækkogamiuglo annorane allikpa, okalunelo: Panîk miklilertiparma kiksartilungalo! Kannera angmarlaurapko Nalekamut, uttertitsungnangilaralo.

36 Tamnale okarpok: Atatâk kannît angmarlaurungne Nalekamut, tagva pinniarviginga sorlo kannernit annilaungmat; Nalekab akkiniarkolaukârlutit omigijingnik Ammonib kittornganginik.

37 Atâtaminullo okarpok: Tamattominga pinniarkolaunga, tunnitsivigilungalo takkengnik maggungnik, attersarkovlunga, majorarkovlungalo kakkanut, uigasungnera kêagiluga ikkingutikka illagællugit.

38 Tamma okarpok: Ailerit; aikolugolo takkengnik mag- 
gungnik. Tagva aivok ikkingutine illagilugit, uigasungninelo kêagiva kakkane.

39 Takkâklo magguk kângilermanik, utterivok atâtaminut. Tamnalo taimailiorpok sorlo angerlaurame; angumiglo tamna illitarksilaungilak. Tamannalo piusiolerlaukpok Israeleme,

40 Israelitit panningit Jahrine tamaine annilauramik Jeftab Gileademiub pannia oggorilugo uvlut sittamat nalugit.

\section{KAPITEL XII. \\ Efraimekut akkerartorningat salagïjauningallo.}

EFraimekullo kattimalerput, ailutiglo avunga, okarlutiglo Jeftamut: Sôg unatadlarnermut Ammonib kittornganginut ailaukît, kaikolaungilaptigullo, aikattaukovluta? Iglokotit illaliudlutit ikkitsomavarut ikkomamut.

2 Jeftab okautivait: Uvanga innukotikkalo angijomik pitjutiksakalaukpogut Ammonib kittornganginut; nipliutilaukpapselo piulilaungilapsingale agganginit.

3 Tækkolauramalo ikkajoromalaunginapse, tarniga pitilaukpara aggamnut, ailungalo Ammonib kittornganginut, Nalekablo tunnilaukpait aggamnut. Sôgle manna mojorarvigivisinga, sorsugiarvigilunga?

4 Jeftablo angutit Gileademetut katterkovait, Efraimekullo sorsugvigivait. Angutillo Gileademiut Efraimekut ullimarpait, tamanna pivlugo, okarmatta: Illipse Gileademiojose, Efraimekut Manassekullo akkorngane, kemmajutitut Efraimekune 1̂tuinaurose.

5 Gileademiullo Efraimekut ingiarlugit, Jordanib ikkarvinga tiguvât, Efraimekullo kemajut, manna okalaurunik: Ikkârlaulanga, tagva angutit Gileademint okautivât: Efraimekumiôvît? Kiolaurunelo tagva: Aukak;

6 Tagva okarkovât: Schibolet, tagva tamna okarpok: Sibolet, okauserijungnarlaungilâlo namaktomik. Tagva tiguvât ullimarlugolo Jordanib ikkârviane; taimak Efraimekunnit ocholaukput nelliutome taipsomane 42000 dit.

7 Jeftalo idluarsailaukpok Israelitinik. Jahrinik 6 inik. Jeftalo Gileademiok tokkolerpok, illijartortaulunelo Gileadib iglugaseksoangita illangane.

8 Tapsoma kingorngane Ebzan Bethlehemiok idluarsailaukpok Israelitinik.

9 Ernekalaukpok 30 ginik, panniniglo 30 ginik tunnijivok, 30 giniglo illelliorivok ernerminut; Israelitiniglo idluarsailaukpok Jahrinik 7 nik,

10 Tokkolunelo illijartortaulunelo Bethleheme. 
11 Tapsomalo kingorngane Elon, Sebulonekomiok idluarsaivok Israelitinik; idluarsailaukporlo Israelitinik Jahrinik tel limaujortunik,

12 Tokkolunelo illijartortaularmelo Ajaloneme Sebulonekut nunangane.

13 Tapsomalo kingorngane idluarsaivok Israelitinik Abdon Hillelib erninga, Pireatonemiok,

14 Tamna ernekalaukpok 40 ginik, kangiakalarmelo 30 ginik, ablangalauktunik siutekoktojut nochangine 70 gine; idluarsailaukporlo Israelitinik Jahrenik 8 inik,

15 Tokkolunelo illijartortaularmelo Pireatoneme Efraimekut nunangane, Amalekiterit kakkangane.

\section{KAPITEL XIII.}

\section{Simsonib okautauninga innulerningalo.}

ISRAELIBLO kittorngangit idluitulligannerput Nalekab sângane; Nalekablo tunnilaukpait Filisterit agganginut Jahrit 40 git nâlugit.

2 Angutekalaukporle Zareame, Danib abvakungita illanganit pijomik, Manoamik attelingmik; nulliangalo kittorngisuilaukpok, ernilaungilarlo.

3 Nalekablo Engelingata arnak tamna sakkervigiva okautilugolo: Ahâk kittorngisuipotit, ernilungilatillo sunamik; singailerniarpotille, erniniarlutillo ernermik.

4 Tagva udsertorit manna, Weinemik pitsartojomiglonêt imiksamik immerkonak, nerrikonaglo ivsornartomik.

5 Singailerniaravit, erniniarlutillo ernermik kipsautingnut pijaujuksaungitomik niakunganut; nukapiak angerutaumajôniarmat Gudemut, anâname timinganit; pigiarniarporlo Israelitit piulilugit Filisterit agganginit.

6 Tagva arnak kaivok uinelo unipkautiva tamattominga, okarlunelo: Gudib angutekotingata tikkipânga, kênangalo tautukalaukpok Gudib Engelingatut, kappianadlartomik; tamanna pivlugo apperilaungilara, nakit pingmangat namungarniarmangallonêt; okautilaungilângalo kannok attekarnerminik.

7 Tapsoma okautivânga: Ahâk singailerniarpotit, erniniarlutillo ernermik. Tagva manna Weinemik immerniarnak, imiksamiglonêt pitsartujomik, nerrinaglo ivsornartomik; nukapiak angerutaumajojuksaungmat Gudemut anâname timinganit, tokkominut tikkilune.

8 Tagva Manoab Nalegak tuksiarvigiva okarlunelo: Nalegak, Gudib angutekotinga tillilauktat, kailaungmile uvapting- 
nut, ajochertuikovlugo uvaptingnik pinniaraksaptingnik nukapiame erniangoniartome.

9 Gudiblo Manoab nippinga tussarpa; Gudiblo Engelingata arnak tikkiniva. Iksivalaukporle peror'sêvingme, uingalo tapsomanêlaungilak.

10 Tagva tuavidlune akpangerpok, unipkarlunelo tamattominga uiminut, okarlunelo: Ahâk angutib uvlome tikkilauktama sakkervigivânga.

11 Manoalo nikovipok malliklunelo nulliaminik, tikkilarmelo angumut tapsomunga, okautilâla: Igvit anguteôvît tamna okarkattaulauktok arnamut omunga? tamna okarpok: Ahaila.

12 Manoalo okarpok: Okauserilauktatit malliklugit pijokarniarpat; kannoêtunik nukapiak illusekartuksauva, pinniartuksaulunelo?

13 Nalekab Engelinga Manoamut okarpok: Tamainik okauserilauktamnik arnamut, ulloreasuktuksauvok;

14 Nerrijuksaungilak nappartomit Weineliksamit pijomik, immertuksaungilarlo Weinemik, immiksamiglonêt pitsartujomik, nerrijuksaungilarlo ivsornartomik; tamaita okauserilauktakka tapsomunga (arnamut) malligaksarivait.

15 Manoalo Nalekab Engelinganut okarpok: Næglingnartotit, ovanesalaurit, angusalloârsumik tuktungajomik akiksoijomavogut illingnut.

16 Nalekable Engelingata Manoa kiova: Ovanekogalloarumga, sulle nerkiksakotitit nerrilungilakka. Nalekamulle tunnergutiksamik ikkoalajaksamik tunnijijomaguvit, tagva taimailiorungnarpotit. Mannoa kaujilaunginame Nalekab Engelegingmago.

17 Manoalo Nalekab Engelinganut okarpok? Kannok attekarkît? nertordlerkovlunuk illingnik, okauserilauktatit malliklugit pijokarniarpat.

18 Nalekable Engelingata okautiva; Sôg attera pivlugo apperivinga, tattamnadlaralloartok?

19 Tagva Manoab angusalloârsuk tuktungajok tiguva, tunnergutiksarlo nerkiksak, tunnilâlo Nalekamut kairtome; tapsomalo (Engelib) sennava tattamnadlartomik. Manoalo nulliangalo tækkonârpuk.

20 Ikkomalo ikkoalangmat Altaremit kôngmut killangmut, tagva Nalekab Engelinga Altarib ikkoalaningagut kôngmuarpok. Manoab nulliangatalo tamanna tækkolauramikko, pamakterpuk nunamut kênatik puvlugik.

21 Nalekablo Engelinga sakkijarungnaipok Manoamut nullianganullo. Tagva Manoa tukkisivok Nalekab Engelegingmago.

22 Manoalo okarpok nulliaminut: Tokkotaksauvoguk illa, Gude tækkolauraptigo. 
23 Nulliangatale kiova: Nalegak issumakarune tokkotsomavlunuk, tagva tunnergut ikkoalajaksak, tunnergullo nerkiksak illelliorajalaungilâk aggaptingnit; taimaitomiglo taimainik sakkertitsiviginajalaunginivâtiguk tussarkonanuglonêt taimaitomik, sorlo manna pilaungmat.

24 Arnablo ernek erniariva, atserarpâlo Simsonemik. Nukapiarlo aglisivallievok, Nalekablo saimarpa.

25 Nalekablo Annerninga pigiarpok tessiorlugo Danekut tangmarvingane, Zareablo Esthaoliblo akkorngangne.

\section{KAPITEL XIV.}

Simsonib pinniarnersoanga, nullianingninga, sagvainingalo nellaupsaraksamik.

SIMSON attersarpok Timnatemut, tækkolunelo arnamik, Filisterit panningita akkornganne.

2 Takpaungaramelo unipkarpok tamattominga atâtaminut, anânaminullo, okarlunelo: Arnamik tækkolankpunga Timnateme, Filisterit panningita akkorngane; tunnitsivigitinga manna taipsominga nulliaksamnut.

3 Atatangalo anânangalo okarpuk tapsomunga: Arnakangilâk manna kattangutivit panningita akkorngane, innukotivillo tamaita akkorngane, aigavit tigusilutillo arnamik Filisterinit kippisimangitunit? Simson okarpok atâtaminut; Tamna tunniuk uvamnut, ijingma pinnaringmakko.

4 Atatangale anânangalo kaujilaungilak, Nalekamit pingmat tamanna; pitjutiksarsiormat Filisterine. Filisterille attanniotivut nelliutome taipsomane Israelitinut.

5 Taimak tagva Simson attersarpok atâtanelo anânanelo illagællugik Timnatemut. Tikkimattalo perorsêvingnut Weineliksanik nappartolingnut, ahâk tagva Loeweâraub errinaloakijarlune pârpa.

6 Nalekablo Amnerningata tikkipa, alliktorpâlo tamna, sorlo angusalloârsuk alliktortaupângmat; sunakaranelonêllo aggangmine: Okalungilarlo atataminut anânaminullonêt pinniarnerilauktaminik.

7 Unnungalo pigame arnak okarkattigiva, Simsoniblo pinnariva ijingminut.

8 Uvlullo illangita kingorngane tikkilerivok nullianigomavlugo, sangulunelo apkomit Loewevinek kemergojomavlugo; ahâk igupsaujartoksoakarpok tagva Loewevinerme orksokalunelo.

9 Tigusilaukporlo tagvangat, nerrilunelo apkome, ailunelo atâtaminut anânaminullo, tunnitsivigivâglotauk nerrikovlugik. Okautingilâgle orksuk tigulauramiuk kellovinermit Loewemit. 
10 Atâtangalo attersarêrmat arnamut tapsomunga, tagva Simsone nulliarêlitainarnerminut nerrimârnekartitsivok, sorlo angutit innûsuktut pivangmatta.

11 Tækkogamitsuglo illakartipât tapsomanetuksanik 30 ginik.

12 Simsonible okautivait: Nellaupsaraksamik okautijomavapse; tamanna nellaupsarupsiuk nellaugupsiuglo, nulliarêlitainarniub uvloksiorviksangine 7 ine tamakkonane, tagva tunnitsivigijomavapse uvinerkunik 30 ginik, annoratsianiglo uvloksiorviksiutinik 30 ginik.

13 Nellaujungnangikupsiugle, tagva illipse tunnitsivigijaksarivapsinga uvinerkunik 30 ginik, annorâtsianiglo uvloksiorviksiutinik 30 ginik. Tapkoalo okautivât: Nellaupsaraksat okautigiuk, tussarkovluta mattominga.

14 Tapsoma okautivait: Nerkiksamik annijokalaukpok nerrijoksoarmit, mammaksaumiglo sangijomit. Uvlunnelo pingasune nellaupsaraksak nellaujungnarlaungilât.

15 Uvlullo 7 ingane Simsonib nullianga okautivât: Uit tillioruk okarkovlugo uvaptingnut nellaupsaraksab tukkinganik, ubvalo igvit atâtavillo iglomiokattingit illaliudlugit ikkomamut ôniarpaptigit. Inalaukpittigut ovunga aklulertitsomavluta? taimaingilârlonêt?

16 Tagva Simsonib nullianga kugviorpok tapsoma sângane, okalunelo: Omigivarma nagligingilarmalo. Innukotima kittornganginut tunnijilaukpotit nellaupsaraksamik, okautilaungilarmalo mattominga. Tapsomale okautiva: Okautigilaungilara atâtamnut anânamnullo, okautigijaksarivigolo illingnut?

17 Tamnalo nulliarêlitainarnimik uvloksiorvingine kugviutsainarlaukpok sângane; uvlulle 7 ingane okautiva, pagvisadlarmago. Tapsomalo nellaupsaraksak okautigiva innukotime kittornganginut.

18 Tagva iglugaseksuit angutingita okautivât uvlut 7 ingane, sekkinek nippilerkârtinago: Suna mammanersauva igupsaujartut orksunginit? Sunalo sanginersauva Loewemit? Tapsomale okautivait: Poagrejarlaungikupse nochakotimnut nellaupsaraksara nellaunajalaungilasse.

19 Nalekablo Annerningata tikkipa, tamnalo attersarpok Askalonemut, angutillo 30 git annaulerpait, tigulaitalo annorângit, annoratsiallo tunnivait tapkonunga nellaupsaraksamik nellautsilauktunut. Ningarnerminelo illungertulerpok, takpaungârlunelo atâtame iglunganut.

20 Simsonible nullianga tunnijaulaukpok tapsoma aiparilauktangita illanganut, illanarijatut attorlauktaminut. 


\section{KAPITEL XV.}

Simsonib Filisterit angïjomik kannoêtokartipait terrianianut, siutełcoktojublo aglerunganut.

UVLULLE illangita kingorngane kattersiviub Weizenik nelliuningane, pijokalaukpok, Simsonib nulliane nellipsarmago, angusalloârsungmik tuktungajomik neksarlune. Issumakaramelo: Nulliamnut aijomavunga sinigvingmut; tagva taipsoma atâtangata itterkojomalaungila.

2 Okarlunelo: Omigijaunasugilaukpara illingnut, tunnilaukparalo illanarijarnut; nukkakarporle ânanaukejanganik, tamna piuk, taipsoma inningane.

3 Tagva Simsonib okautivait: Sullijomik pitjutiksakarpunga Filisterinut; kannoêtokartitsomavapse.

4 Simsonelo aularpok, tigusilunelo terianianik 300 dinik, tigusilunelo aumalingnik, pamiungillo ke:laksorpait attunit aipangata pamiunganut, aumalingmiglo attautsemik pititsivok pamiuk magguk akkonigingnut.

5 Ikkilugillo tapkoa ikkomamut, aitilugillo Filisterit kakkojaksakotingita akkornganut, ikkitipaillo taimak kakkojaksat kippisimajut, kippitallo kippisimangitullo, perorsêvillo Weineliksat, nappartullo orksuggit.

6 Tagva Filisterit okarput: Kina tamattominga pinniarlauka? Tagva okartokarpok: Simson, Thimnatemiub ningaunga; nullianganik aksarlauramiuk, tunnigamiuglo illannarijanganut. Tagva Filisterit takpaungarput, ôpâlo atâtanga illaliudlugo ikkomamut.

7 Simsonible okautivait; Taimailiorlauralloarupse, sulle akkiniaromavapse, kingornganelo nokkaromavunga.

8 Angijomiglo annaulerpait arriangittigut sibvêangittigullo. Attersarporlo kairosuglo kairtomêtok Ethameme innigiva.

9 Tagva Filisterit takpaungarput, Judakullo illumavait, tangmarlutiglo Lehime.

10 Judakullo okautivait: Sôg takpaungarkise sorsugvigiluta? Kiovut: Takpaungarpogut Simson kellaksoromavlugo, pinniarvigilugolo sorlo pinniarvigilaungmattigut.

11 Tagva Judakunnit angutit 3000 dit attersarput kairosungmut Ethamemetomut, okarlutiglo Simsonemut; Kaujimangilatît Filisterit attaniotingmatta uvaptingnut? Sôgle tagva taimaitomik pinniarvigilaukpittigut? Tapsoma okautivait: Sorlo pinniarvigilaungmanga, taimak pinniarvigilaurivakka.

12 Tapkoa okautivât: Attersarpogut kellaksoromavlutit unertotigijomavlutillo Filisterit agganginut. Simsonib okautivait: Tagva angervigimarisinga, agviaromanginapsinga. 
13 Tapkoa okautivât: Agviaromangilaptigit, kellaksoromatuinarpaptigille, tunnilutillo taipkoa agganginut, tokkotsomangilaptigillo. Kellaksorpâllo aklunângnut nutângnut, tessiorlâlo kairtomit takpaunga.

14 Tikkimallo Lehimut Filisterit kaggudlarvigivât kuviasumut. Nalekable Annerningata tikkipa, aklunâglo talligingnelauktuk ivaluksatut îlerpuk, ikkomamut ôtaujotut, kellaksutik aggangine kellarudsarmanik.

15 Nachvarpalo siutekoktojub agleroa nutâk; tagvalo aggane issakpait, tigulâlo, annaulerlaitalo tapsomunga angutit 1000 dit.

16 Simsonelo okarpok: Tagga nellavut kattimgajut: Siutekoktojub aglerunganut angutit 1000 dit annaulersimavakka.

17 Tamakkoalo okauserijarêramigit aglerok egipâ aggangminit, innelo tamanna taiva Ramat-Lehimik.

18 Immerudlaramele Nalegak nipliarvigiva okarlunelo: Igvit taimak angijomik piulijaunermik tunnijilaukpotit kivgavit aggangittigut; mannale tokkojuksauvunga immerungnermut, pijaulungalo kippisimangitut agganginut.

19 Tagva Gudib ittersak Lehimetok koppiva, tagvangallo immek kôlerpok. Immerlunelo annerninga utterpok, nekkoksijaumalunelo. Tamanna pivlugo taijauvok sulle uvlok tamanna tikkilugo tuksiartub puailavinganik Lehimetomik.

20 Idluarsaivorlo Israelitinik Filisterit nelliuningane Jahrinik 20 ginik.

\section{KAPITEL XVI.}

\section{Simsonib kattengninga, ajulerninga, akkiniarningalo kingorlerpâk.}

\section{SIMSON ailaukpok Gasamut, tækkovalo tagvane arnak ang- uniarpaktok, ittervigivalo.}

2 Tagva Gasamiut okautsaulerput: Simson ittersimavok tamaunga. Illumavâllo udsertortaukolugolo unnuak nâlugo iglugaseksuit itterviksoangane, unnuarlo nâlugo innilangalaukput, okarlutiglo: Kaupat, kaumalerpat tokkotsomavavut.

3 Simsonele innilangalaukpok unnuab ketterarninga tikkilugo. Tagva unnuab ketterarningane makkipok, iglugaseksuillo itterviksoangata upkoaksoagik tamarmik tiguvak, nappajugik illaliudlugik, kiviklugiglo senneroangit illaliudlugit, pitilugillo tuingminut, erksuglaitalo kakkab Hebronib sânganetub kânganut.

4 Kingorngane arnak ungagilerpa, kôngme Sorekeme, Delilamik attelik.

5 Tapsomunga Filisterit attaningit takpaungarput, okautilâlo : Tillioruk issumasiorlugolo, naukut taimak angijomik 
pitsartunekarninganik, sunamullo salagijungnarmangaptigo, kellaksorkovluta tapsominga makkutilugolo; tagva attunit tunnitsivigijomavaptigit Silberlingnik 1000 dinik 100 diniglo.

6 Delilalo Simsonemut okarpok: Nagliktara okautilaunga sukutsikut taimak angijomik pitsartunekarnernik, sunamullo kellaksortaujariakarkît makkutijaukovlutit?

7 Simsonib okautiva: Kellaksortaunajaruma aklunanut 7 innut, nappartub ameranganit nutamit pannersimangitomit sennamajunut; tagva sangêlerajarpunga, innuktullo assiptut înajarpunga.

8 Tagva Filisterit attaningita aklunat 7 it nappartub ameranganit nutâmit sennamajut, pannerlungitut sulle âpait tapsomunga, Delilablo kellaksorpa tapkonunga.

9 (Inningatale aiparusingane udsertortokalaukpok tapsominga.) Tapsomalo okautiva: Filisterit illingnut pivut, Simson! Tapsomale aklunât taipkoa kiktorautivait, sorlo aklunarârsuk kiktorangmat îkkomamut pilerpat; tukkisijaulungilarlo pitsartuninga nakit pingmangat.

10 Tagva Delila Simsonemut okarpok: Ahâk mittagvigivarma saglokilungalo; mannale okautilaunga, sunamut kellaksortaujungnarkît?

11 Tamnalo okarpok tapsomunga: Kellaksortaunajaruma aklunânut nutânut, attortaulaungitunut sulle kinamut, tagva sangêlerajarpunga, innuktullo assiptut înajarpunga.

12 Tagva Delilab aklunât nutât tiguvait, kellaksorpâlo tapkonunga, okarlunelo: Filisterit illingnut pivut, Simson! (udsertortokalaukporle tapsominga inningata aiparusingane) tapsomalo kiktorautivait ivaluksatut sorlo.

13 Delilale okarpok tapsomunga: Sulle mittagvigivarma, sæglokilungalo. Nagliktara okautilaunga, sunamut kellaksortaujungnarkît? Tamnalo okarpok tapsomunga: Niakungma nujangit. ingmigolingajut erkesulangajut 7 it pergainajarungne kellaksiutiksamut, atsungertilugolo kikkiangmut.

14 Tapsomalo okautiva: Filisterit pivut illingnut, Simson! tamnale tuppakpok siningnerminit, kellaksutillo pergaimajut, kikkiarlo kellaksiutiksarlo illaliudlugit nutsukpait.

15 Tagva tapsoma okautiva; Kannok okarungnarkît nagligigamga, omattivit illagingigalloarmanga? Pingasuertorlutit mittagvigivarma, okautinangalo sukutsikut taimak angijomik pitsartunekarnernik.

16 Uvlut tamaitale pagvisarmago okauserminut, okumaiksartimagolo, tarninga nukingêrotilerpok tokko tikkilugo.

17 Omattinelo tamât okautigiva tapsomunga okautilugolo: Kipsautingnik pijokalaungilak sulle niakumnut; angernerijangôgama Gudemut anânama timinganit. Sallinajarumga, tagva 
pitsartunera nulerajarpok uvamnit, sangêlerajarlungalo, innuillo assingititut illunaititut înajarlunga.

18 Delila tukkisilerame manna omattine tamât nellonarungnaitilaungmago tapsomunga, tilliklerpok, kaikojaukolugillo Filisterit attaningit, okautsaukolugillo: Ama takpaungarmititse, omattine tamât nellonarungnaitilaungmago uvamnut. Tagva Filisterit attanningit takpaungarput tapsomunga, neksarlutiglo kênaujanik taipkoninga aggangmingne.

19 Sinnikovalo sâmine, kaikojilunelo salliniartomik tapsoma niakungata nujanginik erkesulangajunik 7 nik. Makkutilerpalo. Tagva pitsartuninga nusimajarêrpok tapsomangat.

20 Tapsomalo okautiva: Filisterit illingnut pivut, Simson! Tuppakamelo issumakalerpok: Annijomavunga, sorlo pikattarlaurama nutsugomalungalo; kaujimananelo Nalegak nusimangmat tapsomangat.

21 Filisterille tiguvât, kappitsivigilâllo ijigingnik, attersartipâllo Gasamut, kellaksorpâllo kellaumgujanut magguinut kannujarnut; parngnanaisimavingmelo serkalitsijuksaulaukpok.

22 Niakungatale nujangit, sallijaulauktut perorlerivut.

23 Filisterille attaningit kattimaleramik Guditik Dagon tunnergutiksamik âtsivigijomavlugo, kuviasugomavlutiglo, okarput: Gudipta omigijivut Simson tunnilaukpa aggaptingnut.

24 Taimaktauk, innugiartoksuit tækkogamitsuk, Guditik nakoridlarpât okaramik: Gudipta omigijivut tunnilankpa aggaptingnut, nunaptingnik asseroilauktok, tokkotsilauktorlo unnuktunik uvaptingnit.

25 Omattingillo kuviasudlalaungmatta mana, okarput: Simson aijaule, pingoarkovlugo sâptingne. Tagva Simson aivât parngnanaisimavingmit, pingoarporlo sângine, tapkoalo pitipât nappajuk magguk akkonigingnut.

26 Simsonele nukapiamut tessiortiminut aggangmikut okarpok: Perkolaunga nappajungnik, iglub sukkagingnik aktuikovlunga, egarkovlunga tapkongnunga.

27 Iglole tæattædlalaukpok angutinik arnaniglo. Filisterit attanningit illunatik tagvanelaurivut, iglublo kângane pittakalaurivoktauk angutinik arnaniglo 3000 diokasangnik, tækkonarlauktunik Simsonib pingoarninganik.

28 Simsonible Nalegak nipliarvigiva okarlunelo: Nalegak, Nalegak erkaringa; pitsartutilungalo Gude, ama tamattomane, ijikka pivlugik akkinniarkovlunga Filisterinik.

29 Nappajuglo kerkanetuk, iglublo tungavigijangak sukkagiglo tiguvak, aipanga tallerpingminut, aipalo saumingminut,

30 Okarlunelo: Tokkolanga Filisterit tapkoa illagællugit; okkungalunelo pitsartunerminut tamânut. Tagva iglo immikpok, attanernut innugiartoksoarnullo tamainut illuanêlauktunut. 
Taimak tokkungajut, tokkungane tokkotaulauktut, unnurnersaulaukput tokkolauktaminit innosermine.

31 Tagva kattangutingit, atâtamelo iglomiokotingit illunatik attersarput, tiguvâllo âlâllo takpaunga, illijartorlugolo atâtame Manoab illuvinganut, Zareab Esthaoliblo akkonigingnetomut. Israelitinigle idluarsailaukpok Jahrinik 20 ginik.

\section{KAPITEL XVII.}

\section{Micha kivgartornekartitsivok Gudeungitomik.}

ANGUTEKALAUKPOK Efraimib kakkangine Michamik attelingmik.

2 Tamna ananâminut okalaukpok: Silberlingit 1100 dit tigujaumajut illingnit, okautelugjutigilauktattillo siutingma sângangne, ahâk kênaujat tapkoa uvamnêput, uvanga tigulaukpakkà. Tagva anânanga okarpok: Saimartitaulerit ernîk Nalekamut.

3 Taimak Silberlingit 1100 dit tapkoa tunnilaurivait anânaminut. Anânanga okarpok: Kênaujat tapkoa perkutautilaukpakka aggamnit Nalekamut, ernera pivlugo, adsingoarmik Gudingoarmiglo sennajokarkovlugo; taimaimat tunnilerivakka manna illingnut.

4 Tapsomale kênaujat tunnigivait anânaminut. Tagva anânangata tiguvait Silberlingit 200 dit, tunnilaitalo sabviortemut Goldemik; tamna sennavok tapkonangat adsingoarmik, Gudingoarmiglo, tamana kingorngane Michab iglunganelaukpok.

5 Angullo tamna Micha taimak iglokalaukpok Gudelingmik, sennalunelo ikkiarmik ivsornaitovingmiglo, ernimelo illangat saimarsarpa, ajochertuijokovlugo tapsomunga.

6 Nelliutome taipsomane attanekalaungilak Israeleme, illunatigle attunît pinniarlaukput idluarasugijamingnik.

7 Innuosuktokalaukporlo angumik Bethlehememit Judametomit, Judakut akkorngane, tamna Leviteolaukpok, tujormiolaukporlo tagvane.

8 Aularporle iglugasengnit Bethlehememit, Judametomit, ingergaromavlune pijungnartaminut. Ingergarlaukporlo Efraimib kakkangine, Michablo iglunganut pingmat,

9 Michab apperiva: Nakit pivit? tamna kiovok: Leviteôvunga Bethlehemit Judametomit, ingergarlungalo namut pijungnartamnut.

10 Michable okautiva: Uvamnêtsainarit, atâtaujuksauvotit uvamnut, ajochertuijojuksaulutillo uvamnut; Jahrine tamaine tunnitsivigijomavagit Silberlingnik 10 nik, annoraksarniglo, nerkiksarniglo. Levitelo taimak tagvunga pivok. 
11 Levitelo tamna tagvanêtsomalerpok angutime tapsomane; tapsomalo nukapiak tamna ernermitut pinniarvigilaukpa.

12 Michablo Levite saimarsarpa, ajochertuijokovlugo tapsomunga; taimaglo Michab iglunganêtuarpok.

13 Michalo okarpok: Manna kaujimavunga Nalekab ajungitulliviginiarmanga, ajochertuijokutekarama Leviteôjomik.

\section{KAPITEL XVIII.}

Danekut Laise aksarnigarivait, kivgartornekartitsivullo

tagvane Gudingoarmik.

$\mathrm{N}$

NELLIUTOME taipsomane attanekalaungilak Israeleme. Kittorngarêllo Danekut kingormgutijaksamik nunagijungnartamingnik kennerasuarput; uvlorle tamanna tikkidlugo kingormgutijaksangat nellautaulaungilak sulle tapkonunga, Israelib kittorngarêngita akkorngane.

2 Daniblo kittorngangit abvakungnermingnit, kiglilervingmingnit Zareamit Esthaolemillo tilliklerput angutinik tellimanik sorsugungnartunik, nunna nautsertoriarlugo, kemergolugolo, okautivaillo: Aularitse, nunnalo nautsertoriarsiuk. Tikkipullo Efraimib kakkanginut, Michab iglunganut, sinniktarlutiglo tagvane.

3 Michablo iglomiokattinginêdlutik nukapiab Leviteôjub nippinga illitarivât; sâlerpullo tapsomunga, okautivâllo: Kia ovunga âlaukâtit? Sunamik sullijaksakarkît mâne? Sunakarkîllo mâne?

4 Tapsomalo kiovait: Imâglo, imâglo Michab pinniarvigilaukpânga, attartorlungalo ajochertuijokutaukovlunga tapsomunga.

5 Tapkoa okautivât: Naglingnartotit, Gude apperiuk tukkisilerkovluta, apkosinivut arvertarvigijavut sullinaralloarmangât?

6 Ajochertuijub kiovait: Aularitse ullapirsautekarluse; apkosinise arvertarvigijase idluarpok Nalekab sângane.

7 Tagva angutit tellimat tapkoa aularput, tikkilutiglo Laisemut, tækkolutiglo innuit tagvanetut terlingmatta, sorlo Zidonemiut illusingittitut, uimajaratik terliklutiglo; tagvanelo kiksartitsijokalaungilak tapkoninga, kinakalaungilarlonêt angajokaujomik tapkonunga; ungasidlalaukpullo Zidonemiunit, innuillo sullivigilaungilait.

8 Utterpullo kattangutimingnut Zareamut EsthaolemuHo; kattangutingitalo okautivait: Kannok illingavise? 
9 Tapkoa okarput: Attaik! takpaungalerta; nunale tamna kemmergolaukpavut, ahâglo, ânanaudlarpok. Tamanna pivlugo tuavititse aularluselo, erkinaulugasse, tikkikovluse tagvunga, nuna tamna aksarnigarilugo.

10 Takpaungarupse innukatigêksoarnut terliktunut tikkiniarpose; nunnalo angijôvok, Gudib tunningmago aggapsingnut; nunna imaitok, ajoksarnarlungitok sunamik nunametomik.

11 Tagva aularput tagvangat, Danib abvakunginit, Zareamit Esthaolemillo, angutit 600 dit, oppalungairlutik sakkomingnut unatadlarnermut.

12 Takpaungarpullo, tangmarlutiglo Kireat-Jearimeme Judametome. Tamanna pivlugo inne tamanna tailaukpât Danib tangmarvinganik uvlok tamanna tikkilugo, Kireat-Jearimib ungatanêtok.

13 Tagvangallo aivut Efraimib kakkanginut, tikkilutiglo Michab iglunganut.

14 Tagva angutit tellimat aularlauktut, nunamik Laisemik nautsertorlutik, kiovut, okarlutiglo kattangutimingnut: Kaujimagivise iglune tamakkonane pittakarmat ikkiarmik ivsornaitovingmiglo, adsingoarmiglo, Gudingoarmiglo? Manna issumaksarsiorungnarpose kannok pinniartuksaunipsingnik.

15 Tapkoa tagvunga itterput, tikkilutiglo nukapiab Leviteôjub iglunganut, tagva Michab iglunganut, salutipâllo pitsiarlutik.

16 Tapkoalo 600 dit oppalungairsimajut sakkumingnut, Danib kittornganginit pijut, nangerput upkoaksub sillatâne.

17 Angutillo tellimat nunamik nautsertorialauktut, takpaungarput tikkilutiglo tagvunga, tigusilutiglo adsingoarmik, ikkiarmiglo, ivsornaitovingmiglo, Gudingoarmiglo; taimailiortillugit ajochertuijok nangerpok upkoaksub sillatane 600 dine, oppalungairsimajune sakkomingnut unatadlarnermut.

18 Tapkoa Michab iglunganut pingmatta, tigusingmattalo adsingoarmik, ikkiarmiglo, ivsornaitovingmiglo, Gudingoarmiglo, ajochertuijub okautivât: Suvise ovane?

19 Tapkoa okautivât: Nippangerit, kanîllo epummeruk, aularkattigilutalo, atâtaukovlutit ajochertuijokovlutillo uvaptingnut. Namanersauva illingnut angutib attausênaub iglungane ajochertuijoguvit, Israeliblonêt kittorngarêngita abvakungitalo illangane illuitome?

20 Ajochertuijub tamanna kuviagiva, tigusilunelo ikkiarmik, ivsornaitovingmiglo adsingoarmiglo, ailunelo innugaseksuit akkornganut.

21 Utterlutiglo aularlauramik sorrutsit nergjutillo pivianartokottitiglo sivorlerkovait.

22 Kanningitomêmatta manna Michab iglunganit, tagva angutit iglunit, Michab iglungita kannitanganêtunit, kattimalerput, 
malliklutiglo Danib kittornganginik, nipliutidlarlugillo Danib kittorngangit.

23 Tapkoale kênatik keviarpait, okarlutiglo Michamut: Suniarpît, taimaitomik kaggudlarnekartitsigavit?

24 Tamna kiovok: Gudigijakka sennalauktakka tigulaukpasse, ajochertuijorlo, aularluselo; sunakarkîk manna sulle? Illipselo appertsorpose sulle, suniarnimnik?

25 Danible kittorngangita okautivât: Nippît tussartaukoniarnago uvaptingne, opaktaukonnak angutinut ningartunut; tarnit, iglomiokattivillo tarningit pêjartaukonagit.

26 Taimak Danib kittorngangit sivumuarput apkotimingne. Michalo tækkolaurame songoluarmatta tapsomunga, utterpok tikkimilunelo iglominut.

27 Tapkoale tigusivut Michab sennalauktanganik, ajochertuijomiglo pigilauktanganik, tikkilutiglo Laisemut, innukattigêngnut uimajangitunut, terliktunullo, ullimârpaillo saviksub kênanganut, iglugaseksuillo ikkipait ikkomamut;

28 Piuliklertokalaungilarlo tapkoninga, Zidonemit ungasilaungmatta, innuillo sullivigilaungilait, naksarmelaukpullo BethRehobemetome. Tagvane iglogaselliorput nunakarlutiglo tagvane.

29 Atserarpaillo Danemik, atâtagijatik Dan, Israelib ernerijanga malliklugo. Iglugaseksuillo tapkoa sivorngagut attekalaukput Laisemik.

30 Daniblo kittorngangita, pivlutik adsingoak taimna nappartipât. Jonathanelo, Gersonib Mannasib erningata erninga, erningillo ajochertuijôlaukput kittorngarêt Danekut akkorngane, nelliutok tamna tikkilugo parngnanairsortauvlutik aulartitaukârtinagit nunnamit.

31 Taimaglo adsingoak Michab senajaukolauktanga pitipât akkonamingnut, taimak akkunêrtigijomik Gudib iglungata Silomelaungningatut.

\section{KAPITEL XIX.}

Gibeamiut kangunartunit pitlartaunartuniglo pinniarnekarput.

NELLIUTOME taipsomane attanekalaungilak Israeleme. Angutelo Leviteôjok tujormiolaukpok Efraimib kakkangata sivinganingane, pannervgarnilaukporlo Bethlehemit Judamêtomit.

2 Senniagullo ajortullilaurame kemmavâ, atâtame iglunganut Bethlehememut Judamêtomut, tagvanêlunelo takkit sittamat nâlugit.

3 Uingalo parngnailerpok, malliklugolo, pitsiarlune okarkattigijomavlugo, aijomangmilugo ingminut, aipakalunelo nuka- 
piamik, siutekoktojungniglo maggungnik. Tapsomalo atâtame iglunganut ittertipa. Arnablo atâtangata tækkogamiuk kuviasulerpok illelliorpalo.

4 Sakkingatalo, arnab atâtangata inapa, uvlunik pingasunik uvlilerkovlugo; nerrilutiglo imerlarmiglo siniktarlutiglo unnuarme tagvane.

5 Uvlut sittamangane uvlarme makkijajovut, nikkoviporlo aularomavlune. Tagva arnab atâtanga ningaungminut okarpok: Omattit nekkoksitikâruk niakojârsungmut, kingorngane aulartuksauvose.

6 Ingilaukpuglo nerrikattigêlutiglo imerlarmiglo. Tagva arnab atâtanga angumut tapsomunga okarpok: Nagliktara, unnuak ovanelerit omattillo serrilerle.

7 Angulle makkimat aularomavlune, sakkingata unniksarpa sinniktarkovlugo tagvane.

8 Uvlut tellimangata uvlângôningane makkijajovok aularomavlunelo. Tagva arnab atâtanga okarpok: Nagliktara, omattit nekkoksititsuk, tuavitaililutalo uvloklilerkârtinago; taimaglo nerrikattigêkpuk.

$9 \quad$ Angullo nikkovipok aularomavlune panervgane nukapiako tinelo illagællugik, sakkingatale, arnab atâtangata okautiva: Ahâk uvloklilerpok unnuleromalunelo, unnuak ovanêlerit. Ahâk sinniktarvikarpok sulle uvlome, unnuak ovanelerit, omattillo serrilerle; kaupat makkijajovose aularluselo apkotipsingne iglungnut.

10 Angulle tamna sinniktaromalaungilak, parngnailerporle aularlunelo, tikkilarmelo Jebusib, tagva Jerusalemib sânganut; najortekalaukporlo siutekoktojungnik maggungnik nangmartungnik, pannervgaminiglo aipakarivok.

11 Jebusiblo kannitanginut pigamik uvloklilerpok. Nukapiarlo angajokaminut okarpok: Nagliktara, Jebusiterit iglugaseksoanginut tamakkonunga itterta, sinniktarlutalo unnuak.

12 Angajokangale okarpok tapsomunga: Tækkorngartat, Israelib kittornganginut illingalungitut iglugaseksoanginut itteromangilagut, akkianulle pijomavogut Gibeamut.

13 Okarporlo nukapiakotiminut: Kait, sivumuarta kaglilerkovluta inniksamut sinniktarviptingnut, Gibeamullonêt Ramamullonêt.

14 Sivumuarpullo arvertarlutiglo, sekkinerlo nippivok Gibeab Benjaminemêtub kannitanganêtillugit.

15 Sâlerpullo tagvunga, itteromavlutik sinniktaromavlutiglo Gibeame. Itteramele ingipok iglugaseksuit apkosininganut; sinniktarkojijokalaungimat tapkoninga unuarme iglomine.

16 Ahâglo, tagva angut îtok kaivok sullinerminit perorsêvingmit unnuame; Tamnatauk Efraimib kakkanginit pilauk- 
pok, tujormijolunelo Gibeame; iglugaseksuille innungit Jeminib kittorngarivait.

17 Tækkosaramelo, tækkolunelo tujormijomik apkosinermêtomik iglut akkorngane, okautiva: Namungâromavît, nakillo pivît?

18 Tapsomale okautiva: Bethlehemit Judametomit pivogut, ingergarlutalo Efraimib kakkangata ikkianganut nunakarvimnut; Bethlehemullo Judametomut ailaukpogut, ailungalo manna Nalekab iglunganut, siniktarvikarkojijokangilarlo uvamnik.

19 Ivikarpogut nerkiksakalutalo siutekoktojokotiptingnut, niakojakalutalo, Weinekalaptalo uvamnut kivgarnullo arnamut, nukapiamullo kigavit illagijagingnut; ajoksarniangilagut sunamik.

20 Itôk tamna okarpok: Ullapirsautekarit! Kingomajattit tamaita naipipattit uvamne; unnuakulle iglut akkorngane apkosinermetuinarniarnak.

21 Tessiorpâlo iglominut, siutekoktojuiglo nerripkautiksamik tunnitsivigivak, ittikatiglo ubvarpait, nerrilutiglo immerlarmiglo.

22 Omattingille serridlartillugit manna, ahâk, tagva iglugaseksuit innungit uivêtoâluit tikkiput, iglulo illumavât, avilortarlutiglo upkoamut, okarlutiglo îtomut, iglub angajokanganut: Angut iglungnut tikkitok annitileruk, illitarksilerkovluta tapsominga.

23 Angulle iglub angajokanga annivok tapkonunga, okautilugillo: Aukak kattangutikka, taimak idluitulliniarase; angut tamna itterlaukârmat iglumnut, taimaitomik sillainekartitsiniarase.

24 Ahâk pannekarpunga uigasôjomik sulle, tamnalo pannervgakarpok, tapkoa anititsomavâka illipsingnut, tapkoa unêt sujuktitsungnarpasse, pinniarvigisigiglo pijomajapsingnik; angulle tamna sillainermik taimaitomik pinniarviginiarassiuk.

25 Innuille nalegomalaungilât. Tagva angutib tapsoma pannervgane tiguva annitilâlo, tamna illitarilerpât, attornerluglugolo unnuak nâlugo, kau tikkilugo; uvlogatâlermallo aularkovât.

26 Tagva arnak tamna uvlogatâlertillugo kaivok, pâlaklunelo iglub angajokame najortangata upkoangata sânganut, nellalunelo tagvane kaulermarikârtinnago.

27 Angajokangalo uvlârme makkimat, iglublo upkoanga upkuêrmago, anningmallo aularomavlune; ahâkk, tagva pannervganga nellavok iglub upkoangata sângane, aggangillo kattaub manoanganêlaukput.

28 Tapsomale okautiva: Makkitit, aulartalo. Tamnale kiolungilak. Tagva siutekoktojokotiminut illiva, parngnailerpok aularlunelo nunagijaminut.

29 Angeraramelo manna, savik tiguva, pannervganelo tigumiarlugo pivok, avgorpalo sauningit illaliudlugit 12 ôlivlugolo, tillikliutilaitalo Israelib kiglilervinginut tamainut. 
30 Kinalo tækkolauktok tamattominga, tamna okarpok: Taimaitomik tækkojokalaungilak, pijokalaungilarlo, Israelib kittorngangita aularninganit Egiptenemit, uvlok tamanna tikkilugo. Manna tamanna issumaksarsiorutigisiuk, attannioriarluselo, okarluselo issumapsingnik.

\section{KAPITEL XX.}

\section{Kinguvarêt Benjaminekut nungutaukasalerput.}

TAGVA Israelib kittorngangit Danemit Bersaba tikkidlugo, nunamillo Gileademit, aularput kattimalerlutiglo attautsemut, angutetut attautsetut sorlo, Mizpamut Nalekab sânganut;

2 Israeliblo kinguvarêngita tamaita Gudib illagêktokotingine, innugiartoksoangita angajokangit kattimalerput, angutit pissuktut 400,000 dit saviksoalijarlauktut.

3 Benjaminible kittorngangit tussarlaukput, Israelib kittorngangit takpaungarlaungmatta Mizpamut. Israeliblo kittorngangit okarput: Okaritse, kannok idluineâluk tamanna pilauka?

4 Tagva Levite taimna, arnab tokkotaumajub uinga kiovok okarlunelo: Gibeamut Benjaminemêtomut pilaukpunga panervgara illagællugo, sinniktaromamut tagvane.

5 Tagva Gibeamiut opalaukpấnga, iglomelo unnuarme illumalaukpânga, tokkotsomavlungalo, panervgaralo attornerlulaukpât tokkolerkârtinago.

6 Tagva panervgara tigulaukpara avgorlugolo, tillikliutilugolo Israelib kingormgutijangata nunanginut illunainut; kuksalainermik sillainermiglo pilaungmatta Israeleme.

7 Ahâk illipse kittorngaujose Israelemut illunase manêpose; issumapsingnik, kannorlo pinniaromanipsingnik okaritse.

8 Tagva innugiartoksuit tamaita nikkovilerput angutetut attautsetut sorlo, okarlutiglo: Ittertokartuksaungilak kinamik tupperminut, uttertokartuksaungilarlonêt iglominut,

9 Imagle pinniaromavogut manna Gibeamut:

10 Immerarta; tigusilutalo angutinik tellimaujortunik 100 dinit, 100 diniglo 1000 dinit, 1000 diniglo 10000 dinit, Israelib kinguvarênginit tamainit, tigusikovlugit nerkiksanik innugiartoksoarnut, aikovlugit pinniarkovlugillo Gibeamut Benjaminemêtomut, tamaita sillainingit sakkertilauktangit Israeleme malliklugit.

11 Taimak Israelitit angutingit illunamassiatik kattimalerput angutetut attautsetut sorlo, iglugaseksoarnut taipkonunga, angerkattigêlutiglo.

12 Israeliblo kinguvarêngit tilliklerput angutinik Benjaminib abvakunginut tamainut, okautsaukolugillo: Kannok idluitoksoniôva, piniarnerijaulauktok illipsingne? 
13 Tagva manna angutit taipkoa, uivêtoâluit Gibeamêtut unertotigisigik uvaptingnut, tokkotsikovluta tapkoninga, pêjaikovlutalo idluiniâlungmik mattominga Israelemit. Benjaminible kittorngangita kattangutimik Israelib kittorngangita nippingat nalegomalaungilât.

14 Iglugaseksoarnille kattimalerput Gibeamut, aularomavlutik unatadlarnermut Israelib kittornganginut.

15 Nelliutomelo taipsomane Benjaminib kittorngangit iglugaseksoarnit tamainit kittitaulaukput, angutit 26000 dit saviksoalijartut, Gibeamiut pinagit, tapkoa kittitaulaukput 700 dit, angutit annerijaumajut.

16 Innugiartoksuillo tapkoa akkorngane angutekalaukpok 700 dinik annerijaumajunik, saumiolauktunik, illoreumullo nujak uvigarungnarlaukpât uniornagolo.

17 Israelitille (Benjaminekut illaliutinagit) kittitaulaurivuttauk angutit 400000 dit, saviksoalijartut, sorsugungnartullo illunatik.

18 Tapkoa parngnailerput, takpaungarlutiglo Gudib iglunganut, Gudelo appertsorpât, okarlutiglo: Kikut uvaptingnit aularkârtuksauvât, unatadlarnek pigiarlugo Benjaminib kittornganginut? Nalegak okarpok: Judakut pigiartuksauvut.

19 Taimak Israelib kittorngangit uvlarme parngnailerput, tangmarlutiglo Gibeab sivorânut.

20 Angutillo Israelitit illunatik annivut sorsugomamut Benjaminekunut, oppalungaijailutiglo Gibea sorsugvigilugit.

21 Tagva Benjaminib kittorngangit annisarput Gibeamit, ullimarnilutiglo ochotitsivut nunamut uvlorme taipsomane Israelitit akkorngane 22000 dinik.

22 Innugiartoksuillo, angutit Israelitit maksoatilerput oppalungaijailutiglo ama sorsugomavlutik, tagvane uvlub sivorlingane oppalungairvigilauktamingne.

23 Israeliblo kittorngangit takpaungarput, kugviorlutiglo Nalekab sângane unnuk tikkilugo, Nalegarlo appertsorpât, okarlutiglo: Ama aijuksauvita sorsukluta Benjaminib kittornganginut kattangutiptingnut? Nalegak okarpok: Takpaungarvigisigik.

24 Israeliblo kittorngangita uvlub aipangane Benjaminib kittorngangit najulermattigik,

25 Benjaminekut annisârput Gibeamit, taipkoa pârlugit uvlorme taipsomane, ullimarnilutiglo, ochotitsilutiglo nunamut ama Israelib kittornganginit 18000 dinik, saviksoalijartunik illunainik.

26 Tagva Israelib kittorngangit illunatik, innugiartoksuillo illunatik takpaungarput, kailutiglo Gudib iglunganut, kugviorlutiglo, uvlilutiglo tagvane Nalekab sângane, nerrinatiglo uvlorme taipsomane, unnuk tikkilugo, tunnijilutiglo tunnergutinik ikkoalajaksanik, tunnergutiniglo kujalijutiksanik Nalekab sângane. 
27 Israeliblo kittorngangita Nalegak apperivât, (Gudible angernerilauktangata iklervinga taipsomane tagvanelaukpok;

28 Pinehaselo, Eleaserib Aronib erningata erninga nangerlaukpok tapsoma sângane nelliutome taipsomane) okarlutiglo: Ama aulartuksauvita sorsukluta Benjaminib kittornganginut kattangutiptingnut, nokkartuksauvitalonêt? Nalegak okarpok: Takpaungaritse, kaupat tunnijomavakka aggapsingnut.

29 Israeliblo kittorngangit uttakkijokartitsivut terliarsivingne Gibeab avatâne.

30 Taimaglo Israelib kittorngangit avlut pingajuane takpaungarput Benjaminib kittornganginut, oppalungaijailerlutiglo Gibeamut, sorlo sivorngane maggoêrtorlutik pilauramik.

31 Tagva Benjaminib kittorngangit annisadlalerput innugiartoksuit pârlugit, nulerlutiglo iglugaseksoarnit, pigialerlatiglo ullimarniklutik killerniglutiglo innugiartoksoarnit, sorlo sivorngane maggoêrtorlutik pilauramik, natername, apkosinerne maggungne, aipanga Bethelemut sangmijome, aipanga Gibeamut sangmijome, angutinik Israelitinik 30 ginik.

32 Tagva Benjaminib kittorngangit issumalaukput: Salagijaumavut sâptingne sivurnganetut. Israelible kittorngangit okarput: Kemâlerta nutjikovluta tapkoninga iglugaseksoarnit apkosinermut.

33 Tagva Israelib angutingit illunatik innimingnit annivut, oppalungaijailutiglo Baal-Thamareme. Israeliblo uttakkijokotingit terliarsivingme annivut innimingnit, kairosungmit Gabamik attelingmit,

34 Gibeamullo tikkiput, angutit anerimajut Israelitinit tamainit 10000 dit, unatadlarnek songolaungmat; nelludlalaukpulle kannoêtub tikkiniarmagit.

35 Taimak Nalekab Benjaminekut ullimârpait Israelib kittorngangita sângane, Israelib kittorngangita uvlorme taipsomane asserorlaungmagit Benjaminekunit angutit 25100 dit saviksoalijartut.

36 Benjaminible kittorngangit tækkolaungmatta salagijaunermingnik, Israelib angutingita iniksakartipait; uttakkijut terliarsivingme Gibeametilauktatik sungertutigigamigit.

37 Terliarsivingmêtullo tuavilaurivut, annilutiglo Gibeamut, najutilutiglo, ullimarnilutiglo iglugaseksoarmiunik taimainik saviksub kênanganut.

38 Nellunaikutekartigêlaukpulle Israelib angutingit, terliarsivingmêtullo, saviksoarmut opaklugit, isserluk iglugaseksoarnit kongmuarajarpat.

39 Israeliblo angutingit manna sangulaungmatta unatadlarnerme, Benjaminekullo pigiarmatta ullimarniklutik; killerningmattalo Israelitinit angutinik 30 gekassangnik, issumakarmatta, salagijaumavut sâptingne, sorlo unatadlarnerub tamattoma sivorlinganetut; 
40 Tagva isserluk pikkunga kongmualerpok iglugaseksoarnit, Benjaminekullo keviarput tunnumut; ahâglo, tagva ikkomaub ikkoalaninga kongmuarpok iglugaseksoarnit.

41 Israeliblo angutingit sangulaurivut koaksartilaitalo Benjaminekut; takkolauramik kannoêtub tikkitsomangmagit.

42 Tamanna pivlugo sangulerput Israelib angutingita sângine, apkome sunakajuitomut sangmijome; unatadlarneruble mallikpait; taimaglotauk iglugaseksuit assinginit tamattomunga (unatadlarnermut) pilauktut asserorpait.

43 Illumavaillo Benjaminekut udlapaillo Menuah tikkidlugo, tullerarpaillo Gibeab sivorânut tikkilugo, kittânut.

44 Benjaminekunillo ochovut angutit 18000 dit, illunatik anguteôlauktut sorsugungnartut.

45 Tagva sangulerput kemâlutiglo sunakajuitomut, kairtomut Rimonemut; apkomele tamattomane ullimarpait angutit 5000 dit, mallikpaillo Gideome tikkilugo, ullimarpaillo tapkonangat 2000 dit.

46 Taimaglo ochovut Benjaminekunit uvlorme taipsomane angutit 25000 dit, illunatik saviksoalijartut, illunatiglo angutaulauktut sorsugungnartut.

47 Angutit 600 dituinait sangulerput kemâlutiglo sunakajuitomut, kairtomut Rimonemut, kairtomelo Rimonemêlaukput takkit sittamat nâlugit.

48 Israeliblo angutingit utterivut Benjaminib kittornganginut, ullimarnilutiglo sàviksub kênanganut iglugaseksoarne, tamangnik inungnik nergjutiniglo, naipitaujuniglo tamainik; iglugaseksuillo tamaita naipitaulauktut ikkitauvut ikkomamut.

\section{KAPITEL XXI.}

\section{Kittorngarêt Benjaminekut nulliakartitauninginit.}

\section{I} SRAELIBLE angutingit angermarilaukput Mizpame, okarlutiglo: Kialonêt pannine tunnijaksaringila Benjaminekunut nulliaksamut.

2 Innugiartoksuillo kailaukput Gudib iglunganut, tagvanêlutiglo Gudib sângane unnuk tikkilugo, kagverlutiglo nippimingnik, kêavaksoadlarlutiglo,

3 Okarpullo: Nalegak Israelib Gudinga, sôg tamattominga pijokalauka Israeleme, uvlome Israelib kittorngarêngit attautsenik ikkinersaulersimangmatta?

4 Kaub aipangane innugiartoksuit makkijajovut, sennalutiglo tagvane Altaremik, tunnijilutiglo tunnergutinik ôtaksanik, kujalijutiksaniglo.

5 Israeliblo kittorngangit okarput: Kikkogalloarkât Israelib; 
kittornganginit takpaungarkattaulungitut illagêt illagællugit Nalekamut? Angijomik angernermik pijokalaungmat, takpaungarlungitok Nalekamut Mizpamut tokkotaksaungmat.

6 Israeliblo kittorngangita Benjaminekut, kattangutitik kakkialerutigivât, okarlutiglo: Uvlome kinguvarêt attautsit naviksimavut Israelekunit.

7 Kannok pinniarvigijomavittigik amiakungit nulliakalerkovlugit? Angermarilaurapta Nalekame, panniptingnit tunnitsivigijomanginaptigik nulliaksanik.

8 Okarpullo: Kikkogalloarkât Israelib kittorngarênginit takpaungarlungitut Nalekamut Mizpamut? Ahâglo, tagva pittakalaungilak kinamik illagêt tangmarvingine Jabesemit Gileademetomit.

9 Innugiartoksuit kittilaungmagit, ahâglo tagvanetokalaungilak Jabesemiomik Gileademetomit.

10 Tagva illagêt tagvunga tilliklerput angutinik 12000 dinik, angutinik sorsugungnartunik, perkolugillo, okalarmiglo: Aileritse ullimarniluselo saviksub kênanganut Jabesemiunik Gileademetomiunik, nulliangit kittorngangillo illaliudlugit.

11 Imâgle pijuksauvose: Anguteôjut illunaita, arnallo innangalauktut angume, asseroraksarivasse.

12 Jabesemiunelo Gileademetomiune nachvarput arnanik 400 dinik, uigasojunik, inangalaungitunik angume, tapkoa âpait tangmarvingmut Silomut Kanaanib nunanganetomut.

13 Tagva illagêt illunatik tilliklerput okarkattigêkojilutiglo Benjaminib kittornganginik, kairtome Rimonemetunik, kaikolugillo ullapirsarnartomik.

14 Taimak Benjaminib kittorngangit kailaurivut nelliutome taipsomane, tunnitsivigivaillo arnanik tapkoninga, pilauktamingnik arnanit Jabesemit Gileademetomit, nachvarungnaipullo taimaitunik.

15 Tagva innugiartoksuit Benjaminekut kakkialerutigivait, Nalegak Israelib kittorngarêngine koppakartitsilaungmat.

16 Illagêktullo angajokangit okarput: Sunamik pinniaromavita assingittauk nulliakalerkovlugit? Arnat Benjaminekune pêjarsimangmatta.

17 Okarpulle: Amiakojut Benjaminekunit kingormgutigijatik pigênaraksarivât illa, kinguvarêt attautsit nungutaukonagit Israelemit.

18 Uvagullo panniptingnik tunnitsivigijungnangilavut nulliaksanut. Israelib kittorngangit angerkattigêmarilaungmatta, okarlutiglo: Ominarsijaule tunnijijok nulliaksamik Benjaminekunut.

19 Okarpullo: Ahâk uvloksiorviôvok Nalekamut Silome avanêtome Bethelib akkilinganetome, kittâne, apkosinerme takpaungarviojome Bethelemit Sichemut, Lebonablo sekkerngane. 
20 Benjaminiblo kittorngangit perkovait okautilugillo: Aileritse uttakkiluselo nappartune Weineliksane.

21 Tækkoniarupselo tagva, Silomiut panningit annilermatta imgerlutik okkigêsermut, tagva annisâdlaritse nappartunit Weineliksanit, attunnîllo nulliaksamik tigusileritse Silomiut panninginit, ailuselo Benjaminekut nunanganut.

22 Atâtangille kattangutingillonêt kainiarpatta akkiortaukoluse, okautijomavavut: Napkigisigik pivluta, angutinut illunainut nulliaksamik pilaunginapta unatadlarnerme; illipselo tunnijomalaunginapsigik tapkonunga, passijaksauvose tamattomane.

23 Benjaminiblo kittorngangit taimailiorlaukput, tigusilutiglo nulliaksanik unnurnitik malliklugit, okkigêsertunit, aksarnigarijamingnik, aularlutiglo nunakalarmiglo kingormgutijamingne, iglogaselliorlutiglo iglugaseksoarnik, innigivaillo.

24 Israelib kittorngangittauk taipsomane aularput tagvangat attunît kinguvangukattimingnut illamingnullo, aularlutiglo attunît kingormgutijamingnut.

25 Nelliutome taipsomane attannekalaungiląk Israelekune; attunit pinniarput namagijamingnik.

\section{Ruthib aglangit.}

mantom

\section{KAPITEL I.}

Ruth ingergarpok Naemi illagcellugo Bethlehemut.

NELLIUTOME taipsomane idluarsaijut attanniotillugit, kângnartokalaukpok nuname. Angullo Bethlehemiok Judamêtomiok, nûlaukpok ingergarlune Moabiterit nunanganut, nulliane ernangnelo illagællugit;

2 Attekalauktok Elimelechemik, nulliangalo Naemimik, ernigiglo aipanga Mahlonemik, aipalo Chil-Jonemik, Efratemiongôlaukput Bethlehemit Judametomit. Tikkinamiglo Moabiterit nunanganut tagvanetualaukput. 
3 Elimelechelo Naemib uinga tokkovok, tamnalo amiakolerpok ernangne illagællugik.

4 Tapkoa nullianikpuk arnângnik Moabitereôjungnik. Aipanga attekalaukpok Arpamik, aipa Ruthemik. Tagvanelo nunakalaukârlutik Jahrinik tellimaujortunik,

5 Tamarmik tokkolerpuk, Mahlon Chil-Jonelo; arnarlo amiakolaukpok ernangminit uingminillo.

6 Tagva parngnailerpok ukkuangne illagællugik, aularmilunelo Moabiterit nunanganit; tussarlaurame Moabiterit nunangane, Nalekab innukotine nellipsarlaungmagit, kakkojaksamiglo tunnitsivigingmagit.

7 Annilaukporlo innimit, innigilauktaminit, ukkuakitalo illagivâk. Pissuklunelo apkome, tikkitsomavlune Judakut nunanganut,

8 Ukkoangminut okarpok: Aileritik utterlutiglo attunnit anâname iglunganut. Nalekab napkiningnermik pinniarvigilitik sorlo illiptik tokkungajune, uvamnelo pilauraptik.

9 Nalekab tunnitsivigilitik, innilanganermik nachvarkovlutik, attunit uime iglungane; kunnikpâglo. Tagva nippitik kagvarpâk kêalutiglo,

10 Okautivâglo: Illagællutit nunakotingnut aijomavoguk.

11 Naemile okarpok: Uttileritik pannîka! Sôg illagijomavitinga? Kannok pijomârtome ernekarungnarniarkîk timimne uingolerniartungnik illiptingnut?

12 Uttileritik pannika ailutiglo; manale ningiongoluarpunga uingnigungnarnermut. Okarajaralloarumalo: Nerriungnarpok unuarme tamattomane uingningniarama, kittornganiglo erniniarama,

13 Kannorle uttakkijungnaralloarkitik innumariolerninginik? Kannok mulludlartuksauvitik uingnigatik? Aukak, panîka! naiperkotigidlaraptik; Nalekab aggangita akkerartorlunga tikkidlarmanga.

14 Tagva nippitik kagvarpakik kêanersaulutiglo sulle. Arpablo sakkine kunnikpa; Ruthele tapsomanêtuarpok.

15 Tamnale okarpok: Ahâk angajungoit utterpok innukotiminut Gudiminullo; igvittauk utterit angajungoit malliklugo.

16 Ruth kiovok: Okarviginiarnanga kemmajaksarigapkit, uttertuksaugamalo illingnit. Aivigijarnut, tagvunga aijomagivunga; 'nokkarvigijarne, tagvane nokkaromagivunga; innukotitit innukotigivakka, Gudillo Gudigivara.

17 Tokkoniarvingne, tagvane tokkoniarivunga, tagvanelo illijartortaujomagivunga. Nalekab imâk imâglonêt pinniarvigilinga, tokkub avititaksarivâtiguk.

18 Tæklkogame manna, attangusivlune issumakarmat aiparijomavlugo, okarvigijungnailerpa tamattominga.

19 Taimak tapkoa ingiarkattigêkpuk, Bethlehemut tikki- 
kâratik. Bethlehemullo pingmannik iglugaseksoarmiut illunatik innilangajuêrutigivakit, okarlutiglo: Tamna Naemiôva?

20 Tamnale okarpok: Tainiarassinga Naemimik, taingarsingale Maramik, ajugakangitub angijomik kiksartilaungmanga.

21 Pittakaloriklunga aularlaukpunga, angênarlungale Nalekab angerartilaurivânga. Sôgle Naemimik taivissinga? Nalekab miklilertilauralloarmanga, ajugakangitublo kiksartimanga.

22 Kattersivigle Gerstenik nelliutilaukpok Naemi ukkuangalo Ruth, Moabitereổjok, utterlaungmannik Moabiterit nunanganit Bethlehemut.

\section{KAPITEL II.}

Ruth katiersivok sullugainernit Boasib perorsêvingane.'

ANGUTEKAlaUkPOKTAUK, Naemib uingata illanarijanganik, Elimelechib illanginit, Boasemik attelingmik, anguteôlauktomik perkutelingmik.

2 Ruthelo Moabitereôjok Naemimut okarpok: Perorsêvingmut ailaulanga, sullugainerniglo kattersilunga, tamna malliklugo saimaneksivigijara. Tapsomale okautiva: Ailerit pannîk. 3 Tamna aivok, tikkilune kattersivok perorsêvingme kibluijut malliklugit. Nellautsivorlo perorsêvik tamna perkutaulaungmat Boasemut Elimelechib illanginit pijomut.

4 Ahâglo, Boase Bethlehemit pivok, okarlunelo kibluijunut: Nalekab illagilise! Tapkoa kiovut: Nalekab saimartilitit!

5 Boaselo kivgaminut kibluijunut angajokangortitaumajomut, okarpok: Kia arnak una pigivauk?

6 Kivgarlo angajokangortitaumajok kibluijunut kiovok, okarlunelo: Arnaurok tamna, Noabitereôjok, Naemi illagællugo utterlauktok Moabiterit nunanganit.

7 Okalaungmat: Nægliktara, kattersikolaunga kippitat akkorngane, kibluijut malliklugit; taimaglo tikkipok sullilunelo tagvane uvlamit manna tikkilugo, iglominelo annikitotuinarmik nokkarpok.

8 Tagva Boas Ruthemut okarpok: Tussarkît pannîk? Perorsêviub oma assianut aijuksaungilatit, kattersilutit; nulerniarnaglonêt ovangat, arnakotimnetualerille;

9 Tækkolutillo nane kibluivigijanganik perorsêvingme, tagvunga mallikit. Angutekotikka perkolaukpakka ullapitaukonak kinamut. Immerujarangavillo, ailerit immerkaumut, nukapiakotima kalluvigijanganut, immerlutillo.

10 Tagva pamakterpok kênane pûvlugo, tuksiarlunelo nunamêdlune, okautivalo: Sunamut saimaneksivîk ijikpît sângangne, illitarigamga, tskkkorngartaugalloartunga? 
11 Boase kiovok, okautilâlo: Okautsaumavunga tamainik pinniarnerilauktarnik sakkingnut, uikpit tokkungata kingorngane; atâtait anânaillo, innulervîllo kemalaurangne, innukattigêksoarnullo tikkisimavlutit illitarilaungitarnut sivorngane.

12 Nalekab pinniarnerilauktat akkilêjutigiliuk illingnut, akkilertaunîllo namamarigle Nalekame Israelib Gudingane, tikkivigijarne; esarkungita attâne sungertutiksakaromavlutit.

13 Tamna okarpok: Saimaneksilaunga ijikpit sângangne, Nalegâk; mannigoramga, kivgallo pitsiarlutit okarvigivat, kivgavit illangatitun îlungitungalonêt.

14 Boasib okautiva: Nerriviksaulerpat najutilerit, kakkojaktorlutillo, tamuatillo missuktiuk sêrnartomut. Tamnalo ikterpok kibluijut senianut. Tapsomalo manijervigiva kakkojaksanik jarrasimajunik; tamnalo nerrivok akkeartorlunelo amiakokartitsilarmelo.

15 Nikkovingmallo kattersilune, Boasib nukapiakotine perkovait, okarlunelo: Kippitat akkornganetauk kattersikogisiuk kangusuktiniarnagolo;

16 Kattersimajunittauk amiakokartitsileritse, kemailuselo, kattersikovlugo tamattominga, suartokarniaranelo tapsominga tamanna pivlugo.

17 Taimak kattersivok perorsêvingme unnuk tikkilugo, katjuklugillo kattersorlauktane; Efalo tættækasalaukpok Gerstenik.

18 Kivikpaillo, ailunelo iglugaseksoarnut, sakkingatalo tækkovait kattersorlauktangit. Tagva sagvarpa tunnilâlo tapsomunga amiakogilauktane akkeartornekogilauktaminit.

19 Tagva sakkingata okautiva: Nane uvlome kattersilaukît, nanelo sennalaukit? Saimartitaule illitarksilauktok illingnik. Tapsomale sakkine okautiva, kiname sennavigilauktamine, okarlunelo: Angut sennavigilauktara uvlome, attekarpok Boasemik.

20 Naemile ukkuaminut okarpok: Saimartitaule Nalekamut, takkopsoanine sapkolaunginamiuk, tamangnut innôjunut tokkungajunullo. Naemilo okarpok tapsomunga: Angut tamna illagivavut, kingormgujijuksangôlunelo uvaptingnik.

21 Ruth Moabitereôjok okarpok: Okautilaurivânga: Nukapiakotimnut illautsainartuksauvotit, kattersikârtinagit tamainik perortokotimnik.

22 Naemi Ruthemut, ukkuaminut okarpok: Namakpok pannîk, tapsoma arnakotingitaularkattigigungne, innertertaukonak assiata perorsêvingane.

23 Taimak Boasib arnakotinginut illautsainarpok, kattersilunelo, kattersivik Gerstenik Weizeniglo soraikârtinago; utterlunelo sakkiminut. 


\section{KAPITEL III.}

Ruth winingniarnets pivlugo nellojingnaititanlerpol.

NaEMILo, tapsoma sakkia, okarpok tapsomunga: Pannîk, innilangavikartitsomavagit, innôtsiarungnarkovlutit.

2 Manna Boase, illagijavut arnakotinginevigilauktat, unnuarme tamattomane sanêjaivok Gerstenik katjugvingmine.

3 Tagva ubvarit illingnik, mingoarlutillo, attilutillo annorângnik, attersarillo katjugvingmut; illitarijauniarnagle, nerrijarêrkârtinago immerêrkârtinagolo.

4 Innarniarpalle, tagva ine innangavia kamagiuk, kailutillo, ittiggangillo kebbêjarkit innarlutillo: tagva okautiniarpallukpâtit, pinniaraksarnik.

5 Tamna okarpok: Tamainik okauserijarnik uvamnut, pinniaromavunga.

6 Tamna attersarpok katjugvingmut, pinniarlunelo tamainik, sorlo sakkia perkojilaungmat.

7 Boaselo nerrijarêrame immerêramelo, omattinga serridlalaukpok, kailunelo innarlarmelo, kippitat kakkojaksataggit kallerêktisimajut nellânut; tamnale terrêlune kaivok kebbêjarpaillo ittiggangit, innarlunelo.

8 Unnuak ketterarmat manna, kvaksarpok angut kakkilarlunelo; ahâglo, arnak innangavok ittiggangita kiglingane.

9 Tamnalo okarpok: Kinauvît: Arnak kiovok; Rutheôvunga kivgat, esarokotitik issivikik kivgarnut, kingormgujijôgavit.

10 Angut okarpok: Saimartitaulerit Nalekamut, pannîk; napkiningnermik namanersamik sakkertitsilaukpotit kingorlermik sivorlermit, angutit innuosuktut mallilaunginangne, akluitullonêt, aklujullonêt.

11 Manna pannîk, sivoraniarnak; tamainik okauserijarnik pinniarvigijomavagit; iglugaseksoarmiut innukotikka illunamassiatik kaujimangmatta, arnaugavit illusitsiariktok.

12 Mannalo miksekârtôvok, uvanga kingormguijôgama; pittakarporle attautsemik kanninersaujomik uvamnit.

13 Sinniktarit. Kaupat, nulliagikpattit, namakpok; tussulungikunele tigulutit, tagva uvanga tigujomavagit, sorlo miksekâ̂rtomik Nalegak inôngmat. Sinnilerit kauk tikkidlugo.

14 Tamnalo sinnilaukpok ittiggangine kaumut. Makkilaukporlo illitarksiutijungnarkâratik. Tamnalo issumavok kaujijauniarane, tikkitokalaungmat arnamik katjugvingmut,

15 Okarmilunelo: Ulliksoak attigijat kaitsuk tigumertolerlugolo, tapsomalo tigumertopa. Tamnalo angut ôktulerpok ôktutinik pingasojortunik Gerstenik, illilugillo tapsomunga. Tamnalo angut tikkilerpok iglugaseksoarnut. 
16 Tamnale arnak pivok sakkiminut, tamna okarpok: Kannok illingavît, pannîk? Tamainiglo okautiva, angutib taipsoma pinniarnerilauktanginik tapsomunga.

17. Okarlunelo: Makkoa ôktutit pingasojortut Gerste tunnilaukpait uvamnut, okarmat: Angênarlutit tikkituksaungilatit sakkingnut.

18 Tamnale okarpok: Innilangalerit pannîk, tukkisikârtinak, kannok illingajokarniarnermik; angut tamna innilanganiangimat naggaserkârtinane tamattominga uvlome.

\section{KAPITEL IV.}

Boasib Ruthe nulliagilerpa, saimausertaulunelo.

BOASE takpaungarpok itterviksoarmut, ingilunelo tagvane. Ahâglo, kingormguijuksak taimna kângermat, Boasib okarkattigiva okarlunelo: Kailaurit, ingilutillo maunga, tamaungalônet. Kailaukporlo ingilunelo.

2 Illasearivaillo angutit tellimaujortut, iglugaseksuit angajokanginit, okarlunelo: Ingileritse tamaunga. Tapkoalo ingilerput.

3 Tagva kingormguijuksamut okarpok: Naemib, Moabiterit nunanganit uttersimajub, perorsêvik, kattangutipta Elimelechib perkutigilauktanga niorvgotigijomava.

4 Tamanna pivlugo issumakalaukpunga, kaujititsomavlutit tamattominga, okautitsomavlutillo: Kingormgutijomagungne, tagva pissiariuk iglugaseksoarmiut innukotimalo angajokangita sângine; kingormgutijomangikungne, tagva okautilaunga, kaujimakovlunga tamattominga; kingormgujijokangimat, illingnit assianik, kingunikullo uvamnit. Tamna okarpok: Kingormgutijomavara.

5 Boase okarpok: Uvlorme pissiniarvigijarne perorsêvingmik mattominga Naemib agganginit, Ruthetauk Moabitereôjok, tokkosimajub nulliagilauktanga tigujaksarivat, attekartitsikovlutit tokkosimajomut kingormgutijanganut.

6 Tagva okarpok: Kingormgutijomangilara, asseroinajarkonanga imakkâ kingormgutijamnik. Igvit kingormgutileruk kingormgutijaksarijara; kingormgutijomanginapko.

7 Itsaksoarmille Israelitine illusiolaukpok; Kia perkut kingormgutijomalaungikuniuk, pissiarinagolonêt, tagva, tamanna sokkosijuikovlugo, ittiggagutingme iglua pêrpa, tunnilâlo assiminut; tamanna kigligiudsiniôlaukpok Israeleme.

8 Kingormgujijuksarlo tamna okarpok Boasemut: Igvit pissiariuk; perpâlo ittiggagutime iglua.

9 Boaselo okạpok angajokajanut, innugaseksoarnullo illu- 
nainut: Illipse uvlome kigligiudsijôvose, tamaita pissiarimagapkit, Elimelechib perkutigilauktangit; Chil-Joniblo Mahloniblo perkutigilauktangit tamaita, Naemib agganginit;

10 Tamakkoa tappilugit Ruthetauk, Moabitereôjok, Mahlonib nulliagilauktanga, nullianingniarpara, tokkosimajomut attekartitsikovlunga kingormgutijanganut, attinga pêjartaukonago kattangutime akkornganit, iglugaseksoakotinelo itterviksoanganit; kigligiudsijôvose tamattominga uvlome.

11 Inugaseksuillo itterviksoarmelauktut, angajokajat illagællugit okarput; Kigligiudsijôvogut. Nalekab arnak tamna, iglungnut piniartok Raheletut Leatullo îtiliuk, tamarmik Israelib iglunganik sennalauktutitut, pivalliadlarlelo Efrateme, nertortaulelo Bethleheme.

12 Iglullo Perezib iglungatut ilerle, Thamarib erniarilauktangata Judamut, kinguvâksamit Nalekab tunniniartanganit illingnut, arnamit omangat.

13 Taimak Boasib Ruth tiguva nulliarivlugo. Inangakattigigamiuglo, Nalekab tunnitsivigiva singailerkovlugo, ernermiglo ernilaukpok.

14 Tagva arnat Naemimut okarput: Nalegak nakorijaule, pekartitsitaililaungitok illingnut kingormgujijomik nelliutome tamattomane, attinga Israelemêtsainarkovlugo.

15 Tapsoma nekkoksitiniarpâtit, pattangaitiniarlutillo ningiongônerne, ukkuavit nagligijivit, namanersaujub illingnut ernernit 7 init, erniaringmago.

16 Naemiblo nutarak tiguva, illilâlo sâminut, pairksijôlunelo tapsominga.

17 Sillalingitalo arnat attekartipât, okarlutiglo; Ernek erniangôvok Naemimut, atserarpâllo Obedemik; tamna Isaib Davidib atâtangata atâtagijanga.

18. Tamædsa Perezib kinguvângit: Perezib Hezron ernetariva.

19 Hezronib Ram ernetariva. Ramib Aminadabe ernetariva.

20 Aminadab Nahasson ernetariva. Nahassonib Salma ernetariva.

21 Salmab Boase ernetariva. Boasib Obed ernetariva.

22 Obedib Isai ernetariva. Isaib David ernetariva. 


\title{
Samuelib aglangita sivorlingit.
}

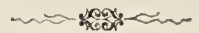 \\ KAPITEL I. \\ Samuelib innulerninganik:
}

ANGUteKalaUKPOK Ramataim Zofimemit Efraimib kakkanginêtomit, Elkanamik attelingmik, Jerohamib erninganik, Elihub erningata, Thohub erningata, Zufib erningata Efratemit pijub.

2 Nulliakalaukporlo maggungnik, aipanga Hannamik attelik, aipanga Penninamik. Penninale kittorngakalaukpok, Hannalo kittorngakalaungilak.

3 Angullo tamna iglugaseksoarnit iglokarviminit majorarlaukpok nelliunermine, tuksiaromavlune tunnergutiksaniglo tunnijilune Nalekamut Silome. Tagvanelaukpugle Nalekab ajochertuijokotigik Hofni, Pinehaselo, Elib ernigik.

4 Uvvlullo illangane pijokalaungmat Elkana tunnergutiksaminik tunnijilaurame, nulliaminut Penninamut tapsomalo erninginut paninginullo illunainut, tunnijivok abvakunik.

5 Hannamulle kiksarlune tunnijivok abvakomik attautsemik; Hamna nagligigamiuk; Nalekable kittorngisuitilaukpâ.

6 Akkerartortingatalo kiksartidlarpa, pingijaivigidlarlâlo, Nalekab kittorngisuitilaungmago.

7 Taimailaukpok Jahrine tamaine majoralaurangat Nalekab iglunganut, kiksartipalo taimak; tamnalo kugviorpok tagva, nerrinanelo sunamik.

8 Elkanable uingata okautiva: Hanna, sôg kêavît, sôglo nerringilatit? Sôglo omattit sænnikteriva? Namanersaungilangale illingnut ernernit tellimaujortunit? 
9 Tagva Hanna nerrilaukârdlune, immerlaukârdlunelo Silome nikkovipok. (Elile ajochertuijok iksivautame iksivavok Nalekab Tempelingata nappajungata seniane).

10 Tamnalo omamut kiksadlalaukpok, tuksiarporlo Nalekamut, kugviorlunelo.

11 Pinniaromajaminiglo angerpok, okarlunelo: Nalegak Zebaoth, kivgavit kannoêninga tækkunârniarungne, issumaginiarumga, kivgallo puigorniangikungne, kivgallo ernermik tunnitsiviginiarungne, tagva Nalekamut tunnijomavara innôsinga nâlugo, pijokartuksaungilarlo kipsautingnik niakunganut.

12 Akkunillo tuksiarmat Nalekab sângane, Elib tapsoma kanninga kamagiva.

13 Hanna omattimigut okarame kangasinâgik kissimik aulavuk, nippingalo tussartaulatungilak. Tagva Elib tallanganasugiva ;

14 Okautivalo: Kannok akkunêrtigijomik tallangajomavit? Weine illungnêtok annikoleruk illungnit.

15 Hannale kiovok, okarlunelo: Aukak, nalegâk, arnauvunga kiksartok. Weinemik immiksamiglo pitsartujomik immerlaungilanga, omattigale navilaukpara Nalekab sângane.

16 Kivgait înasuginiarnago arnatut illuserluktotut; issumajadlarnimnut, kiksadlarnimnullo okarkattarlaukpunga manna tikkilugo.

17 Eli kiovok okarlunelo: Ullapirsautekarlutit aularit; Israelib Gudingata tuksiarkiniarpâtit tuksiararilauktarnik tapsomangat.

18 Tamna okarpok: Kivgait saimaneksile ijikpit sângangne. Taimak arnak aularpok apkosinermine, nerrilunelo, tautorlugungnailunelo.

19 Uvlâkullo parngnailerput, tuksiarlaukârdlutik Nalekab sângane, utterivut tikkilutiglo iglomingnut Ramatemut. Elkanablo nulliane uvinekattigilerpa, Nalekablo issumagiva.

20 Uvlullo illangit kângermatta singailerpok, ernermiglo ernivok, Samuelemiglo atserarpa; okarlunelo: Nalekamit kenuarilaurapko.

21 Angullo Elkana majorarmat paunga iglomiokattine illagællugit tunnergutiksamik tunnijauvaktomik, angerutigilauktaminiglo tunnijijomavlune Nalekamut,

22 Hanna majorarkattaulaungilak, okarporle uiminut: Majorarkattaujomalungilanga nukapiak amâmagungnaikârtinnago, tagva âtsomavara, Nalekab sângane sakkijarkovlugo, tagvanêtsainarkovlugolo sorairane.

23 Elkanab, uingata okautiva: Taimailiorit sorlo namagijaungmat illingnut, nokkangarit amâmaktitsijungnaikârtinnak tâpsominga; Nalekable okauserilauktane nellautsitilerligit. Tai- 
mak arnak paivok, erninelo amâmaktipa, amâmaktitsijungnaikârtinago tapsominga.

24 Amâmaktitsungnairamiuk, âlugo majorarkattigiva, tuktuvait pingasut, Efalo tættælugo senaukamik, publaujarlo Weinetalik illaliudlugo; âpâlo Nalekab iglunganut Silomêtomut. Nukapiarle innusulaukpok sulle.

25 Tuktuvaillo illangat tokkopât, nukapiarlo âpât Elimut.

26 Arnarlo okarpok: Illa nalegâk, sorlo miksekârtomik tarnît inôngmat, nalegâk, uvanga arnauvunga taimna, mâne nangerkattigilauktat, tuksiarlauktorlo Nalekamut,

27 Nukapiak una tuksiarutigilaurapko. Manna Nalekab tuksiaramnik tunnitsivigilaukpânga, tuksiaragilauktamnik tapsomangat.

28 Tamanna pivlugo tunnivara Nalekamut innôsinga nâlugo, Nalekamit tuksiarijaulaungmat. Tuksiarpullo tagvane Nalekamut.

\section{KAPITEL II.}

Hannab tuksiarutinginik kujalijutiksanik. Samuelib innugo:jauninganit. Elib ernitita uivêtoksônigingnik.

Mannalo tuksiarpok okarlunelo: Omattiga kuviasukpok Nalekame, pitsartunera angijorortitaumavok Nalekame, kannera angmarmarikpok omigijikka pivlugit; piuliklernît kuviasutigigapko.

2 Irsornaitokangilak Nalekab assianik; illingnit assekangilak; sapputsauviksakalungilarlo Gudipta assianik.

3 Nertudlartinise, pingijaidlarniselo sorairlit, kannipsingnêtokarungnairle pimariuternermik; Nalegak Gudeôngmat kammatsiartok tamattominga, kajusinerlo taimaitok sullikolungila.

4 Sangijut pittiksingat serkomisimavok, sangêtullo tapserusimavut pitsartunermut.

5 Akkeartorsimalauktut niorvgotigijaumavut nerkiksamut, kâlauktullo kâgungnaiput; kittorngisuitok ernilerkârtinnago 7 nik, kittorngakadlalauktorlo ikigliviokârtinnago.

6 Nalegak tokkotsivok, omartitsilunelo; tessiornikpok kappianartovingmut, uttertitsilunelo tagvangat.

7 Nalegak aklutitsivok, akluititsilunelo, miklilertitsivok, angijorortitsilunelo.

8 Tapsoma ajoksartok kagverpa sannermit, aklujorlo kakkitipa akkilupiamit, pititsomavlugo attannit akkornganut, kingormgujikojomavlugolo opigijaunerub iksivautanganik. Nalekab nunab sukangit pigingmagit, sillaksoarlo pitilaukpa tapkonunga. 
9 Tapsoma ivsornaitokotime ittiggangit sapputiniarpait, Gudekangitulle nungutaujariakarput tâlktome; perkutevaksoakadlarnek ikajorlungimat kinamik.

10 Amnârtortut Nalekamik sutairtuksauvut; kollingine kallertitsiniarpok killangme. Nalekab sillaksub issua erkartoromârpa, attanekotinelo pitsartunekartitsomârpa, kangatartitsomârlugolo saimartitame naksunga.

11 Elkanale ailaukpok Ramatemut iglominut, nukapiarlo kivgartorteôlaukpok Nalekamik, ajochertuijub Elib sângane.

12 Elible ernigik uivêtoksôlaukpuk: tapkoa Nalegak kujanarutigilaukpak;

13 Kamalungilârlonêt ajochertuijut kaitaukojungnartanginik inugiartoksoarnit. Kina tunergutiksamik tunnijijomalaurangat, tagva ajochertuijub kivganga kaivok nerke tertitillugo, kappukautekarlune aggangmine pingasulingmik,

14 Tôrlunelo ukkusingmut, kallutaujamullonêt, êlikavingmullonêt, sabvivingmullonêt, kappukautiminullo amuartanganik ajochertuijok tigusivok. Taimailiorvigivait Israelitit illunamassiatik tagvunga Silomut tikkilauktut.

15 Taimaktauk tunnomik ikkitsikâratik, ajochertuijub kivganga kaivok, okarlunelo tapsomunga tunnergutiksamik kaitsijomut: Nerke kaitsuk uvamnut, jarralugo pikovlunga ajochertuijomut; nerkemik ikkoalammajomik tigusijomangimat illingnit mikkikamigle.

16 Kinalo tagva okalaurangat tapsomunga: Tunno ikkitaule sorlo pijuksaungmat, kingorngane tigusilerit omattivit tussugijanganik; tagva okarpok tapsomunga: Manna tunnijaksarivat uvamnut; pingikuvillo, tagva aksoronermut tigujomavara.

17 Tamanna pivlugo innuosuktûk tapkoa ajorningit angijovaksôlaukput Nalekab sângane; inuit Nalekab tunnergusianga nerkiksak mittautigilaungmatsuk.

18 Samuele kivgartorteôlaukpok Nalekab sângane, nukapiarlo attorlaukpok ikkiamik sâtomik.

19 Tamannalo tappilugo anânangat sennavok tapsomunga ullingmik mikkijomik; takpaungartipâlo tapsomunga, nelliuningane, uine illagilugo takpaungarlaurune tunnergutiksat tunilugit nelliuningane.

20 Eliblo Elkana nulliangalo saimartipak okarlunelo: Nalekab kinguvaksamik tunnitsivigilitit arnamit omangat, tuksiarnek pivlugo tuksiararilauktanga Nalekamit. Aivuglo innigijamingnut.

21 Nalekab Hanna nellipsarpa singailerkovlugo, ernilunelo ernernik pingasunik, panningniglo maggungnik. Samuelele nukapiak aglivalliavok Nalekame.

22 Elile îtudlalaukpok, tussarlunelo tamainik, erningme pinniarninginik Israelitinut illunainut, innangakattigingmattikiglo 
arnat kivgartortut angersimanerub tuppingata upkoangata sângane.

23 Okarporlo tapkungnunga: Sôg taimailiorkitîk? Tussarama idluitulliniptingnik innugiartoksoarnit tapkonangat tamainit.

24 Aukak kittorngâka, tamanna tussaumaniolungilak ânanamik, tussartara. Nalekab innukotingit tappatipasse.

25 Kina idluitullikpat innungmut, tagva idluarsaijok âkiksoijungnarpok tamattominga. Kinale idluitullikpat Nalekamut, kia tuksiarutigijungnarkaûk? Nalelaungilâgle atâtamik nippinganik; Nalegak kajusilaungmat tokkotsomavlugik.

26 Nukapiarle Samuele aglisirok, kuviagijaulunelo Nalekamut innungnullo.

27 Gudible angutekotingata illangat kailaukpok Elimut, okarIunelo tapsomunga: Imâk Nalegak okarpok: Nellojungnaitilaukpunga atâtagijavit iglunganuț, Egiptenemêtillugit sulle, Faraub iglungane;

28 Annerilaukparalo tagvane uvamnut, Israelib kittorngarêngninginit tamainit, ajochertuijônermut, tunnergutiksanik tunijijuksaungmat Altarekotimne, isserluanitiksaniglo ikkitsilune, ikkialijarlunelo sâmne; tunnijilaukpungalo atâtagijavit iglunganut Israelib kittorngangita ikkomanginik tamainik.

29 Sôgle tukarkît tunnerguseamnut, tunnerguseamnullo nerkiksanik, perkolauktamnut pikovlugit iglokotimne? Igvillo ernekotikik opiginersarivakit uvamnit, kuinitigapse innukotima Israelitit tunnergutiksangita nerkiksataggit namanerpânganut.

30 Tamanna pivlugo Nalegak Israelib Gudinga okarpok: Okalaukpunga, iglokotit atâtagijavillo iglunga agvertartuksaungmatta sâmne sorairatik. Mannale Nalegak okarpok: Ungasigle uvamnit, opigosuktorle uvamnik, tamna opigijomagivara; uvamnigle nachojok, tamna nachogijaujuksaugivok.

31 Ahâk, nelliutijomârpok serkomitsijomaviga tallernik, atâtagijavillo iglungata tallinganik, innutokaunekarkonnago iglungne.

32 Tækkoniarpallo akkerartortit iglumne, ajunginekarnerme sunatuinarnik Israelitit pijomârtanginik; innutokaunekaromângilarlo atâtagijavit iglungane sorairane.

33 Innukotingnillo kinalonêt pêjaromangilara Altarekotimnit, ijikik perlerkovlugik, tarnillo issumajarkovlugo; iglokotivillo unnurningit illunatik tokkojuksauvut innumariôningine.

34 Tamanalo nellonaikutarijaksarivat erningnut Hofnimut Pinehasemullo piniartok; uvlorme attautseme tammarmik tokkoniarpuk.

35 Uvangale erniangotitsijomavunga ajochertuijomik nertornartomik, tamna pinniartuksauvok kannok omattima tarnimalo namagijaunigingnik; tapsomungalo iglolioromavunga iglomik sokkosijuitomik, agvertarkovlugo sorairane saimartitama sângane. 
36 Amiakojullo iglungnit kaijomârput, pamakteromârlutiglo taipsoma sângane, kênaujârsuk niakajârsuglo pivlugik, okaromârpullo: Nagliktara, ajochertuijub ikkupianganil pekalaulanga nerrijungnarkovlunga niakojârsungmik.

\section{KAPITEL III.}

\section{Samuelib kaikojauninganik angijorortitauninganiglo.}

Nukapiarlo Samuel kivgartortillugo Nálekamik Elib sângane, Gudib okausingit pittaisalaukput nellautainêsalauklunelo.

2 Pijokalaukporlo nelliutome taipsomane Eli innangavingminetillugo, ijigiglo takpêlerpuk, tækkojungnarungnailaungmat.

3 Samuelelo innarêrpok Nalekab Tempelingane, Gudib iklervingata najortangane, Gudib kollinga kammilerkârttinago.

4 Nalekablo Samuele kaikova. Tamnalo kiovok: Ahâk mânêpunga;

5 Elimullo akpangerpok okarlunelo: Ahâk, mânêpunga, kaikolaukparma. Tamnale okarpok: Kaikolaungilagit; innariartolermit. Ailunelo innalerpok.

6 Nalegak kaikojipsarpok: Samuele! Samuelelo makkipok ailunelo Elimut, okarlunelo: Ahâk, mânêpunga, kaikolaukparma. Tamnale okarpok: Kaikolaungilagit ernîk, innariartolermît.

7 Samuelible Nalegak illitarilaungila sulle, Nalekablo okausinginik nellojungnaititaulaungilak sulle.

8 Nalekablo ama pingajuanik Samuele kaikova. Tamnalo makkipok ailunelo Elimut, okarlunele: Ahâk mânêpunga, kaikolaukparma. Tagva Eli mallugosukpok, Nalekab nukapiak kaikungmago.

9 Okautivalo: innariartolermît, kaikojauniaruvillo, tagva okarit: Nalegak okarit, kivget tussarmat. Samuele aivok innarlunelo innarvingminut.

10 Tagva Nalegak kaivok, tagvungarlunelo, kaikojilunelo sivurnganetut: Samuel! Samuel! Samuelelo okarpok: Okarit: kivget tussarmat.

11 Nalegarlo Samuelemut okarpok: Ahâk sunamik pinniarpunga Israeleme, tamattominga tussartub tapsoma siutigik aviojiniarmannik.

12 Uvlorme taipsomane tikkikojijomavunga Elimut, okauserilauktamnik tapsoma iglunga pivlugo; pigiaromavunga najijomalungalo.

13 Okautilaurapko erkartuijôjomagama taipsoma iglunganut soraijuitomik, idluitullinek pivlugo, kaujimalaungmat kannok ernangne piungitoâlungnik illusekalaungmanik, taipsomalo kingorlugutigilaungilâklonêt. 
14 Tamanna pivlugo Elib iglunga okautigimarilaukpara, Elib iglomiungita idluitulliningit tamakkoa ullapkosertaujuksaungimatta tunnergutiksanut, tunnergutiksanullonêt nerkiksanik soraijunane.

15 Samuelelo innangavok uvlâk tikkidlugo, upkuarlo Nalekab iglungane upkoêrpa. Samuelele sivuravok tækkotitsuk tamanna okautigilugo Elimut.

16 Tagva Elib kaikova, okarlunelo: Ernîk Samuel! tamna kiovok: Ahâk, mânêpunga.

17 Tamna okarpok: Okausek okauserijaumajok illingnut sunauva? Illangallonêt angiariniarnago uvamnut. Gudib imâglonêt imaglonêt pinniarvigilitit, okautigijaumajut illanganik angigutjivigigumga.

18 Tagva Samuelib okautiva tamainik, angigutjivigilungilâlo sunamik. Tamnale okarpok: Nalegauvok, pinniarle namagijaminik.

19 Samuelele pivallialerpok, Nalekablo aipariva, okausingitalo illangallonêt nunamut kattalungilak.

20 Israelitillo illunamassiatik Danemit Bersaba tikkilugo illitarksilerput Samuele nellautaijôngmat nertornartomik Nalekamut.

21 Nalegarlo manamit sakkergannerpok Silome; Nalekab Samuele ingminik nellojungnaitimago Silome, Nalekab okausingittigut.

\section{KAPITEL IV.}

Israelitit salagïauninganit. Angersimanerublo iklervingato aularutijauninganik. Eliblo ernikitalo tokkunginik.

SAMUELIBLO Israelitit illunaita okâlavigilerpait. Israelitillo aularput Filisterit pachrearlugit unatadlarnermut, tangmarterlutiglo Ebenezereme. Filisterillo tangmarsimalaukput Afekeme;

2 Opalungaijarlutiglo Israelitit unatarvigijomavlugit. Sorsungnerlo angijororpok, Israelitillo sâlagijauvut Filisterinut, tapkoalo sorsungnek malliklugo natername angutit 4000 diokassait ullimârpait saviksoarmut.

3 Innugiartoksuillo tangmarvingmut tikkimatta, Israelitit angajokangit okarput: Sôg Nalekab urlome sâlagijaukovâtigut Filisterinut? Nalekab angersimaningata iklervinga Silomit najortigilerlavut, tikkikolugolo akkonaptingnut, piuliklerkovlugo uvaptingnik omigijipta agganginit.

4 Innugiartoksuillo tilliklerput Silomut, aijaukolugolo tagvangat Nalekab Zebaothib, Kerubimib kollâne iksivajub, ikler- 
vinga angersimanetalik. Taikanelaukpuglo Elib ernigik Hofni Pinehaselo, Gudib angersimaningata iklervinga najorlugo.

5 Nalekablo angersimaningata iklervinga tangmarvingmut pingmat, Israelitit illunamassiatik igiakomijarput, nipliadlarnermut nuna immiadlarmat.

6 Filisterillo igiakomijarnerub taimaitub nippivaksoangit tussaramigit, okarput: Igiakomijarnerub taimaitub nippivaksoanga Ebræerit tangmarvingane sunauva? Tukkisigamiglo Nale$\mathrm{kab}$ iklervinga tangmarvingmut tikkisimangmat,

- 7 Erksilerput, okarlutiglo: Gude tangmarvingmut tikkisimavok; okaganerlutiglo: Kappianamêk uvagut! sivorngane taimailaungimat.

8 Kappianamêk uvagut! Kia piulijomavâtigut Gudiôjut sangijoksuit taipkoa agganginit? Tamædsa Gudiôjut taipkoa annaudlalauktut Egiptenemiunik kappianartunut sunatuinarnut sunakajuitome.

9 Tagva maksoaleritse, angutaulerluselo Filisteriôjose, kivgartoriakarkonase Ebræerinik, sorlo kivgartorlaungmasse. Angutauleritse sorsukluselo;

10 Tagva Filisterit sorsukput, Israelitillo salagijaulerput, illunatiglo attunit kemâvut iglomingnut; unatarnerlo angijôlaukpok, Israelitinit ocholaungmatta angutit pissuktut 30000 dit.

11 Gudiblo iklervinga aksarnigarijauvok, Eliblo ernigik Hofni Pinehaselo tokkolerpuk.

12 Tagva Benjaminekut illangat sorsuktokattigêksoarnit akpangerpok, tikkilunelo Silomut uvlome taipsomane, annorânelo alliktorlaukpait, ivjorlo katterarsimavlugo niakominut.

13 Ahâglo, ittermat Eli iksivavok iksivautame, apkosinek tækkojomavlugo; omattinga kappiasudlarmat Gudib iklervinga pivlugo. Angutiblo tapsoma iglugaseksoarnut ittersimagame, okautigiva tamanna; iglugaseksoarmiullo illuêngardlutik avâlakilerput.

14 Eliblo nipliadlarnek tussaramiuk, appertsorpok: Aulajadlarnek tamanna sunauva? Tagva angut tamna tuavilukardlune kaivok, okautigivalo Elimut.

15 (Elile Jahrekalaukpok 98 ginik, ijigiglo takpêsimavuk tækkojungnarane.)

16 Angullo tamna Elimut okarpok: Tikkipunga kemâlungalo uvlome sorsuktokattigêksoarnit. Tamnale okarpok: Kannok tamanna illingava ernîk?

17 Tagva unipkautjijok kiovok okarlunelo: Israelitit Filisterit kemâlaukpait, sorsungnekalaukporlo angijoksoarmik inugiartoksoarne, ernikiglo Hofni Pinehaselo tokkovuk; tamanna tappilugo Gudib iklervinga aksarnigarijauvok.

18 Gudible iklervinga (Elib) erkaramiuk, kingorpiosarlune niverpok iksivautamit, ittertarviksub senniane, kongesêjarlunelo, 
tokkolerlunelo; inutokaulaurame anguteôlunelo okkumaitok. Israelitiniglo idluarsailaukpok Jahrinik 40 ginik.

19 Tapsomale ukkuanga, Pinehasib nullianga singailaukpok, erniviksangalo nelliusimalaukpok. Tapsoma okautigijaumajok tussaramiuk, Gudib iklervinga aksarnigaungmat, sakkinelo uinelo tokkomanik, akkovlêlerpok ernilunelo, ânianartomut sillimanermut tikkitaugame.

20 Tokkolermallo manna, arnat senniane nangertut okarput: Sivoraniarnak, erneârsukarpotit. Tamnale kiolungilak sunamiglonêt, omamiutarksingilarlo tamattominga.

21 Nukapiarlo Ikabodemik atserarpa, okarlunelo: Ananaunek pêrsimavok Israelemit; Gudib iklervinga aksarnigaungmat, sakkingalo uingalo tokkomanik.

22 Okapsarlunelo: Ananaunek pêrsimavok Israelemit, Gudib iklervinga aksarnigaungmat.

\section{KAPITEL V.}

\section{Fïlisterit âniatitauninginils angersimanerub iklervingata pïjau- ninga pivtugo.}

FILISTERIT Gudib iklervinga tiguvât âlugolo Ebenezeremit Asdodemut,

2 Dagonib iglunganut, illilâllo Dagonib sennianut.

3 Asdodemiullo, uvlub aipangane uvlarâsukut makkinamik, Dagone naipipât pamangatillugo netterme, Nalekab iklervingata sângane. Tapkoale Dagone tiguvât, illigivâllo inninganut.

4 Uvlub aipanganele uvlangotillugo makkinamik, Dagon ama pamangatillugo netterme, Nalekab iklervingata sângane, naipipât; niakungale aggangillo igluktut nakkarsimalaukput upkutsab manoangane; timinga kissime illimalaukpok kângane.

5 Tamanna pivlugo Dagonib ajochertuijokotingita, illunatiglo Dagonib iglunganut ittertut, tutilungilât Dagonib iglungata manoanga Asdodeme, uvlok tamanna tikkilugo.

6 Nalekable aggangit okumaidlalaukput Asdodemiunut, asserorpaillo, Asdodemiullo erkânetullo illunaita âniakartipait, timingata tækkojaksaunginingine.

7 Inville Asdodemint tækkogamik taimailiornermik, okarput: Israelib Gudingata iklervinga uvaptingnêtsungnaile, aggangit okkumailuarmatta uvaptingnut, Gudekotiptingnullo Dagonemut.

8 Tilliklerpullo kattikolugillo Filisterit angajokangit illunaita ingmingnut, okarlutiglo: Kannok Israelib Gudingata iklervinga piniarvigijaksarivittigo? Tapkoalo kiovut tagva: Gathemiut Israelib Gudingata iklervinga akkigarlitsuk sunatuinarmut. 
9 Tapkoalo Israelib Gudingata iklervinga akkigarpât sunatuinarmut. Tamnale sunatuinarmut akkigarmatsuk, Nalekab aggangittigut iglugaseksoarne kappianartokalerpok angijoksoarmik, annauvaillo iglugaseksoarmiut tamarmik, mikkijut angijullo, ânianartuniglo pilerput timingita tækkojaksaunginingine.

10 Tagva Nalekab iklervinga tillikliutivât Ekronemut. Gudible iklervinga Ekronemut pingmat, Ekronemiut nipliadlarput: Gudib iklervinga akkigarpât sunatuinarmut uvagut tikkiluta, tokkotsikovlugo uvaptingnik, innukotiptingniglo.

11 Tagva tilliklerput kattimakolugillo Filisterit angajokangit illunaita, okarlutiglo: Israelib Gudingata iklervinga tillikliutilersiuk innigilauktanganut, tokkotsikonnago uvaptingnik innukotiptingniglo. Gudib aggangit kappianartoksoakartitsingmatta iglugaseksoarne illunâne tokkotsinermut.

12 Inuillo tokkolaungitut annaujauvut timingita tækkojaksaunginingine, taimak iglugaseksoarmiut âlaningit kongmuarmatta killangmut.

\section{KAPITEL VI.}

\section{Angersimanerub iklervingata uttertitauninganik Israelitinut.}

T

TAIMAK Nalekab iklervinga takkit 7 it nâlugit Filisterit nunanganêlaukpok.

2 Filisterillo ajochertuijokotitik nellautaijokotitiglo kaikovait, okarlutiglo: Kannok Nalekab iklervinga pinniarvigijaksarivittigo? Ajochertortigut sunakut tillikliutijaksarivittigo innigilauktanganut?

3 Tapkoa okarput: Israelib Gudingata iklervinga tillikliutijomagupsiuk, tagva sunakarane tillikliutiniarassiuk, akkiniaraksarivasse tunnergutiksamik akkiliksêrutiksamik; tagva kanoêtsungnainiarpose, nellojungnaititauniarluselo suna pivlugo tapsoma aggangit nulaungimatta illipsingnit.

4 Tapkoale okarput: Sunamik tunnergutiksamut akkiliksêrutiksamut tunnitsivigijaksarivittigo? Tapkoa kiovut: Nullungoarnik Goldenik tellimanik, nunivakangoarniglo Goldenik tellimanik, Filisterit angajokangita tellimat unurningit malliklugit; ânianartok adsigêlaungmat illunapsingnut, angajokapsingnullo.

5 Taimak manna nullopse adsinginik, nunivakapselo nunapsingnik asseroijut adsinginik sennajariakarpose, nertordlerkovluse Israelib Gudinganik; imakkâ aggangit okkinersauniangimangatta illipsingnut, Gudipsingnullo, nunapsingnullo.

6 Sôg omattise mangaitipissigik, sorlo Egiptenemiut Faraolo omattitik mangaitilaungmagit? Imaingilâk tattamnartullinerminik sakkertitsivigilaungmagit allarpait, aularkolugit? 
7 Tagva tigusileritse manna, sennaluselo aksalloalingnik nutânik, tuktuvângniglo innûtsungnik sarliartungnik maggungnik, annunik attorlaungimariktungnik sulle, annujiluselo tapkungninga aksalloalingnut, nochagiglo kingonigingne iglunginelilk.

8 Tigulugolo Nalekab iklervinga ikkitilugolo aksalloalingnut, pivianartullo Goldemit sennamajut, tunergutiksase akkiliksêrutiksamut tunnijasse tapsomunga, pitisigik iklerviârsungmut, iklerviub senerangata kiglinganut; tillisigiglo, aulartisigiglo.

9 Tækkonârsiuglo. Ailerpannik kiglilervingata apkosiningane Bethsemesemut; tagva tapsoma kappianartunik tamakkoninga tamainik piniarvigilaukpâtigut. Taimaingipanik kaujiniarpogut aggangita âktorlaungimattigut, mânetuinarlutalo tikkitaumatuinarapta tamattomunga.

10 Innuit tapkoa taimailiorput, tigusilutiglo tuktuvângnik maggungnik innûtsungnik sarliartungnik, annulugiglo aksolloalingnut, nochagiglo nûkongilakit iglomit.

11 Nalekablo iklervinga ikkitipât aksalloalingnut, iklerviârsuglo pitalik nunivangoarnik Goldenik, nullungitalo adsingoanginik.

12 Tuktuvâglo ailutik torarluatsiarpuk Bethsemesemut, apkosinerme attautseme, aivuglo nipliadlalutiglo, sangulungilâglo, tallerpingmullonêt, saumingmullonêt. Filisterillo angajokangita mallikpait Beth-Semesib kiglilervinga tikkilugo.

13 Beth-Semesemiulle, kattersivik nelliutingmat, kakkojaksanik Weizenik kibluivut nâksarme, tækkosarlutiglo, iklerviglo tækkovât, kuviasulaukpullo tækkogamitsuk.

14 Aksolloaligle tikkipok Josuab Bethsemesemiub perorsêvikotinganut, nokkarlunelo tagvane. Ujarasuksoakalaukporlo tagvane. Tapkoalo aksalloaliub kejuktanginik koppisivut, tuktuvâglo tunnergutiksautipakit Nalekamut, tunnergutiksamut ôtaksamut.

15 Levitille Nalekab iklervinga akkartipât, iklerviârsuglo sennianelauktok, pittalik pivianartunik Goldenik, illivâllo ujarasuksub kânganut. Bethsemesemiulle tunnijivut uvlorme taipsomane Nalekamut tunnergutiksanik ôtaksanik, tunnergutiksaniglo assianik.

16 Filisterille angajokangit tellimat tækkonârlaukârlutik, utterivut Ekronemut uvlorme taipsomane.

17 Tamakkoangôvulle nullungoat Goldit, Filisterit tunnilauktangit Nalekamut, tunnergutiksamut akkiliksêrutiksamut: Asdod attautsemik, Gasa attautsemik, Askalon attautsemik, Gath attautsemik, Ekronelo attautsemik.

18 Nunivangoallo Goldit, Filisterit angajokangita tellimat iglugaseksoangita unnurningit malliklugit, tamarmik iglugaseksuit avaluliorsimajuit iglugasaillo, ujarasuksoak Abelemik atte- 
lik tikkilugo, Nalekab iklervingata illijauvia tapkonunga; ujarasuglo tagvanêpok Josuab Bethsemesemiub perorsêvingane uvlok tamanna tikkilugo.

19 Bethsemesemiullo illangit annaujauvut tamana pivlugo Nalekab iklervinga tækkolaungmatsuk. Tapsomalo innugiartoksoarnit annauvait 50000 dit 70 gillo. Tagva innugiartoksuit kiksalerput Nalegak taimak unnuktigijunik tokkokojilaungmat innugiartoksuit akkorngane.

20 Innuillo Bethsemesemiut okarput: Kina nangerungnarka Nalekab heiligeôvlune Gudeôjub taimaitub sângane? kinamullo uvaptingnit aijuksauva?

21 Tilliklerpullo kivgartortunik Kiriatjearimemiunut, okautsaukovaillo: Filisterit Nalekab iklervinga uttertilaukpât; attersaleritse ailugolo paunga illipsingnut.

\section{KAPITEL VII.}

Israelitit sagiarninganik salakarninganiglo Filisterinik, Samuelib tuksiarningata kingorngane.

TAIMAK innuit Kiriatjearimemiut kaivut, ailugolo Nalekab iklervinga takpaunga, âlâllo Abinadab Gibeamêtub iglunganut; erningalo Eleaser ivsornaitipât udsertorkovlugo Nalekab iklervinganik.

2 Uvlormillo taipsomanemit Nalekab iklervingata nokkarvinganit Kiriatjearimeme akkunêrpok, Jahrillo 20 git annigorlaukput. Israelitillo illunamassiatik kugviolerput Nalekab sângane.

3. Samuele Israelib iglomiokotinginut illunamassianginut okarpok: Omamut illunânut sâlerupse Nalekamut, tagva Gudingoat tækkorngartat Astarotelo pêjarsigik illipsingnit, omattiselo torartisigik Nalekamut, kivgartortuarisiuglo ; tagva piuliniarpâse Filisterit agganginit.

4 Tagva Israelib kittorngangita pêjarpakit ingmingnit Baalit Astarotelo, Nalegarlo kissiat kivgartorpât.

5 Samuelele okarpok: Israelitit illunamassiatik kattimatisigik Mizpame, tuksiarutjikovlunga illipsingnik Nalekamut.

6 Tikkilutiglo kattimalerput Mizpame, immermiglo kallusivut, kovilâllo Nalekab sânganut, nerritaililutiglo uvlorme taipsomane, okarlutiglo: Nalegak idluitullivigilaukpavut. Taimak Samuelib Israelib kittorngangit idluarsarpait Mizpame.

7 Filisterille tussaramik Israelitit kattimaninganik Mizpame, tagva angajokangit majorarput Israelitit sorsugvigijartorlugit. Israelib kittorngangit tamattominga tussaramik, Filisterit sivurajutigivait, 
8 Okarpullo Samuelemut: Sorairniarnak tuksiarutigiluta Nalekamut Gudiptingnut, piuliklerkovlugo uvaptingnik Filisterit agganginit.

9 Samuele sangârsungmik amâmaktomik tigusivok, Nalekamut tunnergutiksamik ôtaksamik illuitomik tunnijilune, nipliarlunelo Nalekamut Israelitit pivlugit; Nalekablo tussarpâ.

10 Samuelelo tunnergutiksamik ôtaksamik tunnijitillugo, Filisterit tikkilerput Israelitit sorsugvigijomavlugit. Nalegarle kallukarkojivok kalluksoarmik Filisterit kollâne uvlorme taipsomane, koaksartipaillo, sâlagijaukovlugit Israelitinut.

11 Tagva angutit Israelitit aularput Mizpamit, kemâtipaillo Filisterit, ullimarlugillo Bethkarib attâ tikkilugo.

12 Tagva Samuelib ujarak tiguva, nappartilugolo Mizpab Seniblo akkorngangne, atserarpâlo Ebenezeremik, okarlunelo: Maunga tikkilugo Nalekab ikkajorlaukpâtigut.

13 Taimak Filisterit songojungnaititauvut, tikkitsungnailutiglo Israelib nunangata kiglilervianut; Nalekablo aggangita Filisterit akkerartorpait Samuelib innosingane illunane.

14 Taimak Israelitit iglugaseksuit Filisterit aksarnigarilauktangit tapkonangat pilaurivait, Ekronemit Gathe tikkilugo, nunangita kiglilervingit illaliudlugit; Israelitit tapkoa piulivait Filisterit agganginit, Israelitit ullapirsautekalaungmatta Amoriterinut.

15 Samuelible Israelitit idluarsarpait inosermine tamât,

16 Jahrinelo tamaine ingerarpok sunatuinarnut, Bethelemut, Gilgalemullo, Mizpamullo. Israelitillo iglugaseksoarne tamakkonane idluarsarlaurunigit,

17 Uttilerivok Ramatemut, iglunga tagvanelaungmat, tagvanelo idluarsarpait Israelitit, sennalunelo tagvane Altaremik Nalekamut.

\section{KAPITEL VIII.}

\section{Israelitit attanekaromaninganik, Gudiblo namagingininganil tamaitominga.}

SAMUELIBLE innutokaulerame ernangne idluarsaijungortipâk Israelitinut.

2 Erninga erniangokârtok, attekalaukpok Joelemik, tuglialo Abiamik, idluarsaijolaukpuglo Bersabame.

3 Ernigigle agvertarlaungilâk tapsoma apkotigijangane, sangulerpugle pijomatudlarnermut, tigusilutiglo pilliusianik, idluarsainerlo attornerlukpâk.

4 Tagva Israelitit angajokangit kattimalerput, tikkilutiglo Samuelemut Ramatemut, 
5 Okautivâllo: Ahâk itolerpotit, ernekiglo agvertarlungilak apkotigijarne; taimak manna attanekartitigut idluarsaijomik uvaptingnik, sorlo nellojut illunatik pekarmatta.

6 Samuelib tamanna idluigiva, okarmatta: Attanermik tunitsivigitigut idluarsaijomik uvaptingnik. Samuelelo tuksiarpok Nalekamut.

7 Nalekable Samuele okautiva: Inugiartoksuit nippingit nalekit tamaine okauserilauktangine illingnut; tapkoa igviungitok, uvangale singalukogilaukpânga, attaniojuksaunginama tapkonunga.

8 Pinniarvigivâtit, sorlo pinniatsainarlaungmatta uvlormit taipsomangamit Egiptenemit aulartilaurapkit, uvlok tamanna tikkidlugo, kemmalaukpânga, Gudiniglo assimnik kivgartorlaukput.

9 Tagva manna nippingit nalekit. Kigligiutikille nellojungnaitilugillo attanniub attanerijomârtangata illuserijungnartanginik.

10 Samueliblo Nalekab okausingit illunaita okautigivait innugiartoksoarnut, attanermik pijomajunut tapsomangat.

11 Tamakkoa attaniub attaniojomârtub illipsingnut illuserijungnaromârtangit: Ernise tiguniarpait aksalloalikotiminut, ablangajuksanullo horsit kângine, aksalloalikotinganik sivorlerkovlugit pangaliklutik;

12 Angajokangnullo sorsuktuksanut 1000 dinut, 50 ginullo, perorsaijuksanullo perorsêvikotiminik perorsaijunut, kibluijuksanullo kattersivingmine, sappulutakotinganiglo aksaloalikotinginullo attoriakartunik sennakovlugit.

13 Pannisele tiguniarpait âniasiusiorteôkovlugit, igajeôkovlugillo, niakojaliorteôkovlugillo.

14 Perorsêvipse idluarnerpângit, kakkaselo Weineliksat, perorsêviselo orksuktaggit tigujomârpait, tunnilugillo kivgakotiminut.

15 Tamakkoalo tappilugit perorsijaksakotipse, Weineliksakotipselo tellimaujoanik tigusijomârpok, tunnijomârlugillo pimaklertokotiminut kivgakotiminullo.

16 Kivgaselo angutit, kivgaselo arnat, innuosuktokotiselo ânanaunerpât, siutekoktôjokotiselo tigujomârpait, tapkutigûnalo sullijaksane âkiksoromârpait.

17 Omajokotipse kattimgajunginit tellimaujoanik tigusijomârpok, illipselo kivgaujariakarpose tapsomunga.

18 Imakkâ nelliutome taipsomane, attanise annerilauktase illipsingnut nipliarutigijomârupsiuk, Nalekab tussarniangilâse nelliutome taipsomane.

19 Innugiartoksuille kunnulaukput Samuelib nippinga naleklugo, okarpullo; Aukak, attanekartuksauvorle uvaptingnut.

20 Uvaguttauk illingalerkovluta assiptitut nellojutitut illu- 
naititut, attanivut idluarsaikovlugo uvaptingnik, sivorlerkovlugolo uvaptingnik unatariartoraigupta.

21 T'agva Samuelib inugiartoksuit okauserijangit tamaita nalekpait, okautigilugillo Nalekab siutikita sângangne.

22 Nalegarle Samuelemut okarpok: Tapkoa nippinginik nalegit, attannekartitsilutillo tapkonunga. Samuelelo Israelib angutinginut okarpok: Aileritse illunase attunît iglugaseksoapsingnut.

\section{KAPITEL IX.}

Saulib, Gudib annerïangata attannermut, tattamnadlartomik tikkininganits Samuelemut.

ANGUTEKalaUkPORLE Benjaminib kinguvânginit Kisemik attelingmik, Abielsib erningata, Zerorsib erningata, Bechoratsib erningata, Apiab erningata, Jeminib erningata erninganik, tamna angutaulaukpok erkinaitok.

2 Tamna ernekalaukpok Saulemik attelingmik; tamna innuosuktôvlune angutitsiangôlaukpok, ânanaunersakalaungilarlo Israelib kittorngangita akkorngane, niakotut attautsetut takkinersaulaukpok innugiartoksoarnit tamainit.

3 Kisible, Saulib atâtangata siutekoktôjokotine arnet assiojilaukpait, okarporlo ernerminut Saulemut: Nukapitset illangat aipaukoleruk illingnut, parngnailerlutillo siutekoktojullo kenneriartorkit.

4 Aivorlo Efraimib kakkangittigut, nunakullo Salisamik attelikut nenninagillo, aivuk Salimib nunangagut, tagvanêlaungilello; aivuglo Jeminib nunangagut, nenninagillo.

5 Zufible nunanganut tikkimanik, Saule nukapiamut aiparijaminut okarpok: Kailerit angerarmilauluk; atâtama siutekoktôjut nippotivallungmagit pingigilutalo.

6 Tamnale okarpok: Ahâk, pittakarpok iglugaseksoarne tapkonane, Gudib angutekotinganik, okausiovaktomik; okausingit illunaita nellautsivut. Manna tagvungarlauluk; tapsoma apkut attoriakartavuk okautiginiarpallaiva uvaptingnut.

7 Saule nukapiakotiminut okarpok: Aivigigalloaruptigo, sunamik tamna kaitsivigijaksarivittigo? Kakkojakarungnaimat pôptingne, assianiglo tunnergutiksakangilaguk âtsungnartaptingnik Gudib angutinganut tapsomunga. Sunakarpinûk?

8 Nukapiab Saule kiova: Ahâk, najortekarpunga Sekelib Silbereôjub sittamanganik; tamna tunnijomavaruk Gudib angutekotinganut, okautjikovlugo uvaptingnik, apkotiksaptingnik.

9 Itsame aijokalaukpat Israeleme Gude appertsoriartorlugo 
Kap. 9.] I. SAMUEL.

tagva okarpakput: Kaititse, tækkojok aivigilavut. Manna nellautaijunik taijaujut, itsame tækkojunik taijaulaungmatta.

10 Saule nukapiakotiminut okarpok: Namaktomik okalaukpotit; kait, ailauluk. Aitillugiglo iglugaseksoarnut Gudib angutekotingata najuganut,

11 Majorartillugiglo iglugaseksoarnut, niviârsijanik naipitsivuk, annilertunik immermik kallusijartortunik. Tapkonunga okarpuk: Tækkojok tamâneka?

12 Tapkoa kiovakit okarlutiglo: Ahaila, ahâk, tagvanêpok; tuavilerit, uvlome iglugaseksoarnut tikkisimavok, innugiartoksuit uvlome tunnergutinik tunnijijomangmatta kakkarolângme.

13 Iglugaseksoarnut tikkileruptik neniniarpattik, kakkarolângmut majorarkârtinago nerrijomavlune. Innugiartoksuit nerriniangimatta tikkikârtinago; tapsoma tunnergutiksak tuksiarutigikârmago, kingorngane kaikojaujut nerrivut. Taimaimat majoraritik, manna, naipiniaraptiko.

14 Majorartilugiglo iglugaseksoarnut, iglugaseksuillo kerkanêtillugik, ahâk, tagva Samuele annilerpok pachrearlugik, aijomavlune kakkarulângmut.

15 Nalekable Samuelib siutigik nellojungnaitilaukpak uvlub taipsoma ikpeksangane Saule tikkikârtinago, okalaukporlo:

16 Kaupat nelliutome tamattomane, angut Benjaminib nunanganit pijok, tillijomavara illingnut; tamna mingoarlugo attaningortitaksarivat innukotimnut Israelitinut, innukotimnik piuliklerkovlugo Filisterit agganginit. Innukotikka tækkonârlaurapkit, nipliarningillo tikkisimavut sâmnut.

17 Samuele manna trkkosartillugo Saulemik, Nalekab okautiva: Ahâk, tagva angut okautigilauktara illingnut, attaniojuksak innukotimnut.

18 Tagva Saulib Samuele sângariartorpa ittertârvingme, okarlunelo: Okautilaunga, tækkojub iglunga nanêka?

19 Samuelib Saule kiova, okarlunelo: Uvanga tækkojôvunga, sivurlerlutit uvamnik majoralerit kakkarulângmut, uvlome nerrikattigijaksarigaptinga; kaupat aularkojomavagit, omamigijarniglo tamainik okautigijomavagit.

20 Siutekoktôjullo uvlut pingasut sivorngane assiojilauktatit pingiginiarnagit manna, nachvarsimavut. Kialo tamaita ajunginerpât Israeleme piginiarpagît? Illingnut atâtavillo iglomiokotinginut illinganiangilât?

21 Saule kiovok: Jeminib erneringilângâ, Israeliblo kinguvarêngita mikkinerpânginit pingilanga, illakalo ikkinerpaulungilât, Benjaminib kinguvarênginit tamainit? Sôg tagva taimaitomik okautivarma?

22 Samuelible Saule nukapiakotingalo tiguvak tessiorlugiglo iglomut nerrimavingmut, ingitipâglo issukliovlutik kaikojaumajunit, tapkoa anguteôlaukput 30 git. 
23 Samueliblo igaje okautiva: Abvako tunnilauktara illingnut, okautigilauktaralo illingnetitaksarigangne, kaitsuk.

24 Tagva igajib tallek attatigijangalo ittertipa. Illivâlo Saulib sânganut. Samuelelo okarpok: Ahâk tamanna amiakôrok, attuinaruterilerit nerrilutillo, kumergutigijaungmat illingnut, nelliutome tapsomane innuit nerrijartorkolaurapkit. Taimak Saulib Samuele uvlorme taipsomaneme nerrikattigiva.

25 Kakkarulângmillo attersarmatta Samuelib Saule okarkattigiva iglub kângane.

26 Uvlarârsukullo makkiput; kaublo aupallungninga nuilertillugo, Samuelib Saule kaikova iglub kângane, okarlunelo: Makkitit! aularkojikovlunga illingnik. Saulelo parngnailerpok, tapkoalo magguk, aiparêklutik annilerpuk, tamna Samuelelo.

27 Atter'sarlaukârlutik iglugaseksuit issuanut Samuel Saulemut okarpok: Nukapiak okautiuk sirorlerkovlugo uvaptingnik, (sivumuarporlo); igville nokkangalerit, kaujititsikovlunga illingnik, Gudib okauserilauktanginik.

\section{KAPITEL X.}

Saulib mingoartauninganit atíanermut, sakkertitauninganiglo.

\section{$\mathrm{T}$}

AGVA Samuelib publaujak orksuktalik tiguva, kovilugolo tapsoma niakoanut kunnikpa, okarlunelo: Tækkovît Nalekab mingoarsimangmattit, attanniôkovlutit kingormgutigijanganut?

2 Manna aularuvit uvamnit, angutisiniarpotit maggungnik, Rahelib illuvingane, Benjaminib kiglilervingane, Zelzame, okarniartungnik illingnut: Siutekoktôjut keneriartorlauktatit nachvarsimavut; ahâglo, atâtavit siutekoktôjut nippotivait, pingigilutiglo, okarlunelo: Sunamik ernera pitjutigallugo pinniartuksauvîk?

3 Taikangallo sivumuaruvit, tikkiniarpotit Taborib Eichinganut (nappartomut), taingane angutit pingasut naipiniarpâtit majorarasuartut Gudemut Bethelemut. Illangat angusaluârsungnik pingasunik akjarsivok, aipanga kakkojanik pingasunik, pingajuallo publaujamik Weinelingmik.

4 Illanâkarlutiglo salutiniarpâtit, tunnitsiviginiarpâtillo kakkojângnik maggungnik. Tapkoa taipkoa agganginit tigujaksarivâkik.

5 Kingorngane tikkiniarpotit Gudib kakkarulâkotinganut Filisterit tangmarvingata najugânut; tainganelo iglugaseksoarnut tikkileruvit, nellautaijut kattimgajut kakkarulângmit attersarlutik pârmiarpâtit, sivurlerkartut imgerutinik imgertunik, killausijartuniglo, nipsausijartuniglo, Harfesijartuniglo, tapkoalo nellantailutik. 
6 Nalekablo Anerningata tikkilârpâtit, illagilugit nellautailerkovlutit; tagva ablatsangorniarpotit angumut assianut.

7 Nellonaikutat tamakkoa mana tikkiviginiarpattit, tagva pinniarit pitjutekarniarlutit piniarungnartarnik; Gudib aipariniarmattit.

8 Sivunimne attersartuksauvotit Gilgalemut; ahâk, tagvane tikkitsomavagit, tunnijijomalungalo tunnergutiksanik ôtaksanik, tunergutiksaniglo kujulijutiksanik. Uvlut 7 it nâlugit uttakijuksauvotit tikkikârtinanga illingnut, kaujitilutillo pinniaraksarnik.

9 Tunnulermallo, Samuelemit aularomavlune, Gudib omamik assianik tunnitsivigiva, nellunaikutallo taipkoa illunatik tikkilaukput uvlorme taipsomane.

10 Kakkarulângmullo tapsomunga tikkimanik, ahâk, tagva nellautaijut kattimgajut pârpât; Gudiblo Anerningata tikkivigiva, nellautailerporlo tapkoa akkorngane.

11 Illunatiglo sivorngane illitarksilauktut tapsominga, tækkungmatsuk nellautaijut illagilugit nellautailermat, ingmingnut okarput: Sunamut Kisib erninga tiklkitaumava? Sauletauk nellautaijunut illingava?

12 Tagvanetullo illangat kiovlune okarpok: Atâtanga kinauva? Tamanna pivlugo okartokarpakpok: Sauletauk nellautaijunut illingava?

13 Nellautaijarêramelo kakkarulângmut pivok.

14. Saulible kattangutiârsua okarpok tapsomunga, nukapiakotinganullo: Namut ailaukitik? Kiovuk: Siutekoktôjut kenneriartorlugit; tækkogamnuglo tagvanêlaungimatta, Samuelemut tikkipoguk.

15 Tagva Saulib kattangutiârsua okarpok: Okautilaunga, sunamik Samuelib okautilaukâtik?

16 Saulib kattangutiârsune kiova: Okautilaukpâtiguk, siutekoktojut nachvartaumangmatta. Attanniovigle pivlugo okautilungila sunamik.

17 Samuelible inugiartoksuit kaikovait Nalekamnt Mizpamut.

18 Okarlunelo Israelib kittornganginut: Imâk Nalegak Israelib Gudingat okarpok: Uvanga Israelitit Egiptenemit aularutilaukpakka, piulilaukpapselo Egiptenemiut agganginit, attaniovingmiullo illunatik okumaititsilauktut illipsingnik agganginit.

19 Illipselo uvlome Gudise singalukotilaukpasse, ikkajorlauktok illipsingnik kannoênipsingnit, kappiagijapsingnillo, okautilugolo: Attanekartitigut. Atte, tagva manna Nalegak sangariartorsiuk kinguvarêkotise, illagêngniselo malliklugit.

20 Samueliblo manna Israelib kinguvarêngit sakkertimagit Benjaminib kinguvarêkotingit nellautauvut.

21 Benjaminiblo kinguvarêkotingit, illagêngningit illagilugit sakkertimagit, Matrib kittorngarêngit nellautauvut, Saulelo Kisib erninga nellautaurok. Kemerpâllo nachvamagolo. 
22 Tagva Nalegak apperipsarpât: Ovungarniaralloarka? Nalegak kiovok: Ahâk, ijerlaukpok kattaujat akkornganut.

23 Tagva akpangerput, ailugolo tagvangat. Innugiartoksuillo akkornganut pigame, niakotut attautsetut takkinersaulaukpok innugiartoksoarnit illunainit.

24 Samueliblo innugiartoksuit illunaita okautivait: Tagva tækkovose Nalekab annerilauktanganik, nellekangimat innugiartoksoarne tamaine. Tagva innugiartoksuit tamaita igiakomijarput, okarlutiglo: Pilloringnartokarle attanermut!

25 Samuelible innugiartoksuit okautivait attannioviub illuserijanginik, aglalugillo aglangnut, illilugillo Nalekab sânganut. Samueliblo innugiartoksuit aularkovait, illunatik attunit iglomingnut.

26 Saulelo angerarivok Gibeamut; kattimajoksuillo illangita Gudib kiblilauktangita omattingittigut, illagivâtt.

'27 Inuille koksalaitut illangit okarput: Sunamik oma ikajoraksarivâtigut? Nachogivâllo, pilliumiglo âtsivigilaunginivât. Tamnale tussangitsertorpok tapkoninga.

\section{KAPITEL XI.}

Saulib sâlałarninganił sivorlerpâmit Amoniterinit.

NaHASE Amonitereôjok majorarpok, Jabeselo Gileademe illumavait. Angutillo Jabesemiut illunatik Nahasemut okarput: Angerkattigittigut, tagva kivgartoromavaptigit.

2 Nahasible Amonitereôjub kiovait: Tamattominga angerkattigijomavapse, illunase attunit ijipse tallerpia kappijomagapko, sujuktiluselo Israelitit illunatik akkorngane.

3 Tagva angajokaunerôjut Jabeseme okautivât: Tunnitsivigittigut uvlunik 7 nik, kivgartortunik tilliklerkovluta Israelib kiglilervinginut illunainut, tagvalo kinakangipat piuliklerniartomik uvaptingnik, annivigijomavaptigit.

4 Tagva kivgartortut tapkoa tikkiput Gibeamut Saulemut, - okarlutiglo tamattominga innugiartoksuit siutingita sângine. Tagva innuit illunatik niplialerput kêadlarlutiglo.

5 Ahâglo, tagva Saul perorsêvingmit pivok tuktuvait kingornganêdlune, okarlunelo: Inugiartoksuit sujut, kêadlarmatta taimak? Tagva Jabesemiut angutingita, illinganinginik unipkautivât.

6 Tagva Gudib annerningata tikkipa, okautsit taimaitut tussaramigit, ningaumajarningalo illungertudlalerpok.

7 Tuktuvâglo magguk tiguvâk avgorlugiglo, tilliklerlunelo Israelib kiglilervinginut illunainut kivgartortutigut, okautsaukojilunelo: Nelliat aularlungitok Saul Samuelelo malliklugik, 
tapsoma tuktuvakotingit taimailiortitaujuksauvut. Tagva Nalekab sivuranarningata innugiartoksuit tupvigilerpait, aularmatta angutetut attautsetut.

8 Basekemelo opallungaijartauvut; Israeliblo kittorngangit 300000 diolaukput, Judablo kittorngangit 30000 diolaukput.

9 Tillijaujullo tikkisimalauktut okautivait: Imâk angutit Jabesemiut Gileademe okautissigik: Kaupat sekkinek kiakalerpat ikajortamiarpose. Tillijaujut uttermatta, okautigilugolo tamanna angutinut Jabesemiunut, tagva kuviasulerput.

10 Jabesemiullo angutingit okarput (Amoniterinut): Kaupat annijomavogut illipsingnut, issumapsingnik tamainik pinniarvigiluta pikovluse.

11 Uvlublo aipangata uvlangôningane Saulib innugiartoksuit avitipait pingasolilugit, tikkilutiglo tangmarvingmut, udsertortib uvlarme nelliuningane, annaulerpaillo Amoniterit, uvlub kiakarninga tikkidlugo; amiakojulle taimak siamartitauvut, magguglonêt aiparêgungnaimanik.

12 Tagva innugiartoksuit Samuelemut okarput: Kikkôvât okalauktut: Saule attaniojuksauva uvaptingnut? Angutit taipkoa kailersigik, tokkotsikovluta taipkoninga.

13 Saulelo okarpok: Uvlorme tamattomane tokkotaujokartuksaungilak kinamiglonêt; Nalegak uvlome piulijaunermik tunnijilaungmat Israeleme.

14 Samuele innugiartoksoarnut okarpok: Kaititse, Gilgalemut ailerta, attanniôviglo nutângortilerlavut.

15 Tagva innugiartoksuit illunamassiatik Gilgalemut aivut, Saulelo attaningortipât tagvane Gilgaleme Nalekab sângane, tunnijilutiglo tunnergutiksanik kujalijutiksanik Nalekab sângane. Saulelo, Israelib angutingit illunaita illagilugit, kuviasudlalaukpok tagvane.

\section{KAPITEL XII.}

\section{Samuelib idluarsainerminit ipperaininganit, kaiblaininganiglo innugiartolesoarnit.}

$\mathrm{T}$ IAGVA Samuele Israelitinut illunamassianginut okarpok: Ahâk, nippise nalelaukpakka illunaine, okauserilauktapsingne uvamnut, attannermiglo pititsilaukpunga illipsingnut.

2 Mannalo ahâk, tagva attanipse sivorlervigivâse. Uvangale îtomavunga kêrtolarmalo, ernîkalo akkonapsingnêpuk, uvangalo sivurlervigilaukpapse innuosungnimnit uvlok tamanna tikkilugo.

3 Ahâk, manêpunga akkerartorlunga kiogisinga, Nalekab tapsomalo saimartitangata sângane, kia tuktuvakotinga, siute- 
koktojokotingalonêt tigulaungmangapkik? kina aksorornermut aksarlaungmangapko, idluitullivigilaungmangapkolonêt? Kialonêt agganginit pilliusiamik tigusilaungmangarma, ijikalo takpêtitaukolaungmangapkik? Tagva uttertitsomavara.

4 Tapkoa okarput: Aksororvigilaungilaptigut, idluitulliviginatalonêt, tigusilaungilatillo sunamik kia agganginit.

5 Tamna okarpok tapkonunga: Nalegak kigligiudsijôle, saimartitangalo, uvlome uvlorme tamattomane sunasilaunginapse aggamne. Tapkoa okarput: Ahaila, kigligiudsijôjuksauvuk.

6 Samuelelo innugiartoksoarnut okarpok: Illa Nalegak tamna, pingortitsilauktok Mosesemik, Aronemiglo, atâtagijapsingniglo aularutjilauktok Egiptenemit.

7 Taimak ovungaleritse manna erkartuivigiluse pikovlunga Nalekab sângane, Nalekab ajungitullininginik pinniarlauktanginik illipsingnut, atâtagilauktapsingnullo.

8 Jako Egiptenemut tikkisimalaungmat, atâtagilauktase nipliadlarput Nalekamut, tapsomalo Mosese Aronelo tillivâk tessiornikovlugik atâtagijapsingnik Egiptenemit, nunakarkojikovlugiglo tapkoninga tamâne.

9 Tapkoale Nalegak Guditik puigormatsuk, niorvgotigilaukpait Sisserab, sorsuktuksat Hazoreme angajokangita pitsartuninganut, Filisterillo pitsartuninganut, Moabiterillo attaningata pitsartuninganut, tapkoa matadlarvigilaukpait.

10 Amalo niplialerput Nalekamut, okarlutiglo: Idluitullilaukpogut Nalegak kemmalauraptigo, Bâlillo Astarotelo kivgartorlauraptigik; mannale piulitigut omigijipta agganginit, tagva kivgartoromavaptigit.

11 Tagva Nalekab. Jerubâle Bedanelo, Jeftalo, Samuelelo tillivait, piuliluselo omigijipse avatipsingnetut agganginit, nunakarkoluselo ussornarluse.

12 Tækkolaurapsele Nahasib Amonib kittorngangita attaningata kaitsovigingmasse, okalaukpose uvamnut: Igviungitok, attaniuble attanniotivigijaksarivâtigut; Nalegak Gudise attannerilauralloarapsiuk.

13 Manna tagva attannekarpose annerilauktapsingnik, tuksiararilauktapsingniglo; ahâgle, Nalekab attannermik pititsivigimavâse.

14 Nalegak manna sivuraginiarupsiuk, kivgartorlugolo, nippingalo nalengniarlugo, Nalekablo kanninga tappaginiangikupsiuk, tagva tamarmik illipse attanipselo, attannerijapse Nalegak Gudise mallingniarpasse.

15 Nalekable nippinga nalengniangikupsiuk kanningale tappagigupsiuk, tagva Nalekab aggangita illipse atâtagijasselo akkerartorniarpâse.

16 Mannalotauk tamaungaleritse, tækkoluselo pijokartomik angijoksoarmik Nalekab pinniarlârtanganik ijipse sângine. 
17 Perorsijat Weizenit kattersivingat nelliusimangilak? Uvangale Nalegak nipliarvigijomavara kallukojikovlugo, sillalukojikovlugolo, tukkisilerluselo trekkokovluse idluitoksoarmik Nalekab ijikita sângangne pinniarlauktapsingnik, attannermik tuksiarlaurapse.

18 Samueliblo Nalegak nipliarvigingmago, Nalegak kallerkojivok, sillalukojilunelo uvlorme tapsomane. Tagva imugiartoksuit illunamassiatik Nalegak Samuelelo sivoragivakit,

19 Okarlutiglo Samuelemut: Nalegak Gudit tuksiarvigiuk kivgatit pivlugit, tokkolerkonata; ajornivulle illunaita tappilugit, idluitomik tamattominga pilaurivogut, attannermik tuksiarlaurapta uvaptingnut.

20 Samuele innugiartoksoarnut okarpok: Sivuraniarase, illuitunik tamakkoninga tamainik pinnialauralloarpose, kissiane nûniarase Nalegak tunnulugo, kivgartorsiugle omamut tamânut;

21 Sangunaselo suksaungitok malliklugo, piutsengimat, piuliklerungnangilarlo, sunaungitôngmat.

22 Nalekable innukotine kemmalungilait, attine angijoksoak pivlugo; Nalegak pigiarlaungmat illipse innukotingortiluse ingminut.

23 Ungasigletauk uvamnit, taimak idluitullilunga Nalekamut nokkartuksaugama tuksiarutigiluse, ajochertorluselo apkomik idluartomik tamarnangitomiglo.

24 Nalegak sivuragituinarsiuk kivgartorlugolo nertornarluse omamut tamânut; tækkolaurapse kannok angijoksoarmik pinniarvigingmasse.

25 Idluitomigle pinniarniarupse, tagva tamarmik illipse attanniselo assioniarpose.

\section{KAPITEL XIII.}

Jonathanib annaulemingninganik Filisierinik, tapkoalo kaitsoninginit: Israelemui. Saulib tunnïininganik tunnerguitksamik perkojaungitomik.

SAULE Jahre attausek nâlugo attaniolaukpok; Jahriglo magguk nâlugik Israelitit attaniotivigilaukârdlugit,

2 Israelitinit angutit 3000 dit annerivait. Angutit 2000 dit Saule illagilugo Michmasemêlaukput, Betheliblo kakkangane, 1000 dillo Jonathane illagivât Gibeame Benjaminib nunanganêtome; innugiartoksuille assingit aularkovait, illunatik attunit iglomingnut.

3 Jonathanible Filisterit ullimârpait tapkoa tangmarvingine Gibeamelauktome; tamattominga Filisterit kaujititauvut. Saulelo 
tiptulausijarkojivok nuname illunâne, okarkojilunelo: Tamattominga Ebræerit tussarlit.

4 Israelitillo illunamassiatik tussarput okartokarmat: Saulib Filisterit tangmarvingat ullimarlaukpait, Israelitit tippilungmatta Filisterit sângane. Innugiartoksuillo illunamassiatik kaikojauvut Saule malliklugo Gilgalemut.

5 Tagra Filisterit kattimalerput Israelitit sorsugvigijomavlugit, aksalloaggit 30000 dit, ablangajut horsine 6000 dit, innugiartoksuillo assingit, siorkatitut immarbiksub siksanganêtutitut unnuktigijut; majorarpullo tangmarterlutiglo Michmaseme, Bethavenib kittâne.

6 Israelib angutingita tamanna tækkolauramitsuk, kappianartovingmênamik, (innugiartoksuit kappiasudlaramik) ijerput kairosungne, kôrkunelo, kairtunelo, ittersanelo, îttersâlungnelo.

7 Ebræerille aivut Jordane ikârlugo Gadekut nunanganut, Gileademullo. Saulele Gilgalemetuarpok sulle, innugiartoksuillo illunatik tamna malliklugo maksuajungnailerput.

8 Tagva uttakivok uvlut 7 it nâlugit, nelliutok Samuelib okautigilauktanga tikkilugo. Samuele tikkingimat Gilgalemut innugiartoksuit siamarterput tapsomangat.

9 Tagva Saul okarpok: Kaitsileritse uvamnut tunnergutiksanik ôtaksanik, tunnergutiksaniglo kujalijutiksanik. Tunnijivorlo tunnergutinik ôtaksanik.

10 Tunnijijarêrmallo tunnergutinik ôtaksanik, ahâk, tagva Samuele kaivok. Tagva Saulib annilune pachrearpa salutitsomavlugo.

11 Samuele okarpok: Sunamik pinniarlaukît? Saul kiovok: Tækkolaukpunga, innugiartoksuit siamartermatta uvamnit, igvillo kailaunginavit nelliutome okautaumajome, Filisterillo kattimangmatta Michmaseme.

12 Tagva okarpunga: Manna Filisterit attersarviginiarpânga Gilgalemut, uvangalo Nalekab kênanga tuksiarvigilaungilara; tagva oktorlaukpunga tunnijilauklungalo tunnergutiksanik ôtaksanik.

13 Samuele Saulemut okarpok: Sillainartomik pinniarlaukpotit, Nalekablo Gudivit perkojanga, perkolauktanga illingnut, nalelaungilat; manna Nalekab attanio vit Israelemut nakkiterajarmago soraijuitilugo,

14 Mannale attanniovit sokkosijuiniangilak. Nalekab angut kennerêrpa ingminut, omattime namagijanganut, tamna Nalekab perkova attanniokovlugo innukotiminut; igvit Nalekab perkojanga nalelaunginangne.

15 Samulelo aularpok Gilgalemit, ailunelo Gibeamut Benjaminib nunanganêtomut. Saulible innugiartoksuit illagijane kittipait, angutit 600 diokasait.

16 Saulele erningalo Jonathan innugiartoksuillo illagijane 
nokkangavut Benjaminib kakkarulângane. Filisterille tangmarsimavut Michmaseme.

17 Filisterillo tangmarvinganit kattimgajut pingasut aularput nuna asseroromavlugo. Attautsit torarput apkosinermut Oframut sangmijomut, Sualib nunanganut;

18 Aipangit torarput Bethoronib apkosininganut. Pingajuillo torarput apkosinermut, sangmijomut Zeboimib naksanganut, sunakajuitub sennianêtomut.

19 Sabviortemigle naipitsijokalaungilak Israelib nunangane illunâne. Filisterit issumakalaungmatta, Ebræerit saviksoarnik kallugiarniglo sennanajarmatta.

20 Israelitillo illunamassiatik attersariakalaukput Filisterinut, kina poagrejarutekalaurangat, aggajaumiglonêt, ullimaumiglonêt, iviksukattautemiglonêt kênaksartaujariakartomik.

21 Tapkoa aggiakatuinarlaukput kênaksainermut iviksukatautinik, aggajautiniglo, kappukautiniglo, ullimautiniglo.

22 Sorsugviub uvlunga nelliutingmat manna, saviksoarlonêt, kallugiarlonêt, naipitaulaungilak innugiartoksuit Saulib Jonathaniblo illagijangita aggangine; Saule erningalo kissimik sakkokalaukpuk.

23 Filisterillo tangmarsimajut annilerput Michmasib ungatânut.

\section{KAPITEL XIV.}

Jonaihanib piniarnersoanganit nangiartuisartomit. Sauliblo pititsitiassangninganit tapsominga tokkomut, illungertornerminut sillainartomut.

PijokalaukPorlo uvlut illangane, Jonathan Saulib erninga, nukapiaminut, sakkunginik akjarsijomut okarmat: Kait, ailauluk Filisterit tangmarvinganut, akkiptingnêtomut. Okalungilarlo tamattominga atâtaminut.

2 Saulele Gibeametuarlaukpok, Granatit nappartungata iglugaseksuit issunginêlauktub attâne; innugiartoksuillo tapsomanêlauktut, anguteôlaukput. 600 dikassait.

3 Ahialo, Ahitob erninga Ikabodib angajuata, Pinehasib erningata, Elib erningata, ajochertuijôlaukpok Nalekamut Silome, ikkialijarlarmelo. Innugiartoksuit kaujimalaunginivut Jonathan aularsimangmat.

4 Apkotible Jonathanib ainasuarvigijangata Filisterit tangmarvinganut senniane kairtokalaukpok maggungnik nuvulingnik, aipa miksiptingne, aipanga ungatâne; aipa Bozesemik attekalaukpok aipangalo Sennemik. 
5 Aipa sangmilaukpok avangat Michmasemut, aipanga angat Gabamut.

6 Jonathanelo akjarsijokotiminut sakkunginik okarpok: Kait, ailauluk akkiptingnut, kippisimangitut tapkoa tangmarvinganut; imakkâ Nalegak uvaptiguk sulliniarpallukpok; Nalekamut okumailungimat unnuktutigut ikkitutigullonêt ikajorlune.

7 Tagva akjarsijokotingata sakkunginik kiovâ: Pinniarit tamainik omattingnêtunik; ailerit, ahâk, aiparivagit, sorlo omattit pijomangmat.

8 Jonathan okarpok: Atte, akkianut pigumnuk innungnut, sakkânullo pigumnuk;

9 Okarniarpatta tagva: Nokkaritik tikkikârtinata illiptingnut; tagva inniptingne nokkaromavoguk, majorarvigijomangilavuglo.

10 Okarniarpattale: Kaileritik paunga uvaptingnut; tagva majoraromavoguk tapkonunga; tagva Nalekab tunnimavait aggaptingnut. Tamannalo nellonaikutarijomavavuk.

11 Mannalo Filisterit tangmarvingata sakkânut tikkimannik, Filisterit okarput: Ahâk, Ebræerit ittersamingnit ijersimavigilauktamingnit annisimavut.

12 Angutille tangmarvingmêtut Jonathane akjarsijokotingalo sakkunginik, kiovakit, okarlutiglo: Kaileritik paunga uvaptingnut, tagva ajochertoromagalloarpaptik. Tagva Jonathan sakkome akjarsijinganut okarpok: Malliklunga majorarit, Nalekab tunnimavait Israelitit agganginut.

13 Jonathanelo aggangmigut ittikamigullo majoakpok, sakkungatalo akjarsijingata mallikpa. Tagva ochovut Jonathanib sânganut, sakkungitalo akjarsijingat tokkotsikattarpok tapsoma kingorngane;

14 Taimak, Jonathanib sakkungitalo akjarsijingata sorsugvikita sivorlerpângane angutit 20 git tokkotaulaungmatta, perorsêvingme tuktuvâk magguk poagrejarpaktangangne uvlorme attautseme.

15 Tangmarvingmêtullo natername, tangmarviublo innugiartoksoangit illunamassiatik erksinermut tikkitauvut, kattimgajoksuillo aularlauktut sunatuinarmut koaksarputtauk, taimak nuna sajukpilangmat; koaksarniôlaungmat Gudemit pijomik.

16 Sauliblo udsertortingit Gibeame Benjaminib nunanganêtome, tækkovut kattimgajut siamalermatta, sunatuinarmullo akpangermatta, nungutaulermattalo.

17 Saule innugiartoksoarnut illagijaminut okarpok: Kittitsileritse nautsertorluselo kina uvaptingnit aularsimagalloarmangat. Kittitsingmattalo, ahâk, tagva Jonathan sakkungitalo akjarsijingat tagvanêlaungilâk.

18 Tagva Saule Ahiamut okarpok: Gudib iklervinga kaile tamaunga, (Gudib iklervinga nelliutome taipsomane Israelib kittornganginêmat). 
19 Saulelo okartillugo sulle ajochertuijomut, aulajadlarnek, akpangernerlo Filisterit tangmarvingane angivalliavok. Saulelo ajochertuijomut okarpok: Tallit erkititsuk.

20 Saulelo innugiartoksuillo illagijangit illunamassiatik kattimalerput, tikkilutiglo sorsugvingmut: ahâglo, Filisterit illunatik attunit saviksoangit torarput illamingnut, pikabladlarnekarpoilo.

21 Ebraerittauk sivurngane Filisterinêlauktut tapkoalo illagilugit majorarlauktut tangmarvingmut, numamit avatânêtomit, illalutivut Israelitinut, Saulib Jonathaniblo illagijanginut.

22 Israeliblo angutingita illunatik ijerlauktut ingminik Efraimib kakkangane, tussaramik Filisterit kemângmatta, mallikpait sorsuglugit.

23 Taimak Nalekab nelliutome taipsomane Israelitit ikajorpait. Sorsungnerlo pilerpok Bethaven tikkilugo.

24 Israeliblo angutingit nukkingêrotilermatta uvlorme taipsomane, Saulib okautigimarikpait, okarlunelo: Ominarsile, nelliat nerrijok sunamik unnulerkârtinago, omigijikka uvamnut akkiniartaukârtinagit. Tagva immigiartoksuit illunamassiatik nerrilungilat sunamik.

25 Nunakattigêllo illunatik nappartut illuanut pilaukput. Pittakalaukporle igupsaujartut orksuanik nunatuinarme.

26 Innugiartoksuille nappartunut tikkilermatta, ahâk, tagva igupsaujartut orksua korlorpok tagvane; kinalonêlle pititsilungilak illanganik aggangmigut kannerminut, innugiartoksuit okarmaringnek sivoragingmatsuk.

27 Jonathanele tussarlaungilak atâtame inugiartoksuit okautimarilaungmagit, ajaupianelo aggangminut tigumiartane issautiva, nuvualo missukpa igupsaujartut orksuanut, pitilugolo aggangmigut kannerminut, tagva ijigik uitatsialerpuk.

28 Innugiartoksuit illangat kiovok okarlunelo: Atâtavit innugiartoksuit okautimarilaukpait, okalauklunelo: Ominarsile nelliat uvlome nerrijok sunamik. Innugiartoksuille nukingêrotilaukput.

29 Tagva Jonathan okarpok: Atâtama nunakattigêt tammartilankpait; tækkoleritse, kannok ijika uitatsiarmanik, kêtakullungmik igupsaujartut orksuanik ominga oksilaurama.

30 Innugiartoksuille uvlome nerrijuksaulaungimatta omigijimik perkutigilauktanginit nachvarlauktamingnit, tamanna pivlugo sorsungnek Filisterinik anginersaujungnarlaunginivok.

31 Uvlormele taipsomane Filisterit ullimarpait Michmasemit Ajalon tikkilugo. Innugiartoksuillo nukkingêrotidlalaukput.

32 Innugiartoksuillo aksarnigaujut âkiksorpait, tigulugillo saukat, tuktuvaillo, nochaillo, tokkopaillo nunatuinarme, nerrilugillo aukartillugit.

33 Tagva Saule okautsauvok: Ahâk innugiartoksuit idluitullivut, aungmik nerringmatta. Tamna okarpok: Idluitomik 
pinniarlaukpose, ujaraksoak aksakâtisiuk manna tamaunga uvamnut.

34 Saulelo okapsarpok: Innugiartoksuit akkornganut siamartitse, okautisigiglo, illunatik attunit tukturakotimingnik, saugakotimingniglo kaitsikovlugit uvamnut; tokkotsileritselo mâne, nerriluselo, illuitullikonase Nalekamut nerrinermut aungmik. Tagva innugiartoksuit illunatik attunit tukturakotitik aggamingnut kaipait tagvunga unnuarme, tokkopaillo tagvane.

35 Saulelo Altaremik sennavok Nalekamut. Tagva Altare sivurlerpâk tapsoma sennalauktanga Nalekamut.

36 Saulelo okarpok: Attersarta Filisterit malliklugit unnuarme, aksarlugillo kaulerkârtinago, amiakokarkojikonata kinamiglonêt tapkonangat. Tapkoa kiovut: Pinniarit namagijarnik tamainik. Ajochertuijorle okarpok: Tamaunga Gudemut kaglilerta.

37 Saulible Gude apperiva: Attersartuksauvîk Filisterit malliklugit? Israeliblo agganginut tunnijomavigit? Tapsomale kiolaungila nelliutome taipsomane.

38 Tagva Saule okarpok: Inugiartoksuit kattimgajungit illunatik tamaunga kailit, tukkisilerluselo, tækkolapselo kinamut idluitullinek illingamangat nelliutome tamattomane.

39 Taimagle miksekârtigijomik sorlo Nalegak Israelib piulijinga inôngmat, tamna tokkojuksauvok, ernimnullonêt Jonathanemut illingagalloarpallonêt. Inugiartoksoarnillo kialonêt kiolungila.

40 Okarporlo Israelitinut illunamassianut: Illipse mattoma ungatanêleritse; uvanga erneralo Jonathan ovanêtsomavoguk. Innugiartoksuillo Saulemut okarput: Pinniarit namagijarnik.

41 Saulelo okarpok Nalekamut Israelib Gudinganut: Idluarnermik sakkertitsilerit. Tagva Jonathan Saulelo nellautauvuk; innugiartoksuille annigoivut.

42 Saule okarpok: Immeraut attorsiuk pivlunga erneralo Jonathane pivlugo. Tagva Jonathan nellautauvok.

43 Saulelo okarpok Jonathanemut: Okautinga, sunamik pinnialaukit? Jonathanib okautigiva tapsomunga, okarlunelo: Igupsaujartut orksuanik ketârsungmik ôksilaukpunga ajaupiamut aggamnut tigumiarlauktamnut; ahâglo tamanna pivlugo tokkotaksauvunga.

44 Tagva Saule okarpok: Gudib imâk, imâglo pinniarvigilinga; Jonathan tokkojuksauvotit.

45 Innugiartoksuille Saulemut okarput: Jonathane piulijaunartomik, taimak angitigijomik pinniarlauktok Israeleme, tokkojuksauva? Tamanna ungasigle. Sorlo miksekârtomik Nalegak innôngmat, kattaktokartuksaungilak nujarmik attautsemiglonêt niakunganit nunamut; Gude uvlome tapsomûna pinniarlaungmat. Taimak innugiartoksuit Jonathane piulivât, tokkotaujuksaukonnago. 
46 Tagva Saule majorarivok Filisterinit, Filisterillo aivut nunagijamingnut.

47 Saulible Israelib nalegauvinga tigujarêramiuk, omigijine illumajine illunaita sorsugvigilerpait, Moabiterit, Amonib kittorngangit, Edomiterit, Zobamiut attaningat, Filisterillo; sanguvingminelo illunaine sugiarnermik pititsivok.

48 Sorsuktukśoarniglo kattimgajunik âkiksoivok, ullimarlugillo Amalekiterit, Israelitillo piulivait illunatik kappiasuktitsijut tapkoninga agganginit.

49 Saulele ernekalaukpok, Jonathanemik, Iswimiglo Malchisuamiglo. Pannigiglo imaitungnik attekalaukpuk: angajudlek, Merobemik, nukardlerlo Michalemik.

50 Sauliblo nullianga, Ahimâzib panninga, attekalaukpok Ahinoamemik. Sorsuktuksaksoakotingitalo angajokangat, Nerib erninga, Saulib kattangutiârsua attekalaukpok Abneremik.

51 Saulible Kise atâtagiva; Nerele Abnerib atâtanga Abielsib ernerilaukpa.

52 Songojomigle sorsungnekatsainarlaukpok Filisterinut Saulib innosingane tamât. Nanelo Saule angumik nukkelingmik songojomiglo tækkolaurangame, tamna pitipa ingminut.

\section{KAPITEL XV.}

Saulib salakarninganik Amalekiterinit; nalengininganiglo Gudemik, ajektortauninganiglo.

SAMUELE Saulemut okarpok: Nalekab tillilaukpânga mingoaraksarigapkit attanermut innukotinginut Israelitinut; tagva tussarit manna Nalekab okausingita. nippinganik.

2 Imâk Nalegak Zebaoth okarpok: Erkaimavunga, sumik Amalek pinniarlaungmat Israelitinut, kannok terliarvigilaungmagit apkome, Egiptenemit aulartillugit.

3 Tagva manna ailerit, ullimarlugillo Amalekiterit, nungulugillo pigijangit tamaita illaliudlugit. Nikkaginiarnagit; tokkotsilerille tamangnik angutinik arnaniglo, sorrutseniglo amâmaktuniglo, tuktuvângnik saukaniglo, Kamêlinik, siutekoktojuniglo.

4 Sauliblo innugiartoksuit kattitipait, kittilaitalo Telaimeme, angutit pissuktut 200000 dit, angutillo 10000 dit Judamit.

5 Saulelo Amalekiterit iglugaseksoanginut tikkiname terliarsiviksalliorpok kôb kiglingane.

6 Keniterillo okautsaukovait: Aileritse nûluselo attersaritse Amalekiterinit, tapkonunga illaliudluse, illipsingnik nungutsi. 
konanga; illipse Israelitit illunaita takkopsoagilaurapsigik Egiptenemit aularlauktillugit. Taimak Keniterit nûlerput Amalekiterinit.

7 Tagva Saulib Amalekiterit ullimarpait Hevilamit Sur, Egiptenib sivuranêtok tikkilugo.

8 Agaglo Amalekiterit attanningat tiguva ômatillugo, innukattigêksuillo illunamassiatik nungupait saviksub kênanganut.

9 Saulible innugiartoksuillo Agag nikkagivât, saugatsiallo, tuktuvaillo kuinijut, saugârkallo, illunaitalo ânanaujut, nungutsomalaungilaillo; kannoêtulle piungitullo, tamakkoa nungupait.

10 Tagva Nalekab okausingata Samuele tikkipa, okarlunelo:

11 Kakkialerpunga Saule attaningortilaurapko; tunnusimangmanga, okautsikkalo nellautsitilaungimagit. Tamanna Samuelib issumajarutigilerpa, niplialunelo Nalekamut unnuak nâlugo.

12 Samuele uvlarârsukut parngnailerpok Saule uvlarme pâromavlugo. Okautsauvorlo Saule Karmelemut tikkisimangmat, nappartitsimangmallo tagvane ingminut sâlakarnerub nellonaikutanganik, ingerarlaungmallo sunatuinarmut, tikkisimangmallo unnunga Gilgalemut.

13 Samuele manna Saulemut tikkimat, Saule okarpok tapsomunga: Nalekab saimartilitit! Nalekab okausingit nellautsitilaukpakka.

14 Samuelible Saule kiova: Sunauvâle saukat nipliadlarningit siutimne, tuktuvaillo kallingoadlarningit tussartakka?

15 Saule okarpok: Amalekiterinit neksarlaukpait; innugiartoksuit illunatik saukat tuktuvaillo ânanaunerpât nikkagilaungmagit, tunnergutiksat Nalekamut Gudingnut pivlugit; assingit nungulaukpavut.

16 Samuelible Saule kiova: Okautsaulerit; sunamik Nalekab okauserilauktanganik uvamnut unnuarme tamattomane. Tamna okarpok: okarit!

17 Samuel okarpok: Imaingilâk, mikkijolauktillutit ijikpit sângangne, angajokangortitaulaukpotit Israelib kinguvarêngita akkorngane, Nalekablo mingoarlaukpâtit attaningortilutit Israelitinut?

18 Nalekablo tillilaukpâtit apkosinermut, okarlunelo: Ailerit idluitullijullo Amalekiterit nungukit, sorsugvigilugillo nungutsikârtinak taipkoninga.

19 Sôg Nalekab nippinga nalelaungikiuk, sangulutillo aksarnigaujanut, idluitullilutillo Nalekab ijikita sângangne?

20 Saulib Samuele kiova: Nalekab nippinga nalelauralloarpara, ailungalo apkomut Nalekab tilliklerutigilauktanganut uvamnik. Agaglo Amalekiterit attaningat âlaukpara tamaunga, Amalekiterillo nungulaukpakka;

21 Inugiartoksuille aksarnigaujut saukat tuktuvaillo, âna- 
naunerpât nungutaksat akkorngane, tigulaukpait tunnergutigijomavlugit Nalekamut Gudingnut Gilgaleme.

22 Samuelele okarpok: Issumavit Nalekab tunnergutiksat, tunergutiksallo ôtaksat kuviaginersaringmagit nalengnermit Nalekab nippinganik? Ahâk nalengnek namanersauvok tunnergutiksamit, kammatsiarnerlo namanersauvok saukat angutingita orsuanit.

23 Nalenginek angekokterniôngmat, akkerartornerlo kivgartorniôvok Gudeungitunik, Gudingoaniglo. Mannalo Nalekab okausingit singalukogilaurangne, tapsoma singalukogilaurivâtit attanniokonak Israelitinut.

24 Tagva Saul Samuelemut okarpok: Idluitullilaukpunga, Nalekab perkojanga okautsitillo tappagilaurapkit; innugiartoksuit illimagilaurapkit, nippingillo nalelaukpakka.

25 Mannalo idluitullinermik tamattominga issumagijungnaivigilaunga, utterkattigilungalo, Nalekamut tuksiarkovlunga.

26 Samuele Saulemut okarpok: Utterkattigijomangilagit; Nalekab okausingit singalukogilaurangne, Nalekablo igvit singalukogingmattit attaniôkonnak Israelitinut.

27 Samuelelo tunnulermat aularomavlune, tapsoma tiguva ullingata terrekungagut, tamnalo allikpok.

28 Tagva Samuele okarpok tapsomunga: Nalekab uvlome Israelib attaniovinga aksarnigarilaukpa illingnit, tunnimalugolo innukattingnut idluarkijarnut.

29 Sâlakarte Israeleme sæglolunginivok, kakkialingilarlo; innuôlunginame suna kakkialerutigilugo pijuksaungmat.

30 Tamnale okarpok: Idluitullilaukpunga; opigilaungale mana innukotima angajokangita Israelitillo sầngine; utterkattigilungalo, Nalegak Gudit tuksiarvigilugo pikovlunga.

31 Taimak Samuele utterpok, malliklunelo Saulemik, Saule tuksiarkovlugo Nalekamut.

32 Samuel okarpok: Agag Amalekiterit attaningat kaitaule uvamnut. Agagiblo sivuranane aivigiva, okarlunelo: Taimak tokkub kassilinarninga pêrtaujariakarpok.

33 Samuel okarpok: Sorlo saviksoarpit arnat aksarlaungmagit kittornganginik, taimak anânat aksartaujuksaugivok kittorngaminik arnat akkorngane. Taimak Samuelib Agag ullimarpa avgorlugo Nalekab sângane Gilgaleme.

34 Samuelelo aivok Ramatemut; Saulele majorarpok iglominut Gibeamêtomut.

35 Samueliblo Saule manamit tækkojungnaipa tokkome uvlunga tikkilugo. Samuelible Saule oggurilauralloarpa, Nalegak kakkialilaungmat Saule attaningortilaungmago Israelitinut. 


\section{KAPITEL XVI.}

Davidib mingoariauninganit Samuelemut aitanermut. Sauliblo annûdlartub kuviasuktitauninganik Davidib Harfesijarninganut.

$\mathrm{N}$

ALEGARLO Samuelemnt okarpok: Kanok akkunêrtigijomik ogguarkît Saul singalukogimajara pivlugo, attaniojuksaungimat Israelitinut. Naksukotit orksukaut illulertoruk orksumik, ailutillo, tillijomavagit Bethlehemiomut Isaimut; tapsoma erningita illangat tækkungiarapko, attanniokovlugo.

2 Samuele okarpok: Kannok taikungartuksauvîk? Saulib tussarniarpa tokkoniarlungalo. Nalegak okarpok: Tuktuvângnit nocharmik tigusilerit illingnut, okarillo: Tikkipunga tunnergutiksamik tunnijijomavlunga Nalekamut.

3 Isailo kaikojaksarivat tunnijivingmut tunergutiksamik, tagvane kaujititsomavagit pinniaraksarnik, mingoarsikovlutit okautiginiartamnik illingnut.

4 Samuel pinniarpok sorlo Nalekab okautilaungmago, tikkiporlo Bethlehemut. Tagva iglugaseksuit angajokangit kakkilarput, pachrearpâllo, okarlutiglo: Ullapirsautekarka tikkinavit?

5 Tamna okiarpok: Ahaila, tikkipunga Nalegak tunnergutiksamik tunnitsivigijomavlugo; ivsornaititse illipsingnik, kailuselo illagilunga tunnijivingmut tunnergutiksamik. Isailo erningillo ivsornaitipait kaikolugillo tunnijivingmut tunnergutiksamik.

6 Tagvalo manna ittermatta Eliabe tækkonârpa issumakarlunelo, tamnaumangat Nalekab saimartitanga.

7 Nalegarle Samuelemut okarpok; Tækkonârniarnak tautuanik angininganiglonêt; uvanga nachogigapko. Innub tækkoningatut pilungimat. Innuk tautukpok ijit sanginêtunik, Nalekable omat nautsertorpa.

8 Tagra Isaib Abinadabe kaikova, sângagorkolugolo Samuelib. Okarporlo: Nalekab tamna annerilunginiva.

9 Tagva Isaib Sama sângagorkova. Samuele okarpok: Nalekab tamna annerilunginiva.

10 Tagva Isaib ernine 7 it sângagorkovait. Samuelele okarpok Isaimut: Nalekab tapkoa nelliallonêt annerilaungila.

11 Samuelelo Isaimut okarpok: Nukapitset tapkoatuangôvât? Tamna okarpok: Nukkârdlerpâk amiakovok sulle; ahâglo saukat mianerivait. Tagva Samuel Isaimut okarpok: Tilliklerit tagvunga, aijaukolugolo; inginianginapta tikkikârtinaago ovunga. 
12 Tagva tilliklerpok tagvunga aijaukolugolo. Aupalangalaukporlo, ijitsiariklunelo, taututsiariklarmelo. Nalegarlo okarpok: Atte! mingoarleruk, tamnauvok.

13 Tagva Samuelib naksukotine orksuktalik tiguva, mingoarpâlo kattangutingita akkorngane. Nalekablö Anerningata Davide tikkivigiva uvlormit taipsomangamit kingornganelo. Samuelele parngnailerpok, ailunelo Ramamut.

14 Nalekable Anerninga nûlerpok Saulemit, annernerublo idluitub Nalekamit pijub uimajartipa.

15 Tagva Saulib kivgangit okarput tapsomunga: Ahâk, annernerub idluitub Gudemit pijub uimajartidlarpâtit.

16 Nalegapta kivgane, sângane nangertut okautiligit, kennerkovlugit angumik Harfesijatsiarungnartomik; Harfesijarkovlugo aggangminut tikkitauleraikuvit annernermut idluitomut Gudemit pijomut, nakkornersaulerkovlutit.

17 Tagva Saule kivgaminut okarpok: Kênajaleritse angumik, nokakseralingmik sungiusimatsiartomik, kaitsiuglo uvamnut.

18 Tagva nukapitset tapkoa illangat kiovok, okarlunelo: Ahâk, tækkolaukpara Isaib Bethlehemiub erningita illangat, tamna nokakseralingmik attutsiarungnarpok, anguteôjok erkinaitok, sorsugungnartok, sillatôjorlo sunatuinarne, pinnarnartorlo, Nalekablo illagiva.

19 Tagva Saulib kivgartortut tillivait Isaimut, okautsaukovalo: Ernît Davide saukanêtok tillilauruk uvamnut.

20 Tagva Isaib siutekoktôjok tiguva, kakkojarlo illulerkuteloWeinetalik, angusalluârsuglo tuktungajok illaliudlugit, tillikliutilaitalo Saulemut ernermigut Davidekut.

21 Taimak Davide Saulemut tikkipok, kivgartorlunelo sângane, tapsomalo nagligidlalerpa, akjarsijungorporlo sakkunginik.

22 Saulelo tilliklerpok Isaimut, okautsaukolugolo: Davide sâmnêtsainarkolauruk, saimaneksingmat ijingma sângangne.

23 Manna Gudib annerningata Saule tikkigaikpago, tagva Davidib Harfe tiguva, attorlugolo aggangminut; tagva Saule nekkoksilerpok, namanersaulerlunelo, annernerlo idluitok nûpok tapsomangat.

\section{KAPITEL XVII.}

\section{Filisterit unatadlarninganik Israelitinut, Davidib illorear- ninganik Goliathemik.}

\footnotetext{
$\mathrm{F}$

ILISTERIT sorsuktuksaksoakotitik kattitipait sorsungnermut, kattimalerpullo Suchome Judametome, tangmarterlutiglo Suchob Asekablo akkorngangne, Daminib nâningane.
} 
2 Saulele Israeliblo angutingit kattimalerput, tangmarterlutiglo naksarme nappartolingme Eichenik, oppalungaijarlutiglo Filisterit sorsugvigijomavlugit.

3 Filisterillo nangerput kakkame ungatâne, Israelitillo kakkame miksâne, naksakalaungmat akkorngane.

4 Tagva annijokarpok Filisterit tangmarvinganit innukpamik, Goliathemik attelingmik, Gathemit pijomik, tallitut 6 tut, aggaktullo attautsit sennimututiginingititut, poktutigijomik;

5 Nessalugakalaukporlo kannujarmik niakomine, sagvingmiutakalarmelo kavisiliktun itomik; sagvingmiutangatalo kanujaub okumaininga Sekeletut 5000 ditut okumaitigijôlaukpok.

6 Kannujarmiglo karlekalaukpok niungmine, sappulutakalarmelo kannujarmik tuingmingne.

7 Kallugiakotingatalo ippua umiab napparutangatut îlaukpok; kallugiakotingatalo kikkiektanga Sekeletut 600 ditut okumaitigijôlaukpok, aksarsijokotingatalo sappulutanganik sivorlerpa.

8 Nangerporlo nipliarlunelo Israelib sorsuktokattigêkotinginut, okarvigilugillo: Sôg aularlaukise oppalungaijarluse sorsugomamut? Uvanga Filistereôlungilanga, illipselo kivgaungilase Saulemut? Annerosugitse illapse illanganik attersarniartomik uvamnut.

9 Sappingikune sorsugvigilunga, ullimarumgalo, tagva kivgaujomavogut illipsingnut, uvangale sappingikupko, ullimarlugolo, taga illipse kivgaujuksauvose uvaptingnut, kivgartorkovluse uvaptingnik.

10 Filistereôjorlo okarpok: Uvlome, uvlorme tamattomane Israelib sorsuktokattigêngningit mittautigilaukpakka; tunnitsivigisinga attautsemik, sorsugutikovlunuk.

11 Saulelo Israelitillo illunamassiatik Filistereôjub oma okauserijanginik tussaramik, kakkiladlarput sivoradlalutiglo.

12 Davidele angutib Efratemiub Bethlehemêtub, Judab nunangane, Isaimik attiggub erneriva; tamna ernekalaukpok 8 inik, itolunelo Saulib nelliuningane, inutokaulunelo angutit akkorngane.

13 Isaible erningit angajudlit pingasut Saule illagællugo aularlaukput sorsungnermut, attekarlutiglo, erniangokârtok Eliabemik, tuglia Abinadabemik, pingajuak Sammamik.

14 Davidele nukârdlerpaulaukpok. Angajudlịlle pingasut Saule illagsellugo aularmatta unatadlarnermut,

15 Davide aularivok Saulemit, atâtame saugakotinginik mianersikovlugo Bethleheme.

16 Filisterele sakkerpok uvlakut, unnulerninganelo, sakkertilunelo uvlut 40 git nâlugit.

17 Isaile ernerminut Davidemut okarpok: Tigukit angajungnut oktût una Efa tættælugo kakkojaksat jarrasimajut, kakkojallo tellimaujortut ukkua, akpangerlutillo tangmarvingmut angajungnut, 
18 Punneôjartullo tellimaujortut nutât ukkoa, âlugillo angajokanganut, angajutille nellipsarkit kannoêngitsiarmangatta, tigusilutillo sunamik perkojikpatta.

19 Saulele, tapkoalo, Israelitillo angutingit illunatik naksarmelaukput nappartolingme Eichenik, sorsugvigilaitalo Filisterit.

20 Tagva Davide uvlarârsukut parngnailerpok, saukaniglo kemailerpok udsertortemut, akjarsilunelo taikunga, sorlo Isaib perkolaungmago, tikkilunelo tallunut kamutingnut senamajunut. Sorsuktokattigêllo aularsimalaukput oppallungaijarsimalutiglo, nipliadlarlutiglo sorsungnerme.

21 Israelitit parngnailaungmatta, Filisterit parngnailaurivuttauk tapkonunga.

22 Tagva Davidib illulerkut aksartanga kemmakpa illulerkutit udsertortinganut, akpangerlunelo sorsuktunut, ailunelo salutilaitalo angajune.

23 Okartillugolo sulle tapkonunga, ahâk, tagva sakkerpok Filisterit sorsuktokattigêngninginit innukpak, Goliathemik attelik, Filistereôjok Gathemiok, okarlunelo sorlo sivurngane pilaurame; Davidele tussarpok tamattominga.

24 Angutille Israelitit nelliata angut tamna tækkoguniuk kemâjutigiva, sivoradlarlunelo.

25 Israelitillo illunatik attunît okarput: Angut taimna . sakkertok tækkolaukpissiuk? sakkersimangmat Israelitit mittautigijomavlugit. Attaniublo tamna ullimarniktok tapsominga akluititsomava tunnitsivigijomalâlo panniminik; atâtangatalo iglomiokattingit kivgaujungnaititsomavait Israeleme.

26 Tagva Davide angutinut nangerkattigijaminut okarpok: Sunamik tamna pinniarvioniarka Filisteremik ominga ullimarniktok, kangusuktitaunermiglo tamattominga pejaijok Israelemit? Kinauvâle Filistere kippisimangitok una, Gudib omatsainartub sorsuktuksaksoakotingit mittautigingmagit?

27 Tagva innugiartoksuit okautivât sivornganetut: Taimailiorvioniarpok tamna ullimarniktok tapsominga.

28 Eliablo kattangutingita angajudlerpângata tussarpa angutit okarkattigingmagit, illungertolerlunelo Davide ningaumajarvigiva, okarlunelo: Sôg attersarsimavît? Sôglo saukat ikkitut taikane innukajuitome kemalaukpigit? Angutaunasuginît omattivillo kassêninga illitarigalloarpâka. Attersarsimavotille sorsungnek tækkojomagangne.

29 Davidele kiovok: Sunamigle piniarlaukik? Perkojaumalungilanga tamattominga?

30 Allarpâlo, sâlunelo assianut, okarlunelo sivornganetut. Tagva innugiartoksuit kiovât sivornganetut.

31 Okautsillo, Davidib okauserijangit tussaramigit, okautigivait Saulemut; tapsomalo aijaukova.

32 Davidelo Saulemut okarpok: Innub kialonêt omattinga 
mungoniarane taimna pivlugo, kivgait aijuksauvok Filistere tamna sorsugvigilugo.

33 Saulele Davidemut okarpok: Igvit aijungnangilatit Filisteremut omunga, sorsugvigilugo, igvit nukapiangôgavit, tamnale sorsuktuksangôvok inuosungnerminit.

34 Davidele Saulemut okarpok: Kivgavit atâtame saugakotingit mianerilaukpait, tikkitokalaukporlo Loewemik aklamiglo, aksarnigakarporlo kingmiarlune saugarmik kattimgajunit.

35 Udlatorparalo annaulerlugolo, piulilaukparalo siggunganit; opaktormangalo tigulaukpara umingitigut, annaulugolo tokkopara.

36 Taimak kivgavit tamarmik Loewelo aklarlo annaulaukpak. Taimaglo manna Filistere tamna kippisimangitok îtuksauvok taipkoa illangatut; Gudib omatsainartub sorsuktuksaksoakotingit mamailisarlaungmagit.

37 Davidelo okarpok: Nalekab piulilauktima Loewemit aklamillo, tapsoma piuliniarivângatauk Filisteremit omangat.

38 Saulelo Davidemut okarpok: Ailerit, Nalekab illagilitit. Sauliblo Davide attitipa annorâminik, nessarpâlo nesssâlukamik kannujarmik niakoanut, sagvingmiutamiglo attitipa.

39 Davidiblo tapseruserutigiva saviksoanga annorâme sillatâgut, pissugiarlunelo, ôktorlautsenginame sulle taimaitomik. Tagva Davide Saulemut okarpok: Taimaidlunga pissugungnangilanga, sungiusimalunginama; pêrpaillo ingminit.

40 Tiguvâlo ajaupiane aggangminut, annerivaillo ujarkat tellimat manniktut kôngmit, pitilugillo ikpiarsukotiminut attortaminut, mianersijut attorpaktanganut, pôngmullo, illoereullo tiguva aggangminut ailunelo Filisteremut.

41 Filisterelo kailaurivok, kaglilerlunelo Davidemut, akjarsijokotingatalo sappulutanganik sivurlerpa.

42 Filistere manna tækkosarame, Davidelo tækkonârlugo pigame, nachogiva, nukapiangôngmat, aupalangajôvlune' pinnarnarlunelo.

43. Filisterelo Davidemut okarpok: Kingmiôvîk ajaupialijarlutit kaivigigamga? Okarnerlutigivâlo Davide, Gudigijane tailugo.

44 Okarporlo Davidemut: Kait uvamnut, uvinît tunnijomavara tingmitsanut killaub attânetunut, omajunullo naternamêtunut.

45 Davidele Filisteremut okarpok: Igvit kaivigivarma saviksoalijarlutit, kallugialijarlutillo, sappulutalijarlutillo; uvangale kaivigivagit Nalekab Zebaothib, Israelib sorsuktuksaksoakotingita Gudingata mittautigilauktavit attingane.

46 Uvlorme tamattomane Nalekab unertotiginiarpâtit aggamnut, annaunikovlunga illingnik, pêrsikovlungalo niakungnik illingnit; tunnijikovlungalo Filisterit kattimgajokotingita timing- 
inik anernengêrtunik tingmitsanut killaub attânêtunut, omajunullo nujuartunut nunamêtunut, nunamiut illunatik tukkisilerkovlugit Israele Gudekarmat,

47 Illagêktullo ukkoa tukkisilerkovlugit, Nalegak ikajorlungimat saviksoakullonêt, kallugiaksoakullonêt; Nalekab sorsungnek nangmineringmago, tunniniarpâselo aggaptingnut.

48 Tagva manna Filistere parngnailermat kailunelo, Davidelo kaglilermago, Davide tuaviatorpok akpangerlunelo kattimgajoksoakattigênit Filisteremut.

49 Davidiblo aggane pitipait ikpiârsungmut, ujarkallo illangat tiguva, illoerearlunelo uvigarpâlo Filistere kaungagut, ujarak ittermat kaunganut, tamnalo ocholermat nunamut kênaminut.

50 Taimak Davidib Filistere sâlagiva illoereumut, ujarkamullo, ullimarlugolo tokkopâla. Davidelo saviksoakalunginame aggangmine,

51 Akpangerpok Filisterelo tikkipa, saviksoangalo amuva kenailitanganit, tokkopâlo, niakungalo nakkarpa tapsomunga. Filisterille tækkogamik, songonerpâkotigijatik tokkungmat, kemâvut.

52 Israeliblo Judablo angutingit parngnailerput, nipliarlutiglo, Filisterillo udlapait naksamut Ekroniblo ittertarvinginut tikkilutik, Filisterillo ullimartauvlutik ochovut apkome ittertar.vingnut sangmijome, Gathelo Ekronelo tikkilugit.

53 Israeliblo kittorngangit utterput udlaningnermit Filisterinik, tangmarvingatalo perkutingit aksarnigarivait.

54 Davidible Filisterib niakoa tiguva, âpâlo Jerusalemut, sakkungille iglominut illivait.

55 Saulib Davide Filisteremut aijok tækkogamiuk, okarpok sorsuktuksaksoakotime angajokanganut Abnermut: Kia ernerivaûk nukapiak tamna? Abnerele okarpok: Sorlo miksekârtomik tarnît inôngmat attanersoak, nellovunga.

56 Attanek okarpok: Tagva apperkutigiuk, kia erneringmangago innuosuktok tamna.

57 Davidele uttermat unatadlarnermit Filisterinik, Abnerib tiguva âpâlo Saulib sânganut; Filisteriblo niakoa tigumiarpa aggangminut.

58 Sauliblo okautiva: Nukapiak, kia ernerivâtit? Davide okarpok: Kivgavit Isaib Bethlehemiub ernerivânga.

\section{KAPITEL XVIII.}

Jonathanib Davidiblo illanarêngnigingnik; Sauliblo annerudsininganik Davidemik, tolkotsinasuarninganiglo tapsominga.

OKARERMALlo Saulemut Jonathanib omattingata Davidib omattinga angerkattigiva, Jonathaniblo nagligilerpa omattinetut nangmininetut. 
2 Sauliblo tiguva uvlorme taipsomane, utterkojungnailugolo atâtangata iglunganut.

3 Jonathanelo Davidelo angerkattigêkpuk; nagligingmago omattinetut nangmininetut.

4 Jonathaniblo ulline attortane pêrpa, tunnivâlo Davidemut, taimaktauk ulliksoakotine, saviksoakotinelo, pittiksikotinelo, tapserutekotinelo.

5 Davidelo aularkattarpok namut tillijauvingminut Saulemut, sillatojomiglo illusekarlune. Sauliblo angajokangortipa sorsuktuksaksoakattigênut; namagijauvorlo innugiartoksoarnut illunainut, Sauliblo kivganginuttauk.

6 Pijokalaukporle, uttermat unatadlarnermit Filisterinik, arnat Israelitit iglugaseksoanginit illunainit annisimalaungmatta attanek Saule pachreartorlugo, imgerlutik okigêserlutiglo, kuviasuklutiglo, nipliajorlutiglo.

7 Arnallo maggoilimajut nellekarlutik imgerkattigêkput, nipliajorlutiglo, okarlutiglo: Saulib 1000 dit ullimarlaukpait, Davidible 10000 dit.

8 Tagva Saule ningaumajadlalerpok, okauserlo tamanna idluigiva, okarlunelo: Davidemut tunnijilaukput 10000 dinik, uvamnullo 1000 dinik; attaniovik pigijomâriva.

9 Sauliblo Davide annujutigiva uvlormit taimangamit kingornganelo.

10 Uvlublo aipangane annernerub idluitub Gudemit pijub Saule tikkipa, nellautaivorlo iglomine. Davidele nokakseralingmik attorpok aggangminut, sorlo uvlut tamaita pivalaurame. Sauliblo kallugiak tigumiarpa aggangminut,

11 Naukiarpalo, issumakarlunelo: Davide mangiptaromavara iglub senneranganut. Davidible maggoêrtorlune ullorearpa.

12 Sauliblo Davide sivurajutigiva; Nalekab illagingmago, Saulemille nusimalaukpok.

13 Tagva Saulib nuktipa ingminit, angajokangortilalo angutinut 1000 dinut; tamnalo aularkattarpok kailunelo innugiartoksuit sivurlerlugit.

14 Davidelo sillatôjomik illusekarpok pinniarnermine illunaine, Nalekablo illagiva.

15 Saule manna tækkogame, taimak sillatôjunik illusekarninganik, illeragiva.

16 Israelitille illuêngardlutik Judakullo Davide nagligivât, aularlutiglo utterlutiglo sivurlervigingmagit.

17 Saulelo Davidemut okarpok: Ahâk, panniga angajudlek Merobe tunnijomavara illingnut, nulliangôkovlugo illingnut; sivurajuituinarit, aulatsilerlutillo Nalekab unatadlarninginik. Saule issumakalaurame: Aggama âktoraksaringilât, Filisterille aggangita.

18 Davidible Saule kiova: Kinauvîk uvanga? Innoseralo 
atâtamalo kittorngarêngningit sunauvât Israeleme, uvanga ningaungolertuksaugama attannermut?

19 Nelliunerle nelliusimalermat Merobib Saulib panningata tunnijaksauvia Davidemut, tunijauvok Adrielemut Meholatemiomut, tapsomunga nulliangokovlugo.

20 Michalible Saulib paningata Davide nagligiva. Saule okautsaulaurame tamattominga okarpok: Tamanna namakpok;

21 Tunnijomavara tapsomunga, kattaktitaujutiksanganut illingakovlugo, Filisterit agganginut tikkitaukovlugo. Okarlunelo Davidemut: Uvlome taipsoma tuglianut ningaungolertuksauvotit uvamnut.

22 Sauliblo kivgane perkovait: Angigutsivluse Davide okarvigisiuk, okarluselo: Ahâk, attaniub kuviagivâtit, kivgangitalo illunatik nagligivâtit, taimaimat manna ningaungôlerit attanermut.

23 Sauliblo kivgangita okautsit taimaitut okauserivait Davidib siutikita sângangne. Davidele okarpok: Tamanna mikkijonasugivissiuk ningaungôlerlune attanermut? Uvangale anguteôvunga aklujok pijariakitorlo.

24 Sauliblo kivgangita tamanna unipkautilaurivât, okarlutiglo: Okautsit taimaitut Davidib okauserilaukpait.

25 Saule okarpok: Imâk Davidemut okaritse: Attanek tunnergusiamik pilliusiamik tussulungilak, kissiane Filisterit 100 dit amingita nuvunginik, attaniub omigijingit akkiniartaukovlugit. Saulib Davide kattaktinasuarmago Filisterit aggangitigut.

26 Tagva kivgangita Davide okautivât okautsinik taimaitunik, Davidiblo tamanna idluarasugiva ningaungôlerkovlugo attanermut. Nelliutorlo nalaungilak sulle.

27 Tagva Davide parngnailerpok, angutekotinelo illagilugit aularpok, ullimarnilunelo Filisterit akkorngane angutinik 200 dinik. Davidiblo amingita nuvungit kaipait, unnurningillo namaksitipait attanermut, ningaungolerkovlugo attanermut. Tagva Saulib pannerminik tunnitsivigiva nulliangokovlugo tapsomunga.

28 Saulelo tækkovok mallugosularmelo Nalekab Davide illagingmago. Michaliblo Saulib panningata nagligiva.

29 Tagva Saulib Davide sivuragiluarpa sulle, omigilâlo innôtsine nâlugo.

30 Filisterillo angajokangit aularmatta, Davide sillatunersauvlune pinniarlaukpok Saulib kivganginit tamainit, aularangatta; taimak attinga nertortaudlalaungmat. 


\section{KAPITEL XIX.}

Davidib annigoininganik; Saulib pinnasuarkattarninganik tokkotsomavlugo.

SAULIBLE ernine Jonathan, kivganelo illunaita okarvigilerpait, tokkotsikovlugit Davidemik. Jonathanible Saulib erningata Davide nagligidlarpa.

2 Kaujitipalo tamattominga, okarlunelo: Atâtama Saulib pijugakpâtit. Taimaimat udsertorit illingnik kaupat tikkilugo, ergusimalutillo ijerit illingnik.

3 Annijomavungale atâtagalo nangerkattigijomavara natername innigijarne, pivlutillo atâtaga okarkattigijomavara, tækkoniartamniglo nellojungnaititsomavagit.

4 Jonathanelo idluarnerpâmik okarpok atâtaminut Saulemut Davide pivlugo, okautivâlo: Attaniub kivgane Davide idluitullijutiginiarnago ; idluitullivigilaungimattit, pinniarningalo piutsiarpok illingnut.

5 Innôtsinelo ivlerilaungila, Filisterelo ullimarlaukpa, Nalekablo piulijaunermik angijoksoarmik Israelitit illunaita pinniarvigilaukpait. 'Tamanna tækkolaukpat, kuviasutigidlalaukpallo. Sôgle auk passijaksaungitok idluitullivioijomaviuk, Davide pitjutekarnak tokkotsomagangne?

6 Tagva Saulib Jonathanib nippinga nalekpa, okarmariklunelo: Sorlo miksekârtomik Nalegak inôngmat, tokkotaujuksaungilak.

7 Tagva Jonathanib Davide kaikova, okautsillo tamakkoa tamaita okautigilerpait tapsomunga, âpâlo Saulemut, tapsoma sânganekovlugo sivurnganetut.

8 Sorsungnekalaurivorle ama; Davidiblo aularlune Filisterit sorsugvigilerpait, unnuktullo ullimarpait, kemâjutigingmatsuk.

9 Annerneâluble Nalekamit pijub Saule tikkipa, iksivavorlo iglomine, kallugiarlo tigumiarpa aggangminut; Davidele nokakseralingmik attorpok aggangminut.

10 Saulelo pinnasuarpok Davide kallugiarmut mangiptaromavlugo iglub senneranganut. Tapsomale tuavilukarlune Saule kemmakpa, kallugiarlo iglub senneranganut kapputivok. Davidele kemâvok, annigoilunelo unnuarme taipsomane.

11 Saulele kivgartortunik tilliklerpok Davidib iglunganut, udsertorkovlugit tapsominga, tokkotsikovlugillo tapsominga uvlub kaulerningane. Nulliangatalo Michalib Davide nellojungnaitipa tamattominga, okarlunelo: Unuarme tamattomane tarnît piuliniangikungne, tagva kaupat tokkojuksauniarpotit. 
12 Tagva Michalib igalâkut ningitipa, ailerkovlugo, kemmâkovlugolo, annigoikovlugolo.

13 Michalib innungoak tiguva, illilâlo sinigvingmut, tuktungajublo aminga illiva niakoanut, ullìkpâlo annorangnik.

14 Tagva Saul kivgartortunik tilliklerpok aiklerkovlugit Davidemik. Michalle okarpok: Kannimavok.

15 Saulele kivgartortunik tillikTerpok Davide tækkojartorlugo, okarlunelo: Kailersiuk paunga uvamnut, aumitit illaliudlugit, tokkotaukovlugo.

16 Tillijaujut manna tikkimatta, ahâk tagva innungoak nellavok sinnigvingme, tuktungajublo aminga niakoane,

17 Tagvale Saule Michalemut okarpok: Sôg uivêrivinga, aulartilugolo omigijara, anigoikovlugo? Michal Saulemut okarpok: Tapsoma okautilaukpânga: Aularlanga, tokkoniarpagillonêt. 18 Davidele kemâvok, annigoilunelo, tikkiporlo Samuelemut Ramamêtomut, okautivâlo tamainik Saulib pinniarlauktanginik tapsomunga. Samuelelo aularkattigiva, Najotemêtuarlutiglo.

19 Saulelo okautsaulerpok: Ahâk Davide Najotemêpok Ramamêtome.

20 Tagva Saule kivgartortunik tilliklerpok tagvunga, aiklerkovlugit Davidemik. Tapkoalo tækkovait nellautaijut kattimgajut maggoit, nellautaitillugit, Samuelelo aulatsijolaukpok tapkoninga. Tagva Gudib annerningata Saulib tillijangit tikkipait, nellautailerkovlugittauk.

21 Saule tamattominga okautsaulermat, tilliklerpok kivgartortunik taipkoa assinginik, tapkoalo nellautaigivut.

22 Tagva nangminek aivok Ramamut, tikkinamelo immektarviksoarmut Sekumetomut, appertsorpok okarlunelo: Samuelelo Davidelo nanêkâk? Tagva okautsauvok: Ahâk, Najotemêpuk, Ramamêtome.

23 Aivorlo tagvunga Najotemut Ramamêtomut. Gudiblo Anerningata tikkipatauk, agvertarporlo nellautailunelo tikkikârane Najotemut Ramamêtomut.

24 Annorânelo pêrpaittauk, nellautailunelotauk Samuelib sângane, ussingavlunelo pamakterkattarpok uvlok nâlugo, unuarlo nâlugo. Tamanna pivlugo okartokarpakpok: Sauletauk nellautaijunut illingava?

\section{KAPITEL. XX.}

\section{Davidiblo Jonathaniblo illanarêngnigingnik.}

DAVIDELE kemâvok Najotemit Ramamêtomit, tikkilunelo, okalarmelo Jonathanib sângane: Sunamik pinniarlaukîk? Sunamik namangitullilaukik? Sunamik idluitullilaukik atâtarnut, innôsera pinnasuarmago? 
2 Tamnale okarpok tapsomunga: Tamanna ungasigle, tokkotaujuksaungilatit. Ahâk, atâtaga pinniarlungilak sunamik, angijomiglonêt mikkijomiglonêt, kaujitilungitaminik siutimnut; sôg atâtama tamattominga angigutijaksarivânga? Taimaipallungilak.

3 Tagva Davide okamarigannerpok, okarlunelo: Atâtait kaujimatsiarpok saimaneksigama ijikpit sângangne, tamanalo pivlugo issumavallakpok: Jonathan kaujijuksaungilak tamattominga, issumajarutiginajarmago. Ahamarik sorlo miksekârtomik Nalegak innôngmat, sorlolo miksekârtomik tarnit innôngmat, ablorniôtuinarpok attautsemik uvangalo tokkublo akkonaptingne.

4 Jonathan Davidemut okarpok: Pinniarvigijomavagit omattivit tussugijanganik. -

5 Davide okarpok tapsomunga: Ahâk, kaupat takkêlauvok, attannek illagilugo iksivakattaujuksauviga nerrimârvingme; ailanga tagva, natername ijerkovlunga uvluk pingajuak unnunga tikkidlugo.

6 Atâtavit apperkutigigumga, tagva okarit: Davidib kennuvigilaukpânga akpangerkojaujomanermik iglugaseksoakotiminut Bethlehemut; illanyit illunatik tagvane tunnergutiksamik tunnijingmatta, Jahrine tamaine tunnijauvaktomik.

7 Okarniarpat: Namakpok, tagva kivgat illingatsiarpok. Ningaumajalerniarpalle, tagva mallugosungniarpotit idluitomik kajusimalaungmat.

8 Taimak manna napkigosungnermik kivgait pinniarvigiuk; uvanga, kivgait angerkattigilauramga Nalekame. Idluitullinekarpalle illumne, tagva igvit tokkunga; sôgle âtsomavinga atâtarnut?

9 Jonathan okarpok: Tamanna ungasigle uvamnit, mallugosuktuksaugama, atâtaga kajusimangmat idluitomik pititsivigijomavlutit okautijaksarinaglo.

10 Davidele okarpok: Kia okautijartoraksarivinga, atâtavit okumaitomik kioniarpattit?

11 Jonathan Davidemut okarpok: Kait, anilunuk naternamut ailauluk. Tammarmiglo naternaliarpuk.

12 Jonathanelo Davidemut okarpok: Nalegak Israelib Gudinga, atâtaga issumasiorupko kaupat uvluglo pingajuangne, kannoêngitokarpallo Davide pivlugo, tilliklerlungikumalo, tamanna kaujititaukovlugo siutingnut;

13 Tagva Nalekab Jonathane pinniarvigiliuk imaitomiglonêt, imaitomiglonêt. Atâtamale idluitok namagikpago pivlutit, tagva kaujititaukojomagivara siutingnut, kemâlutillo ullapirsautekarlutit aularkovlutit. Nalekablo illagilitit sorlo atâtaga illagilaungmago. 
14 Taimailiorlungikuma, tagva Nalekab napkigosungninganik pinniarviginiarnanga innôtillungalonêt tokkotillungalonêt.

15 Nalekablo Davidib omigijingit pêjarniarpagit attunit nunamit; tagva igvit napkigosungnît pêrniarnago iglomiokattimnit soraijuitomik.

16 Taimak Jonathanib Davidib iglomiokattingit angerkattigivait, okarlunelo: Nalegak kaitaukojile Davidib omigijingita agganginit.

17 Jonathanelo piganerpok, okautimariklugolo Davide, taimak angitigijomik nagligidlarpa; nagligigamiuk tarninetut nangmininetut.

18 Jonathanelo okarpok tapsomunga: Kaupat takkêlauvok, tagva apperkutigijauniarpotit; kingomajauniaravit iksivavigivaktarne.

19 Uvlugle pingajuangne kaisalaurit kanunga, ailutillo tagvinga ijervigilauktarnut sennaviub uvlungane, ingilutillo ujaraksub Aselemik attiggub tungânut.

20 Tagva senniane karkjutinik pingasunik pittiktaromavunga, sorlo nellonaikutamut pittiktarajaruma.

21 A hâglo nukapiak tillijomavara okarlunga: Ailerit karkjutit keneriartorkit. Nukapiamut okarniaruma: Ahâk karkjutit miksiptingnêput tunnungne, aikit; tagva kailerit ullapirsautekarmat nangiarnartokangilarlo, sorlo miksekârtomik Nalegak inôngmat.

22 Inuosuktomut okarniarumale: Ahâk karkjutit ikkanêput sivurângne; tagva ailerit, Nalekab aularkongmattit.

23 Sunamigle igvit uvangalo okarkattigilauktaptingne, tagva Nalegak, igvit uvangalo akkonaptingnêpok soraijunane.

24 Davide ijerpok natername. Takkêlaulermallo attannek ingilerpok sâmut nerrimârvingmut, nerrijomavlune.

25 Attannerlo ingilerêrmat innigivaktaminut illuserivaktamitut iglub senneranganut; Jonathane nikkovilerpok, Abnerele ingilerpok Saulib sennianut. Davidelo kingomajauvok innigivaktangane.

26 Saulelo uvlorme taipsomane okalungilak sunamik; issumakalaukporle: Tikkitaumavallukpok sunamut, ivsornailungimat.

27 Takkêlaub uvlungata tugliane Davide kingomajaungmat iksivavigivaktangane, Saule ernerminut Jonathanemut okarpok: Sôg Isaib erninga tikkilungilâk nerrimarvingmut ikpeksarlonêt, uvlomelonêt?

28 Jonathanib Saule kiova: Kennuvigilaukpânga Bethlehemut aijomagame,

29 Okarlunelo: Ailangatôk, illagêngnivut tunnergutiksamik tunnijijariakarmatta iglugaseksoarne; angajungmalo nangminek perkolaukpânga; saimaneksiguma manna ijikpit sângangne, tagva 
aularomavunga kattangutikka tækkojartorlugit. Tamanna pirlugo tikkilungilak attaniub nerrimârvinganut.

30 Tagva Saulib illungertorlune Jonathane ningaumajarvigiva, okarlunelo tapsomunga: Tappatovlutit idluitoâlôjotit! nellungigalloarpunga, igvit Isaib erninga annerimagangne, igvit anânavillo piungitub nachogijaujutiksaptingnut.

31 Taimagle akkunêrtigijomik Isaib erninga innôngmat nuname, igvit attaniovîllo illagællutit îtsungnarniangilatit. Taimaimat tilliklerit manna, aijaukolugolo maunga uvamnut; tokkojuksaungmat.

32 Jonathanib atâtane Saule kiova okautilugolo: Suna pivlugo tokkojuksauva? Sumik pinniarlauka?

33 Tagva Saulib kallugiak naukiutiva tapsomunga kapputijomavlugo. Tagva Jonathan tukkisilerpok atâtanga kajusimamaringmat Davide tokkotsomavlugo,

34 Illungertorlunelo ningaumajarlune nikkovipok nerrimarvingmit, takkêlaublo uvlungata tugliane taipsomane nerrilungilak nerkiksamik; Davide paksarigamiuk; atâtangata taimak pitlaraksaunerarmago.

35 Uvlâkut Jonathane annilerpok naternamut, Davidib tillijauvianut tapsomunga, nukapiablo mikkijub aipariva.

36 Okarporlo nukapiamut: Akpangerit, karkjutillo pittiksautigijakka kenneriartorkit. Nukapiarlo akpangertillugo, karksok attausek pittikserutigiva kollâgut.

37 Nukapiarlo tikkimat tagvunga Jonathanib karkjutinik pittikservigijanganut, Jonathanib nipliutiva okarlunelo: Karkjutit ikkanêput sivurangne.

38 Nipliutipsarpâlo: Tuavitit nokarniarnaglo. Tagva Jonathanib nukapiakotingata karkjutit abvorpait, âlugillo angajokaminut.

39 Nukapiarlo nellolaukpok tamattoma tukkinganik, Jonathanele Davidelo kissimik kaujimavuk.

40 Tagva Jonathanib sakkune tunnivait nukapiakotiminut, okarlunelo tapsomunga: Ailerit akjarlugillo iglugaseksoarnut.

41 Nukapiak aulartuarmat, Davide nikkovipok innigijaminit sekkernganêtomit, kênanelo pûvlugo pamakterpok nunamut, pingasuêrtorlunelo opigosukpok, kunniktigêklutiglo, kugviorkattigêlarmiglo; Davidele piluarlune.

42 Jonathanele Davidemut okarpok: Ullapirsautekarlutit aulaurit. Tamamnuk okautigimarilauktavuk Nalekab attingane, okarlunuglo: Nalegak uvanga igvillo akkonaptingnele, kinguvakotimalo kinguvakotivillo akkorngane; tamanna sokkosijuile naksaungitomut.

43 Parngnailerporlo aularlunelo, Jonathanelo iglugaseksoarnut tikkipok. 


\section{KAPITEL XXI.}

Davidib kiângninganik, nerrininganiglo lickkojanik trekkojaksanik; tigusininganiglo Goliathib saviksoagilaulitanganik, kemâninganiglo Filisterinut.

DAVIDELE Nobemut tikkipok, ajochertuijomut Ahimelechemik attelingmut. Ahimelechelo kakkilarpok Davide pachrearamiuk, okautivâlo: Sôg innutôvlutit illakarnaglo angumik tikkikit?

2 Davide ajochertuijomut Ahimelechemut okarpok: Attaniub sunamik perkolaukpânga, okautilungalo: Kaujijauniarane kinamut, suna pivlugo tillilaurapkit, perkolauktamniglo illingnut. Nukapiakotikka tillimagapkittauk maunga ikkungalonêt.

3 Pekarkît manna sunamik attuinaujomik? Tagva tunnitsivigilaunga aggamnut kakkojanik tellimanik, ubvalo nachvarniartarnik.

4 Ajochertuijub Davide okautiva okarlunelo: Kakkojatuinarmik attuinaujakangilanga, kakkojamigle ivsornaitomik; nukapitset aglertuinarajalaurunik nulliamingnik.

5 Davidib ajochertuijok okautiva: Uvlut pingasut nâvlugit arnat avisimalaukput uvaptingnit aularkârnanga, nukapitsello illulerkutingit ivsornailaukput; apkulle tamanna ivsornailungipat, tagva uvlome ivsornaititauvok illulerkumut.

6 Tava ajochertuijub tunnitsivigiva ivsornaitunik, assianik kakkojakalaungimat kakkojanit takkojaksanit komergutigijaumajunik Nalekab sângane, kakkojat assingit nutât illijaukovlugit uvlorme taipsomane, taipkoa tigujauvigilauktanganut.

7 Uvlormele taipsomane angutekalaukpok kamane palangairutsaumajomik Nalekab sângane, Saulib kivgangita illanganik, Doegemik attelingmik, Edomitereôjomik, angajokaunerôjomik Saulib mianerijokotinginit.

8 Davidelo Ahimelechemut okarpok: Mâne aggangne kapputekangilâk saviksoakangilârlonêt? Saviksoara sakkukkalo neksarlungilakka; attaniub perkojangit tuavinarlaungmatta.

9 Ajochertuijok okarpok: Goliathib Filisterib ullimarlauktavit naksarme nappartolingme Eichenik, saviksoanga ovanêpok, immusimavlune ulliksoarmut, ikkiab ungatâne. Tamna pijomagungne, tagva tiguleruk, assianik pittakangimat mâne tapso-mangat. Davide okarpok: Adsekangilak, tunnilauruk uvamnut.

10 Davidelo parngnailerpok kemâlunelo Saulemit, tikkilunelo Achisemut Gathemiut attanninganut. 
11 Achisible kivgangit okarput tapsomunga: Davideôvok taimna nunab attaninga, imgerutigilauktangat, okarlutiglo: Saul ullimarnilaukpok 1000 dinik, Davidele 10000 dinik.

12 Davidelo okautsit tamakkoa omamiutarilerpait, sivuragivalo Achise Gathemiut attaningat,

13 Tautunelo ablatsautipa tapkoa tækkoane, aksakâtilunelo ingminik tapkoa aggangita attâne, aporlunelo upkoamut ittertarviksoarmêtomut, nuvângalo korliarpok uminginut.

14 Tagva Achise okarpok kivgaminut: Ahâk tækkovose angut tamna kaujimangojarmat; sôg âpissiuk uvamnut?

15 Kaujimangojartokarkîk ikkiluartunik, tamna âlaurapsiuk ovunga, senimne tattamajarkovlugo? Tamna ittertuksauva iglumnut?

\section{KAPITEL XXII.}

\section{Saulib tokkotsininganik Doegekut ajochertü̈unik 85 ginik.}

DAvidelo aularpok tagvangat, kemâlunelo Adullamib kairosunganut. Kattangutingita atâtamelo iglomiokattingita illunatik tamanna tussaramitsuk, tikkilerput tapsomunga kannunga taikunga.

2 Angutillo sunatuinait, kappianartomêlauktut, akkiliksakartullo, omattekartullo kiksartunik, kattimalerput tapsomunga, tamnalo angajokagivât; angutillo 400 diokassait tapsomanelaukput.

3 Davidelo aularpok tagvangat Mizpemut Moabiterit nunanganêtomut, okarlunelo Moabiterit attaninganut: Atâtaga anânagalo itterlit annilillo illipsingne, tukkisilerkârtinanga sumik Gudib pinniarvigijomârmangmânga.

4 Tagvanekovaillo Moabiterit attaningane, tapsomanêkovlugit Davide kairosuksoarmêtillugo.

5 Nellautaijorle Gade Davidemut okarpok: Kairosuksoarmêtsainarniarnak, aularille tikkilutillo Judab nunanganut. Tagva Davide aivok, tikkilunelo nappartunut Haretemik attelingnut.

6 Saulelo tussarpok Davide angutillo illagijangit sakkermatta. Saulelo nunakarame Gibeame nappartolingme Ramametome, kallugiak tigumiarpa aggangminut, kivgangillo illunatik nangerput seniane.

7 Tagva Saule okarpok kivgaminut senniane nangertunut: Illipse Jeminib kittorngangit tussaritse, Isaib erningata illunase tunitsiviginiaralloarpasse perorsêvingnik, kakkaniglo Weineliksanik, angajokangortiniarluselo 1000 dinut, 100 dinullo? 
8 Angerkattigêlaurapse akkerartorlunga, kaujititsijokangilarlo tamattominga siutimnut,ernermatauk Isaib erninga angerkattigilaungmago. Akkunapsingnêtokangilâk kinamik kangârtomik pivlunga nellonaijaikovlugo siutimnut? Ernerma kivgara akkerartortimago uvamnik; unangmiklerkovlugo uvamnik, sorlo sakkersimangmat.

9 Tagva Doeg Edomiok, Saulib kivgangita senniane nangertok kiovok okarlunelo: Isaib erninga tækkolaukpara, Nobemut Ahimelechemut, Ahitob erninganut tikkilaungmat.

10 Taipsoma Nalegak apperilaukpa pivlugo, tunnitsivigilâlo nerkiksanik, Goliathiblo Filistereôjub saviksoanganik.

11 Tagva Saule tilliklerpok taikunga, kaikojaukolugolo Ahimeleche ajochertuijok Ahitob erninga, atâtangatalo iglomiokattingit ajochertuijut Nobeme illunaita. Kaivullo illunatik attannermut.

12 Saulelo okarpok: Tussarit igvit Ahitob erninga. Tamna okarpok: Mânepunga Nalegâk.

13 Saulelo okarpok tapsomunga: Sôg angerkattigêlaukitik akkerartorlunga, igvit Isaiblo erninga, kakkojamiglo saviksoarmiglo tunnitsivigilaurangne, Gudelo apperilaurangne pivlugo, makkitatilugolo, unangmiklerkovlugo uvamnik, sorlo sakkersimangmat?

14 Abimelechib attannek kiova, okarlunelo: Kinalo kivgavit illunatik akkorngane Davidetut nertornartôva, ningaungôvlune attannermut, naletsiatsainarlutillo pijok, opigijaulunelo iglungne?

15 Uvlomele pigiartainarlaukîk Gude apperilugo tamna pivlugo? Tamanna ungasigle uvamnit. Attannek taimaitomik illijiniarane kivgaminut atâtamalonêt iglomiokattinginut; kivgait tamakkoninga tamainik, mikkijomiglonêt, angijomiglonêt kaujilaungimat.

16 Attannerle okarpok: Ahimelech tokkotaksauvotit, igvit atâtavillo iglomiokattingit illunamassiatik.

17 Attannerlo okarpok sorsuktuksakotiminut senimine nangertunut: Sanguleritse tokkotsiluselo Nalekab ajochertuijokotinginik; aggangitatauk Daride illagingmatsuk; kaujimalauramiglo kemâninganik, nellojungnaitilaungilânga tamattominga. Attaniuble kivgangita aggatik illijomalaungilait Nalekab ajochertuijokotinginut tapkoa ullimârlugit.

18 Tagva attannek Doegemut okarpok: Igvit sangulerit ullimârlugillo ajochertuijut. Doeg Edomitereôjok sangulerpok ullimarpaillo ajochertuijut, taimak uvlorme taipsomane angutit 85 it ikkialijartut sâtunik tokkungmatta.

19 Ajochertuijullo iglugaseksoangit Nobemik attiggit ullimârpait saviksub kênanganut, tamarmik angutit arnallo, sorrutsillo, amâmaktullo, tuktuvait siutekoktôjullo, saukallo. 
20 Ahimelechible Ahitob erningata, erningita illangat Abjatharemik attelik annigoivok, kemâlunelo Davide malliklugo;

21 Nellojungnaitilugolo Saulib Nalekab ajochertuijokotingit tokkolaungmagit.

22 Davidele Abjatharemut okarpok: Kaujimalauralloarpunga uvlorme taipsomane Edomiok Doeg tagvanêlaungmat, Saule tamattominga okautiniarajarmago: Uvanga passijaksauvunga atâtavit iglomiokattingita tarninginut tamainut.

23 Uvamnêtuarit sivuraniarnaglo; pijugaklertuble inosimnik, innôtsittauk pijugakpa, illagællungalo piulijaujuksauvotit.

\section{KAPITEL XXIII.}

Davidib piuliklerninganik iglugaselisoarnik Kegilamik attelingnik Filisterinit, 7iggotig̈̈janinganiglo, piulïjauninganiglo. D AVIDELO okautsaulerpok: Ahâk Filisterit Kegilamiut sorsugvigilerpait, aksarnigarilugillo kakkojaksalliorvingit.

2 Tagva Davidib Nalegak apperiva okarlunelo: Aijuksauvîk tagvunga, Filisterillo taipkoa ullimârlugit? Nalegarlo Daridemut okarpok: Ailerit tagvunga, Filisterillo ullimârniarpattit, Kegilamiullo piuliniarpattit.

3 Angutille Davidemêlauktut okautivât: Ahâk, sivuravogut tamâne Judame, aijomalutalo Kegilamut Filisterit kattimgajoksoanginut.

4 Tagva Davidib Nalegak ama apperiva; Nalekablo kiova okarlunelo: Atte, attersarit Kegilamut, Filisterit tunnijomagapkit aggangnut.

5 Taimak Davide angutekotine illagilugit aularpok Kegilamut, Filisterillo sursugvigivait, nergjutekotingillo aularutivait, ullimarpaillo angijomik. Taimak Davidib Kegilamiut piulivait.

6 Abjatharele, Ahimelechib erninga kemâgame Kegilamut Davidemut, ikkiak neksarpa unnunga.

7 Tagva Saule okautsaulerpok, Davide Kegilamut tikkisimangmat, okarlunelo: Gudib tunnimava aggamnut, pallangairudsaumangmat, iglugaseksoarnut tikkisimangmat, upkoaksoalingnut pallangairuservingnut senneroangnullo pallangairtaumajunut.

8 Sauliblo innugiartoksuit illunaita kaikojaukovait sorsungnermut unnunga Kegilamut, illujikovlugit Davidemik angutekotinginiglo.

9 Davidele mallugosulerame Saulib idluitomik issumagingmago, okarpok ajochertuijomut Abjatharemut: Ikkiak kaitsuk. 
10 Davidelo okarpok: Nalegak Israelib Gudinga, kivgait tussarlaukpok, Saule pinnasuarmat tikkitsomavlune Kegilamut, iglugaseksuillo asseroromavlugit pivlunga.

11 Kegilamiut pititsomarpânga taipsoma agganginut? Saulelo attersarniarkâ sorlo kivgait tussarlaungmat? Tamattominga Nalegak Israelib Gudinga kivgait nellojungnairtilauruk. Nalegarlo okarpok: Attersarniarpok.

12 Davide okarpok: Kegilamiulle uvanga angutekotikkalo pitiniarpattigut Saulib agganginut? Nalegak okarpok: Ahaila.

13 Tagva Davide parngnailerpok, angutekotine illagællugit 600 diolauktut, aulârlutiglo Kegilamit, agvertarlutiglo namut sappingitamingnut. Saule manna okautsaulerame Davide Kegilamit annigoilermat, aularane nokkangalerpok.

14 Davidele sunakajuitomêtuarpok kairosungme, kakkametuarlunelo Sifib sunakajuitungane. Saulible kennitsainarpa innôtsine nâlugo, Gudible tunnilaungila tapsoma agganginut.

15 Davidelo tækkovok, Saule aularsimangmat inôsinga nungutsomavlugo. Davidele Sifib sunakajuitunganêlaukpok nappartune.

16 Tagva Jonathan Saulib erninga parngnailerpok, ailunelo Davidemut nappartunut, aggangillo pitsartutipait Gudeme;

17 Okarlunelo tapsomunga: Sivuraniarnak, atâtama Saulib aggangita nachvarniangilâtit, attaningoromârpotillo Israelitinut, tagva uvanga tugliojomavunga illingnut; tamattominga atâtaga kaujimatsiarivok.

18 Angerkattigêkpuglo Nalekab sângane; Davidelo nappartunetuarpok, Jonathanele angerarivok.

19 Sifemiulle majorarput Saulemut Gibeamut, okarlutiglo: Davide ijersimangilâk uvaptingne kairosuksoarme nappartunetome, Hachilab kakkarolângane, tallerpianetome sunakangitome?

20 Taimak attanek manna kailune attersarle omattime pijomajanganut; tagva uvagut unertutigijomavavut attaniub agganginut.

21 Tagva Saule okarpok: Saimartitauleritse Nalekamut, napkigilaurapsinga.

22 Taimak aileritse manna, nellunginersaulerasnaritselo sulle, kaujimakovluse, tækkoluselo nane ittiggangita innigilauktanginik, kialo tækkolaungmangago ; okautsaumagama pingitsertormat.

23 Ullernairtuileritse, nautsertorluselo inninik illunainik ijervigijanginik, kailermititselo uvamnut kaujimamarikupse; tagva illagijomavapse. Nunamepat tagva kênajaromavara 1000 diokattartut Judametut akkorngane.

24 Tagva parngnailerput ailutiglo Sifemut Saulib sivurngane. Davidele angutingillo Maonib sunakajuitunganêlaukput, natername sunakajuitub tallerpiane.

25 Saule angutekotingillo manna aularmatta kenneromar- 
lutik, tagva Davide okautsaulerpok tamattominga, attersarporlo kairtomut, nokkangalunelo Maonib sunakajuitungane. Saulib tamanna tussaramiuk, Davide udlapa Maonib sunakajuitungane.

26 Saulelo angutekotine illagilugit aivok kakkab uvinganingata igluane, Davidelo angutekotinelo illagilugit kakkab uvinganingata ittiviane. Davidele tuavilukarmat Saule annigoromavlugo, tagva Saulib angutekotingitalo Davide angutekotingillo illumav: it, tigujomavlugit.

27 Tikkitokarporle tillijaujomik Saulemut, okartomik: Tuavitit, kailutillo, Filisterit ittâsimangmatta nunamut.

28 Tagva Saule uttilerpok udlaningnermit Davidemik, ailunelo Filisterit pachrearlugit; tamanna pivlugo nuna tamna taijauvok Sela Mahelkot.

\section{KAPITEL XXIV.}

\section{Davidib nikkasungninganik Saulemik kairosungme.}

DAVIDELo aularlune tagvangat majorarpok, Engediblo kairosunganêtuarpok.

2 Saulelo uttermat Filisterinit tagva okautsaulerpok: Ahâk, Davide sunakajuitomêpok Engedimik attelingme.

3 Sauliblo angutit innuosuktut 3000 dit Israelitinit illunainit tiguvait, ailunelo taikunga, Davide angutekotingillo kenneriartorlugit tuktungajut kairtungine.

4 Tikkinamelo saukat mianerijauvinganut apkosiniub sennianetunut kairosukarpok tagvane, Saulelo itterpok, ittikkane ulligomavlugit; Davidele angutekotingillo kairosub kinguane iksivavut.

5 Tagva Davidib angutekotingit okarput tapsomunga: Ahâk, tagva uvlok, Nalekab okautigilauktanga illingnut: Ahâk, omigijit tunnijomavara aggangnut, pinniarvigilugo pikovlutit namagijarnut. Davidelo nikkovipok, terrêsarlunelo Saulib ullingata terrekunganik kippisivok.

6 Kingornganele omattinga tiglitâlerpok, Saulib ullingata terrekunganik kippisimagame.

7 Okarlunelo angutekotiminut: Tamanna Nalekab ungasiktiliuk uvamnit, taimailiortuksaugama, aggakka illilugit angajokamnut, Nalekab saimartitanganut; Nalekab saimartitaringmago.

8 Davidib angutekotine innerterpait okautsinut, akkerartorkolungilaillo Saulemut. Saulele parngnailerpok kairosungmit annilune, pissuglunelo apkosinerme;

9 Kingorngane Davidetauk parngnailerpok, anilunelo kairosungmit, Saulelo kingornganit nipliarvigiva okarlunelo: Angajokâk attanek! Saule kêviarpok tækkolunelo kingunerminut. Davidiblo kênane pûpa nunamut, opigosuklunelo; 
10 Okarlunelo Saulemut: Sôg innuit okausingit nalekpigit okartut: Davidib kannoênît pinasuarpâ?

11 Ahâk, uvlorme tamattomane ijikilk tækkovuk, Nalekab uvlome tunnilaungmattit aggamnut kairosungme, okartokalaukporlo, tokkotaksarigapkit. Kissiane nikkagijaulaukpotit okalaurama: Aggakka illijomalungilakka angajokamnut, Nalekab saimartitaringmago.

12 Atâtak, tækkolerittôk ullikpît terrekovininganik aggamne, tokkotsomalaungilagit ullikpît terrekunga kippilaurapko. Illitarksilerit tækkolutillo, idluitokalungimat, tappanermiglonêt aggamne. Idluitullivigilaungilagittauk; igvillo tarniga udlapat pêjaromavlugo.

13 Nalegak idluarsaijôniarpok uvangalo igvillo akkonaptingne, pivlungalo kiglugviginiarpâtit; aggangmale aktoraksaringilâtit.

14 Sorlo okausiôvaktok nutaungitok malliklugo okartokarmat: Gudekangitunit illutsit piungitut pivut. Aggamale aktoraksaringilâtit.

15 Kina malligasuarpiûk Israelib attanninga? Kina udlakiuk? Kingmek tokkungajok, komaujak attausênak.

16 Nalegak idluarsaijôle, idluarsailunelo uvangalo igvillo akkonaptingne, trkkosarlunelo, illinganeralo âkiksorliuk, piulilingalo aggangnit.

17. Davidiblo okautsit taimaitut okauserijarêrmagit Saulemut, Saule okarpok: Tamanna igvit nippigingikiuk ernîk Davide? Saulelo niplilerpok kêalunelo,

18 Okarlunelo Davidemut: Igvit idluarnersauvotit uvamnit. Igvit idluartullivigilaukparma, uvangale idluitullivigilaukpagit.

19 Uvlomelo kaujitilaukparma kannok ajungitullivigilauramga, Nalekab pitilaungmanga aggangnut, igvillo tokkolaunginamgalonêt.

20 Kannok nelliata omigijine naipiguniuk aularkonajaralloarkauk apkotikut kanoêngitokut? Nalekab ajungitomik akkiniarlitit, uvlome piniarlauktat uvamnut pivlugo.

21 Ahâglo manna, nellungilanga attaningoromâravit, Israeliblo attaniovinga aggangnêpok.

22 Tagva manna okautimarilaunga Nalekab sângane, pêjaikonak kinguvamnik kingunapkut, attimniglo nungutsikonak, atâtama iglomiokattinginit.

23 Davidiblo Saule okautimarilerpa. Tagva Saule angerarpok. Davidele angutekotingillo majorarput kairtunit imnaksoarnut. 


\section{KAPITEL XXV.}

Samuelib tolkhnganik. Davidib 7iksartitauninganik Nabale mut, ullapirsartauninganigle Abigailemut. Davidiblo nulliangningninganik.

SAMUELELO tokkovok, Israelitillo illunamassiatik kattimalerput oggorilugolo, illijartorlâllo iglungane Ramamêtome. Davidele parngnailerpok, attersarlunelo Paranib sunakajuitunganut.

2 Angutekalaukporlo Maoneme, pinniarvikartomik Karmeleme; angullo tamna perkutevaksoakalaukpok, saugakutekarpok 3000 dinik, tuktungajokutekalarmelo 1000 dinik. Pijokalaukporlo manna saugakotine sallingmagit Karmeleme.

3 Attekalaukporlo Nabalemik, nulliangalo attekarpok Abigailemik, arnaulaukporlo sillatôjok kênatsiariktorlo; angulle taimna mangaitolaukpok kassêtolunelo pinniarnermine, Kaleblo kinguvanginit pilaukpok.

4 Davidelo sunakajuitome tussarame Nabalib saugakotine sallingmagit;

5 Tilliklerpok innuosuktunik tellimaujortunik, okarlunelo tapkonunga: Majoraritse takpaunga Karmelemut; Nabalemullo tikkigupse tagva pivlunga illanakarluse salutisiuk,

6 Okarluselo: Pilloringnartokarle, ullapirsautib illagilitit, iglomiokattitillo illunaita, pigijattillo tamaita!

7 Tussarlaukpunga sallijekaravit saukanik. Mianerijokotitit pigijatit, illagilaukpâtigut, mittautigilaungilavut, kingumaklilaungilello sunamik unnurninginit, nunakarlutik Karmeleme.

8 Innuosukotitit apperikit tamanna pivlugo, tapkoa okautiniarpâtit; innuosuktullo ukkoa saimaneksilit ijikpit sângangne, tikkisimagapta uvlorme idluartome. Kivgatit ernillo Davide tunnitsivigikit aggakpit nachvarungnartanginik.

9 Davidiblo innuosuktokotingit tikkinamik tagrunga, okautsillo tamakkoa tamaita okauserijarêramigit Davide pivlugo Nabalemut, nippangerput.

10 Nabalible Davidib kivgangit kiovait okarlunelo: Davide taimna kinauva? Isaiblo erninga taimna kinauva? Kivget tapkoa unnuksivalliavut manna angajokamingnit kemâjut.

11 Kakkojakotikka, immerlo nerkillo sallijokotimnut tokkolauktakka, tigujaksarinajarpakka, tunnilugillo innungnut tapkonunga illitaringitamnut, nakit pingmangatta?

12 Tagva Davidib innuosuktokotingit sangulerivut apkotigijamingnut, tikkinamitsuglo, tamakkoninga tamainik okautivât. 
13 Tagva Davide angutekotiminut okarpok: Illunase attunit saviksoase tapseruserutigisigik makkitipsingnut. Illunatiglo saviksoatik tapseruserpait. Davidelo tapseruserpok saviksoaminik makkitiminut, angutillo 400 dit aularlutik mallikpât takpaunga; 200 dille paivut perkutine.

14 Inuosuktulle illangata Abigaile Nabalib nullianga okautiva okarlune: Ahâk Davide kivgartortunik tilliklilaukpok sunakajuitomit, angajokavut salutilugo; tapsomale kinangagilaukpait.

15 Taipkoalo piutsialauralloarput uvaptingnut, mittautigilaungilâtigut, kingomaklilaungilagut sunamik kattimgojokotipta, unurninginit taipkonane agvertarluta naternamêdluta.

16 Avaluksoatitut illingalaukput uvaptingnut uvlotillugo unuarmelo, saukanik mianersitilluta taipkonane.

17 Kammatsiarit manna, trkkolutillo pinniaraksarnik, nellonangilak kannoêtokarniarmat angajokaptingnut, iglunganullo illunânut; angutauvorlo kuksalaitok, okautjanêtok.

18 Tagva Abigaile tuavilerpok tigulugillo kakkojat 200 dit, illulerkutiglo magguk Weinetalik, saukallo igamajut tellimat, oktutillo tellimat sennaugaktagit, Weiniblo paungangit kattingajut pannertut 100 dit, Feigillo 200 dit, ikkitipaillo siutekoktôjunut.

19 Inuosuktokotiminullo angutinut okarpok: Sivorlilersinga, ahâk, kingorngane kainiarivunga. Uinelo Nabal okautilaungila tamattominga.

20 Siutekoktôjomelo ablangatillugo, attersartillugolo kakkab tachaliningane; ahâk, tagva Davidib angutekotingitalo pârpât, tikkivigivaillo.

21 Davidele okalaukpok: Atte, sullinanga sapputilaukpakka tamaita taipsoma pingit sunakajuitome tamattomane, kingomaklilaungimat sunamik perkutiminit; taipsomale ajungitullinera akkiniarutigiva uvamnut idluitomut.

22 Gudib tamattominga, anginersamiglo Davidib omigijingit pinniarvigiligit, taimna kaulerkârtinago amiakokarkogupko attautsemik innungmik omajomiglonêt, perkutinginit pigijanginit tamainit.

23 Abigailiblo manna Davide tækkogamiuk akkasârpok siutekoktôjomit, pamakterlunelo Davidib sângane, kênane pûvlugo, opigosuklunelo nuname.

24 Pamakterlunelo ittigganginut, okarlunelo: Adjai, nalegâk, idluitullinek tamanna uvanga pigilago, kivgaillo okalerle siutikpît sângangne, kivgavillo okausingit tussarkit.

25 Nalekama omattine torartiniarnago Nabalemut omunga, angumut kuksalaitomut, sillaitôngmat sorlo attinga tukkekarmat, sillainerlo taipsomanepok. Uvangale kivgait nalekama innuosuktokotingit tækkolaungilakka tillilauktatit. 
26 Mannale nalegâk, sorlo miksekârtomik Nalegak inôngmat, sorlolo miksekârtomik tarnît inôngmat, Nalekab agviarlaukpâtit, tikkikonak aungmut tamattomunga, aggaktillo piulilaukpait. Mannalo Nabaletut îlertuksauvut omigijitit, idluitullijomajullo nalekamnut.

27 Mânepok saimanartok kivgavit kaitanga nalekamnut, tamanna innuosuktunut tunnileruk nalekama agvertarkattigijanginut.

28 Kivgait issumagijungnaivigiuk idluitullinermik tamattominga. Nalekab nalegara sennaviginiarmago iglomik sokkosijuitomik, Nalekab unatadlarninginik aulatsigavit; idluitomiglo nachvartokarniarane illingne, innôtsit nâlugo.

29 Inungmiglo makkitajokarniarpat unangmijomavlutit, tarnîllo pinnasuarlugo; tagva nalekama tarninga kellaksorkassiutijaumaniarpok innôjut kippitanganut Nalekame Gudingne; omigijivillo tarninga ajuktartaujomârpok ajuktaumut.

30 Nalekab nalegara ajungitunik tamainik okautigilauktaminik illingnut pinniarviginiarpago, perkojiniarpallo attanniokovlutit Israelemut,

31 Tagva nalekama omattinganut apporviksauniangilak, sinaungajutiksauniangilarlo, aungmik kovisilaunginavit pitjutekarnak, illingniglo nangminernik ikajorlaunginavit. Tagva Nalekab nalegara ajungitulliviginiarpa, kivgello erkaumajomârpat.

32 Tagva Davide Abigailemut okarpok: Nalegak Israelib Gudinga nertortaule, uvlorme tamattomane tilliklerlauktok illingnik, pachrearkovlutit uvamnik.

33 Okalânitillo saimanartôlît, igvillo saimartitaulerit uvlome agviarlauramga, tikkikonnanga aungmut, piuliklerkonnanga uvamnik, aggamnut nangminimnut.

34 Ahamarik, sorlo miksekârtomik Nalegak inôngmat agviartiga, idluitullikonnanga illingnut, tuavilutit pachrearajarlaungikumga, tagva Nabalemut amiakokarajangilak uvlârme tamattomane attautsemik innungmik omajomiglonêt.

35 Taimak Davidib tiguvait tapsoma agganginit kailauktangit tapsomunga, okautilugolo: Ullapirsautekarlutit majorarit iglungnut; ahâk, nippît nalelaukpara, kinaunîllo kamagivara.

36 Abigaile Nabalemut tikkimat, ahâk, tagva nerrimartitsijarêrpok iglomine, attanniub nerrimaraksangatun itomik, omattingalo serridlalaukpok illumine, talladlarporlo. Tapsomale okautilungila sunamik, mikkijomiglonêt angijomiglonêt, kaulerkârtinago.

37 Kaulermalle Weinelo Nabalemit annisimangmat tagva nulliangata okautiva taimaitomik. Tagva omattinga timingane tokkolerpok, ujarkatun îlerdlune.

38 Uvlullo tellimaujortolermatta Nalekab annauva tokkokovlugo. 
39 Davidele tamattominga tussarame Nabale tokkolerêrmat okarpok: Nalegak nertortaule mittautigijaunimnik akkiniarutekalauktok Nabalemik, kivganelo sapputilaungmago idluitullinermit; Nalekab Nabalib idluitullininga tamana akkiniarutigilaungmago niakoanut. Davidelo tilliklerpok Abigailelo okautsaukova nullianigomagamiuk.

40 Davidible kivgangit Abigailemut tikkinamik Karmelemut, okarkattigivât, okarlutiglo: Davidib tillilaukpâtigut, nullianigomangmattit.

41 Tamna nikkovilerpok, kênanelo pûvlugo nunamut, opigosukpok okarlunelo: Ahâk kivget mânepok, kivgartorkovlugo nalekama kivganginik, ittigganginiglo ubvarkovlugo.

42 Abigailelo tuavilaukpok parngnailerlunelo siutekoktôjorlo ablangavigiva, uigasuillo tellimat kivgarijane illagilugit, malliklugillo Davidib tillilauktangit, nulliangolerlunelo tapsomunga.

43 Davidiblo Ahinoametauk Jesreelmiok nullianikpa, tamarmiglo nulliarilaukpâk.

44 Saulible pannine Michale, Davidib nullianga, tunniva Faltimut, Laisib Galimiub erninganut.

\section{KAPITEL XXVI.}

\section{Davidib tigusiningani\% Saulio kallugianganit ermgusiârsung- aniglo; nikkasungninganiglo Saulib inôsinganit.}

SIFEMIULLE tikkiput Saulemut Gibeamêtomut okarlutiglo: Davide ijersimangilâk Hachilab kakkarulângane sunakajuitub sivurâne?

2 Tagva Saule parngnailerpok, attersarlunelo Sifib sunakajuitunganut, angutit innuosuktut 3000 dit Israelemit illagilugit, Davide kenneromavlugo Sifib sunakajuitungane.

3 Tangmarterporlo Hachilab kakkarulângane sunakajuitub sivuranêtome, apkosiniub tungâne. Davidele sunakajuitomêtuarpok. Tækkogamelo Saulib mallingmago sunakajuitomut;

4 Nautsertortuksanik tilliklerpok, tukkisilerlunelo Saule nellâgut tikkisimangmât.

5 Davidelo parngnailerpok, tikkilunelo tagvunga Saulib tangmarvigijanganut, tækkolunelo Saulib innangavigijanganik, Abner Nerib erninga sorsuktuksaksoakotingita angajokangat illagilugo. Saule tallut illuanêlaukpok kamutinut sennamajut, sorsuktuksoakotingillo avatinganêdlutik.

6 Tagva Davide kiovlune, okarpok Ahimelechemut, Hethemiomut, Abisaimullo Zerujab erninganut, Joabib nukanganut: Kia attersarkattigijomavânga Saulib tangmarvinganut? Abisai okarpok: Uvanga attersarkattigijomavagit. 
7 Taimak Davide Abisailo tikkipuk unuarme inugiartoksoarnut. Ahâglo, Saule innangavok sinniklunelo tallut kamutit senamajut illuane, kallugiangalo kapposimavok niakungata tungâne nunamut; Abnerele inugiartoksuillo inangavut avatâne.

8 Tagva Abisai Davidemut okarpok: Gudib omigijit uvlome pitimava aggangnut; taimak uvanga kallugiarmut attausiarlunga mangiptaromavara nunamut, aipanganik pijariakarkonago.

9 Davidele Abisaimut okarpok: Asserorniarnago pît; kiale aggane illijomavagit Nalekab saimartitanganut pitlartauniaranelo?

10 Davide okapsarivok: Sorlo miksekârtomik Nalegak inôngmat, Nalekab annaulungipago, piviksangalonêt tikkilungipat tokkokovlugo, sorsungnermullonêt ailune tokkotaulungipat,

11 Nalekab uvamnit ungasiktiliuk, aggakka illijaksarigapkit Nalekab saimartitanganut. Taimak tigukik manna kallugiak niakungata tungânêtok ermgusiârsuglo, aularlauluglo.

12 Taimak Davidib kallugiak ermgusiârsuglo, Saulib niakungata tungânêtuk, tiguvâk aularlunelo; tækkojokalungilarlo, mallugosuktokalungilarlonêt tamattominga, tuppalertokangilarlonêt; siningnermut ittijomut, Nalekamit pijomut tikkitaulaungmatta.

13 Davidelo manna akkianut tikkisimagame, nangeriartorpok kakkab nuvuanut allivasiktomit, sivitolaungmat akkorngane;

14 Kaggudlarvigivaillo innugiartoksuit Abnerelo, okarlunelo: Tussarlungilatît Abner? Abnerelo kiovok okarlunelo: Kinauvît, attanek taimak kaggudlarvigigangne?

15 Davidelo Abneremut okarpok: Angutaulungilatit? Kialo nelligivâtit Israeleme? Sôgle sapputilaungikiuk angajokajet attanek? Innugiartoksuit illangat ittersimangmat, angajokajet attanek asseroromavlugo.

16 Namalungilarle taimailiorlauravit. Sorlo Nalegak miksekârtomik innôngmat, kittorngauvose tokkomut, angajokâse Nalekab saimartitanga sapputilaunginapsiuk. Tækkolerit manna manêpuk attaniub kallugianga ermgusiârsungalo, niakungata tunganêlauktuk.

17 Tagva Saulib Davidib nippinga illitarilerpa, okarlunelo: Tamanna igvit nippigingikiuk ernîk Davide? Davide okarpok: Nippiga tagva, nalegâk attanek.

18 Okapsarlunelo: Sôg nalekama kivgane taimak unangmivaûk? sunaliorlaukik? Sunamiglo idluitomik pittakarka aggamne?

19 Taimak tussarittôk manna, nalegâk attanek, kivgame okausinginik: Nalekab kajungertipattit akkerartorlunga, tagva tunnergutiksamik nerkiksamik naimalerle; innuble kittorngangit taimailiorpatta, tagva ominarsijaulit Nalekab sângane, uvlome ajektoraminga, Nalekab kingormgutijanganêtsungnanginama, okarlutiglo: Ailerit kivgartorlutillo Gudib assinginik. 
20 Tagva manna auga kovijauniarane nunamut Nalekab kênangata sângane. Israelib attaninga aularsimagame komaujak attausênak kenneromavlugo, sorlo niksartok tupjartauvangmat kakkane.

21 Saulelo okarpok: Idluitullilaukpunga, kailaungmît ernîk Davide; mattoma kingorngane idluitullivigijomairpagit; tarniga uvlorme tamattomane ivlerijaulaungmat, ijikpît sângangne. Ahâk sillaidlunga illisimaidlarlungalo pinniarlaukpunga.

22 Davide kiovok okarlunelo: Ahâk, attaniub kallugiakotinga mânêpok; innuosuktut illangat ovungalerle ailugolo.

23 Nalekable illunaita attunît akkiniaromârpait, idluarningit okperningillo malliklugit, Nalekab uvlome tunnilaungmattit aggamnut, uvangale aggakka illijomalaungilakka Nalekab saimartitanganut.

24 Sorlolo uvlome tarnît angijônasugijaulaungmat ijimnut, taimak tarniga angijônasugijaule Nalekab ijikita sângangne, piulilingalo kappianartunit tamainit.

25 Saule Davidemut okarpok : Saimartitaulerit ernîk Davide; pinniarniarpat namaksitiniarlugolo. Davidele aularpok apkosinermine, Saulelo utterivok nunaminut.

\section{KAPITEL XXVII.}

Davidib nunakarninganit Zilklageme, Filisterit nunanganêtome. D

DAVIDELE omattimine issumalerpok; Uvlut illangane pinniarpunga Saulib agganginut; namanersakalungilak uvamnut, kemâniarnimnit Filisterit nunanganut, Saule nippotjikovlugo uvamnik, kennerungnainiarlungalo Israelib kiglilervingine tamaine; tagva annigoiniarpunga agganginit.

2 Parngnailerporlo, ailunelo angutit 600dit, tapsomanêlauktut illagilugit Achisemut Maochib erninganut, Gathemiut attaninganut.

3 Taimak Davide angutekotine illagilugit Achisemetuarpok Gathemetome, illunatik illatik illagilugit; Davidelotauk nulliakotingne Ahinoame Jesreelemiok, Abigailo Nabalib nulliagilauktanga Karmelemiok illagilugik.

4 Saulelo okautsaulerame Davidib kemâninganik Gathemut, kennerungnaipa.

5 Davidelo okarpok Achisemut: Saimaneksiguma ijikpît sângangne, tagva tunnitsiviokolaunga inniksamik iglugaseksuit illangane nuname, tagvane nunakarkovlunga; suna pivlugo kivgait illagilutit attanersub iglugaseksoakotingane iglokartuksauva?

6 Tagva Achisib uvlorme taipsomane tunnitsivigiva Zikla- 
gemik. Tamanna pivlugo Ziklage Judab attaninganut illingavok, uvlok tamanna tikkilugo.

7 Nelliunerle Davidib nunakarninga Filisterit nunangane, Jahreôvok attausek, takkillo sittamat.

8 Davidele angutekotine illagilugit majorarpok ittârlunelo Gesuriterit, Girsiterillo, Amalekiterillo nunanginut, tapkoa itsangmit nuna tamana nunakotigilaungmatsuk, Suremit Egiptene tikkilugo.

9 Davidible nuna sâlagingmago, angutillonêt arnallonêt innốkolaungilait, saukallo tuktuvaillo, siutekoktôjullo, Kamêlillo, anorâllo tiguvait uttermilunelo, tikkilunelo Achisemut.

10 Achiselo okalaurangat: Uvlome ittarlaungilasse? Tagva Davide okarpok: Aunga Judab sekkernganut, Jerahmeeliterillo sekkernganut, Keniterillo sekkernganut.

11 Davidible angutillonêt arnallonêt innôvlutik tikkikolaungilait Gathemut, issumakarporlo, taipkoa okausiksakarungnarajarput, okallajungnarajarlutiglo pivluta. Taimailiorpok Davide, tamannalo illuseritsainarlaukpa nunakardlune Filisterit nunangane.

12 Tamanna pivlugo Achisib Davide okperiva, issumakalunelo: Ingminik tippiluktilaukpok inukotiminut Israelitinut, tamanna pivlugo kivgautsainartuksauvok uvamnut.

\section{KAPITEL XXVIII.}

Saulib unatadlarnerme Filisterinut ikajortiksasiorninganit angakome arname, koaksartitauvlunelo nellojungnaititauninganik

\section{kanoêniarnerminik.}

PIJOKalaukPorlo nelliutome taipsomane Filisterit sorsuktuksaksoakotitik kattitimagit, Israelitit sorsugvigijartoromavlugit. Achiselo Davidemut okarpok: Kaujijuksauvotit igvit angutekotitillo aularkattaujuksaugapse illagilunga sorsuktuksakattigêksoarnut.

2 Davide Achisemut okarpok: Atte, tukkisijuksaurotit kivgavit pinniarungnarninginik. Achis Davidemut okarpok: Tamanna pivlugo udsertortingortitsomavagit niakumnik, innôsera nâlugo.

3 Samuelele tokkolaukpok, Israelitillo illuêngardlutik oggurilaukpât, illijartorlaukpâllo iglugaseksoakotingane Ramame. Sauliblo nellautainerluktut angakullo pêjarlaukpait nunamit.

4 Manna Filisterit kattimalermatta, tikkilutiglo tangmarterlutiglo Sunememe; Saulibtauk Israelitit illuêngardlutik kattitipait, tangmarterlutiglo Gilboame.

5 Saulible Filisterit kattimgajoksoakotingit tækkogamigit erksilerpok, omattingalo mungudlalerpok. 
6 Tapsomalo Nalegak apperiva; Nalekable kiolungila, sinnektomanekullonêt, kaumaniutiksakullonêt nellautaijokullonêt.

7 Tagva Saule kivgaminut okarpok: Kenneritse arnamik annernelingmik nellautaijungnartomik, aikovlunga taipsomunga, apperilugolo. Kivgangillo okarput tapsomunga, âhak Endoreme arnakarpok annernelingmik nellautaijungnartomik.

8 Sauliblo annorâne pêrpait, assingillo attivait, ailunelo taikunga, assikitalo magguk illagivak, tikkilutiglo unnuarme arnamut, okarlunelo: Nagliktara, nellautaivigilaunga annernermut nellautaijungnartomut, sakkêvigilungalo taipsominga okautigijamnik illingnut.

9 Arnak okarpok tapsomunga: Ahâk, kaujimagalloarpotit Saulib pinniarnerilauktanginik, kannok nellautainerluktut angakullo pêjarlaungmagit nunamit; sôgle tagva tarniga pititsomaviuk nullutsanut tokkotaukovlunga?

10 Saulible okautimarilerpa Nalekame, okarlunelo: Sorlo miksekârtomik Nalegak inôngmat, tamana idluitulliningortitaujuksaungilak illingnut.

11 Tagva arnak okarpok: Kinamik sakkêvigijaksarivigit? Tamna okarpok: Samuele sakkertiuk.

12 Arnablo manna Samuel tækkogamiuk nipliakivok, okarlunelo Saulemut: Sôg uivêrivînga? Sauleôvatit.

13 Attanerlo okautiva: Sivuranak, sunamik tækkovît? Arnak Saulemut okarpok: Gudinik tækkovunga nuijunik nunamit.

14 Tamna okarpok: Kannok tautukarka? Arnak okarpok: Itok nuivok, annorarsimajok ullingmik Seideôjomik. Tagva Saule tukkisivok Samueleôngmat, kênanelo pûpa nunamut, opigosuklunelo.

15 Samuelele Saulemut okarpok: Sôg ullapilaukpinga majoakogamga paunga? Saule okarpok: Kappiasudlarpunga, Filisterit sorsugvigivânga, Gudelo nûsimavok uvamnit, kiungilângalo, nellautaijokullonêt, sinnektomanikullonêt; tamanna pivlugo kaikojaukolaukpagit, kaujititsikovlutit uvamnut pinniaraksamnik.

16 Samuel okarpok: Sôg uvanga appertsoromavinga, Nalegak nûsimangmat illingnit, omisuktungormallo illingnik?

17 Nalekab pinniarviginiarpâtit, sorlo uvapkut okalaungmat, attanioviglo aksarnigariniarpa aggangnit, tunniniarlugolo Davidemut innukattingnut;

18 Nalekab nippinga nalelaunginangne, ningaumajarningatalo illungertorninga namaksitilaunginangne Amalekiterinut; tamanna pivlugo Nalekab taimailiorvigilaukpâtit manna.

19 Tamanalo pivlugo Nalekab Israelitittauk illaliudlutit tunniniarpait Filisterit agganginut. Kaupat igvit ernivillo illaginiarpapsinga. Taimaktauk Nalekab Israelib sorsuktokattigêk* soangit tunniniarpait Filisterit agganginut. 
20 Tagva Saul tagvainak ochovok nettermut takkininga illunât malliklugo, koaksarutigidlalaitalo Samuelib okausingit, nukkingêrotilerporlo illumine; uvlok nâlugo unnuarlo nâlugo nerrilaunginame sunamik.

21 Arnarlo itterpok Saulemut, tækkolunelo angijomik koaksangangmat, okarporlo tapsomunga: Ahâk kivgavit nippit nalelaukpa, tarnigalo pitilaukpara aggamnut, okautsitit okauserilauktatit uvamnut nalelaurapkit;

22 Taimak igvitteuk mana kivgavit nippinga naleguk. Tamuamik kakkojamik sâmiutsivigijomavagit, nerrikovlutit, nukkiksarkovlutillo aijungnarkovlutillo apkotigijarnut.

23 Tamnale kunnulaukpok, okarlunelo: Nerrijomangilanga. Tagva kivgangita arnablo innapât, nippinginik nalekovlugo. Makkiporlo nettermit ingilunelo sinnigvingmut.

24 Arnarlo nochakalaukpok kuinititaumajomik iglomine, tagva tuavidlune tokkopa, tigusilunelo sennaukamik, senalugolo, sêrnakaranelo igava,

25 Kaipalo Saulib kivgakitalo sânginut. Nerrijarêramiglo nikkoviput, aularlutiglo unnuarme.

\section{KAPITEL XXIX.}

\section{Davidib uttertitauninga Filisterinut.}

FILISTERILLE sorsuktokattigêksoakotitik illunaita kattitipait Afekeme. Israelitillo tangmarterput Aineme Jesreelemêtome.

2 Filisterillo angajokaksoangit attunit agvertarput $100 \mathrm{dit}$ 1000 dillo illagilugit; Davidele angutekotingillo kingurlerput Achiseme.

3 Tagva Filisterit angajokaksoangit okarput: Ebræerit tapkoa suksauvât? Achisib okautivait: Tamna Davideungilâk Saulib, Israelitit attaningata kivganga, uvamnêlauktok manna Jahrelo uvlullo nâlugit, sunasilaungilangalo idluitomik tapsomane taimangat kemâninganit mana tikkidlugo?

4 Filisterille angajokaksoangita ningaumajarvigivât, okautilugolo: Angut taimna utterkoleruk nokkangakolugolo nunamine, tillijauvigijamine illingnut, illagiluta attersarkonago sorsungnermut, akkerangorkonago uvaptingnut sorsungnerme. Sunamulle namagijaunersaujungnarajarka angajokaminut, pititsininganit tapsomunga angutit ukkoa niakunginik?

5 Davideungilâk taimna, imgerutigilauktangat okigêserme: Saulib 1000 dit ullimarlaukpait, Davidible 10000 dit?

6 Tagva Achisib Davide kaikova, okautilugolo: Sorlo miksekârtomik Nalegak inôngmat, uvanga nellagorasugivagit, aular- 
nîllo itternîllo illagilunga sorsuktokattigêksoarne namagivara, idluitomiglo mallugosulaungilanga illingne, nelliutomit taimanganit tikkilauravit uvamnut manna tikkidlugo; angajokaksuille namagilungilâtit.

7 Taimaimat utterit manna, ailutillo ullapirsautekarlutit, idluitullikonak Filiserit angajokangita ijingita sângine.

8 Davidele ekarpok Achisemut: Sunamik pinialaukik, sunamiglo mallugosulaukit kivgarne, nelliutomit taimangat sângnelaurama, manna tikkidlugo, kaijuksaunginama, sorsuktuksaunangalo angajokama attanniub omigijinginut?

9 Achis kiovok, okarlunelo Davidemut: Kaujimagalloarpunga, ijingmalo. namagivâtit Gudib Engelingatut sorlo. Filisterille angajokâksoangit okalaukput: Majorarkattaukoniarnago illagiluta sorungnermut.

10 Taimaimat kaupat uvlarârsukut parngnailerit igvit angajokavillo kivgangit, tikkikattigijatit; uvlarârsukullo, parngnailerêrupse, kaulerpallo tagva aularitse.

11 Taimak Davide angutekotingillo uvlarârsukut parngnailerput, aularomavlutik uvlangotilugo, tikkimilutiglo Filisterit nunanganut. Filisterille majorarput Jesereelemut,

\section{KAPITEL XXX.}

\section{Davidib salakarninganik Amalekiterinik, aitorituninganiglo aksarnigaujurite.}

TAGVA manna Davide uvluk pingajuangne angutekotine illagilugit Ziklagemut tikkimat, Amalekiterit ittâlaukput annģa, Ziklagemullo; Ziklagemiullo sâlagilaukpait, iglullo ikkilugit ikkomamut.

2 Arnallo mikkijut angijullo aularutilaukpait; tokkotsilaungilelle kinamik, aularutilaukpaille, aularsimalaukpullo apkotimikut.

3 Davidelo angutekotine illagilugit iglugaseksoarnut tikkiname, tækkolunelo ikkomamut ikkisimangmatta, nulliakotitiglo ernekotitiglo pannikotitiglo parngnairsortaumangmatta,

4 Davide innugiartoksuillo illagijangit nipliakivut, kêadlarlutiglo kêajungnarungnaikâratik.

5 Davidible nulliagik parngnanêrsortaulaurivuk, Ahinoame Jesereelemiok, Abigailelo Nabalib Karmelemiub nulliagilauktanga.

6 Davidelo angijomik kappiasulaukpok, inugiartoksuit milloromangmatsuk, innugiartoksuit illunatik tarningit annulaungmatta, nelliat ernine panninelo pivlugit. Davidele pitsartutipok ingminik Nalekame Gudimine; 
7 Okarlunelo Abjatharemut ajochertuijomut, Ahimelechib erninganut: Kaitsivigilaunga ikkiamik. Abjathariblo ikkiak kailaungmago Davidemut,

8 Davidib Nalegak appertsorpa, okarlunelo: Sorsuktuksat udlajaksarivakka, anguniarpakkalo? Okarpok: Udlakit, anguniarpattit, piuliklerniarlutillo.

9 Tagva Davide aularpok, angutillo 600 dit illagijangit; tikkinamiglo Besorib kônganut illangit nokkarput.

10 Davidele angutillo 400 dit udlanikput; angutille 200 dit nokkartut merngortorluarlaukput Besorib kônga ikârungnarlugo.

11 Angumiglo Egiptenemiomik naipitsivut natername, tamna tessiorpât Davidemut, tunnitsivigivâllo kakkojamik, nerrikovlugo, imipâllo immermik.

12 Tunnitsivigivâllo Feigib illanganik, Weiniblo paunganginik kattingajunik pannertunik maggoinik. Nerrijarêramelo annerninga utterivok; uvlut pingasut, unnuallo pingasut nâlugit nerrilaungimat sunamik, immermiglo immerlaungimat.

13 Davidelo okarpok tapsomunga: Kia pigivâtit? Nakillo pivît? Okarpok: Nukapiangôvunga Egiptenemiok, kivgaulungalo Amalekiteremut; angajokamalo kemalaukpânga kannimalaurama, uvlut pingasut mattoma sivurngane.

14 Ittâlaukpogut aunga Kretimut, Judamullo Kaleblo sekkernganut, Ziklagelo ikkilaukpavut ikkomamut.

15 Daviaib okautiva: Attersartitsomavinga ununga sorsuktuksanut taipkonunga? Okarpok: Okautimarilaunga Gudeme, tokkotsomanginamga, pititsomanginamgalonêt angajokama agganginut; tagva attersartitsomavagit, sorsuktuksanut taipkonunga.

16 Tessiorpaillo ununga. Ahâglo, siamarsimalaukput nuname illunâne, nerrilutiglo immerlarmiglo, okigêserlutiglo aksarnigaujut unnuktovaksuit aksarnigarilauktatik Filisterit Judablo nunanginit pivlugit.

17 Davidiblo ullimarpait allajalernermit uvlub aipangata unnunga tikkilugo, kemâjokalaungimat attautsemiglonêt, angutit inuosuktut 400 dit assinginik, Kamelinut ikkisâdlartut kemâlutiglo.

18 Taimak Davidib Amalekiterit aksarnigarilauktangit tamaita piulivait nuliiakotingnelo;

19 Kingomajaujokalaungilarlo mikkijomiglonêt, angijomiglonêt, ernermiglonêt, pannermiglonêt, perkomiglonêt sunamiglonêt ivajârlauktanginik. Davidib tamaita uttertipait,

20 Davidiblo saukat, tuktuvaillo tiguvait, nergjutillo ungorpait, okarpullo: Tamadsa Davidib aksarnigarijangit.

21 Davidelo angutinut 200 dinut merngortorluarlauktunut Davide malligungnarlugo, Besoriblo kôngane nokkarlauktunut tikkimat, annilerput Davide innugiartoksuillo illagijangit pach- 
reartorlugit. Davidiblo innuit kaglilerpait salutilugillo illana* karlune.

22 Tagva innuit kassêtut kuksalaitullo, Davidib illagilauktangita akkornganêtut, kiovut okarlutiglo: Aularkattigilaungimattigut, tunitsiviojuksaungilet aksarnigaujunit piulilauktaptingnit. Nelliatale nulliane kittornganelo tessiorligit aularlunelo.

23 Tagva Davide okarpok: Taimailiortuksaungilase kattangutikka Nalekab tunnilauktangit uvaptingnut pitjutigællugit, sapputsilauktub uvaptingnik, tunnijilauktublo sorsuktuksoarnik taipkoninga tikkilauktunik sorsugvigijomavluta, aggaptingnut.

24 Kia tamattomane naleksarivâsse? Sorlo tapkoa sorsungnermut attersarlauktut ningersiangititut, taimaituksaugivut tapkoa perkutine nokkangajut ningersiangit, adsigêktomiglo aviktaujuksauvut.

25 Tamanna nelliutomit taimanganit kingornganelo illusiôlaukpok idluarniôlunelo Israeleme, uvlok tamanna tikkidlugo.

26 Davidelo Ziklagemut tikkiname, aksarnigavinit illangit tillikliutivait angajokajanut Judame illanarijaminut, okarlunelo: Ahâk, tagva pilliusiakarpose Nalekab omigijingita aksarnigavininginit.

27 Tapkoale tilliklervigivait, Bethlehemêtut, Ramotemêtut âne, Jatiremêtut,

28 Aroeremêtut, Sifamotemêtut, Estemoamêtullo,

29. Rachalemêtut, taipkoalo Jerahmeliterit iglugaseksoanginêtut, Keniterillo iglugaseksoanginêtut,

30 Taipkoalo, Harmametut, Bor-Asanemêtut, Atachemêtut,

31 Taipkoa Hebronemêtut, illunanêtullo Davidib agvertarvigilauktangine angutekotine illagilugit.

\section{KAPITEL XXXI.}

Saulib kappininganik ingminik sorsungnerme; erningitalo pingasut tokkotauninganik.

FILISTERILLE Israelitit sorsugvigivait; Israeliblo angutingita Filisterit kemâjutigivait, ocholutiglo ullimartauvlutik Gilboab kakkangane.

2 Filisterillo Saule erningillo unangmivait, ullimarpaillo Jonathan, Abinadabelo, Malchisualo, Saulib erningit.

3 Sorsungnerle songudlalaukpok Saulemut, pittiksertullo pittikpât uvigarlugolo, ikkilertaumalaukporlo angijomik pittiksertunut.

4 Tagva Saule akjarsijokotiminut sakkunginik okarpok: Saviksuit amuleruk, kappilungalo tapsomunga, kippisimangitut 
taipkoa kaikonnagit kappilungalo, mittautigilungalo. Aksarsijokotingale sakkunik, pijomalungilak, angijomik sivuragame. Tagva Saulib saviksoak tiguva, ochovigivâlo.

5 Aksarsijokotingalo sakkunik tækkogame Saule tokkungmat, ochotipoktauk saviksoarminut, tokkokattigilugolo.

6 Taimak Saule erningillo pingasut akjarsijokotingalo sakkunginik, angutekotingillo tokkovut attautsekut, uvlorme taipsomane.

7 Israelible angutingit naksab ungatâne, Jordaniblo akkianêlauktut trekkogamik Israelib angutingit kemângmatta, Saulelo erningillo tokkungangmatta, iglugaseksuit kemmakpait, kemâlutiglotauk; taimaglo Filisterit kaivut iglogilaitalo.

8 Uvlub aipangane Filisterit kaivut, ullimarsimajut mattâromavlugit, naipipaillo Saule erningillo pingasut nellarlutik Gilboab kakkangane;

9 Niakungalo nakkarpât, sakkungillo pêrpait tapsomangat, tillikliutivaillo Filisterit nunanganut sunatuinarnut, okautigijaukovlugo Gudingoakotimik iglungane, innugiartoksuillo akkorngane.

10 Sagvingmiutangalo illivât Astarotib iglunganut, timingale tokkungajok nivingatipât Bethsanib avaluksoanginut.

11 Jabesemiut Gileademe tussaramik Filisterit pinniarlauktanginik Saulemut;

12 Parngnailerput angutit sorsugungnartut, ingergarlutiglo unnuak nâvlugo, tigulugillo Saulib erningitalo timingit tokkungajut Bethsanib avalunginit, âlugillo Jabesemut, ikkipaillo tagvane.

13 Sauningillo tiguvait, illijartorlugillo nappartub Jabese. mêtub attâne, nerritaililutiglo urlut 7 it nâlugit. 


\section{Samuelib aglangita aipangit.}

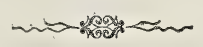

\section{KAPITEL I.}

Davidib pitlarningninganik sceglovlune okartomik tokkotsigame Saulemik. Tapsomalo imgerutinginite ogguarsiutinik Saul Jo" nathanelo pivlugit.

SAULIB tokkungata kingorngane, Davide unatadlarnermit Amalekiterinik utterlaungmat, maggungniglo uvlilaukârtillugo Ziklageme;

2 Ahâk, tagva uvluk pingajuangne angut Saulib sorsukattigêksoakotinginit tikkilerpok, annorakarlune alliksimajunik, ivjokalunelo niakomine. Davidemullo tikkiname pamakterpok nunamut, opigosuklunelo.

3 Davidible okautiva: Nakit pivît? Tapsoma okarvigiva: Israelib sorsukattigêkssoakotinginit kemâlaukpunga.

4 Davidib okautiva: Okarviginga, kannok illingava? Tamna okarpok: Innugiartoksuit kemâlaukput sorsungnermit, innuillo unnuktut ochosimavut; tappilugillo Saulelo erningalo Jonathan tokkovûktauk.

5 Davidib innuosuktok okautjine tamattominga okautiva: Nakit kaujimavît, Saulelo erningalo Jonathan tokkungmannik?

6 Innuosuktok okautjinga tamattominga okarpok: Maungatuinak tikkilaukpunga Gilboab kakkanginut; ahâglo, Saule egangavok kallugiakotiminut, kemuksillo, horsillo kângine ablangajut udlavât.

7 Keviarlaukporlo, tækkolungalo, kaikolarmalo. Uvangalo okarpunga: Mânepunga.

8 Tapsomalo okautivânga: Kinauvit? Okautivara: Amalekitereôvunga. 
9 Tamnalo okarpok uvamnut: Nangeriartorit uvamnut, tokkolungalo, illunane kappiasuktitaugama, innôseralo illumnềmarikpok sulle.

10 Tagva aivigilaukpara, tokkolugolo, kaujimamarilaurama innojungnangininganik ocholaungningata kingorngane; niakutelo tigulaukpara niakoanit, tallermiutarlo tallinganit, kaimavâkalo tamaunga illingnut nalekamnut.

11 Tagva Davidib annorâne tiguvait, alliklaitalo, angutillo tapsomanêlauktut illunatik taimailiorput.

12 Ogguarlutiglo kêalarmiglo, nerritailivullo unnuk tikkilugo, Saule, erningalo Jonathan pivlugik, Nalekablo innukotingit pivlugit, Israeliblo iglomiokotingit pivlugit, saviksoarmut ochotitaulaungmatta.

13. Davidiblo innuosuktok okautjine tamattominga okautiva: Nakit pivît? Tamna okarpok: Tækkorngârtab, Amalekiterê̂jub ernerivânga.

14 Davide okarpok tapsomunga: Suvît sivuralaunginavit, aggaktit illilugit Nalekab saimartitanganut, asserorlugo!

15 Davidiblo innuosuktokotime illangat okautiva: Maungalerit ullimârlugolo. Tapsomalo ullimârpa, tokkokovlugo.

16 Tagva Davidib okautiva: Aut niakungnut illingale; kannît nangminît pivlutit okalalaungmat, okarmallo: Nalekab saimartitanga tokkolaukpara.

17 Davidiblo ogguarsiutit tamakkoa okâlatigilaukpait, Saule erningalo Jonathane pivlugik.

18 Perkojivorlo Judab kittorngangit ajochertortaukovlugit pittiktarnermik. Ahâk, aglaksimavut nellagortut aglakotingine:

19 Angajokaunerôjut Israeleme pôktojokotingne ullimartaumavut. Kannok songojoksuit ochosimavut!

20 Okarniarase tamattominga Gatheme, unipkautiginiarassiuglo apkosinerme Askalonib iglungita akkorngane, Filisterit panningit kuviasulerkonnagit, kippisimangitut panningit serridlarkonnagit.

21 Kakkaujose Gilboame, mitsuksoakarniarane, sillalukarniaranelo illipsingnut, perorsêvikarniaranelo illipsingne, kaitsijunik tunnergutiksanik pilliusijanik; sôngojut sappulutangit tagvane pêjartaulaungmatta, Saulib sappulutanga, sorlo mingoarsimanajangipat orksomut.

22 Jonathanib pittiksinga uniorsilaungimarikpok, Sauliblo saviksoanga uttilaungimarikpok aukejartaunane ullimarsimajut aunganut, orksokijaunanelo sôngojoksuit orksunginut.

23 Saule Jonathanelo erdlingnarlutik innerkonarlutiglo innôsigingne, tokkomingnetauk aviutingilâk; okinersauvuk nektoralingmit, sanginersaulutiglo Loewemit.

24 Illipse Israelib panningit Saule kêagisiuk, annorakartitsilauktok illipsingnik aupaloriktunik pinnarnartunik, ânanausê- 
lauktorlo illipsingnik annorapsingne pinnarnarsautinut Goldelingnut.

25 Kannok sôngojoksuit taimak ochomavut sorsungnerme! Jonathan pôktojokotingne ullimartaumavok.

26 Kiksautigivagit kattangutîk Jonathan; angijomik kuviasutigilaukpagit alliagilutillo; naglingningnît kuviaginersarilaukpara arnab naglingninganit.

27 Kannok sôngojoksuit ochomavut, sorsugungnartullo tokkotaumavut!

\section{KAPITEL II.}

Davidib attaningortitauninganik Judakunut. Isbosethib attan ningortitauninganik kinguvarêt assinginut, salagïjauninganiglo unatadlarnerme.

Pijokalauktut tamakkoa kingorngane Davidib Nalegak apperiva, okarlunelo: Majorartuksauvîk paunga Judab iglu-gaseksoangita illanganut? Nalekablo okautiva: Majorarit paunga. Davide okarpok: Namut? Tamna okarpok: Hebronemut.

2 Taimak Davide tagvungarpok, nulliangne Ahinoame Jesreelemiok, Abigailelo Nabalib Karmelemiub nulliagilauktanga illagællugik.

3 Angutillo tapsomanêlauktut illautilugit Davidib takpaungartipait, illunatik attunit iglomiokotingit illagilugit; iglokalerlutiglo Hebronib iglugaseksoangine.

4. Judablo angutingit tikkilerput, Davidelo mingoarpât tagvane, attaningortilugo Judakunut. Davidelo okautsaulerame, Jabesemiut Gileademêtut Saule illijartorlaungmatsuk;

5 Kivgartortunik tilliklervigivait, okautsaukolugillo: Saimartitauleritse Nalekamut, napkiningnermik taimaitomik pinniarlaurapse angajokapsingnut Saulemut, illijartorlaurapsiuk.

6 Taimak Nalekab manna napkiningnermik nertornarnermiglo pinniarvigilise; uvangalo "ajungitullivigijomagivapse, taimailiorlaurapse.

7 Taimaglo manna aggase pitsartutitaulit, sivoraniaraselo; angajokase Saule innôjungnaimat, tagva Judakut mingoarlaukpânga attaningortilunga ingmingnut.

8 Abnerible Nerib erningata, Saulib sorsuktuksaksoakotingita angajokangata, Isboseth Saulib erninga tiguva, âpâlo Mahanaimemut,

9 Attaningortilâlo Gileademut, Assurimullo, Jesreelemullo, Efraimemullo, Israelitinullo illunainut. 
10 Isbosethelo Saulib erninga Jahrekalaukpok 40 ginik attanningortitautillugo Israelitinut, attanniotilunelo Jahrik magguk nâlugik. Judakulle Davide ungagivât.

11 Nelliutorlo Davide attanniotillugo Hebroneme Judakunut, Jahriôvut 7 it takkillo pingasojortut.

12 Abnerelo Nerib erninga, Isbosethib Saulib erningata kivgangit illagællugit aularpok sorsuktuksaksoakattigênit, ingergalunelo Gibeonemut;

13 Joabelo Zerujab erninga aularpok Davidib kivgangit illagilugit; ingmingnullo tikkiutivut Gibeonib tessingane, tangmarterpullo tapkoa miksâne, taipkoalo tetsib akkiane.

14 Abneriblo Joabe okautiva; Nukapitset parngnailerlit, pingoartigêlerlillo sâptingne. Joabe okarpok: Taimailiolerlit.

15 Tagva parngnailerput ailutiglo 12 it Benjaminekunit, Isbosethemut Saulib erninganut illingajunit, 12 illo Davidib kivganginit.

16 Illunatiglo attunît ingmingnut tigutivut niakungitigut, saviksoatiglo kapputivait senneranginut ochokattigêkpullo. Tamana pivlugo inne tamanna taijauvok Helkat Hazurim Gibeonemêtok.

17 Sorsungnekalerporlo uvlorme taipsomane sôngojomik. Abnerele Israeliblo angutingit sâlagijaulaukput Davidib angutinginut.

18 Tagvanetokalaukporle Zerujab erninginik pingasunik: Joabe, Abisai, Asahelelo. Asahelele okkilalaukpok tuktungajotut naternamêtotut;

19 Asaheliblo Abner udlapa, nûlungilarlo tallerpingmullonêt, saumingmullonêt Abneremit.

20 Tagva Abner kêviarpok okarlunelo: Asaheleôvît? Tamna okarpok: Ahaila.

21 Abnerib okautiva: Pêrit tallerpingmullonêt saumingmulslonêt, nukapitsello tapkoa illangat piuk illingnut, aksarlugolo sagvingmiutanganik. Asahelible kemmagomalaungila.

22 Tagva Abnerib Asahele okautipsarpa; Pêrit uvamnit. Sôg pijomavit ullimartaunernik uvamnut, ochokovlutit nunamut? Kanorlo kênara allortitsungnarajarpigo angajuvit Joabib sângane?

23 Kunnulaukporle nûvigilugo. Tagva Abnerib kingumut kallugiaminut kappiva nângagut, kallugiak tunnuagut annilermat; ochovorlo tagvane, tokkolerlunelo tapsoma sângane, nelliallo tikkitok tagvunga Asahalib tokkungavlune nellavigijanganut nokkarpok.

24 Joabible Abisaiblo Abner udlavâk sekkerngub nippininga tikkilugo. Tikkinamiglo Amab kakkarulânganut Giab sânganêtomut, apkome sangmijome Gibeonib sunakajuitunganut; 
25 Benjaminib kittorngangit kattilerput Abnerib kingorngane, kattimgajoârsolerlutiglo, kakkarulaillo illangata kânga aivigivât.

26 Abneriblo Joabe nipliutiva, okarlunelo: Saviksoak sorairane nungutsijuksauva? Nellovît kingorngane ogguanartokarnersaunajarmat? Kannok akkunêrtigijomik innugiartoksuit okautijomangipigît allarkovlugit kattangutimingnit?

27 Joabe okarpok: Sorlo miksekârtomik Gude innôngmat, uvlome uvlâkut taimak okarajalauruvit, innugiartoksuit illunatik attunit allarajarlaukput kattangutimingnit.

28 Joabiblo tiptulaut tiptulausijautigiva, innugiartoksuillo illunatik nokkarput, udlanigungnailutiglo Israelitinik, sorsugungnailutiglotauk.

29 Abnerible angutekotingitalo unnuak tamana nâlugo naternaksoak pissugvigivât, Jordanelo ikârpât, Bithronelo illunât ingergavigivât, tikkilutiglo tangmarvingmut.

30 Joabele tunnulerpok Abneremit, innugiartoksuillo illunaita kattitipait; Davidiblo kivganginit angutit 19 it kingomajauvut, Asahelelo.

31 Davidible kivgangit Benjaminekut Abneriblo angutekotingita akkorngane ullimarnilaukput, angutit 360 git tokkotaulaungmatta.

32 Asahelelo kivikpât, illijartorlâlo atâtangata illuvinganut Bethlehemêtomut. Joabelo angutekotinelo illagilugit, unuak nâlugo ingergakattigêkput, kagujauvlutik Hebroneme.

\section{KAPITEL III.}

\section{Abnerib illagêlerninganik Davidemut, tokkotauninganiglo Joabemut.}

AKKUNÊRTOMIGLE sorsungnekalaukpok Saulib iglomiokotingita Davidiblo iglomiokotingita akkorngane. Davidele agver tarpok pivallialunelo; Saulible iglomiokotingit agvertarput ikiglivallialutiglo.

2 Davidemullo nutakkanik erniangojokalaukpok Hebroneme: Ernerikârtanga Amnon, Ahinoamit Jesreelemiomit;

3 Tuglia Kileabe, Abigailemit, Nabalib Karmelemiub nulliagilauktanganit; pingajuat Absalome, Maachab, Thalmaib attaniub Gesureme panningata erninga.

4 Sittamangat Adonia, Hagithib erninga; tellimangat Safatja Abitalib erninga.

5 Pingasojoat, Jetream Eglamit Davidib nullianganit. Tapkoa Davidemut erniangôlaukput Hebroneme. 
6 Sorsungnekartillugo manna Saulib iglomiokotingita Davidiblo iglomiokotingita akkorngane, Abnerib Saulib iglomiokotingit pitsartutipait.

7 Saulelo panervgakalaukpok Rizpamik attelingmik, Ajab panninganik. Isbosethelo Abneremut okarpok: Sôg atâtama pannervganga sinnikattigiviûk?

8 Tagva Abnerib Isbosethib okausingit tamakkoa ningaumajadlarutigivait, okarlunelo: Kingmiub niakungatut illingavîk, Judakut akkerartorlugit Saulib atâtavit iglomiokotinginut, tapsomalo kattangutinginut, illanarijinginullo, napkigosangnermik sakkertitsijunga, pititsilaungitungalo illingnik Davidib aggang:inut? Igvillo uvlome passivarma idluitullinermik arnak pivlugo?

9 Gurlib Abner imâglo, imâglo pinniarvigiliuk, pinniarlungikuma, sorlo Nalekab Davide okautimarilaungmago,

10 Attaniovik Saulib iglunganit pêjartaujuksaungmat, Davidiblo iksivautaksoanga makkitâtitaujuksaungmat, Israelemut Judamullo, Danemit Berseba tikkilugo.

11 Tagva mattoma kingorngane kiojungnangilâ okautsemik attautsemiglonêt, taimak sivuragiva.

12 Abnerelo kivgartortunik tilliklerpok Davidemut, okautsaukolâlo: Kia nuna perkutigivâ̂k? Okarporlo: Angerkattiginga; ahâk, aggama illagijaksarivâtit, sangutitsikovlunga illingnut Israelitinik illunamassianginik.

13 Tamna okarpok: Atte, angerkattigijomavagit. Attauserle tuksiarpara illingnit, kênamnik tækkokonak, kissiane kaititsikârtillutit uvamnut Michalemik, Saulib panninganik, kainiaruvit kênara tækkojomavlugo.

14 Davide kivgartortunik tilliklilaurivoktauk Isbosethemut, Saulib erninganut, okautsaukolâlo: Nulliakotimnik Michalemik tunnitsiviginga angerutigilauktamnik uvamnut Filisterit 100 dit uviningita aminginut.

15 Isbosethelo tilliklerpok tagvunga, tigujaukolâlo angumit taipsomangat Paltielemit, Laisib erninganit.

16 Uingatalo kannilârpa, kêalunelo kingorngane Bahurime tikkilugo. Tagva Abnerib okautiva: Uttilerit, ailutillo. Uttilerporlo.

17 Abneriblo Israelitit angajokaunerôjungit okarkattigivait, okarlunelo: Illipse mattoma sivurngane akkunît Davide pinnasuarlaukpasse attanningorkovlugo illipsingnut.

18 Taimailioleritse manna, Nalekab Davide okautigilaungmago: Innukotikka Israelitit piulijomavakka kivgama Davidib aggangittigut, Filisterit agganginit omigijingitalo illunatik agganginit.

19 Abner okarivoktauk Benjaminekut siutingita sângine; okariartorlunelotauk Davidib siutikita sângangne Hebroneme, tamainik Israelitit Benjaminekullo illunatik namagijanganik. 
20 Abnerelo mana Hebronemut Davidemut tikkilermat, angutit 20 git illagallugit, Davidib nerrimârterpait.

21 Abnerelo Davidemut okarpok: Parngnaileromavunga aijomalungalo Israelitit illunamassiatik kattitilugit angajokamnut attanermut, angerkattigivlutit pikovlugit, attaniokovlutit tarnivit tussugijanganut. Taimak Davidib Abner nûlerkova ingminit, aularkovlugo ullapirsautekarlune.

22 Ahâglo, Davidib Joabiblo kivgangit tikkilerput sorsuktokattigênit, neksarlutiglo aksarnigaujunik unnuktunik. Abnerele manna Davidemêtsungnailaukpok Hebroneme; nûlerkolaukpâle, ullapirsautekarlune aularlaungmat.

23 Joabele sorsuktuksaksoakattigêllo illunatik illagællugo tikkisimangmat, okautsaulerpok, Abner Nerib erninga tikkisimalaungmat attannermut, nûlerkolaungmagolo ingminit, ullapirsautekarlune aularlaungmat;

24 Tagva Joabib attanek ittervigiva, okarlunelo: Sunaliorlaukît? Ahâk, Abnerib tikkilaukpâtit; sôg nûlerkolaukiûk illingnit, aularkovlugo?

25 Abner, Nerib erninga illitaringipiûk? Tikkisimagame tillioromavlutit, illitarksilerkovlugo itterningnik, anningnerniglo, timeroilerkovlugolo pinniarnernik tamainik.

26 Joabelo Davidemit annigame, kivgartortunik tilliklerpok Abnerib kingorngane, aiklermikovlugit tapsominga Borhasiramit; Davidelo kaujimalaungilak tamattominga.

27 Abnerelo manna tikkipsarmat Hebronemut, Joabib tessiorpa ittertarviksub kerkânut, angigutsivlune okarkattigijomavlugo; kappivâlo tagvane nângagut, tokkolerkovlugo, nukame Asahelib aunga pivlugo.

28 Davide tamattominga kingorngane tussarame, okarpok: Passijaksaungilanga attaniovigalo Nalekab sângane soraijuitomik, Abnerib Nerib erningata aunga pivlugo;

29 Joabible niakoanut illingalerle, taipsomalo atâtangata iglomiokotinginut illunainut; pittakangitsainarniaranelo Joabib kinguvângine, ajualingmik marnekatsainartomik, kallakadlartomiglo, ajaupialijarlunelo pissugiakartomik, saviksoarmullo ochotitaujomik, ajoksartomiglo nerkiksamik.

30 Taimak Joabib angajuatalo Abisaib Abner tokkopâk, tamanna pivlugo nukagik Asahele tokkolaungmago unatadlarnerme Gibeoneme.

31 Davidele Joabemut innugiartoksoarnullo illunainut tapsoma illagijanginut okarpok: Annorase alliktorsigik, pôngniglo tapseruseritse illipsingnut, Abnerelo oggurilugo pititse. Attanniublo pissuklune iklervik mallikpa.

32 Abnerelo illijartormatsuk Hebroneme, attannek nipliarkivok, kêalunelo Abnerib illuvingane, innugiartoksuillo illunatik kêavuttauk. 
33 Attanniublo Abner ârusutigiva, okarlunelo: Abner tokkolerlaungilak sillaitub tokkolerningatut.

34 Aggaktit kellaksorsimalaungilet, ittikkatillo kippitjutinut illijaungilet; ochosimavotit, sorlo nelliat uivêtoâlungnut ochotitaungmat. Tagva innugiartoksuit illunatik kêagiluarpât sulle.

35 Innugiartoksuit illunatik manna ittermatta Davide nerrikattigijomavlugo uvlomariktillugo sulle, Davide angermarikpok, okarlunelo: Gudib imâglonêt imâglonêt pinniarvigilinga, kakkojaktoruma, sunamiglonêt ôksiguma sekkinek nippilerkârtinnago.

36 Inugiartoksuillo illunatik tukkisilerpât, attanniublo pinniarningit tamaita namagijauvat inügiartoksuit illunamassiatik jijingita sângine.

37 Inugiartoksuillo illunatik Israelitillo illunamassiatik tukkisilerput uvlorme taipsomane, attannermit pilaungimat Abner Nerib erninga tokkotaulaungmat.

38 Attaniublo kivgane okautivait: Kaujimangilase uvlorme tamattomane attannek, pijariakôrtojorlo ochosimangmat Israeleme?

39 Uvangale sangêpunga, attanniolungalo mingoarsimajok. Angutigle Zerujab kittorngagik, sinaungavuk uvamnut. Nalekab tamna idluitullijok akkinniarvigiliuk, idluitullininga malliklugo.

\section{KAPITEL IV.}

\section{Isbosethib tokkotauninganik. Davidiblo alkiniarninganik tokkotauninganit.}

SAULIBLE erninga tussarame Abnerib tokkotauninganik Hebroneme, aggangit nukkingêrotilerput, Israelitillo illunamassiatik koaksarput.

2 Sauliblo erninga angutekalaukpok maggungnik angajokaujungnik sorsuktuksanut; aipanga attekalaukpok Baenamik, ajpanga Rechobemik, Rimonib Berothemiub ernigingnik Benjaminib kittornganginit pijub. Beroth Benjaminekunut illingalaungmattauk.

3 Berothemiullo kemâlaukput Gethaimemut tujormiangolutiglo tagvane, uvlok tamanna tikkilugo.

4 Jonathane, Saulib erninga ernekalaurivok, tamna ittikkamine tussialaukpok, Jahrekalaukporlo tellimanik, pijokalauktut Saule Jonathanelo pivlugik tussartautillugit Jesreelemit, pairijingatalo kivikpa kemâlunelo; tuavilukârtillugolo kemâlunelo, pâlakpok tussialerlunelo; attekalaukporlo Mefibosethemik.

5 Taimak manna Rimonib Berothemiub ernigik Rechobe Baenalo aivuk, tikkilutiglo Isbosethib iglunganut, uvlub kiakarpangoningane; innangavorlo innarvingmine ketterartillugo. 
6 Tikkipuglo iglub illuanut, kakkojaksanik Waizenik aiklepomavlutik, kappivâglo nângagut kemâlutiglo.

7 Iglomut ittermannik taimna nellavok sinigvingmine inarvingmine, kappivâglo tokkolermat, niakungalo nakkarpâk, niakungalo tiguvâk, aularlutiglo apkosinerme naternaksoakut pijome unnuak nâlugo.

8 Apâglo Isbosethib niakunga Davidemut Hebronemut, okarlutiglo attannermut: Ahâk tagva Isbosethib, Saulib erningata, omigijivit, tarningnik unangmiklerlauktub niakunga; Nalekab uvlome angajokara attannek akkiniarutigilaukpa Sauleme tapsomalo kinguvângine.

9 Tagva Davidib kiovak: Sorlo Nalegak miksekârtomik inôngmat, tarnimnik piuliklerlauktok kappianartunit tamainit,

10 Taimna tigulaukpara nellonairsijok uvamnik, okartorlo: Saule tokkungavok, issumakalauktorlo pijokartunik ajungitunik unipkarame, tokkolaukparalo Ziklageme, akkilertauniarasugitillugo uvamnut, unipkautsine pivlugit.

11 Inûglo idluitoâluk ukkoa angut idluartok tokkolaukpâk iglungane, inangavingane. Illa auk tamanna kaikojaksaringipigo aggapsingnit, illiptiglo pêrlutik nunamit?

12 Davidiblo innuosuktekotine perkovait; tapkoa tokkopakit, aggangillo ittiggangillo nakkarpait nivingatilugiglo tetsib kiglingane Hebronemêtome. Isbosethible niakunga tigurât illijarm torpallo Abnerib illuvinganut Hebronemêtomut.

\section{KAPITEL V.}

Davidib mingoartaupsarninganik attanermut Israelitinut illtnainut; salakarninganiglo Jerusalemiunik Filisteriniglo.

KINGUVARÊLLO Israelitit illunatik tikkilerput Davidemut Hebronemut, okarlutiglo: Ahâk, saunekattigivaptigit, uvinekattigilutillo.

2 Taimaglotauk mattoma sivurngane Saule attanniotillugo uvaptingnut, igvit Israelitit aulartilugillo ittertilaukpattit. Nalekablo okautilaukpâtit: Igvit innukotikka Israelitit pairijaksarivattit attaniojuksaulutillo Israelitinut.

3 Angajokaunerôjullo Israelitine illunatik tikkilerput attanermut Hebronemut. Attaniublo Davidib angerkattigivait Nalekab sângane, tapkoalo Davide mingoarpât attanningortilugo Israelemit.

4 Davide Jahrekalaukpok 30 ginik attaningortitaugame, attanniotilunelo Jahrit 40 git nâlugit.

5 Hebroneme attanniotilaukpok Jahrit 7 it takkillo 6 it nâv- 
lugit Judakunut; Jerusalemele attanniotilaukpok Jahrit 33 git nâvlugit, Israelitit illunamassianginut Judakunullo.

6 Attannerlo angutekotine illagællugit aularpok Jerusalemut, Jebusiterit nunakalauktut tagvane sorsugvigijomavlugit. Tapkoale Davide okautivât: Ovunga itterniangilatit; tautungitulle tussiatullo agviarniarpâtit. Issumakalaukpulle Davide tagvunga itterungnarniangimat.

7 Davidible Zionib igloksoanga sôngojok aksarnigariva, tagva Davidib iglugaseksoangit.

8 Tagva Davide uvlorme taipsomane okarpok: Jebusiterinik salakartok, tikkitorlo iglut kânginut, tussiætunut tautungitunullo, Davidib tarningata omigijanginut, tamna aulatsijungortitaujuksauvok angajokangortitaujuksaulunelo. Tamanna pivlugo okartokarpakpok: Ittertokarniarane iglomut tussiætomiglonêt tautungitomiglonêt.

9 Taimak Davidib igloksoak sôngojok iglogiva, tailugolo: Davidib iglogaseksoangit. Davidelo igloliorpok avatâgut Millomit, illuanelo.

10 Davidelo agvertarpok pivallialunelo, Nalekablo, Gudib Zebaothib illagiva.

11 Hiramiblo Tirusemiut attannerijangata Davide kivgartortunik tilliklervigiva, nappartuniglo Cederinik, iglub seneranganut illingajunik, igluliorteniglo, ujaralerijuniglo, iglomik igluliorkovlugit Davidemut.

12 Lavidelo tukkisilerpok Nalekab attanermut Israelitinut nakkiterlaungmago, attaniovingalo angijorortimago innukotine Israelitit pivlugit.

13 Davidelo nulliaitsarinersauvok, panervgaksarinersaulunelo sulle Jerusaleme, Hebronemit tikkilaukârdlune; ernetarnersaulunelo sulle pannetarnersaularmelo.

14 Tamakkoalo erniangolauktut tapsomunga Jerusaleme attingit: Sammua, Sobabe, Nathan, Salomo.

15 Jebehar, Elisua, Nefeg, Jafia.

16 Elisama, Eliada, Elifaleth.

17 Filisterillo tussaramik Davide attanningortilugo mingoartaulaungmat, illunatik paunga majorarput Davide kenneromavlugo. Davide tamattominga tussarame attersarpok igloksoarmut avalulingmut.

18 Filisterille tikkilerput tangmarterlutiglo Refaimib naksangane.

19 Davidiblo Nalegak appertsorpa, okarlunelo: Paunga majorartuksauvîk Filisterit akkerartorlugit? Aggamnullo tunnijomavigit? Nalegak Davidemut okarpok: Majorarit takpaunga, Filisterit tunnijomavakka aggangnut.

20 Davidelo tikkipok Baal-Prazimemut, ullimarpaillo tagvane, okarlunelo: Nalekab omigijikka sivunamne siamartilaukpait sorlo 
imiksuit suppingmatta. Tamanna pivlugo nuna tamna taijaulaukpok Baal-Prazim.

21. Gudingoakotitiglo kemmakpait tagvunga; Davidible angutekotingitalo tiguvait.

22 Filisterille ama majorarput paunga, tangmarterlutiglo Refaimib naksangane.

23 Davidiblo Nalegak apperiva; tamna okarpok: Majorartuksaungilatit paunga; kingorngattigulle tikkikit, tikkikovlutit tapkonunga nappartut Maulberiggit sâdlugit.

24 Tussarniaruvillo nappartut Maulbeeriggit pappingita kêaksungningat pigialermat, tagva tuavitit, Nalegak annisimangmat sivunerne, Filisterit kattimgajoksoakotingit annauleromavlugit:

25 Davide taimailiorpok, sorlo Nalekab perkolaungmago, ullimarpaillo Filisterit Gebamit Gaser tikkilugo.

\section{KAPITEL VI.}

Angersimanerub iklervingata ittertauninganik Jerusalemut kuviasungnermut.

DAvIDIBLO ama angutit innuosuktut Israeleme illunaita kattitipait, 30,000 dit.

2 Parngnailerporlo, ailunelo innugiartoksuit illagijane Judakunit illunatik illagilugit, aikleromavlune takpaunga Gudib iklervinganik, attekartomik tukkelingmik imaitomik: Nalekab Zebaothib attinga kângane innekarpok, Kerubimib kollâne.

3 Tapkoalo Gudib iklervinga ikkitipât aksalloalingmut nutâmaringnut, ailugolo Abinadab Gibeonemêtub iglunganit. Usable Ahioblo, Abinadab ernikita aksalloalik nutâk aulatipait.

4 Tapkoalo Gudib iklervinga illaliudlugo anningmatsuk Abinadab Gibeoneme nunakalauktub iglunganit, Ahiolo iklerviub sivorngane pissuktillugo;

5 Davide, Israeliblo iglomiokotingit illunamassiatik nokakseralingnik sunatuinarnik, kejungmit keblariktomit sennamajunik, attorput Naiekab sivurngane, Harfinik, Psalteriniglo killautiniglo, sivaniârsungniglo Cimbeleniglo.

6 Tikkimattalo Nachonib kakkojaksalliorvinganut, Usab issakpa najumilugolo Gudib iklervinga, tuktuvait sangulermatta.

7 Tagva Nalekab Usa illungertorlune ningaumajarvigilerpa, Gudiblo annauva tagvane idluitullininga pivlugo, tokkokovluga Gudib iklervingata tungâne.

8 Tagva Davide kiksalerpok, Nalekab Usa taimak sugauti. laungmago; innelo tamna taiva Perez-Usamik, uvlok tamanna tikkilugo. 
9 Davidiblo uvlorme taipsomane Nalegak sivuragiva, okarlunelo: Kannok Nalekab iklervinga uvamnut kaijuksauva?

10 Ingminullo âtaukojomalaungila Davidib iglugaseksoanginut, âtaukovâle Obed-Edomib Gathemiub iglunganut.

11 Nalekablo iklervinga takkit pingasut nâlugit Obed-Edomib Gathemiub iglunganetuarmat, Nalekab pitsartutipa, iglomiokattingillo illunaita.

12 Attannerlo Davide okautsaulerpok tamattominga, Nalekab Obed-Edomib iglomiokotingit, tamaitalo pigijangit pitsartutimagit, Gudib iklervinga pivlugo. Tagva aivok aiklerlunelo Gudib iklervinganik Obed-Edomib iglunganit, takpaungartilugo Davidib iglugaseksoakotinganut, kuviasudlarlune.

13 Tapkoalo Nalekab iklervinganik akkigartut sivumualaukârlutik ablornernik pingasojortunik, tunnergumik tunnijijokarpok tuktuvangmik attautsemik, saukamiglo koinijomik attautsemik.

14 Davidelo illungertorlune okigêserpok Nalekab sivurngane, tapserusersimalaukporlo ulliksoarmik sâtomik.

15 Davidiblo Israelitillo illunamassiatik Nalekab iklervinga takpaungartipât, igiakomijarlutik, tiptulausijarlutiglo.

16 Nalekablo iklervinga Davidib iglugaseksoanginut tikkilermat, Michal Saulib panninga nuitavok igalakut, tækkovâlo attannek Davide kiggertartillugo okigêsertillugolo Nalekab sivurngane, nachogivâlo omættimine.

17 Nalekable iklervinga ittertingmatsuk, illivât inniksanganut, tuppiub Davidib tuppertorlauktangata tapsomunga kerkânut. Davidelo tunnijivok tunnergutinik ôtaksanik kujalijutiksaniglo Nalekab sângane.

18 Davidiblo tunnergutit ikkoalajaksat, tunnergutillo kujalijutiksat tunnijarêramigit, innugiartoksuit saimartipait Nalekab Zebaothib attingane.

19 Aitortorpaillo innugiartoksuit illunaita, Israelib unnurningit, tamarmik angutit arnallo attunit niakojârsumik sâtomik, abvakomiglo nerkemik, Weinemiglo oktut tættælugo. Tagva innugiartoksuit illunatik sangulerput attunît iglomingnut.

20 Davidele uttermat iglomiokotine saimartitsomavlugit, tagva Michalib Saulib panningata pachreartorpa, okarlunelo: Kannok ânanaudlalaukpok uvlome Israelitit attanningat, kivgame kivgangita arnat sângine anorairsimalauktok, sorlo innuit illuserluktut annorairpangmatta.

21 Davidele Michalemut okarpok: Harfesijaromavunga Nalekab sângane, annerosulauktub uvamnik atatarnit taipsomalo iglomiokotinginit illunainit, perkolaungmanga attanniojuksaukovlunga Nalekab innukotinginut Israelitinut;

22 Mikkinersauleromavungalo sulle tamattomangat, pijariakitonasugijomavungalo ijipkut, kivgello arnat okautigilauktatit illagilugit nertortaujomavlunga. 
23 Michalle Saulib paninga kittorngakalaungilak tokkome uvlunga tikkilugo.

\section{KAPITEL VII.}

\section{Davidib senajomaninganik Tempelemik, angervioninganiglo Messiasemik. Tuksiarninganiglo.}

\section{ATTANNEK manna iglomine iksivagame, Nalekab mergoêr-} serviksamik tunnitsivigilaungmago omigijinginit avatanêtunit tamainit;

2 Nellautaijomut Nathanemut okarpok: Ahâk, uvanga iglo Cederinit sennamajok innigivara, Gudiblo iklervinga innekarpok itsat attâne.

3 Nathanib attannek okautiva: Ailerit, tamaita omattingnêtut pinniarniarkit, Nalekab illagingmattit.

4 Unuarmele Nalekab okausinga tikkipok Nathanemut, okarlunelo:

5 Ailerit okarlutillo kivgamnut Davidemut: Imâk Nalegak okarpok: Igvit iglolioraksarivinga takkamane innekarkovlunga?

6 Iglome innekalaungitunga sulle uvlormit taipsomangat Israelitit Egiptenemit aularutilaurapkit, uvlok tamanna tikkilugo, agvertarlaukpungale tupperme, innekartomelo.

7 Namullonêt Israelib kittorngangit illunaita illagilugit agvertarlaurama; Israelib kinguvarêngita nelliat okarkattigilauralloarpigit, perkolugit innukotikka Israelitit pairilugit, okarlungalo: Sôg igloliorlungipissinga Cederelingmik?

8 Taimak manna imâk okartuksauvotit kivgamnut Davidemut: Imâk Nalegak Zebaoth okarpok: Tigulaukpagit saukat mianerijauvinganit attanniojuksaukovlutit innukotimnut Israelitinut;

9 Aiparilaukpagillo namut aivigilauktarnut, omigijitillo illunaita sivunerne pêjarlaukpakka, attekartilaukpagillo angijomik, sorlo angijoksuit nunamêtut attíngititut.

10 Uvangalo innukotikka Israelitit innekartitsomavakka, illijomavakkalo, tagvane nunakarkovlugit, tamarnartovingmelo agvertarungnaikovlugit, kassênerublo kittorngangit tetjarungnaikovlugit tapkoninga sivurnganetut.

11 Nelliutomillo taipsomangamit idluarsaijunik pititsilaurama innukotimnut Israelitinut; merngoêrsernermiglo tunnitsivigijomavagit omigijingnit illunainit. Nalekablo nellojungnaitipâtit, Nalekab igvit iglomik iglolioromangmattit.

12 Manna nelliunît annigoromârpat, atâtagijattit illagællugit nellavlutit sinnigomâruvit, kinguvaksat, timingnit pijuksak kingunerne makkititsomavara, taimna attaniovinganik nakkitervigijomavara. 
13 Taipsomalo attera iglolioraksariva, uvangalo attaniovingata iksivautanga sokkosijuititsomavara soraijuitomik.

14 Uvanga atâtaujomavunga taipsomunga, taimnalo erniojuksauvok uvamnut. Idluitullinermik piniarpat, innuit issakautinginut, innuillo kittorngangita annautanginut sugiaromavara;

15 Napkiningnerale pêrtaujuksaungilak taipsomangat, sorlo pêrlaurapko Saulemit, sivunerne pêjarlauktara.

16 Igluille, attanniovîllo sokkosijuituksauvuk issokangitomut sângne, iksivautallo soraijuituksauvok issokangitomut.

17 Nathanib okautsit tamakkoa illunaita, tækkotitsûglo tamana tamât okautigijarêrmagit Davidemut;

18 Tagva Davide attannek kaivok, nokkangalunelo Nalekab sângane, okarlunelo: Kinauvîk Nalegak Nalegak! iglomiokatikkalo sunauvât, manna tikkidlugo âlauramga?

19 Tamannalo tappilugo tamanna suglugilaukpat Nalegak Nalegak! kivgaville iglunga okarvigilaukpat sulle kanningitut pijomârtullo pivlugit. Tamanna illusiôvok innub illusingatut, Gudeôjub Nalegaujublo.

20 Sunamiglo Davidib anginersamik okarkattigijaksarivâtit? Kivgait illitarivat Nalegak, Nalegak!

21 Okautsitit pivlugit, omattekotillo malliklugo taimaitunik pijariakortôjunik pinniarlaukpotit, kivgait nellojungnaitigangn $\theta$ tamakkoninga.

22 Tamanna pivlugo angijônasugijauvotit; illiktut îtokangimat, Gudekangilarlo illiktun îtomik; tamaita siutiptingnut tussarlauktavut malliklugit.

23 Nanele nuname innukattigêksoakaka innukotiktut Israelitititut? tapkoa pivlugit Gude ailaungmat innukotiksaminik piulikleromavlune, attekartitsomavlune ingminut, pinniarnerniglo. taimaitunik angijoksoarnik kappianartuniglo pinniarlune nunakotingne, innukotivit sângine, piulilauktavit illingnut Egiptenemit, nellojunit, Gudingoanginillo.

24 Igvillo innukotitit Israelitit innukotingortilaukpattit illingnut soraijuitomik; igvillo Nalegak Gudiôlerlaukpotit tapkonunga.

25 Taimak manna Nalegak Gude okautsit tamakkoa okauserilauktattit kivgait iglungalo pivlugik nakkiterkit issokangitomut; pinniarlutillo sorlo okalauravit.

26 Tagva attit angijororniarpok issokangitomut, okartokaromârkovlugo: Nalegak Zebaoth Gudeôvok Israelitinut, Kivgavillo Davidib iglunga sokkosijuijomârpok sângne.

27 Igvit Nalegak Zebaoth Israelitit Gudingat, kivgavit siutinga angmarpat okarlutillo: Uvanga iglomik iglulioromavagit. Tamanna pivlugo kivgavit omattine nachvarpa, tuksiarnerit ta. makkoa tuksiarutigilugit illingnut pikovlugo. 
28 Manna Nalegak Nalegak! Igvit Gudeôvotit, okautsitillo miksekârnioniarput. Igvit ajungitut taimaitut okautigilaukpattıt kivgarnut.

29 Taimak pigiarit manna, kivgavillo iglunga pitsartutilauruk, sângnetsainarkovlugo soraijuitomik; igvit Nalegak Nalegak! okautigilaurangne, saimartitsinernullo kivgavit iglunga, saimartitaujomârpok soraijuitomik.

\section{KAPITEL VIII.}

Davidib salakarninginik sunatuinarnik. Pinniarkojaunerillo pijariakortônerpât âkiktauninginik.

PiJokalaukPORLo mattoma kingorngane Davidib Filisterit sâlagingmagit, nukkingêrotipaillo; sappulutaksoarlo sôngojok kakkarulângme Amamik attelingme aksarnigariva Filisterit agganginit.

2 Moabiterit ullimarlaurivait taimak, pingajuingit magguit tokkomut pitingmagit, pingajuingillo attautsit innukongmagit. Taimak Moabiterit kivgangortitauvut Davidemut, âtsilutiglo pilliutinik tapsomunga.

3 Davidib Hadad-Eser, Rehobib erninga Zobamiut attanningat sâlagigiva, ingergarame, pitsartunine aingmilugo, kôngmut Fratemut.

4 Davidiblo tapkonangat tiguvait 1700 dit horsit kângine ablangajut, 20000 dillo pissuktut, aksalloalingillo illunaita attoraksaujungnaitipait, aksalloaggillo 100 dit amiakokovait.

5 Siriamiullo Damaskusemit kaivut Hadad-Eser, Zobamiut attaningat ikajoromavlugo; Davidiblo Sireamiut angutit 22000 dit ullimârpait.

6 Sorsuktuksaniglo pititsivok Damaskusemut Siriamêtomut. Taimak Siriamiut kivgaulerput Davidemut, pilliutinik âtsilerkovlugit tapsomunga. Nalekab Davide ikajormago namut ainingine illunaine.

7 Davidiblo sappulutat Goldeôjut Hadad-Eserib kivganginut illingalauktut tiguvait, âlaitalo Jerusalemut.

8 Betamille Berothaimemillo Hadad-Eserib iglugaseksoanginit attanek Davide tigusivok kannujarmik unnudlartomik.

9 Toile attaniôjok Hematheme tussarame Davidib HadadEserib sorsuksoakotingit illunaita ullimarlaungmagit;

10 Ernine Joram tilliva Davidemut, illanakardlunelo salutitaukova, saimartitaukolâlo Hadad-Eser sorsugvigilaungmago sâlagilaungmagolo, (Toi unatadlarnekarmat Hadad-Eseremut) najortekarporlo pivianartunik Silbereôjunik, Goldeôjuniglo, kakkorliôjuniglo; 
11 Tamakkoa attaniub Davidib perkutautipaittauk Nalekamut, Silbere Goldelo assingit Nalekamut perkutautilauktangit nellojunit illunainit nalektilauktaminit, illaliudlugit:

12 Siriamiunit, Moabemiunit, Ammonib kittornganginit, Filisterinit, Amalekiterinit, Hadad-Eseriblo Rehub erningata, Zobamiut attaningata aksartaujutigijanginit.

13 Taimaktauk Davidib attine angijorortipa, utterame Siriamiullo 18000 dit ullimaramigit sioraujaliub naksangane.

14 Sorsuktuksaniglo pititsivok Edomiut nunanganut, Edomiullo illunamassiatik David nalekpât, Nalekab Davide ikajormago, namut ainingine illunaine.

15 Taimak Davide attanniolaukpok Israelitinut illunamassianginut, idluarnermiglo idluarsainermiglo sakkertitsivok innugiartoksoarnut illunamassianginut.

16 Joabe, Zerujab erninga angajokaulaukpok sorsuktuksaksoakattigênut; Josafatele, Ahiludib erninga aglakteôlaukpok attanioviub pijokarninginik.

17 Zadok Ahitob erninga, Ahimelechelo, Abjatharib erninga ajochertuijersoangôlaukpuk; Seraja aglakpakteôlaukpok.

18 Benaja Jojadab erninga angajokaulaukpok Krethinut Pletinullo. Davidib erningit attannertuijôlaukput.

\section{KAPITEL IX.}

\section{Davidib ajungitullininganik Jonathanib erninganut.}

DAVIDELO okarpok: Amiakokariva sulle kinamik Saulib iglunganit, napkiningnermik pinniarkovlunga tapsomunga Jonathane pivlugo?

2 Kivgakalaukporle Saulib iglunganit Zibamik attelingmik, tamna kaikovât Davidemut. Attanniublo okautiva: Zibaôvît? Tamna okarpok: Ahaila, kivgait.

3 Attannek okarpok: Pittakarka sulle kinamik Saulib iglunganit, Gudib napkigosungninganik pinniarvigilugo pikovlunga? Ziba okarpok attannermut: Pittakarpok sulle Jonathanib erningita illanganik ittikamigut tussiatomik.

4 Attanniub okautiva: Nanekâ? Ziba okarpok attannermut: Ahâk Lodabaremêpok, Machirib Amielib erningata iglungane.

5 Tagva attannek Davide tilliklerpok tagvunga, aijaukolâlo Lodabaremit Machirib Amielib erningata iglunganit.

6 Manna Mefiboseth Jonathanib, Saulib erningata erninga, Davidemut tikkiname, pamakterlune kênane pûpa, opigosuklunelo. Davidele okarpok: Mefiboseth! Tamna okarpok: Mânepunga kivgait. 
7 Davidib okautiva: Sivoraniarnak; takopsoanermik pinniarvigijomagapkit, Jonathan, atâtait pivlugo; atâtatsiavillo Saulib perorsêvikotigilauktanginik illunainik tunnitsivigijomagivagit; igville uvlut tamaita nerrimavikotimne nerrikattautsainartuksauvotit.

8 Tamnale opigosukpok, okarlunelo: Kinauvîk uvanga kivgait, sâleravit kingmemut tokkungajomut, sorlo uvanga illingagama?

9 Tagva Davidib Ziba Saulib kivganga, kaikova okautilâlo: Tamaita Saulib iglomiokotingitalo illunatik perkutigilauktangit tunnimavakka angajokavit erninganut.

10 Taimak manna perorsêvikotinga sennalersiuk igvit, kittorngakotivillo, kivgakotivillo, kattitilugillo perortangit, innogutiksaukovlugit angajokavît erninganut, nerkiksakarkovlugo; Mefibosethele angajokavit erninga, uvlut taimaita nerrikattaujuksauvok nerrimavikotimne. Zibale ernekalaukpok 15 enik kivgakalarmelo 20 ginik.

11 Zibalo attannermut okarpok: Tamainik, sorlo angajokama attanniub kivgane perkolaungmago, taimak kivgait pinniartuksauvok. Mefibosethelo nerrijuksauvok nerrimavikotimne sorlo attanniub kittorngangita nelliatitut.

12 Mefibosethelo erniârsukalaukpok, tamna attekalaukpok Michamik. Zibable iglomiokotingita illunatik Mefibosethe kivgartorpât.

13 Mefibosethele iglokalaukpok Jerusaleme, nerrigame uvlut tamaita attanniub nerrimavikotingane, tussialaukporlo ittikkamigut igluktut.

\section{KAPITEL $\mathrm{X}$.}

\section{Ammoniterit idluitullininganik Davidib tillijanginut, salagïauninginiglo.}

MATTOMALO kingorngane pijokalaukpok Ammonib kittorng-
angita attanningat tokkolermat, erningalo Hanon attaningor-
pok taipsoma innigilauktangane.
2 Tagva Davide okarpok: Napkiningnermik pinniaroma-
vunga Hanonemut Nahasib erninganut, sorlo taipsoma atâtangata
napkiningnermik pinniarvigilaungmanga. Tilliklerporlo tag-
vunga, mannigortaukolâlo kivgaminut atâtanga pivlugo. Da-
vidib kivgangit manna tikkimatta Ammonib kittorngangita
nunanganut,
3 Tagva Ammonib kittorngangita angajokaunerôjungit okar-
put Hanonemut, angajokamingnut: Issumavit Davidib atâtait 
opigingmago ijikpît sângangne, manigoijunik tilliklervigilaungmattit? Issumalungilatit tamanna pivlugo kivgane tillilauramigit illingnut, iglugaseksuit nautsertoromagamigit, tukkisinasuarlugillo, serkomilugillo?

4 Tagva Hanonib Davidib kivgangit tiguvait, umingillo kippivait koppilugit, annorângillo kipjaumiktorpait ketterarlugit tapserut tikkilugo, aularkolaitalo.

5 Davide tamattominga okautsaumagame, tilliklerpok pachreartaukovlugit; angutit taipkoa angijomik kangusuktitaulaungmatta. Attaniublo okautsaukovait: Jerichometuaritse, umise perorsearilugit, kingorngane uttileritse.

6 Ammonible kittorngangit tækkogamik tippilungorsimagamik Davidib sângane, tilliklerput kaikojilutiglo Siriamiunik, Rehobib iglomiokotinginik, Siriamiuniglo Zobamiunik, angutinik pissuktunik 20000 dinik, attannermillo Maachamit angutinik 1000 dinik, Istobemillo 12000 dinik.

7 Davidib tamanna tussaramiuk, Joabe tilliva sorsuktuksaksoakattigêt illunamassiatik illagilugit.

8 Ammoniblo kittorngangit aularput, parngnailerlutiglo sorsungnermut ittertarviksub upkoangata sillatâne. Siriamiulle Zobamit, Rehobemillo, Istobemillo, Maachamillo, kissimik naternamelaukput.

9 Joabelo manna tækkogame sorsungnek tapsomunga torarmat sivurânit kingornganillo; Israelitit angutinginit innuosuktunit illunainit annerosukpok, parngnailunelo Siriamiut akkerartoromavlugit.

10 Inugiartoksuillo assingit pitipait angajume Abisaib agganginut, parngnailerkovlugo Ammonib kittorngangit akkerartorlugit.

11 Okarlunelo: Siriamiut ajugauniarpatta uvamnut, tagva ikajoriartorlaunga; Ammonible kittorngangit ajugauniarpatta illingnut, tagva uvanga ikajoriartoromavagit.

12 Mannigorit, pitsartulertalo innukotivut Gudiptalo iglugaseksoangit pitjutigællugit; Nalegarle pinniarle namagijaminik.

13 Joabiblo innukotine illagijine illagilugit Siriamiut kagli lerpait sorsugvigilugit; taipkoalo kemâvât.

14 Ammoniblo kittorngangit tækkogamik Siriamiut kemângmatta, nangminektauk Abisai kemâvât, itterlutiglo iglugaseksoarnut. Taimak Joabe uttilerpok Ammonib kittornganginit, tikkilunelo Jerusalemut.

15 Siriamiullo tækkogamik salagijaugamik Israelitinut, kattilerput attautsemut,

16 Hadad-Eserelo tilliklerpok aijautipaillo Siriamiut kôksub akkianêtut, tikkipullo Helamemut, Sobachiblo Hadad-Eserib sorsuktuksaksoakotingita angajokangata sivorlervigivait. 
17 Davide tamattominga okautijaumagame Israelitit illuêngarlutik kattitipait, ingergalunelo Jordan ikârlugo, tikkilunelo Helamemut. Siriamiullo parngnailerput Davide akkerartorlugo sorsugvigijomavlugo.

18 Siriamiulle Israelitit kemâvait; Davidiblo Siriamiunit aksalloaggit 700 dit innungit, horsillo kângine ablangajut 18000 dit tokkopait; tapkoa illaliudlugit Sobache sorsuksoakattigêt angajokangat ullimarpa tokkokovlugo tagvane.

19 Attannille Hadad-Eserib nalektingit tækkogamik salagijaunermingnik Israelitinut, ulapirsautiksaliorput Israelitinut, naleklutiglo tapkoninga. Siriamiulle sivuravut Ammonib kittorngangit ikajornersarilugit.

\section{KAPITEL XI.}

\section{Davidib asseroininganik nulliarêngnermik, tokkotsininganiglo.}

J AHRELO annigormat, nelliutome attannît aularvigivaktangane, Davidib Joabe kivganelo illagællugit, Israelitillo illuêngarlutik tillivait, asseroikovlugit Ammonib kittornganginik, Rabbalo illuvait. Davidele Jerusalemetuarpok.

2 Pijokalaukporlo Davide unnub nelliuningane makkilerame innangavingminit, pissuklunelo attanniub iglungata kângane, kânganillo tækkova arnak ubvartok ingminik, arnarlo tamna taututsiarilaukpok.

3 Davidelo tilliklerpok tagvunga, arnarlo taimna apperkutigijaukova, okarkojilunelo: Bathsebaungilâk taimna, Eliamib pannia; Uriab Hetiteriôjub nullianga?

4 Davidelo kivgartortunik tilliklerpok tagvunga, aijaukolâlo. Tapsomalo ittervigingmatsuk, sinnikattigiva. Tamnalo arnak sujungaitipok ingminik sujungnerminit, utterlunelo iglominut.

5 Arnarlo singailerpok, tilliklerlunelo, Davidelo nellojung naititaukova okautsaukolâlo: Singaititaumavunga.

6 Davidele tilliklerpok Joabemut: Tilliklerviginga Uriamik Hetitereôjomik. Joabiblo Uria tilliva Davidemut.

7 Uriablo tikkingmatsuk, Davide appertsorpok Joabe innugiartoksuillo sorsungnerlo pivlugit, illingatsiaralloarmangât?

8 Davidiblo Uria okautiva: Attersarit iglokotingnut, ubvarlutillo ittiggangnik. Urialo anningmat attanniub iglunganit, attanniub pilliutinga mallikpok tapsominga.

9 Urialo innariarpok attanniub iglungata upkuangata sângane, angajokame kirgangita illunatik innangavinganut, attersartaililunelo iglominut.

10 Davidele okautijaulerame: Uria attersarlungilak iglomi- 
nut; Davidib okautiva: Nunab assianit tikkisimangilatit? Sôg attersarlaungilatit iglokotingnut?

11 Uriale Davidemut okarpok: Iklervik, Israelitillo Judamiullo tuppernetualerput, angajokaralo Joabe, angajokamalo kivgangit unnatadlarnermeput; uvangalo iglumnut aijuksaunajarkik, nerrikovlunga, immerkovlungalo, nulliaralo innangakattigilugo? Sorlo miksekârtomik innôgavit, tarnîllo inôngmat, taimailiorlungilanga.

12 Davidib Uria okautiva: Taimak uvlometauk ovanetuarit, kaupat aularkojomavagit. Taimak Uria uvlorme taipsomane, aipanganelo Jerusalemetuarpok.

13 Davidiblo kaikova, sângane nerrikovlugo immerkovlugolo; tallatipalo. Unnungmallo annivok, innariarlune sinnigomamut innangavingminut, angajokame kivgangit illagilugit; attersarlungilarlo iglominut.

14 Uvlarme Davide aglangnik aglakpok Joabemut, tillikliutilaitalo Uriakut.

15 Aglakporle aglangne tapkonane imâk: Uria pitisiuk sorsungnerub sugaluluarvianut, tunnulugolo allarsiuk, ullimartaukovlugo, tokkokovlugolo.

16 Joabiblo manna iglugaseksuit illumangmagit, Uria innekartipa kaujimajaminut angutekarmat tagvane sorsugungnartunik.

17 Iglugaseksuillo angutingit annidlarmatta Joabelo sorsugvigingmatsuk, innugiartoksuit Davidib kivgangita illangit ochovut, Urialo Hetitere tokkogivok.

18 Tagva Joabe tilliklerpok, Davidelo okautsaukova tamainik, sorsungnerub illuserilauktanginik.

19 Tillijarlo perkova, okarlunelo: Sorsungnerub illuserijanga tamât okautigijarêrungne attanermut,

20 Tækkoguvillo attannek ningarosulermat, okautikpattillo: Sôg sorsungnerme taimak iglugaseksuit kanileriartorlaukpissigik? Kaujimangilase kannok pittiksertokarpangmat avalut kânganit?

21 Kia Abimeleche Jerubesetib erninga annaulaukauk? Arnab serkalitsiviub ujaraktangata illakunga egilaungikauk avalnt kânganit tapsomunga, tokkolugo Tebezeme? Sôg avalut taimak kaninariartorlaukpissigik? Tagva okartuksauvotit: Kivgait Uria Hetitereôjok tokkungagivok.

22 Tillijarlo aivok tikkilunelo, okarlunelo Davidemut tamainik, tillijaujutigijaminik Joabemut.

23 Tillijarlo Davidemut okarpok: Angutit ajugarilaukpavut, annidlarlutiglo uvaptingnut naternamut, uvagulle tapkoa tunganelaukpogut ittertarviksub upkoangata sânga tikkilugo.

24 Pittiksertullo pittiktarlaukput kivgarnut avalut kânganit, tokkolaitalo illangit, attaniub kivganginit; tapkoalo illagællugit kivgait Uria Hetitereôjok tokkogivok. 
25 Davide tillijamut okarpok: Imâk Joabemut okartuksauvotit: Tamanna kuvianaijutiginiarnago pît, saviksoak nungutsingmat manna ominga, manna ipsominga. Katsungaileritse sorsungnerme iglugaseksoarnik, serkomitsikovlutit tapkoninga; mannigorillo.

26 Uriablo nullianga tussarame uine Uria tokkungmat, uine ogguriva.

27 Ogguarêrmalle, Davide tilliklerpok aijaukolâlo iglominut, nulliangolerporlo tapsomunga, ernilunelo tapsomunga ernermik. Nalekable pinniarnek tamanna Davidib pinniartanga idluigiva.

\section{KAPITEL XII.}

Nathanib okâlaninganik kakkialerkojïjomits; Davidib kakkialerninganik; nellojungnaititauninganik Gudib issumangnarungnaininganik. Salomonib innulerninganik. - Ammoniterit salagijauninganik.

N ALEKABLO Nathan tilliva Davidemut. Tapsomalo tikkigamiuk, okautiva: Iglugaseksuit illangane angutekalaukpok maggungnik, aipa akluilaukpok, aipa akluvok.

2 Akluitok saugakuttekadlalaukpok, tuktuvakuttekalarmelo.

3 . Aklujorle sunakalaungilak saugârsukotituane pissiarilauktame assianik; nerritipalo angijororkovlugo tapsomane, kittorngangillo illagilugit; nerrilaukpok tamuanganit, immerlunelo ermgusiârsukotinganit, sinnilarmelo tapsoma sângane, panniktullo kamagiva.

4 Tikkitokalaungmalle nerrimarkattaujomik angumut akluitomut, tagva saugakotine tuktuvakotinelo ivlerivait, tapkonangat nerrimarkattaujok nerkiksaliorlugo, aklujublo saugakotinga tiguva âkiksorlâlo angumut tikkitiminut.

5 Tagva Davidib illungertulerlune angut taimna taimailiorlauktok ningaumajarvigidlarpa, okarlunelo Nathanemut: Sorlo Nalegak miksekârtomik inôngmat, angut taimna taimailiorlauktok tokkotaksauvok.

6 Tamannalo tappilugo saugak taimna sittamairtorlune akkileraksariva, tamattominga pinniarlaungmat ivlersilaungimallo.

7 Tagva Nathan Davidemut okarpok: Igvit anguteôvotit taimna. Imâk Nalegak Israelib Gudinga okarpok: Uvanga mingoarlutit attaningortilaukpagit Israelemut, piulilaukpagillo Saulib agganginit.

8 Tunnitsivigilaukpagillo angajokavit iglunganik, tappilugolo nullianginik sângnut, Israelekullo Judakullo tunnilaukpakka il- 
lingnut, tamannalo suglunarpat tagva mattominga ipsomingalo illautitsijomavunga sulle tamattomunga.

9 Sôgle tagva Nalekab okausingit nachogilaukpigit, idluitoâlungmik taimaitomik pinniaravit tapsoma ijikita sângangne? Uria Hetitere ullimarlaukpat saviksoarmut; nullianga tigulaukpat nulliarilugo, taimnale tokkolaukpat Ammonib kittorngangita saviksoangagut.

10 Manna saviksoak nûlertuksaungilak iglokotingnit soraijuitomik; nachogilauramga, Uriablo Hetitereôjub nullianga tigulaurangne, nulliangôkovlugo illingnut.

11 Imâk Nalegak okarpok: Ahâk kannoêtunik sakkertitsivigijomavagit iglokotingnit nangminerijarnit pijunik, nulliakotitillo tigujomavakka ijikpît sângangne tunnijomalugillo innukattingnut, nulliakotitit sinikattigivlugit pikovlugo sekkerngub kaumamaringningane.

12 Igvit tamattominga sagvangitome pinnialauravit, uvangale tamattominga pinniaromavunga Israelitit illunamassiatik tækkoane, sekkinekarninganelo.

13 Tagva Davide Nathanemut okarpok: Idluitullilaukpunga Nalekamut. Nathanib Davide okautiva: Tagva Nalekab ajornit pêjarsimagiva, tokkoniangilatit.

14 Nalekable omigijingit pijokartomut omunga mittautekartilaurangne, tamanna pivlugo ernek erniangomajok illingnut tokkolerniarpok.

15 Nathanelo angerarpok. Nalekablo nutarak Uriab nulliagilauktangata erniarilauktanga Davidemut annauva, kannimadlarkovlugo tokkojungnarlune.

16 Davidiblo Gude tuksiarvigiva nukapiarârsuk pivlugo, nerritaililunelo itterporlo, pàmangalunelo unnuak nâlugo netterme.

17 Tagva iglungata angajokaunerôjungit makkiput makkititsomavlugo netter'mit; pijomalaungilarle, nerrikattinginivaillo.

18 Uvlulle 7 ingane nutarak tokkolerpok. Davidiblo kivgangit sivuralaukput tamanna okautigilugo Davidemut nutarak tokkungmat. Issumakaramik: Ahâk nutarak innôtillugo sulle okarkattigilaukpavut, tapsomalo nippivut nalelaungila; kannok angiluartomik issumajalerniarpok okarupta: Nutarak tokkungavok.

19 Davidele tækkogame kivgane issivsormatta mallugosuleramelo nutarak tokkungmat, kivgane okautivait: Nutarak tokkungava? Tapkoa okarput: Ahaila.

20 Tagva Davide makkipok nettermit ermiklunelo, mingoarlarmelo, annorangniglo assianik attivok, ailunelo Nalekab iglunganut, opigosularmelo. Angerarame niakojamik kaitaujojilerpok nerrilunelo.

21 Tagva kivgangita okautivât: Sunauva tamanna piniartat? 
Nutarak innôtillugo nerritaililaukpotit kêalutillo; mannale tokkungalermat makkipotit nerrilutillo?

22 Tamna okarpok: Nutarak nerritailijutigilaukpara, kêagilugolo innôtillugo; issumakalaurama: Imakkâ Gudib saimarviginiangimangângma nutarak innôkojaukovlugo.

23 Mannale tokkungangmat, suna pivlugo nerritailijuksauvîk? Uttertitsungnaralloarpigô? Aivigijomâralloarpara, tamnale utterniatsengilak ama uvamnut.

24 Davidiblo nulliane Bathseba manigorlaukârdlugo ittervigiva sinnikattigilugolo. Ernermiglo ernivok tapsomunga, tamna atserarpa Salomomik. Nalekablo nagligiva.

25 Pitipâlo Nathanib nellautaijub agganginut. Tapsoma atserarpa Jedidjamik Nalegak pivlugo.

26 Taimak manna Joabib Rabba Ammonib kittorngangita iglugaseksoakotingit sorsugvigivait, aksarnigarivaillo attaniub iglugaseksoakotingit,

27 Tilliklerlunelo kivgartortunik Davidemut, okautsaukovâlo: Rabbamiut sorsugvigilaukpakka, sâlagilaurivakkalotauk iglugaseksuit immiksoaggit.

28 Tagva igvit manna innugiartoksuit assingit kattitikit, iglugaseksuillo illumakit, salagilugillo, uvanga salakarkonanga tapkoninga, atteralo tamanna pivlugo taijaukonago.

29 Taimak Davidib innugiartoksuit illunaita kattitilugit tiguvait, aularlunelo tagvunga, Rabbamiullo sorsugvigivait, sâlagilaitalo.

30 Taipkoalo attaningata niakotinga, Talentetut okumaitigijotut Goldekalauktok, ujaraliglo pivianartunik tiguva tapsoma niakoanit, illijaulunelo Davidib niakunganut; aksarnigaujullo unnuktovaksuit neksarpait iglugaseksoarnit.

31 Inugiartoksuille takkamanetut annitipait, illilaitalo kiblut kikkiengôjut, kapputillo, kuksautello kikkiengôjut attânut, ikkilaitalo kieksautine igaliksalliorvingne. Taimailiorvigivait Ammonib kittorngangita iglugaseksoangit illunaita. Tagva Davide innugiartoksuillo illunatik uttilerput Jerusalemut.

\section{KAPITEL XIII.}

Amnonib ajortullininganik najaminut. Absalonib̧ tokkotsikojininganik tapsominga, kemâninganiglo.

T

TAMAKKOALO kingorngane pijokalaukpok Absalome Davidib erninga najakarmat ânanamik, Thamaremik attelingmik; Amnoniblo Davidib erningata alligartulerpa.

2 Amnonelo kanimasertorpok, Thamar najane, pivlugo. 
Uigasolaungmat, Amnoniblo okumainasugiva tamna sunamik pinniarvigilugo.

3 Amnonele illanarijekalaukpok, Jonadabemik attelingmik, Simeab Davidib angajuata erninganik; Jonadabelo tamna anguteolaukpok sillatudlartok.

4 Tamna okarpok tapsomunga: Sôg igvit attaniub erninga uvlub aipanganit aipanganut salludlarpalliavît? Tamattominga okautijomangipinga? Tagva Amnonib okautiva: Thamar, nukkama Absalonib najanga alligartorpara.

5 Jonadab okautiva: Inariartorit sinnigvingnut kanimasertorlutillo. Tagvalo atatait kainiarpat tækkojomavlutit, tagva okautiuk: Nagliktara, najara Thamar kailaule nekkoksititsikovlugo uvamnik, nerkiksalliorkovlugo sâmne tækkonârtillunga, uvangalo taipsoma agganginit nerrikovlunga.

6 Taimak Amnon innariarpok kanimasertorlunelo. Attannerlo manna tikkilermat tækkojomavlugo, Amnon attanermut okarpok; Nagliktara, najara Thamar kailaule, sâmne șennakovlugo uvamnut nerkiksamik sâtomik attautsemik, maggungniglonêt, nerrikovlungalo taipsoma agganginit.

7 Tagva Davide tilliklerpok Thamaremut iglomut, okautsaukolâlo: Ailerit annivît Amnonib iglunganut, nerkiksalliorlugolo.

8 Thamar aivok annime Amnonib iglunganut, tamnale nellavok sinnigvingmine. Tapsomalo sennaugak tiguva, akkulugolo immermut, sennalugolo; sâtuniglo sennavok ijikita sângangne, igalunelo sâtunik tapkoninga.

9 Tapsomalo (Thamarib) nerkiksak tamanna tiguva, nâvilugolo sânganut; nerrinermigle kunnulaukpok: Amnonelo okarpok: Illunatik annilerlit uvamnit. Illunatiglo annivut tapsomangat.

10 Tagva Amnonib Thamar okautiva: Nerkiksak kaileruk aiparusermut, nerrikovlunga aggangnit. Tagva Thamarib sâtut igalauktane tiguvait, âlugillo anniminut Amnonemut aiparutsiub illunanut.

11 Amagolo tamanna tapsomunga, nerrikovlugo, tigura, okautilâlo: Kailaurit najâk, sinnikattigilungalo.

12 Tapsomale okautiva: Taimangungitok annîk, sangêtiniarnanga, taimailiortokarpangimat Israeleme; sillainartomik taimaitomik pinniarnak.

13 Namut kangusungnera pivlugo aijuksauvîk? Igvillo sillaitutitut Israelemetutitut îniarpotit. Attannerle okarkattigiuk, tapsoma ivlerkutiniangilâtit uvamnik.

14 Tapsomale nalegomalaungila, sâlagivâlo, sangêtipâlo innangakattigilugolo.

15 Amnoniblo angijoksoarmik omigilerpa, taimak omisung- 
nek manna anginersaulaungmat, naglingnermit sivurngane. Amnoniblo okautiva: Parngnailerit annilutillo.

16 Tapsomale okautiva: Idluitullinek tamanna, ajektoramga anginersauvok assianit pinniarlauktarnit uvamnut. Nippingale tussarlaungila,

17 Nukapiakotinele kivgarijane kaikova, okarlunelo: Una annitiuk uramnit, upkoarlo kingorngane parngnanaileruk.

18 Pattigosijarlaukporlo tarsepatâmik, taimaitunik attanniub panningit pattigosijarlauramik uigasôvlutik. Kivgangatalo annitilaukârtillugo, upkoarlo kingorngane pallangersimangmago;

19 Thamarib argsat egipait niakominut, pattigotelo tarsepatâk attortane alliktorpa, agganelo illivait niakominut, pissuklunelo kêaviok.

20 Anningatalo Absalomib okautiva: Annit Amnon illingnelauka? Unêt najâk, nipp»ngerit, annigivat, tamanna taimak omamiutariniarnago. Taimak Thamar uigasovlune Absalomib annigijame iglunganêtuarpok.

21 Attannerlo Davide tamattominga tamât tussarame, ningalerpok angijoksoarmik. Absalomible Amnon okarkattigingila ajortomiglonét ajungitomiglonêt.

22 Absalomible Amnon omigiva, najanga Thamar sangêtilaungmago.

23 Jahrigle magguk nângmannik, Absalome sallijekalaukpok saugakotiminik Baalhazoreme Efraimib nunanganêtome; Absalomiblo attanniub kittorngangit illunaita kaikovait,

24 Tikkilerlunelo attannermut, okarlunelo: Ahâk kivgait sallijekarpok saukanik; attaniub kivgane illagilugit kivgane illagijomaliuk.

25 Attanniub Absalome okautiva: Aukak, ernîk, illunata ainiarata, pagvisarkonata illingnik. Innagalloarmagolo, aijomalaungilak sulle, saimartipale.

26 Absalome okarpok: Angajugale Amnon illaujuksaungilâk uvaptingnut? Attanniub okautiva: Sôg taipsoma illagijaksarivâtit?

27 Tagva Absalomib innapa, illaukojikovlugo Amnonemik, attaniublo kittornganginik illunainik.

28 Absalomible nukapiakotine perkovait, okarlunelo: Kamatsiaritse, Amnon Weinemut kuviasulerpat, uvangalo okautigupse: Amnone annaulersiuk, tokkolugolo, sivurakonnase, perkolaurapse; sivurajuileritse omaridlarluselo.

29 Taimak Absalomib nukapiakotingita Amnon pinniarvigivât, sorlo Absalomib perkolaungmagit. Tagva attanniub kittorngangit nikkoviput, illunatiglo attunît ikkivut siutekoktôjokotimingnut, kemmâlutiglo.

30 Apkomêtillugillo sulle, Davide tussaumanermut tikkitau. 
vok, Absalomib attanniub kittorngangit illunaita tokkolaungmagit, amiakokalungimallo tapkonangat attautsemiglonêt.

31 Tagva attannek nikkovipok, annoranelo alliktorpa, pamakterlunelo nettermut; kivgangitalo avatingane nangertut illunatik annoratik alliktorpait.

32 Tagva Jonadabe, Simeab, Davidib angajungata erninga kiovok okarlunelo: Angajokara issumaniarane, nukapitset attaniub kittorngangit illunatik tokkungangmatta; Amnonele kissime tokkungavok. Absalome issumanerlulaungmat uvlormit taipsomanemit najanga sangêtilaungmago.

33 Taimaimat manna angajokangma attaniub, tamanna omamiutariniarnago, attaniub kittorngangit illunaita tokkonasugællugit; Amnonele kissime tokkovok.

34 Absalomele kemâvok. Nukapiarlo udsertorvingme ijiminut tækkosarpok, tækkolunelo; ahâglo innugiartoksuit aggerput nellekârdlutik apkosinerme kakkab sivinganingane.

35 Tagva Jonadabe attannermut okarpok: Ahâk attaniub kittorngangit kaivut; sorlo kivgait okalaungmat, taimailingalaukpok.

36 Okarêrmallo, ahâk tagva attanniub kittorngangit tikkilerput, niplialerlutiglo kêalarmiglo. Attannek kivgangillo illunatik kêavaksoadlalaurivuttauk.

37 Absalomele kemâvok, ailunelo Talmaimut Amihudib erninganut, attanniôjomut Gesureme. Tapsomale ernine oggurilaukpa uvlut tamaita.

38 Taimak Absalome kemmâvok, ailunelo Gesuremut, Jahrillo pingasut nâlugit tagvanêtuarlaukpok.

39 Attaniublo Davidib tarninga paingulerpok, aularomavlune Absalomemut; allijilaurame ernime Amnonib tokkunganik.

\section{KAPITEL XIV.}

Absalomib saimarvigïauninganik, arnab tulsiutsininga tapsominga pivlugo.

JOABELE Zerujab erninga mallugosulerpok attaniub omattinga torarmat Absalomemut,

2 Tilliklerlun lo Tekoamut aiklerkojilunelo tagvangat arnamik sillatojomiik, okautivâlo: Ogguarit annorâniglo ogguarsiutinik attitilerit, mingoarniarnaglo illingnik orksumut, arnaujalerille akkunît ogguarlauktomik tokkungajok pivlugo.

3 Attanermullo ittertuksauvotit, okarkattigilugolo imaitomik imaitomiglo. Joabiblo kaujitipa sunamik okartuksauninganik.

4 Arnablo Tekoamiub attannek okarkattigijomagamiuk, pa- 
makterpok nettermut kênane pûvlugo, opigosulunelo, okalarmelo: Attannek ikkajorlaunga!

5 Attanniub okautiva: Suvît? Tamna okarpok: Uigarniôvunga, arnak ogguartok, uigalo tokkolerlaukpok.

6 Kivgaillo arnak ernekalaukpok maggungnik, tapkoa naternamêdlutik aivakattigêlaukpuk, piuliklertokalaungimallo, aipangata aipa annaudlugo tokkolaukpa,

7 Ahâglo, manna illagêt illunatik kivgait akkerartorvigilerpât, okarlutiglo: Ernît tokkotsilauktok kattangutiminik kaitsuk, tokkotsikovluta taipsominga, kattangutingata tokkolauktangata tarningata akkiniarutiksanganut, kingormgojijuksarlo pêjaromavavut; ingniârsukotigalo amiakojok sulle, kamitsomavât, uigilauktamnit amiakokarkonago attermiglonêt sunamiglonêt nuname.

8 Attanniub arnak okautiva: Angerarit, pivlutit perkojijomavunga.

9 Arnablo Tekoamiub attannek okautiva: Nalegâk attannek, idluitullinek tamanna illingalerle uvamnut, atâtamalo iglomiukotinginut, attannerle iksivautaksoangalo passijaksaungilik.

10 Attannek okarpok: Kina akkerartorlutit okausekartok, tamna kaitsuk uvamnut; tagva aktuijungnaituksauvok illingnik.

11 Tamna (arnak) okarpok: Attannek, Nalegarnik Gudingnik erkaumalerle, aungmik akkiniartut unnulualerkonnagit, asseroilutik, erneralo pêjartaukonnago taipkonunga. Tamna okarpok: Sorlo miksekârtomik Nalegak inôngmat, nujak attauserlonêt kattaktuksaungilak ernerpît niakunganit nunamut.

12 Arnarlo akarpok: Kivgait nalekamnut attannermut sunamik okarle. Tamna okarpok: Okarit.

13 Arnak okarpok: Sôg taimaitomik issumakalaukît Gudib innukotingit akkerartorlugit, attannek taimaitomik okalaungmat, idluitullingmat, ajektortanelo utterkolungimago?

14 Uvagut tokkoleriakarapta, illingalutalo immektut nunamut kivilertotut, nokkartitaujungnangitotut; Gudiblo innôsek pêromangila issumaksarsiorporle, ajektortaumajok ajektortaumikonnago tapsomunga.

15 Taimak manna tikkisimavunga nalegara attannek okarkattigijomavlugo; innugiartoksuit kappiasuktimanga. Kivgait issumakalaurame: Attannek okarkattigijomavara; imakkâ pinniarniarpok kivgangata okausekarninganik.

16 Kivgane arnak tussarniarmago, piuliklerkovlugo uvamnik, illunatik agganginit pêjaijomajut uvamnik, ernimniglo, Gudib kingormgutijanganit.

17 Kivgaillo issumakalaukpok: Nalekama attanniub okausingit manigoiniôjuksauvut uvamnut; nalegara attannek Gudib. Engelingatut îmat, tussarungnarmat sunamik idluarmangat idluimangallonêt. Tamanna pivlugo, Nalekab Gudivit illaginiarpâtit. 
18 Attannek kiovok, okarlunelo arnamut: Missiarviginiarnanga apperkotiginiartamnik illingnik, Arnak okarpok: Nalegara attannek okarle.

19 Attannek okarpok: Joabib aggangita illagingilâtit tamakkonane tamaine? Arnak kiovok okarlunelo: Sorlo miksekârtomik tarnît inôngmat nalegara attannek, taipsoma assekangilak tallerpingmêlonêt saumingmêlonêt, nalekama attanniub okautigimajanganit. Kivgavit Joabib perkolaungmanga tamattominga, taipsomalo taimaitomik tamât kivgait arnak illumertilaukpa;

20 Illusek tamanna taimak sangutitaksarigapko, tamanna kivgavit Joabib âkiksorlaukpa. Nalegarale sillatôvok Gudib Engelingata sillatuningatut, mallugosukovlugo pijokartunik tamainik nuname.

21 Tagva attanniub Joabe okautiva: Ahâk taimailiorlaukpunga; taimaimat ailerit manna, nukapiarlo Absalome uttertilaungmiuk.

22 Tagva Joabe pamakterpok nettermut kênane pûvlugo, opigosuklunelo, attannerlo kujagiva, okarlunelo: Uvlome kivgait tukkisilerpok, saimaneksimagama ijikpît sângangne angajokara attannek; attannek pinniarmat kivgame okauserijanganik.

23 Taimak Joabe parngnailerpok, ailunelo Gesuremut, âlâlo Absalome Jerusalemut.

24 Attannerle okarpok: Itterile iglominut, kênaralo tækkoniarnago. Taimak Absalome tikkilerivok iglominut, tækkotaililugolo attanniub kênanga.

25 Israeleme illunane angutekalaungilak taimak ânanautigijomik Absalometut, taimaglo okautaulaukpok illunainut; ittiggangita allugingnit kabsinga tikkilugo kannoêtokalaungimarikpok tapsomane.

26 Niakungatalo nujangit kippijaulaukpatta, (tamannalo Jahrine tamât pivalaukpok, nujangille okumailuarlaukput kippijaujariakarmatta) tagva niakungata nujangit Sekeletut 200 ditut okumaitigijôvalaukput, attanniub Sekelingita okumainingit malliklugit.

27 Absalomemullo erniangolaukput ernit pingasut, pannerlo attausek, tamna attekalaukpok Thamaremik, arnaulaukporlo taututsiariktok.

28 Taimak Absalome Jahrik magguk nâlugik Jerusalemetuarpok, tækkolugane attanniub kênanganik.

29 Absalomelo tilliklerpok Joabemut, tillijomavlugo attannermut, taimnalo kaijomalaungilak tapsomunga. Tamnale tilliklipsarpok, sullelo kaijomalaungilak.

30 Tagva kivgaminut okarpok: Ahâk, Joabib perorsêvikotinga perorsêvikotima senianêpok, perortokarporlo Gerstenik; 
taimak aileritse ikkilugillo ikkomamut pititse. Tagva Absalomib kivgangita perorsêvik tamna ikkipât.

31 Tagva Joabe parngnailerpok tikkilunelo Absalomemut iglub illuanut, okautivalo: Sôg kivgavît perorsêvikotiga ikkomamut ikkilaukatsuk?

32 Absalomelo Joabemut okarpok: Ahâk tilliklerlaukpunga illingnut okautsaukolaukpagillo: Kait tamaunga, tilliklerkovlunga illingnik attannermut, okautsaukolugo, sôg Gesuremit utterlaukîk? Namanersaunajarpok, tagvanenajaruma sulle. Taimak manna attanniub kênanga tækkolaulago: idluinekarpalle uvamne, tagva tokkunga.

33 Joabelo itterpok attannermut, okautivâlo tamattominga. Absalomelo kaikova, itterkovlugo attannermut; tamnalo kênaminik pûlune nettermut opigosukpok, attanniub sângane; attaniublo Absalome kunnikpa.

\section{KAPITEL XV.}

\section{Absalomib attaningoromaninganik. Davidib kemâninganik.}

PiJOKALAUKPORLO tamakkoa kingorngane Absalome âkiksoikojilaungmat ingminut aksalloalingnik horseniglo, angutiniglo 50 ginik, sivurlerpaktunik tapsominga.

2 Absalomelo taimak uvlâkut annijajovok, nangeriartorlunelo apkosiniub kiglinganut ittertârviub kannitanganut. Kinalo passiklernekalaurangat, attanniub idluarsaivianut aijomalaurangat; Absalomib kaikova ingminut, okarlunelo: Iglugaseksuit nellianit pivit? Tamnalo tagva okalaukpat: Kivgait Israelib kinguvarêngita illanganit pivok;

3 Tagva Absalomib okautiva: Ahâk illiornît idluarpok, namatuinarlunelo, kiglissiniartekangilatille attannerme.

4 Absalomelo okarpok: Kannoktôk uvanga idluarsaijungortitaunajaruma nuname, nelliat sunakartok idluarsartaujutiksakartorlo tikkilerajarkovlugo uvamnut, uvanga ikajorajarkovlunga tapsominga, idluarnermik pikovlugo!

5 Kiale kaglilerangago opigijomavlugo, tagva aggane issakpait tigulâlo, kunniklâlo.

6 Absalome taimailiorpok Israelitinut illunamassianginut, kailerangatta attanermut idluarsartaujomavlutik, tiglikpaillo taimak Israelitit angutingita omattingit.

7 Jahrit 40 git kingorngane Absalome attannermut okarpok: Aijomavunga angerneralo nellautsititsomavara Hebroneme, Nalekamut angernerilauktara.

8 Kivgait angerlaungmat, Gesuremêdlunga Siriame, okarlungalo: Nalekab ama tikkiutipanga Jerusalemut, tagva Nalegak kivgartoromavara. 
9 Attanniub okautiva: Ullapirsautekarlutit aularit. Parngnailerporlo, ailunelo Hebronemut.

10 Absalomele nautsertortuksanik tilliklilaukpok Israelitit kinguvarênginut illunainut, okautsaukojilunelo: Tiptulautib nippinganik tussarniarupse, tagva nipliaritse: Absalome attanningortitaumavok Hebroneme.

11 Angutille 200 dit Jerusalemit kaikojaumajut Absalome illagivât; issumalingasuêrlutigle aivut, kaujimanatiglo pijokartomik tamattominga.

12 Absalomele tilliklilerivoktauk Ahitofelemut Gilomiomut, Davidib attannertuijokotinganut, iglogaseksoanganit Gilomik attelingnit pijomut. Manna tunnergutinik tunnijingmat, angerkattigênek pitsartulerpalliavok, innugiartoksuillo kailutik unnuksivalliavut Absalomeme.

13 Tagva attausek tikkilerpok, tamna okautjivok Davidemik tamattominga, okarlunelo: Israelitit illunatik attunit omattingita Absalome mallikpât.

14 Davidele kivgaminut tapsomanêlauktunut Jerusaleme illunainut okarpok: Atte kemâlerta, mâne annigoiviksakarniangimat Absalomemit; tuavititse aularkovluta, oppaluijaukonnata tapsomunga, tigusilunelo uvaptingnik, pijaksaungitomiglo pititsivigivluta, iglugaseksuillo ullimârlugit saviksub kênanganut.

15 Tagva attanniub kivgangit okarput tapsomunga: Ahâk kivgatit oppalungêrsimavut, pinniarlutik sunamik, angajokama attanniub annerosungninganik.

16 Attannerlo pissuklune annivok iglomiokattine illunatik illagilugit. Arnalle tellimaujortut pannervgat nokkarkovait, paikovlugit iglomik.

17 Attannerlo innugiartoksuillo illunatik pissuklutik annijarêramik, nokkarput iglub kanningitangane.

18 Kivgangillo illunatik senianêlutik pissukput; taimaktauk Kretit Pletillo, Gathemiullo illunatik, augutit 600 dit pissuklutik Gathemit tikkilauktut, attannek sivurlervigivât.

19 Attannerlo Itaimut Gathemiomut okarpok: Sôg igvittauk illagivittigut? Utterit attannermêtuarlutillo; tujormiangôgavit, nunagilauktarnillo aularlutit tamaungarpotit.

20 Ikpeksak tikkisimavotit, uvlomelo kajusivotit aularkattigijomavluta. Uvangale aijomavunga aivigijungnartamnut. Utterlaurit, kattangutitillo illagællutit napkigijaunermik nertornarnermiglo pilit.

21 Itai kiovok okarlunelo: Sorlo miksekârtomik Nalegak inôngmat, sorlolo miksekârtomik angajokâra attannek inôngmat, nane angajokama attanniub innekarniarningane, tokkomut illinganiarmangat innôtsemullonêt, kivgait tagvanêniarivok.

22 Davide okarpok Itaimut: Tagva kait, aularkattigilutalo. 
Taimak Itai Gathemiok angutekotingillo illunatik aikattauvut, sorrutsillo illunatik illagijangit.

23 Nunamiullo illunatik kêadlarput nippekortôjomut, innugiartoksuillo illunatik kannilârpait. Attannerlo aivok kôb Kidronib akkianut, innugiartoksui'lo illunatik sivurlerput, apkosinerme sunakajuitomut sangmijome.

24 Ahâglo Zadok tagvanêlaurivok, Levitillo illunatik tapsomanêlauktut, akkigarlutiglo Gudib angerningata iklervinganik, illilâlo tagvunga. Abjatharelo kagverpok innugiartoksuit illunatik iglugaseksoarnit annikârtinnagit.

25 Attanniuble Zadoke okautiva: Gudib iklervinga iglugaseksoarnut âlaungmiuk. Saimaneksiniaruma Nalekab sângane, tagva ainiarivânga, tækkokoniarpângalo ominga iglominiglo.

26 Okarunele imâk: Kuviagingilagit; ahâk mânepunga. Tapsoma pinniarvigilinga namagijaminut.

27 Attanniublo ajochertuijok Zadok okautiva: Igvit tækkojôlungilatit? Utterit iglugaseksoarnut ullapirsautekarlutit, illagællutiglo ernîktik, Ahimaaz ernît, Jonathanelo Abjatharib erninga.

28 Ahâk natername sunakajuitome uttakkijomavunga, kivgartortomik tikkitokarkârtinnago illiptingnit, okautsaukârtinangalo.

29 Taimak Zadokib Abjathariblo Gudib iklervinga âlerivâk Jerusalemut, tagvanêtuarlutiglo.

30 Davidible kakkak orksuktalik majorarpa kêalunelo, niakungalo ulliksimalaukpok, kammilârlunelo pissukpok. Taimaktauk innugiartoksiut illunatik illagijangita attunnit niakotik ulliksimalaukpait, majorarpullo kêalutiglo.

31 Davidelo okautsaulerame, Ahitofelib Absalome illagingmago, okarpok: Nalegak, Ahitofelib attanertuininga sillainingortilauruk.

32 Davidelo kakkab kânganut tikkimat, tagvunga, Gudemik tuksiarviovaktomut, ahâk tagva Husaib Arachemiub pârpa, ulliglijarlune alliksimajomik; ivjokarlunelo niakomine.

33 Davidiblo okautiva: Igvit illagigumga okumaijutiksauniarpotit uvamnut.

34 Iglugaseksoarnulle ailerajaruvit okarajarlutillo Absalomemut: Kivgarivarma, attannermut illingajomavunga, atâtarnut kivgaulauktunga nelliutome taipsomane, kivgaujomavunga manna illingnut; tagva idluarkutiksamnut Ahitofelib attannertuininga asserorajarpat.

35 Taimak Zadokib Abjathariblo ajochertuijuk illagivâtit. Tamaita tussarniartatit attaniub iglunganit okauseriniarpattit ajochertuijungnut Zadokemut Abjatharemullo.

36 Ahâk tapkungnanêpuk ernikik, Ahimaaz Zadokib, Jonathanelo Abjatharib erninga. Taipkungnûna okautsaukojungnarparma tussarniartarnik. 
37 Taimak Husai, Davidib illanârijanga, tikkilerpok iglugaseksoarnut; Absalomelo tikkilerpok Jerusalemut.

\section{KAPITEL XVI.}

Zibab mangatsininganik Mefibosethemik. Simeib okarnerlotjininganit Davidemit. Absalomib mallingninganit Ahitofelib attannertuininganik piungitoâlungmik.

DAVIDELo kakkab pôktojoninganit kêtamik attersarêrmat; ahâk tagva Zibab Mefibosethib kivgangata pârpa, siutekoktojunik kapsinik iksivavilingnik neksarlune; tapkoalo kângine niakojakarpok 200 dinik, Weiniblo paunganginik kattingajunik pannertunik 100 dinik, Feiginiglo 100 dinik, publaujamiglo Weinelingmik.

2 Tagva attannek Zibamut okarpok: Sunamut ukkoa attoromavigit? Ziba okarpok: Siutekoktôjut attanniub illanginut illingajuksauvut, kângine ablangakovlugit, niakojallo, Feigillo nukapitsanut nerkiksaujuksauvut, Weinelo imiksauvok tapkonunga, merngortorniarpatta sunakajuitome.

3 Attannek okarpok: Angajokavit erninga nanêka? Ziba attannermut okarpok: Ahâk nokkangalaukpok Jerusaleme; okarame: Uvlome Israelib iglomiokotingita atâtama nalegauvinganik tunnitsiviginiarivânga.

4 Attannek Zibamut okarpok: Ahâk Mefibosethib perkutingit tamaita perkutaujuksauvut illingnut. Ziba okarpok: Opigosukpunga, saimaneksilaunga sângne angajokâra âttanerijaralo.

5 Attannerle Davide tikkimat Bahurim tikkilugo, ahâk tagva angut tagvangat annilerpok, Saulib iglungata kinguvânganit pijok, Simeimik attelik, Gerab erninga, tamna annilerpok okarnerluklunelo.

6 Millorpâlo Davide ujarkanut, attanniublo Davidib kivgangit illunaita. Innugiartoksuit illunatik sôngojokotingillo illunatik, tapsoma tallerpianelaungmatta saumianelo.

7 Imâgle Simei okarpok, okarnerlutjilune: Annit, annit, kingmiôjotit aungmik nerrijok, anguteâlôjotit!

8 Nalekab akkiniarutigimavâtit Saulib iglomiokotingita illunatik aungit pivlugit, tamna inangêrlugo attaningorsimagavit. Manna Nalekab attanniovik tunnimava ernerpît Absalomib agganginut; ahâglo manna kannoêtokotingnêpotit, kingmiôgavit aungmik nerijok.

9 Abisaile Zerujab erninga okarpok attannermut: Kingmiub tokkungajub oma angajokara attannek okarnerlotitaksarivaûk? Aivigijomavara, niakunganiglo nussuijomavara. 
Kap. 16.] II. SAMUEL.

10 Attannek okarpok: Illipse Zerujab kittorngangit sugivissinga? Okautekalulerle; Nalekab perkungmago: Davide okarnerlotiuk. Kina manna okarungnarka?' Sôg taimailiorkît?

11 Davidelo Abisaimut kivgaminullo illunainut okarpok: Ahâk, ernima, timimnit pimajub, pijugakpânga, suna pivlugo Jeminib erninga una manna taimailiortuksaunginiva? Agviarnago taimailiorle, okarnerlukovlugo, Nalekab perkolaungmago.

12 Imakkâ Nalekab ajulernera tækkonârniarpa, pitsiarnermullo akkinniarlunga tapsoma okarnerlotjininga uvlomênitak pivlugo.

13 Taimak Davide innukotine illagilugit sivumuarpok apkosinerme; Simeile kakkab uvinganingane pissukpok senniane, okarnerluklunelo, ujarkello ivjullo kattingajut milloriutivait tapsomunga.

14 Attannerlo innugiartoksuillo illunatik; Israelib angutingit illagijangit illagilugit, merngortorlune tikkipok. Ajelimut, nekkoksilarmelo ingminik tagvane.

15 Absalomele innugiartoksuillo, Israelib angutingit illunatik Jerusalemut tikkilerput, Ahitofeliblo illagiva.

16 Husaible Arachemiub Davidib illanârijangata Absalome itteramiuk, Absalome okautiva: Pilloringnartokarle, nalegak attannek! Pilloringnartokarle, nalegak attannek!

17 Absalomible Husai okautiva: Tamannauva napkiningnît illannarijingnut? Sôg illannârijît illagilaungikiûk?

18 Husaible Absalome okautiva: Taimangungitok, Nalekable innugiartoksuillo ukkoa, angutillo illunatik Israeleme anerijanganut perkutaujomavunga, tapsomanêtsainaromalungalo.

19 Amalo kina kivgartoraksarivigo? Erningata sângane kivgartortuksaungilanga? Sorlo atâtavît sângane kivgartorlaurama, taimailingajomagivunga sângne.

20 Absalomelo Ahitofelemut okarpok: Attannertuileritse, sunamik pinniartuksauvita?

21 Ahitofelelo okarpok Absalomemut: Atâtavit pannervgangit, kemalauktangit paikovlugit iglomik, uvinikattigikit; tagva Israelitit illunamassiangit tussarniarput atatait tippeluktilaurangne, illunatiglo aggangit illingnêtut nangiartuinersaulerniarput.

22 Tagva tuppek napparterpât Absalomemut iglub kângane, Absalomiblo atâtame pannervgangit uvinekattigivait, Israelitit illunamassiatik ijingita sângine.

23 Nelliutome taipsomane, Ahitofele attannertuilaurangat, tamanna illingalaukpok sorlo Gude apperijaunajalaukpat; taimailaukput Ahitofelib attannertuiningit illunaita, tamangne Davideme Absalomemelo. 


\section{KAPITEL XVII.}

Ahitofelib attannertuiningata sillatojub, unatadlarnek pivlugo, agviartauninganik. Tapsoma 7evilerninganik kimingninganiglo ingminik.

A HITOFELO Absalomemut okarpok: Angutit 12000 dit annerijomavakka, parngnaileromalungalo, Davidelo udlajomavara unnuangotillugo,

2 Opingâromavaralo merngortortillugo ajalutillugolo. Tagva koaksartigupko, innugiartoksuillo illagijangit illunatik kemâlerpatta, tagva attannek kissiat ullimaromavara.

3 Innugiartoksuillo illunaita uttertitsomagivakka illingnut. Illunatiglo illingnut âtaulerêrniarpatta sorlo tussugavit, tagva innugiartoksuit illunatik ullapirsautelingmetuarniarput.

4 Absalomib tamanna idluarasugiva, angajokaunerôjullo Israelemêtut illunatik.

5 Absalomele okarpok: Husai Arachimiok kaikojaulaungmile, tussartalo kannok tapsoma okarninganik tamanna pivlugo.

6 Husai ittermat Absalomemut, Absalomib okautiva: Imaitunik Ahitofele okausekalaukpok; igvit okalaurit, taimailiortuksauvita, taimailiortuksaungilagullonêt?

7 Tagva Husai okarpok Absalomemut: Attannertuinek Ahitofelib sakkertanga tamattomane, attannertuiniôlungilak namatsiartok.

8 Husailo okapsarivok: Atâtait illitaritsiaralloarpat, innukotingillo sôngojoksôngmatta, ningaumajadlarmattalo issumamikut aklatut piarkaminik aksartaumajotut natername; tamannalo tappilugo atâtait angutiôvok unatadlarnermik sungiusimajok, siglotoniangilarlo innugiartoksuit illagællugit.

9 Ahâk, imakkâ ijerpallaivok manna ittersat illanganut, assianullonêt inniksamut. Pijokarajarpat tagva, sivurlerpamik pijaungimat, nipliartokarajarpallo okartomik: Sorsungnekalaukpok innugiartoksuit Absalomemik malliktut akkorngane;

10 Tagva illunatik attunit maksoajungnailerajarput, sorsugungnartorloctauk aglat, omattekartorlo Loewetut. Israelitit illunamassiatik kaujimangmatta atâtait sangijoksôngmat, illagijangillo sorsugungnartôngmatta.

11 Tamattomingale attannertuivunga, kattersikovlutit illingnut Israelinik illunamassianginik, Danemit Bersaba tikkilugo, taimak unnuktigijunik siorkatitut immarbiub siksanganêtutitut; igvillo nangminek akkornganêdlutit aularkattigilugit pikovlutit. 
12 Tagva opingâromavavut nâne nennisivigijaptingne taipsominga, tikkidlaromavavullo sorlo mitsuksoak nunamut kattangmat, amiakokarkojikonnata tapsominga, angutekotinginillo attautsemiglonêt.

13 Iglugaseksuillo illanganut tikkisimalerpat, tagva Israelitit illuêngarlutik iglugaseksuit tamakkoa aklunânut napperaksarivait, aksorortilugillo kôngmut, nachvartokarungnaikovlugo tagvane, ujarârsumik attautsemiglonêt.

14 Tagva Absalome Israelitillo illunamassiatik okarput: Husaib Arachimiub attannertuininga namanersauvok Ahitofelib attannertuininganit. Nalekable taimailingatipa Ahitofelib attanertuininga sillatôjok agviartaukovlugo, Nalegak kanoêtomik pititsikovlugo Absalomemut.

15 Husailo Zadokemut Abjatharemullo ajochertuijungnut okarpok: Imâglo, imaglo Ahitofelib Absalome angajokaunerôjullo Israeleme attannertorlaukpait; uvangale imâk imâglo attannertuilaukpunga.

16 Taimak manna tuavi tillikleritik, Davidelo okautsaukolertikko, okarlutiglo: Nokkanganiarnak unnuak nâvlugo sunakajuitub naternangane, ikârasualerille, attannek êjaukonago innugiartoksuit illagijingit illagrelugit.

17 Jonathanele Ahimaazelo nangerpuk immektarvingme Rogelemik attelingme, arnablo kivgaub okautijartorpâk tamattominga. Tapkoalo aivuk tagvunga, attannerlo Davide okautijartorpâk tamattominga; tækkotijuksaungimanik, iglugaseksoarnut tikkinajarlutik.

18 Nukapiable tækkovak, Absalomelo okautiva tamattominga. Tapkoale tuavilukarlutik aivuk, tikkilutiglo Bahurimemut, angutit illangata iglunganut; taimna avalutekotingita illuane immektarvikarpok, tapkoalo akkarpuk takkamunga.

19 Arnablo kebbik tigulugo issivilâlo immektarviub pângata kollânut, kakkojaksallo serkalimajut issivipait oma kânganut, taimak mallugijaujungnarlungimat.

20 Absalomib kivgangit manna arnamut, iglub illuanut tikkinamik, okarput: Ahimaaz Jonathanelo nanêkak? Arnab okautivait: Kokulluk una ikârlaukpâk. Kenneramiglo, nennisinatiglo, utterivut Jerusalemut:

21 Taipkoalo aularêrmatta, kagverpuk immektarvingmit, ailutiglo, Davidelo attannek okautijartorpâk, okarlutiglo Davidemut: Parngnaileritse, tuavidluselo kôk ikârsiuk; imâglo, imâglo Ahitofele attannertuilaungmat akkerartorluse.

22 Tagva Davide parngnailerpok innugiartoksuillo illagijangit illunatik, Jordanelo ikârpât kaulermarikârtinago, amiakokalaungilarlo attautsemiglonêt ikârlaungitomik Jordanemik.

23 Ahitofele tækkogame attannertuinine malliktaulungimat siutekoktôjokotine iksivariktârpa, parngnailunelo, angerarlunelo 
iglugaseksoarnut iglokarvigijaminut, iglunelo âkikpa, kimiklunelo tokkolarmelo, illijartortauvorlo atâtame illuvekotinganut. 24 Davidelo tikkilerpok Mahanaimemut. Absalomiblo Jordane ikârpa, Israelitillo angutingit illunatik illagivât.

25 Absalomiblo Amasa Joabib inningane angajokangortilaukpa sorsuktuksakattigêksoarnut. Amasale erniolaukpok angumut Jethramik attelingmut, Israeliteôjomut, taipsoma Abigail Nahasib pannia, Zerujab Joabib anânangata nukanga aipariva.

26 Israelitille Absalomelo tangmarterput Gileademe.

27 Davide Mahanaimemut tikkisimangmat, tagva Sobi, Nahasib erninga Rabbatemit, Ammonib kittorngangita iglugaseksoakotinganit, Machirelo Amielib erninga Lodabaremit, Barsillailo Gileademiok Roglimit, kaitsivut,

28 Aumitinik, pôgutaniglo, illulerkuteniglo macharnik, Weizenik, Gerstenik, sennaukamiglo, Gersteniglo jarasimajunik, Bohneniglo, Linsiniglo, Grützeniglo,

29 Igupsaujartullo orksuanik, immublo orksuanik, punnaujartuniglo sennamajunik saukat tuktuvaillo immunginit, Davidemut, inugiartoksoarnullo tapsomanêtunut, nerkiksakarkovlugit. Issumakaramik, inugiartoksuit nellonangitomik kâkput merngortorlutiglo immerudlalarmiglo sunakajuitome.

\section{KAPITEL XVIII.}

Absalomib sorsuksoakotingita salagïauninginit Joabemut; $A \bar{b}$ salomib kappijauninganit, nivingatillugo nappartome Eichemik attelingme.

Davidiblo innugiartoksuit illagijine oppalungaijarpait, angajokakartilaitalo 1000 dinut, 100 dinullo.

2 Tillivaillo, innugiartoksuit pingajungit attautsit, Joabe angajokagilugo; pingajungillo attautsit, Abisai angajokagilugo; pingajungillo attautsit Ithai Gathemiok angajokagilugo. Attannerlo innugiartoksoarnut okarpok: Uvangatauk aularkattigijomavapse.

3 Innugiartoksuille okarput: Aulartuksaungilatit; uvagulle kemâniaralloarupta, ketterarlutalonêt tokkolerniarupta, kamaginiangilâtigut; igville îpotit sorlo 10000 ditut uvaptingnit; taimak manna namanersauvok ikajoruptigut iglugaseksoarnit.

4 Attanniub okautivait: Namagijapsingnik pinniaromavunga. Attannerlo ittertarviksub tungânut nangeriartorpok, innugiartoksuillo illunatik annilerput, 100 dit kattigêklutik, 1000 dillo kattigêklutik.

5 Attanniublo Joabe, Abisailo, Ithailo perkovait, okauti- 
Kap. 18.] II. SAMUEL.

lugillo: Sugautitaililugo nukapiak Absalome pinniarvigisiuk. Innugiartoksuillo illunatik tussarput attanniub angajokait illunaita perkungmagit Absalome pivlugo.

6 Innugiartoksuillo sillatânut naternamut tikkimatta, Israelitit pachrearlugit; tagva sorsungnek pigiarpok Efraimib nappartungine.

7 Innugiartoksuillo Israelitit sâlagijauvut tagvane Davidib. kivganginut, uvlorme taipsomane ullimartaunekalaungmat angijomik, angutinik 20000 dinik.

8 Sorsungnerlo siamarsimalaukpok tagvane nuname illunâne; nappartulle nungutsinersaulaukput innungnik uvlorme taipsomane, saviksoarmit.

9 Absalomiblo Davidib kivgangit pârpait, ablangavlune horseojartub kângane. Horseojartorlo nappartoksub silliktub Eichemik attiggub attânut tikkimat, niakunga nâpok Eicheme, nivingalunelo killaub nunablo akkorngangne, horseojarkotingale tapsoma attânit pangalidlarlune aularpok.

10 Angutit illangata tamanna tækkogamiuk, okautigiva Joabemut, okarlunelo: Ahâk Absalome tækkolaukpara nivingatillugo Eicheme.

11 Joabiblo angut tamna okautjine tamattominga okautiva: Ahâk, tamattominga tækkolaukît? Sôg annaulaungikiuk, nunamut ochotilugo? Tagva pivlunga Silberlinginik 10 nik, tapserumiglo tunnitsivigijomanajarpagit.

12 Angut tamna Joabemut okarpok: Silberlinginik 1000 dinik tunnitsivigigalloarumga aggamnut, sulle aggakka illijomanajangilakka attanniub erninganut. Attanniub igvit perkolaungmattit. Abisailo Ithailo siutiptingnut tussartilluta, okarlunelo: Udsertoritse, kinalonêt pinniarlukonnago nukapiamut Absalomemut.

13 Ubvalo sunamik idluitullinajalauruma nangiarnartomik tarnimnut, attannek sunamiglonêt angigutijaulungimat; tagva igvit nangminek akkerartorajarparma.

14 Joabe okarpok: Taimak akkunêrtigijomik nokkarungnangilanga illingne. Tagva Joabib kallugitset pingasut tiguvait aggangminut, kapputilaitalo $\Lambda$ bsalomib omattinganut innotillugo sulle Eicheme.

15 Nukapitsello tellimaujortut, Joabib sakkunginik akjarsijut, tikkilutik illumavât, annaulerlugolo tokkopât.

16 Tagva Joabib tiptulaut tiptulausijautigiva, innugiartoksuillo utterkovait, udlanigungnaikovlugit Israelitinik; Joabib innugiartoksuit ivlerijomangmagit.

17 Absalomelo tiguvât, egilugolo nappartut akkornganut ittersâlungmut, ujarkello unnuktut perorliutivait kânganut. Israelitillo illuêngarlutik attunît kemâvut iglokotimingnut.

18 Absalomele nappajoksoamik nangminerijaminut nappartitsilaukpok inôvlune sulle; tamna napparsimavok attaniub 
naksakotingane. Okalaurame: Ernekangilanga, tamana pivlugo, tamanna erkaumajaksaujuksauvok attimnut; atserarpâlo nappajok attine malliklugo, taijauvorlo uvlok tamanna tikkilugo, Absalomib inninganik.

19 Ahimaaz Zadokib erninga okarpok: Nagliktara akpangerlangatôk, attannerlo nellojungnaitilugo, Nalekab piulijaunermik sakkertitsivigingmago omigijingita agganginit.

20 Joabible okautiva: Igvit uvlome taigoraksamik ajungitomik âtsiniangilatit. Uvlub tamattoma assiane taigotaksamik âtsiniarpotit, uvlomeloungitok; attanniub erninga tokkungmat.

21 Kusimulle Joabe okarpok: Attannek okautijartoleruk tækkolauktarnik. Kusiblo Joabe opigiva, akpangerlunelo.

22 Ahimaazele Zadokib erninga okapsarpok Joabemut: Kannok uvangatauk akpangerajaruma Kusi malliklugo? Joabe okarpok: Sôg akpangeromavît ernîk? Kait ovunga, taigoraksamik ajungitomik âtsiniangilatit.

23 Kannok akpangerajaruma? Tapsoma okautiva: Tagva akpangerit. Taimak Ahimaaz akpangerpok apkome naggonerpâme, Kusilo sivurngitorpa.

24 Davidele iksivavok upkoaksoak magguk akkorngangne. Udsertortelo majoarpok upkoaksub avalunetub kânganut, ijingminullo tækkosarpok, tækkolunelo angumik, inutôlune akpangertomik.

25 Nipliarporlo, attannerlo okautiva tamattominga. Attanerle okarpok: Innutôgune, tagva taigotaksak ajungitok tapsoma kanninganêpok. Tamnalo tuavilukarlune kannilertillugo,

26 Udsertortib angutib taipsoma assia akpangertok tækkova, nipliarlunelo upkoaksoarmut, okarlunelo: Ahâk angut innutôlune akpangerpok. Attannek okarpok: Tamnatauk kivgartorteôvok ajungitok.

27 Udsertorte okarpok: Sivurliub akpangerninga tækkovara Ahimaazib Zadokib erningata akpangerningatun îmat. Attannerlo okarpok: Angutaurok tamna idluartok, ajungituniglo taigotaksanik kaitsivok.

28 Ahimaazele nipliarpok, okarlunelo attannermut: Ullapirsaut! Opigosuklunelo attanniub sângane kênane pûlugo nunamut, okarlunelo: Nertortaule Nalegak Gudit, innuit aggamingnik issaktitsilauktut nalekamnut attannermut akkerartorlugo unertutigijingat.

29 Attannek okarpok: Nukapiak Absalome kanoêlungigalloarka? Ahimaaze okarpok: Angijomik aulajadlarnermik tækkolaukpunga, attanniub kivgangata Joabib uvanga kivgait tillingmanga, nellovungalo sunaulaungmangat.

30 Attannek okarpok: Avatâgorit nangeriartorlutillo maunga. Avatâgorporlo nangeriartorlunelo tagvunga.

31 Ahâk tagva Kusi tikkilerpok, okarlunelo: Mâne taigo- 
raksakarpok ajungitomik, nalegara attannek! Nalekab uvlome piulijaunermik sakkêvigilaukpâtit, illunatik akkerartorlauktivit agganginit.

32 Attannerle Kusimut okarpok: Nukapiak Absalome kanoêlungigalloarka? Kusi okarpok: Nalekama attanniub omigijingit illunaita illingajuksauvut nukapiab oma illinganingatut; illunaitalo akkerartortut illingnik idluitullijomavlutik.

33 Tagva attanek kiksalerpok, ailunelo iglomut angijomut ittertârvingmêtomut, kêalunelo, pissuklunelo okarpok imâk: Ernik, Absalom, ernîk, ernîk Absalome! Gude pijomaletôk uvanga tokkojuksaunimnik inningne! Absalomekotîk, ernîk, ernîk!

\section{KAPITEL XIX.}

Davidib uttertitauninganit Judakunut. Tapsomalo issumatoninganik kujalidlarninganiglo.

JOABELO okautsaumalerpok: Ahâk attannek kêavok, ogguarlunelo Absalome pivlugo.

2 Sâlakarnermillo uvlorme taipsomane, kiksarnekalerpok innugiartoksuit illunamassiatik akkorngane, innugiartoksuit illunamassiatik tussarlaungmatta, attaniub ernine issumajarutigingmago.

3 Inugiartoksuillo angigutsivlutik, aularasuarput uvlorme taipsomane, iglugaseksoarnut ittertaililutik, sorlo inugiartoksuit angigutsivlutik aularpangmatta, kangusuktitaumajut sorsungnerme kemâlaukpatta.

4 Attaniublo kênane ulliksimalaukpa, okausertosarlunelo nipliavok: Ernîk Absalome, Absalome, ernîk, ernîk!

5 Joabible attannek iglub illuanut tikkipa okarlunelo: Uvlome kivgatit, uvlome piuliklerlauktut tarningnik, ernivîllo, pannivillo, nulliavillo panervgakotivillo tarninginik, illunaita kangusuktilaukpattit;

6 Omigijitit nagligigangne, nagligijitillo omigigungne, mallugosukojigavit uvlome angajokajet kivgatit kamaginginangne. Mallugosugalloarama uvlome, Absalome innotuinarajarpat, uvagullo uvlome illunata tokkonajarupta, idluarasugituinarajarpat tamanna.

7 Tagva parngnailerit manna annilutillo, illanâkarlutillo kivgatit okarkattigilerkit. Okautimarikapkit Nalekame: Anniniangikuvit, angut attauserlonêt, illingnêtuarniangilak unnuarme tamattomane Tamanna sugalunersauniarpok illingnut, kappianartunit tamainit tikkitautigijarnit innuosungnernit manna tikkilugo. 
8. Tagva attannek parngnailerpok, ingilunelo ittertarviub. illuanut. Inugiartoksuillo illunaita okautsaulerput: Ahâk attannek iksivavok ittertarviksub illuane. Tagva innugiartoksuit illunatik tikkilerput attanniub sầnganut. Israelitille kemmâlaukput illunatik attunît iglomingnut.

9 Innugiartoksuillo illunatik aivakattigêlerput Israelib kinguvarêngine illunaine, okarlutiglo: Attanniub piulilaukpâtigut omigijipta agganginit, piulilaukpâtigullo Filisterit agganginit, kemmâjariakalaukporlo nunamit Absalome pivlugo.

10 Absalomelo mingoarlauktavut attanniokovlugo uvaptingnut tokkolaukpok unatadlarnerme. Sôg manna kikkarkise taimak, attannek aitaililugo pigapse?

11 Attannerlo tilliklerpok Zadokemut Abjatharemullo ajochertuijungnut, okautsaukovâglo: Angajokaunerôjut Judakune okarkattigisigik, okarlutiglo: Sôg illipse kingurlerpaujomavise attanek aimilugo iglunganut? (Israelitit illunatik okausingit tikkisimalaungmatta attanniub sânganut iglunganut.)

12 Illipse kattangutigivapse, saunekattigiluse uvinekattigivapse; Sôg taimaimat illipse kingurlerpaujomavise, attannek aimilugo?

13 Amasamullo okaleritik: Saunekarkattigingilagit uvinekarkattigingilagillo? Gudib imâglo imâglo pinniarvigilinga, angajokaksoangojuksaungikuvit sorsuktuksakattigêksoarnut sâmne, Joabib iningane, innôtsit nâlugo.

14 Judakullo angutingita illunatik omattingit sangutipait, sorlo angutib attautsib omattingatut. Tilliklerpullo attanermut: Kailaungmît, igvit kivgakotitillo illunamassiatik.

15 Taimak attannek utterivok, Jordanemullo tikkimat, Ju$\mathrm{dab}$ angutingit Gilgalemut tikkisimalaukput, attersaromavlutik, attannek pachrearomavlugo, attannerlo ikârtilugo Jordanib akkianut.

16 Simeilo, Gerab erninga, Jeminib erningata Bahurime nunakalauktok, tuavilaukpok, Judablo angutingit illagallugit attersarpok attannek pachrearlugo.

17 Angutillo 1000 dit Benjaminekunit illagivât, taimaktauk Ziba, kivgak Saulib iglunganit pijok, ernine 15̃ it, kivganelo 20 git illagilugit, Jordanelo ikârpât attannek sivurlervigilugo.

18 Umiarlo oppalungaijarpât, attaniub illangit ikârtilugit, pinniarlutiglo kuviagijanginik. Simeile Gerab erninga attanniub sânganut pamakterpok Jordane ikârmago,

19 Okarlunelo attannermut: Nalekama idluitullinermik tamattominga illingatiniarnangatôk, erkainiaranelo kivgavit idluitullivigilaungmatit uvlorme taipsomane, nalegara attannek annitillugo Jerusalemit, attaniublo omamiutariniarnagotôk.

20 Kivgait illitarksilermat idluitullidlalaurama. Ahâglo 
uvlome tikkitunit Josefib iglomiukotinginit tamainit sivurliokauvunga, nalegara attannek pachrearomavlugo.

21 Abisaile Zerujab erninga kiovok okarlunelo: Simeilo tamanna pivlugo, tokkotaksaunajangilâk, Nalekab saimartitanga okarnerlotigilauralloarmago?

22 Davidele okarpok: Uvanga sugivissinga illipse Zerujab kittorngangit, uvlome Satanasingoromagapse uvamnut? Uvlome kina tokkotaujuksaunajarka Israeleme? Issumavit kaujimalunginama, uvlome attanningortitaumagama Israelitinut?

23 Attannerlo Simeimut okarpok: Tokkojuksaungilatit. Attaniublo angermarikpa.

24 Mefibosethe Saulib ernguta kailune attersarivok, attannek pachrearlugo. Ittikkanelo uminelonêt ipêrutilaungilait, annoranelo ergortorlaungilait uvlormit taipsomangat, attanniub aularvianit, uvlok taimna ullapirsautekarlune uttermat tikkilugo.

25 Tagva manna Jerusalemut tikkiname attannek pâromavlugo, attanniub okautiva: Sôg aularkattigilaungipinga Mefiboseth?

26 Tamnalo okarpok: Nalegara attannek! kivgama uivêrilaukpânga. Kivgait issumakalaungmat, siutekoktôjut illangat iksivaviktâromavara, ablangajomalungalo tapsoma kângane, attannermullo ingergalunga; kivgait tussiangmat.

27 Tamannalo tappilugo taipsoma kivgait passilauriva attanerme. Nalegarale attannek Gudib Engelingatut îpok; pinniarit namagijarnik.

28 Atâtama iglomiokotingit illunatik sunaulautsengimatta, assianik, innungnit tokkotaksanit, nalekama attanniub sângane; taimak kivgait ingitilaukpat taipkoa nerrimarkattaujut nerrimavingne akkornganut; sunamik assianik idluartomik pitjutiksakarkîk attannek nipliarvigilugo?

29 Attanniub okautiva: Sunamik unnurnersanik okarkît sulle illuserilauktat pivlugo? Okautigilaukpara: Igvillo Zibalo perorsêvik avingniarpattik illiptingnut.

30 Mefiboseth attanermut okarpok: Taipsoma illuêngarlugo tiguliuk, nalegara attannek ullapirsautekarlune angerarsimalerêrmat.

31 Barsilailo Gileademiok attersarpok Roglimit, attannerlo tessiorpa Jordanib akkianut, Jordaneme kannilaromavlugo.

32 Barsilailo innutokaudlalaukpok Jahrekarlune 80 ginik; tapsoma attannek pattangaitilaukpa, Mahanaimetillugo, angutitsiangolaurame.

33 Attannerlo Barsilaimut okarpok: Ikârkattigijaksarivarma, pattangaititsomavagit uvamne, Jerusaleme.

34 Barsilaile attannermut okarpok: Sunauva sulle inôneri- 
niartara, uvanga attannek illagællugo takpaungartuksaugama Jerusalemut?

35 Uvlome Jahrekarpunga 80 ginik. Kannok uvanga illitarksijuksaunajarkik, sunamik idluarmangât, idluimangâllonêt, ikpigilungalonêt sunaumangât nerrijara, imertaralo, tussarlungalonêt sunaumangatta imgerpaktut angutit imgertullo arnat imgerutingit? Sôg kivgavit nalegara attannek akkunênersamik pagvisaraksarivaûk?

36 Kivgavit attannek kêtamik kannilaraksariva Jordane ikârlugo. Sôg attaniub taimaitomik nelileromavânga?

37 Kivgait utterkoleruk, tokkolerkovlunga iglugaseksoarne iglokarvigijamne atâtama, anânamalo illuvikita tungâne. Ahâk ovanêpok kivgait Kimeham, tamna nalegara attannek illagællugo ailerle, pinniarvigilugolo namagijarnik.

38 Attannek okarpok: Kimehamib aiparijaksarivânga akkianut, uvangalo pinniarvigijomavara kuviagijarnik; tamainiktauk annerijarnik uvamnit, tamakkoninga pinniaromavunga.

39 Innugiartoksuillo illunatik Jordane ikarêrmatsuk, attanniublotauk, attanniub Barsillai kunnikpa saimartilâlo; tamnalo utterivok nunakotiminut.

40 Attannerlo ingergavok Gilgalemut, Kimehamiblo ingergakattigiva. Innugiartoksuillo Judakut illunamassiatik attannek ikârtilaukpât, innugiartoksuille Israelitit ketterkatuinangit tagvanêlaukput.

41 Ahâglo, tagva Israelitit angutingit illunatik tikkilerput attannermut, okautivâllo: Sôg kattangutipta Judakut angutingita tiglilaukâtit, attannerlo iglomiokotingillo, ikârtilaukagit Jordanekut, Davidiblo angutekotingit illunaita illagælugo?

42 Tagva Judakut angutingita Israelitit kiovait; attannek kannidlartomik illagivavut, sôg tamanna ningarosujutigivissiuk? Attanermit nerkiksamik pilliusianiglonêt pilaurasugivittigut?

43 Taimak tagva Israelitit angutingita Judamit pijut, kiovait okarlutiglo: Uvagut tellimaujortuêrtorluta pitjuţekarnersauvogut attanermut, taimaktauk Davidemut, illipsingnit. Sôgle tagva uvagut taimak sunaungitonasugivittigut, okautsivut sivurliotitaulaungimatta, attanivut ailugo? Judable angutingit songonersanik okausekalaukput Israelitit angutinginit.

\section{KAPITEL XX.}

Pikabladlartub Sebab unangmëjauninganik, tokkotauninganiglo. Amasab tokkotauninganik.

ANGUTEKALAUKPORLO tagvane illitarijaujomik kuksalaitomik, Sebamik attelingmik, Bichirib, angutib Jeminib kingu- 
vânginit pijub erninganik; tapsoma tiptulaut tiptulausijautigiva okarlunelo: Illangiutivikalungilagut Davidemik, kingormgutijaksakaratalonêt Isaib erningane. Israelitiôjut illunamassiatik attunît ailerlit iglokotimingnut!

2 Tagva Israelitit illunamassiatik Davide allarpât, mallikpâllo Seba Bichrib erninga. Judable angutingita attannekotitik attâvigivât Jordanemit Jerusaleme tikkilugo.

3 Attanerle Davide angerarsimalerame Jerusalemut, pannerrget tellimaujortut kemmalauktane iglomik paikovlugit tiguvait, pitilaitalo pairijauvingmut, pattangaitilugillo, sinnikattigilungilaille. Taimaglo parngnanairsortaulaukput tokkotik tikkilugit, innôlutiglo uigarniojutitut.

4 Attannerlo Amasamut okarpok: Angutit Judame, illunaita kaikokit, kaikovlugit uvluk pingajuane; igvillotauk tamâne nangertuksauvotit.

5 Amasalo aivok Judamiut kaikolugit; nelliutorle tailauktanga tapsomunga kangiupa.

6 Tagva Davide Abisaimut okarpok: Manna Seba Bichrib erningata anginersamik kiksartiniarpâtigut Absalomemit. Igvit angajokavit kivgangit tigukit, udlalugolo, iglugaseksoarnik sôngojunik ingminut nachvarkonnago, kemâkonnagolo ijiptingnit.

7 Tagva aularput tamna malliklugo Joabib angutingit illunatik, taimaktauk Kretillo Pletillo, sôngojullo illunatik. Aularpulle Jerusalemit, Seba Bichrib erninga udlalugo.

8 Ujarasuksoarmêtillugille Gibeonemêtome Amasab, sivurngitorpait. Joabele tapserusersimalaukpok annorame attortame kângagut, tapsomalo kângagut tapserutemiutekalaukpok saviksoarmik, tamna kenailitamêdlune nivingavok tapsoma makkitingane, sivumuartillugolo tamna amuvok kattaklunelo.

9 Joabiblo Amasa okautiva: Ullapirsautekalerit kattangutîk! Joabiblo aggangminut tallerpingminut Amasa tiguva umingittigut, kunnigomavlugo.

10 Amasablo saviksoak Joabib agganginêtok kamatsoliorpa; tapsomalo tamattomunga kappiva nângagut, erchavingit kattangmatta nunamut, kappipsarlaungilâlo, tokkolaukporlo. Joabible angajuatalo Abisaib Seba Bichrib erninga udlalaukpâk.

11 Jo: biblo angutekotingita illangata nangeriartorpa sennianut, okarlunelo: Nelliat Joabemik kuviasutekartok, Davidemullo illingajok, mallilerle Joabemik!

12 Amasale nellavok aungme aksasimavlune apkosiniub kerkane. Illangalle tækkogame innugiartoksuit illunatik tagvane nokkarmatta, Amasa nûpa apkosinermit perorsêvingmut, egitsilunelo anorânik tapsoma kânganut, tækkogame, illunatik tikkitut tapsomunga nokkarmatta.

13 Manna apkosinermit pêrsimangmat, illunatik attunît Joabe mallikpât, Seba Bichrib erninga udlajomavlugo. 
14 Ingergarorlo Israelib kinguvarêngittigut illunaitigut, Ảbelemut, Beth-Maachamullo, Habarimemullo illunânut; tapkoalo kattimalerput mallikpâllo.

15 Tikkilutiglo, illumavâllo Abeleme Bethmaachemelo, tallumiglo illijivut iglugaseksuit avatâtigut, avalunullo tikkiput, innugiartoksuillo illunatik Joabib illagijangit aksorotjivut avalullo ochotitsomavât.

16 Tagva arnak sillatôjok iglugaseksoarnit kaigarsukpok: Nalegitse! nalegitse! Joabe okautisiuk, tamaungarkovlugo; okarkattigijomavara.

17 Tikkimagolo, arnak okarpok: Joabeovît? Tamna okarpok: Ahaila. Arnab okautiva: Kivgavit arnab okausingit tussarkit. Tamna okarpok: Tussarpunga.

18 Arnak okarpok: Itsak okartokarpakpok: Kina appertsutekarpat, tamna appertsorle Abeleme; taimaglo idluartomik illinganekarpalaukpok.

19 Uvanga iglugaseksoarnutkennukajuitunut nertornartunullo Israelemetunut illingavunga; igvillo iglugaseksuit anânalo Israeleme asseroromavigit? Sog Nalekab kingormgutijanga nungutsomaviuk?

20 Joabe kiovok okarlunelo: Tamanna ungasigle, tamanna ungasigle uvamnit, nungutsijuksaunajarama, asseroijuksaunajaramalo; taimailingalungilak.

21 Angutible Efraimib kakkanginit pijub, Sebamik attiggub, Bichrib erningata, attannek Davide pikablavigilugo akkerartorlaukpa. Tamna kissimiovlune tunnilersiuk, tagva iglugaseksoarnit nûtsomavunga. Arnab Joabe okautiva: Ahâk niakoa egitaujuksauvok illingnut avalut kollâtigut.

22 Arnarlo kamunga tikkipok innugiartoksoarnut illunainut sillatunerminut. Tapkoalo Sebab Bichrib erningata niakunga nakkarpât, egilugolo Joabemut. Tagva tapsoma tiptulaut tiptulausijautigiva, tapkoalo siamalerput iglugaseksoarnit, illunatik attunit iglomingnut. Joabele uttilerivok Jerusalemut attanermut.

23 Joabele angajokaulaukpok Israelib sorsuktuksaksoanginut illunainut. Benajalo Jojadab erninga angajokaulaukpok Kretinut Pletinullo.

24 Adoramelo pimaklerteôlaukpok, Josafatelo Ahiludib erninga, aglakteolaukpok attanioviub pijokarninginik.

2う Seja aglakpakteôlaukpok. Zadok Abjatharelo ajochertuijolaukpuk.

26 Taimaktauk Ira Jairemiok attanertuijolaukpok Davidemut. 


\section{KAPITEL XXI.}

\section{Kângnartomil Jahrit pingasut nâvlugit Saule pivlugo. Davidib} unatadlarninganik sâlakarninganiglo Filisterinili.

\section{DAVIDIB nelliuningane kângnartokalaurivoktauk Jahrine tug-} lerêne pingasune. Davidiblo Nalekab kênanga kennerpa. Nalegarlo okarpok: Saule pivlugo, iglolo aulik pivlugo Gibeonemiut tokkolaungmagit.

2 Tagva attanniub Gibeonemiut kaikojaukovait okautilugillo: (Gibeonemiut Israelib kittornganginit pilaungilet, amiakolankpulle Amoriterinit; Israelible kittorngangita angerkattigimarilaukpait, Sauliblo ullimarasuarlaukpait illungertornermine Israelib kittorngangit Judakullo pivlugit.)

3 Tagva Davide manna Gibeonemiunut okarpok: Sunamik piniarvigijaksarivapse? Sunamullo ullapkoseraksarivapse, saimartitsikovluse Nalekab kingormgutijanganik?

4 Gibeonemiut okautivât: Goldemiglonêt, Silberemiglonet pijomalungilagut Saulemit, tapsomalo iglunganit, pijomalunginivogut kinalonêt tokkotaukovlugo Israeleme. Tamna okarpok: Sunamigle okarkise pinniartuksaunimnik illipsingnut.

5 Tapkoa attannek okautivât: Angut taimna asseroilauktok nungutsilauktorlo uvaptingnik, nungutaksarivavut taipsomangat amiakokarkonago sunamiglonêt Israelib erkâne illunaine.

6 Tunnitsivigitigut angutinik 7 nik, taipsoma iglunganit pijunik, kimitsikovluta taipkoninga Nalekamut, Saulib Gibeakotingane, Nalekab annerilauktangata. Attannek okarpok tunnijomavakka.

7 Attanniuble Mefiboseth, Jonathanib, Saulib erningata erninga ivleriva, Nalekab angermaringninga pivlugo, tapkungnangnelauktok tagva Davideme Jonathanemelo Saulib erningane.

8 Rizpable Ajab panningata ernigik, ernilauktagik Saulemut, Armoni, Mefibosethelo; taimaktauk Michalib Saulib panningata erningit tellimat, ernilauktangit Adrielemut Barsilaib erninganut, Mahalomiomut, attaniub tiguvait,

9 Tunnilaitalo Gibeonemiut agganginut; tapkoa kimikpait kakkame Nalekab sângane. Taimak 7 it tapkoa ochovut attautsekut, tokkolutiglo kattersiviub sivurlerpângata nelliuningane, kattersiviub Gerstinik pigiarningane.

10 Tagva Rizpab Ajab panningata pôksaijak tiguva issivilâlo kairtub kầnganut kattersiviub pigiarningane, immek killangmit kôlerkârtinago tapkonunga; uvlotillugolo tingmitset killaub attânetut mikolaungilait tapkoa kângine, unnuarmelonêt omajut naternamêtut tapkonungarkolaungilait. 
11 Davidelo okautsaulerpok, sunamik Rizpab Saulib pannervgangata pinniarlauktanganik.

12 Davidelo tagvungârpok tigusilunelo Saulib taipsomalo erningata Jonathanib sauninginik, Jabesemiunit Gileademetomit, taipkoa tiglilauktanginik, Bethsanib apkosininganit. Filisterinut nivingartauvinginit nelliutome taipsomane Filisterit Saule ullimarmatsuk Gilboab kakkangane;

13 Kaipaillo tagvangat takpaunga, kattitilugillo kimmitaumajut sauningit illaliutilugit;

14 Illijartorpaillo Saulib tapsomalo erningata Jonathanib sauningit Zelame Benjaminib nunanganêtome, atâtangata Kisib illuvinganut; pinniarlutiglo tamainik, sorlo attannek perkojilaungmat. Taimak Gude tamakkoa kingorngane ullapkosersimalaurivok nunamut.

15 Amale Filisterit unatadlarnermik pigiarlaurivut Israelitinut; Davidelo attersarpok, kivgangitalo illagilugo, Filisterillo sorsugvigivait. Davidelo merngortorlaukpok.

16 Jesbilo Nobemetok, (Rafab kittorngangita illangat, kallugiangalo kanmujakalaukpok Sekelititut 300 ditut okumaitigijunik, sakkokalaukporlo nutânik) tamna issumakalaukpok Davide ullimâromavlugo.

17 Abisaible Zerujab erningata ikajorpa, Filisterelo ullimarlugo tokkopa. Tagva Davidib angutekotingita okautimarikpât okarlutiglo: Aularungnaituksauvotit illagiluta sorsungnermut, kaumaute Israeleme kamitaukonago.

18 Mattoma kingorngane ama unatadlarnekalaurivok Gobeme Filisterinut. Tagva Sibechaib Husathemiub, Saf Rafatib kittorngangita illangattauk, ullimârpa.

19 Amalo unatadlarnekalaurivok Gobeme Filisterinut. Tagva Elhananib Jaere Orgimib erningata Bethlehemiojub Goliathe Gathemiok ullimarpa, kallugiakalauktok ipulingmik, umiab napparutangatut îtomik.

20 Amalo unatadlarnekalaukpok sulle Gatheme. Tagvane angutekalaukpok takkijomik, tamna innugangoakalaukpok 6 inik aggangmine ittikkaminelo, tagva kattilugit 24 giovut; tamnalo Rafamittauk erniangolaukpok.

21 Israelitillo mittautigingmagit, Jonathanib Simeab Davidib angajuata erninga ullimarpa.

22 Tapkoa sittamat erniangolaukput Rafamut Gathemiomut, ochotitaulutiglo Davidib tapsomalo kivgangita agganginut. 


\section{KAPITEL XXII.}

\section{Davidib imgerutinginik nertordlerutiksanik, piulijaunine omigi-} jiminit pivlugo.

D AVIDIBLO imgerutiksab tamattoma okausertangit okauserivait Nalekab sângane, nelliutome taipsomane, Nalekab piulilaungmago, omigijingita illunatik agganginit, Sauliblo agganginit, okarporlo:

2 Nalegak kairtorivara, sernigijiksakautigivaralo, piulijigilugolo.

3 Gude torkorviksakautigivara tettigijara, sappulutaksarijara, piulijauviksamalo sakkotinga, sapputsijiga kemâvigijaralo, piuli.jiga, piuliklertotit uvamnik idluitullivionermit.

4 Nalegak nertoromavara, nipliarvigijomalugolo; tagva omigijimnit piulijanniarpunga.

5 Tokkub ânianarningita illumalaungmanga, Belialiblo kôngita koaksartilaukpânga.

6 Kappianartoviub kellerutingita napperlaukpânga, tokkublo aklunangita sâlagilaukpânga.

7 Kappiasuleraiguma, tagva Nalegak nipliarvigivara, kaigarsuklungalo Gudimnut; tagva Tempelekotiminit nippiga tussarpa, kaigarsungneralo sânganut pivok siutikingnut.

8 Nuna sajukpilulaukpok, aulatitaulunelo, killaub tungavingit aulavut, sajukpilutiglo ningaumajartillugo.

9 Iserluk kongmuarlaukpok kinganganit, ikkomalo nungutsijok kanninganit, kaumalakarmat tamattomangat.

10 Killak nakiglitilaukpa akkarlunelo, tâktokalaukporlo ittiggangita attâne.

11 Kerubiblo kângane ablangavok, tingilunelo tagvunga, ikkiakilaukporlo annorib êsarungine.

12 'Tuppingalo avatâne tâktoksoniolaukpok, nuvujangolunelo kernertunik silliktuniglo.

13 Keblidlarnermut sânganêtomut, ikkoalalaukpok kaumalarlune.

14 Nalegak kallukarkojilaukpok killangmit, kogvasinerpangôjublo kalluksoakotine annitilaukpait.

15 Kargjutekotine pittiksautilaukpait, siamartilaitalo; kaumalaktitsilaukpok koaksartipaillo.

16 Tagva imiksuit kôdlartut tækkojaulaukput, nunaksublo tungavinga ulligêrtaumalaukpok, Nalekab suadlarninganut, kingangata annerninganut, annerteridlarninganullo.

17 Tamna tilliklilaukpok kôtsiktomit, ailungalo, kakkilaukpângalo immaksoarnit. 
18 Piulilaukpânga omigijimnit sangijoksoarnit, omigijimnit pitsartuluadlalauktunit uvamnut,

19 Sâlakarlauktunit uvamnik, kanoênima nelliuningane, Nalegarlo sungertuteolaukpok uvamnut.

20 Tapsoma annitilaukpânga innitôjomut; nussulaukpânga kuviagilauraminga.

21 Nalekab ajungitullivigivânga idluarnera malliklugo, akkiniarpânga aggangma ivsornainingat malliklugo.

22 Uvanga Nalekab apkosiningit attorapkit, idluitullilungilangalo Gudimnut.

23 Idluarijangit illunaita ijingma sângangnêput, perkojangillo egilungilakka uvamnit.

24 Idluinekalungilangale sângane, ulloreasuklungalo ajortunik.

25 Tamanna pivlugo Nalekab akkinniarvigivânga idluarnera malliklugo, ivsornainera ijikita sângangne malliklugo.

26 Heiligeôjune heiligeôvotit, idluartunelo idluarpotit.

27 Ivsornaitune ivsornaipotit, mumiksimajune mumiksimavotit.

28 Innuit ajulertut ikajorangne, ijingnullo piojorijut miklilertipattit.

29 Igvit Nalegak kaumautigigapkit. Nalekab tângnera kaumaksarpa.

30 Aiparilutit sorsuktut egilugit serkomitsungnarapkit; Gudigalo aiparilugo karmaksuit abtusarvigijungnarpakka.

31 Gudib apkosiningit kanoêtokatsengilet, Nalekab okausingit illakangilet. Tamna sappulutauvok illunainut tettekartunut tapsominga.

32 Nanele Gudekarka Nalegak pinago? Nanelo torkorviksakautekarka Gudivut pinago?

33 Gudib nekkoksitipânga pitsartunermut, apkosinermiglo unertutivânga tamarnangitomik.

34 Ittigakka tuktut ittiggangititut îtipait, pitilungalo poktojokotimnut.

35 Tapsoma aggakka ajochertorpait sorsungnermik, talleralo ajochertorpa pittikse kannujaujok kellulugo.

36 Tunnitsivigivarmalo piuliklernivit sappulutanganik. Nikkanarsartigumgalo angijorortiparma.

37 Attimne inniksamik âkiksoivotit pissungnermut, kamgâka wniorterkonagik.

38 Omigijikka udlatsomavakka, nungutsomalugillo, utteromalungilangalo tokkotsijarêrkarnanga tapkoninga.

39 Tokkotsomavakka egilugillo, serkomitsomavakka, akkerartoraksaringilânga; ittikkama attânut ochojuksauvut.

40 Pitsartunekartitsungnarparma sorsungnermut, akkerartortikka ochotitsungnarpattit attimnut. 
41 Omigijikka kemâtipattit, asseroikovlunga omisuktunik uvamnik.

42 Keviarput, piuliklertekangilarle; Nalekamut, tapsomale kiolungilait.

43 Toggarlugit serkallititsomavakka sannektitut nuname, allorluktitut apkosinerme serkallititsomavakka, siamartitsomalugillo.

44 Piulilaukparma innukotima annartorninginit, sapputilungalo angajokangorkovlunga nellojut akkorngane; innugiartoksuit illitarilaungitama kivgartorpânga.

45 Tujormitsat sappidlalaukpânga, naletsiarpângalo siutinut naletsiartunut.

46 Tujormitsat perlersimavut, nimajalutiglo kellerutimingne.

47 Nalegak omavok, kairtoralo torkorviksakautiga, Gudigalo nertortaule, piuljauviksama kairtunga nakorijaule.

48 Gude tamna, akkiniarnermik tunnijijok uvamnut, innukattigêksoarniglo ochotitsijok attimnut.

49 Tapsoma ikajorpânga omigijimnit. Igvit angijorortiparma taipkonangat akkerartortimnit; igvit ikajorparma koksalaitunit.

50 Tamanna pivlugo kujagijomavagit Nalegak, nellojut akkorngane, akkillo imgerutigilugo kujalijutiksanik,

51 Attannekotiminut sakkertitsijok, piulijaunermik angijoksoarmik, ajungitullijorlo saimartitaminut Davidemut, tapsomalo kinguvânginut soraijuitomik.

\section{KAPITEL XXIII.}

Davidib okausingita kingorlinginit; tapsomalo songojokotinginik pinniarninginiglo.

TamaDsA Davidib okausingita kingorlingit: Davide Isaib erninga okalaukpok, angut tamna angijorortitaumajok, Jakob Gudingata saimartitanga, Israelib imgerutiksalliortangat tussarnidlartunik okarpok:

2 Nalekab annerninga uvapkut okalaukpok, tapsomalo okalâninga okapkut okautigijaulaukpok.

3 Israelib Gudinga okalaukpok uvamnut, Israeliblo kairtunga okâlalaukpok, attanniôjok idluartok innuit akkorngane, attanniôjok sivuranerme Gudemik.

4 Uvlâb kaumaningatut itsomârpok, sekkinek uvlarme nuilertillugo nuvujakangipat, sillalunguerub kingorngane kaumadlarnermut iviksukat nuilerpatta nunamit.

5 Igluga taimailingalungigalloarmat Gudeme, angernermigle tunnitsivigilaukpânga soraijuitomik, akiksoijomik tamainik, sok- 
kosijuitomiglo, piulijaujutigijamniglo tamât, tussugijamniglo, sulle perorsakolungigalloarmago.

6 Belialille illunatik Dornititut nutsuksimajutitut îput, aggangnut tigujaujungnangitutitut;

7 Tigusijomajorle tapkoninga, kikkiangmik, kallugiablo ippuanik aggangminejariakarpok, ikkomamullo ikkitaujomârput, tagvane najortamingne.

8 Tamædsa Daridib sôngojokotingita attingit: Jasabeame Hachmonib erninga, pijariakortonerpaulaukpok pingasut akkorngane; kallugiakotine kongmuartipa, angutillo 800 dit ullimarpait attautsekut.

9 Tapsoma kingorngane Eleasarib Dodob Ahohib erningata erningata Davidib sôngojokotingita pingasut akkorngane Davide illagiva. Filisterit okarnerlotigingmattigik, kattimalaungmattalo sorsungnermut, Israeliblo angutingita majoarmatta paunga.

10 Tagva tamna nangerpok ullimarpaillo Filisterit, aggangit merngortornermut keratalerkârtinagit saviksoarme. Nalegarlo piulijaunermik angijomik tunnijilaukpok taipsomane, innugiartoksuit uttermatta tamna malliklugo aksarnilutik.

11 Tapsoma kingorngane Samma Agab Harariub erninga îlaukpok. Filisterit kattimalertillugit kattimgajunut attautsinut, perorsêvikalaukporlo tagvane perorsijalingmik Linsinik, innugiartoksuillo Filisterit kemâvait;

12 Tagva tamna nangeriartorpok perorsêviub kerkânut, piulilalo, annaulerlaitalo Filisterit, Gudelo sâlakarnermik angijomik tunnijilaukpok.

13 Tapkoalo pingasut pijariakortônerpaujut 30 git akkorngane, attersarlutik tikkiput Davidemut kattersiviub nelliuningane Adullamib kairosunganut, Filisterillo kattimgajut tangmarsimalaukput Refaimib naksangane.

14 Davidele taipsomane kairtoksoarne igloksoatut avaluliorsimajotut îtunelaukpok. Filisterille innugiartoksoangit tangmarsimalaukput Bethleheme.

15 Davidelo ikligosulaukpok, okarlunelo: Kia imiksaitoromavânga immektarviub Bethleheme ittertarviub attânetub imminganit?

16 Tagva songojoksuit pingasut tapkoa Filisterit tangmarvingat ittârpât, kallulutiglo immermik immektarvingmit Bethlehemêtomit ittertarviub attâne, akjartorpâllo, âlugolo Davidemut. Tapsomale immeromalaungilâ, kovilâle Nalekamut,

17 Okarlunelo: Nalekab tamanna ungasiktiliuk uvamnit, taimailiorkovlunga. Aungôlungilâk angutinit tapkonangat, innosermingnik ivlersilaungitunit, ailauktunillo tagvunga? Immeromalaungilâlo. Songojoksuit pingasut tapkoa taimailiorlaukput.

18 Abisai Joabib angajua angajokaunerôlaurivoktauk pinga- 
sut akkorngane. Kallugiakotine kongmuartipa ullimarpaillo 300 dit; okausionginarlaukpoktauk pingasut akkorngane.

19 Opigijaunarnerpângolaukporlo pingasut akkorngane, angajokaulaukporlo tapkonunga; pingasulle taipkoa innorpait.

20 Benajalo Jojadab Ishailib erningata erninga, Kabzeelemiub pinniarnersoakalauktub. Tapsoma angutik Amonitcrik Loewetut îtuk ullimarpak, attersarlunelo Loewelo tokkopa imektarvingme aputekarvingme.

21 Egiptenemiorlotauk ullimarpa, angut erksinadlartok, tamna kallugiamik tigumiarpok aggangminut. Tapsomale attersarlune aivigiva ajaupialijarlune, Egiptenemiorlo aksarpa aksorornermut kallugiarmik agganginit, tokkopâlo kallugiakotinganut nangminerijanganut.

22 Taimailiorlaukpok Benaja Jojadab erninga, okausiongînarlaukporlo songojoksuit pingasut tapkoa akkorngane.

23 Opigijaunarnersaulaukporlo 30 ginit; pingasulle taipkoa innorpait. Davidiblo attannertuijungortipa.

24 Asahel, Joabib nukka, 30 git tapkoa akkornganêpok. Elhanan Dodab erninga, Bethlehemiub.

25 Samma, Haradamiok. Elika, Haradamiok.

26 Helez, Paltemiok. Ira, Jekezib erninga, Tekoamiok.

27 Abieser, Antotemiok. Mebunai, Husatemiok.

28 Zalmon, Ahohimiok. Maherai, Netofatemiok.

29 Helebe, Baenab erninga, Netofatemiok. Ithai, Ribaib erninga, Gibeamiok, Benjaminib kittorngangita iglugaseksoanganit.

30 Benaja, Pirgatonemiok. Hidai, Gaasib kônginit pijok.

31 Abialbon, Arbatemiok. Asmaveth, Barhumemiok.

32 Eljaheba, Salbonemiok. Jasonib, Jonathaniblo kittorngangit.

33 Samma, Hararemiok. Ahiam, Sararib erninga, Hararemiok.

34 Elifelet, Ahasbaib, Macheatib erningata erninga. Eliam, Ahitofelib Gilonemiub erninga.

35 Hezrai, Karmelemiok. Paerai, Arbitemiok.

36 Jegeal, Nathanib erninga Zobamiok. Bani, Gademiok.

37 Zelek, Amonitereôjok. Naharai, Beerothemiok, Joabib Zerujab erningata sakkunginik akjarsijok.

38 Ira, Jethremiok. Garebe, Jethremiok.

39 Uria, Hethitereôjok. Tapkoa illunatik kattilugit 37 giôvut. 


\section{KAPITEL XXIV.}

Davidib kittitsikojininganik innugiartoksoarnik, pitlartauninganiglo tamanna pivlugo.

$\mathrm{N}_{\mathrm{H}}$

ALEKABLO ningaumajarninga illungertulerlaurivok ama Israelitinut, Davidelo kajungersarpa tapkoa akkorngane, okarkovlugo: Ailerit, Israelitillo Judakullo kittijartorkit.

2 Attannerlo Joabemut sorsuktuksaksoakotime angajokanganut okarpok: Israelib kinguvarêngita illunatik akkorngattigut ailerit, Danemit Berseba tikkilugo; innugiartoksuillo kittikit; kaujilerkovlunga kapsiomangatta.

3 Joabe attannermut okarpok: Nalegak Gudit illautitsilerle innugiartoksoarnut tapkonunga, sorlo manna illingangmatta 100 deêrtortunik unnurnersanik sulle, nalegara attannek, ijingme kuviasutiksanganik tækkokovlugo tapkønane; kissiane sôg nalekama attanniub tamanna kuviasutigivaûk?

4 Attanniuble okausingit ajugaulaukput Joabemut, sorsuktuksello angajokanginut. Taimak Joabe aularpok, sorsuktuksello angajokangit attannermit, innugiartoksuit Israelitit kittijartorlugit.

5 Ailutiglo Jordane ikarpât, tangmarlutiglo Aroereme, iglugaseksuit tallerpiane, Gadib kôngata kiglinganetome, Jaeseremelo;

6 Tikkilutiglo Gileademut, Hadsiblo nunanganut atsiktometomut; tikkilutiglo Danjaanemut, Zidoniblo avatânut;

7 Tikkipullo iglugaseksoarnut songojunut Tirusemik attelingnut, Heviterillo, Kanaaniterillo iglugaseksoanginut illunainut, tikkilutiglo Judab sekkernganut, Bersebamut;

8 Taimaglo nuna illunât ingergavigivât, tikkilutiglo Jerusalemut takkit 9 it uvlullo 20 git aularvingmik kingorngane.

9 Joabiblo attanermut tunnivait innugiartoksuit kittitaumajut unnurningit. Israelemelo angutekalaukpok 800000 dinik sôngojunik, saviksoarmik amuarsijungnartunik, Judamelo angutinik 500000 dinik.

10 Davidiblo omattinga tiglilerpok innugiartoksuit kittitaulaukârtillugit. Davidelo Nalekamut okarpok: Angijoksoarmik idluitullilaukpunga, tamattominga pinnialaurama; mannalo Nơlegak kivgavit idluitullininga pêjalauruk, sillaidlartomik pinniarlaurama.

11 Davidelo uvlarme makkimat, Nalekab okausinga Gademut, nellautaijomut, Davidib tækkojokotinganut, pivok okarlunelo:

12 Ailerit tagrunga Davidelo okarvigiuk: Imâk Nalegak 
okarpok: Pingasunik adsigêngitunik kaitsivunga illingnut; annerosulerit tapkoa illanganik, taimailiorkovlunga illingnut.

13 Gadelo Davidemut tikkipok, okautigivâlo tamanna tapsomunga, okautivâlo: Pijomavît Jahrit 7 it nâlugit kângnartokarkovlugo nunakotingne? Ubvalo takkit pingasut nâlugit akkerartortitit kemâjariakarangne, taipkoalo unangmiklerkovlugit illingnik? Ubvalo uvlut pingasut nâlugit nusilingnartokarkovlugo nunakotingne? Tagva kamatsialerit manna nautsertorlutillo, sunamik okartuksaumangarma tapsomunga tillilauktimnut.

14 Davide Gademut okarpok: Kappiasudlarpunga, kissiane Nalekab agganginut kattangniarta, tapsoma napkigosungninga angijongmat; innuit agganginut kattagomalungilanga.

15 Taimak Nalegak nusilingnartomik tikkitokarkojivok Israelitinut, uvlâmit nelliutok tamna taijaumajok tikkidlugo, innugiartoksoarnit Danemit Berseba tikkilugo angutit 70000 dit. tokkolermatta.

16 Engeliblo aggane issangmagit Jerusalemib kollânut asseroromavlugit; Nalegak kakkialerpok idluinermik tamattominga, okarlunelo Engelemut, asseroijomut innugiartoksoarnik: Namaksivok, aggaktit erkitikit. Nalekable Engelinga Arafnab Jebusitereôjub kakkojaksaliorvinganelaukpok.

17 Davidible Engele innugiartoksoarnik tokkotsijok tækkogamiuk, okarpok Nalekamut: Ahâk, uvanga idluitullilaukpunga, uvanga idluitullinermik ominga piniarlaukpunga; sunamik saukat ukkoa pinniarlaukât? Aggaktit torarlit uvamnut akkerartorlunga, atâtamalo iglunganut.

18 Gadelo Davidemut tikkipok nelliutome taipsomane okautivalo: Majorarit takpaunga, Altaremiglo sennalerit Nalekamut Arafnab Jebusitereôjub kakkojaksalliorvingane.

19 Taimak Davide majorarpok takpaunga, sorlo Gade okalaungmat, sorlolo Nalegak perkojilaungmat.

20 Arafnalo keviarame, attannek kivgane illagilugit kaitillugo tækkova, kênanelo pûlugo nunamut opigosukpok,

21 Okarlunelo: Sôg nalegara attannek kivgaminut kaiva? Davide okarpok: Kakkojaksaliorvik una pissiarilugo illingnit, Altaremiglo sennalunga Nalekamut, pagvisartoâluk innugiartoksoarne soraikovlugo.

22 Arafnale Davidemut okarpok: Nalegara attannek tigusilerle, tunnergumiglo tunnijile namagijaminut; ahâk tagva tuktuvakarpok tunnergutiksamut ôtaksamut, tuktuvaublo annungita kejuktangit ikkomaksamut.

23 Tamaita Arafnab attanniojub tunnivait attannermut. Arafnalo okarpok attannermut: Nalekab Gudivit namagijautilitit ingminut. 
24 Attannerle Arafnamut okarpok: Taimangungitok, pissiarijomavarale illingnit kênaujanut akkiluanga malliklugo; Nalekamnut Gudimnut tunnergumik ôtaksamik tunnijomalunginama, akkekangitomik pilauktamnik. Taimak Davidib kakkojaksaliorvik, tuktuvâglo pissiarivâk Sekelinut Silbereôjunut 50 ginut.

25 Sennalunelo tagvunga Altaremik Nalekamut, tunnijilunelo tunnergutinik ôtaksanik, tunnergutiniglo kujalijutiksanik. Nalegarlo kennuvigijauvok nuna saimarvigilugo; Nalegarlo ullapkosertaulaukpok nunamut, pagvisartoâluglo sorailaukpok innugiartoksuit Israelitit akkorngane.

\section{Attannit aglangita sivorlingit.}

maxteran

\section{KAPITEL I.}

Salomob mingoartauvlune saimartitauvlunelo attanningortitauninganik.

A TTANNERLO Davide innutokaugame, Jahrekarlunelo unnuktunik, aukanerungnarlaungilak, annorangnut ulliktaulauralloarmat.

2 Tagva kivgangita okautivât: Nalegara attannek pivlugo kennerlit innuosuktomik uigâsungmik, attanniub sângane nangerniartomik, pairksiniartomiglo tapsominga, talligingnelo sinningniartomik ivaniartomiglo nalekamnik attannermik.

3 Israelitillo kiglilervingine illunaine uigârsuk ânanak kennerasuarpât, nachvarpâllo Abisag Sunemiok, âpâllo attannermut. 
4 Tamnalo uigarsôlaukpok ânanaudlartok, pairivâlo attannek, kivgartorlugolo. Attanniuble uvinekattigilungilâ.

5 Adoniale Hagithib erninga angijorortipok, okarlunelo: Attaningoromavunga; sennakojilunelo ingminut kammutingnik aksalloalingnik, angutiniglo ablangajunik horsine, angutiniglo 50 ginik sivurlertunik tapsominga.

6 Atâtangatalo kuvianailisaromalaungila nelliunermine, okarajarlune: Sôg taimailiorkît? Tamnalotauk angutitsiangolaurivok, anânangatalo erniarilaukpa tugliovlune Absalomemut.

7 Attannertortigivaglo Joabe Zerujab erninga, Abjatharelo ajochertuijok. Tapkoa Adonia ikajorpâk.

8 Zadokible ajochertuijub, Benajablo Jojadab erningata, Nathaniblo nellautaijub, Simeiblo, Reiblo, Davidiblo sôngojokotingita Adonia illigilungilât.

9 Adoniablo saukat, tuktuvaillo, nergjutillo koinititaumajut tunnergutautigamigit ujarasuksub Soheletemik attiggub, immektarviub Rogelemik attiggub, sennianêtub tungâne, kattangutine attaniub erningit illunaita kaikovait, Judablo angutingit attanniub kivgangit illunaita.

10 Nellautaijorle Nathan, Benajalo, sôngojullo, Salomolo nukkane kaikolaungilait.

11 Tagva Nathan okarpok Bathsebamut Salomob anânanganut: Tussarlaungilatit Adonia Hagithib erninga, attaningorsimangmat, nalegavullo Davide nellomarikpok tamattominga?

12 Tagva kailaurit manna attannertoromavagit, tarningnik ernerpillo Salomob tarninganik piuliklerkovlutit.

13 Ailaurit itterlutillo kammunga attannermut Davidemut, okautilugolo: Nalegâk attannek, kivgait arnak angermarilaungikiûk, okarvigilugolo: Ernît Salomo kingunamne attanningortuksauvok, iksivajuksaulunelo iksivautakotimne? Sôgle manna Adonia attanningorsimava?

14 Ahâk tagvanêtillutit sulle, attannermullo okartillutit, kingunangne itteromavunga, okausiksatillo okautigijarêromavakka.

15 Bathsebalo itterpok attannermut kammunga aiparusermut. Attannerlo innutokaudlalaukpok, Abisagiblo Sunemiub attannek kivgartorpa.

16 Bathsebablo okkungalune attannek opigiva: Attannerle okarpok: Suvît?

17 Tapsoma okautiva: Nalegâk, igvit kivgait angermarilaukpat Nalegarne Gudingne: Ernît Salomo attanningortuksauvok kingunamne, iksivajuksaulunelo iksivautakotimne.

18 Mannale ahâk, Adonia attanningorsimavok; igvillo nalegara attannek nellovat tamanna.

19 Taipsoma angusalluit, tuktuvaillo, nergjutillo koinititaumajut tunnergutautilaukpait, attaniublo erningit illunaita 
kaikolaukpait, taimaktauk Abjathåre ajochertuijok, Joabelo sorsuktuksaksoakattigêt angajokangat; kivgelle Salomo kaikolaungila.

20 Illingnullo nalegara attannek Israelitit ijingit illunatik torarput, nellojungnaititsikovlutit taipkoninga, kina iksivajuksaumangât nalekama attanniub iksivautaksoangane tapsoma kingorngane.

21 Imaitsomârporlo, nalegara attannek atâtagijane illagilugit sinnisimalerpat, tagva uvanga, erneralo Salomo, idluitullijonasugijauniarpoguk.

22 Ahâglo, tamna okartillugo sulle attannermut, nellautaijok Nathan kailune itterpok.

23 Attannerlo okautivât tamattominga: Ahâk nellautaijok Nathan mânepok. Kammungalo itterame attanniub sânganut, kênane pûlugo nunamut, attannek opigiva.

24 Okarlunelo: Nalegara attannek okalaukît: Adonia kingunamne attaniojuksauvok, iksivajuksaularmelo iksivautakotimne?

25 Uvlome attersarmat, tuktuvaillo, nergjutillo koinitisimajut, saukallo unnuktovaksuit tunergutautilaukpait, attaniublo erningit illunaita kaikolaukpait, angajokâjello, taimaktauk Abjathar ajochertuijok. Ahâglo nerrivut, immerlutiglo taipsoma sângane, okarlutiglo: Pilloringnartokarle attannermut Adoniamut!

26 Uvangale kivgait, Zadokelo ajochertuijok, Benajalo, Jojadab erninga, kivgaillo Salomo, kaikolaungilâtigut.

27 Tamanna nalekamnut attannermut perkojaumalauka? kivgatillo kaujilaungilet kina nalekama attanniub iksivautangane tapsoma kingorngane iksivajuksaumangât?

28 Tagva attannek Davide kiovok okarlunelo: Bathseba kaikolersiuk uvamnut. Tamnalo itterpok attanniub sânganut, attannerlo sângerpa.

29 Attannerlo angermarikpok, okarlunelo: Sorlo Nalegak miksekârtomik innôngmat, tarnimnik piuliklerlauktok kannoêtunit tamainit,

30 Uvlome pinniaromavunga sorlo angermarilaurapkit Nalekame Israelib Gudingane, okarlunga, ernît Salomo kingunamne attaniojuksaungmat; tamnalo iksivajuksauvok iksivautakotimne innangêrdlunga.

31 Tagva Bathseba okkungalerpok kênane pûlugo nunamut, opigivâlo attannek, okarlunelo: Pilloringnartokarle nalekamnut attannermut Davidemut sorairane.

32 Attannerlo Davide okarpok: Ajochertuijok Zadok kaikolersiuk uvamnut, nellautaijorlo Nathan, Benajalo Jojadab erninga. Ittermattalo attanniub sânganut,

33 Attanniub okautivait: Angajokapse kivgangit illasearisigik, erneralo Salomo ikkitisiuk horseojârtokotimnut, attersartilugolo unnunga Gihonemut. 
34 Ajochertuijublo Zadokib, aipakarlune nellautaîjomik Nathanemik, mingoarliuk tagvane attaningortilugo Israelitinut. Tiptulausijaritselo tiptulautinut, okarluselo: Pilloringnartokarle attannermut Salomomut!

35 Tamnalo malliklugo majoraritse kailuselo, tagva iksivajuksauvok iksivautakotimne, attanniojuksauvorlo innangêrdlunga; uvangalo perkojomavara attanniokovlugo Israelitinut, Judakunullo.

36 Tagva Benajab Jojadab erningata attannek kiova, okarlunelo: Amen, Nalegak nalekama Gudinga okarletauk taimak!

37 Sorlo Nalekab nalegara aiparilaungmago, taimak Salomotauk aipariliuk, iksivautanga anginersaukovlugo Davidib iksivautanganit.

38 Tagva ajochertuijok Zadok, nellautaijorlo Nathan, Benajalo Jojadab erninga, Kretillo Pletillo, attersarput kannunga, Salomolo ikkitipât attanniub Dovidib horseojartokotinganut, attersartilugolo Gihonemut.

39 Zadokiblo ajochertuijub nakjuk orksukaut tiguva tuppermit, mingoarpâlo Salomo, tiptulausijarpullo tiptulautinut, innugiartoksuillo illunatik okarput: Pilloringnartokarle attannermut Salomomut!

40 Innugiartoksuillo illunatik malliklugo majorarput, innugiartoksuillo nipsausijarput nipsautinut, kuviasudlarlutiglo, nuna nippersoanginut imiadlarmat.

41 Adoniablo tussarpa illunatiglo kaikolauktangita, najortigijangita; nerrijarêrlaukpullo. Joablo tiptulautib nippinga tussaramiuk okarpok: Kannok nipliadlarnek, aulajadlarnerlo iglugaseksoarne tukkekarkat?

42 Okartillugolo sulle, ahâk, tagva Jonathan Abjatharib ajochertuijub erninga kaiłok. Adonialo okarpok: Kailaurit kammunga, angutaugavit nellagôrtok, taigotaksaniglo ajungitunik kaitsivotit.

43 Jonathanele kiovok, okarlunelo Adoniamut: Illa nalegapta attanniub Davidib ernine Salomo attanningortilaukpa.

44 Illagællugolo tillilaukpait ajochertuijok Zadok, nellautaijorlo Nathan, Benajalo Jojadab erninga, Kretillo Pletillo: ikkitilaukpâllo attaniub horseojartokotinganut.

45 Ajochertuijublo Zadokib nellautaijok Nathan aiparilugo mingoarlaukpa attaningortilugo Gihoneme, majorarlaukpullo tagvangat kuviasuklutik, iglugaseksuit aulajadlarmatta; tamannauvok nipliadlarnek tussarlauktasse.

46 Taimaglo Salomo iksivavok attanniub iksivautangane.

47 Attanniublo kivgangit ittersimavut kammunga, nalegavut Davide saimartilugo, okalaukpullo: Gudivit Salomob attinga anginersautiliuk atternit, iksivautangalo anginersautiliuk iksivautarnit. Attannerlo opigosulaukpok innangavingme. 
48 Attannerlo okalaurivok imâk: Nertortaule Nalegak Israelib Gudingat, uvlome iksivakojimajok kinamik iksivautamne, jjikka tækkokovlugik tamattominga.

49 Tagva koaksarput parngnailutiglo illunatik Adoniamut kaikojaulauktut, aularlutiglo attunît apkotigijamingnut.

50 Adoniable Salomo sivuragiva, parngnailerporlo ailunelo Altariblo nakjugik tigumiarpâk.

51 Salomolo okautsaulerpok: Ahâk, Adoniab attannek Salomo sivuragiva; ahâglo, Altarib nakjugik tigumiarpâk, okarlunelo: Attanniub Salomonib angermarilinga uvlome, kivgane tokkotsomangimago saviksoarmut.

52 Salomo okarpok: Nellagôrniarune, tagva nujak attauserlonêt tapsomangat kattaktuksaungilak nunamut, idluitomigle nachvarviôgune, tokkotaksaurok.

53 Attannerlo Salomo tilliklerpok tagvunga, aijaukolâlo Altaremit. Tikkinamelo attannek Salomo opigiva. Salomole okarpok tapsomunga: Ailerit iglokotingnut.

\section{KAPITEL II.}

Davidib pijomajangita kingorlingat, tokkungalo. Salomonib mallingninga tapsominga, idluarsainingalo nertornartok.

$\mathrm{MI}$ ANNA nelliutok Davidib tokkoviksanga kaglilermat; ernine Salomo perkova, okarlunelo:

2 Uvanga sillaksoarmiut illunamassiatik apkotingat aiviginiarpara; tagva maksoalerit, angutaulutillo.

3 Kamagikillo kamagijaksat Nalegak Gudit pivlugo, agvertarkovlutit tapsoma apkosiningine, kamatsiarkovlutillo illusiokojanginik, perkojanginiglo, idluarijanginiglo, kigligiudsininginiglo, sorlo aglaksimangmat Mosesib perkojangine, sillatôkovlutit piniarnerne illunaine, namullo sangulervigijarne;

4 Nalegak okautsiminik okauserilauktaminik pivlunga nellautsititsikovlugo, okarame: Kittorngavit apkoserijatik udsertoromârpagit, sâmne nertornarlutik, omamullo tamânut, tarnemullo tamânut agvertarkovlugit; tagva angumik illingnit pijomik Israelib iksivautanganetomik ajoksartokarniatsengimarikpok.

5 Kaujimatsiarpotittauk sumik Joab Zerujab erningata pinniarvigilaungmanga, sunamik pinniarlauktanganik Israelib sorsuktuksaksoakattigêkotingita angajokagingnut, Abneremut Nerib erninganut, Amasamullo Jetherib erninganut, tokkolauktamingnut, kovisilunelo unatadlarnerub aunganik, ullapirsautekartillugo, unatadlarnerublo aunganik pititsivok tapserutekotiminut, makkitimigut attorlauktaminut, ittiggagutiminullo ittikaminelauktunut. 
6 Pinnialerit sillatonît malliklugo, nujangillo kêrtojut ullapirsautekarlutik unnungakoniarnagit tokkungajut inninganut.

7 Barsilaible Gileademiub kittorngangit napkoarnermík pinniarvigijaksarvattit nerrimârkattaukovlugit nerrimarvingne. Taimailiorvigilaungmanga angajoat Absalome kemâlaurapko.

8 Ahâglo najortigivat Simei, Gerab erninga, Jeminib erningata Bahurimemiok, okarnerlotjilauktok uvamnik mamaitoâlungmik, nelliutome taipsomane aivigijamne Mahanaimemut. Taipsomale attersarlune pachrearlaukpânga Jordanemut. Tagva angermarilaukpara Nalekame okarlunga: Tokkotsomangilagit saviksoarmut.

9 Mannale passijaksaungitokoniarnago, angutaugavit sillatôjok, kaujimatsiarniarpotillo sunamik pinniarvigijaksarimangangne, nujangit kêrtojut aungmut unnungartilugit tokkungajut inninganut pikovlutit.

10 Taimak Davide sinnisilerpok, ailune atâtagijaminut, illi-jartortaulunelo Davidib iglugaseksoakotingine.

11 Nelliutorle Davidib attanniovia Israelitinut Jahreôvut 40 git. Jahrit 7 it nâlugit attanniolaukpok Hebroneme, Jahrit 33 nâlugit Jerusaleme.

12 Salomolo iksivavok atâtame Davidib iksivautakotingane, attannioningalo sokkosijuilaukpok.

13 Adoniale Hagithib erninga kailaukpok kamunga Bathsebamut Salomob anânanganut. Tamnalo okarpok: Ullapirsautekarlutit tikkigalloarivît? Tamna okarpok: Ahaila.

14 Okarlunelo: Okausiksakarpunga illingnut. Arnak okarpok: Okarit.

15 Tamna okarpok: Nellungilatit attanniovik nangminerilaurapko, Israelitillo illuêngarlutik torarvigilaukpânga, uvanga attaniojuksaugama; mannale attaniovik sangutitaumavok, nukamnullo illingatitaumavok, Nalekamit pigimava.

16 Manna tuksiarnek attausek kennuarivara illingnit, kippilungniarnanga. Tamna arnak okarpok: Okalerit.

17 Tamna okarpok: Attannek Salomo okarkattigilauruk, kippilungniangimattit, tunnijikovlugo uvamnut Abisagemik Sunemiomik, nulliangokovlugo uvamnut.

18 Bathseba okarpok: Taimaile, pivlutit attannek okarkattigijomavara.

19 Bathsebalo itterlune tikkipok kammunga attannermut Salomomut, okarkattigijomavlugo Adonia pivlugo. Attannerlo nikkovilerpok pachreartorpâlo, opigilugolo, ingilunelo iksivautaminut. Iksivautarlo kaitaulaukpok attanniub anânanganut, ingilerkovlugo tapsoma tallerpianut.

20 Tamnalo arnak okarpok: Tuksiarârsungmik kennuvunga illingnit, kippilungniangilarma. Attanniub okautiva: Tuksiarit anânak, kippilungniangilagit. 
21 Tamna arnak okarpok: Abisag Sunemiovinek tunnijaule angajungnut Adoniamut, nulliangokovlugo tapsomunga.

22 Tagva attannek Salomo kiovok, okarlunelo anânaminut: Sôg Abisag Sunemiovinek kennuariviuk Adoniamut? Attanioviktauk kennuarigiuk tapsomunga, angajuklerpaungmat kattangutimnit, illigijaulunelo Abjatharemut ajochertuijomut, Joabemullo Zerujab erninganut.

23 Attannerlo Salomo angermarikpok Nalekame, okarlunelo: Gudib mattominga ipsomingalo pinniarvigilinga, Adonia okalaungipat tamattominga akkerartorlune innôserminut.

24 Mannalo, sorlo Nalegak miksekârtomik inôngmat nakkítertok uvamnik, iksivakojijorlo uvamnik atâtama Davidib iksivautangane, iglomiglo sennalauktok uvamnut, sorlo okalaungmat; Adonia uvlome tokkotaujuksaurok.

25 Attannerlo Salomo tilliklerpok taipsomunga Benajamik Jojadab erninganik; tapsoma ullimârpa tokkodlugo.

26 Ajochertuijomullo Abjatharemut attannek okarpok: Ailerit Anatotemut perorsêvikotingnut, tokkotaksaugavit. Uvlomele tokkotsomangilagit, Nalekab iklervinga akjarlaurangne atâtama Davidib sivorngane, siorniorkattaulauravillo atâtaga siorniudlartillugo.

27 Taimak Salomob ajochertuijok Abjathar ajæktorpa, ajochertuijokonnago Nalekamut, nellautsikovlugit Nalekab okausingit, okauserilauktangit Elib iglomiokotingit pivlugit Silome.

28 Tussaumajarlo tamanna pivok Joabemut; Joabib Adonia ungagilaungmago, Absalomeleungitok. Tagva Joabe kemâvok Nalekab tuppinganut, tigumiarpâglo Altarib nakjugik.

29 Attannerlo Salomo okautsaulerpok Joabe kemâsimangmat Nalekab tuppinganut; ahâglo nangerpok Altareme. Tagva Salomob Benaja Jojadab erninga tilliva, okarlunelo: Ailerit ullimârlugolo.

30 Benajalo Nalekab tuppinganut pigame, okautiva: Imâk attannek okarpok: annit. Tamna okarpok: Mâne tokkojomavunga. Benajablo tamanna okautigiva attannermut, okarlunelo: Taimak okalaukpok, taimaglo kiolaukpânga.

31 Attanniub okautiva: Pinniarit okauserilauktangatut, ullimârlugolo, illijartorlugolo; aungmik, Joabib sullinane kovilauktanganik pêjaikovlutit uvamnit, atâtamalo iglomiokotinginit.

32 Nalekablo akkiniarutigiliuk aunganik niakoanut, angutik tokkolaungmagik, idluarkijagik, namakijagiglo, innuarlaukpâglo saviksoarmut, atâtaga Davide nellotillugo tamattominga, Abner, Nerib erninga, Israelitit sorsuktuksaksoakattigêngita angajokangat, Amasalo Jetherib erninga, Judakut sorsuktuksaksoakattigêngita angajokangat.

33 Tapkoa augik akkiniarutigijaukovlugik Joabib niakoanut, kinguvângitalo soraijuitomik; Davidele kinguvângillo, iglungalo, 
iksivautangalo, ullapirsautekarkovlugit soraijuitomik Nalekab sângane.

34 Benajalo Jojadab erninga majorarpok, ullimârpâlo tokkolâlo. Illijartortauvorlo iglomine sunakajuitome.

35 Attanniublo Benaja Jojadab erninga angajokangortipa sorsuktuksanut, taipsoma inninganut; Zadokelo ajochertuijok, attanniub pitipa Abjatharib innigilauktanganut.

36 Attannerlo tilliklerpok, Simeilo kaikojaukova, okautivâlo: Igloliorit igluksarnik Jerusaleme, nunakarlutillo tagvane, anniniarnaglo tagvangat, ikkungalonêt taikungalonêt.

37 Uvlut nelliane anniviginiartarne kôb Kidronib akkianut, tagva kaujimalerit tokkotaujuksaugavit; aut niakungnut pile.

38 Simei okarpok attannermut: Okarnît idluarpok; sorlo nalegara attannek okalaungmat, kivgait taimailiomiarpok. Taimak Simei nunakalaukpok Jerusaleme akkunît.

39 Pijokarlaukporle Jahrit pingasut kingorngane Simeib kivgagik kemângmannik Achisemut Maechab erninganut Gathemiut attanninganut. Simeilo okautsaulerpok: Ahâk, kivgakotitik Gathemêpuk.

40 Tagva Simei parngnailerpok, siutekoktôjokotinelo iksivaviktariva aularlunelo Gathemut Achisemut, kivgane kenneromavlugik. Tagrungalo tikkiname kivgane âpâk Gathemit.

41 Salomolo okautsauvok Simei aularlaungmat Jerusalemit Gathemut, uttersimangmallo.

42 Tagva attannek tilliklerpok, kaikojaukolâlo, okautilugolo: Angermarilaungilagit Nalekame, kigligiudsivigilutillo, okautilutillo, uvlut nelliane aularajarvingne ikkunga, taikungalonêt kaujijuksaugavit tokkotaksaunernik? Igvillo okautilaukparma: Okautsitit tussartakka idluartôvut.

43 Sôgle Nalekab angermaringninga, perkojarlo tunnilauktara illingnut, mallilaungipigik?

44 Attannerlo okarpok Simeimut: Nellungilet kassênek tamât, omattivit kaujimajanga, pinniarlauktat atâtamnut Davidemut; Nalekab kassênît akkiniarutigimava niakungnut.

45 Attannerlo Salomo pitsartutitaumavok, Davidiblo iksivautanga sokkosijuijomârpok Nalekab sângane sorairane.

46 Attanniublo Benaja Jojadab erninga perkova, tamna annivok ullimârpâlo tokkolugo, Attannioviglo aulajuititaulaukpok Salomob agganginut, 


\section{KAPITEL III.}

Salomob nullianingninganik, tuksiarninganiglo illissimanermik; idluarsainingariglo sillatôjomit.

SALOMOBLo Farao Egiptenemiut attanningat illanârilerpa, nullianilunelo Faraob panninganik, âpâlo Davidib iglugaseksoanginut, igloliorkârtinago iglominik Nalekablo iglunganik, avaluksoarniglo Jerusalemib avatâne.

2 Innugiartoksuille tunnergutinik tunnijilaukput sulle pôktojune; nelliutok taipsoma tikkidlugo igloliortokalaungimat sulle iglomik Nalekab attinganut illingajomik.

3 Salomoblo Nalegak nagligiva, agvertarlunelo atâtame Davidib illuserilauktangit malliklugit; kissiane pôktojune tunnergutinik tunnijivok isserluanititsilunelo.

4 Attannerlo aivok Gibeonemut, tagvane tunnergutinik tunnijijomavlune; tamanna pôktojolaungmat ânanaudlartok. Salomolo tunnijivok tunnergutinik ôtaksanik 1000 dinik Altareme tapsomane.

כ็ Nalekablo Salomo sakkervigiva Gibeoneme sinnektomanekut unnuarme, Gudelo okarpok: Tuksiarit sunamik, tunnijaksamnik illingnut.

6 Salomo okarpok: Igvit atâtaga Davide kivgait napkoarnermik angijomik pinniarvigilaukpat, agvertarlaungmat sângne miksekârnerme, idluarnermelo, omattiblo nellagôrningane; napkoarnermiglo tamattominga sokkosijuivigilaukpat, ernermiglo tunnitsivigilaukpat iksivautangane iksivajomik, sorlo manna illingangmat.

7 Mannalo Nalegak Gudiga, kivgait attanningortilaukpat atâtama Davidib inningane. Uvangalo nukapiarârsovunga kaujimanangalo kannok anninimnik itternimniglonêt.

8 Kivgaillo innugiartoksuit annerilauktavit akkornganêpok, taimak unnuktigijôjut kittitaujungnangimatta, aglagutigijaujungnangimattalo unnumut.

9 Taimak kivgait omamik naletseriartomik tunnitsivigilauruk, innukotingnik idluarsaijungnarkovlugo, tukkisilerkovlugolo sunamik idluarmangât, idluimangâllonêt. Kinale sappingilâk innugiartoksoakotitit taimak unnuktigijôjut idluarsarungnarlugit?

10 Tamanna Nalekab namagiva, Salomo taimaitomik tuksiarmat.

11 Gudiblo okautiva: Taimaitomik tuksiaravit, tuksiarlaunginavillo innôtsemik sivitôjomik, akluinermiglonêt, omigijivit tarninginiglonêt, sillatunermigle idluarsaijungnarlutit;

12 Ahâk tagva okautsitit malliklugit pinniarpunga. Ahâk 
omamik illissimajomik, sillatôjomiglo tunnitsivigimavagit, illiktut îtokalaungimat sivunerne, pittakarniangimallo kingunerne.

13 Taimaktauk tuksiararingitarnik tunnitsivigimagivagit, imaitunik, aklúinermik, nertortaunermiglo, adsekarkonnak attautsemiglonêt attannit nelliunernetut akkorngane.

14 Apkosinimnelo agvertarniaruvit, illusiokojakka, perkojakkalo mallingniarungne, sorlo atatait Davide agvertarlaungmat; tagva tunnitsivigijomavagit innotsemik sivitôjomik.

15 Salomolo tuppalermat, ahâk, tagva tamanna sinnektomaniolaukpok. Tikkiporlo Jerusalemut, nangeriartorlunelo Nalekab angersimaningata iklervingata sânganut, tunnijilunelo tunnergutinik ôtaksanik, kujalijutiksaniglo, âkiksoilunelo nerrimâraksamik angijomik kivgaminut illunainut.

16 Nelliutome taipsomane anguniarpaktûk kaivuk attannermut, nangeriartorlutiglo sânganut.

17 Arnâglo aipanga okarpok: Nalegâk, uvanga arnarlo una kattimgalaukpoguk iglome attautseme; uvangalo innangakattigilaukpara iglome.

18 Ernilaungnimalo uvlut pingasut kingorngane, tamna ernilaurivok. Uvaguglo aiparêlaukpoguk, taimak tujormijakangimat iglome uraguk assiptingnik.

19 Arnablo oma erninga tokkovok unnuarme; sinniklune nennerlugo tokkolaungmago.

20 Unuarmelo makkipok tiguvâlo ernera sennimnit, kivgait arnak sinniktillugo, illilâlo talliminut, erninelo tokkungajok illiva tallimnut.

21 Uvlâkullo makkilaurama ernera amamaktitsomavlugo, ahâk tagva tokkungalaukpok. Uvlarmelo kemmergolaukpara, ahâglo, ernerilaungitara erniliaringitaralo.

22 Arnâglo aipa okarpok: Taimangungitok, ernera innôvok, ernîllo tokkungavok. Tamnale okarpok: Taimangungitok ernît tokkungavok, erneralo innôvok. Taimaglo okarpuk attanniub sângane.

23 Attannerlo okarpok: Una okarpok: Ernera innôvok, ernîllo tokkungavok; tamnalo okarpok: Taimangungitok, ernît tokkungavok, erneralo innôvok.

24 Attannerlo okarpok: Saviksoarmik aiklerutigissinga. Saviksoarlo kaitaulerêrmat attanniub sânganut,

25 Attannek okarpok: Nutarak innôjok aviksiuk maggolitilugo, koppangalo tunnisiuk omunga, koppangalo aipa tapsomunga.

26 Tagva arnak tamna ernelik innôjomik, okarpok attannermut, (omattinga illungertudlalermat ernine pivlugo): Nalegâk sorruksek innôvlune tunnisiuk tapsomunga, tokkoniarassiuglo. Arnâgle aipanga okarpok: Uvamnullonêt illingnullonêt illinganiarnago, aviktaule. 
27 Tagva attannek kiovok, okarlunelo: Sorrusek innôvlune tunnisiuk omunga, tokkoniarassiuglo, tamna anânaungmat.

28 Idluarsainerlo tamanna, attanniub attorlauktanga tussaumajaulerpok Israelitit illunamassianginut, attannerlo sivoragivât; tækkogamil Gudib illissimaninga tapsomanêmat, idluarsainermik attorlune.

\section{KAPITEL IV.}

Salomob pimaklertokotingit, ânanauningalo, illissimaningalo.

It

AIMAK Salomo attanniôlaukpok Israelitit illunamassianginut。 2 Angajokakotigilauktangillo tapkoangôvut: Asarja Zadokib ajochertuijub erninga.

3 Elihoref, Ahijalo Sisab erningik aglakpaktôlaukpuk. Josafat Ahiludib eminga angajokaulaukpok aglakpaktunut.

4 Benaja Jojadab erninga angajokaulaukpok sorsuktuksaksoakattigênut. Zadokelo, Abjatharelo ajochertuijolaukpuk.

5 Asarja, Nathanib erninga angajokaulaukpok pimaklertunut. Sabude Nathanib ajochertuijub erninga, illanângolaukpok attannermut.

6 Ahisar aulatsijolaukpok attanniub iglunganik, Adonirame Abdab erninga aulatsijolaukpok perorsevikotinginik.

7 Salomolo pimaklertekutekalaukpok 12 inik Israelitit illunamassianginut, pattangaititsijunik attannermik iglomiokotinginiglo. Tapkoa attunit Jahreme attautseme, takkek attausek nâlugo pattangaititsijariakalaukput.

8 Imâglo attekalaukput: Hurib erninga, Efraimib kakkangine;

9 Dekerib erninga Makazeme, Saalbimemelo, Bethsemesemelo, Eglonemelo, Bethananemelo;

10 Hesedib erninga Arubotheme, Suchomiglo pekalaurivok, Heferiblo nunanganik illunânik;

11 Abinadib erninga Nafat-Doreme illunâne, Tofathelo Salomob pannia nulliariva;

12 Baena Ahiludib erninga Thanacheme, Megidomelo, Bethseanemelo illunâne, Zarthanab sennianetome, Jesreelib attâne, naternak Meholamik attelik tikkidlugo; Jakmeamib ungatanut tikkilugo.

13 Gerib erninga Ramotheme Gileademêtome, pigilaukpaillo Jairib Mannassib erningata iglugas ekotingit Gileademêtut, Argublo Basanemêtub erkâ, iglugaseksuit angijut 60 git, karmaksoarnik avaluggit, kannujarniglo senneroagit;

14 Ahinadabe, Iddob erninga, Mahanaimeme;

15 Ahimaze Naftalime, tamnatauk nullianikpok Salomob paninganik Basmatemik; 
16 Baena Husib erninga, Assereme, Alothemelo;

17 Josafath Paruab erninga, Isaschareme;

18 Simei Elab erninga, Benjamineme;

19 Geber Urib erninga, nuname Gileademe, Sihonib Amoriterit attaningata, Ogiblo Basanemiut attaningata nunagilauktagingne; pimaklerte attausek nuname tamattomanelaukpok.

20 Judakulle Israelitillo taimak unnuktigijôlaukput siorkattitut imarbiksub siksanganêtutitut, nerrirullo, immerlutiglo, serridlalaularmiglo.

21 Taimaglo Salomo nalegaulaukpok attaniovingnut illunainut, kôksoarmit Filisterit nunangat, Egiptenemiut kiglilervingat tikkidlugik; pilliutinik kaitsivut tapsomunga, nalekpâlo inôsingane tamât.

22 Salomolo uvlut tamaita pijariakalaukpok nerkiksaminut Korit 30 git tætælugit sennaukamik kakkortamik; Koriniglo 60 ginik sennaukamik assianik;

23 Tuktuvângnik tellimaujortunik koinititaumajunik; tuktuvângniglo 20 ginik, natername iviksukatalingme mianerijaulauktunik, saukaniglo 100 dinik; tuktut, tuktungajullo tuktuojartullo, nergjutillo koinititaumajut taijaukassiutinagit.

24 Attaniotigame nunab illunaksoanganut, kôksub miksânetomut, Tifsamit Gasa tikkilugo, attaniovingnut illunainut, Jôksub miksânétunut, ullapirsautekalaukporlo illunainit avatânêtunit.

25 Taimak Judakut Israelitillo ussornarnarlutik nunakalaungmatta, nelliat nappartokotime Weineliksab attâne, Feigeliksablo attâne, Danemit Berseba tikkidlugo, Salomob inosingane tamât.

26 Salomolo horsekutekalaukpok kammutingnut illingajunik 40000 dinik, sorsuktuksakutekalarmelo horsit kângine ablangajunik 12000 dinik.

27 Pimaklertillo taipkoa Salomo, illunaitalo tapsoma nerrimarvinganut illingajut pattangaitipait, attunît takkermingne, ajoksarkojilungilello sunamiglonêt.

28 Gerstelotauk ivillo horsinut, pangalidlartunullo illingajut, kaipait nunamut najortanganut, attunît illusekarkojaunitik malliklugit.

29 Gudiblo Salomo tunnitsivigiva illissimanersoarmik, illitarsinersoarmiglo, sillatônermiglo, siorkattitut imarbiub siksanganetutitut.

30 Salomob illissimaninga anginersaulaungmat, kittorngat taunanêtut illunatik, Egiptenemiullo illissimaninginit illunainit;

31 Illissimanersaulaurivoktauk imgerusiortunit, Ethanemit Esrahitemiomit, Hemanemillo, Kalkolemillo, Dardamillo, Maholib ernikingnit; tussaumajautsiarlaukporla nellojunut avatanêlauktunut illunainut. 
32 Ajochertutsit adsiojut 3000 dit okauserilaukpait, imgerutingillo 1005 eôlaukput.

33 Nappartullo okautigivait Cederemit Libanonemêtomit, Isope tikkilugo perorsajok iglub sennerangane. Nergjutillotauk okautigivait, tingmitsello, pamgortullo, mingeritsello.

34 Innukattigêksoarnillo tamainit tikkitokalaukpok Salomob illissimaninganik tussaromajunik, attannernit illunainit nunamêtunit, illissimaninganik tussarlauktunik.

\section{KAPITEL. V.}

\section{Sulomob illanôrêlerninga Hiramemut, oppalungä̈ainingalo igloliornermut Tempelemil.}

Miramelo Tirusemiut attanningat tilliklerpok kivgaminik Salomomut; tussarlaurame mingoarlaungmatsuk, attanningortilugo atâtangata inningane. Hiramib Davide illanâritsainarlaungmago innosermine tamât.

2 Salomolo tilliklerpok Hiramemut okautsaukolâlo:

3 Kaujimavotit atâtaga igloliorungnalaungimat iglomik $\mathrm{Na}$ lekab Gudime attinganut illingajomik, unatadlarnek illumajaujutigijanga pivlugo; Nalekamut illijaukârtinagit allungita attânut.

4 Mannale Nalekab Gudima merngoêrsernermik tunnitsivigimavânga avatimne, akkerartortokarungnangimat, agviarutiksakarungnaimallo idluitomik.

5 Ahâk, tagva issumavunga iglolioromavlunga iglomik Nalekab Gudima attinganut illingajomik, sorlo Nalegak okausekalaungmat atâtamnut Davidemut, okalaungmallo: Ernivît, inangêrlutit ingititsomajama iksivautarnut, tapsoma attera iglolioraksariva.

6 Taimaimat, perkojilerit manna, Cederinik Libanoneme nakkartsijokarkovlugo, uvamnut illingajuksanik; kivgakotitit illaukovlugit kivgakotimnut. Kivgakotivillo ikkajusiksanginik tunnitsivigijomavagit, tamainik sorlo okarniaravit. Kaujimagavit uvaptingnêtokangimat, kejungnik nakkartsijungnartunik Zidonemiutitut.

7 Hiramele Salomob okausinginik tamakkoninga tussarame, kuviasudlalerpok, okarlunelo: Uvlome Nalegak nertortaule, tunnijilauktok Davidemut ernermik sillatutsiartomik, innugiartoksoarnut taipkonunga attanniôjomik.

8 Hiramelo tilliklerpok Salomomut, okautsaukovalo: Tussarlaukpunga okautsaukojarnik uvamnut, pinniaromavunga pijomajat tamât malliklugo, kejuit Cederit Cipressillo pitjutigællugit. 
9 Kivgakotima attersautijaksarivait Libanonemit immarbiub kiglinganut; kallingortitaukojomavakkalo, âtaukojomalugillo immarbikut nunamut taijaukoniartarnut uvamnut; kellarudjartankojomavakkalo tagvane, iguptertaukolugillo; igvillo aijaukoniarpattit. Igville pijomajara pinniaraksarigivat, nerkiksaniglo tunnijijuksauvotit kivgakotimnut.

10 Taimak Hiramib Salomo tunnitsivigiva kejungnik, Cederinik Cipressiniglo, pijomajanga tamât malliklugo.

11 Salomoble Hirame tunnitsivigiva Korinik 2000 n dinik Weizenik, nerkiksanik tapsoma kivganginut. Koriniglo 20 ginik orksumik togarsimajomik. Taimaitunik Salomo tunnijivok Hiramemut Jahrine tamaine.

12 Nalekablo Salomo sillatunermik tunnitsivigiva, sorlo okarvigilaungmago. Hiramelo Salomolo ullapirsautekalaukpuk ingmingnut, angerkattigêklutiglo.

13 Salomolo annerosulerpok sennajuksanik Israelitit illunamassianginit, sennajuksallo angutaulaukput 30000 dit.

14 Tillivaillo Libanonemut, takkit nelliane 10000 dit, taimak takkek attausek nâlugo Libanonemelaungmatta, takkiglo nâlugik nunamingnêdlutik. Adoniramelo angajokaulaukpok sennajuksanut tapkonunga.

15 Salomolo angutekutekalaukpok 70000 dinik akjarsijunik, 80000 diniglo ullimarniktunik kakkame;

16 Salomob angajokakotingit pinagit, aulatsijungortitaumajut sullijaksamik tamattominga, imâgle, 3300 dit aulatsilauktut innugiartoksoarnik sennajunik.

17 Attannerlo perkojivok ujaraksoarnik angijunik pivianartuniglo pêrsikovlugit ullimartauniartunik, iglub tungavianut illingajuksànik.

18 Salomoblo igloliortekotingit, Hiramiblo igloliortekotingit, Giblimiullo kejuit ullimarlugit avikpait, âkiksoilutiglo kejungnik ujarkaniglo igloliornermut iglomik.

\section{KAPITEL VI.}

\section{Tempetib sennajauninga Jahrine 7 ne.}

ISRAELIB kittorngangita aularningata Egiptenemit kingorngangata Jahringita 480 ingane, Salomob attanioningata Jahringita sittamangane, takkerme Sifemik attelingme, tagva takkib aipanga, Nalegak igloliolerpa iglomik.

2 Iglole Salomob igloliortanga Nalekamut, tallitut 60 gitut takkitigijolaukpok, tallitullo 20 gitut sennimutotigijolaukpok, tallitullo 30 gitut poktotigijolaukpok.

3 Tempeliblo sângane torksumik sennavok, tallitut 20 gitut 
takkitigijomik, iglub sennimutuninga malliklugo, tallitullo tellimaujortutitut sennimututigijomik, iglub sivurâne.

4 Igalângniglo sennavok iglome; illuane nerrotôjunik, sillatanelo nerrokitunik.

5 Iglublo sennerangata sillatâne aiparusermik agvertarvingnik kallerêktunik sennavok, avatâne illunane, Tempelib avatâne, aiparusingatalo avatâne; inniniglo sennavok sennerâne avatâne illunane.

6 Agvertarviglo allerpâk tallitut tellimatut senimututigijôlaukpok, tuglialo tallitut pingasojortotut sennimututigijôlaukpok, pingajoello tallitut 7 itut sennimututigijôlaukpok. Iglernangnik sennakojilaungmat iglub sennerangata sillatane avatâne, egangakonnagit iglub senneranganut.

7 Iglolo igloliortautillugo, ujarkanit, ullimarsimajarêrtunit igloliortauvok, taimak igloliornerme ujaratsiat katjungningit, ullimautillo ullimarningit, sakkullonêt kikkiangojut pergvallungningit tussartaulaungimatta.

8 Upkoakalaukporle iglub kerkane tallerpiane, majutitigut siuteroktut îtutigut majorarvikarkovlugo agvertarviub allerpânganit akkulermut, agvertarvingmillo akkulermit pingajuanut.

9 Taimak iglo igloliorpa pijarêrtilâlo; kâkartipâlo iglo sâtujanik, ikkângniglo Cedereôjunik.

10 Sennavorlo agvertarvingnik kallerêktunik iglub avatâne illunane, tallitut tellimatut pôktutigijunik, tungavullo iglome ikkângnut Cedereôjunut.

11 Nalekablo okausingit Salomomut pivut, okarporlo:

12 Iglo igloliortat pivlugo, perkojakka malliklugit agvertarniaruvit, namagijakka malliklugit pimmiarniaruvit, perkojakkalo illunaita mallingniarungne; tagva okautsikka nellautsitiniarpakka illingne, sorlo atatait Davide okarvigilaurapko;

13 Innekaromavungalo Israelib kittorngangita akkorngane, innukotikkalo Israelitit kemmagomangilakka.

14 Taimak Salomob iglo igloliorpa, pijarêrtilâlo.

15 Iglublo sennerangita illungit illupiarutserpait sâtujanik Cederiôjunik; iglub nettinganit kollâ tikkidlugo illupiarutserpa satujanik; iglublo nettinga netsisertorpa satujanik Cipressiojunik.

16 Iglublo kinguane sennavok upkomik sâtujanit Cedereôjunit, tallitut 20 itut takkitigijomik, nettermit kollâ tikkidlugo; sennavorlo tamattominga, illuane ivsornaitopângokovlugo;

17 Igluble, tagva Tempelib sivua tallitut 40 gitut takkitigijolaukpok.

18 Illuane iglo illunane Cedereotuinaulaukpok sennangoaraktaggit, kuglungajungoanik, nuvugalangoaniglo siggimajungoanik; ujaraglo attauserlonêt trekkuksaulaungilak.

19 Igluble kinguata illua âkiksorpa, Nalekab angersimaningata iklervinga âtaukovlugo takkamunga. 
20 Innelo kinguane tallitut 20 gitut takkitigijolaukpok, tallitullo 20 gitut sennimututigijovlune, tallitullo 20 gitut poktotigijolaukpok, illupiarutserpâlo Goldemik pivianadlartomik; Altarelo sillaparpa sâtujanik Cederiojunik.

21 Salomoblo iglo illuane illupiarutserpa Goldemik pivianartomik; kellaumgujallo Goldeôjut siakitipait upkub, Goldemik illupiarusimajub sânganut.

22 Iglolo illunât, illunanemarik illupiarutserpa Goldemik; Altarelo illunât upkub sanganelauktok sillaparpa Goldemik.

23 Kamanelo inneme kinguanetome, Kerubingoakartitsivok maggungnik. Kerubingoaglo sennavak, kamanetuksak inneme kinguanetome, tallitut tellimaujortutitut poktutigijuk, nappartub orksuliuk kejunganit.

24 Kerubingoaglo esarungit attunit tallitut tellimatut takkitigijolaukput, taimak tallitut -tellimaujortutitut îlaungmat tapsoma esarungata nuvuanit igluata nuvunganut.

25 Taimaglo Kerubingoak aipanga, tallitut tellimaujortutitut îlaurivok, anginigiglo taimaikoarêlaukpuk, adsigêktomiglo illusekarpuk.

26 Kerubingoaglo aipa tallitut tellimaujortutitut poktotigijolaukpok, aipangalo taimailaurivok.

27 Kerubingoaglo pitipak kamunga iglub illuanut. Kerubingoaglo esarungit issivijauvut Kerubingoak aipangata esarunga aktualermat iglub senneranganut, aipatalo êsarunga aktuavok iglub senneranganut akkianêtomut; igluble kerkane esarkuk aktuatigêkpuk.

28 Kerubingoaglo sillaparpâk Goldemik.

29 Iglublo sennerangit illûnane illuane sillatânelo sennangoarakarkovait Kerubingoanik, Palmingoanik, nuvugolangoaniglo siggimajunik.

30 Iglublo nettinga sillaparpa Goldemik illuane sillatênelo.

31 Ittervingmullo innemut kinguanêtomut sennavok upkoangnik maggungnik, nappartub orksuliub kejuanit, nappajungillo kattablo kollâ kattilugit sennerkab tellimauningatut angitigijolaukput.

32 Sennangoarkaniglo Kerubingoanik, Palmingoanik, nuvugolangoaniglo siggimajunik ivertitsikojivok tapkonunga; sillaparpaglo Goldemik.

33 Taimaglotauk Tempelib itterviane nappajunik sennavok, nappartub orksoliub kejuanit, sennerkab sittamauningatut silliktigijunik.

34 Upkoangniglo maggungnik, kejungmit Cipressemik attelingmit; upkoaglo attunît maggolimalaukpuk iglugêngnik attatigêngnik kevertautimingnut.

35 Sennangoarkaniglo kipperoksimajunik, Kerubingoanik, Palmingoaniglo, nuvugolangoaniglo siggimajunik, pitetservigi- 
vait, sillaparpaillo Goldemik, taimamarik sorlo perkojaulaungmat.

36 Illulo kamanênersak avaluliorsimajok sennagiva, ujarkanit ullimarsimajunit, pingasunik kallerêktitsivlune, kânganelo kejukarlune kejungnik Cedereôjunik saviksimajunik attautsemik.

37 Jahrit sittamangane takkerme Sifemik attelingme tungavik Nalekab iglunganut illijaulaukpok,

38 Jahrillo 11 ingane takkerme Bulemik attelingme, (tagva takkit 8 ingat) iglo pijarêrtaumalaukpok, sorlo illingajuksaulaungmat, Jahrillo 7 it nâlugit igluliorasuarpât.

\section{KAPITEL VII.}

Attanniub iglokotingita igloliortauningat; perkutillo Tempelemut illingajuksaí sennajauningit.

IGLOKOTIMINIGLE Salomo Jahrit 13 it nâlugit igloliorlaukpok; piiarêrmarikpâlo.

2 Sennalaurivatauk iglo nappârtut Libanonib iglunganik attelik, tallitut 100 ditut takkitigijok, tallitut 50 gitut sennimututigijok, tallitullo 30 gitut pôktotigijok, nappajune tukkilerêngne sittamaine, ikâlingne kângane.

3 Kâkartitaulaukporlo, innît nappajunêtut kângine kêjungmik Cedereôjomik, nappajut tapkoa 45 giôlaukput, 15 it tukkilerêngne attautsine.

4 Igalâkalaukporlo tukkilerêngne pingasune akkilerêngnik, pingasut, akkilekarlutik pingasunik.

5 Upkuallo nappajullo illunatik iktokerilaukput, taimaktauk igalât kaumajorlo akkilekarlune kaumajomik.

6 Torksumiglo sennagivok nappajunit, tallitut 50 gitut takkitigijomik, tallitullo 30 gitut sennimututigijomik; amalo torksumik oma sângane, nappajunit koakjulingnillo silliktunit.

7 Sennagivoktauk torksumik erkartuiviub iksivautanganut, erkartuiviokovlugo, netsisertorpâglo Cederinik.

8 Iglungalo iglogijanga avaluliorsimajub assianetok torksub kinguane, taimailuatsiamik illusekarlune sennajaulaurivok; sennalaurivoktauk iglomik torksutut îtomik Faraob panninganut, Salomob nullianilauktanganut.

9 Tapkoalo tamaita ujaraulaukput pivianartut, iktokeriksaut malliklugo ullimârtaulauktut, kiblutinuliło kiblormiktortaulauktut illuane sillatânelo, tungavinginit kângit tikkidlugit, taimaglotauk sillata avalulingme angijome;

10 Tungavigle ujaraulaurivoktauk pivianartut angijullo, tallitut tellimaujortut takkitigijut sittamaujortullo silliktigijut. 
11 Tapkoalo kângine ujarakalaukpok pivianartunik, iktokeriksaut malliklugo ullimartaumajunik, Cederiniglo.

12 Avaluliorsimajorle angijok, avatâne tukkilerêktokalaukpok pingasuinik ujaralingnik, tukkilerêngniglo attautsinik satujanik Cedereojunik; taimailingalaurivoktank avaluliorsimajok kamanênersak Nalekab iglungane, torksuglo Nalekab iglungane.

13 Attannerlo Salome tilliklerpok aijaukolâlo Hirame Tirusemiok,

14 Uigarniub erninga Naftalib kinguvanginit, atâtangalo angutaulaukpok Tirusemiok, kanutjerijolauktok; tamnalo illumersimamarikpok illisimanermik sillatunermiglo, sappinginermiglo sennalune sunatuinarnik kannujarmit; tamnalo tikkipok attannermut Salomomut, sennalunelo tapsoma pinniarkojanginik tamainik.

15 Nappajuglo sennavâk kannujarmit, attunît tallitut 18 tut poktotigijuk, nimerlo tallitut 12 tut takkitigijok namatuinaulaukpok avatâgut, nappajuk attunît sillingnigingnut.

16 Niakojartuglo kovivak kannujarmit nappajûk kangangnêtuksâk; niakojartuglo attunnit tallitut tellimatut poktotigijolaukpuk.

17 Niakojartûglo kângine nulluangoakalaukpok, kellaumgujakalaukporlo pergaimajunik 7 inik aipane, 7 niglo aipangane.

18 Paungâlungoaniglo Granatinik attilingnik sennilerêktunik maggoinik pititsivok niakojartungnut avatânut, niakojartuk ulliktaukovlugik tapkonunga.

19 Niakojartuglo nappajuk kânginetuk nuvugolaktut Liliemik attckartut îlaukpuk, taipkotitunak torksungmetutitut, tallitut sittamatut silliktigijovlutik.

20 Paungâlungoallo Granatit sennilerêktut avatâne 200 diolaukput kotsiktome sennilerêktune, atsiktomelo niakojartub puvioninganingata avatanelauktungne, niakojartungne tamangne, nappajuk kânginelauktungne.

21 Nappajûglo nappartipak Tempelib torksungata sângane. Nappartitanelo tallerpingmut Jachinemik atserarpa; nappartitanelo saumingmut, Boasemik atserarpa.

22 Taimaglo nappajuk kângangne Lilietut îlaukpok. Taimaglo nappajuk pijarêrtaumalaukpuk.

23 Immarbingoarlo kovilugo sennava, tallitut tellimaujortutitut silliktigijomik sinânit aipanganit aipanga tikkilugo, avatâne illunane, tallitullo tellimatut pôktutigijomik nimmerlo tallitut 30 gitut takkitigijomik namatuinarlaukpok sillingninganut avatâne.

24 Immarbingoablo, tallitut tellimaujortutitut silliktigigolauktub, sinâne kuglungajungoakalaukpok imârbingoab kiglingane illunane; kuglungajungoallo tapkoa sennilerêklutik maggoilivlutik kovijaumalaukput. 
25 Nappavorlo tuktuvangoane 12 ine, tapkonangat pingasut sângmivut avunga, pingasut paunga, pingasut aunga, pingasullo taununga, immarbingoarlo kânginelune, okpatingit illunatik illumut torarmatta.

26 Ivjuningalo aggaktitut igluinartut sennimutoningititut îlaukpok, sinâlo ermgusiarsub sinâtut îlaukpok, Lilietut siggimajotut îllune; oktutillo Bathenik attiggit 2000 dit illulekarungnarlaukpok.

27 Sennalaurivoktauk ikkovraksanik tellimaujortunik kannujarmit, attunit tallitut sittamatut takkitigijunik, sennimututigijuniglo, tallitullo pingasutut poktutigijunik.

28 Ikkovraksallo imâk sennajaulaukput, annekarmatta okaloraujat akkorngane.

29 Senneragingne okaloraujat akkorngane Loewingoakalaukpok tuktuvangoaniglo, Kerubingoaniglo, okaloraujanelo taimaitokalaurivok kôtsiktomê, Loewingoallo tuktuvangoallo attâne suvlolikalaukpok nivingajotun îtunik.

30 lkkovraksallo attunit aksaloakalaukput sittamanik kannujarnik; terrekunginelo sittamane tuengoakalaukpok kovijaumajunik, attunit akkilerêkput, egangalutiglo ukkusingmut.

31 Kongesingoalle ikkovraksat kerkangine tallertut attautsetut pôktotigijolaukput, angmalokitauvlutik, tallertut igluinartut ketterkangatullo silliktigijolaukput, kongesingoanelo katsiktokalaukpok iktokeriktunik, angmalokitaungitunik.

32 Aksalloajullo sittamat attâne senneranginelaukput, ikkangillo ikkovrangnelaukput, aksalloajut attunît tallitut igluinartut ketterkangatullo pôktotigijolaukput.

33 Aksalloajullo tapkoa aksalloaliub aksalloajungititut îlaukput. Ikângillo aksalloavingillo, issagutângillo avalungillo illunaita kovijaumalaukput.

34 Tuêngoallo sittamat ikkovraksat terrekungine sittamanelaukput; ikkovranêlaukput tuengoangit.

35 Ikovrello kongesingine tallib ketterkangatut poktotigijome okaloraujakalaukpok, annekalunelo avatâne illûnane.

36 Tapkoalo anninginut, okaloraujanullo ittersaliorkojivok Kerubingoanik, Loewingoaniglo, nappartungoaniglo, Palminik attilingnik; sorlo attunît inniksakalaungmatta avatâne illunane.

37 Taimak illusekarlutik ikkovraksat tellimaujortut sennalaukpait, kovilugit, anginingit illusingillo adsigêmariklutik.

38 Ukkusillo tellimaujortut kannujarmit sennavait, attunit Bathinik 40 ginik illulekarungnarlauktut; tallitullo sittamatut silliktigijolaukput; ikkovrello attunît kangine ukkusikalaukpok attautsemik.

39 Ikkovrello tellimat illivait iglub terrekunganut tallerpingmut, assingillo tellimat, terrekungmut saumingmut; immarbingoarle illiva iglub sivurânut tallerpianut sekkernganut. 
40 Hiramiblo sennavaittauk ukkusit, poagillo itterngujallo; pijarêrtipaillo sennajaksat tamaita attanniub Salomonib sennajaukolauktangit, Nalekab iglunganut.

41 Tagva nappajuk, niakojartuglo nappajuk kângangne, nulluangoaglo maggoit niakojartuk nappajungnêtuk ulliksanginut;

42 Paungâlungoallo 400 dit Granatit nulluangoanut illingalauktut, scmnilerêt maggoit nulluangoane attautsene, aksaujak niakojartuk kângangnêtuk ulliksanginut;

43 Taimaktauk ikkovret tellimaujortut, ukkusillo tellimaujortut, ikkovret kânginelauktut;

44 Imarbingoarlo, tuktuvângoallo 12 ît attâne;

45 Ukkusillo poagillo itteringojallo, illulerkutillo tamakkoa illunaita Hiramib sennajangit Salomomut, Nalekab iglunganut illingajut, kannujatuinarmit sennajaulaukput.

46 Jordanib erkâne Sochotib Zarthaniblo akkorngangne attanniub kovijaukovait macharmut.

47 Salomoblo illulerkutit tamaita kiktitautingnut oktortaukolaungilait, kannujab unnudlarninga pitjutigællugo.

48 Salomoblo perkutit illunaita sennalaukpait Nalekab iglunganut illingajut: tagva Altare Goldeôjok, sâlo Goldeôjok kakkojaliksak tækkojaksanik;

49 Nennergoavît tellimat tallerpingmut, nennergoavîllo tellimat saumingmut, inniub kammanenerpaub sivurâne, Goldemit illakangitomit, nuvugolangoallo, kollillo, takkutillo Goldemit;

50 Itteringojallo poagitillo, sâvillo allupsautillo, kallutaujallo isserluanititsijutit, komigautillo Goldemit pivianartomit, upkoamelo ivsornainerpângojome, Tempeliblo upkoangane kevertaucit Goldeolaurivut.

51 Taimak sennajaksat Salomob sennajangit Nalekab iglungane pijarêrtitaumavut. Salomoblo ittertipait kamunga, tamaita atâtame Davidib ivsornaitilauktangit, Silberelo Goldelo, illulerkutillo; illilugillo Nalekab Tempelingata pivianartokoting inut.

\section{KAPITEL VIII.}

Angersimanerub itulervingata âtauninga Tempelemuit; Tempetib attortautainarninga.

I'AGVA attanniub Salomob ingminut kattikovait angajokaunerôjut Israelitine, kinguvarêt angajokangit, atâtaujullo lsraelitit akkorngane attanningit illunaita Jerusalemut, Nalekab angersimaningata iklervinga âlugo takpaunga Davidib iglugaseksoanganit, tagva Zionemit.

2 Angutillo Israeleme illunatik kattilerput attannermut 
Salomomut, uvloksiorvingme, takkerme Ethanemik attelingme, tagva takkit 7 ingane.

3 Israelitillo angajokaunerojungit illunatik tikkimatta; ajochertuijut Nalekab iklervinga kivikpât,

4 Apâlo takpaunga; taimaglotauk angersimanerub tuppinga, ivsornaitoviublo perkutingit illunaïta, tuppermêlauktut, ajochertuijut Levitillo takpaungartipait.

5 Attannerlo Salomo, illagêllo Israelitit, kattimalerlauktut tapsomunga, aikattigilaukpât iklerviub sivorngane, tunnijilutiglo tunnergutinik, saukanik tuktuvangniglo, taimak unnuktigijunik kittiksaulaungimatta, kittitaujungnangimattalo.

6 Taimak ajochertuijut Nalekab angersimaningata iklervinga âpât inniksanganut, innemut iglub kinguanêtomut, ivsornainerpangôjomut, Kerubingoâk esarungita attânut.

7 Kerubingoâk esarotik issivingmagit inniub iklerviub makkitavigijangata kollâne, ullikpâlo iklervik akkerautingillo kollânit.

8 Akkerautingillo taimak takkitigijolaukput, issungit tækkojaulaungmatta ivsornaitovingmit, inniub kinguanêtub sângane, igluble sivurânit tækkojaulaungilet; tagvanêtsainarpullo uvlok tamanna tikkilugo.

9 Pittakalaungilarlo sunamik iklervingme, aglagvîk ujaraujuk assigingnik, Mosesib illilauktakita kamunga Horebeme, Nalekab Israelib kittorngangit angerkattigilaungmagit, Egiptenemiut nunanganit aularsimalauktillugit.

10 Ajochertuijulle annilertillugit ivsornaitovingmit nuvujab Nalekab iglunga nâmalerpa;

11 Ajochertuijullo nangerungnarlaungilet tagvane pinniaraksatik pinniarlugit, nuvujak pivlugo. Nalekab ânanauningata Nalekab iglunga nâmangmago.

12 Tagva Salomo okarpok: Nalegak okarpok, tâktome innekaromagame.

13 Uvanga iglo igloliorlaukpara igluksarnut, inne innigijaksarijat soraijuitomik.

14 Attanniublo kênane allarpa saimartilaitalo illagêt Israelitit illunamassiangit; illagêllo Israelitit illunatik nangerput tagvane.

15 Tamnalo okarpok: Nalegak Israelib Gudingat nertortaule, kannerminut atâtamnut Davidemut okalauktok, aggangminullo nellautsititsilauktok, okalauktorlo:

16 Uvlormit taipsomanemit innukotimnik Israelitinik aulartitsivigilauktamnit Egiptenemit, iglugaseksuit nellianik annerosulaungilanga Israelib kinguvarêngita nellianit, iglo igloliortaukovlugo pivlunga, attera tagvanêkovlugo; Davidele annerilaukpara angajokaukovlugo innukotimnut Israelitinut. 
17 Atâtagalo Davide issumakalauralloarpok, iglo iglolioromavlugo Nalekab Israelib Gudingata attinganut illingajuksak.

18 Nalegarle okalaukpok atâtamnut Davidemut: Issumakaravit attera iglolioromavlugo, namaktullilaukpotit tamattominga kajusilauravit.

19 Igville iglo tamanna iglolioraksaringilat, erniville illingnit pinniartub, tapsoma attera iglolioraksariva.

20 Nalekablo okautsine okauserilauktane nellautsitimavait. Uvanga atâtaga Davide innangêrlaurapko, iksivalungalo Israelib iksivautangane, "sorlo Nalegak okalaungmat, iglolo igloliorlaukpara Nalekab Israelib Gudingata attinganut.

21 Akiksoilaukpungalo tagvane inniksamik iklervingmut Nalekab angersimaninganik pittalingmut, angerutigilauktanganik atâtagilauktaptingnut, Egiptenemiut nunanganit aulartilauramigit.

22 Salomoblo Nalekab Altarekotinga sângerpa, illagêt Israelitit illunamassiatik najortillugit, agganelo issangmivait killangmut,

23 Okarlunelo: Nalegak Israelib Gudinga, illiktut Gudekangilak, takpânelonêt killangme, attânelonêt nuname sokkosijuititsijomik angernermik napkiningnermiglo kivgakotingnut agvertarlauktunut sângne omamut tamânut.

24 Sokkosijuititsilauktotit atâtamnut Davidemut, okauserilauktarnik tapsomunga. Kannernut okauserilaukpat, aggangnullo nellautsitilaukpat, sorlo uvlorme tamattomane îmat.

25 Mannalo Nalegak Israelib Gudingat! kivgait atâtaga Davide sokkosijuititsivigilauruk okauserilauktarnik tapsomunga, okarlutit: Ajoksartuksaungilatit angumik sâmne iksivajomik Israelib iksivautaksoangane, ernitit kammatsiartuinarpatta apkotigijamingnik, agvertarkovlugit sâmne, sorlo igvit agvertarlauravit sâmne.

26 Manna Israelib Gudinga, okautsitit miksekârtotikit, okauserilauktattit kivgarnut atâtamnut Davidemut.

27 Gudele nellâgut innekaromaralloarka nuname? Ahâk, killaub, killaillo killangita illunamassiata illulungilâtit. Kannorle tagva iglo una igloliolauktara pinniarungnarajarka tamattominga?

28 Sâlaurilletôk kivgavit tuksiarninganut, kennuninganullo, Nalegak Gudik, tussarkovlutit nipliarnermik, tuksiarnermiglo, kivgavit uvlome tuksiarnerijanganik sângne;

29 Ijikik uitatsainarkovlugik uvlotillugo unnuarmelo iglomut omunga, innemut okautigilauktarnut: Attera tagvanêtuksauvok; tussarkovlutit tuksiarnernik kivgavit tuksiarnerijanginik mâne inneme tamattomane;

30 Taimak tagva tussalaurit kivgavit innukotivillo Israelitit kennuninginik kennuneriniartanginik mâne; tussalerit tamatto- 
minga inneme, innigijarne killangme, tussalaurit issumagijungnailugillo ajornerit.

31 Kina idluitullilerangat innukattiminut, kigligiksartuksaugunelo angermariktuksaugunelo, taimaimat sorlo okarame angermaringnerlo âtaulerpat Altarekotivit sânganut, iglome omanetub;

32 Tagva igvit tussalaurit killangme, pinniarlutillo, erkartorlugillo kivgatit, uivêtok passijaksaunerarlugo, agvertarningalo pitilugo niakunganut; idluartorlo idluartonerarlugo, tunnitsivigilugolo idluarninga malliklugo.

33 Innukotitit Israelitit sâlagijaulerangatta omigijimingnut, idluitullilauramik illingnut, sâviginiarpattillo, attillo kigligiudsivigilugo pikpatta, tuksiarpattalo kennulutiglo illingnut iglome omane;

34 Tagva igvit tussalaurit killangme, issumagijungnailugillo innukotivit Israelitit ajorningit, uttertiniarlaitalo nunamut atâtagilauktanginut tunnilauktarnut.

35 Killaglo parngnanairsimalerangat, sillaluluganelo, idluitullilaungmatta illingnut; tuksiarniarpattalo inneme omane, attillo kigligiutilugo pinniarpatta, ajornermingnillo sâpatta, pitlarangne;

36 Tagva igvit tussalaurit killangme, issumagijungnailugillo kivgavit innukotivillo Israelitit ajorningit, apkosinermik ajungitomik agvertarvigijaksarijanganik unnertutilaukârlugit, tunnijilutillo sillalungnermik nunakotingnut, tunnilauktarnut innukotingnut, innigijaukovlugo tapkonunga.

37 Kâkalerangat, nusilinartokalerangallonêt, perortullonêt kakkojaksat kernersilerangatta, piglertaujartokalerangallonêt nerrijoksoarnik, omigijingitalonêt nuname ittertarvingit illumarangagit, pagvinartokalerangallonêt, kannimasekalerangallonêt, nellianik;

38 Tuksiartut kennujullo tamaita, innuit nellîllonêt, innukotitit Israelitillonêt, tukkisilerpatta attunît omattimik pagvinarninganik, aggatiglo issangniarpattigik iglomut omunga;

39 Tamanna igvit tussalauruk killangme, inneme inigijarne, issumagijungnainilutillo, pinniarlutillo, tunnijilutillo nellianut, agvertarnerilauktanga tamât malliklugo, sorlo omattinga illitarigangne; igvit kissivit innuit kittorngangita illunatik omattingit illitarigangne;

40 Sivoratsainarkovlugit illingnik nelliutune tamaine, innotillugit nuname, atâtagijaptingnut tunnilauktarne.

41 Tækkorngartattauk nelliat, innukotingnut Israelitinut illingalungitok, tikkilerangat nunamit kanningitomêtomit attit pivlugo ;

42 (Tussarniarmatta atternik angijoksoarmik, aggangniglo pitsartujoksoarnik, tallerniglo issamijomik), kailunelo tuksiarpat iglub oma sângane, 
43 Tagva igvit tussarit killangme inneme, innigijarne, pinniarlutillo tamainik tækkorngartab nipliarutigijanganik illingnut; innukattigêksuit nunametut illunatik illitarksilerkovlugit atternik, sivorakovlugittauk illingnik, innukotititut Israelititut, tukkisilerkovlugillo attit malliklugo iglo tamanna igloliolauktara taijaungmat.

44 Innukotitit aularniarangatta unatadlarnermut omigijitik sorsugvigilugit apkome tilliklerutigijarne tapkoninga; tuksiarniaraikpatta Nalekamut, iglugaselssuit annerilauktatit sâdlugit, iglolo atternut igloliolauktara sâdlugo.

45 Tagva igvit killangme tussalaurit tapkoa tuksiarninginik, kennuninginiglo, idluartomiglo sakkêvigikit.

46 Idluitulliniaraikpatta illingnut, (innukangimat idluitullilungitomik) igvillo ningarosugutigigungne, tunnilugillo omigijinginut, sâlagijingitalo aularutikpagit omisuktut nunanganut, kanningitomullonêt; kannitomullonêt;

47 Omamiutarilerpatsuglo nuname parngnanairsimavigijamingne, sâlerpattalo tuksiarlutiglo illingnut, nuname parngnanairsimavigijamingne okarlutiglo: Idluitullilaukpogut, tammarlauklutalo uivêtolaukpogut;

48 Taimaglo sâlerpatta illingnut omamut tamânut, tarnermullo tamânut, omigijimik, aularutijimik nunangane, tuksiarpattalo illingnut, sâlutik nunagijamingnut atâtagijanginut tunnilauktarnut, iglugaseksoarnut annerilauktarnut, iglomullo igloliorlauktamnut atternut.

49 Tagva tussalaurit tuksiarninginik, kenuninginiglo, killangme, inneme innigijarne, idluartomiglo sakkêvigikit;

50 Innukotitillo issumagijungnaivigikit idluitullininginik illingnut, tappaninginiglo tamainik, tappajutigilauktanginik illingnik; napkiningneksilaulillo salagijimingne, napkiningnikovlugit tapkoninga.

51 Innukutaungmatta perkutaulutiglo illingnut, aularutilauktatit Egiptenemit kiaksaumit kikkiangmit;

52 Ijikik uitakovlugik kivgavit innukotivillo Israelitit kennuninginut, tussarkovlutit tapkoninga, nipliarutiginiartangit illingnut tamaita pivlugit.

53 Igvit apterlaurangne innukotingnut, nunab innukattigêksoanginit tamainit, sorlo okalauravit kivgakut Mosesekut, atâtagijavut aularutilaurangne Egiptenemit, Nalegak Jehova!

54 Salomoblo tuksiarnerit kennunerillo tamakkoa tamaita nâgamigit Nalekab sângane, tagva makkipok Nalekab Altarekotinganit, serkortornermit aggane issangmilugit killangmut.

55 Nangeriartorporlo tagvunga, saimartilaitalo illagêktut Israelitit illunamassiatik nippekortôjomut, okarlunelo:

56 Nertortaule Nalegak, innukotiminut Israelitinut merng- 
oêrsernermik tunnijilauktok, sorlo okalaurame. Okausek attauserlonêt tikkingilungilak okausinginit ajungitunit tamainit, kivgamigut Mosesekut okauserilauktanginit.

57 Nalekab Gudipta illagilittigut, sorlo atâtagijavut illagilaungmagit. Tapsoma kemmangniarattigut agganelo erkitiniarnagit uvaptingnit,

58 Omattivut sangutilugit tapsomunga, agvertarkovluta apkosiningine tamaine, mallikovlutalo perkojanginik, illusiokojanginiglo, idluarijanginiglo, perkolauktanginik atâtagijaptingnut.

59 Okautsikkalo tamakkoa Nalekab sângane kennunerilauktakka, najorlit Nalekamut Gudiptingnut uvlotillugo unnuamelo, kivgane idluartomik sakkêvigilugo pikovlugo, innukotinelo Israelitit, uvlut tamaita, sorlo pijariakaramik;

60 Innukattigêksuit nuname illunatik illitarksikovlugit, Nalegak Gudeôngmat, assekangimallo.

61 Omattiselo illuêngarlutik illingalaulit Nalekamut Gudiptingnut, agvertarkovluse, tapsoma illusiokojangit malliklugit, mallikovluselo tapsoma perkojanginik, sorlo pigapse nelliutome tamattomane.

62 Attannerlo Israelitillo illuêngarlutik tamna illagiluga tunnergutinik tunnijivut Nalekab sângane.

63 Salomolo tunnergutinik tunnijivok kujalijutiksatut tunnijaminik Nalekamut, tuktuvanguik 22000 dinik, saukaniglo 120000 dinik. Taimaglo attanniub Israeliblo kittorngangita Nalekab iglunga attortainarpât.

64 Uvlorme taipsomane attanniub inne avaluliorsimajok Nalekab iglungata sivurânetok ivsornaitipa; tagvane tunnergutit ôtaksat, tunnergutillo nerkiksat, tunnergutillo ôtaksat orksungit tunningmagit; Altare kannujaujok Nalekab sânganêtok, mikkiluarlaungmat tunnergutinut ôtaksanut, tunnerjutinullo. nerkiksaujunut, tunnergutillo ôtaksat orksunginut.

65 Taimaglo nelliutome taipsomane Salomob uvloksiorvik uvloksiorpa, Israelitillo illuêngarlutik illagællugo, kattimajut unnuktovaksuit Hemathemit Egiptenib kônga tikkialugo, Nalekab Gudipta sângane; uvlut 7 it nâlugit, amalo uvlut 7 it nâlugit; tagva uvlôvut 14 it.

66 Uvlullo 8 ingane innugiartoksuit aularkovait. Tapkoalo attannek saimartipat, ailutiglo tuppermingnut, kuviasuklutik maksoalutiglo ajungitut tamaita Nalekab pinniarlauktangit kivgaminut Davidemut, innukotiminullo Israelitinut pivlugit. 


\section{KAPITEL IX.}

Nalekab saklipsarninga Salomomut. Salomonib tunnijininga ighugaseksoarnik 20 ginit Hiramemut. Umialitorningalo.

$\mathrm{S}$

ALOMOBLO Nalekab iglunga, attanniublo iglunga, tamaitalo tussugijane, kajungerijanelo sennajomavlugit pijarêrtilauramigit;

2 Nalekab sakkervigiva aipanganik, sorlo sakkervigilaungmago Gibeoneme.

3 Nalekablo okautiva: Tuksiarnit, kennunillo, kennunerilauktat uvamnut, tussarlaukpara, iglolo tamanna igloliorlauktat, attimnik pititsikovlunga tagvunga soraijuitomik, ivsornaiviotilaukpara; ijikkalo omattigalo tagvanêtuksauvut nelliutune tamaine.

4 Igvillo agvertarniaruvit sâmne, sorlo atâtait Davide agvertarlaungmat, omamut nellagortomut, annerpanaijuitomullo, pinniarkovlutit taimailuatsiamik, sorlo pêrkolaurapkit; perkojakkalo idluarijakkalo mallingniarungne;

5 Tagva attanionivit Israelitinut iksivautaksoanga sokkosijuititsomavara soraijuitomik; sorlo atâtait Davide okarvigilaurapko, okarlunga: Ajoksartuksaungilatit angumik Israelib iksivautaksoanganêtomik.

6 Allarniarupsingale illipse ernipselo, mallingnagillo perkojakka, idluarijakkalo, mannilauktakka illipsingnut, ainiarluselo Gudib assingit kivgartorlugit, tuksiarvigilugillo pigupse;

7 Tagva Israelitit pêjarniarpakka nunamit tunnilauktamnit tapkonunga ; iglolo ivsornaiviotilauktara attimnut, pêjaromavara kênarma sânganit; Israelitillo okausiovaktungoromârput, okangoarutingorlutiglo innukattigêksoarnut illunainut.

8 Iglolo tamanna poktojoksogalloarlune, illunainut sennerkotsijomârtunut kâkkiladlarutiksaujomârpok, supporomârput okarlutiglo: Sôg Nalekab nuna tamanna, iglolo tamanna taimailiorvigilaukpagit?

9 Okartokaromârporlo: Tamanna pivlugo, Nalegak Guditik kemmasimangmatsuk, atâtagijanginik aularutitsilauktok Egiptenemiut nunanganit, Gudiblo assingit illalliormagit, tuksiarvigingmagillo, kivgartorlaitalo; tamanna pivlugo Nalekab tikkitaukolaukpait kannoêtomut mattomunga tamât.

10 Jahrit 20 git nângmatta, Salomob attorlauktangit, iglûk, Nalekab́ iglunga, attanniublo iglunga, igloliorlugik;

11 (Hiramib Tirusemiut attaningata Salomo tunnitsivigilaukpa tapkungnunga, nappartunik Cederinik, Cipressiniglo, Goldemiglo, taimaluatsiamik sorlo tussulaungmat) tagva Salomob 
Hirame iglugaseksoarnik 20 ginik nuname Galilæamêtunik tunnitsivigiva.

12 Hiramelo aularpok Tirusemit, iglugaseksuit taipkoa tækkojartorlugit, Salomob tunnilauktangit tapsomunga; namagilaungilaillo.

13 Okarporlo: Iglugaseksuit tunnilauktatit uvamnat kanoêlingavat, kattangutîk? Atserarpaillo nunamik Kabulemik, uvlok tamanna tikkidlugo.

14 Hiramiblo attannek tillikliutjivigiva Goldemik Talentinik 120 ginik.

15 Tamannalo pitjutauvok Salomob sennajuksat annerilaungmagit, Nalekab iglunga iglokotinelo, Millolo Jerusalemiblo avalungit, Hazorelo, Meggidolo Gaserelo sennajomalaungmagit.

16 Farao Egiptenemiut attanningat takpaungarlaukpok, sâlagilaukpaillo Gaser, ikkilugillo ikkomamut, Kanaanemiullo iglugaseksoarnêlauktut tokkopait; iglugaseksuillo pilliutigilaukpait panniminut, Salomob nullianganut.

17 Salomoblo Gaser sennavait, Bethhoronelo atsiktok,

18 Baalatelo, Tadmorelo, sunnakajuitome nuname.

19 Iglugaseksuillo kemmatullıviojut Salomob pigijangit, iglugaseksuillo kammutingnut illingajut, iglugaseksuillo sorsuktuksanut ablangajunut horsit kângine illingajut, tamaitalo tussugijane sennajomavlugit, Jerusaleme, Libanoneme, attanniovimelo nunangane illûnane.

20 Innugiartoksuit amiakovinit Amoriterinit. Hethiterinillo Feresiterinillo, Heviterinillo Jebusiterinillo,. Israelib kittornganginit pilaungitut;

21 Taipkoa amiakolauktut nuname kittorngangit, Israelib kittorngangita nungutsungnarlaungitangit, tapkoa Salomob sennajuksautipait uvlok tamanna tikkidlugo.

22 Israelible kittornganginit kivgangortitsilaungilak, sorsuktuksaulaungmatta, kivgaulutiglo tapsomunga, angajokakotigilaitalo, sorsuktuksaulutiglo kamutingne, angajokaulutiglo kammutekotinginut, ablangajunullo horsit kângine.

23 Aulatsijullo Salomob sullijaksanginik, 550 giolaukput, angajokaulauktut innugiartoksoarnut sennalauktunut sullijaksame tamattomane.

24 Faraoblo pannia nûlune takpaungarpok Davidib iglugaseksoanganit iglominut, Salomob igloliorlauktanganut tamna pivlugo. Taipsomane Millo sennalauriva.

25 Salomolo pingasoêrtorlune Jahreme attautseme tunnijivok tunnergutinik ôtaksanik, kujalijutiksaniglo, Altareme sennalauktamine Nalekamut, isserluanititsilunelo kângane, Nalekab sângane. Iglolo pijarêrtipa.

26 Salomolo umîjorpoktauk umiaksoarmik, Ezon Gebereme, 
Elothib immarbiub iviggub suvlolingnik sikjanganêtub kannitanganêtome. Edomiterit nunangane.

27 Hiramiblo kivgane, umiaktornermik sungiusimajut, immarbiksoarmiglo illitarksijut tillivait umiaksoakut omuna Salomob kivgangit illagilugit.

28 Tapkoalo tikkiput Ofiremut, aiklerlutiglo tagvangat Goldemik Talentinik 420 ginik, atsilutiglo tamakkoninga attannermut Salomomut.

\section{KAPITEL $\mathrm{X}$.}

Arabiamiut altanningata arnab nellipsainnnganik Salomomik.

Arabiamiullo attaningata arnab Salomonib tussaumajanga, Nalekab attinga pitjutigællugo tussaramiuk, kaivok oktoromavlugo nellaupsarutiksanut.

2 Jerusalemullo tikkipok unnuktunik najortekarlune, Kamelinik, mingoarutiksanik pivianartunik, Goldeniglo unnuktunik, ujarkaniglo pivianartunik, nangmartunik. Salomomullo tikkipok, okarvigivalo tamainik omamigijaminik.

3 Salomoblo okautiva tamainik apperkutigijanginik; sumiglonêt nellonartokalaungilak attannermut, okauserilungitanganik tapsomunga.

4 Arabiamiullo attaningat arnak tækkogame Salomob illisimaninganik tamât, iglomiglo igloliorlauktanganik.

5 Nerkiksaniglo tapsoma nerrimarvianut illingajunik, kivgangitalo iglunginik, naipertutsiarninginiglo, annoranginiglo, imiksalliortokotinginiglo, tunnergutinginiglo ôtaksanik tunnijanginik Nalekab iglungane; tagva uimamajotun-1̂lerpok,

6 Okarlunelo attannermut: Miksekârtôvok tussalauktara nunakotimne, 1llinganît illissimanîllo pivlugik.

7 Okperijomalaungilaralo tikkikârtinnanga, tækkolugolo ijimnut. Ahâglo, koppanganiglonêt okautsaulaungilanga, illissimanelionersauvotit ajunginelionersaulutillo tussaumajamnit tussarlauktamnit.

8 Pilloridlarput innukotitit, nangitsainartut sângne, tussarlutiglo illissimanernik.

9 Nertortaule Nalegak Gudit, ingitilaungmattit Israelib iksivautanganut, Nalekab Israelitit nagligingmagit soraijuitomik, tamanna pivlugo igvit attanningortilaukpâtit, namanermik idluarnermiglo sakkertitsikovlutit.

10 Tapsomalo attannek tunnitsivigiva Goldemik Tallentinik 120 ginik, mingoarutiksaniglo tippitsiariktunik unnuktovaksoarnik ujarkaniglo pivianartunik. Mingoarutiksat taimak unnukti- 
gijut Arabiamiut attaningata - tunnilauktangititut attannermut Salomomut tikkiutjaujungnailaukput.

11 Hiramiblo umiaksoakotinga Goldetortok Ofiremit, kaitsivoktauk Ofiremit nappartunik Sandelemik attelingnik unnuktunik, ujårkaniglo pivianartunik.

12 Attanniub!o sennakojivok kejungmit Sandelemik attelingmit kattagêkutingnik tigumiarvingnik Nalekab iglungane attanniublo iglungane; Harfeniglo, nokakseralingniglo imgerpaktunut. Kejuillo Sandeleojut taimaitut tikkiutjaujungnailaukput, tækkojaujungnailutiglo taimangat, uvlok tamanna tikkidlugo.

13 Attanniublo Salomob Arabiamut attanningat arnak tunnitsivigiva tamainik tussugijanganik tuksiararijanganiglo, tunnijane tapsomunga pijarællune tappilugit. Allârporlo aulârlunelo nunakotiminut kivgakotine illagilugit.

14 Golrele tikkiutjaulauktok Salomomut Jahreme attautseme okumainingat Talenteôlaukput 666 git.

15 Tamannalo niuvertinit niuveriarniartunillo, innugiartoksuillo (angerlauktut Salomomut) attanninginit, aulatsijunillo nunnane pijok, taijaukassiutinago.

16 Attannerlo Salomo sennakojilaukpok, sappulutaksanik 200 dinik Goldemit namanerpâmit; Sekelititut 600 ditut okumaitigijut Golde attorpa sappulutaksamut attautsemut.

17 Sappulutaksaniglo angijunik, Tartschenik attelingnik, 200 dinik; Paunditullo pingasutut okumaitigijok Golde attorpa Tartschemut attautsemut. Attanniublo pitipait igloksoarmut, nappartut Libanonemit igloksoanganik attelingmut.

18 Attannerlo iksivautaksoarmik sennavok Elefantib tôganginit, sillapârpâlo Goldemik pivianarnerpangôjomik.

19 Iksivautaksoarlo tamna, tungmerakalaukpok pingasujortunik, iksivautablo tunnuata kollâ kattigoliungalaukpok. Iksivavingatalo senneragik iglugêngnik egangavikalaukpuk tallingnut, Loewingoaglo nangerlaukpuk egangavikita tungangne.

20 Loewingoallo 12 it nangerlaukput tumerangne pingasojortune ittivigêngne. Taimaitomik semnamajokalautsengilak attanniovît nelliane.

21 Attanniublo Salomob illulerkotingit immiksakautit Goldiolankput, illulerkutillo illunaita Libanonib nappartungita ig- lunganêlauktut Goldetuinaulaukptut; Silbere Salomonib nelliuningane sunaunasugijautsengimat.

2. Attannek umiaksoakalaungmat immarbingme, Hiramib umiaksoakotinga aiparællugo Tarsisemut umiaktortok; attausêardlune Jahrine pingasune umiaksoak utterkattarpok, tikkiutjilunelo Goldemik, Silberemiglo Elefantillo tôganginik, pissuktuniglo itjuartunik, tingmianiglo Fauenik attelingnik.

23 Taimak attannek Salomo akluinersaulaukpok, illissimanersaulauklunelo attannernit illunainit nunamêtunit. 
24 Nunaggillo illunatik tussudlarput Salomo tækkojomavlugo, sillatuninga, Gudib tunnilauktanga tapsoma omattinganut tussaromavlugo.

25 Illunatiglo âtsivigivât pilliutinik, perkutinik Silbereôjunik Goldeôjuniglo, annorangniglo, sakkuniglo tauvlukutiksaniglo, horseniglo, horseojartuniglo, Jahrine tamaine.

26 Salomolo kattersoivok kamutingnik aksalloalingnik, sorsuktuksaniglo horsit kângine ablangajunik, pekalaukporlo kamutingnik aksalīoalingnik 1400 dinik, sorsuktuksaniglo ablangajunik horsit kângine 12000 dinik, innekarkovaillo iglugaseksoarne aksalloalingnut illingajune, Jerusalemelo attanniub najugâne.

27 Attaniublo Silberit taimak unuktigijotipait Jerusaleme ujarkatitut, Cederillo nappartutitut Sikomorinik attekartutitut, naksarne perortutitut.

28 Salomolo âtsiviomavok horsenik Egiptenemit, niuviaksaniglo sunatuinarnik, attanniublo niuvertekotingita niuviaksat tamakkoa pissiarivait,

29 Kaitsivullo Egiptenemit, aksalloalingmik attautsimik Sekelinut 600 dinut Silberemik; horsemiglo attautsemik Sekelinut 150 ginut. Taimaglo âtaulaurivuttauk Hethiterit attaninginut, Siriamiullo attaninginut, taipkoa aggangittigut.

\section{KAPITEL XI.}

Salomob nulliangií; kivgartorningalo Gudingoanit, tokkungalo.

A

ATTANNIUBLE Salomob arnat unnuktut Judit nunangata assinginit pijut nagligivait, Faraob panniata senniane arnat Moabiterinit, Amoniterinillo, Edomiterinillo, Zidonemiunillo, Hethiterinillo,

2 Innukattigêksoarnit taimaitunit Nalekab okautigilauktanginit: Aiviginiarassigik, tikkitailitissigiglo illipsingnut; nellonangitomik omattise sangutiniarpait, Gudingoakotimingnut. Taimaitut Salomob ungagivait naglingnermut.

3 Nulliakalaukporlo 700 dinik attannit panninginik, pannervgakalarmelo 300 dinik. Nulliangitalo omattinga sangutipât.

4 Salomolo innutokaulermat nulliakotingita omattinga sangutipât Gudingoanut, omattingalo illuêngardlune illingalaungilak Nalekamut Gudiminut, sorlo atâtangata Davidib omattinga pilaungmat.

5 Taimak Salomo agvertarpok Astorete, Zidonemiut Gudingat, Milkomelo Amoniterit makkojungnartokotingat malliklugik.

6 Salomoblo Nalekab kuviagingitanga pinniarpa, mallingna- 
nelo illuêngardlune Nalekamik, sorlo atâtanga Davide pinniarlaungmat.

7 Taipsomane Salomob poktojok senavâ Kamos Moabiterit makkojungnartokotingat pivlugo, kakkame Jerusalemib nellânetome, Molechelo Amoniterit makkojungnartokotingat pivlugo.

8 Taimailiorlaukpok Salomo nulliane tækkorngartanit pijut illunaita pivlugit, Gudingoakotimingnut isserluanititsijut, tunnijijullo tunnergutinik.

9 Tagva Nalekab Salomo ningarilerpa, omattinga Nalekamik Israelib Gudinganik allaingmat, maggoêrtorlune sakkerlauktomik tapsomunga,

10 Perkojilauktomiglo tapsomunga, Gudib assingit malliklugit agvertartuksaungimat; mallilaungilaille perkojaunine Nalekamut.

11 Tamanna pivlugo Nalegak okarpok Salomomut: Taimailiortokalaungmat illingnut, angernera, perkojakkalo, perkolauktakka illingnut, mallilaunginangne, attanniovik aksarnigarijomagivara illingnit, tunnijomalugolo kivgakotingnut.

12 Innôtillutille taimailioromalungilanga atâtait Davide pivlugo; ernivîlle agganginit aksarnigarijomavara.

13 Attanniovigle illuêngardlune aksarnigarijomangilara, kinguvarêt attautsit tunnijomavakka ernernut, Davide kivgara. pivlugo, Jerusalemelo pivlugit annerilauktakka.

14 Nalekablo Salomo akkerartortekartipa Hadademik Edomiomik, attanniub Edomeme kinguvanganit pijomik.

15 Davidele Edomemêlauktillugo Joabelo sorsuktuksaksoakattigêt angajokangat takpaungarame ullimarsimajut illijartoromavlugit, angutaujut Edomeme illunamassiatik ullimarpait.

16 Joabe takkit 6 it nâlugit Edomemêtuarame; Israelitillo illuêngardlutik, nungutsikârtinane angutaujunik illunainik Edomeme.

17 Tagva Hadade kemâvok illagællugolo Edomiut angutingita, atatangata kivgangita illangit, Egiptenemut tikkitsomavlutik. Hadadele nukapiangolaukpok sulle.

18 Aularpullo Midianemit, tikkilutiglo Paranemut, neksarpullo innungnik Paranemit, tikkilutiglo Egiptenemut Faraomut Egiptenemiut attanninganut. Tapsoma tunnitsivigiva iglomik, innugutiksamiglo, nunamiglo.

19 Hadadelo napkoaneksivok angijomik Faraome, nulliamiglo tunnitsivigiva, nulliame Tachpenesib nukkanganik.

20 Tachpenesiblo nukâ ernivok tapsomunga erninganik Genubathemik; Tachpenesiblo innugoiva Faraub iglungane; Genubathelo Faraub iglunganetsainarlaukpok, attanniub erningita akkorngane.

21 Hadadelo Egipteneme tussarame, Davide atâtagijangit illagællugit sinnisimangmat, Joabelo sorsuktuksaksoangita anga- 
jokangat tokkungmat, Faraomut okarpok: Aularkolaunga, nunakotimnut aimikovlunga.

22 Faraub okautiva: Sunamik kingumaklerkît uvamne nunakotingnut nûtsomagavit? Tamna okarpok: kingumaklerlungilanga sunamik, aularkolaungale.

23 Gudiblo akkerartortekartipa sulle assianik, Resonemik, Eljadab erninganik, angajokaminit Hadadeseremit Zobamiut attanninganit kemâlauktomik.

24 Angutiniglo kattersoivok ingminut, angajokaulaukporlo sorsuktuksat illanginut, Davide taipkoninga (Siriamiunik) salakarlauktillugo; aularpullo Damaskusemut, nunagivâllo attaniôtilutiglo Damaskuseme.

25 Tamnalo kanoêtokartitsinine tappilugo akkerartortêolaukpok Israelitinut Salomob innosingata nâninga tikkidlugo; attanniolaukporlo Siriamiunut.

26 Jerobeamelotauk Nebatib erninga Efratemiok Zaredamit, Salomob kivganga, (anânangalo Zerugaulaukpok uigarnek) tamna aggangminik kongmuartitsivok attannermut akkerartorlune.

27 Tamannalo pitjutauvok aggane kongmuartimagit attanek akkerartorlugo: Salomob Millo sennalaungmago, kingek Davidib. atâtame iglugaseksoangane simmikpa.

28 Jerobeamelo angutaulaukpok erkinaitok. Salomoblo innusuktok tækkogamiuk sullidlarmat, aulatsijungortipa sennajuksanik Josefib iglunganit pijunik illunainik.

29 Pijokalaukporlo nelliutome taipsomane, Jerobeame aulartillugo Jerusalemit, Ahiab Silomiub nellautaijub naipipa apkosinerme, ulliksoalijarporlo nutâmaringmik, tapkoalo magguk inutolaukpuk tagvane.

30. Ahiablo ullik nutâk attortane tiguva alliktorlâlo, allingningit 12 ôlivlugit,

31 Okarlunelo Jerobiamut: Alliktut tellimaujortut tigukit illingnut. Imâk Nalegak Israelib Gudingat okarmat: Ahâk attanniovik aksarnigarijomavara Salomob agganginit, kinguvarêllo tellimaujortut tunnijomavakka illingnut.

32 Kinguvarêt attautsit pigênaraksarivait kivgara Davide pivlugo, iglugaseksuillo Jerusalem pivlugit, annerilauktakka Israelib kinguvarênginit illunainit.

33 Tamanna pivlugo allarlaungmanga, Astarote Zidonemiut Gudingoangat, Kamoselo, Moabiterit Gudingoangat, Milkomelo, Amoniterit Gudingoangat tuksiarvigilaungmagit; agvertarlaungimattalo apkosinimne, pinniaratiglo kuviagijamnik, perkojamnik, idluarijamniglo Davidetut atâtangatut.

34 Attanioviuble illûnanga tigujomangilara agganginit, attanniotsainarkojomavara innosinga nâlugo, Davide kivgara annerilauktara pivlugo, perkojamnik idluarijamniglo mallilauktok. 
35 Erningata agganginit attaniovik tigujomavara, illingnullo kinguvarêt tellimaujortut,

36 Erninganullo kinguvarêt attautsit tunnijomavakka, Davide kivgara kaumautekatsainarkovlugo sâmne iglugaseksoarne Jerusaleme annerilauktamne attera pitilugo tagvunga.

37 Igvillo tigujomavagit manna, attanniotilerkovlutit tamainut omattivit tussugijanginut, attanniojuksaulutillo Israelitinut.

38 Naletsiarniaruvillo manna tamainik perkoniartamnik illingnut, agvertarniaruvillo apkosinimne, pinniarlutillo kuviagijamnik, idluarijakka perkojakkalo malliklugit piguvit, sorlo kivgara Davide pinniarlaungmat; tagva aiparijomavagit, iglolioromavlutillo sokkosijuitomik, sorlo Davide igloliorlaurapko, tunnitsivigijomavlutillo Israelemik;

39 Davidiblo kinguvângit nikkanarsartitsomavakka tamanna pivlugo, soraijuitomigleungitok.

40 Salomoble pijugakpa. Tagva Jerobeame parngnailerpok kemâlunelo Egiptenemut, Sisakemut, Egiptenemiut attaninganut. Egiptenemêturiporlo Salomob tokkunga tikkilugo.

41 Unipkautsille tamakkoa assingit sulle Salomo pivlugo, pinniarlauktangit tamaita, illissimaningalo, aglaksimavut Salomob pinniarningita aglangine.

42 Salomoblo attannioningata Jerusaleme Israelitit illunamassianginut, nelliuninga Jahriovut 40 git.

43 Salomolo sinnisilerpok ailune atatagijaminut, illijartortaulunelo atâtame Davidib iglugaseksoangine. Erningalo Rehabeame innangêrlugo attanningorpok.

\section{KAPITEL XII.}

\section{Kinguvarêt 10 it apterningit Rehabeamemit. Jerobeamiblo} atianningortitauninga, kivgartorningalo Gudingoanik. REHABEAMELO Sichememut aivok, Israelitit illuêngarlutik Sichememut tikkisimagamik attanningortitsomavlugo.

2 Jerobeamelo, Nebatib erninga tussarpok tamattominga Egiptenemêdlune sulle, kemâvigijamine attannek Salomo pivlugo, Egiptenemêtuarporlo.

3 Tilliklerpullo taikunga, kaikojaukolâllo. Tagva Jerobeame Israelitillo kattimajut illunatik tikkiput Rehabeame okarkattigijomavlugo, okarpullo:

4 Atâtavit nangmariakartavut okumailuartilaukpa, taimaimat igvit manna kivgartornek okumaitok, nangmagarlo siorniornartok illilauktanga uvaptingnut okinersautilikik: tagva nalegomavaptigit.

5 Tapsomale okautivait: Aileritse uvlut pingajuat tikkidlugo, tagva kaipsarniaritse uvamnut. Innugiartoksuillo aularput. 
6 Attanniublo Rehabeamib angajokajat atâtame Salomob sângane nangerlauktut innôtillugo sulle, kakimiolerkattigivait, okarlunelo: Kannok attannertuivise innugiartoksuit taipkoa kiolugit?

7 Tapkoa okautivât: Uvlome innugiartoksuit taipkoa kivgartorniarungne, pijomajangillo mallingniarungne, tussarlugillo, illanâmartuniglo okausekarvigigungne, tagva nalengniarpâtit innotsit nâlugo.

8 Tapsomale angajuklit attannertuiningat tunnilauktangat tapsomunga kemmakpâ, kakimiolerkattigivaillo innuosuktut, ikkingutine sânganelo nangertut.

9 Okautivaillo: Kannok illipse attannertuivise, sunamik kiojuksaugapta innugiartoksoarnik, okautjigilauktamnik: Nangmagak atâtavit illilauktanga uvaptingnut okinersautiuk?

10 Innuosuktullo, ikkingutingita okautivât: Innugiartoksuit okautjigilauktatit: A tâtavit nangmagaksavut okumailuartilaukpa, igvit ckinersautiuk, imâk okautijaksarivattit: Erkekokotiga sillinersaujuksauvok atâtama koktoragingnit.

11 Illa atâtama nangmiulaukpâse okumaitomik, uvangale illautitsijomavunga sulle tamattomunga; atâtama ipperautanut ipperartorlaukpâse, uvangale Scorpionenut sugiaromavapse.

12 Taimak Jerobeame innugiartoksuit illuêngârdlutik illagællugit tikkipok Rehabeamut, uvlut pingajuane, sorlo attannek okausekalaungmat okarlune: Tikkipsaritse uvamnut uvlut pingajuane.

13 Attanniublo innugiartoksuit kiovait okumaitomik, attannertuinerlo angajuklit tunnilauktangat tapsomunga kemmakpâ,

14 Okarvigivaillo innuosuktut attannertuiningat malliklugo, okarlunelo: Atâtama nangmagaksase okumaitilaukpait, uvangale illautitsijomavunga sulle; atâtama ipperautanut ipperartorlaukpase, uvangale Scorpioninut sugiaromavapse.

15 Attanniublo innugiartoksuit nalelungilait; illingatitaungmat taimak Nalekamut, okautsiminik nellautitsikovlugo kauserilauktaminik Ahiakut Silomiokut Jerobeamemut, Nebatib erninganut.

16 Israelitille illunamassiangit tækkogamik attanniub tussaromangimagit, innugiartoksuit attannek kiovâ.t okarlutiglo: Sunamik illangiutivikarkita Davidemik? Isaiblo erninga pigingilavut, Israelitiôjose aileritse tuppekotipsingnut! Taimak manna igvit Davidekotivît iglunga tækkonâruk. Taimak Israelitit aivut tupperkotimingnut,

17 Israelible kittornganginut Judakut igloksoangine nunakartunut, tapkonunga Rehabeam attanniolaukpok.

18 Attanniublo Rehabeamib Adorame pimmaklerte tillingmago tagvunga, Israelitit illunamassiatik millorpât ujarkanut 
tokkolugo. Attannerle Rehabeame tuavidlune ikkivok aksalloalingnut, kemâlunelo Jerusalemut.

19 Taimak Israelitit apterput Davidib iglunganit, uvlok tamanna tikkilugo.

20 Israelitit illuêngarlutik tussaramik manna Jerobeame uttersimangmat, tikkilerput, kaikojaukolâlo illagêktunut, attanningortilâlo Israelitit illunamassianginut; kinguvarêllo Judakut assinginik, malliktokalaungilak attautsemiglonêt Davidib iglunganik.

21 Rehabeamelo Jerusalemut tikkiname Judakut illuêngardlutik, kinguvarêllo Benjaminekut kattertipaị, angutit innuosuktut sorsugungnartut 180000 dit, Israelitit kinguvarêngit sorsugvigijomavlugit, attannioviglo ama sattortautilugo Rehabeamemut Salomob erninganut.

22 Gudible okausinga pivok Semajamut Gudib angutekotinganut, okarlunelo:

23 Rehabeame Salomob erninga Judakut attanningat, Judakullo illunamassiatik, Benjaminekullo, innugiartoksuillo assingit, okautikit okarlutillo:

24 Imâk Nalegak okarpok: Takpaungartuksaungilase kattangutise Israelib kittorngangit sorsugvigilugit; angutit illunatik attunît iglomingnut angerarlît, tamanna uvamnit pingmat. Nalekpaillo Nalekab okausingit, utterlutiglo, ailarmiglo sorlo Nalegak okarlaungmat.

25 Jerobeamible Sicheme Efraimib kakkangine iglugaselliorpait, nunagivaillo; aularporlo tagvangat Pnuelo iglugaselliorpait.

26 Jerobeamele issumertuivok omattiminut: Attanniovik manna utterniarivok Davidib iglunganut;

27 Innugiartoksuit ukkoa takpaungartuksaupatta, tunnergutiksaniglo tunnijilutik Nalekab iglungane Jerusaleme; tagva innugiartoksuit ukkua omattingit sâlerniarivut angajokamingnut Rehabeamemut Judakut attaninganut, tokkoniarpângalo, sâlerniarlutiglo Rehabeamemut Judakut attaninganut.

28 Tagva Jerobeame kakimiojokarkojivok, sennalunelo nochangoangnik Goldeojungnik, okautivaillo: Ajornarpok illipsingnut majorarluse Jerusalemut, ahâk, Israelitiôjose tagva Gudise aularutjilauktut illipsingnik Egiptenemit.

29 Aipangalo nappava Betheleme, aipalo Daneme.

30 Tamannalo idluitullijutaulaukpok; innugiartoksuit aipa sângeriartormatsuk (opigilugo) Dane tikkidlugo.

31 Iglomiglo igloliorpoktauk poktôjune, ajochertuijungortitsivorlo innugiartoksoarnit erkanangitonerpaujunit, Levib kittornganginit pingitunik.

32 Uvloksiorvingmiglo akiksoivok takkit 8 ingata uvlungita 15 ingane, uvloksiorviub Judame adsinganik, tunnergutiniglo 
tunnijivok Altareme. Taimailiorpok Betheleme, tunnergutit tunnijaukovlugit nochangoangnut sennalauktangangnut; âkiksoilunelo Betheleme ajochertuijunik poktôjunut sennalauktaminut.

33 Tunnergutiniglo tunnijivok Altarib sennalauktame kângane Betheleme, takkit 8 ingata uvlungita 15 ingane, omattiminut issumertorlauktangane; akiksoilunelo taimak uvloksiorvingnik Israelib kittornganginut; tunnijilunelo tunnergutinik Altarib kângane, isserluanititsilunelo.

\section{KAPITEL XIII.}

Jerobeamib aggangita tokkungalerningit, âkiktauningillo nellautaijub tuksiarutingmago. Nellautaijub tokkotauninga Loewemut nalengininga Gudemik pivlugo.

AHAGLO, Gudib angutekotingita illangat tikkipok Judamit Bethelemut, Nalekab okausingagut, Jerobeame nangertillugo Altarib kiglingane isserluanititsivlune.

2 Nipliarporlo Altaremut Nalekab okausingagut, okarlunelo: Altare, Altare! Nalegak imâk okarpok: Ahâk, ernermik, erniangojokaromârpok, Davidib iglunganut, Josiamik attekaromârtomik, tamna tunnijijomârpok kângne pôktojub ajochertuijokotinginik, isserluanititsijunik kângne, innuillo sauningit ikkitso-mârpait kângne.

3 Nellonaikutamiglo tunnijivok uvlorme taipsomane, okarlunelo: Tamanna nellonaikutauvok, Nalekab tamanna okauserilaungmago: Ahâk, Altare koppiniarpok, arkjello kânganetok navitauniarput.

4 Attanniublo Gudib angutekotingata nipliartub Altaremut Bethelemetomut okausingit tussaramigit, aggane issakpait Altarib senniane, okarlunelo: Tigusiuk. Aggangillo issangmijangit tapsomunga tokkungalerput, ergitijungnangerpaillo.

5 Altarelo koppilerpok, arkjællo navitauvut Altaremit, nellonaikutak Gudib angutekotingata tunnilauktanga Nalekab okausingagut malliklugo.

6 Attannerlo kiovlune okarpok Gudib angutekotinganut: Nalekab Gudivit kênanga kennuvigiuktôk, tuksiutilungalo, aggakka âkiktaukovlugit. Tagva Gudib angutekotingata Nalekab kênanga kennuvigiva, attanniublo aggangit âkilerput sivurnganetullo îdlutik.

7 Attanniublo Gudib angutekotinga okarvigiva: Kailaurit angerarkattigilaunga, nekkoksitilutillo, pilliumik tunnitsivigijomavagit. 
8 Gudible angutekotingata attannek okautiva: Iglokotivît koppanganik tunnitsiviginajaralloarumga, sulle aikattiginajangilagit; inneme tamattomane niakojaktoromanginama, immeromalungilangalonêt immermik.

9 Imâk perkojaumagama Nalekab okausingagut, okautsaulungalo: Niakojaktortuksaungilatit, immertuksaunaglo, uttertuksaungilatillo apkosinekut attorlauktakut.

10 Aularporlo apkosiniub assiagut, utterlungilarlo apkome aivigilauktamine Bethelemut.

11 Betheleme nellautaijok innutokaujok nunakalaukpok; tapsomalo erningata tikkipa, unipkautilâlo pinniarnernik tamainik, Gudib angutekotingata taipsoma pinniarlauktanginik uvlorme tapsomane Betheleme, okausinginiglo Altaremut.

12 Atâtangatalo okarvigivait: Apkosinit nelliagut aularlaukka? Erningit tækkolaungmatta apkosinît nelliagut Gudib angutekotinga, Judamit tikkilauktok, aularlaungmat.

13 Tapsomalo ernine okautivait: Siutekoktôjok iksivaviktarsiuk pivlunga. Siutekoktôjorlo iksivaviktarpât tapsomunga, tapsomalo ikkivigiva,

14 Mallikpâlo Gudib angutekotinga, naipipâlo iksivatillugo nappartub Terebintemik attiggub attâne, okautivâlo: Igvit tamnauvît Gudib angutekotinga Judamit tikkilauktok? Tamna okarpok: Ahaila.

15 Tapsoma okautiva: Kait, angerarkattigilaunga, niakojaktorlutillo.

16 Tamnale okarpok: Utterkattigijungnangilagit, aiparijungnangilagillo, niakojaktorkattigijungnangilagit, immertorkattigini-arnaglonêt mâne inneme tamattomane.

17 Nalekab okausingittigut okautsaugama: Tagvane niakojaktortuksaungilatillonêt immertuksaunaglonêt uttertuksaungilatillo apkosinekut attorlauktakut tamaunga.

18 Tapsoma okautiva: Uvanga nellautaijôgivungatauk illiktut, Engeliblo okarkattigilaukpânga Nalekab okausingagut, okautilungalo: Uttertitsuk iglokotingnut, niakojartorkovlugo, immerkovlugolo. Sæglokipâle.

19 Tagva utterłattigivâ, niakojartorlunelo, immerlarmelo iglungane.

20 Iksivatillugolo nerrimarvingme, Nalekab okausinga tikkipok nellautaijomut uttertitsilauktomut tapsominga;

21 Kaigarsugvigivâlo Gudib angutekotinga Judamit tikkilauktok, okarlunelo: Imâk Nalegak okarpok: T'amanna pivlugo Nalekab kanninga nalelaunginangne, perkojarlo Nalekab Gudivît perkojutigilauktanga illingnut mallilaunginangne,

22 Uttersimagavillo, niakojartorlutillo imerlarpillo inneme okarutigilauktangane illingnut: Niakojaktortuksaungilatit, immertuksaunaglonêt; tamanna pivlugo timît tokkungajok atâtagijavit illuvinganut pijuksaungilak. 
23 Niakojaktorlaukârtillugolo immerlaukârdlumelo, siutekoktôjok iksivavik târpâ nellautaijomut uttertilauktaminut.

24 Aulartillugolo Loewib naipipa apkosinerme, tokolâlo; timingalo tokkungajok egitaumalaukpok apkosinermut, siutekoktojorlo nangerpok senniane, Loewelo nangerpok timib tokkungajub senniane.

25 Ahâglo innuit sennerkoput tækkolutiglo timemik tokkungajomik egisimajomik apkosinermut, Loewemiglo nangertomik timib tokkungajub senniane, tikkilutiglo okalarmiglo tamattominga iglugaseksoarne nellautaijub innutokaujub iglokarvigijangine.

26 Nellautaijorlo uttertitsilauktok taipsominga, tamattominga tussarame okarpok: Angutauvok Gudib pigijanga, Nalekab kanninganik nalelaungitok; tamanna pivlugo Nalekab tunnilankpa Loewemut, tapsoma alliktorlaukpâ tokkolâlo, okautsit malliklugit, Nalekab okauserilauktangit tapsomunga.

27 Okarporlo ernerminut: Siutekoktôjok iksivaviktarsiuk pivlunga. Iksivaviktarêrmatsuglo,

28 Aularpok tagvunga, nachvarpâlo timinga tokkungajok, egisimajok apkosinermut, siutekoktojorlo Loewelo timib tokkungajub sennianetillugik. Loewe nerrilaungimarikpok timemit tokkungajomit, siutekoktojorlo alliktorlaungila.

29 Tagva nellautaijub Gudib angutekotingata timinga tokkungajok kivikpa, illilâlo siutekoktôjomut uttertipâlo; nellautaijorlo innutokak tikkipok iglugaseksoarnut, ogurilugo illijartorlugolo.

30 Timelo tokkungajok illiva illuvekotiminut; ogurivâllo okarlutik: Kiksarnamêk kattangutik!

31 Illijartorêrmatsuglo ernerminut okarpok: Tokkojomâruma, tagva illijartorsinga illuvermut, Gudib angutekotingata illijartortauvigijanganut, saunikkalo illiniarsigik tapsoma sauningita sennianut.

32 Pijomârmat, sorlo kaigarsulaungmat Altaremut Bethelemêtomut Nalekab okausingagut, pôktôjullo iglunginut illunainut, Samariab iglugaseksoanginnêtunut.

33 Pijokartuble tamattoma kingorngane Jerobeamib apkotigijane idluitok allârlungila, amale ajochertuijungortitsivok innungnit erkanangitonerpângôjunit; nelliallo pijomajok ajochertuijungortitaurok, iglunut poktôjunêtunut.

34 Tamannalo illingalaukpok Jerobeamib iglungata idluitullininganut, asserortaukovlugo pêjartaukovlugolo nunamit. 


\section{KAPITEL XIV.}

Ahiab nellautaininga Jerobeame pivlugo. Jerobeamib toklunga. Rehabeamib attanniotininga, idluitulliningit, pitlartauningalo tamakkoa pivlugit.

NeLliUTOME taipsomane Abia Jerobeamib erninga kannimalaukpok.

2 Jerobeamelo nulliaminut okarpok: Parngnailerit, pissertorlutillo, kinalonêt mallugosulerkonago Jerobeamib nulliaringmattit, ailutillo Silomut. Ahâk nellautaijok Ania tagvanepok, okautjigilauktara, attanniojuksaugama innugiartoksoarnut tapkonunga ;

3 Neksarlutillo niakojanik tellimaujortunik, sâtuniglo, illulerkomiglo tættætomik igupsaujartut orksunginik, aivigilugolo, okarkovlugo illingnut, kannok nukapiab illinganiarianganik.

4 Jerobeamiblo nullianga taimailiorpok, parngnailerlunelo ailarmelo Silomut, tikkiporlo Ahiab iglunganut. Ahiale tautugungnarlaungilak, ijigik innutokaunermut aulasuêrmannik.

5 Nalekable Ahia okautiva: Ahâk Jerobeamib nullianga kaivok, suna apperkutigijomavlugo illingnit, ernine pivlugo, tamna kannimangmat. Tagva manna okautiniarpet imâk imâglo. Itteramelo manna tækkorngartausertorpok.

6 Ahiable ittiggangita kikkergallijarningat tussarmago ittertillugo upkoakut, okarpok: Kailerit kamunga, igvit Jerobeamib nullianga, sôg tækkorngartausertorkit? Tillijaumavunga illingnut, taigotaksamut okumaitomut.

7 Ailerit Jerobeamelo okautiuk: Imâk Nalegak Israelib Gudingat okarpok: Angijorortilaukpagit innugiartoksoarnit, attanningortilutillo innukotimnut Israelitinut;

8 Attannioviglo Davidib iglunganit aksarnigarilaukpara, tunnilaukparalo illingnut. Igville kivgaptut Davidetut îlaungilatit, perkojamnik mallilauktotut, agvertarlauktotullo malliklunga omamut tamânut, pinniarlune ijingma idluarijanginik kissiane;

9 Sugaluluartomiglo pinniarlaukpotit tamainit sivunernelauktunit, ailaukpotit Gudiblo assinginik, Gudingoanik kovimajunik sennalutit illingnut, ningaksaromavlunga, tunnungnullo egimavarma,

10 Taimaimat, ahâk, kanoêtomik pititsijomavunga Jerobeamib iglunganut, pêjaijomavungalo Jerobeamemit, innungniglo omajuniglo tamainik, kivgaujomik kivgaungitomiglo Israeleme; Jerobeamiblo iglungata kinguvângit pêjaromavakka, sorlo aktarnerlukut pêjartauvangmatta, nungumarikârtinago.

11 Kina Jerobeamekunit tokkoniartok iglugaseksoarne king- 
minut nerrijaujuksauvok, kinalo natemame tokkolertok tamna tingmitjet sillamêtut nerrijaksarivât; Nalekab tamanna okauserilaungmago.

12 Igvillo parngnailerit angerarlutilllo. Ittikkatillo ablorniarpatta iglugaseksoarnut, sorrusek tokkoniarpok.

13 Israelitillo illuêngarlutik oguriniarpât, illijartorniarlâllo. Tamna kissime Jerobeamekunit illuvermut pinniarmat, tapsomane sunamik Nalekab Israelib Gudingata kuviagijanganut illingajomik nachvartaungmat, Jerobeamib iglungane.

14 Nalegarle attannekartitsijomârpok Israelitinut, pêjaijomârtomik Jerobeamib iglunganik uvlorme taipsomane. Sunalo pilerêrka manna?

15 Nalekablo Israelitit annauleromârpait, aulakovlugit, sorlo ivik suvlolik immâne aulataungmat, Israelitillo nutsugomârpait nunamit ajungitomit mattomangat tunnilauktaminit atâtanginut, siamârtitsomârpaillo kôksub ungâtânut, tamanna pivlugo nappartokotimingnik (Gudingoalingnik) sennalaungmatta, Nalegak ningaksaromavlugo.

16 Israelitillo tunnijomârpait, Jerobeamib idluitullilauktub, Israelitiniglo idluitullititsilauktub ajorningit pivlugit.

17 Tagva Jerobeamib nullianga parngnailerpok, ailunelo, tikkilarmelo Tirzamut. Iglublo manoanganut tikkitillugo nukapiak tokkovok.

18 Illijartorpâllo, Israelitillo illuêngarlutik ogurivât, Nalekab okausingit malliklugit okauserilauktangit kivgamigut Ahiakut nellautaijokut.

19 Unipkautsillo tamakkoa assingit sulle Jerobeame pivlugo, kannok unatadlalaungmat, attanniotilaungmallo, ahâk, tamanna aglaksimavok Israelitit attanningita aglangine.

20 Jerobeamible attannioningata nelliuninga Jahreôvut 22 it. Sinnisilerporlo atâtagijaminut ailune, erningalo Nadabe attanningorpok innangêrlugo.

21 Taimak Rehabeame Salomob erninga attanniolaukpok Judame. Rehabeame Jahrekalaukpok 41 ginik attanningorame, attanniotilaukporlo Jahrit 17 it nâlugit Jerusaleme iglugaseksoarne Nalekab annerilauktangine Israelib kinguvarênginit tamainit, atterminik pititsikovlugo tagvunga. Anânanga attekalaukpok Naemamik Amonitereôjok.

22 Judakullo pinniarlaukput Nalekab idluigijanginik, ningaksarnersarivâllo tamainit atâtagijangita pinniarlauktanginit, idluinermingnut pinniarlauktamingnut.

23 Sennalaukpullotauk ingmingnut poktôjunik, nappajuniglo, nappartuniglo, kakkarulangne pôktojune illunaine, nappartullo tungojortut illunatik attâne.

24 Ajortullijokalaukpoktauk nuname; nellojullo Nalekab pê- 
jarlauktangita Israelib kittorngangita sivorngane makkojungnartulliningit attorpait.

25 Attanniuble Rehabeamib Jahringita tellimangane Sisak Egiptenemiut attaningat takpaungarpok, Jerusaleme sorsugvigilugit.

26 Tiguvaillo perkutit piơianartut Nalekab iglunganit, attanniublo iglunganit, tamaitalo tigujaujungnartut, tiguvaillo sappulutaksat Goldeôjut Salomob sennajaukolauktangit.

27 Tapkoalo inninginut attaniub Rehabeamib sappulutaksat kannujarmit sennajaukovait, tunnivaillo sorsuktuksat angajokangita, attanniub iglungata upkuanganik udsertortut agganginut.

28 Attannerlo Nalekab iglunganut itterlaurangat, sorsuktuksat akjarkattarpait, uttertikattarlaitalo kingorngane sorsuktuksat iglunganut.

29 Unipkautsille tamakkoa assingit sulle Rehabeame pivlugo, pinniarlauktangillo tamaita, ahâk, tamanna aglaksimavok Judakut attanningita aglangine.

30 Unatadlarnekatsainarlaukporle Rehabeamib Jerobeamiblo akkorngangne innositik nâlugik.

31 Rehabeamelo sinnisilerpok, atâtagijaminut ailune, illijartortaulunelo atâtagijaminut Davidib iglugaseksoangine. Anânangalo attekalaukpok Naemamik Amonitereôjok. Erningalo Abiame attanningorpok innangêrlugo.

\section{KAPITEL XV.}

\section{Judakut attanningit Abiame, Assa, Josufatelo. Israelitillo attannigit Nadabe, Baesalo.}

JEROBEAMIB Nebatib erningata Jahringita 18 ingane Abiame attanningorpok Judakunut,

2 Attanniotilunelo Jerusaleme Jahrit pingasut nâlugit. Anânanga attekalaukpok Maechamik Abisalomib panninga.

3 Tamnalo agvertarpok atâtame idluitulliningine tamaine piniarlauktangine tapsoma sângane, omattingalo illingamarilaungilak Nalekamut Gudiminut, sorlo atâtangata Davidib omattinga-îlaungmat.

4 Davidele pivlugo, Nalekab Gudingata kaumautekarkova Jerusaleme, erninga pitilaungmago tapsoma kingorngane, Jerusalemelo sokkosertailitingmagit.

5 Davide pinniarlaungmat Nalekab idluarijanganik, allailaungimallo tamainit perkolauktanginit tapsomunga, innôsermine tamât, pijokartok Uriamut Hethitereôjomut pivlugo kissianeungitok. 
6 Unatadlarnekatsainarlaukporle Rehabeamib Jerobeamiblo akkorngangine innôsingane tamât.

7 Unipkautsille tamakkoa assingit sulle Abia pivlugo, pinniarlauktangillo tamaita, ahâk tamanna aglaksimavok Judakut attanningita aglangine. Unatadlarnekatsainarlaukporle Abiablo Jerobeamiblo akkorngangne.

8 Abialo sinnisilerpok, atâtagijaminut ailune, illijartorpâllo Davidib iglugaseksoangine. Erningalo Assa attanningorpok innangêrlugo.

9 Jerobeamib Israelitit attanningata Jahringita 20 ingane Assa attanningorpok Judakunut;

10 Attanniotilaukporlo Jerusaleme Jahrit 41 git nâlugit. Anânanga attekalaukpok Maechamik, Abisalomib pannia.

11 Assalo pinniarpok Nalekab idluarijanganik, atâtangatut Davidetut.

12 Ajortullijullo pêjarpait nunamit, Gudingoallo atâtagijangita sennalauktangit illunaita tagvanêtsungnaitipait.

13 Taimaktauk anânane attanniojungnaitipa, Gudingoamik sennalaungmat ingminut nappartune. Assablo Gudingoakotinga pêjarpa, ikkipâlo kôb Kidronemik attiggub naksangane.

14 Poktojulle pêjartaulaungilet. Assable omattinga nellagorlune Nalegak ungagiva innosermine tamât.

15 Silberelo Goldelo, illulerkutillo, atâtangata irsornaitilauktangit, nangminerlo ivsornaitilauktane ittertipait Nalekab iglunganut.

16 Unatadlarnekatsainarlaukporlo Assablo Baesablo Israelitit attaningata akkorngangne innôsermingne tamât.

17 Baesale Israelitit attaningat takpaungarpok Judakut sorsugvigivlugit, Ramalo igluliorsavait, kinalonêt aularungnartuksaukonnago aijungnartuksaukonnagolonêt Assab Judakut attanningata miksânut.

18 Tagva Assab Silbere Goldelo Nalekab iglungata perkutetalinganelauktut sulle, attanniublo iglungata perkutitalinganelauktut tiguvait tunnilaitalo kivgame agganginut, tillivaillo Benhadademut Tabrimonib Hesionib erningata erninganut, attanniôjomut Siriame, iglokalauktomut Damașkuseme, okautsaukovâlo:

19 Angerkattigêngnekarpok uvamne illingnelo, atâtamnelo atâtangnelo; tamanna pivlugo pilliumik tujoivigivagit, Silberemik Goldemiglo, angerkattigêngnek illingnêtok Baesamêtorlo Israelitit attaningane serkomilugo pikovlutit, tamna aularkovlugo uvamnit.

20 Benhadadiblo attannek Assa nalekpa, angajokâkotinelo tillivait Israelitit iglugaseksoanginut, ullimârpaillo Ijon, Danelo, Abelbetmaechalo, Kinerothelo illûnât, Naftalikut nunangane tamât. 
21 Baesab tamanna tussaramiuk nokarpok igluliorlune Ramamik, utterivlunelo Tirzamut.

22 Attanniuble Assab Judakut illunamassiangit kaikojaukovait, attauserlonêllo tikkingitsariakalaungilak, ujarkello, kejuillo Baesab igloliorutigilauktangit tiguvait akjatorlaitalo Ramamit; attanniublo Assab tamakkonangat Geba Mizpalo Benjaminekune igluliorpait.

23 Unipkautsit tamakkoa assingit sulle Assa pivlugo, sôngoningalo tamât, pinniarlauktangillo tamaita, iglugaseksuillo iglugaseliorlauktangit, ahâk tamanna aglaksimavok Judakut attanningita aglangine. Innutokaunime nelliuningane kissiane ittiggalerilaukpok.

24 Assalo sinnisilerpok atâtagijaminut ailune, illijartortaulunelo atâtagijaminut, Davidib, atâtagijame iglugaseksoangine. Josafatelo erninga attanningorpok innangểrlugo.

25 Nadabele Jerobeamib erninga attanningorpok Israelitinut, Assab Judakut attanningata Jahringita aipangane, attanniotilunelo Israelitinut Jahrik magguk nâlugik,

26 Pinniarporlo Nalekab idluigijanginik, agvertarlunelo atâtame apkosiningine, idluitullininginelo, idluitullititsijutigilauktangine Israelitinik.

27 Baesale Ahiab erninga Isascharekunit pijok, angerkattigêngnekartitsivok pivlugo, ullimârpâlo Gibethoneme Filisterit nunanganêtome. Nadab Israelitillo illuêngarlutik Gibethon illumangmagit.

28 Taimak Baesab tokkopa Assab Judakut attanningata Jahringita pingajuane, attanningorlunelo innangêrlugo.

29 Attanniôgamelo manna, Jerobeamib iglomiokotingit illunaita ullimarpait, amiakokarkojilungilarlo Jerobeamekunit annernelingmik, nungutsikârtinago tapsominga, Nalekab okausingit okauserilauktangit kivgamigut Ahiakut, Silomiokut, malliklugit;

30 Jerobeamib idluiningit pivlugit, idluitullinerilauktangit, idluitullititsijutigijangillo Israelitinik, kajungersainermut ningaksaijutigijangillo Nalekamik Israelib Gudinganik.

31 Unipkautsille tamakkoa assingit sulle Nadabe pivlugo, pinniarlauktangillo tamaita, ahâk tamanna aglaksimavok Israelitit attanningita aglangine.

32 Unatadlarnekatsainarlaukporlo Assab Baesablo Israelitit attanningata akkorngangne innôsermingne tamât.

33 Assab Judakut attanningata Jahringita pingajuane, Baesa Ahiab erninga attanningorpok Israelitit illunamassianginut, Tirzame, Jahrit 24 git nâlugit,

34 Pinniarlaukporlo Nalekab idluigijanginik, agvertarlunelo Jerobeamib apkutigijangine idluitullininginelo idluitullititsijutigijangine Israelitinik. 


\section{KAPITEL XVI.}

Baesa, Ella, Simri, Anri, Ahabelo attanniolaukitut Israelitimut.

Ninte Nalekable okausinga tikkipok Jehumut Hananib erninganut Baesa pitjutigællugo, okarlunelo:

2 Tamanna pivlugo sannermit makkitilaurapkit, attanningortilutillo innukotimnut Israelitinut, igvillo agvertaravit Jerobeamib apkotigijangane, idluitullititsilutillo innukotimnik Israelitinik, ningaksaramgalo tapkoa idluitullininginut;

3 Ahâk tamanna pivlugo Baesab kinguvangit iglokotingatalo kinguvangit pêjaromavakka, iglokotillo illingatitsomavara Jerobeamib Nebatib erningata iglungatut.

4 Kina Baesakunit tokkojok iglugaseksoarne tamna kingmit nerrijaksarivât, kinalo natername tokkojok tamna tingmitjet sillamêtut nerrijaksarivât.

5 Unipkautsille tamakkoa assingit sulle Baesa pivlugo, pinniarnerijangillo, sôngoningalo, ahâk, tamanna aglaksimarok Israelitit attanningita aglangine.

6 Baesalo sinnisilerpok atâtagijaminut ailune, illijartortaulunelo Tirzame. Erningalo Ella attanningorpok innangêrlugo.

7 Nalekab okausingittauk tikkiput nellautaijokut Jehukut Hananib erningagut Baesamut iglunganullo, idluinek tamât pivlugo pinniarnerijanga Nalekab sângane, ningaksarlugo aggangme pinniarninginut, Jerobeamib iglungatut îlerkovlugo; taimaglotauk tamanna pivlugo, taipkoa ullimârlaungmagit.

8 Assab Judakut attanningata Jahringita 26 ingane, Ella Baesab erninga attanningorpok Israelitinut, Tirzame Jahrîk magguk nâlugik.

9 Kivgangale Simri, angajokaujok kammutit ketterkanginut, angerkattigêngnekartitsivok akkerartorlugo. Tirzamelaukporle Weinetorlunelo tallalunelo Arzab pimaklerteôjub Tirzame iglungane.

10 Simrile itterpok, ullimârpâlo tokkolugo, Assab Judakut attanningata Jahringita 27 ingane, attanningorlunelo innangêrlugo.

11 Attanniogamelo, iksivalunelo taipsoma iksivautangane, Baesab iglomiokotingit illunaita ullimârpait, amiakokarkojingilarlo tapsomangat kinamiglonêt, illanganiglonêt, illanarijanganiglonêt.

12 Taimak Simrib Baesab iglomiokotingit illunaita pêjarpait, Nalekab okausingit malliklugit, okauserilauktangit Baesa pivlugo nellautaijokut Jehukut;

13 Baesab idluiningit illunaita, erningatalo Ellab idluitulli. 
ningit pivlugit, pinniarlauktangit, Israelitiniglo idluitullititsivlutik, Nalegak Israelib Gudinga ningaksarlugo, kivgartornermingnut Gudingoanik.

14 Unipkautsille tamakkoa assingit sulle Ella pivlugo, tamaitalo pinniarlauktangit, ahâk tamanna aglaksimavok Israelitit attanningita aglangine.

15 Assab Judakut attanningata Jahringita 27 ingane Simri attanningorpok Tirzame uvlut 7 it nâlugit. Innugiartoksuillo tangmarsimalaukput Gibetonit Filisterit nunanganêtut sivurâne.

16 Innugiartoksuille tangmarvingne tussaramik okartokarmat Simri angerkattigêngnekartitsimangmat, attannerlo ullimârmago ; tagva Israelitit illuêngarlutik uvlorme taipsomatsainaujome Amri sorsuktuksaksoakattigêt angajokangat attanningortipât tangmarvingme.

17 Amrilo Israelitillo illuêngarlutik illagællugo majorarput Gibetonemit, illumavaillo Tirza.

18 Simrile tækkogame iglugaseksuit sâlagijauniarmatta, itterpok attanniub igloksoangata sôngoninganut, ikkipâlo attanniub igloksoanga, tokkolunelo,

19 Ajornine pinniarlauktane pivlugit, pinniarame Nalekab idluigijanginik, agvertaramelo Jerobeamib apkot gijangane, tapsomalo idluiningine, pinniarlauktangine, Israelitit idluitullitilugit.

20 Unipkautsit tamakkoa assingit sulle Simri pivlugo, kannorlo angerkattigêngnekartitsilaungmat, ahâk, tamanna aglaksimavok Israelitit attanningita aglangine.

21 Taipsomane innugiartoksuit aviutivut maggolivlutik; ketterkangita aipaingita Tibni Ginatib erninga ungagivât, attanningortilugo, ketterkangitale aipaingita Amri ungagivât.

22. Innugiartoksuille Amrib ungagijingit ajugaulaukput innugiartoksoarnut Tibnib Ginatib erningata ungagijinginut. Tibnilo tokkovok, tagva Amri attanningorpok.

23 Assab Judakut attanningata Jahringita 31 ingane, Amri attanningorpok Israelitinut, attanniotilunelo Jahrit 12 it nalugit, Tirzamêdlune attanniôtivok Jahrit 6 it nâlugit.

24 Kakkarlo Samariamik attelik pissiariva Semeremit Tallentîngnut maggungnut Silberemik, iglugaselliorlunelo kakkab kângane, iglugaseksuillo iglugaselliortane atserarpait kakkab innuata Semerib attinga malliklugo Samariamik.

25 Amrilo pinniarpok Nalekab idluigijanginik, sugaluluarlaukporlo sivorlerijaminit tamainit.

26 Agvertarlwnelo Jerobeamib Nebatib erningata apkotigijangine illunaine, idluininginelo, idluitullititsijutigijangine Israelitinik, Nalegak Israelib Gudinga ningaksarlugo kivgartornermingnut Gudingoanik.

27 Unipkautsille tamakkoa assingit sulle Amri pirlugo pin- 
niarlauktangillo tamaita, sôngoningalo attorlauktanga, ahâk tamanna aglaksimavok Israelitit attanningita aglangine.

28 Amrilo sinnisilerpok atâtagijaminut ailune, illijartortauvorlo Samariame. Erningalo Ahabe attanningorpok innangêrlugo.

29 Assab Judakut attanningata Jahringita 38 ingane Ahabe Amrib erninga attanningorpok Israelitinut; attanniotivorlo Israelitinut Samariame Jahrit 22 it nâlugit.

30 Ahabelo Amrib erninga pinniarlaukpok Nalekab idluigijanginik, sugaluluartomik illunainit sivorlerilauktaminit.

31 Suglugilaukpa agvertarame Jerobeamib Nebatib erningata idluiningine, nullianikpâlotauk Isebele Etbaalib Zidonemiut attanningata pannia; Baalelo kivgartoriartorpâ, tuksiarvigivâlo.

32 Altarelo sennava Baal pivlugo, Baalib iglungane sennalauktamine tapsomunga Samariame.

33 Nappartuniglo illijivok; Ahabelo sugaluluartomik pinniarpok Nalegak Israelib Gudinga ningaksarlugo, Israelitit attanninginit illunainit sivorlerilauktaminit.

34 Nelliutome taipsomane Hielib Bethelemiub Jericho iglugaselliorpait. Erninga angajuklek Abirame akkiliutaulaukpok tungavik illilauramiuk, erningalo nukârdlek Segube, akkiliutaulaukpok upkoaksuit illilauramigit; Nalekab okausingit malliklugit, okauserilauktangit Josuakut Nunib erningagut.

\section{KAPITEL XVII.}

\section{Nellautaijub Eliab pinniarningit.}

Elialo Tisbitemiok Gileademiut illangat okarpok Ahabemut: Sorlo miksekârtomik Nalegak Israelitit Gudingat, sângiartara innôngmat, Jahrine tamakkonane mitsungniangilarlonêt, sillalungniangilarlonêt, kissiane uvanga okaruma.

2 Nalekablo okausingata tikkipa, okarlunelo:

3 Aularit ovangat, kingmuarlutillo kaulerviub nellânut, ijerlutillo kôngme Kritemik attelingme, kôktome Jordanemut;

4 Kôngmillo immertuksauvotit; tullukello perkomavakka pattangaititsikovlugit illingnik tagvane.

5 Tagva aivok taikunga, pinniarlunelo Nalekab okausingit malliklugit, aivorlo ingilunelo kôngme Kritemik attelingme, Jordanemut kôktome.

6 Tullukello atsivigikattarpât kakkojamik, nerkemiglo uvlâkut, unnukullo, immerporlo kôngmit.

7 Pijokalaukporlo uvlut kapsit kingorngane, kôk ipterilingmat; sillalulaungimat nuname.

8 Tagva Nalekab okausingata tikkipa, okarlunelo: 
9 Parngnailerit ailutillo Zarpatemut, Zidonib kamnitanganêtomut, tagvanêtuarlutillo; tagvane uigarnerit illangat perkomagapko pattangaititsikovlugo illingnik.

10 Parngnailerporlo ailunelo Zarpatemut. Tikkimallo iglugaseksuit upkoaksoanginut, ahâk, tagva uigarnekarpok tagvane kejungnik kattersoijomik. Tapsomalo kaikova, okarlunelo: Immiârsungmik aiklerutilaunga illulerkume, immerkovlunga.

11 Aitillugolo aikleromamut, nipliarvigiva okarlunelo: Tamuamik niakojamik kaitsivigikassiutilaungatauk.

12 Arnak okarpok: Sorlo miksekârtomik Nalegak inôngmat, igamajokalungilanga sumiglonêt, tigumiakamik sennaukamik kissiane kattangme, orksuârsungmiglo publaujarme. Ahâglo kejuit illanginik kattersimavunga, itterlungalo, âkiksoromavlugo pivlunga, erneralo pivlugo, nerrikovlunuk, tokkolunuglo.

13 Eliab okautiva: Sivuraniarnak, ailerit pinniarlutillo, sorlo okarlauravit; sivurlermigle sâtoârsungmik igalerit tapkonangat pivlunga, annitsivigilungalo tamattominga, pivlutille ernillo pivlugo sennalerit kingorngane;

14 Imâk Nalegak Israelib Gudinga okarmat: Sennaugak katangme ketaulertuksaungilak, publaujarlo orksutalik ajoksartuksaungilak, uvlok taimna Nalekab sillalukojivia nuname tikkilugo.

15 Arnak aivok, pinniarlunelo, sorlo Elia okalaungmat. Tamnalo nerrivok, arnarlo iglomiokatingillo uvlut kapsit nâlugit.

16 Sennaugak katangme kêtaunersaupallialaungilak, publaujarlo orksuktalik ajoksarlaungilak, Nalekab okausingit okauserilauktangit Eliakut malliklugit.

17 Pijokartullo tamakkoa kingorngane arnab iglomêvigijangata erninga kannimalerpok, kannimasingalo taimak sôngotigijolaukpok annernekarungnailermat tapsomane.

18 Tapsomalo arnab Elia okautiva: Suvigivagit igvit Gudib angutekotinga? Tikkisimavarma idluinikka erkartaukovlugit, emeralo tokkotaukovlugo.

19 Tapsomalo okautiva: Ernît tunniuk uvamnut. Tiguvâlo sânganit, majorarlunelo innemut iglogijaminut, illivâlo sinnigvingminut.

20 Nalegarlo nipliarvigiva okarlunelo: Nalegak Gudîk, vigarnek iglomêvigijara, taimak idluitullivigilaukiuk erninga tokkogangne?

21 Tessiterporlo pingasuêrtorlune sorrutsib kângane, nipliarlunelo Nalekamut okarlunelo: Nalegak Gudîk, sorrutsib oma tarninga utterkolauruk tapsomunga.

22 Nalekablo Eliab nippinga tussarpa; sorrutsiblo tarninga utterpok tapsomunga omarlunelo. 
23 Eliablo sorrusek tiguva, âlâlo unnunga iglomut, tunnivâlo anânanganut, okarlunelo: Ahâk tagga, ernît innôvok.

24 Arnablo Elia okautiva: Manna illitarksivunga Gudib angutekotigingmattit, Nalekablo okausinga kannerne miksekârtongmat.

\section{KAPITEL XVIII.}

\section{Eliab tokkotsininga Baalib ajochertuijokotinginik; okarningalo sillalungniarmat.}

\section{NELLIUTORLO sivitôjok kângermat, Nalekab okausingata Elia tikkipa, Jahrit pingajuane, okarlunelo: Ailerit tækkotilutillo Ahabemut, sillalukojikovlunga nuname.}

2 Elialo aivok tækkotilune ingminik Ahabemut. Kângnartoksoakarporle Samariame.

3 Ahabelo Obadja, pimaklertokotine kaikova. (Obadjable Nalegak sivoragiva angijomik.)

4 Isebelible Nalekab nellautaijokotingit nungutilaungmagit, Obadjab nellautaijut 100 dit tiguvait, ijerpaillo kairosungmut, mâne 50 git, ikkane 50 git, pattangaitilaitalo kakkojanik, imermiglo.

5 Taimak Ahab Obadja okautiva manna: Nuna ingerarvigiuk, immektarvingnut, kôngnullo illunainut, iviksukanik nachvarungnarmangapta, horsillo, horsiojartullo piulilugit, nergjutit illunamassiatik tokkokonnagit.

6 Nunalo avikpâk ingmingnut ingergarvigijomavlugo. Ahabe innutolune ingerarpok apkosinît illangane, Obadjalotauk innutolune apkosiniub tapsoma assiane.

7 Obadjalo manna apkosinermêtillugo, ahâk, tagva Eliab pârpâ; illitarilerpâlo, pamakterporlo kênane pûlugo okarlunelo: Igvit Eliaungilatit Nalegara?

8 Tamna okarpok: Aukak; angajokait okautijartoruk: Ahâk Elia mânêpok.

9 Tamnale okarpok: Sunamik idluitullilaukik, kivgait tunnijomagangne Ahab agganginut, tokkotsikovlugo uvamnik?

10 Sorlo miksekârtomik Nalegak Gudit inôngmat, innukattigêksoakangilak, attanniovikaranelo, nalekama tilliklervigilaungitanganik, kennerlutit. Okalaukpattalo: Mânêngilak, tagva angermaringnermik tigusilaukpok attanniovingmit mattomangat, innukattigêksoarnillo taipkonangat, nachvartaulunginavit.

11 Mannalo igvit okarpotit: Angajokait okautijartoruk: Ahâk Elia mânêpok. 
12 Ainniarajarumalo manna illingnit, tagva Nalekab annerningata nutinajarpâtit kaujimangitamnut, kainiarumalo Ahabelo okautilugo tammattominga, tapsomalo nachvarlungipattit; tagva tokkoniarpânga. Kivgavillo Nalegak sivoragigalloarpâ innuosungnimnit.

13 Nalegara okautsaumalungilâk pinniarlauktamnik Isebele tokkotsitillugo Nalekab nellautaijokotinginik? Nalekab nellautaijokotingit 100 dit jjerlaurapkit, mâne 50 git, ikkanelo 50 git kairosungmut, pattangaitilugillo, kakkojamik immermiglo?

14 Igvillo manna okarpotit: Angajokait okautijartoruk: Elia manêpok, tokkotsikovlugo uramnik.

15 Elia okarpok: Sorlo miksekârtomik Nalegak Zebaoth sangiartara inôngmat, uvlome tækkotitsomavunga tapsomunga.

16 Tagva Obadjab Ahabe pachrearpa, okautilâlo tamattominga. Ahablo Elia pachrearpa.

17 Ahablo Elia tækkogamiuk, Ahab okautiva: Igviovit Israelitinik asseroijotit?

18 Tamnale okarpok: Uvanga Israelitinik asseroilungilanga, igville, atâtavillo iglomiokatingit, Nalekab perkojangit kemmalaurapsigik, agvertarapselo Baale malliklugo.

19 Atte manna tilliklilaurit Israelitit illuêngarlutik kattimalerkokit uvamnut kakkame Karmeleme, Baaliblo nellautaijokotingit 450 git, taimaktauk nappartut nellautaijokotingit 400 dit Isebelib nerrimavianit nerrijut.

20 Taimaglo Ahabe tilliklerpok Israelib kittorngangita illunatik akkornganut, nellautaijullo taipkoa kattimakovait kakkame Karmelemik attelingme.

21 Tagva Eliab innugiartoksuit illunamassiangit nangeriartorvigivait, okarlunelo: Kannok akkunêrtigijomik tussiarkise iglugêngne? Nalegak Gudeokpat, tagva tamna malliklugo agvertaritse; Baaleokpalle, tagva tamna malliklugo agvertaritse. Innugiartoksuillo kiolungilât.

22 Tagva Eliab innugiartoksuit okautivait: Uvanga innutôlunga amiakovunga, nellautaijovlunga Nalekamut, Baalible nellautaijokotingit 450 geôvut.

23 Tagva manna tunnitsivigitigut tuktuvângnik maggungnik, tapkoa annerosulerlit tuktuvâk aipânik, avgorlugolo, illilugolo kejuit kânginut ikkomakartailile, tagva uvanga tuktuvâk aipânga tigujomavara illijomalugolo kejuit kânginut, ikkomakartitsomanginivakka.

24 Tagva illipse Gudipse attinga nipliarvigisiuk, uvangalo Nalekab attinga nipliarvigijomavara. Gudîglo nelliak ikkomamut kioniartok, tamna Gudeôle. Innugiartoksuillo illuêngarlutik kiovut: Taimak idluarpok.

25 Eliablo Baalib nellautaijokotingit okautivait: Tuktuva- 
kotise annerisiuk, sivorliolerluselo, illipse unnuktogapse, Gudipselo attinga nipliarvigisiuk, ikkomakartiniarnagole.

26 Tapkoalo tuktuvâk tiguvât tunnijanga tapkonunga, tunnergutautipâllo, Baabliblo attinga nipliarvigivât uvlângmit uvlub ketterarninga tikkidlugo, okarkattarlutiglo: Baal tussartigut! Tagvanele nippekalaungilak, kionekalaungilarlo. Tussiarpullo Altare sennalauktatik avatagorvigilugo.

27 Ketterarmallo manna, Eliab mittautigilerpait, okarlunelo: Nippekortôvluse kaggudlaritse, Gudeôngmat issumaksarsiorpallaivok, sullijaksakarporlonêt, assinuarporlonêt, sinnikporlonêt, tuppalerkovlugo.

28 Tapkoalo nippekortôvlutik kaggudlarput, killerlutiglo ingminik savingnut nuvutsiariktunullo illutsitik malliklugit, aunârkârtinagit.

29 Ketterarmallo nellautaivut, tunnergutiksak nerkiksak tunnijaukârtinago; tagvanele nippekalaungilak, kionekaranelonêt, kamanekangilarlonêt.

30 Tagva Elia innugiartoksuit illunamassianginut okarpok: Kaileritse tamaunga uvamnut! Innugiartoksuillo nangeriartorvigingmatsuk, Nalekab Altarekotinga serkomitaumajok âkiksorpa.

31 Ujarkallo 12 it tiguvait Jakob kittorngangita kinguvarêngita unnurningit malliklugit, (Nalekab okausingita okarvigijangata imâk: Israelemik attekartuksauvotit.)

32 Ujarkanillo tapkonangat Altaremik sennavok Nalekab attingane, ittersalliorlunelo Altarib avâtâne, oktutut magguktut kângasutiksaliktitut silliktigijomik,

33 Kejuillo ketjiorpait, tuktuvaglo avgorpa, illivâlo kejuit kânginut,

34 Okarlunelo: Katet sittamat tættælugit immermik ailersigik, kovilugillo tunnergutiksamut ikkoalajaksamut kejungnullo. Okarporlo: Taimailiupsaritse. Taimailiupsarpullo. Tamnalo okarpok: Pingajuanik taimailioritse. Tapkoalo pingajuanik taimailiorput.

35 Immerlo kôkpok Altarib avatâne, ittersarlo ullipkilaurivok immermik.

36 Tunnergutekarviublo nerkiksanik nelliuningane, Elia nellautaijok tagvungariarpok okarlunelo: Nalegak Abramib, Isaublo, Israeliblo Gudingat, uvlome kaujijaule igvit Gudeôgavit Israeleme, uvangalo kivgaugama illingnut, taimailiorlauramalo okautsitit malliklugit.

37 Tussarlaunga, Nalegak tussarlaunga, innugiartoksuit tapkoa kaujilerkovlugit, igvit Nalegak Gudeôgavit, tapkoa omattinginik sagiartitsikovlutit kingorngane!

38 Tagva Nalekab ikkomanga kattakpok pângat, nerrivaillo tunnergutiksak ôtaksak, kejuillo, ujarkello, ivjorlo immerlo ittersangmêtok alluktorpa. 
39 Innugiartoksuillo tamanna tækkogamitsuk, pamakterput okarlutiglo: Nalegak Gudeôvok, Nalegak Gudeôvok!

40 Eliable okautivait: Baalib nellautaijokotingit tigulersigik, tapkoa illangallonêt kemâkonnago. Tiguvaillo. Eliablo attersartipait kôngmut Kisonemik attelingmut, tokkopaillo.

41 Eliablo Ahabe okautiva: Paunga majorarit, nerrilutillo immerit sillaludlarnerub mapkullungninga tussarapko.

42 Ahablo takpaungartillugo nerrijomavlune, immeromavlunelo, Elia majorarpok Karmelib kemerdluata nuvuanut, okkungalunelo nunamut, niakonelo pitipa serkotingme akkorngangnut,

43 Okarlunelo kivgaminut: Majorarit paunga tækkosarlutillo immarbingmut. Tamna majorarpok, tækkosarlunelo, okarporlo: Pittakangilak sunamik tagvane. Elia okarpok: Aipsarkattarit tagvunga 7 êrtorlutit.

447 inganelo, okarpok: Ahâk nuvujârsuk kongmuarpok immarbingmit, angutib aggangita sillingningatut itok. Elia okarpok: Takpaungalerit Ahabelo okautiuk: Parngnairit attersarlutillo, sillalungmut tikkitaukonnak.

45 Annikitoârsuglo killak kernertaumavok nurujanut, annoremullo, sillalungnekarporlo angijomik. Ahabele kemukserpok, ailunelo Jesreelemut.

46 Nalekablo aggangita Elia tikkipât, tapsomalo makkitine tapseruserpa, akpangerlunelo Ahab sivurngane, Jesreel tikkidlugo.

\section{KAPITEL XIX.}

Eliab kemâninga Isebel pivlugo; Gudib sakkerninga Eliamut; Elisab kaikojauninga.

A HABLO Isebele okautiva tamainik Eliab pinniarninginik, kannorlo Baalib nellautaijokotingit illunaita saviksoarmut tokkolaungmagit.

2 Tagva Isebele tillijaksamik tilliklerpok Eliamut, okautsaukovalo: Gudit immâglonêt, immâglonêt, pinniarvigilinga, kaupat nelliutome tamattomane innôtsit pinniarvigilungikupko, taipkoa attunît innôsingititut.

3 Tamattomingalo tækkogame, parngnailerpok, ailunelo innôtsine pivlugo, tikkilarmelo Bersebamut Judamêtomut, kivganelo kemmakpa tagvunga.

4 Tamnale sunakajuitomut aivok ingmeriaktut kanningitigijomik, tikkiporlo ingilunelo kigutangernaujakutit, attânut, tuksiarporlo tokkonerminik, okarlunelo: Namaksivok, taimak manna Nalegak tarniga tigulauruk; nekkornersaulungilanga atâtagijamnit. 
5 Inarlunelo sinnilarmelo kigutangernaujakutit attâne. Ahâglo, Engelib aktorpa, okautilalo: Makkitit nerrilutillo.

6 Tækkosarporlo, ahâglo, niakungata tungâne niakojakarpok jarrasimajomik, publaujakarlunelo immalingmik. Nerrijarêramelo immerêrlarmelo innapsarpok.

7 Nalekablo Engelinga tikkipsarpok aipanganik, aktorpalo, okarlunelo: Makkitit nerrilutillo: apkosinek attoriakartat sivitôngmat.

8 Makkiporlo, nerrilunelo immerlarmelo, pissukporlo nerkiksab tamattuma nekkoksititsininganut, uvlut 40 git unnuallo 40 git nâlugit, Gudib kakkanga Horebe tikkilugo.

9 Itterporlo tagvane kairosungmut, siniktarlunelo takamane. Ahâglo, Nalekab okausingata tikkipa, okautilâlo: Sunasiorkît mâne Elia?

10 Tamna okarpok: Illungertorlaukpunga. Nalegak Gude Zebaoth pivlugo; Israelib kittorngangita angernerilauktat kemangmatsuk, Altarekotitillo serkomilaukpait, nellautaijokotitillo tokkolaukpait saviksoarmut, uvangalo amiakotôvunga, pinnasuarpullo innôsimnik, pêjaivigijomavlunga.

11 Tamnalo okarpok: Annilerit, nangeriartorlutillo kakkame Nalekab sângane. Ahâglo Nalegak kângerpok, annorelo angijoksoak sôngojorlo, kakkanik kopluijok, kairtuniglo serkomitsijok sivurlerpok Nalekamik; Nalegarle annoremêlaungilak. Annorible kingorngane nunab sajukpiludlarninga; Nalegarle nunab sajukpiludlarninganêlaungilak.

12 Nunab sajukpiludlarningata kingorngane ikkoma; Nalegarle ikkomamelaungilak. Ikkomablo kingorngane sulluksuârsuk tussarnertok.

13 Eliab tamanna tussaramiuk, tagva kênane ullikpa ulliksoarminut, annilunelo, nangeriartorlarmelo kairosub pânganut. Ahâglo, nippib tikkipa, okarlunelo: Sunamik sullijaksakarkît mâne Elia?

14 Tamna okarpok: Nalegak, Gude Zebaoth pivlugo illungertorlaukpunga; Israelible kittorngangita angernerilauktat kemmalaukpât, Altarekotitit serkomilaukpait, nellautaijokotitit tokkolaukpait saviksoarmut, uvangalo amiakotôvunga, pinasuarpullo innôsimnik pêjaivigijomavlunga.

15 Nalekablo okautiva: Uttilermit, ailutillo apkotigijarne sunakajuitokut Damaskusemut, itterlutillo Hasaelelo mingoaruk attanningortilugo Siriamut,

16 Jehulo Nimsib erninga, attanningortilugo Israelitinut, Elisalo Safatib Abelmeholamiub erninga, nellautaijungortilugo innangêrlutit.

17 Pijokartuksauvorlo, kina Hasaelib saviksoanganik kemaijok, tamna Jehub tokkotaksariva, Jehublo sariksoanganik kemaijok, tamna Elisab tokkotaksariva. 
18 Uvangalo amiakokarkojijomavunga Israelitine 7000 dinik, serkunik illunainik serkortorlaungitunik Baalib sângane, kannerniglo illunainik kunnilaungitunik tapsominga.

19 Aularporlo tagvangat, Elisalo Safatib erninga naipipâ makpiteritillugo nunamik tuktuvangnut iglngêngnut 12 inut, tamnalo nangminek 12 it tapkoa akkornganelaukpok. Eliablo aivigiva ulliksoakotinelo egipa tapsoma kânganut.

20 Tagva tuktuvait kemmaklugit, akpangerdlunelo Elia mallikpa, okarlunelo: Atâtagalo anânagalo kunigiartorlaulakka, tagvalo malligomavagit. Tapsoma okautiva: Ailerit, utterlutillo! sunamigle pinniarlaukpagit?

21 Utterporlo tapsomangat; tuktuvâglo iglugêk tiguvâk tokkolugiglo, nerkingillo ikkoalavait kejungnut tuktuvâk annunginêtunut, tunnivaillo innungnut, nerrikovlugit. Parngnailerporlo mallikpâlo Elia, kivgartorlâlo.

\section{KAPITEL XX.}

\section{Ahabib unnatadlarninga Benhadademut.}

BEnHADADIBLO Siriamiut attaningata sorsuktuksakotinne illunaita kattitipait, attannîllo 32 git illagivât, horsillo, aksalloagillo; paungalo majorarpok, Samarialo illumavait sorsugvigilugillo.

2 Tillijaksaniglo tilliklerpok Ahabemut Israelib attanninganut, iglugaseksuit illuanut,

3 Okautsaukolâlo: Imâk Benhadade okarpok: Silberekotit, Goldekotillo perkutigivakka, nulliakotitillo kittorngakotitillo idluarnerpât perkutigigivakka.

4 Israelib attanningat kiovok okarlunelo: Nalegâk attannek, sorlo okalauravit, perkutaumavunga illingnut, tamaitalo pigijakka.

5 Tillijallo tikkipsarput, okarlutiglo : Imâk Benhadade okarpok: Tilliklervigilaurapkit, okautsaukolaurapkillo: Silberekotit Goldekotillo, nulliakotitillo, kittorngakotitillo tunnijaksarivattit uvamnut;

6 Tagva kaupat nelliutome tamattomane kivgakotikka tillijomavakka illingnut, iglokotingnik attannerijivillo iglunginik nellipsaikovlugit, innerkogijatillo tigujaksarivait aggamingnut aksarnigarijaksarilaitalo.

7 Tagva Israelitit attanningata nunab angajokaunerôjungit illunaita kaikovait, okarlunelo: Illitarksileritse tækkoluselo idluiluktomik kajusimangmat. Tilliklervigilaukpânga, nulliakotikka, kittorngakotikkalo, Silberekotikkalo, Goldekotikkalo pivlugit, tamakkoninga kippilugvigilaungilara. 
8 Tagva angajokaunerôjut illunatik, innugiartoksuillo illunamassiatik okautivât: Nalektuksaungilatit, angertuksaunaglonêt.

9 Benhadadiblo tillijanginut okarpok: Nalegara, attannek okautisiuk: Tamaita sivurlermik okautigijaukolauktattit kivgarnut, pinniaromavakka; tamannale sapperpara. Tillijallo ailerput okarlutiglo tamattominga.

10 Tagva Benhadad tilliklervigiva okautsaukolâlo: Gudit imâk, imâglo piniarvigilinga, Samariab sanningit namaktuksaulerpatta, innugiartoksuit malliktut uvamnik attunit tigumiarkamik pikovlugit.

11 Israelitille attanningat kiovok, okarlunelo: Okaritse: Sagvimiutamik attijok nertortuksaungilak ingminik, mattârtotut tamattominga.

12 Benhadad tamattominga tussarame, tupperne attannit immerkattigilugit pilune, kivgaminut okarpok: Parngnaileritse. Tapkoalo parngnailerput iglugaseksuit sorsugvigilugit.

13 Ahâglo, nellautaijut illangata Ahabe Israelitit attanningat aivigiva, okarlunelo: Imâk Nalegak okarpok: Kattimgajoksuit unnuktoksuit ukkoa tækkolaukpigit? Ahâk, uvlome tunnijomavakka aggangnut; nellojungnailerkovlutit uvanga Nalegaugama.

14 Ahabe okarpok: Kinakut? Tamna okarpok: Nunab angajokangita innuosuktokotingittigut. Ahabe okarpok: Kina sorsungnermik pigiartuksauva? Tamna okarpok: Igvit.

15 Tagva nunab angajokangita innuosuktotoktingit kittipait, tapkoalo 232 giolaukput. Tapkoalo kingorngane innugiartoksuit illunaita Israelib kittorngangit illunaita kittipait angutit 7000 dit.

16 Tapkoalo annivut uvlub ketterarningane. Benhadadele immerpok, tallamalauklunelo tupperme attanit 32 it tapkoa ikkajoriartorsimajut tapsominga illagilugit.

17 Nunablo angajokangita innuosuktokotingit annikârput. Benhadadele tilliklerpok nautsertortuksanik tapkoalo okautivât, okarlutiglo: Angutit annivut Samariamit.

18 Tamna okarpok: Annisimakpatta ullapirsautekaromamut tigusigik innôtillugit, annisimakpattalo unatadlaromamut tigusigik innotillugit.

19 Nunable angajokangita innuosuktokotingit annivut iglugaseksoarnit, sorsuktuksello tapkoa kingorngane.

20 Illunatiglo attunnit ullimârnikput tikkitamingnik. Siriamiullo kemâvut, Israelitillo udlapait. Benhadadele Siriamiut attanningat kemâvok horsekut, horsit kângine ablangajut illagilugit.

21 Israeliblo attanningat aularpok ullimârpaillo horsit kamutillo, taimaglo Siriamiune sâlagijaunekartitsivok angijoksoarmik.

22 Tagva nellautaijub Israelib attanningat aivigiva okauti- 
lâlo: Ailerit pitsartutilutillo, kamatsiarlarpillo, tækkolutillo pinniaraksarnik. Siriamiut attanningat majoraromârivok sorsugvigivlutit, Jahre tamanna nâkpat.

23 Siriamiullo attaningata kivgangita okautivât: Taipkoa Gudingit Gudiôvut kakkanut, tamanna pivlugo ajugaulaukput uvaptingnut. Naternamele sorsugvigijomavavut, ajugauniangimangapta taipkonunga.

24 Imailiorit: Attannit nûkokit, attunît inninginit, inangêrtaukokillo aulatsijunut nunamik,

25 Sorsuktuksaksoarniglo akiksoilerit pivlutit taipkotitunak assiojilauktatitut, horsiniglo aksalloalingniglo taipkotitunak, sorsugvigilavullo natername. Illâlo ajugaujomavogut tapkonunga. Tapsomalo tapkoa nippingit nalekpait taimailiorlunelo.

26 Jahrelo tamanna nâsimangmat Benhadadib Siriamiut oppalungaijarpait, majorarlunelo Afekemut Israelitit sorsugvigijomavlugit.

27 Israeliblo kittorngangit oppalungaijartaulaukputtauk pattangaititaulutiglo, aularlarmiglo taipkoa pachrearlugit, tangmarpullo akkingerlugit sorlo tuktungajutitut kattimgajutitut ikkitutitut maggoititut. Siriamiulle nunamik najimavut.

28 Tagva Gudib angutekotinga tikkipok, okarlunelo Israelitit attaninganut: Imâk Nalegak okarpok: Siriamiut okalaungmatta: Nalegak Gudeôvok kakkanut, Gudeôlungilarlo nâksanut; tamanna pivlugo, kattimgajoksuit tapkoa illunamassiatik tunnijomavakka aggangnut, illipse tukkisilerkovluse uvanga Nalegaugama.

29 Tangmarsimavlutiglo sangautivut uvlut 7 it nâvlugit. Uvlullo 7 ingane sorsungnek pigiarpok; Israeliblo kittorngangita Siriamiut pissuktut 100000 dit ullimarpait uvlorme attautseme.

30 Assingillo kemâvut Afekemut, iglugaseksuit illuanut, avalungillo (karmangit) ochovut amiakolauktunut, angutinut 27000 dinut. Benhadadelo kemâvoktauk iglugaseksuit illuanut, nûkattarlunelo innemit attautsemit aipanganut.

31 Tagva tapsoma kivgangita okautivât: Ahâk tussarlaukpogut, Israelitit attaningit, attanniongmatta napkigosuktut; tagva pôngnik tapseruserta makkitiptigut, aklunaniglo nimerdlotjita niakuptingnut, annilerlutalo Israelitit attanninganut, imakkâ innokoniarpâtit.

32 Tapkoalo makkititik tapseruserpait pôngnik, aklunâllo nimerdlotivait niakomingnut, kailutiglo Israelitit attanninganut, okarlutiglo: Benhadadib kivgavit okautsaukovâtit: Nagliktara innokolaunga. Tamnale okarpok: Innovâ sulle? tagva kattangutigivara,

33 Angutillo tapkoa okausek tamanna illaliorsarpât, tukkekartilâlo ingmingnut, okarlutiglo: Ahaila kattangutit Benha- 
dad. Tamna okarpok: Kaileritse kailersiuglo. Tagva Benhadadib annivigiva, tapsomalo ikkikova aksalloalingmut (ingminut.) 34 Benhadadiblo okautiva: Iglugaseksoarnik atâtama aksarnigarilauktanginik atâtarnit sattorsititsomavagit; apkosinerniglo pivlutit sennalerit Damaskuseme, sorlo atâtaga pinnialaungmat Samariame. Uvangalo angerkattigivlutit aularkojomavagit. Tapsomalo angerkattigêngnekarvigiva, aularkolâlo.

35 Tagva angutit illangata nellautaijut erninginit sillaline okautiva Nalekab okausingagut: Nagliktara annaulaunga! Tamnale kunnulaukpok annaulugo.

36 Tagva okautiva: Tamanna pivlugo, Nalekab nippinga nalelaunginangne; ahâk aularuvit uvamnit, tagva Loewib annauniarpâtit. Aularmallo tapsomangat Loewib naipipa, annaulâlo. 37 Tapsomalo angutib taipsoma assia naipipa okarlunelo: Nagliktara annaulaunga! Angutiblo tapsoma annauva killerlugo.

38 Tagva nellautaijok tamna aivok, attannerlo uttakiva apkosiniub senniane, kênaminiglo pissertorpok argsanut.

39 Attannerlo kângermat, attannek nipliutiva, okarlunelo: Kivgait aularsimalaukpok sorsugviub kerkânut, ahâglo, angutit illangat tikkilune angumik atsivigivânga, okarlunelo: Angut una udsertoruk; kingomajauniarpat tagva innôtsit akkiliutiksaujuksauvok innosinganut, ubvalo Talentemik Silberemik akkilêjuksauvotit.

40 Kivgaillo sullijaksakartillugo mâne, ikkanelo, tagva tagvanêtsungnailaukpok. Israelitit attanningata okautiva: Tagga illinganit nangminek okautigivet.

41 Tagva tuavidlune argsak pêrpa kênaminit; Israelitillo attanningata illitarilerpa nellautaijut illagingmatsuk.

42 Tapsomalo okautiva: Imâk Nalegak okarpok: Tamanna pivlugo angut taimna, tokkotaksaunerartara aularkolaurangne illingnit, innôtsit taipsoma innôsingata inninganêtuksauvok innukotitillo taipsoma innukotingita inninginêdlutik.

43 Israelitillo attanningat kuvianaililunelo ningarlarmelo angerarpok iglominut tikkilunelo Samariamut.

\section{KAPITEL XXI.}

Nabotib tokkotauninga passijaksaunane; Ahablo okautsauninga tamanna pivlugo Eliamut, iglungata pêjartaujuksauninganit.

\section{$P$} PIJOKaRTULLO tamakkoa kingorngane pilerpok, Nabote Jesreelemiut illangat perorsêvikarmat nappartolingnik Weineliksanik Jesreeleme, Ahabib attanniub Samariame igloksoangata kiglingane.

2 Ahabiblo Nabote okarkattigiva okarlunelo: Perorsêvikotit nappartolik Weineliksanik tunnilauruk uvamnut, perorsêvingor- 
titsomavara perorsijanut makpertalingnut; kannidlarmat iglumnut. Perorsêvingmik nappartolingmik Weineliksanik namanersamik tunnitsivigijomavagit tamattomunga; ubvalo namaksiguvit Silberemik tunnitsivigijomavagit taimak unnuktigijunik akkiluangatut.

3 Nabotele Ahabemut okarpok: Tamattominga Nalegak ungasigle uvamnit, kingormgutijara atatagijamnit tunnilugo illingnut.

4 Tagva Ahabe angerarpok ningaumajarlune okausek tamanna pivlugo, Nabotib Jesreelemiub okauserilauktanga tapsomunga, okarlunelo: kingormgutijara atâtagijamnit tunnijomangilara illingnut. Innarporlo sinigvingminut, kênanelo keviartipa, kakkojaktoranelo.

5 Tagva nulliangata Isebelib tikkipa, okarvigivâlo: Sôgle annernît annudlarka, nerringilatillo kakkojamik?

6 Tamna okarpok: Nabote Jesreelemiok okarkattigilaukpara, okautilugolo: Perorsêvikotingnik Weineliksalingnik tunnitsivigilaunga kênaujarnut; ubvalo namagijaukpat illingnut perorsêviub Weineliksaliub assianik tunnitsivigijomavagit tamattomunga. Taimnale okarpok: Perorsêvikotimnik Weineliksalingmik tunnitsivigijomangilagit.

7 Tagva nullianga Isebele okarpok tapsomunga: Taimailiorlutit attanniotivit Israelitinut? Makkitit, kakkojaktorlutillo, maksoaniarlutillo. Uvanga Nabotib Jesreelemiub perorsêvikotinga Weineliksalik nangminerijautitsomavara illingnut.

8 Aglangniglo aglakpok Ahabib attingane nakkiterpaillo Ahabib nakkitsutekotinganut, tillklintivaillo innutokaunerôjunut angajokaunerôjunullo, taipsoma iglugaseksoakotinginetunut Nabotib najuganêtunut.

9 Imâglo aglakpok aglangne tamakkonane: Nerritailinermik akpartitsikojileritse, Nabotelo sivorliovlune ingitissiuk innugiartoksoarne;

10 Angutiglo uivêtuk ingitissigik tapsoma sânganut, passiklerkovlugik tapsominga, okarlutiglo: Gudelo attannerlo okarnerlotigilaukpâkik. Annitissiuglo millorlugolo tokkolugo.

11. Taipsomalo iglugaseksoakotingita innutokaunerôjungit angajokangillo, iglugaseksoakotingine iglokalauktut, taimailiorput sorlo Isebele perkungmagit, sorlo taimna aglangne tillikliutigijamine aglalaungmat;

12 Nerritailinermiglo akpartitsikojivut, Nabotelo sivorliovlune iksivakovât innugiartoksoarne.

13 Tagva angutik uivêtuk sangeriartorpâk, innugiartoksuillo sângine Nabote passivâk okarlutiglo: Nabotib Gude attannerlo okarnerlotigilaukpak. Tagva tessiorpât iglugaseksuit sillatânut milliorpâllo tokkolugo.

14 Tagva tilliklerput Isebelemứt, okautsaukovâllo: Nabote millorsimavok tokkungalunelo. 
15 Isebele tussarame, Nabote, millorsimangmat tokkungangmallo, Ahabemut okarpok: Atte Nabotib perorsêvikotinga Weineliksalik, kunujutigilauktanga tunnilugo illingnut kênaujanut, nangminiliutiuk. Nabote innôtsengimat, tokkungavorle.

16 Ahabe tussarame Nabote tokkungangmat, tagva parngnailerpok attersaromavlune Nabotib Jesreelemiub perorsêvikotinganut Weineliksalingmut, nangminiliutijomavlugo.

17 Nalekable okausingata Elia Tisbitemiok tikkipa, okarlunelo:

18 Parngnailerit attersarlutillo Ahabe Israelitit attanningat Samariame pachrerarlugo. Ahâk, Nabotib perorsêvikotinganêpok, attersarsimavok tagvunga nangmineliutijomavlugo.

19 Okarvigiuglo okarlutillo: Imẩk Nalegak okarpok: Innuarlaukpotit, nangmineliutjilutillotauk. Okarvigiuk okarlutillo: Imâk Nalegak okarpok: Kingmit alluktorvingane Nabotib aunganik, kingmittauk aut alluktoraksarivât.

20 Ahabelo Eliamut okarpok: Nachvarpinga omisukteojotit uvamnik? Tamna okarpok: Ahaila nachvarpagit, niorvgotigigavit pinniartuinaromavlutit Nalekab ijikita idluigijanginik.

21 Ahâk kannoêtomik kaitsivigijomavagit, kinguvakotitillo pêjaromavakka; Ahabemillo pêjainiaromavunga tamainik kivgaujuniglo kivgaungituniglo Israeleme.

22 Iglokotillo taimailingatitsomavara Jerobeamib Nebatib erningata iglungatut, Baesablo Ahiab erningata iglungatut; ningaksainek, ningaksaijutigijat uvamnik, Israelitiniglo idluitullititsijutigijat pivlugo.

23 Isebelelotauk Nalekab okautigiva, okarlunelo: Kingmit Isebele nerrijaksarivat Jesreelib avalungita kiglingane.

24 Ahabekunit tokkoniartok iglugaseksoarne kingmit nerrijaksarivat; naternamelo sillame tokkoniartok, tamna tingmitjet killaub attânetut nerrijaksarivât.

2ら Pittakalaungilak Ahabetut ingminik niorvgotigijomik idluitullilune Nalekab sângane; nulliangata Isebelib uiveringmago taimailiorkovlugo.

26 Makkojungnartullivorlo unnuktunik, agvertarlunelo Gudingoat mallikiugit, taimaluatsiavlune sorlo Amoriterit, Israelib kittorngangita sivorngane, Nalekab pêjarlauktangit pinniarlaungmatta.

27 Ahabible okautsit taimaitut tussaramigit, annorane allikpait, pôngmiglo tapserusêvok timiminut, nerritaililunelo, sinnilarmelo pôngme, sukkailisarlunelo pissukpok.

28 Tagva Nalekab okausingata Elia Tisbitemiok tikkipa, okarlunelo:

29 Tækkovit kannok Ahabe nikkanasarmat ingminik sâmne? Tamanna pivlugo nikkanarsarmat ingminik sâmne, kannoêtok tikkikojomalungilara tapsoma uvlungine; erningatale uvlungine kannoêtok tikkerkojomavara iglunganut. 


\section{KAPITEL XXII.}

Ahabib tokkotauninga unnatadlarnerme. Josafat attanniojok Judakunut; Ahasja attanniojol Israelitinut.

J AHRILLO pingasut kângerput unatadlarnekarane Siriamiut Israelitillo akkorngane.

2 Jahrille pingajuane Josafat Judakut attanningat attersarlune tikkipok Israelitit attanninganut.

3 Israelitillo attanningat okarpok kivgaminut: Kaujimangilase Ramoth Gileademêtut perkutigigaptigik? uvagullo kikkarpogut, aksarnigarilungilavullo Siriamiut attanningata agganginit?

4 Josafatemullo okarpok: Illagijomavinga unnatadlarnermut Ramothemut Gileademêtomut? Josafate Israelitit attanninganut okarpok: Illiktut itsomavunga, innukotikkalo innukotititut, horsekotikkalo horsekotititut.

5 Josafatelo okarpok Israelitit attanninganut: Uvlome Nalekab okausinga appertsolauruk.

6 Tagva Israelitit attanningata nellautaijut 400 deôkasait kattimakovait, okautilaitalo:-Ramotemut Gileademêtomut aijuksauvîk sorsugvigilugit, nippotijaksarivakkalonêt? Tapkoa okarput: Majorarit takpaunga, Nalekab tunniniarpait attanniub agganginut.

7 Josafatele okarpok: Pittakarungnaika Nalekab nellautaijokotingita illanganik, tapsomuna appertsorungnarajarluta?

8 Israelitit attanningat okarpok Josafatemut: Angutekaralloarpok sulle attautsemik, appertsorungnarutigijaptingnik Nalekamik, Michamik Jemlab erninganik. Kissiane omigivara, nellautaivigingimanga ajungitomik, idluitomigle kissiane. Josafate okarpok: Attannek taimak okaniarane.

9 Tagva Israelitit attanningata angajokait illangat kaikova, okarlunelo: Micha Jemlab erninga kaisaruk ovunga.

10) Israelitille attanningat Josafatelo Judakut attanningat iksivavuk attunît iksivautaksoakotimingne, annorarsimavlutik attanneksiutinik inneme Samariab itterviksoangata sillatânetome; nellautaijullo illunatik nellautaivut tapkoa sângane.

11 Zedekialo Knaenab erninga sennalaukpok ingminut naksungnik kikkiangnik, okarlunelo: Imâk Nalegak okarpok: Ukkungnunga Siriamiut nakjungmigarniarpattit, nungutsikârtinak taipkoninga.

12 Nellautaijullo illunatik taimak nellautaivut, okarlutiglo: Majorarit takpaunga Ramotemut Gileademêtomut, sullidlarlutillo, Nalekab tunninarmagit attanniub agganginut.

13 Tillijablo aimalauktub Micha kaikolugo, okautiva: Ahâk nellautaijut okalaningit illunatik adsigêktomik attanniub idlu- 
arkutinganut illingavut, taimaimat okautsitit taimailingalaulittauk taipkoa okausingititut, okarlutillo ajungitomik.

14 Micha okarpok: Sorlo Nalegak miksekârtomik innôngmat, okaromavunga Nalekab okauseriniartanginik uvamnut.

15 Attannermullo tikkimat, attanniub okautiva: Micha, Ramotemut Gileademêtomut aulartuksauvita, sorsungniarluta, nippotijaksarivittigolonêt? Tapsoma okautiva: Ahaila, majorarit takpaunga, sullidlarlutillo, Nalekab tunniniarpait attanniub agganginut.

16 Attanniub okaupsarpa: Kapseartorlunga okautimarijariakarpagit, assianik okausekarkonnak uvamnut, Nalekab attingane miksekarnermit.

17 Tamna okarpok: Israelitit illunamassiatik tækkolaukpakka siamarsimatillugit kakkat kângine saukattitut mianerijekangitutitut. Nalegarlo okarpok: Ukkoa angajokakangilet, illunatik attunît angerarlit iglomingnut ullapirsautekarlutik.

18 Tagva Israelitit attanningat okarpok Josafatemut: Okautilaungilagit ajungitomik nellautailungimat pivlunga, idluituinarmigle?

19 Tamnalo okarpok: Tamanna pivlugo tussarkit manna Nalekab okausingit: Nalegak tækkolaukpara iksivatillugo iksivautaksoarmine, killangmiugaseksuillo illunamassiatik nangertillugit senniane, tallerpiane, saumianelo.

20 Nalegarlo okarpok: Kia Ahabe tillioromavaûk, majorarkovlugo takpaunga, ochokovlugolo Ramoteme Gileademêtome? Illangallo imâk okarpok, assialo imâk.

21 Tagva annernit illangat kaivok, Nalegarlo sangerpa, okarlunelo: Uvanga tillioromavara. Nalekab okautiva: Sunamut?

22 Tamna okarpok, aularomavunga, annerniojomalungalo nellagôngitok taipsoma nellautaijokotingita kanningine illunaine. Tamnalo okarpok: Igvit tillioraksarivat, sullidlarniarpotillo, aularit taimailiorlutillo.

23 Ahâglo manna, Nalekab annernek nellagôngitok tunnimava, nellautaijokotivit kanninginut illunainut, Nalekablo idluitomik okarutigilaukpâtit.

24 Tagva Zedekia Knaenab erninga tikkilerpok tamaunga, ulloarokpâlo Micha, okarlunelo: Kannok? Nalekab annerninga nûsimava uvamnit, okarkovlugo illingnut?

25 Micha okarpok: Ahâk tækkojomârpat uvlorme taipsomane, innimit attautsemit kemâkattarniaruvit assianut ailutit, ijerlutit.

26 Israelitit attanningat okarpok: Micha tiguleruk, âlugolo Amonemut, iglugaseksuit angajokanganut, Joasemullo attanniub erninganut, 
27 Okarlutillo: Imâk attannek okarpok: Una pitisiuk parngnanairsimavingmut, nerritilugolo kakkojamik, immertilugolo immermik kiksarnartomik, utterkârtinanga ullapirsautekarlunga.

28 Micha okarpok: Ullapirsautekarlutit utterniaruvit, tagva Nalegak uvapkut okalaungilak. Okarporlo: Tussaritse innugiartoksojose illunase!

29 Taimak Israelitit attanningat, Josafatelo Judakut attanningat majorarput takpaunga Ramotemut Gileademêtomut.

30 Israelitillo attanningat okarpok Josafatemut: Pissertorlunga sorsungnermut aijomavunga, igville annorakotitit attikit. Taimaglo Israelitit attanningat pissertorpok ingminik annoraminut, tikkilunelo sorsungnermut.

31 Siriamiulle attanningata aksalloalikotime 32 ôlauktut angajokangit perkovait okarlunelo: Sorsuktuksaungilase mikkijunullonêt, angijunullonêt. Israelitille attanninganut kissiane.

32 Aksalloagillo angajokangita Josafate tækkogamitsuk aulajitigivat attannionasugivlugo Israelitinut tunarivâllo sorsuklugo; Josafatele nipliadlarpok.

33 Aksalloagillo angajokangit tækkogamik attanniolungimat Israelitinut allarpât.

34 Angutille illangata maungatuinak pittikse kelluva, pittiklugolo Israelitit attanningat, sagvingmiutab pêrniublo akkorngagut. Tamnalo okarpok kemuksertokotiminut: Sangulerit nûtilungalo sorsungnermit, ikkilersimagama.

35 Sorsungnerlo sugalulerpok uvlorme taipsomane, attannerlo nangertitaumalaukpok aksalloaliub kângane Siriamiut akkiane, tokkolunelo unnungme. Auglo ikkimit kôkpok aksalloaliub illuanut.

36 Tagva akpartaukojauvut tangmarvingme sekkinek nippitillugo, okartokarlune: Illunatik attunnit iglugaseksoarmingnut nunagijamingnullo ailit.

37 Taimak attannek tokkorok, âtaulunelo Samariamut. Illijartorpâllo Samariame.

38 Aksalloaliglo ubvarmatsuk tetserme Samariamêtome, kingmit aunga alluktorpât; anguniarpaktulle ubvarpât, Nalekab okausingit okauserilauktangit malliklugit.

39 Unipkautsille tamakkoa assingit sulle Ahabe pivlugo, pinniarlauktangit, iglolo igluliorlauktanga Elefantit tôganginit, iglugaseksuillo sennalauktangit, ahak tamakkoa aglaksimavut Israelitit attanningita aglangine.

40 Ahabelo sinnisilerpok, atâtagijaminut ailune; erningalo Ahasja attanningorpok inangêrlugo.

41 Josafatelo Assab erninga attanningorpok Judakunut, Ahab Israelitit attanningata Jahringita sittamangane.

42 Jahrekalaukporlo attanningorame 35 ginik, attaniotivorlo 
Jahrinik 25̆ ginik Jerusaleme. Anânanga attekalaukpok Asubamik Silhib panninga.

43 Agvertarporlo atâtame Assab apkosiningane tamât, senniagôrlungilarlo, pinniarporlo Nalekab idluarijanganik.

44 Poktojulle ipperarlaungilait, innugiartoksuillo tunnergutinik tunnijivut, isserluanititsilutiglo sulle pôktojune.

45 Josafatelo ullapirsautekarpok Israelitit attanninganut.

46 Unipkautsille tamakkoa assingit sulle Josafate pivlugo, sôngoninga, pinniarlauktangillo, kannorlo sorsuglaungmat, âhak, tamakkoa aglaksimavut Judakut attanningita aglangine.

47 Ajortullijullo amiakolauktut sulle atâtame Assab nelliuninganit pêjarpait nunamit.

48 Attannekalaungilarlo Edomiterit nunangane.

49 Josafatelo umiaksoarnik sennakojilaukpok, immarbiksoakut Ofiremut umiaktortuksaulauktunik Goldemik aiklertuksauvlutik. Aularlaungilelle, serkomitaulaukpulle Ezeongebereme.

50 Taipsomane Ahasja Ahabib erninga okarpok Josafatemut: Kivgakka umiaktorkattaukolaukit kivgakotingnut umiaksoakut. Josafatele pijomalaungilak.

51 Josafatelo sinnisilerpok atâtagijaminut ailune, illijartortaulaukporlo atâtagijaminut, atâtame Davidib iglugaseksoakotingine; Joramelo erninga attanningorpok innangêrlugo.

52 Ahasja, Ahabib erninga attanningorpok Israelitinut Samariame, Josafatib Judakut attanningata Jahringita 17 ingane; attanniotivorlo Israelitinut Jahringnik maggungnik.

53 Pinniarporlo Nalekab idluigijanganik, agvertarlunelo atâtame anânamelo apkosinigingne, Jerobeamiblo Nebatib erningata, Israelitinik idluitullititsilauktub apkosiningane;

54 Baalelo kivgartorpa, tuksiarvigilalo; ningaksarpalo Nalegak Israelib Gudinga, taimaluatsiak sorlo atâtanga pinniarlaungmat. 


\section{Attannit aglangita aipangit.}

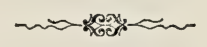

KAPITEL I.

Ahasjab kannimaninga, toklkungalo. Tapsoma innukotingita nungutauningat ikkomamut liilangmit pïomut, nellautä̈ub Eliab okausingit malliklugit.

$\mathrm{M}_{0}$ jungnaimat.

2 Ahasjalo kattakpok killapataujakut iglomine Samariame, kannimalerlunelo, tillijaksaniglo tilliklerpok, okautivaillo: Apperiartoritse Baal-Sebubemik Ekronemiut Gudinganik, kannimasermit tamattomangat nekkoksiniarmangarma?

3 Nalekable Engelingata Elia Tisbitemiok okarvigiva: Parngnairit, attanniublo Samariamiub tillijangit pârniarkit, okautilugillo: Gudekangilâk Israelitine, Baal-Sebube Ekronemiut Gudingat apperiartorapsiuk?

4 Tamanna pivlugo Nalegak okarpok: Innangavingnit, nellariarvigijarnit makkitsungnaituksauvotit; tokkojuksaulutillē. Elialo aularpok.

5 Tagvalo tillijaujut uttermatta tapsomunga, tapsoma okautivait: Sôg utterpise?

6 Tapkoa okautivât: Angutib majorarlune pârlaukpâtigut, okautilutalo: Uttermititse attannermut tillijigilauktapsingnut, okautilugolo: Imâk Nalegak okarpok: Gudekangilâk Israelitine, tillikleravit Baal-Sebube Ekronemiut Gudingat apperiartorlugo? Tamanna pivlugo makkitsungnaituksauvotit innangavingmit nellariarvigilauktarnit, tokkojuksauvotille.

7 Tapsoma okautivait: Kanoêtomik angut taimna tautukalauka pârsijok illipsingnik, okautjiselo tamattominga?

8 Tapkoa okautivât: Angutaulaukpok merkolingnik annoralik, tapseruteliglo makkitimigut amivinermik. Tamnale okarpok: Eliaôvok, Tisbitemiok.

9 Tagva tilliklervigiva angajokamik 50 ginut, 50 git tapkoa illagilugo. Tikkinamiuglo takpaunga, ahâk, tagva iksivavok kakkab kângane. Tapsomalo okautiva: Igvit Gudib angutinga, attannek okarpok: Attersarit. 
10 Eliab angajokak 50 ginut kiova, okautilugolo: Gudib angutigikpanga, tagva ikkoma kattagle killangmit, nerrilitillo 50 gikotitillo. Tagva ikkoma kattakpok killangmit, nerrivâlo, 50 gikotingillo.

11 Tilliklipsarpoṛlo angajokab assianik 50 ginut, 50 gillo illagilugo, tapsomunga. Tapsoma kiovlune, okautiva: Igvit Gudib angutinga, imâk attannek okarpok: Attersarit.

12 Elia kiovok okarlunelo: Gudib angutekotigikpanga, tagva ikkoma kattagle killangmit, nerrilitillo 50 gikotitillo. Tagva Gudib ikkomanga kattakpok killangmit, nerrivâlo, 50 gịkotingillo.

13 Tagva ama angajokait pingajuat 50 ginut, tilliva, 50 gitlo illagilugo. Tamna tikkiname tapsomunga takpaunga, Elia serkortorvigiva tuksiarvigilugolo, okautilâlo: Igvit Gudib angutekotinga, innosera, 50 gillo tapkoa innosingit akkitôlit ijikpit sângangne.

14 Ahâk ikkoma kattalaukpok killangmit, nerrilaukpaillo sivorlîk angajokâk, 50 gik tingillo; mannale innnosera akkitôle ijikpît sângangne.

15 Tagva Nalekab Engelingata Elia okautiva: Attersarkattigiuk, erksiginiarnagolo. Tagva parngnailerpok attersarkattigivâlo attannermut.

16 Okarporlo tapsomunga: Imâk Nalegak okarpok: Tamanna pivlugo tillijanik tilliklilauravit, Baal-Sebubelo Ekronemiut Gudingat appertsorkolaurangne, Gudekarajangimat sorlo Israeleme, tapsoma okausingit appertsortaujungnarmatta, tagva inangavingnit nellariarvigilauktarnit makkitsungnaituksauvotit, tokkojuksauvotille.

17 Taimak tokkovok Nalekab okausingit, Eliab okauserilauktangit malliklugit. Joramelo attanningorpok tamna innangêrlugo, Joramib Josafatib erningata Judakut attanningata Jahringita aipangane; ernekalaungimat.

18 Unipkautsit tamakkoa assingit Ahasja pivlugo sulle, pinniarlauktangit, ahâk tamakkoa aglaksimavut, Israelitit attanningita aglangine.

\section{KAPITEL II.}

\section{Eliab killaliarninga; Elisab tattamnartitangit.}

NALEKABLE Elia kongmuarkojomalaungmago killangmut, Elia Elisalo aularpuk Gilgalemit.

2 Eliablo Elisa okautiva: Nagliktara ovanetualaurit! Nalekab tillingmanga Bethelemut. Elisale okarpok: Sorlo mik- 
sekârtomik Nalegak inôngmat, tarnîllo, kemangniangilagit. Taimak attersarpûk unnunga Bethelemut.

3 Tagva nellautaijut erningit Bethelemêlauktut annilerput Elisamut, okautivâlo: Kaujimagalloarkît Nalekab angajokait uvlome aularutiniarmago niakovit kollâgut? Tamnale okarpok: Kaujimagalloarivara, nippangertuinaritse.

4 Eliablo okautiva: Elisa, naglingnartotit ovanetualaurit, Nalekab tillingmanga Jerichomut. Tamnale okarpok: Sorlo miksekârtomik Nalegak innôngmat, tarnîllo, kemangniangilagit. Taimaglo Jerichomut tikkipuk.

5 Tagva nellautaijut erningita Jerichomelauktut Elisa tikkipât, okautilâlo: Kaujimagalloarkît Nalekab angajokait uvuvlome aularutiniarmago niakovit kollâgut? Tamnale okarpok: Kaujimagalloarivara, nippangertuinaritse.

6 Eliablo okautiva: Naglingnartotit ovanetualaurit; Nalekab tillingmanga Jordanemut. Tamnale okarpok: Sorlo miksekârtomik Nalegak innôngmat, tarnillo, kemangniangilagit: Taimaglo aikattigêkpuk.

7 Angutille 50 git, nellautaijut erninginit aivut, nessipirlutiglo kaningitomit; tapkoale nangerpuk Jordaneme.

8 Tagva Eliab ulliksoane tiguva, immulâlo, immerlo annauva, tamanalo tagva avikpok avunga ikkungalo, tammarmiglo ikkârpuk ipteriliktokut.

9 Akkianullo tikkingmanik Eliab Elisa okautiva: Tuksiarit sunamik pinniartuksaunimnik illingnut, nûtitaukârtinanga illingnit. Elisa okarpook: Annernît uvamnêkovlugo maggoêrtorlune.

10 Tamna okarpok. Pijariakortôjomik tuksiarkauvotit; tækkoniarumgale nûtitautillunga illingnit, tagva taimainiarpok, tækkoniangikumgale, taimainiangilak.

11 Pissukattigêktillugiglo, okartillugolo; ahâk, tagva tikkilerpok aksalloalik ikkomalik, horselîk ikkomaggit, avikpâglo; Eliało ullajujaksoakut kongmuarpok killingmut.

12 Elisale tækkovok tamattominga, nipliadlarlunelo: Atâtâk, atâtâk! Israelib aksalloalikotingit, ablangajokotingillo horsine! Tækkojungnaipâlo. Anoranelo tiguvait allikpaillo, maggolivlugit.

13 Eliablo ullinga, kattalauktanga kivikpâ utterlunelo; nangeriartorlunelo Jordanib sikjanganut.

14 Tiguvalo Eliab ullinga, kattalauktanga, immerlo annauva, okarlunelo: Nanekâ manna Nalegak Eliab Gudinga? Immerlo annaungmago tagva avikpok avunga ikkungalo, Elisalo pissukpok tagvûna.

15 Nellautaijullo erningita, akkingertangita Jerichome, tækkovât, okarlutiglo: Eliab annerningata Elisa innilangavigiva; pachreartorpâllo akkungavigivlugolo nunamut opigivât; 
16 Okautivâllo: Ahâk kivgakotivit akkorngane angutekarpok こ̆0 ginik sôngojunik, tapkoa aularlit, angajokait kenneriartorlugo; imakkâ Nalekab annerningata aularutilaukpago, pitilugolo kakkat nelliata kânganullonêt, nâksat nellianullonêt. Tamnale okarpok tilliklerniarase.

17 Innapâlle akkunît, merngortorkârtinago, okarlunelo: Tillik!eritse. Tilliklerpullo angutinik 50 ginik, tapkoa kennerpât uvlut pingasut nâlugit, nenninagolo.

18 Utterpullo tapsomunga, tamnale Jerichomêtualaukpok, okarporlo tapkonunga: Okautilaungilapse aijuksaunginapse?

19 Iglugaseksuillo angutingita Elisa okautivât: Ahâk iglugaseksoarne makkonane innekarominarpok sorlo nalegara tækkungmat, kissiane imilukarpok ovane, nunalo alliptornarpok.

20 Tamna okarpok: Kaitsivigisinga pogutamik nutâmik, pitetservigilugolo sioraujamik. 'Tapkoalo âtsivigivât tamattominga.

21 Annivorlo puailavingmut, sioraujallo egipait illuanut, okarlunelo: Imâk Nalegak okarpok: Immek tamanna kanoêtsungnaitipara, mannamit tokkomiglonêt alliptuamiglonet pijokarungnaituksauvok tamattomangat.

22 Taimak immek tamanna atsuilingorpok uvlok tamanna tikkilugo, Elisab okausingit, okauserijangit malliklugit.

23 Majorarporlo takpaunga Bethelemut. Apkosinermelo majorartillugo nukapiarârsuit annilerput iglugaseksoarnit, milłorpâllo ujarkanut, okautikattarpâllo: Nujaêrsimajotit majorarlutit kait! Nujaêrsimajotit majorarlutit kait!

24 Keviarporlo, tækkonamigillo okarnerlutigivait Nalekab attingane. Tagva aklâk tikkilerpuk nappartunit, alliktorlugillo sorrutsit 42 git tapkonangat.

25 Tagvangallo majorarpok kakkamut Karmelemik attelingmut; tagvangallo utterpok Samariamut.

\section{KAPITEL III.}

Joramib attanniotininga Israelitinut. Unatadlarninga, sâlaTarningalo Moabuterinit.

JORAME Ahab erninga attanningorpok Israelitinut Samariame. Josafatib Judakut attanningata Jahringita 18 ingane; attanniotilunelo Jahrinik 12 inik.

2 Pinniarporlo Nalekab idluigijanginik, atâtamituungitorle, anânamituungitorlo. Baalib nappajunga atâtangata sennajaukolauktanga pêjarpa.

3 Jerobeamible Nebatib erningata Israelitinik idluitullititsi- 
lauktub idluiningit ungagitsainarpait, allailungilarlo tapkonangat.

4 Mesale Moabiterit attanningat saugakutekalaukpok unnuktunik, akkilêvigijariakarpâlo Jahrine tamaine Israelitit attanningat, saugârsuit 100000 dit, angusalluillo 100000 dit merkunginik.

5 Ahable tokkungangmat, Moabiterit attanningata Israelitit attanningat nalegungnaipa.

6 Tagva attannek Jorame nelliutome taipsomane aularpok Samariamit oppalungaijailunelo Israelitit illunamassianginik.

7 Tilliklerlunelo Josafatemut Judakut attanninganut okautsaukovâlo: Moabiterit attanningata nalegungnaipânga, kailaurit sorsukattigilungalo Moabiterinik. Tamna okarpok: Majorarlunga takpaungaromavunga, uvanga illiktut-ipunga, innukotikkalo innukotititut, horsekotikkalo horsekotititut.

8 Okarlunèlo: Apkosinît nelliatigut takpaungaromavita? Taimna okarpok: Apkosinekut Edomiterit sunakajuitunginêtutigut.

9 Taimak aularput tagvunga, Israelitit attanningat, Judakut attanningat, Edomiterillo attanningat. Imerianiglo 7 inik ingerarlaukârmatta, sorsuktuksaksoakattigêt, nergjutillo najortangit immekangilet.

10 'Tagva Israelitit attanningat okarpok: Kappianamêk! Nalekab attannit ukkua pingasut kaikolaukpait tamaunga, tunnijomangmagit Moabiterit agganginut.

11 Josafatele okarpok: Nalekab nellautaijokotingita illangat ovanêngilâk, Nalekamik appersorkovluta tapsomuna? Tagva Israelitit attanningata kivgangita illangat kiovok okarlunelo: Elisa Safatib erninga, kovisilauktok immermik Eliab agganginut mânêpok,

12 Josafate okarpok: Nalekab okausingit taipsomanêput. Taimak attersarput taipsomunga, Israelitit attanningat, Josafatelo Edomiterillo attanningat.

13 Elisable Israelitit attanningat okautiva: Igvit suvigivinga? Ailerit atâtavit nellautaijokotinginut, anânavillo nellautaijokotinginut. Israelitit attanningata okautiva: Aukak, Nalekab attannit pingasut ukkoa kaikungmagit tunnijomavlugit Moabiterit agganginut.

14 Elisa okarpok: Sorlo miksekârtomik Nalegak Zebaoth sângiartara inôngmat, Josafate, Judakut attanningat issumaginajangikupko, igvit tækkosarajangilagit, kamaginajangilagillonêt.

15 Tagva kaitsivigisinga manna nipliajortomik. Angullo nokakseralingmik attortillugo Nalekab aggangita tikkipa;

16 Okarporlo: Imâk Nalegak okarpok: Sunatuinarne ittersallioritse naksarme tamattomane.

17 Nalegak imâk okarmat: Annoremiglonêt, sillalungmig- 
lonêt tækkoniangilase, sulle naksak tamanna nâmaniarpok immermik, illipse, illaselo, nergjutekotiselo immerungnarkovluse.

18 Tamannalo mikkiluarpok sulle Nalekab sângane, Moabiterit tunniniarivait aggapsingnut.

19 Iglugaseksuillo sôngojut illunaita, iglugaseksuillo annerimajut illunaita ullimarniarpasse, nakkartsiniarluselo nappartunik ajungitunik illunainik, puailavillo immermik illunaita simingniarpasse, perorsêvillo namanersat illunaita asserorniarpasse ujarkanut.

20 Uvlarmelo tunnergutit tunnijauviane, ahâk immersoak kôkpok Edomemit, nunalo nâmalerpok immermik.

21 Moabiterillo tussaramik, attannit majorarmatta sorsugvigijomavlugit, kaikojivut illunainik attorungnarnermut sakkunik namaktunik Jahrekartunik, innotokaunersaniglo, nangeriartorlutiglo kiglilervingmut.

22 Uvlarârsukullo parngnaimatta, sekkinerlo nuilermat immersub kângane, Moabiterit immek aupallugasugivât auktut;

23 Okarlutiglo: Aungôvok; attannit asserortaumavut saviksoarmut, ullimartigêkpallaivut. Atte, Moabitereôjose manna aksarnigakaritse.

24 Israelitille tangmarvinganut tikkingmatta, Israelitit parngnailerput ullimarpaillo Moabiterit, tapkoalo kemâvait. Israelitillo nunamut tikkiput ullimârpaillo Moabiterit.

25 Iglugaseksuit serkomipait, illunatiglo attunit ujaratik egipait perorsêvingnut namanerpânut tættætipaillo, puailavillo illunaita simikpait, nappartullo ajungitut illunaita nakkarpait, amiakokakârtinagit kissiane iglugaseksuit ujarangit Kirharaseteme. Illoereartullo illumavait, illoerearlugillo.

26 Moabiterillo attanningat tækkogame, sorsungnek sôngoluarmat tapsomunga, angutit 700 dit saviksoarmik amusijut ingminut pitipait pittarutigomamut Edomiterit attanninganut; sappidlalaukpulle.

27 Tagva taipsoma erninga ernerikârtanga innangêrlugo attanniojuksaulauktok tiguva, tunnilâlo tunnergumut ôtaksamut avalut kângine. Tagva ningarnekalerpok angijomik Israelitit pivlugit, allarpaillo utterlutiglo nunamut.

KAPITEL IV.

\section{Elisab tattamnartulliningit tellimat.}

NellautaiJullo erningita nulliangita illangata Elisa nipliutiva okarlunelo: Kivgait, uiga tokkungavok, kaujimavotillo, kivgavit Nalegak sivoragilaungmago, mannalo akkiliksakarvinga tikkipok kittorngâka tigujomavlugik, kivgangortitsomavlngiglo ingminut. 
2 Elisab okautiva: Sunamik pinniarvigijaksarivagit? Okautinga, sunamik pittakarkît iglungne? Tamna okarpok: Kivgait arnak sunakalungilak iglome publaujab orksukautib assianik.

3 Tamna (Elisa) okarpok: Attarsijartolerit sillalingnit arnanit illunainit illulerkutinik illulekangitunik ikkitolungitunik.

4 Itterlutillo, tunnungnelo ernikpîllo tunnukingne upkoak pallangairuk, kovisilutillo illulerkutinut illunainut, tætætullo âtaulìt assianut.

כ́ Arnak aivok, upkuarlo tunnumine erningmelo tunnukingne pallangairpa; tapkoa illulerkutit âpait tapsomunga, tamnalo kovisivok kamunga.

6 Illulerkutillo tættælaungmatta, ernine okautiva: Illulerkumik attautsemik sulle issautiivigilaunga. "Tapsoma okautiva: Illulerkutekarungnaipok mâne. Tagva orksuk nokarpok.

7 Tamnalo arnak aivok, okarlunelo tamattominga Gudib angutekotinganut. Tamna okarpok: Ailerit orksuglo niorvgotigiuk, akkiliksakarvillo akkilêvigiuk; igville ernikpillo amiakunga innogutigisiuk.

8 Nelliutomelo taipsomane pijokalaukpok, Elisa Sunememut aingmat. Tagvane arnakalaukpok akluitomik, tapsoma innapa nerrikovlugo tapsomane. Tagvûnarlaurangamelo ittervigitsainnarpa nerrikattigilâlo.

9 Tamnalo arnak uiminut okarpok: Ahâk tækkovunga Gudib angutekotinga ovûnatsainartok ivsornaidlarmat.

10 Inniliorlaulavuk mikkijomik iglupta kollâne, innarvingmiglo, sâmiglo, iksivautamiglo, nenergoabvingmiglo pititsilauluk takkamunga, tikkilerangatiguk itterkovlugo takkamunga.

11 Pijokalaukporlo uvlut illangane, itterame tagvunga, innarpok kollâne inneme sinniklunelo takkamane.

12 Kivgaminullo Gehasimut okarpok: Sunamemiok arnak kaikolauruk. Kaikungmagolo sângerpa.

13 Tapsomalo (Elisab) Gehasi okautiva: Okautiuk: Ahâk, kivgartornermik tamattominga tamainik pinniarvigilaukpâptiguk, sunamik uvanga pinniarvigijaksarivagit? Okausiksakarkît attannermut, sorsuktuksallonêt angajokanganut? Tamna (arnak) okarpok: Nunakarpunga innukotima akkorngane.

14 Tamna (Elisa) okarpok: Sunamigle pinniarviojungnarka? Gehasi okarpok: Atso illa, ernekangilak, uingalo itôvok.

15 Tamna okarpok: Kaikoleruk. Kaikungmagolo, upkuêrdlune nangeriartorpok.

16 (Elisalo) okarpok: akkiâgo nelliutome tamattomane ernermik kuningniktuksauvotit. Tamna (arnak) okarpok: Aukak illa, nalegâk, angutaujotit Gudemut, kivgait arnak sæglokiniarnago!

17 Arnarlo tamna singailerpok ernilunelo ernermik nelliu- 
tome taimaluatsiame akkâgolermat, sorlo Elisab okautilaungmago.

18 Soruserlo angijororame pijokarpok uvlut illangane, annilerame atâtaminut kippisijunut (kakkojaksanik),

19 Okarporlo atâtaminut: Kappianamêk niakora, niakora! Tamna okarpok nukapiakotiminut: Anânanganut âleruk.

20 Tapsomalo tiguva âlâlo anananganut. 'Tapsomalo (anânangata) sârliarpa uvlub ketterarninga tikkilugo, tagva tokkovok.

21 Tamnalo (arnak) majoarpok kollânut, illivâlo Gudib angutekotingata innarvianut, pallangairsivok annilunelo,

22 Uinelo kaikova, okarlunelo: Nukapitset illanganik tilliklervigilaunga siutekoktôjomiglo; Gudib angutekotinganut aijomavunga utteromalungalo.

23 Tamna okarpok: Sôg aivigijomaviûk? Uvlome takkêlaulungimat Sabbateolungilarlonêt. Tamna (arnak) okarpok: Nipportuinarit.

24 Siutekoktôjorlo ilksivaviltarpa okarlunelo nukapiamut: Ungortuinarit katsungailutit, nokartailitilungalo ablanganerme sivumut, kissiane okautigupkit.

25 T'aimak aularpok, tikkilunelo Gudib angutekotinganut kakkamêtillugo Karmelemik attelingme. Gudible angutekotingata kanningitomit tækkogamiuk, okarpok nukapiakotiminut Gehasimut: Ahak arnak Sunememiok tikkilerpok.

26 Taimak manna akpangerdlutit pachreartoruk, apperilugolo, tamna nangminek, uingalo erningalo kanoêngitsiarolloarmangatta? Tamna okarpok: Ahaila kannoêngitsiarpogut.

27 Tikkinamele Gudib angutekotinganut kakkab kânganut, ittiggangit erkisimiarpait; Gehasile kaivok ajagomavlugo. Gudible angutekotinga okarpok: Taimailingale, tarninga kiksadlarmat; Nalekablo angigutilaukpânga tamattominga, nellojungnaitilaungilângalo.

28 Arnak okarpok: Kânga ernermik tuksiarlaukîk nalekamnit? Okalaungilanga, uiverijaksaringilarma?

29 Tamna (Elisa) okarpok Gehasimut: Makkitingnik tapseruserit, ajaupiakotigalo tiguleruk aggangnut, aivigilugolo; pârtauguvit kinamut, tagva salutiniarnago pit; salutitaugalloaruvillo, tagva kioniarnak, ajaupiakotigalo illiuk nukapiab kênanganut.

30 Nukapiable anânanga okarpok: Sorlo miksekârtomik Nalegak inôngmat tarnîllo, allarniangilagit. Tagva parngnailerpok mallikpalo (arnak).

31 Gehasile tapkoa sivorngangne aimalaukpok tagvunga, ajaupiarlo illiva nukapiab kênanganut; tagvanele nippekalaungilarlonêt ikipiginekangilarlonêt. Utterporlo pachrearlugo, nellajungnaitipâlo, okarlunelo: Nukapiak tuppalungilak. 
32 Elisalo iglomut tikkimat, ahâk tagva nukapiak tokkungavlune nellavok innarvingane.

33 Itterporlo, upkoarlo pallangairpa, tuksiarlunelo Nalekamut,

34 Kagvalerporlo pallungerlunelo sorrutsib kânganut, kanninelo illiva sorrutsib kanninganut, ijingnelo ijekingnut, agganelo tapsoma agganginut, taimaglo tessiterpok tapsoma kânganut, sorrutsib timinga aukanerkovlugo.

35 Tamnale (Elisa) makkipok uttertarlunelo iglome, kagvarlunelo tessiterporlo tapsoma kânganut. Tagva nukapiak tagioksavok 7 êrtorlune, kingorngane nukapiak ijingne uitipâk.

36 Elisablo Gehasi kaikova, okarlunelo: Arnak Sunememiok kaikoleruk. Kaikovâlo. Tapsomalo tikkipa. Tamna okarpok: Tagga ernît tiguleruk.

37 Tagva (arnak) tikkilerpok, pamakterlunelo ittigganginut, opigosulunelo netterme, erninelo tiguva annilunelo.

38 Elisale tikkingmat Gilgalemut, kâvikarpok nuname, nellautaijullo erningit, nunakalaukput tapsoma sângane. Tapsomalo kivgane okautiva: Ukkusiksoak oklertorvigiuk, igalutillo perortunik nellautaijut erningita nerkiksanginik.

39 Tagva tapkoa illangat naternamut aivok, perortunik kattersijomavlune, naipitsilunelo perortunik, manetuinartome perortunik, nunivarlunelo tapkonangat paungâlukullungnik, Kolokwintenik attelingnik, anorakotine tættædlugo, tikkinamelo okliutivait savingmut ukkusingmut; illitarilungimattigik.

40 Navingmagolo nerrijaukovlugo angutinut, nerrikamiglo nerkiksamit tamattomangat, tagva nipliarput okarlutiglo: Igvit Gudib angutekotinga, tokko ukkusingmêpok! Nerrilugo sapperamik.

41 Tamnale (Elisa) okarpok: Sennaukamik kaitsileritse. Pitipâlo ukkusingmut, okarlunelo: Naviuk innuit sânginut nerrikovlugit. Tagva idluitokalaungilak ukkusingme.

42 Tikkitokarporle angumik Baalsalisamit, kaitsivigilugolo Gudib angutekotinga niakojanik kakkojaksanit innersimakârtunit, niakojanik 20 ginik Gerstemit, kakkojaksaniglo togarsimajunik ikpiârsukotimine. Tamnale okarpok: Tunnikit innungnut nerrikovlugit.

43 Kivganga okarpok: Kannok angutit 100 dit tunnitsivigijaksarivakka tamakkonangat? Tamna okarpok: Innugiartoksuit tunnitsivigikit nerrikovlugit. Nalegak imâk okarmat: Nerrijokarniarpok, amiakokarniarlunelo.

44 Tagva illivait tapkoa sânginut, nerrikovlugit; amiakokarporlo sulle, Nalekab okausingit malliklugit. 


\section{KAPITEL V.}

Naemanib Siriamiub kallakartub kallakarungnaititauninga Elisamut. Gehasib kallakartitanninga, pijomatudlarninga pivlugo.

$\mathrm{N}$

NaEman Siriamiut attanningata sorsuktuksaksoakotingita angajokangat, angutitsiangolaukpok angajokame sângane, opigijaulunelo; tapsomuna Nalegak sâlakarnermik tunnijingmat Siriame. Angutaudlalaukporlo, kallaliolunele.

2 Sorsuktukselle Siriame aularlaukput, niviârsiarlo aularutilaukpât Israelitit nunanganit, tamna kivgaulaukpok Naemanib nullianganut.

3 Tamna angajokaminut arnamut okarpok: Kannoktôk nalegara nellautaijomênajarune Samariame, taipsoma pejaiviginajarpa kallanginik.

4 Tagva itterpok angajokaminut okautilâlo tamattominga, okarlunelo: Imâglo, imâglo niviârsiak Israelitit nunanganit pijok okalaukpok.

5 Siriamiut attanningat okarpok: Tagva ailerit tagvunga, Israelitit attanningat aglagvigijomavara. Aivorlo tagvunga, neksarlunelo Talentinik 10 nik Silberemik, Sekeleniglo 6000 dinik Goldemik, annorangniglo 10 nik uvloksiorviksiutinik;

6 Atsilunelo aglangnik tamakkoninga Israelitit attanninganut, imâk okartunik: Aglait ukkua tikkipatta illingnut, ahâk, tagva kaujilerit kivgara Naeman tillilaurapko illingnut, kallakarungnaitilugo pikovlutit.

7 Israelitillo attanningata aglait tamakkoa attuaramigit, annorakotine allikpait, okarlunelo: Gudeovîk, tokkotsijungnarajarlunga omartitsijungnarajarlungalo, tilliklervigingmanga, angut tamna kallangêjarlugo pikovlunga? Kamatsiaritse kannok pitjutiksarsiormat uvamne.

8 Elisab Gudib angutekotingata tamanna tussaramiuk, attanniub annorakotine allilaungmagit, tilliklervigiva, okautsaukolâlo: Sôg annorakotitit allilaukpigit? Uvamnut kailerle tukkisilerkorlugo nellautaijokarmat Israeleme.

9 Taimak Naeman kaivok aksalloalikut horseliktutigut, nokarlunelo Elisab iglungata sângane.

10 Tagva Elisab tillijaksak tilliva tapsomunga, okautsaukovâlo: Ailerit ubvarlutillo illingnik 7 êrtorlutit Jordaneme, tagva uvinît âkiktauniarpok kallakarungnainiarlutillo.

11 Tagva Naeman ningalerpok, aularlunelo okalarmelo: Issumalaukpunga anniviginiarmanga, nangeriartorlunelo tamaunga, 
Nalekablo Gudime attinga nipliarvigilugo, aggangminullo sukkutsera tillaijarlugo, taimaglo kallak pêjarniarmago.

12 Koksoak Amana Farfarelo Damaskusemêtuk namanersaungilâk immeksoarnit illunainit Israelemetunit? Tapkonane ubvarungnangilanga kallangêjarlungalo? Allaivorlo aularlunelo ningardlune.

13 Tagva kivgangita tikkipât, okarvigilugolo, okarlutiglo: Nagliktavut atâtak, nellautaijub pijariakortôjomik pinniarkolaukpattit, pinniarajangilatit tamattominga? Kannok angiluartomik, okautilaungmattit: Ubvarit, tagva kallangêrotiniarpotit.

14 Tagva niovok, missiklunelo Jordanemut, akkarlunelo 7 êrtorlune, sorlo Gudib angutekotinga okalaungmat: uviningalo âkiktaumavok nukapiab innuosuktub uviningatut-idlune, ivsornailunelo.

15 Utterivorlo Gudib angutekotinganut, illagijane illunatik illagælugit. Itteramelo sângerpa, okarlunelo: Ahâk illitarksimavunga, Gudekangimat nunane illunaine, Israeleme kissiane; mannalo pilliumik tigusilaurit kivgarnit.

16 Tamna okarpok: Sorlo miksekârtomik Nalegak sangiartara innôngmat, tigulungilara. Innapâlo tigusikovlugo; pijomalaungilarle.

17 Tagva Naeman okarpok: Kivgait tunnitsiviojungnangilâk nunab oma ivjunganik, taimak angitigijomik horseojartuk magguk neksarungnartagingnik? Kivgait tunnergutiksanik, tunnergutiniglo ikkoalajaksanik tunnijijomairpok Gudeungitunut, Nalekamulle;

18 Nalekab kivgait tamattomane saimarvigilugo pikovlugo, tuksiaraikuma Rimonib iglungane, angajokara Rimonib iglunganut tuksiariartoraikune, aggakkalo egangavigivlugit pileraikune, tuksialeraikumalo Rimonib iglungane, tagva Nalekab kivgait issumagijungnaivigilugo pile.

19 Tapsomalo okautiva: Aularit ullapirsautekarlutit. Aularporlo tapsomangat, apkosiniub takkiningata illanganik.

20 Tagva Gehasi, Eliab, Gudib angutekotingata kivganga okarpok: Ahâk angajokama Siriamiok Naeman una nikkagilaukpa, tigusilaungimat sunamik taipsoma agganginit kailauktanginik: Sorlo miksekârtomik Nalegak innôngmat, udlagomavara, tigusijomalungalo sunamik taipsomangat.

21 Taimak Gehasib Naeman udlapa, Naemanelo tækkogame udlaningmat, niovok aksalloalingmit, pachreartorpa, okarlunelo: Illunane idluarka?

22 Tamna okarpok: Ahaila. Kissiane angajokama tillilaukpânga, okautsaukolutillo: Ahâk, tikkitaumavunga, nukapiangnut, Efraimib kakkanginit, nellautaijut erninginit; tunnitsivigilaukik Talentemik Silberemik, annorangniglo maggungnik uvloksiorviksiutinik. 
23 Naeman okarpok: Nagliktara tigusilaurit Silberemik Talentingnik. Innapâlo, poksilunelo Talentingnik maggungnik Silberemik, pôngnut maggungnut, annorângniglo uvloksiorviksiutinik, tunnivaillo kivgamingnut, tapkoa akjarsivuk tapkoninga sivorngane.

24 Ofelemullo tikkiname, tiguvait tapkoa agganginit, illivaillo iglomut, angutiglo aularkovak.

25 'Tapkoalo aularêrmanik, nalegane sângerpa. Elisablo okautiva: Gehasi nakit pivît? Tamna okarpok: Kivgait ikkungalonêt, tagvungalonêt ailaungilak.

26 Tapsomale okautiva: Omattiga agvertarlaungilâk, angut taimna uttermat aksalloalikotiminit, pachreartorlutit? Tamanna nelliutijolauka, Silbere anorâllo, perorsêvillo orksuktaggit, perorsêvillo Weineggit, saukallo, tuktuvaillo, kivgello angutit, kivgello arnat tigulugit?

27 Naemanible kallangata atâvigênarniarpâtit kinguvakotitillo soraijuitomik. Tagva annilerpok tapsomangat, kallakarlune apputitut.

\section{KAPITEL VI.}

Elisab poktartitsininga kikkiengmik; nollojungnaititsinengalo attannermik Siriamiut pinniarninginit. Kâvitssoarlo Samariame.

$\mathrm{N}$

EellaUtaiJUT erningita Elisa okautivât: Ahâk inne innigijarut mâne sângne nimniuluarpok uvaptingnut.

2 Jordanemut aikolertigut, attunîllo tagvangat aiklerkotigut nappartunik ikkâksanik, igloliorkovluta tagvane, inniksariniartaptingnik. Tamna okarpok: Aileritse tagvunga.

3 Tapkoalo illangat okarpok: Nagliktara kivgakotitit illagikit. Tamna okarpok: Illagijomavapse.

4 Illagivaillo. Jordanemullo tikkinamik nappartunik nakkartsivut.

5 Illangallo nappartomik nakkartsigame, ullimaut kattakpok kongmut. Nipliadlarporlo okarlunelo: Kappianamêk Nalegâk! tappilugo attartugauvok.

6 Gudible angutekotinga okarpok: Nane kattaksimava? Kattagvigilauktangalo unertutigingmago tapsomunga, kejungmik kippisivok, tongnilunelo tagvunga. Tagva ullimaut pokilarpok.

7 Okarporlo: Kiviguk. Tagva aggane issakpait, tigulâlo.

8 Siriamiullo attaningat unatadlarnekarpok Israelitinut, kivganelo issumaksarsiorkattigivait, okarlunelo: Ikane tagvanelo tangmaromavogut.

9 Gudible angutekotinga tilliklerpok Israelitit attanning- 
anut, okautsaukolugolo: Udsertorit, inne taimna kamagitaililugo pikonak, Siriamiut attersaromangmatta tagvunga.

10 Israelitillo attanningat tilliklerpok tagva innemut tagvunga, Gudib angutekotingata unertutigilauktanganut tapsomunga, tennersarlugo, udsertorpâlo; taimailiortuinarlungilarlo attausêardlune, maggoêrtorlunelonêt.

11 Tagva Siriamiut attanningata omattingata tamanna annûjutigiva, kivganelo kaikovait, okautilugillo: Okautijomangipissinga, illapta nelliata Israelitit attanningat illagingmangago?

12 Tagva kivgangita illangat okarpok: Taimangungitok angajokara attannek; Elisale nellautaijok Israeleme, tamainik okarpok Israelitit attanninganut, okauserijarnik aiparuserkotingne innarvikotivit najugâne.

13 Tamna okarpok: Tagva aularitse, tækkojartorluselo, nanêmangat, tilliklerniarkovlunga tagvunga, aijaukolugolo. Nellojungnaitipâllo, okarlutiglo: Ahâk, Dotanemêpok.

14 Tagva tilliklerpok tagvunga horsinik aksalloalingniglo sorsuktuksaksoarniglo kattimgajoksoarnik, tikkipullo tagvunga unnuarme, illumavaillo iglugaseksuit.

15 Gudiblo angutekotingata kivganga uvlarârsukut makkingmat, annilermallo; ahâk, tagva kattimgajoksuit iglugaseksuit illumavait, horselijarlutik aksalloalijarlutiglo. Tagva kivgangata okautiva: Kappianamêk nalegâk, kannok pinniaromavinuk manna?

16 Tamna okarpok: sivuraniarnak; tapkoa unnurnersaungmatta uvaptingnêtut, tapkonangat tapkonanêtunit.

17 Elisalo tuksiarpok, okarlunelo: Nalegak ijigik uitikik tækkokovlugo. Nalekablo nukapiab ijigik uitipâk tækkokovlugo. Ahâglo, kakkak nâmalaukpok horsenik aksalloalingniglo ikkomalingnik Elisab avatâne.

18 Attarsimangmatta tapsomunga, Elisa tuksiarpok, okarlunelo: Nalegak innugiartoksuit ukkoa tikkitaukokit tautunginermut. Tapsomalo tautunginermut tikkitaukovait, Elisab okausingit malliklugit.

19 Elisablo okautivait: Apkosinek una tamnaulungilak, iglugaseksuillo tapkoangunginivut. Malliksinga, uvangalo tessioromavapse angumut kennertapsingnut. Tessiorpaillo Samariamut.

20 Samariamullo tikkilermatta, Elisa okarpok: Nalegak tapkoa ijingit uitikit tautukovlugit. Nalekablo ijingit uitipait tautukovlugit; ahâglo tagva Samariab: (iglugaseksuit) kerkanginêmarilaukput.

21 Israelitillo attanningata Elisa okautiva tækkogamigit: Atâtâk saviksoarmut ullimaraksarivakka?

22 Tamna okarpok: Ullimaraksaringilattit. Angujattit saviksoakotingnut, pittiksekotingnullo, taipkoa ullimarkit. Sâmi- 
utsivigikit kakkojanik immermiglo, nerrikovlugit immerkovlugillo, aularkolugillo angajokakotinganut.

23 Tagva nerkiksaliorpait angijomik. Nerrijarêrmattalo, immerêrlutiglo, aularkovait, aikovlugit angajokamingnut. Taimangat Siriamiut sorsuktuksangit tikkitsungnaiput Israelitit nunanganut.

24 Tamakkoalo kingorngane pijokalaukpok, Benhadadib Siriamiut attanningata sorsuktuksaksoakotine kattitimagit majorarlunelo takpaunga, Samarialo illumavait.

25 Kâviksoakadlarporlo Samariame. Iglugaseksuit illumavait, siutekoktôjub niakunga akkekakârtinago kênaujanik, Silberlingemik attelingnik, 80 ginik. Taubillo annangit oktutib Kabemik attiggub sittamauliviningat akkekakârtinagit Silberlinginik 5 nik.

26 Israelitillo attanningat avallunut aimat, arnat illangata nipliarvigiva okarlunelo: Ikajorlaunga nalegara attannek!

27 Tamna okarpok: Nalekab ikajungipattit, sunamut uvanga ikajoraksarivagit? kakkojaksat tullerartauvinganit, Weineliksallonêt iptejaivinganit?

28 Attanniublo okautiva: Suvit? Tamna okarpok: Arnab oma okautilaukpânga: Ernit tunnilauruk uvlome nerrikovlunnuk, kaupat ernera nerrijomavavuk.

29 Taimak ernera nerrilaukpavuk. Uvangalo okautilaukpara uvlub aipangane: Ernit tunnileruk, nerrilauluglo; jjersimavâle.

30 Attanniub arnab oma okausingit tussaramigit, annorane allikpait ailune avalunut. Tagva innugiartoksuit illunatik tækkovât pôlijarmat timimine.

31 Okarporlo: Gudib imâk, imâglo pinniarvigilinga, Elisab Safatib erningata niakunga uvlome tapsomanêtsainarpat.

32 Elisale iksivavok iglomine, Israelitillo angajokangita iksivakattigivat. Attannerlo tilliklerpok angumik sivunermine. Tillijarle tikkikârnago tapsomunga, okarpok angajokaunerôjunut: Tækkolaukise kannok innuartub erninga una tilliklilaungmat tamaunga, niakumnik nussuksijomavlune? Tækkoleritse, tillijak tikkiniarpat, tagva upkoak pallangairsiuk, ajagissiuglo upkoamut; ahâk, angajokangata ittiggangita kikkergallijarningita mallikpât.

33 Taimaglo okartillugo sulle, ahâk, tagva tillijak tikkipok unnunga tapsomunga, okarlunelo: Ahâk, kanoêtok taimaitok pivok Nalekamit; sunamik sulle nerriuktuksauvik Nalekamit? 


\section{KAPITEL VII.}

Elisab nellautaininganik ivlub aipangane nerliksat akkekitojuksaungmatta.

ELisale okarpok: Nalekab okausingit tussarsigik: Imâk Nalegak okarpok: Kaupat nelliutome tamattomane, oktut attausek sennaukamik kakkiortamik, akkekaniarpok Sekkelemik attautsemik, oktutiglo Gerstenik Sekkelemik attautsemik, Samariab itterviksoangata attâne.

2 Tagva angajokaksuit illangata, attanniub aggangita ajækpagangata Gudib angutekotinga kiova, okarlunelo: Nalegarlo igalangnik sennanajarune killangme, kannok tamanna pijungnarajarka? Tamna okarpok: Ahâk ijingnut tækkoniarpet, nerriniangilatille tamattomangat.

3 Angutekalaukporlo sittamanik kallalingnik upkoame itterviksub sillatâne, tapkoa ingmingnut okautiutivut: Suna pivlugo ovanêtsomavita tokkokârtinata?

4 Iglugaseksoarnut itterungnarasuginajaralloarupta, tagva kâviksoakarpok iglugaseksoarne, tokkojariakarajaralloarpogullo tagvane: ovanêtuarupta tokkojariakarivogut. Taimak manna kaileritse, Siriamiullo tangmarvinganut akpangerta. Innokopattigut, tagva innôvogut, tokkopattigullo, tagva tokkunganiarpogut.

5 Tavligarârsukullo parngnaiput, tikkitsomavlutik Siriamiut kattimgajoksoakotinginut. Siriamiullo tangmarvingata issuanut tikkingmatta, ahâk, tagva innukatsengilak tagvane.

6 Nalekab Siriamiut tussarkolaungmagit horsit kikkergaillijarninginik, kemuksillo aksalloaggit kallergallijarninginik, kattimgajoksuillo kikkergallijarninginik; ingmingnut okalaungmatta: Ahâk, Israelitit attanningata kaikolaukpait Hetiterit attanningit, Egipteniblo attannertangit, sorsugvigivluta pikovlugit, opingartaukovlutalo taipkonunga.

7 Parngnailerpullo kemâlutiglo tavligarârsukut; kemailutiglo tuppermingnik, horsekotimingnik, siutekoktojokotimingniglo tangmarvingmetunik sokkoseratik, kemâlutiglo innôsermingnik piulikleromavlutik.

8 Kallakartut tapkoa manna tangmarvingmut tikkinamik, tuppit illanganut itterput, nerrivut immerlutiglo, tigusilarmiglo Goldemik Silberemiglo, annorangniglo, ijersijartorlutiglo tamakkoninga, utterpullo, itterlarmiglo tuppiub assianut, tigusilutigle tapsomangat ijeriartorpaillo.

9 Tagvale ingminut okautiutivut: Taimailiorniarata, uvlok tamanna uvlôvok taigotaksalik ajungitomik; angigutiguptigo 
tamanna uttakilutalo kaulermarikârtinago, tagva pitlartauniksarsiniarpogut. Tagvalo manna kaititse, ailerlutalo, nellojungnaititsilutalo tamattominga attanniub iglungane.

10 Tikkinamiglo nipliarput iglugaseksuit upkoaksoangane, okautilaitalo udsertortut, okarlutiglo: Siriamiut tangmarvinganut tikkisimavogut; ahâglo innukangilak tagvane, nippekalungilarlonêt innungmit; horsille siutekoktojullo pittuksimatillugit, tuppillo sokkoseratik tagvanêput.

11 Upkoaksublo udsertortingit kaikovait, okariartorkovlugit tamattominga attanniub iglungane.

12 Tagva attannek makkipok unnuarme, okarlunelo kivgaminut: Okautijomavapse, sunamik Siriamiut piniarvigingmattigut: Kaujimavut kâdlarapta, ailaukpullo tangmarvingmit, ijeriartoromamut ingmingnik natername, issumatillugit: Taipkoa annilerniarpatta iglugaseksoarnit, tagva innôtillugit tigujomavavut, ittajomalutalo iglugaseksoarnut.

13. Tagva kivgangita illangat kiovok okarlunelo: Horsit tellimat amiakojut sulle kamane, (ahâk kamane amiakovut Israelitit umnurninginit sakkijarungnaitunit) tapkoa tillikliutilavut, tækkolutalo.

14 Tagva kammutik maggoit aksalloalik horsit illaliutilugit tiguvait! attanniublo tillivait Siriamiut kattimgajoksuit malliklugit okarlunelo: Aileritse tækkoluselo.

15 Mallikpaillo Jordan tikkidlugo; ahâglo, tagva apkosinek nâmalaukpok anorângnik perkutiniglo, Siriamiut egilauktanginik, tuavilukalauramik. Tillijallo utterput okarlutiglo tamattominga attannermut.

16 Tagva innugiartoksuit annilerput aksarpâllo Siriamiut tangmarvingat. Oktullo attausek sennaukamik kakkortamik akkekarpok Sekelemik attautsemik, oktutiglo magguk Gerstenik akkekarivuk Sekelemik attautsemik, Nalekab okausingit maliklugit.

17 Attanniuble angajokak taimna aggangme ajækparvigijangait tilliva ittertarviksub attânnut. Innugiartoksuillo tullerarpât ittertarviksoarme, tokkolugolo sorlo Gudib angutekotinga okalaungmat, attannek attersartillugo tapsomunga.

18 Pijokalaukporle Gudib angutekotinga okartillugo attannermut okarlunelo: Kaupat nelliutome tamattomane oktutik Gerstenik akkekarniarpuk Sekelemik attautsemik, oktullo attausek sennaukamik kakkortamik Sekelemik attautsemik Samariab ittertarviksoangata attâne.

19 Tagra angajokab oma Gudib angutekotinga kiova okarlunelo: Ahâk, Nalegak igalangnik sennanajarune killangme, kannok tamanna pilerungnarajarka? Tamnale okarpok: Ahâk ijingnut tækkoniarpat, nerriniangilatillo tamattomangat. 
20 Taimailingaluatsialaurivok, innugiartoksuit tullerajarmatsuk ittertarviksoarme, tokkovorlo.

\section{KAPITEL VIII.}

Kâviksoakarnek Jahrit 7 it nâlugit. Siriamiut assianik attannekarningat. Joramelo, Ahasjalo attanniojuk Judame.

EI

LISABLO arnak tamna okarvigiva, omartilauktame ernerub anânanga, okarlunelo: Parngnailerit iglomiokattitillo illagælugit aularit, tujormijangolutillo sappingitarne; Nalegak kâvingmik kaikojiniarmat, tamanna nunamut tikkiniarpok Jahrit 7 it nâlugit.

2 Arnak tamna parngnailerpok taimailiorlunelo, sorlo Gudib angutekotinga okalaungmat, tujormiangolaukporlo Filisterit nunangane, Jahrit 7 it nâlugit.

3 Jahrille 7 it tapkoa kangermatta, arnak tamna utterivok Filisterit nunanganit; aularporlo attannek nipliarvigilugo, iglokotine perorsêvikotinelo pivlugit.

4 Attanniuble Gehasi Gudib angutekotingata kivganga okarvigiva okarlunelo: Unipkautilaunga pinniarnersoarnik tamainik Elisab pinniarlauktanginik.

5 Unipkartillugolo attannermut, kannok tokkungajok omartilaungmago; ahâk tagva namâkilune arnak tamna tikkipok tagvunga, omartitaumajub ernerub anânanga, attannerlo nipliarvigiva iglokotine pivlugo, perorsêvikotinelo pivlugo. Tagva Gehasi okarpok: Nalegâk attannek, tagva arnak tamna; tagvalo erninga Elisab omartilauktanga.

6 Attanniublo arnak apperiva; tapsomalo arnab unipkautiva tamattominga. Tagva attanniub pimaklertit illangat illaukova tapsomunga; okarlunelo: Sattorsitiuk tamainik nangminerijanginik, tappilugillo perorsêviub perortungita tamaita akkinginik, taimangat nuna aularlaungmago manna tikkilugo.

7 Elisalo Damaskusemut tikkipok. Tagvane Benhadad Siriamiut attanningat kanimavlune nellalaukpok; okautsauvorlo, okartokarlunelo: Gudib angutekotinga tamaungarsimavok.

8 Tagva attannek Hasaelemut okarpok: Pilliutiksanik najortekarlutit, Gudib angutekotinga pachrearuk, Nalegarlo apperiuk taipsomûna, okarlutillo kannimasermit mattomangat nekkoksiniaralloarmangarma.

9 Hasaeliblo pachrearpa pilliutiksaniglo neksarpok, perkutiniglo sunatuinarnik Damaskusemit, nangmagaksanik 40 ginik Kamêlinut. Tikkinamelo sângerpa okarlunelo: Ernivit Benhadadib attanniub Siriame tillimavânga illingnut, okautsaukovâtillo: Nekkoksijungnaralloarkik kannimasermit omangat? 
10 Elisab okautiva: Okautijartoruk: Nekkoksiniarpotit; Nalekable nellojungnaitilaukpânga, tokkoniarmat.

11 Gudiblo angutekotingata okkijiva akkunit kêalunelo.

12 Tagva Hasael okarpok: Sôg nalegara kêava? Tamna okarpok: Kaujimavunga kannok igvit Israelib kittorngangit idluitullivigijomârangne. Iglugaseksoangit songojut ikkomamut ikkitsomârpattit angutekotingit innuosuktut tokkotsomârpattit saviksoarmut, sorrusiârsukotingillo tokkotsomârpattit, arnakotingillo singaijut ullimârlugit avgoromârpattit.

13 Hasaele okarpok; Kivgait kingmek sunauva, taimaitunik pijariakortôjunik pinniartuksaunajarmat? Elisa okarpok: Nalekab nellojungnaitilaukpânga, igvit attannioniaravit Siriamiunut.

14 Tamnalo aularpok Elisamit, tikkilunelo angajokaminut, tapsoma okautiva: Sunamik Elisab okautilaukâtit? Okarpok: Okautilaukpânga: Nekkornersauniarpotit.

15 Kaulermallo ullik pergaimajok tiguva missuklugolo immermut, issivilâlo tapsoma kânganut; tagva tokkovok. Hasaelelo attanningorpok innangêrlugo.

16 Joramib Ahabib erningata Israelitit attanningata Jahringita 5 ingane, Joram Josafatib erninga attanningorpok Judakunut.

17 Jahrekalaukpok 32 ginik attanningorame, attanniotilunelo Jahrit 8 it nâlugit Jerusaleme.

18 Agvertarlunelo Israelitit attanningita apkosiningine, sorlo Ahabekut pilaungmatta; Ahabib pannia nulliarigamiuk; piniarporlo Nalekab idluigijanginik.

19 Nalekable Judakut asseroromalaungilait kivgakotine Davide pivlugo; sorlo okautilaungmago, tunnitsivigitsainaromavlugo kaumautiksamik kittorngangita akkorngane soraijuitomik.

20 Tapsoma nelliuningane Edomiterit nalegungnailaukput Judakunik, attannekartitsilutiglo ingmingnut.

21 Jorame Zairekut aularlaungmat, aksalloaggillo illunatik illagivlugo, unnuarmelo parngnailaukpok, Edomiterillo illumajine ullimârlaukpait, taimaglotauk kamutit angajokangit, innugiartoksuillo kemâvut iglomingnut.

22 Tamanna pivlugo Edomiterit nalegungnaiput Judakunik uvlok tamanna tikkilugo. Nelliutome taipsomane Libna nalegungnailaurivut.

23 Unipkautsit assingit Jorame pivlugo, tamaitalo pinniarlauktangit, ahâk tamanna aglaksimavok Judakut attanningita aglangine.

24 Joramelo sinnisilerpok atâtagijaminut ailune, illijartor- 
taulunelo atatagijaminut, Davidib iglugaseksoakotingine, Ahasjalo erninga attanningorpok inangêrlugo.

25 Joramib Ahabib erningata Israelitit attanningata Jahringita 12 ingane, Ahasja Joramib erninga attanningorpok Judakunut.

26 Ahasja Jahrekalaukpok 22 ginik attanningorame, attanniotilunelo Jahre attausek nâlugo Jerusaleme. Anânanga attekalaukpok Ataliamik, Amrib Israelitit attanningata panninga.

27 Agvertarporlo Ahabekut apkosiningane, pinniarlunelo Nalekab idluigijanginik Ahabekutitut, sakkeolaungmat Ahabekunut.

28 Joramelo Ahabib erninga illagælugo aularpok Ramothemut Gileademetomut, Hasael Siriamiut attanningat sorsugvigijomavlugo; Siriamiulle Jorame sâlagivât.

29 Tagva Jorame attannek utterpok mamititaujomavlune Jesreeleme ikkiminit, pilauktaminit Siriamiunut Ramame, Hasael Siriamiut attanningat sorsugvigingmago. Ahasjalo Joramib Judakut attanningata erninga attersarpok, Jorame Ahab erninga tækkojartoromavlugo Jesreeleme; kannimangmat.

\section{KAPITEL IX.}

Jehub mingoartauninga attanningortitauvlune; tokkotsiningalo Joramemik, Israelitit attanninganik, Ahasjamiglo Judakut attanninganik. Isebelib tokkotauninga.

E okautilugolo: Tapseruserit makkitingnik, publaujarlo orksukaut una najortigiuk, ailutillo Ramothemut Gileademêtomut.

2 Tagvungalo tikkiniaruvit, tagvane Jehu Josafatib Nimsib erningata erninga tækkoniarpat. Itterit nikkovikolugolo kattangutime akkornganit; tessiorlugolo innemut kammanênerpangôjomut,

3 Publaujarlo orksukaut tiguleruk, kovilugolo niakunganut, okarlutillo: Imâk Nalegak okarpok: Mingoarlutit attanningortilaukpagit Israelitinut. Upkoarlo upkoêraksarivat, kemajuksaulutillo siglutonaglo.

4 Nellautaijublo innuosuktokotinga, nukapiak aivok Ramothemut Gileademêtomut.

5 Ittermallo, ahâk tagva sorsuktuksat angajokangit iksivavut tagvane. Okarporlo: Illingnut angajokaujotit okausiksakarpunga. Jehu okarpok: nelliptingnut? Tamna okarpok: Illingnut angajokaujotit.

6 Tagva nikkovipok itterlunelo. Tapsomale orksuk koviva 
niakoanut, okautivâlo: Imâk Nalegak Israelib Gudinga okarpok: Mingoarlutit attanningortimavagit Nalekab innukotinginut Israelitinut.

7 Igvillo Ahabib angajokavit iglomiukotingit ullimaraksarivattit, nellautaijut kivgakotima aungit akkiniarutigijomavakka Isebelemut.

8 Ahabekullo illunamassiatik asserortaujuksauvut, Ahabekunillo pêjaromavakka tamaita kivgallo kivgaungitullo Israeleme.

9 Ahabiblo iglomiukotingit illingatitsomavakka Jerobeamib Nebatib erningata iglomiokotingittitut, Baesablo Ahiab erningata iglomiokotingittitut.

10 Kingmillo Isebele nerrijaksarivât perorsêvingme Jesreeleme, kinamullo illijartortaujuksaungilak. Upkoarlo upkoêrpa kemâlunelo.

11 Jehulo annilermat angajokame kivganginut, okautsaurok: Illinganekatsiarka? Sôg uimajartok taimna tikkilauka illingnut? Tapsoma okautivait: Angut taimna illitarigalloarpasse okauserijangillo.

12 Tapkoa okarput, tamanna miksekârtolungilak, okautitigulle. Tamna okarpok: Imâglo imâglo, okautilaukpânga, okarlaukporlo: Imâk Nalegak okarpok: mingoarlutit attanningortimavagit Israelitinut.

13 Tagva tuavidlarput, attunnillo annoratik tiguvât illilugillo tapsoma attânut tumerangnut, tiptulautelo tiptulausijautigivat, okarlutiglo: Jehu attanningorpok!

14 Taimak Jehu Josafatib Nimsib erningata erninga angerkattigêngnekartitsivok Jorame akkerartorlugo. Joramele Israelitit illunatik illagilugit Ramothib Gileademêtub sângane tangmarsimalaukpok, Hasaele Siriamiut attanningat pivlugo.

15 Joramele attannek uttersimalaukpok mamititaujomavlune Jesreeleme, ikkiminit pilauktaminit Siriamiunut, Hasaele Siriamiut attanningat sorsugvigingmago. Jehulo okarpok: Taimak issumagupse, tagva kinalonêt kemâjuksaungilak iglugaseksoarnit, ainajarlune unipkautigilugolo Jesreeleme.

16 Jehulo ikkivok aularlunelo Jesreelemut, Jorame tagvane nellangmat; taimaglo Ahasja Judakut attanningat attersarlaukpok tagvunga Jorame tækkojomavlugo.

17 Udsertortuble nuvoguserme Jesreeleme nangertub Jehub kattimgajokotingit aggertut tækkovait, okarlunelo: Kattimgajut tækkovakka. Tagva Jorame okarpok: Ablangajok horsib kângane kaikoleruk tillilugolo pachrearlugit, okarlelo: Ullapirsautekarka?

18 Ablangajorlo horseme pachrearpâ, okarlunelo : Imâk attannek okarpok: Ullapirsautekarka? Jehu okarpok: Igvit ullapirsaut sugiviuk? sangulerit tunnumnut. Udsertorte kaujititsivok okarlunelo:Tillijab tikkimavait, utteranelo.

19 Tagva ablangajub horseme assianik tilliklerpok. Tamna 
tikkiname tapsomunga, okarpok: Imâk attannek okarpok: Ullapirsautekarka? Jehu okarpok: Ullapirsaut sugiviuk? sangulerit tunnumnut.

20 Tagva udsertorte nellojungnaititsivok okarlunelo: Tikkisimavait, utterungnaiporlo. Perajarniôvorlo Jehub Nimsib erningata perajarningatut; perajarmat, issumairotijotut.

21 Tagva Jorame okarpok: Annujileritse. Aksalloalikotingalo parngnaitipât; aularpuglo, Jorame Israelitit attanningat, Ahasjalo Judakut attanningat, attunit aksalloalikotimingne, Jehu pachrearomavlugo; naipipaglo Nabotib Jesreelemiub perorsêvigilauktangane.

22 Joramiblo Jehu tækkogamiuk, okarpok: Jehu ullapirsautekarka? Tamnale okarpok: Ullapirsaut suna? anânavit Isebelib anguniarningit, angakoktorningillo pivalliatuinartillugit?

23 Tagva Jorame allaivok tapsomangat, kemâlunelo, okalarmelo Ahasjamut: Pikkablajoksonekarpok Ahasja.

24 Jehuble pittikse tiguva, pittikpâlo Jorame tallikita akkorngatigut, kargjub omatterungmago, ochovorlo kammutekotimine.

25 Okarporlo angajokangmut Bidekaremut: Tiguleruk egilugolo Nabotib Jesreelemiub perorsêvigilauktanganut. Erkaigama igvit uvangalo kemukserlauramnuk aksalloalingme attautseme atâtangata Ahabib kingorngane, Nalekab nangmagak tamanna illilaungmago tapsomunga.

26 Ahamarik, Nalegak okalaukpok, Nabotib aunga, erningitalo aungit ikpeksak tækkolaukpakka, uvangalo kigluvigijomavagit tamattominga perorsêvingme tamattomane. Taimak tiguleruk manna egilugolo perorsêvingmut tamattomunga, Nalekab okausingit malliklugit.

27 Ahasja Judakut attanningat tamattominga tækkogame, kemâvok apkosinerme, perorsêviub iglunganut sângmijome. Jehuble udlava, okarlunelo: Ullimarsiuktauk kamutingne! Pilaukpok majokame Guremut Jeblaamib kanitanganêtome. Kemâvorlo Meggidomut, tokkolunelo tagvane.

28 Kivgangitalo kemuksiutivat Jerusalemut, illijartorpâllo tagvane illuvinganut, atâtagijanginut, Davidib iglugaseksoakotingine.

29 Ahasja attanningorlaukpok Judakunut Joramib Ahabib erningata Jahringita 11 ingane.

30 Jehulo Jesreelemut tikkimat, Isebelelo tussarame tamattominga, kênane mingoarpa, pinnarnartitsilunelo niakominik, nuitalunelo igalakut.

31 Jehulo ittertarviksub attânut tikkimat, Isebele okarpok: Simri, angajokaminik innuarlauktok illingatsialauka?

32 Tamnalo arlorpok igalangmut, okarlunelo: Kina uvamnêka mâne? Tagva magguk pingasullonêt sâpât. 
33 Tamna okarpok: Kattaktisiuk unnunga. Kattaktipâllo; aungalo serkerarpok iglub senneranganut horsinullo; tullerajarpâllo.

34 Itterêramelo nerrilaukârdlunelo, immerlaukardlarmelo, okarpok: Tækkojartorsiuk ominarsijaujok taimna, illijartorsiuglo, attanniub pannigingmago.

35 Tækkojartoramitsugle sunasilungilet tapsomangat niekungata, ittiggangitalo aggangitalo assianik;

36 Utterivullo okautilugolo mattominga. Tamnale okarpok: Tamannauvok Nalekab okauserilauktanga kivgamigut Eliakut, Tisbitemiojokut okalauklunelo: Perorsêvingme Jesreelemetome kingmit Isebelib uvininga nerrijaksarivât.

37 Isebeliblo timinga tokkungajok perorsaijotut natername, nellajuksauvok perorsêvingme Jesreelemetome; okarungnartokarkonago: tagva Isebele.

\section{KAPITEL X.}

Ahabib kinguvangita. Baaliblo kivgangita nungutauningit.

\section{Jehublo tokkunga.}

AHABELO ernekalaukpok 70 ginik Samariame. Jehulo aglangnik aglakpok, tillikliutivaillo Samariamut, iglugaseksuit Jesreelib angajokanginut, angajokaunerôjunut Ahabiblo kittorngangita pairijinginut, tapkoa imâk okarput:

2 Aglait ukkoa illipsingnut tikkipatta, najortekarapse angajokapse erninginik, kamutinginiglo, horsekotinginiglo, iglugaseksoarniglo sôngojunik, sakkuniglo.

3 Tagva tækkungiarsiuk namanerpâk, pikkaringnerpâglo angajokapse erninginit, pitisiuglo angajokapse iksivautaksoanganut, angajokapselo iglomiokotingit sorsugutisigik.

4 Taipkoale sivoradlarput, okarlutiglo: Ahâk, attannîk magguk nangerungnarlaungilâk sângane, kannok uvagut tagva nangeromavita?

5 Iglublo iglugaseksuillo angajokangit, pairksijullo tilliklerput Jehumut, okautsaukovâllo : Kivgauvogut illingnut, tamainik pinniaromavogut okauserijarnik uvaptingnut; uvagut attanningortitsijomangilagut kinamik; pinnialaurit namagijarnik.

6 Tagva aglait ạipainginik aglagvigivait, imầk okartunik: Illagigupsinga, nippigalo nalekupsiuk, tagva angajokapse erningita niakungit tigulersigik, kailugillo uvamnut, kaupat nelliutome tamattomane, Jesreelemut, (Attanniuble erningit, angutaulaukput 70 git. Iglugaseksuillo angajokaunerungita nullasavait.)

7 Aglait tapkoa manna tapkonunga tikkimatta, tiguvait 
attanniub erningit, tokkopaillo angutit 70 git, niakungillo illivait amargaujanut, tillikliutivaillo tapsomunga Jesreelemut.

8 Tillijarlo tikkipok nellojungnaitilâlo, okarlunelo: Attanniub erningita niakungit âlaukpait ovunga. Tamna okarpok: Illisigik, maggoililugit kallerêktisigik, itterviksub upkoangata sângane kaupemut.

9 Uvlakullo annigame, nangeriartorpok tagvunga, okarlunelo innugiartoksoarnut illunainut: Illipse idluartovose. Ahâk uvanga angerkattigêngnekartitsilaukpunga angajokara akkerartorlugo, tokkolaukparalo; kiale ukkoa ullimarlaukagit?

10 Tagva illitarksileritse Gudib okausingita okauserilauktangita Ahabe pivlugo illangallonêt kattaksimangimat nunamut; Nalegarlo pinniarlaukpok, sorlo okalaurame kivgamigut Eliakut.

11 Jehublo amiakojut Ahabekunit Jesreeleme illunaita ullimarpait, angajokakotingit illunaita, illangillo, ajochertuijokotingillo, amiakokakonago attautsemiglonêt.

12 Parngnailerporlo, aularlunelo tikkilarmelo Samariamut. Apkosiniublo senniane iglokalaukpok mianersijunut illingajomik.

13 Tagvane Jehub Ahasjab Judakut attanningata kattangutingit naipipait, okarlunelo: Kikovise? Tapkoa okarput: Ahasjab kattangutigivâtigut, attersarpogullo attanniub kittorngangit, attanniublo nulliangata kittorngangit sallutitsomavlugit.

14 Tamnale okarpok: Tigusigik innôtillugit. Tiguvaillo innôtillugit, tokkopaillo immertarvingme mianersijut iglungata sennianetome, angutit 42 git, amiakokarkojilungilarlo tapkonangat attautsemiglonêt.

15 Tagvangallo sivumuarpok, naipipalo Jonada Rechabib erninga, pârsijok tapsominga; salutipâlo okautilâlo: Omattit nellagôrka, sorlo omattiga illingangmat omattingnut? Jonada okarpok: Ahaila. Taimaipat tagva aggaktit tunnikit uvamnut. Agganelo tunnivait tapsomunga. Ikkikovâlo ingminut aksalloalingnut;

16 Okarlunelo: Aiparilaunga tækkolutillo illungertornimnik Nalegak pivlugo. Taimaglo kemuksiutitaukova illagælugo kammutekotimine.

17 Samariamullo tikkiname Ahabekut amiakungit Samariame illunaita ullimârpait nungumariktitsikârtinane tapkoninga, Nalekab okausingit, okauserilauktangit Eliamut, malliklugit.

18 Jehublo innugiartoksuit illunaita kattikovait, okautsaukolaitalo: Ahabib Baal kêtatuinarmik kivgartorlaukpa, Jehub namanersamik kivgartoromavâ.

19 Mannalo Baalib nellautaijokotingit illunaita, kivgakotingillo illunaita, ajochertuijokotingillo illunaita kaikosigik uvamnut, tikkitailijokarniarane; tunijijariakarama Baalemut tunnergutiksamik angijomik. Tikkitailijok innôjuksaungilak. Jehule taimailiorpok pingitsertorlune Baalib kivgangit tokkotsomavlugit. 
20 Jehulo okarpok: Baalib uvloksiorvinga ivsornaitisiuk, akpautigijaukosiuglo.

21 Jehulo tilliklerpok Israelitinut illunainut, tikkerkojilunelo Baalib kivganginik illunainik, amiakokalungimat attautsemiglonêt tikkilaungitomik. Tikkipullo Baalib iglunganut, Baaliblo iglunga tættæmalaukpok issuanit issuanut, aipânut.

22 Okarporlo pimaklertunut iglomik annorâktalingmik: Baalib kivgangit illunaita annitsivigisigik annorângnik. Annorângniglo kaitsivut tapkonunga.

23 Jehublo Jonada Rechabib erninga itterkattigiva Baalib kattimavinganut, okarlunelo Baalib kivganginut: . Nautsertoleritse tækkoluselo mâne pittakangimangat akkonapsingne Nalekab kivgangita nellianik, Baalible kivganginik kissênik.

24 Itterpullo tunnergutinik, tunnergutiniglo otaksanik tunnijijomarlutik. Jehuble sillatâne angutit 80 git perkolaukpait, okalauklunelo: Angutit tapkoa aggapsingnut tunnijama nelliat kemâkpat, tagra innôsise akkiksaujuksauvut, tapsoma innôsingata inningane.

25 Tunnergutiksarlo ôtaksak tunijaulerêrmat, Jehu sorsuktuksanut angajokangnullo okarpok: Itteritse, ullimarlugillo illunaita, nelliallonêt annitailitisiuk. Ullimârpaillo saviksub kênanganut. Sorsuktuksello angajokaillo egipait sillamut, ailutiglo iglugaseksoarnut Baalib kattimavingata najugânut,

26 Amnipaillo nappajut Baalib kattimavingata illuanit, ikkilaitalo.

27 Baaliblo nappajunga, kattimavinga illaliudlugo serkomipât, annagvingortipâllo uvlok tamanna tikkilugo.

28 Taimak Jehub Baale pêjarpa Israelemit.

29 Jerobeamible Nebatib erningata, Israelitinik idluitullititsilauktub, idluiningit, tagva nochangoâk Goldik Betheleme Danemelo allarlungilak.

30 Nalegarlo Jehumut okarpok: Tamanna pivlugo kunnutuilauravit, pinniarlutit namagijamnik, Ahabekunelo pinnialauravit tamainik, omattimnelauktunik, iksivajokartuksauvotit Israelitit iksivautaksoangane kittorngakotingnit, kinguvarêt sittamangat tikkidlugit.

31 Jehuble kamagingila Nalekab Israelib Gudingata perkojangit malliklugit agvertarlune omamut tamâmut; Jerobeamib Israelitinik idluitullititsilauktub idluiningit allârlungimagit.

32 Nelliutome taipsomane Nalegak pigiarpok Israelitit illangerlugit; Hasaeliblo sâlagivait Israelitit kiglilervingine illunaine,

33 Jordanemit sekkerngub nuijarviata nellânut, nuna Gileademik attelik illunât, Gadekullo nunangat, Rubenekullo nunangat, Manassekullo nunangat, Aroeremit kongmêtomit Arnonib kannitanganêtomit, Gileadelo Basanelo.

34 Unipkautsit makkoa assingit Jehu pivlugo, pinniarlauk- 
tangit, sôngoningalo tamât, ahâk, tamanna aglaksimavok Israelitit attanningita aglangine.

35 Jehulo sinnisilerpok atâtagijaminut ailune, illijartorpâllo Samariame. Erningalo Joahas âttanningorpok innangêrlugo.

36 Jehuble attanniotivinga Israelitinut Samariame, Jahreôvut 28 git.

\section{KAPITEL XI.}

Athaljab pinniarlungningit. Joasib attanningortitauninga Judakunut. Athaljab tokkotauninga.

ATHALJALE Ahasjab anânanga, tækkogame ernine tokkungmat, parngnailerpok, tokkotsilunelo attanniub kinguvânginik illunainik.

2 Josebable attanniub Joramib panningata Ahasjab najangata tiguva Joase, Ahasjab erninga, tiglikpâlo attanniub kittornganginit tokkotaujunit pairijinga aiparællugo innime sinnigvingme; ijerpâlo Athalja pivlugo, tokkotaukonnago;

3 Ijertaumalaukporlo tamna (pairijine) aiparællugo Jahrit 6 it nâlugit. Athaljale attanniolaukpok nunamè.

4 Jahrille 7 ingane Jojada tilliklerpok, angajokajallo 100 dinut, kappitallo sorsuktuksallo illagællugit tiguvait, itterkolugillo tapsomunga Nalekab iglunganut, angerkattigivaillo, angermarikovaillo Nalekab iglungane, attanniublo erninga tækkojautipa tapkonunga,

5 Perkolugillo okalarmelo: Tamannauvok piniaraksase: Illipse pingasoliluse, pingajuise attautsit nangeriartortuksauvut Sabbatib uvlungane, udsertortuksauvlutiglo attanniub iglungane;

6 Pingajuiselo attautsit, itterviksoarmêtuksauvut Suremik attelingme, pingajuiselo attautsit itterviksoarme sorsuktuksat tunnuanetome, taimaglo iglo udsertorsiuk, agviarluselo.

7 Pingajuisele maggoit, Sabbateme nûlertose, udsertortuksauvose Nalekab iglungane attanniub avatâne;

8 Illumajaksarivasse, attunît sakkokotipsingnik tigumiarluse aggapsingnut; kinalo kerdlerotijok siæktut akkorngatigut tamna tokkotaujuksauvok; attannermeleritselo annileraikpat itteraikpallo.

9 Angajokajallo 100 dinut pinniarput tamainik, sorlo Jojadab ajochertuijub perkungmagit, pitipaillo attunît ingmingnut angutekotitik, Sabbateme tikkitut, Sabbatemelo nûlertut, tikkilutiglo ajochertuijomut Jojadamut.

10 Ajochertuijublo angajokait tunnitsivigivait kallugiarnik, 
sappulutaniglo, attanniub Davidib pigilauktanginik, Nalekablo iglunganetunik.

11 Sorsuktuksallo nangerput attanniub avatâne, attunît sakkomingnik tigumiarlutik, iglub sennerangata tallerpianit, iglub senerangata saumia tikkidlugo, Altaremelo, iglomelo.

12 Tapsomalo attanniub erninga annitipa; niakoserpâlo, perkojaniglo tunnitsivigiva, attaningortipâlo, mingoarlâlo aggatiglo pattiktipait nipliarlutiglo: Attannek innôle!

$13 \Lambda$ thaljablo sorsuktuksat, innugiartoksuillo nipliarningit tussaramigit, tikkilerpok innugiartoksoarnut Nalekab iglunganut.

14 Tækkosarlunelo, ahâglo, attannek nangerpok nappajome, sorlo illusiovalaungmat, angajokaunerôjullo tiptulausijartullo attannek nangerkattigivât, nunablo innungiartoksoangit illunatik kuviasudlalaukput, tiptulausijarlutiglo. Athaljable annorane allikpait nipliarlunelo: Pikkablojoksônek, pikkablajoksônek!

15 Ajochertuijuble Jojadab angajokajat 100 dinut sorsuktuksanut angajokangortitaumajut perkovait, okautilugillo: Annitisiuk siaktat akkornganut, malliktorlo tapsominga tokkosiuk saviksoarmut. Ajochertuijok okalaungmat: Tokkotauniarane Nalekab iglungane.

16 Aggatiglo illivait tapsomunga; aivorlo apkosinerme horsit aivigivaktangine attanniub iglunganut, tokkotauvorlo tagvane.

17 Jodadalo angerkattigêngnekartitsivok Nalekab attanniublo innugiartoksuillo akkorngane, Nalekab innukotigijaksaringmagit; taimaktauk attanniub innugiartoksuillo akkorngane.

18 Tagva nunab innugiartoksoangit illunamassiatik itterput Baalib kattimavinganut, imitipâllo Altarekotingit, Gudingoakotingillo serkomipait; Matthanelo Baalib ajochertuijokotinga tokkopât Altarit sângine. Ajochertuijorlo udsertortokartitsivok Nalekab iglungane.

19 Tiguvaillo angajokat 100 dinut. kappitallo, sorsuktuksallo, nunablo innugiartoksoangit illunaita, attannerlo attersartipât Nalekab iglunganit, tikkilutiglo apkosinerme, sorsuktuksat itterviksoanganit pijome, attanniub iglunganut; ingiporlo attanniub iksivautaksoanganut.

20 Innugiartoksuillo nuname illunamassiatik kuviasudlalaukput, iglug iseksuillo innilangalaukput. Athaljale tokkopât saviksoarmut attanniub iglungane.

21 Joaselo Jahrekalaukpok 7 nik attanningorame. 


\section{KAPITEL XII.}

\section{Joasib attanniub Judame attanniotininga.}

JEHÜB Jahringita 7 ingane Joase attanningorpok, attanniotilunelo Jahrit 40 git nâlugit Jerusaleme. Anânanga attekalaukpok Zibeamik, Bersabamioviniôjok.

2 Joaselo pinniarlaukpok idluartomik, Nalekablo kuviagijanganik, taimak akkunêrtigijomik, ajochertortautillugo ajochertuijomut Jojadamut;

3 Kissiane pôktojut pêjartaulaungilet; innugiartoksuille tunnergutinik tunnijivut, isserluanititsilutiglo sulle poktôjune.

4 Joaselo okarpok ajochertuijunut: Kênaujat illunaita ivsornaititaulertut, ittertitaujut Nalekab iglunganut, kênaujat attortauvaktut, kênaujat illunatik attunît tunnijariakartangit tarnitik pivlugit, kênaujallo illunaita, nelliata tunnijangit perkojaunane omamut kunutuitomut Nalekab iglunganut,

5 Tamakkoa ajochertuijut tigujaksarivait ingmingnut, attunît illitarijamingnit. Tamakkonangat âkiksoraksarivait sujuksimanerit Nalekab iglungane, sujuksimanerit illunaita nachvartaulertut tagvane.

6 Ajochertuijullo attanniub Joasib Jahringita 23 ingane sujuksimanerit iglome akiksortaukolaungimattigik;

7 Attanniub Joasib ajochertuijok Jojada kaikova, ajochertuijullo assingit, okautivaillo: Sôg sujuksimanerit iglome âkiksorlungipissigik? Mannalo kênaujat tigusijaksaringilase illitarijapsingnit, tunnijaksarilaukpassele iglub sujuksimaninginut.

8 Tagva ajochertuijut angerput tigusijungnairomavlutik kênaujanik innugiartoksoarnit, sujuksimanerillo iglome âkiksornagit.

9 Tagva ajochertuijub Jojadab iklervik tiguva, puttorlugolo killakartipa kânga, illilâlo Altarib sennianut tallerpianut. Nalekab iglungata ittertauviata sennianut. Ajochertuijullo upkoamik udsertortut, takkamunga pititsivut kênaujanik illunainik, Nalekab iglunganut kaitaumajunik.

10 Tækkopattalo kênaujakadlarmat iklervingme, tagva attanniub aglapaktokotinga, ajochertuijoksoarlo majorarlutik tikkilerpuk, pôlerlugillo, kittipaillo kênaujat naipitaujut Nalekab iglungane.

11 Kênaujalle kittitaumajut tunnivait sennajut sullijaksamik tamattominga, perkojaumajut aulatsikovlugit Nalekab iglunganik agganginut; tapkoalo tunnivait igloliortunut sennajunullo Nalekab iglungane,

12 Tagva karmaliortunut, ujarkaniglo ullimârtunut, keju- 
niglo ujarkaniglo ullimarsimajunik pissiniartunut, sujuksimanerit Nalekab iglungane akiksortaukovlugit, tamaitalo nachvartangit akiktaujariakartut Nalekab iglungane.

13 Sennakojilungilelle kênaujanit kaitaumajunit Nalekab iglunganut, itteringujanik Silberinik, savingniglo, pogutaniglo tiptulautiniglo, illulerkutillonêt nellianik Goldeôjomik Silbereôjomiglonêt;

14 Tapkoale sullijaksamik sennajut tunnitsiviovut tamattominga, akiksoikovlugit sujuksimanernik Nalekab iglungane.

15 Angutille tunnitsiviojut agganginut kênaujanik tunnijikovlugit taipkonunga sennajunut, apperijaulaungilet kingorngane tapkoa pivlugit; nertornarlutik pinniarmatta.

16 Kênaujalle tunnergutinit akkiliksêrutiksanit, tunnergutanillo ajortoêrutiksanit, Nalekab iglunganut âtaulungilet, ajochertuijunut illingangmatta.

17 Nelliutome taipsomane Hasael Siriamiut attanningat majorarpok, Gathelo sorsugvigivait, sâlagilaitalo. Hạsaeliblo kênane sâtipa majoraromavlune Jerusalemut;

18 Tagva Joasib Judakut attanningata ivsornaititaumajut taimaita tiguvait, atâtagijame Josafatib, Joramiblo, Ahasjablo Judakut attanningita ivsornaitilauktangit, Goldelo tamât nachvartaumajok Nalekab iglungata perkutitalingane, tillikliutivaillo Hasaelemut Siriamiut attanninganut, tagva aularpok Jerusalemit.

19 Unipkautsillo tamakkoa assingit Joase pivlugo, pinniarningillo tamaita, tamanna aglaksimavok Judakut attanningita aglangine.

20 Kivgangillo pikablavut, angerkattigêngnekartitsivullo, annaulerpâllo Millob iglungane unnunga sangmijome Sillamut.

21 Josabarib Simeatib erningata, Josabadiblo Somerib erningata annaulerlugo tokkopâk. Illijartortauvorlo atâtagijaminut Davidib iglugaseksoangine. Amazialo erninga attanningorpok innangêrlugo,

\section{KAPITEL XIII.}

\section{Joahasib Joasiblo aitanniojuk Israelitinut attanniotinigik. Elisab tokkoninga.}

JOASIB Ahasjab erningata Judakut attanningata Jahringita 23 ingane, Joahas, Jehub erninga attanningorpok Israelitinut Samariame, attanniotilunelo Jahrit 17 it nâlugit.

2 Pinniarporlo Nalekab idluigijanganik, agvertarlunelo Jerobeamib Nebatib erningata Israelitinik idluitullititsilauktub idluiningit malliklugit, allailungilarlo tapkoninga. 
3 Nalekablo ningausınga illungertulerpok Israelitit pivlugit, tunnilaitalo Hasaelib Siriamiut attanningata agganginut, Benhadadiblo Hasaelib erningata agganginut, innôsingit nâlugit.

4 Tagva Joahas tuksiarpok Nalekamut, Nalekablo tussarpa; Israelitit okumaiksartitauningat tækkogamiuk, Siriamiut attanningata okumaiksartimagit.

5 Nalekablo Israelitit piuliklertemik tunnitsivigivait, tessiortemik tapkoninga Siriamiut pitsartuninganit, Israelib kittorngangit iglomingne innekarmikovlugit sivurnganetut.

6 Sullele Jerobeamib Israelitinik idluitullititsilauktub iglomiokotingita idluitulliningit allarlaungilait; agvertarpulle tapkonane. Nappartullo (Gudingoataggit) Samariame, tagvanetsainarlaurivut.

7 Taipsoma Joahase unnurnersanik amiakokarkolaungimago ablangajunit 50 ginit horsit kângine, kamutinillo aksalloalingnit 10 nit, angutinillo pissuktunit 10,000 dinit. Siriamiut attanningata tokkolaungmagit, illingatilaukpaillo sanniktitut kakkojaksat tullertautilugit sorlo.

8 Unipkautsit tamakkoa assingit sulle Joahas pivlugo, tamaitalo pinniarlauktangit, pitsartuningalo, ahâk, tamanna aglaksimavok Israelitit attanningita aglangine.

9 Joahaselo sinnisilerpok, atâtagijaminut ailune, illijartortauvorlo Samariame. Erningalo Joase attanningorpok innangêrlugo.

10 Joasib Judakut attanningata Jahringita 37 ingane, Joas, Joahasib erninga attanningorpok Israelitinut Samariame, attaniotilunelo Jahrit 16 it nâllugit;

11 Pinniarporlo Nalekab idluigijanginik, Jerobeamiblo, Nebatib erningata Israelitinik idluitullititsilauktub idluitulliningit allarlaungilait, agvertarporle tapkonane.

12 Unipkautsillo assingit sulle Joas pivlugo, pinniarlauktangillo, pitsartuningalo, kannok Amazia Judakut attanningat sorsugvigilaungmago; ahâk, tamanna aglaksimavok Israelitit attanningita aglangine.

13 Joaselo sinnisilerpok atâtagijaminut ailune. Jerobeamelo ingipok iksivautanganut. Joasele illijartortauvok Samariame Israelitit attanninginut.

14 Elisale kannimalerpok, kanimaserme tokkojutigijamine. Joasiblo Israelitit attanningata attersarvigiva, kugviorlunelo sângane okarlunelo: Atâtâk, atâtâk! Israelitit aksalloalikotingit, ablangajokotingallo horsine!

15 Elisable okautiva: Tigusilerit pittiksemik, kargjutiniglo. Tigusivorlo pittiksemik kargjutiniglo.

16 Tamnalo okarpok Israelitit attanninganut: Aggangnut pittikse kelluleruk; kelluvâlo aggangminut. Elisablo aggane illivait attanniub aggangita kânginut, 
17 Okarlunelo: igalâk unnunga sangmijok angmaruk. Tapsomalo angmarpa. Elisalo okarpok: Pittikserit; tamnalo pittikserpok. Elisale okarpok: Kargjut attausek sâlakarnartok Nalekamit; kargjut attausek piulijaunartok Siriamiut akkerartorlugit; igvillo Siriamiut ullimarniarpattit Afekeme, nungutaukârtinagit.

18 Okarporlo: Kargjutit tigukit. Tapsomalo tiguvait. Tamnalo okarpok Israelitit attanninganut: Nuna annauleruk. Annaulerpâlo pingasuêrtorlune, nokarlunelo.

19 Tagva Gudib angutekotingata ningarilerpâ, okarlunelo: Tellimêrtorlutit pingasojortoêrtorlutillonêt annaulernilauruvit, tagva Siriamiut sâlaginiarajarpattit nungutaukârtinagit, mannale pingasuêrtorlutit salaginiarpattit.

20 Elisale tokkovok, illijartortaulunelo. Moabiterillo sorsuktuksangit ittâvut nunamut Jahreme taipsomane.

21 Pijokalaukporlo, angut illijartormatsuk, sorsuktuksalle tækkungmattigik, angut tamna egipât Elisab illuvinganut. Tamnalo unnungarame, Elisablo sauningit âktoramigit, omârpok tullerarlunelo ittikaminut.

22 Hasaelible Siriamiut attanningata Israelitit okumaiksartilaukpait Joahasib innôsingane tamầt.

23 Nalekable saimarvigivait napkigilaitalo, sâlugillo, angersimanine Abrahamut, Isaangmullo, Jakomullo pivlugo, asseroromalaungilaillo, ajæktorlaungilaillo kênaminit manna tikkilugo.

24 Hasaelelo Siriamiut attanningat tokkovok, erningalo Benhadad attanningorpok innangêrdlugo.

25 Tagva Joasib Joahasib erningata iglugaseksuit tigulerivait Benhadadib, Hasaelib erningata agganginit, taipsoma tigulauktangit Joahasib atâtame agganginit unnatadlarnerme. Pingasuêrtoriune Joasib sâlagiva Israelitillo iglugaseksoagilauktangit sattortautipait.

\section{KAPITEL XIV.}

Amazia Asarjalo, attannîk Judakune; Jerobeame attannek Israelitine.

J OASIB Joahasib erningata Israelitit attanningata Jahringita aipangane, Amazia Joasib erninga attanningorpok Judakunut.

2 Jahrekalaukporlo 25 ginik attanningorame, attanniotilunelo Jahrit 29it nâlugit Jerusaleme. Anânanga Joadanemik attekalaukpok, Jerusalememiok.

3 Pinniarporlo Nalekab namagijanganik, atâtamitulle Davidetuungitok, taimaluatsiarle sorlo atâtanga Joase pinniarlaungmat, taimailiorivoktauk. 
4 Pôktojut pêjartaulungimatta, innugiartoksuille tunnergutinik tunijivut isserluanititsilutiglo pôktojune.

5 Pijokalaukporlo attanniovingmine sôngogame, kivgane ullimarpait atâtanganik tokkotsilauktut.

6 Innuartulle kittorngangit tokkolaungilait; sorlo aglaksimangmat Mosesib aglangine perkojaktalingne, Nalegak perkojilaungmat, okalaungmallo: Atâtait tokkotaujuksaungilet kittorngat pivlugit, kittorngallo tokkotaujuksaungilet atâtait pivlugit, nellialle ajornine pivlugit tokkojuksauvok.

7 Edomiterillo ullimârpaittauk nâksame sioraujaktalingme 10000 dit, iglugaseksuillo Selamik attiggit sâlagivait unatadlarnermut; atserarpaillo Jakteelemik, uvlok tamanna tikkidlugo.

8 Taipsomane Amaziab tillijat tillivait Joasemut Joahasib Jehub erningata erninganut, Israelitit attanninganut, okautsaukovâlo: Kait tækkonautilauluk.

9 Joasele Israelitit attanningat tilliklerpok Amaziamut, Judakut attanninganut, okautsaukolâlo: Orpik Dorne Libanonemêtok, tilliklerpok Cederemut Libanonemêtomut, okautsaukovalo: Pannît tunnileruk ernimnut nulliangokovlugo tapsomunga. Omajulle nujuartut natername Libanoneme, orpik Dorne tullerajakpât tullerlugolo serkomipât.

10 Edomiterit salagilaukpattit, tamanna omattivit piojorijutigiva. Nertornaut tamanna pigiuk, nunangnêtuarlutillo, sôg kannoêtoksiorkît, ochokovlutit Judæalo illagællutit?

11 Amaziale nalelungilak. Tagva Joas Israelitit attanningat majorarlune takpaungarpok; tækkonautivuglo tamna Amazialo Judakut attanningat, Bethsemeseme Judæametome.

12 Judakulle sâlagijauvut Israelitinut, illunatiglo attunît kemâvut iglomingnut.

13 Joasiblo Israelitit attanningata tiguva Amazia Judakut attanningatJoasib Ahasjab erningata erninga Bethsemeseme; tikkiporlo Jerusalemut, Jerusalemiblo avaluksoangit serkomipait, itterviksoarmit Efraimemik attelingmit terrekub itterviksoanga tikkidlugo, tallitut 400 ditut takkitigijomik;

14 Tiguvaillo Goldit tamaita, Silberillo tamaita, illulerkutillo tamaita nachvartaulertut Nalekab iglungane, attanniublo iglungata perkutitalingane, tappilugillo sorrutsit sattoraksauvlutik, utterlunelo Samariamut.

15 Unipkautsille makkoa assingit Joase pivlugo pinniarlauktangit, sôngoningalo, kannorlo Amazia Judakut atttanningat sorsugvigilaungmago, ahâk, tamanna aglaksimmavok Israelitit attanningita aglangine.

16 Joaselo sinnisilerpok atâtagijaminut ailune, illijartortaulunelo Samariame Israelitit attanninginut. Erningalo Jerobeam attanningorpok innangêrdlugo. 
17 Amaziale Joasib Judakut attanningata erninga, innôlaukpok Joasib Joahasib erningata Israelitit attanningata tokkungata kingorngane Jahrinik 15 nik.

18 Unipkautsille tamakkoa assingit Amazia pivlugo, tamanna aglaksimavok Judakut attanningita aglangine.

19 Jerusalemelo angerkattigêngnekartitsivut tamna akkerartorlugo, tamnale kemâvok Lachisemut. Tapkoalo tilliklerput malliklugo Lachisemut, tokkopâllo tagvane.

20 Apâllo horsekut, illijartortauvorlo Jerusaleme atâtagijamine Davidib iglugaseksoangine.

21 Innugiartoksuillo Judakut illuêngarlutik Asarja Jahrekartillugo 16 nik tiguvât attanningortilâllo atâtangata Amaziab inningane.

22 Tapsoma Elathe iglugaseliorpait, sattortautilaitalo Judakunut, attannek sinnisilaukârdlune atâtagijane illagællugit.

23 Amaziab erningata Judakut attanningata Jahringita 15 ingane, Jerobeame Joasib erninga attanningorpok Israelitinut Samariame, (attanniotilunelo) Jahrit 41 nâlugit.

24 Pinniarporlo Nalekab idluigijanginik, allailungilarlo Jerobeamib Nebatib erningata, Israelitinik idluitullititsilauktub idluitullininginik tamainik.

25 Tapsomale Israelitit kiglilervingit sattortautipait Israelitinut Hemathemit, immarbik naternamêtok tikkilugo, Nalekab Israelib Gudingata okausingit malliklugit, okauserilauktangit kivgamigut Jonakut Amithaib erningagut, nellautaijokut, Gad Heferemit pijokut.

26 Nalekab Israelitit kappianarningat illapsungnartok tækkungmago, illunaita pijaulaungmatta, kivgaujut, kivgaungitullo, ikajortekalaungimallo Israelitine.

27 Nalegarlo okalaungilak Israelitit attingit pêjaromagamigit killaub attâne; taimaglo ikajorpait Jerobeamekut, Joasib erningagut.

28 Unipkautsit tamakkoa assingit Jerobeame pivlugo, tamaitalo pinniarlauktangit, sôngoninga, kannorlo sorsuglaungninga, Judakunut Israeleme sattortautilaungmagit Damaskuse, Hematelo, ahâk, tamanna aglaksimavok Israelitit attanningita aglangine.

29 Jerobeamelo sinnisilerpok, atâtagijane Israelitit attanningit illaliudlugit. Erningalo Sacharja attanningorpok innangêrlugo. 


\section{KAPITEL XV.}

Asarja Jothamelo attannîk Judakune. Sacharja, Sallume; Menaheme, Pekaja, Peka, Hosealo, attannit Isralitine. Israelitit aularudsauningita Assiriamut pigiarningat.

JEROBEAMIB Israelitit attanningata Jahringita 27 ingane attanningorpok Asarja, Amaziab Judakut attanningata erninga; 2 Jahrekalaukporlo 16 nik attanningorame, attanniotilunelo Jerusaleme, Jahrit 52 git nâlugit. . Anânanga attekalaukpok Jechaljamik, Jerusalemiojok.

3 Pinniarporlo Nalekab namagijanganik, taimaluatsiak atâtanetut Amaziatut;

4 Pôktojulle pêjartaulaungilet, innugiartoksuille tunnergutinik tunnijivut isserluanititsilutiglo sulle pôktojune.

5 Nalekablo attannek annauva, kallakarporlo, tokkome uvlunga tikkilugo, innekalunelo iglome ingmigolingajome. Jothamele attanniub erninga iglomik aulatsivok idluarsailunelo innugiartoksoarnik nuname.

6 Unipkautsille tamakkoa assingit Asarja pivlugo, tamaitalo pinniarlauktangit, ahâk, tamanna aglaksimavok Judakut attanningita aglangine.

7 Asarjalo sinnisilerpok atâtagijaminut ailune, illijartortauvorlo atâtagijamine Davidib iglugaseksoangine. Erningalo Jothame attaningorpok innangêrlugo.

8 Asarjab Judakut attanningata Jahringita 38 ingane Sacharja Jerobeamib erninga attanningorpok Israelitinut Samariame, attanniotilaukporlo takkit 6 it nâlugit;

9 Pinniarporlo Nalekab idluigijanginik, sorlo atâtagijangit pinniarlaungmatta. Jerobeamib Nebatib erningata Israelitinik idluitullititsijub idluitulliningit allarlaungilait.

10 Sallumelo Jabesib erninga angerkattigêngnekartitsivok akkerartorlugo, annaulerpâlo innugiartoksuit tækkoane, tokkolâlo, attanningorlunelo innangêrdlugo.

11 Unipkautsit tamakkoa assingit Sacharja pivlugo, ahâk, tamanna aglaksimavok Israelitit attanningita aglangine.

12 Tamannauvorlo Nalekab okauserilauktanga Jehumut: Igvit kittorngakartuksauvotit Israelitit iksivautaksoangane iksivajunik, kittorngarêt sittamangat tikkidlugo; taimailaurivok.

13 Sallumele Jabesib erninga attanningorpok, Asarjab Judakut attanningata Jahringita 39 ingane, attanniotivorlo takkek attausek nâlugo Samariame.

14 Tagva Menaheme Gadib erninga majorarlune takpaung- 
arpok Tirzamit, tikkilunelo Samariamut, ullimarpâlo Sallume Jabesib erninga Samariame, tokkopâlo, attanningorlunelo innangêrdlugo.

15 Unipkautsit tamakkoa assingit Sallume pivlugo, angerkattigêngnekartitsiningalo, ahâk, tamanna aglaksimavok Israelitit attanningita aglangine.

16 Taipsomane Menahemib Tifsa, illunaitalo tapkonanêlauktut kiglilervingillo Tirzab miksâne ullimârpait, itterkojomalaungimatsuk; singaijungillo illunaita ullimarpait, alliktorlaitalo.

17 Asarjab Judakut attanningata Jahringita 39 ingane Menaheme Gadib erninga attanningorpok Israelitinut, attanniotilunelo Jahrit 10 it nâvlugit Samariame.

18 Pinniarporlo Nalekab idluigijanginik. Inôsermine tamât Jerobeamib Nebatib erningata Israelitinik idiluitullititsijub idluitulliningit allârlaungilait.

19 Fulelo Assiriamiut attanningat tikkipok nunamut. Menahemiblo Fule tunnitsivigiva Talentinik 1000 dinik Silberemik, illilertuikovlugo tapsominga, attannioviglo sokkosijuitilugo tapsomunga pikovlugo.

20 Menahemelo perkojivok akluinersat Israeleme angutit attunît tunijikovlugit Sekelinik 50 ginik Silberemik, tunijikovlugo tamakkoninga Assiriamiut attanninganut. Taimak Assiriamiut attanningat aularlune angerarpok, nunamêtuarlungilarlo.

21 Unipkautsille tamakkoa assingit Menaheme pivlugo, tamaitalo pinniarlauktangit, ahâk, tamanna aglaksimavok Israelitit attanningita aglangine.

22 Menahemelo sinnisilerpok atâtagijaminut ailune, Pekajalo erninga attanningorpok innangêrdlugo.

23 Asarjab Judakut attanningata Jahringita 50 ingane, Pekaja Menahemib erninga attanningorpok Israelitinut Samariame, attanniotivorlo Jahrîk magguk nâlugik;

24 Pinniarporlo Nalekab idluigijanginik, Jerobeamib, Nebatib erningata, Israelitinik idluitullititsilauktub idluitulliningit allarlaunginamigit.

25 Peka, Remaljab erninga, angajokakotinga angerkattigêngnekartitsivok akkerartorlugo, ullimarpâlo Samariame, attanniub igloksoangane, Argobe, Arielo illaliudlugit, angutillo 50 git Gileadib kittornganginit illagivât, tokkopâlo, attanningorlunelo innangêrlugo.

26 Unipkautsit tamakkoa assingit Pekaja pivlugo, tamaitalo pinniarlauktangit, ahâk, tamanna aglaksimavok Israelitit attanningita aglangine.

27 Asarjab Judakut attanningata Jahringita 52 ingane, Peka Remaljab erninga attanningorpok Israelitinut Samariame; attanniotilunelo Jahrit 20 git nâlugit;

28 Pinniarporlo Nalekab idluigijanginik; Jerobeamib, Ne- 
batib erningata Israelitinik idluitullititsilauktub idluitulliningit allarlaunginamigit.

29 Pekab Israelitit attanningata nelliuningane, Tiglat Pilesser Assiriamiut attanningat tikkipok, tigulaitalo Hion, Abel, Bethmaecha, Janoha, Kedes, Hazor, Gilead, Galilæalo, Naftalikullo nunangat illunât, aularutivaillo Assiriamut.

30 Hosealo, Elab erninga angerkattigêngnekartitsivok Peka Ramaljab erninga akkerartorlugo, annaulerlugolo tokkopa, attanningorlunelo innangêrlugo, Jothamib Usiab erningata Jahringita 20 ingane.

31 Unipkautsille tamakkoa assingit Peka pivlugo, tamaitalo pinniarlauktangit, tamanna aglaksimavok Israelitit attanningita aglangine.

32 Pekab Remaljab erningata Israelitit attanningata Jahringita aipangane, Jothame Usiab Judakut attanningata erninga attanningorpok,

33 Jahrekalaukporlo 25 ginik attanningorame, attanniotilunelo Jahrit 16 it nâlugit Jerusaleme. Anânanga attekalaukpok Jerusamik, Zadokib pannia.

34 Pinniarporlo Nalekab namagijanganik, taimaluatsiak sorlo atâtanga Usia pinniarlaungmat.

35 Kissiane pôktojut pêjartaulungilet, innugiartoksuille tunnergutinik tunnijivut, isserluanititsilutiglo sulle pôktojune. Tapsoma itterviksoak kogvasiktok Nalekab iglungane sennava.

36 Unipkautsit tamakkoa assingit Jothame pivlugo, tamaitalo pinniarlauktangit, ahâk, tamanna aglaksimavok Judakut attanningita aglangine.

37 Nelliutome taipsomane Nalegak pigiarpok tilliklerlune Judamut Rezinemik Siriamiut attanninganik, Pekamiglo Remaljab erninganik.

38 Jothamelo sinnisilerpok atâtagijaminut ailune, illijartortaulunelo atâtagijamine Davidib atâtagijame iglugaseksoangine. Ahaselo erninga attanningorpok innangêrlugo.

\section{KAPITEL XVI.}

\section{Ahas Judakut attanningat.}

PEKAB Remaljab erningata Jahringita 17 ingane, Ahas, Jothamib Judakut attanningata erninga attanningorpok.

2 Ahase Jahrekalaukpok 20 ginik attanningorame, attanniotilunelo Jahrit 16 it nâlugit Jerusaleme. Pinniarlungilarlo Nalekab Gudime namagijanganik, atâtagijamitut Davidetut.

3 Agvertarporle Israelitit attanningita apkosiningine, erni- 
nelo ikkomakorkova, nellojut Nalekab pêjarlauktangita Israelib kittorngangita sivorngane, makkojungnartulliningit malliklugit;

4 Tunnergutiniglo tunnijivok isserluanititsilunelo poktojune, kakkarulangnelo, nappartullo tungojortat illunatik attâne.

5 Taipsomane Rezin Siriamiut attanningat, Pekalo Remaljab erninga Israelitit attąnningat, aularlutik takpaungarpuk Jerusalemut sorsugvigijomavlugit, illumavâglo Ahase; salagijungnarlaungilaille.

6 Nelliutome taipsomane Rezinib Siriamiut attanningata Siria sattorsitipa Elathemik, Judillo ajæktorpait Elathemit; Siriamiulle tikkilutik innigivaillo uvlok tamanna tikkilugo.

7 Ahasible tillijat tillivait Tiglat Pilesseremut Assiriamiut attanninganut okautsaukovâlo: Kivgauvunga erniolungalo illingnut; majorarlutit kailaurit tamaunga ikajorlungalo Siriamiut attanningata, Israelitillo attanningata agganginit, akkerartortuk uvamnik.

8 Ahasiblo Silbere Goldelo Nalekab iglungane, attanniublo iglungata perkutitalingane nachvartaulauktut tiguvait, tujorpâlo Assiriamiut attanningat pilliutinik.

9 Assiriamiullo attanningata nalekpâ, aularlunelo Damaskusemut, sâlàgivaillo, aularutivaillo (Damaskusemiut) Kiremut, tokkopâlo Rezine.

10 Attanniublo Ahasib Tiglat Pilesser Assiramiut attanningat aularlune pachrearpa Damaskusemut. Altarelo Damaskusemêtok tækkogamiuk; attanniub Ahasib Altarib adsinga oktutingalo sorlo tamna sennajaulaungmat, tillikliutiva ajochertuijomut Uriamut.

11 Uriablo ajochertuijub Altare sennava, taimaluatsiak sorlo Ahasib attanniub tilliklervigilaungmago Damaskusemit taimak sennava, attannek Ahase tikkikârtinago Damaskusemit.

12 Attannerlo Damaskusemit tikkiname, Altarelo tamna tækkogamiuk, tunnergutinik tunnijivok kângane.

13 Ikkilugillo kângane tunnergutine ikkoalajaksat, nerkiksallo, kovilugolo kânganut tunnergutine immiksak tunnergutillo kujalijutiksat tunnijame aungit serkijartaukovait Altarib oma kânganut.

14 Altarele kannujangôjok Nalekab sângane nappajok nûktipa inninganit, nappakonago Altarib nutâb Nalekablo iglungata akkorngangne; napparpâlo Altarib sennianut tachanut.

15 Attanniublo Ahasib Uria ajochertuijok perkova, okarlunelo: Altareme angijome ikkitsijuksauvotit tunnergutinik ikkoalajunik uvlarme, tunnergutiniglo nerkiksanik unnungme, attanniublo tunnergutinginik ikkoalajaksanik, nerkiksaniglo, innugiartoksuillo nunamiut tunnergutinginik ikkoalajaksanik, tunnergutinginiglo nerkiksanik, tunnergutinginiglo imiksanik, tunnergutillo ikkoalajaksat, tunnergutillo assingita aungit tamaita 
serkijaraksarivattit kânganut; Altarelo kannujangôjok pivlugo sulle issumaksarsioromavunga.

16 Urialo ajochertuijok tamainik pinniarpok, perkojaunerminik attannermut Ahasemut.

17 Attanniublo Ahasib ikkovrat sennerangit serkomipait, ukkusillo akkârtipait; immarbingoarlo tuktuvangoanit kannujangojunit tapsoma attânetunit akkârtipa, illivalo nettermut ujaraujomut.

18 Taimaktauk torksuk, Sabbatib torksunga kâlik, sennajaulauktok iglome, attanniublo torksunga sillatanelauktok, nuktipak Nalekab iglunganut, Assiriamiut attanningat pivlugo.

19 Unipkautsille tamakkoa assingit Ahase pivlugo, pinniarlauktangit, ahâk, tamanna aglaksimavok Judakut attanningita aglangine.

20 Ahaselo sinnisilerpok atâtagijaminut ailune, illijartortaulunelo atâtagijamine Davidib iglugaseksoangine. Hiskialo erninga attanningorpok innangêrlugo.

\section{KAPITEL XVII.}

Kinguvarêt 10 it aularutijauningit Salmanassermut, Assiriamiut attanninganut, Hosea attanniotillugo. Tokkorngartat nunakarput Israelitit nunangane.

AHASIB Judakut attanningata Jahringita 12 ingane, Hosea Elab erninga attanningorpok Israelitinut Samariame, attanniotilunelo Jahrit 9 it nâlugit.

2 Pinniarporlo Nalekab idluigijanginik, Israelitille attanningitituungitok sivorlimetut.

3 Tapsomunga Salmanasser Assiriamiut attanningat tikkilerpok sorsuklune. Hosealo kivgangorpok tapsomunga, tunnitsivigivâlo pilliutinik.

4 Assiriamiulle attanningat tukkisilerpok Hosea angerkattigêngnekartitsingmat akkerartorlugo, tillijaniglo tilliklerlaungmat Somut Egiptenemiut attanninganut, pilliutiniglo tunnijijungnaimat Assiriamiut attanninganut sivornganetut Jahrine tamaine. Tamanna pivlugo Assiriamiut attanningata illumara, pitipâlo kellaksorsimatillugo parngnanairsimavingmut.

5 Assiriamiullo attanningat tikkilerpok nunab illunanganut, Samariamullo, illumavaillo Jahrit pingasut nâvługit.

6 Hoseablo Jahringita 9 ingane Assiriamiut attanningata Samaria salagivait, Israelitillo aularutivait Assiriamut, nunakartipaillo Halame, Haboremela Gosanib kôngane, Mederillo iglugaseksoangine. 
7 Tamannalo pilaukpok, Israelib kittorngangit idluitullilaungmatta Nalekamut Gudemingnut, tessiorlauktomut tapkoninga Egiptenemit, Faraub Egiptenemiut attanningata agganginit, Gudiblo assingit sivoragilaungmattigik;

8 Agvertarlaukpullo nellojut, Nalekab pêjarlauktangita Israelib kittorngangita sivorngane illusingit malliklugit, sorlolo Israelitit attanningit pinniarlaungmatta.

9 Israeliblo kittorngangit angigutsivlutik illusekarput namalungitunik Nalekab Gudimik sầngane, pôktojuniglo sennavut ingmingnut iglugaseksoakotimingne illunaine, nuvugosingnelo iglugaseksoarne sôngojune.

10 Nappajuniglo nappartitsivut, Gudingoaniglo kakkarulangne pôktojune illunaine, nappartullo tungojortat illunatik attâne;

11 Isserluanititsilaukpullo tagvane poktojune illunaine nellojutitut, Nalekab tapkoa sivorngane pêjarlauktangititut. Pinniarlutiglo idluitoâlungnik, Nalekab ningaksartaujutiksanginik.

12 Kivgartorlutiglo Gudeungitunik, Nalekab okarutigilauktanginik tapkonunga: Taimaitomik pinniartuksaungilase!

13 Nalekablo Israelitit Judakullo tennersarlaukpait nellautaijokotimigut illunaitigut, tækkojokotimigullo, okautsaukovaillo: Utteritse apkotipsingnit idluitunit, mallikluselo perkojamnik, innatsinimniglo; perkojat illunatik malliklugit atâtagijapsingnut perkolauktakka, tillikliutilauktakkalo illipsingnut kivgakotipkut nellautaijutigut;

14 Nalelaungilelle kongesetigle keratatipait atâtagijamiktut, okperlaungitutitut Nalekamut Gudemingnut.

15 Perkojangillo angerningalo, atâtagijanginut angerlauktanga, tennersariningillo tunnilauktangit tapkonunga, nachogivait, agvertarlutiglo Gudingoat suksaungitut malliklugit, pinniarlutiglo suksaungitomik, nellojut malliklugit avatimingne nunakalauktut, Nalekab perkojijutigijangit tapkoninga, taipkotitunak pinniartuksaungimatta.

16 Nalekable Gudemik perkojangit illunaita kemmakpait, sennalutiglo ingmingnut nochangoangnik kovijaumajungnik, nappartuniglo; tuksiarlutiglo killaub kattimgajoksoanginut illunainut kivgartorlarmiglo Baalemik;

17 Ernitiglo pannitiglo ikkomakôrkovait, nellautailuklutiglo angakoktorlarmiglo; iggisimavullo ingminik tunnilutik Nalekab idluigijanginik, ningaksarlugo.

18 Tagva Nalekab Israelitit ningaridlalaukpait, pêrpaillo kênaminik; amiakokalungilak kinguvarêt Judakut assinginik.

19 Judakuttauk Nalekab Gudimik perkojangit mallilaungilait, agvertarpulle Israelitit illusingit, sennalauktangit malliklugit.

20 Tamanna pivlugo Nalekab Israelib kinguvângit illunaita 
ajæktorpait, tetjarlugillo, tunnilaitalo arktaijut agganginut, egitsikârane tapkoninga kênaminit.

21 Israelitit pêrsimalaukput Davidib iglunganit; Jerobeamelo Nebatib erninga attanningortipât, tapsomalo Israelitit tunnutipait Nalekamit, idluitullitilaitalo angijoksoarmik.

22 Taimak Israelib kittorngangit agvertarlaukput Jerobeamib idluiningine sennalauktangine tamaine, allarlaungilaillo,

23 Nalegak Israelitinik pêjaikârtinago kênaminit, sorlo okalaurame kivgamigut illunaitigut nellautaijutigut Taimak Israelitit nunamingnit aularutitaulaukput Assiriamut uvlok tamanna tikkilugo.

24 Assiriamiullo attanningat tikkitokarkojivok innungnik Babelemit, Kutamillo, Avamillo, Hemathemillo, Sefarfaimemillo, pitipaillo Samariab iglugaseksoanginut, Israelib kittorngangit innangêrlugit. Tapkoalo Samaria nangminiutipât iglugaseksoangillo innigilaita.

25 Nunakarnimigle pigiarningane Nalegak sivuragilaungimatsuk, Nalegak tilliklerpok Loewinik tapkoa akkornganut, tapkoa tokkotsivut akkorngane.

26 Assiriamiullo attanningat okautsaukovât: Innugiartoksuit aularutilauktavit, pitilauktavillo Samariab iglugaseksoanginut, nunab tamattoma Gudingata illusinga nellovât, tamanna pivlugo Loewit tillilaukpait akkornganut, ahâglo, tapkoa tokkopait, nunab tamattoma Gudingata illusinga illitaringimatsuk.

27 Tagva Assiriamiut attanningat perkojivok, okarlunelo: Atsileritse tagvunga ajochertuijut aularutjigilauktapse illanganik, ailerluselo nunakarluselo tagvane, tapsomalo ajochertorlerligit nunab Gudingata illusinganik.

28 Tagva ajochertuijut taipkoa Samariamit aularutilauktangita illangat tikkilerpok, nunakarlunelo Betheleme, ajochertorpaillo, kannok Nalegak sivuragijaksaringmatsut.

29 Innukattigêksuille illunatik attunît Guditik sennavait, pitipaillo pôktojut iglunginut Samariamiut sennalauktanginut, innukattigêksuit illunatik iglugaseksoamingne nunagijamingne.

30 Babelemiovinît Suchot Benoth sennavât. Kutemiovinît Nergele sennavât. Hemathemiovinît Assima sennavât.

31 Avamiovinît Nibehase Tartakelo sennavâk. Sefarfaimimiovinit ernitik ikkipait Adrameliche Anamelechelo Sefarfaimemiut Gudingoakotigik pivlugit.

32 Nalegarlo sivuragikassiutivat, ajochertuijungortitsivullo nellimingnik pivlutik, poktôjunetuksanik, tapkoa tunnergutinik tunnijivut pivlugit iglune pôktojunetune.

33 Taimak Nalegak sivuragivât, Gudingoakotitiglo kivgartorkassiutivait, innukattigêksuit taipkoa aularutjauvigilauktamik tamaunga illusingit malliklugit. 
34 Uvlorlo tamanna tikkilugo taimailiorput, illutsit nutaungitut malliklugit Nalegak sivuragijatuarilungilât, pinniaratiglo illuserivalauktatik, piuserivalauktatiglo malliklugit, pinniaratiglo illusiokojaujut perkojallo malliklugit, Nalekab perkolauktangit Jakob, atserartaulauktub tapsomunga Israelemik, kittornganginut;

35 Nalekab angerkattigingmagit, perkolaitalo, okarlunelo: Gudib assingit sivuraginiarassigik tuksiarviginagillo, kivgartorassigiglo, tunijitaililuselo tunnergutinik tapkonunga;

36 Nalegarle tessiortigijase Egiptenemit, pitsartunersoarmut tallerminullo issamijomut, tamna sivuragisiuk, tuksiarvigisiuglo, tunnergutiniglo tunnitsivigisiuk;

37 Illutsillo, idluarnerillo, innatsinerillo, perkojallo aglagutigilauktangit pivluse, tapkoa malligaksarivasse, pinniatsainarlugillo; Gudiblo assingit sivuraginagit,

38 Angerkattigêngnerlo angernerilauktanga illipsingnut puigorniarassiuk, Gudib attingit sivuragilugit pikonase;

39 Nalegarle Gudise sivuragijaksarivasse, tapsoma piuliniarpâse omigijipse illunatik agganginit.

40 Tapkoale nalelaungilet, illutsitigle illuserilauktatik malliklugit pinniarlaukput.

41 Taimak innukattigêt tapkoa Nalegak sivuragivat, kivgartorkassiutilaitalotauk Gudingoakotitik. Taimailiorlaurivuttauk taipkoa kittorngangit erngutingillo uvlok tamanna tikkilugo, sorlo atâtagilauktangit pinniarlaungmatta.

\section{KAPITEL XVIII.}

Hiskia attannek Judakunut. Jerusalemib illumajauninga Sanheribemut.

HoseAB Elab erningata Israelitit attanningata Jahringita pingajuane, Hiskia Ahasib Judakut attanningata erninga attanningorpok;

2 Jahrekalaukporlo 25 ginik attanningorame attanniotilunelo Jahrit 29 it nâlugit Jerusaleme. Anânanga attekalaukpok Abimik, Sacharjab pannia.

3 Pinniarporlo Nalekab namagijanganik, atâtagijangatut Davidetut.

4 Pôktojut attorungnaitipait, nappajullo serkomipait, nappartullo pêjarpait, kuglugiangojartorlo kannujangôjok, Mosesib sennalauktanga tugarlugo serkaliva; nelliutok taipsoma tikkilugo Israelib kittorngangita isserluanititsivigilaungmatsuk, atserartauvorlo Nehustanemik.

5 Tapsoma Nalegak Israelib Gudinga tettigiva, kingornga- 
nelo Judakut attanningita illunatik akkorngane adsekalaungilak, sivurnganelauktullo akkornganêlonêt.

6 Tapsoma Nalegak ungagiva uniorksivigingilalo, perkojangillo mallikpait, Nalekab perkolauktangit Mosesemut.

7 Nalekablo aipariva; illunanelo namut aivigijamine pillorilaukpok. Assiriamiullo attanningat allârpa nalegungnailugolo.

8 Filisterillo ullimarpait Gasa tikkidlugo, asserorlugillo erkangit, udsertortib nuvugusinganit iglugaseksuit sôngojut tikkilugit.

9 Hiskiab Judakut attanningata Jahringita sittamangane, tagva Hoseab Elab erningata Israelitit attanningata Jahringita 7 ingane, Salmanasser Assiriamiut attanningat aularlune takpaungarpok Samariamut, sorsugvigilugit, illumavaillo,

10 Sâlagivaillo Jahrit pingasut kingorngane, Hiskiab Jahringita 6 ingane, tagva Hoseab Israelitit attanningata Jahringita 9 ingane Samaria sâlagijauvok.

11 Assiriamiullo attanningata Israelitit aularutivait Assiriamut, nunakartipaillo Holame Haboremelo Gosanib kôngane, Mederillo iglugaseksoangine.

12 Tamanna pivlugo Nalekab Gudimik nippinga nalelaungimatsuk, angerningalo; tamaitalo Mosesib Nalekab kivgangata perkolauktangit tappagilaungmattigik, nalelaungimattigiglo, pinniarlaungimattigiglonêt.

13 Attanniuble Hiskiab Jahringita 14 ingane Sanherib Assiriamiut attanningat aularlune takpaungarpok, Judakut iglugaseksoangit sôngojut illunaita sorsugvigilugit, aksarnigarivaillo.

14 Tagva Hiskia Judakut attanningat tilliklerpok Assiriamiut attanninganut Lachisemut, okautsaukovalo: Idluitullilaukpunga, aularit uvamnit; tunnijaukojattit uvamnut tunnijomavakka. Tagva Assiriamiut attanningata Hiskia Judakut attanningat tunnijikova Talentenik 300 dinik Silberemik, Talentiniglo 30 ginik Goldemik.

15 Taimak Hiskiab Silberit tamaita Nalekab iglungane, attanniublo iglungata perkutitalingine nachvartaumajut tunnivait.

16 Nelliutome taipsomane Hiskiab Goldit Nalekab iglungata upkoanginit nappajunillo, Hiskiab Judakut attanningata sillapartaukolauktanginit pêrpait, tunnilaitalo Assiriamiut attanninganut.

17 Assiriamiulle attanningata Tartane, Rabsariselo Rabsakelo tillivait Lachisemit, illakarlutik kattimgajoksoarnik attannermut Hiskiamut Jerusalemut, majorarlutiglo takpaungarput. Tikkinamiglo nokkarput tetsib kogvasiktub, ergortuijut perorsêvingata apkosingata sennianêtub kôvingane,

18 Attannerlo nipliarvigivât. Tagva Eliakimib Hilkiab erningata, aulatsijub, attanniub iglunganik, Sebenablo aglakpak- 
tub, Joablo Assafab erningata angajokaujub aglakpaktunut annivigivait.

19 Rabsakiblo okautivait: Okaritsetôk attannermut Hiskiamut: Imâk attannersoak Assiriamiut attanningat okarpok: Tettekarnek tettigijat sunauva?

20 Okarpotit, (okautsitille okausetuinauvut): Attannertuinekarpunga, sôngonekalungalo sorsungnermut. Kinale sungertutigiviuk nalegungnailauramga?

21 Ahâk tettiksakarpotit ipsominga ajaupiamik suvlulingmik, napvaujersimajomik Egiptenemik. Tapsoma kia ajækpakuniuk aggangit kappiniarpait puttorniarlaitalo. Taimailingavok Farao Egiptenemiut attanningat, illunainut tettekartunut tapsominga.

22 Okautigupsingalo: Jehova Gudivut tettigivavut. Tamnale tamnaungilâk, Hiskiab pejaivigijanga pôktojokotinginik, Altarekotinginiglo, okalauklune Judakunut Jerusalemiunullo: Altarib oma Jerusalemêtub sângane tuksiartuksauvose?

23 Mannalo attetôk angajokara Assiriamiut attanningat angervigiuk: horsinik 2000 dinik tunnitsivigijomavagit, ablangajokartitsijungnaruvit tapkonunga.

24 Kannok uttertitsijomagalloarkît angajokangma aulatsijokotingita mikkinerpângita illanganik? Egiptenemigle sungertutekarpotit, kammutit ablangajullo horsit kângine pivlugit?

25 Issumavîlle, uvanga Nalegak pinago tamaungarama iglugaseksuit tamakkoa asserorlugit pikovlunga? Nalekab okautilaukpânga: Majorarit takpaunga nunamut omunga, asserorlugolo.

26 Tagva Eliakime Hilkiab erninga Sebenalo Joalo okarput Rabsakemut: Kivgatit Siriamiut okausingit attorlugit okarvigikit, tukkisigaptigik; Judillo okausingit attorlugit okarviginiaratigut, innugiartoksuit avaluksuit kânginêtut siutingita sângine.

27 Rabsakible okautivait: Angajokangma tillilaukpingale angajokângnut illingnullonêt okausekarkovlunga taimaitunik? Angutinulloungitok avaluksuit kânginetunut, illagiluse annarmingnik nerrikovlugit, itteromingniglo immerkovlugit?

28 Tagva Rabsake nangeriartorpok tagvunga, nipliarlunelo nippekôrtojomut, Judit okausingit attorlugit, okarlunelo: Tussaleritse attannersub Assiriamiut attanningata okausinginik.

29 Imâk attannek okarpok: Uiverijautailititse Hiskiamut piulijungnangermasse aggamnit.

30 Mannigortautailititselo Hiskiamut Nalegak tettigivlugo pijuksaugapse, okartillugo: Nalekab piuliniarpâtigut, iglugaseksuillo makkoa tunnijauniangilet Assiriamiut attanningata agganginut.

31 Hiskia nalengniarassiuk. Imâk Assiriamiut attanningat okarmat: Ullapirsautekarkattigissinga, annivigilungalo, tagva illunase attunit, nerrijuksauvose nappartokotipsingnit Weine- 
liksamit, Feigeliksamillo, immertuksauluselo immektârvikotipsingnit;

32 Tikkikârtinanga ailuselo nunamut, nunapse adsinganut, pittalingmut kakkojaksanik, Weineliksablo paungangita iptinganik, kakkojamiglo, perorsêvingniglo nappartolingnik Weineliksanik, nappartuniglo orksulingnik, orksumiglo, igupsaujartullo orksunginik, innokovluse tokkokonnaselo. Hiskia nalengniarassiuk, tapsoma uiveringmasse, okarlune: Nalekab piuliniarpâtigut.

33 Innukattigêksuit assipse Gudingita attunît nunakotitik piulilaurivattigik Assiriamiut attanningata agganginit?

34 Hemathemiut Arpamiullo Gudingit nanêkat? Sefarfaimemiut Henamiut, Iwamiullo Gudingit nanekât? Samaria piulilaurivattigik aggamnit?

35 Nelliangovât illunatik Gudingita akkorngane, nunakotimingnik piuliklerlauktut aggamnit, Nalekab Jerusalem piulijaksariniarmagit aggamnit?

36 Innugiartoksuille niplilaungilet, kiolungilâllo sunamik; attannek perkojilaungmat, okalaungmallo: Kioniarassiuk.

37 Tagva Eliakimib Hilkiab erningata aulatsijub attanniub iglunganik, Sebenablo aglakpaktub, Joab Assafib erningata angajokaujub aglakpaktunut, Hiskia tikkipât annoralijarlutik alliksimajunik, unipkautivâllo Rabsakib okausinginik.

\section{KAPITEL XIX.}

\section{Assiriamiut annaujauningit Nalekab Engelinganut.}

A TTANNIUBLE Hiskiab tamakkoa tussaramigit, anorâne allikpait, pôngmiglo attitipok, ailunelo Nalekab iglunganut,

2 Tillivaillo Eliakime aulatsijok attanniub iglunganik Sebenalo aglakpaktok, ajochertuijut angajuklit illagilugit, pôngnik attisimavlutik, nellautaijomut Jesaiasemut, Amozib erninganut,

3 Tapkoalo okautivât: Imâk Hiskiase okarpok: Tamanna uvlôvok kappianartolik, suartauneliglo, okarnerlungneliglo; nutakkat tikkisimavut erniviksak tikkilugo, nukkekalungilarlo ernijungnarnermut.

4 Imakkâ Nalekab Gudivit tussaromangmangagit Rabsakib okausingit illunaita, tillijaumajub angajokaminut Assiriamiut attaninganut, Gude omatsainartok mittautigilugo, suarlugolo okautsinut, Nalekab Gudivit tussarlauktanginut. Tagva igvit amiakojut sulle innugiartoksoarnit tuksiarutigilaukit.

5 Taimaglo attanniub Hiskiab kivgangit Jesaiasemut tikkiput.

6 Jesajasiblo okautivait: Angajokase okautisiuk: Imâk 
Nalegak okarpok: Okautsit tussarlauktatit Assiriamiut attanningata nukapiakotingita okarnerlutigijangit uvamnik, sivuraginiarnagit pît.

7 Ahâk annernermik tunnitsivigijomavara, tussaumajamiglo tussartuksauvok, uttertuksaulunelo nunaminut; saviksoarmullo ochotitsomavara nunangane.

'8 Rabsakelo utterpok, Assiriamiullo attanningat naipipa sorsuktillugo Libnamut; tussarlaurame Lachisemit aularsimaninganik.

9 Tagva Tirhaka Aetiopiamiut attanningat pivlugo tussarpok: Ahâk aularpok sorsugvigijomavlutit. Tagva ama tillijat tillivait Hiskiamut, okautsaukovalo:

10 Imâk Hiskia Judakut attanningat okautisiuk: Uiverijautailitit Gudekotingnut tettigijarnut okarlutit: Jerusaleme tunnijauniængilet Assiriamiut agganginut.

11 Ahâk, tussarlaukpotit Assiriamiut attanningita pinniarninginik nunanut illunainut, kannok nungutilaungmattigik; igvillo piulijaujuksaunajarkît?

12 Innukattigêksuit assipse, atâtagilauktama asserorlauktangita Gudingita piulilaukpattigik? Gosanemiut, Haranemiut, Rezefemiut, Edeniblo kittorngangita, Telassaremelauktut?

13 Nanêkât Hemathemiut attanningat, Arpademiullo attanningat, iglugaseksuillo Sefarfaimemik attiggit attannertangat, Hanablo, Ivablo?

14 Hiskiablo aglait tamakkoa tiguvait tillijab agganginit, attuarpaillo, majorarlunelo Nalekab iglunganut, issivipaillo Nalekab sânganut:

15 Hiskialo tuksiarpok Nalekab sângane okarlunelo: Nalegak Israelib Gudinga, Kerubimit kângine iksivajotit, igvit Gudetôvotit attanniovingnut nunamêtunut illunainut, igvit killangmik nunamiglo pingortitsilaukpotit.

16 Nalegak siutikik mannikik tussalaurillo, ijikik uitilaukik tækkolutillo, tussarlaukillo Sanherib okausingit, tillikliutilauktangit tamaunga, Gude omatsainartok mittautigijomavlugo.

17 Nellagorpok Nalegak, Assiriamiut attanningita innukattigêksuit nunangillo, asserorlaukpait saviksoarmut,

18 Gudingillo egilaukpait ikkomamut; Gudeolaungimatta, innuille aggangita pinniarnerivait, kejungôvlutik ajarauvlutiglo; taipkoa nungutilaukpait.

19 Mannale Nalegak Gudivut ikajorlauktiguttôk taipsoma agganginut, attanniovît nunamêtut illunamassiatik illitarksilerkovlugit, igvit Nalegak Gudetôgavit.

20 Tagva Jesaiase, Amozib erninga, tilliklerpok Hiskiamut, okautsaukolâlo: Imâk Nalegak Israelib Gudinga okarpok: Tuksiarnerilauktatit uvamnut, Sanheribe Assiriamiut attanningat pivlugo, tussarlaukpakka. 
21 Imaipullo Nalekab okauserijangit tamna pivlugo: Uigasub Zionib panningata nachogivâtit, mittautigilutillo; panniojub Jerusalemib kingunerne, illekettamarutigivâtit.

22 Kina mittautigilaukiuk, okarnerlutigilugolo? Kina nippekarutigilaukiuk? Ijikik torartilaukpakik kôngmut Heiligeojomut Israelemetomut.

23 Tillijakotikut Nalegak mittautigilaukpat, okalauklutillo: Kamutekotimnut unnuktojunut, kakkat pôktoninginut majorarpunga Libanonib sivinganingane; Cederekotingillo pôktojut, Cipressekotingillo annerimajut nakkarpakka, tikkitsomalungalo kiglilervingita sinniktarvinganut tikkilugo, Karmelib nappartunganut.

24 Uvanga poagrearpunga immerlungalo imiksanik tækkorngârtanik, ittikkamalo tullerninginut Egiptenemiut kôksoangit illunaita ipteriliktipakka.

25 Tussarlaungilatille uvanga tamanna itsaksoarme taimailingatilaurapko, pigiarnermelo kajusimautigiumijarapko? Manna tikkikolaukpara, asseroikovlutit iglugaseksoarnik sôngojunik, ujarangortilugit kallerêktunik patsalungajunik,

26 Innungillo nukkingêrotitauvlutik mungungalaukput kangusuktitaulutiglo; iviksukatitut naternametutitut-îlerlaukput, perorsijatitullo tungojortutitut, iviksukatitullo iglut kânginêtutitut, kunnulertutitut innerkârtinagit.

27 Nunakarnîlle, annivaktarnillo, itternerivaktarnîllo kaujimavakka, pikablâvigigamgalo.

28 Pikablâvigigamgale, pingijainîllo kôngmuarsimangmat siutimnut, tamanna pivlugo suvlolikotimnik illijijomavunga kingarnut, kingmiarakotimniglo kannernut, uttertitsomavagillo apkotigilauktakut tamaunga.

29 Tamannalo nellonaikutiksarivat: Jahreme tamattomane nerrijuksauvose ingminik perorlauktunik; Jahriblo aipangane ingminik nuijomârtunik; Jahriglo pingajuangne karngasoileritse kattersoilerluselo, Weineliksaniglo illiorkaileritse, nerriluselo paunganginik.

30 Piulijaumajullo, amiakullo Judakunit ama amâkarniarivut attimingne, paungakaromârlutiglo kollimingne.

31 Jerusalemit anniniarmatta amiakut, piulijaumajullo Zionib kakkanganit. Nalekab Zebaothib illungertorninga tamattominga pinniarpok.

32 Tamanna pivlugo Nalekab Assiriamiut attanningat okautigiva imâk: Tikkituksaungilak iglugaseksuit makkoa illuanut, kargjullo attauserlonêt pittiksautijaksaringila illuanut, sappulutaksarlo attauserlonêt tikkituksaungilak sivurânut, talluliortuksaungilarlo avatane;

33 Apkomele tikkivigijamine tamaunga uttertuksaurok, tik- 
kituksaungilarlo iglugaseksuit tamakkoa illuanut, Nalegab okauserilaukpa.

34 Uvangalo iglugaseksuit makkoa serngnigijomavakka piulijomalugillo pivlunga, kivgaralo Davide pivlugo.

35 Unnuarmelo tapsomane Nalekab Engelinga annivok annaulernilunelo Assiriamiut tangmârvingane, angutinik 185000 dinik. Uvlarârsukullo makkilaungmatta, ahâk, tagva nellajokalaukpok illunane timinik tokkungajuinarnik.

36 Tagva Sanheribe Assiriamiut attanningat parngnailerpok, aularlunelo utterlarmelo, nokkangalunelo Niniveme.

37 Gudimelo Nisrochib iglungane tuksiartillugo ernekita Adramelechib Sarezeriblo ullimarpâk saviksoarmut, kemâvuglo nunemut Araratemik attelingmut. Erningalo Asserhadon attanningorpok innangêrlugo.

\section{KAPITEL XX.}

Hiskiab kannimaninganik, âkiktauninganiglo tattamnadlartomik. Tokkunganiglo.

Nelliutome taipsomane Hiskia kannimalaukpok tokkoajarlune. Nellautaijublo Jesaiasib, Amozib erningata tikkipa, okautilâlo: Imâk Nalegak okarpok: Iglokotit oppalungaijaruk tokkoniaravit, nekkornersauniangilatillo.

2 Tapsomale kênane sâtipa iglub sennerânut, tuksiarlunelo Nalekamut, okarlunelo:

3 Erkailaurittôk Nalegak! sângne nertornarlunga agvertarlaurama, omamullo nellagôrtomut, pinniarlauramalo namagijarnik! Hiskialo kugviudlalaukpok.

4 Jesaiasele iglugaseksuit kerkanginit annilerkârtinago sulle, Nalekab okausingata tikkipa, okarlunelo:

5 Utterit, Hiskialo innukotima attanningat okautiuk: Imâk Nalegak atâtavit Davidib Gudinga okarpok: Tuksiarnit tussarlaukpara, kugvitillo tækkolaukpakka. Âhâk, pijungnaititsomavagit; uvlut pingajuane majorarlutit takpaungalârpotit Nalekab iglunganut;

6 Innôserilauktarnullo illautitsijomavunga Jahrinik 15 nik, piulijomavagillo, iglugaseksuillo tamakkoa, Assiriamiut attanningata agganginit, iglugaseksuillo tamakkoa serngnigijomavakka pivlunga, kivgaralo Davide pivlugo.

7 Jesaiaselo okarpok: Kaitsileritse Feigemik makpasimajomik; kaitsivullo, illilâlo soggomut; nekkoksilaukporlo.

8 Hiskiable Jesaiase okautiva: Kannoêtomik nellonaikutekarka Nalekab pijungnaitiniarmanga, uvlut pingajuane takpaunga Nalekab iglunganut itterlârama? 
9 Jesaiase okarpok: Tamanna nellonaikutariuk Nalekamit, Nalegak pinniarmat okauserijamitut: tachak tittarnik tillimaujortunik uttertuksauva, sivumuartuksauvalonêt?

10 Hiskia okarpok: Ajornangilak tachak sivumuarkovlugo tittarnik 10 nik; tamanna pijomalungilara, uttimuartuksauvorle tittarnik 10 nik.

11 Tagva nellautaijub Jesaiasib Nalegak nipliarvigiva, tapsomalo tachak uttimuartipa tittarnik 10 nik, akkakârlauktok Ahasib sekkerngoakotingata tikkutâne.

12 Nelliutome taipsomane Berodachib Baladanib, Baledamib erningata erningata Babelemiut attanningata aglait pilliutiksallo tillikliutivait Hiskiamut, tussarlaurame Hiskia kannimalaungmat.

13 Hiskiable kuviasukattigivait, iglolo perkotitalik tamât tækkojautipa tapkonunga, Silbere Goldelo, mingoarutiksallo, orksuglo pivianartok, innelo sakkutalik, tamaitalo perkotitalikotiminelauktut. Pittakalaungilarlo sunamik tapsoma iglungane nalegauvinganelo tamât, Hiskiab tækkojautilaungitanganik tapkonunga.

14 Tagva Jesaiase nellautaijok tikkipok attannermut Hiskiamut, okautilâlo: Kannok angutit taipkoa okalaukat? Nakillo tikkilaukâtit? Hiskia okarpok: Nunamit ungasiktomit tikkilaukpânga, Babelemit.

15 Tamna okarpok: Sunamik tækkolaukat iglungne? Hiskia okarpok: Tamaita iglumnêtut tækkolaukpait; perkutitalikotimnelo, pittakangilak sunamik, tækkojautilaungitamnik taipkonunga.

16 Tagva Jesaiasib Hiskia okautiva: Nalekab okausingit tussarkit:

17 Ahâk, nelliutijomârpok, tamaita iglungnêtut atâtagilauktavillo kattersorlauktangit uvlok tamanna tikkilugo, aularudsaujomârmatta Babelemut; amiakokarkojaujomângilarlo sunamik, Nalegak okarpok.

18 Taimaktauk ernitit illingnit pijut, ernitarijomârtatit, tigujaujomârput kivgaukovlugit Babelemiut attanningata igloksoangane.

19 Hiskiale okarpok Jesaiasemut: Nalekab okausingit okauserimajangit namakput. Okarivorlo: Ullapirsautekartuinarletôk, nertornarnekarlelo innôtillunga!

20 Unipkautsit tamakkoa assingit Hiskia pivlugo, sôngoningalo tamât, pinniarlauktangillo, tetserlo, kôgvillo koktitsijutigilauktangit immermik iglugaseksoarnut, ahâk tamanna aglaksimavok Judakut attanningita aglangine.

21 Hiskialo sinnisilerpok, atâtagijaminut ailune. Manasselo erninga attanningorpok innangêrlugo. 


\section{KAPITEL XXI.}

\section{Manasse, Amonelo attanniôjuk Judame.}

M ANNASSE Jahrekalaukpok 12 inik attanningorame, attanniotilunelo Jahrit 55 git nâlugit Jerusaleme. Anânanga attekalaukpok Hefzibamik.

2 Pinniarporlo Nalekab idluigijanganik, innukattigêksuit Nalekab pêjarlauktangita Israelib kittorngangita sirorngane, makkojungnartulliningit malliklugit;

3 Pôktojullo atâtangata asserorlauktangit sennapsarivait, Altarillo sennavait Baalemut, nappartuniglo Gudingoalingnik âkiksoivok, sorlo Ahabe Israelitit attanningat pinnialaungmat, killangmiogaseksuillo tamaita (uvloritsat) tuksiarvigivait kivgartorlaitalo.

4 Altarillo sennavait Nalekab iglungane, Nalekab okautigilauktangane: Jerusalemut attera pititsomavara.

5 Uvloriagaseksoarnullo illunainut Altarinik sennavok Nalekab iglungata innigingne avaluliorsimajungne tamangne.

6 Erninelo ikkomakôrkova, tingmitjello nipjarningit nellonaikutallo kammagivait, angakoktorlunelo nellautainarluklarmelo, pinniarporlo unnuktunik Nalekab idluigijanginik, ningaksarlugo.

7 Gudingoarlotauk sennalauktane pitipa iglomut tapsomunga, Nalekab okautigilauktanganut Davidemut, Salomomullo: Iglomut tamattomunga, Jerusalemullo, annerimajamnut Israelib kinguvarênginit tamainit, attera pititsomavara soraijuitomik,

8 Israelitillo ittiggangit aularkojomairpakka nunamit tunnilauktamnit atâtagilauktanginut, kissianele kammatsiarpatta, pinniarpattalo tamaita perkolauktakka malliklugit, perkojallo tamaita malliklugit, kivgama Mosesib perkolauktangit tapkonunga.

9 Nalelaungilelle; Mannassible uiverivait sugaluluarkovlugit innukattigêksoarnit, Nalekab pêjarlauktanginit Israelib kittorngangita sivorngane.

10 Tagva Nalegak okalavok kivgamigut nellautaijutigut okarlunelo:

11 Taimaimat Manassib Judakut attanningata makkojungnartut tamakkoa pinniarlaungmagit, sugaluluartut Amoriterit sivornganelauktut makkojungnartullininginit tamainit, Judakullo idluitullitilaungmagittauk Gudingoakotiminut.

12 Tamanna pirlugo Nalegak Israelib Gudinga okarpok imâk: Ahâk, kannoêtomik kaitsijomavunga Jerusalemut, Judamullo, tamattominga tussarniartub tapsoma siutigik aviojiniartuksaungmanik. 
13 Jerusalemelo nokakservalleromavakka Samariab nokakservallautinganut, Ahabiblo iglungata nausaikotinganut; Jerusalemelo nâvitsomavakka, sorlo pogutak nâvitaungmat, pussititsomavakkalo;

14 Kingormgutijamalo amiakungit kemagomavakka; tunnijomalaitalo omigijingita agganginut, aksarnigaukovlugit, ivajarijaukovlugillo omigijinginut illunainut.

15 Tamanna pivlugo, pinniarlaungmatta idluigijamnik, ningaksarlaukpângalo uvlormit taipsomangamit atâtagilauktangit Egiptenemit aularlaungmatta uvlok tamanna tikkilugo.

16 Manasselo kovisilaurivoktauk aungnik passijaksaungitunik unnuktunik, nâmatitsilerkârtinnago Jerusalemik tamattomunga; idluitulliningita senniatigut idluitullititsijutigijangita Judakunik, pinniarkovlugit Nalekab idluigijanginik.

17 Unipkautsit tamakkoa assingit Manasse pivlugo, tamaitalo pinniarlauktangit, ahâk, tamakkoa aglaksimavut Judakut attanningita aglangine.

18 Manasselo sinnisilerpok atatagijaminut ailune, illijartortaulunelo perorsêvingme iglome kiglinganêtome, tagva Usab perorsêvingane. Erningalo Amon attanningorpok innangêrlugo.

19 Amon Jahrekalaukpok 22 ginik attanningorame, attanniotilunelo Jahrîk magguk nâvlugik Jerusaleme. Anânanga attekalaukpok Mesulemetemik, Harozib pannia, Jatbamiovinek.

20 Pinniarporlo Nalekab idluigijanginik, sorlo atâtanga Manasse pinniarlaungmat.

21 Agvertarmarikporlo apkome, atâtame agvertarvigilauktangane; kivgartorpaillo Gudingoat atâtame kivgartorlauktangit, tuksiarvigilaitalo.

22 Kemmakpâlo Nalegak, atâtagilauktame Gudingat, agvertarlungilarlo Nalekab apkosiningane.

23 Kivgangillo angerkattigêngnekartitsivut Amon akkerartorlugo, tokkopâllo attannek iglungane.

24 Innugiartoksuille nuname ullimârpait illunaita angerkattigêngnekartitsilauktut attannek Amon akkerartorlugo. Nunablo innugiartoksoangita erninga Josia attanningortipất innangêrlugo.

25 Amoniblo pinniarningita assingit, ahâk, tamakkoa aglaksimavut Judakut attanningita aglangine.

26 Illijartortauvorlo illuvikotiminut, Usab perorsêvikoting anêtomut. Erningalo Josia attanningorpok innangêrlugo. 


\section{KAPITEL XXII.}

\section{Attannele Josia. Tempelib sujungaititauninga.}

JOSIA Jahrekalaukpok sittamaujortunik attanningorame, attanniotilunelo Jahrit 31 git nâlugit Jerusaleme. Anânanga attekalaukpok Jedidamik, Adajab pannia Bazkatemiovinek.

2 Pinniarporlo Nalekab namagijanganik, agvertarporlo atâtagijame Davidiḩ apkosiningane tamât, senniagôrlungilarlo tallerpingmullonêt saumingmullonêt.

3 Attanniublo Josiab Jahringita 18 ingane, attanniub tilliva Safan, Azaljab Mesulamib erningata erninga, aglakpaktok, Nalekab igiunganut okarlunelo :

4 Majorarit ajochertuijersoarmut Hilkiamut, tunnijikovlugo kênaujanik Nalekab iglunganut âtaumajunik, upkoat udsertortingita kattersorlauktanginik innugiartoksoarnit;

5 Tunnijaukovlugillo aulatsikojaujut sullijaksanik Nalekab iglungane, agganginut, tunnijikovlugit tapkoninga, sennajunut Nalekab iglungane, iglub sujuksimaningit âkiksorlugit pikovlugit;

6 Tagva igluliortunut, sennajunut, karmaliortunullo, pissiniartuksaujunullo kejungnik ujarkaniglo ullimarsimajunik, iglub âkiktaujutiksanganut illingajunik;

7 Kissiane appertsortaukonnagit kenaujat agganginut tunnijaujut pivlugit, nertornarlutik pinniarmatta.

8 Tagva Hilkiab ajochertuijoksub Safan, aglakpaktok okautiva: Aglait perkojaktaggit nachvarlaukpakka Nalekab iglungane. Hilkiablo aglait tapkoa tunnivait Safanemut attuarsikovlugo tapkoninga.

9 Safaniblo aglakpaktub âpait attannermut, unipkautivâlo mattominga, okarlunelo: Kivgakotivit kênaujat iglumelauktut navilaukpait, tunnilaukpaillo aulatsijut sullijaksanik agganginut, perkojaumajut tamattomunga Nalekab iglungane.

10 Safaniblo aglakpaktub attannek unipkautiva okarlunelo: Hilkiab ajochertuijub aglangnik tunitsivigilaukpânga. Safaniblo attuarpait attanniub sângane.

11 Attanniuble aglait perkojaktaggit okausertangit tussaramigit, annorane allikpait.

12 Attanniublo Hilkia ajochertuijok, Ahikamelo Safanib erninga, Achborelo Michajub erninga, Safanelo aglakpaktok, Asajalo attanniub kivganga perkovait, okarlunelo:

13 Nalegak apperiartorsiuk pirlunga innugiartoksuillo pivlugit, Judakullo illuêngarlutik pivlugit, aglait tapkoa nachvartaumajut okausertangit pitjutigællugit; Nalekab ningausinga illungertorutigijarut angijoksongmat, tamanna pirlugo, atâtagi- 
javut nalelaungimatta aglait tapkoa okausertanginik, pinniaratiglo tamainik aglaksimajunik tapkonane.

14 Tagva Hilkia ajochertuijok, Ahikamelo, Achborelo, Safanelo, Asajalo aivut nellautaijomut arnamut Huldamut, Sallumib, Tikvab erningata Harhamib udsertortub anorânik erningata nullianganut, (iglokarporlo Jerusalamib abvakungata aipangine) okarvigivâllo.

15 Tapsomalo okautivait: Imâk Nalegak Israelib Gudinga okarpok: Angut tillijise uvamnut okarvigisiuk:

16 Imâk Nalegak okarpok: Kannoêtomik kaitsijomavunga iglugaseksoarnut makkonunga, innunginullo, perkojat Judakut attanningata attuarlauktangita okausertanginik tamainik.

17 Tamanna pivlugo, kemmalaungmanga, Gudiblo assingit isserluanititsivigilaungmattigik, ningaksaromavlunga aggarmik pinniarninginut tamainut; tamanna pivlugo illungertornera iglugaseksuit tamakkoa pivlugit, ikkoalavok, kamitauniangilarlo.

18 Judakulle attanningat tillijise Nalegak appertsorlugo, imâk okautijaksarivasse: Imâk Nalegak Israelib Gudinga okarpok :

19 Tamanna pivlugo, omattit auksititaulaungmat okautsinut tussarlauktarnut, nikkanarsarlauravillo illingnik Nalekab sângane, tussarlauravit okauserilauktamnik, iglugaseksuit tamakkoa innungillo pitjutigællugit, asserortaujuksaungmatta, ominarsijaujuksaungmattalo, annorakotitillo allilaurangne, kugviorlauravillo sâmne; tagva uvanga tussarlaurivara, Nalegak okarpok.

20 Tamanna pivlugo atâtagijarnut illaliutitsomavagit, ullapirsautekarlutillo illuvingnut illautitaujuksauvotit, ijikpillo kannoêtut tamaita tækkojaksaringilait tikkerkojomârtakka iglugaseksoarnut tamakkonunga. Tapkoalo tamakkoa okautigivait attannermut.

\section{KAPITEL XXIII.}

Josiab attuartaukojininga perkojanik, serkomitsiningalo Gudingoanik; tokkungalo.

A TTANNERLO tilliklerpok, kattikovaillo ingminut Judakut Jerusalemiullo angajuklingit illunaita.

2 Attannerlo majorarpok takpaunga Nalekab iglunganut, Judakullo angutingita Jerusalemiullo illunatik illagivât, ajochertuijut nellautaijullo, innugiartoksuillo illumatik, mikkijut angijullo; siutingitalo sângine aglait angerkattigêngniggit, nachvartaumalauktut Nalekab iglungane okausertangit illunaita attuartauvut. 
3 Attannerlo nangerpok nappajut illangane, angerkattigêngnekartitsivorlo Nalekab sângane, Nalegak malliklugo agvertaromagamik, perkojangillo, illusiokojangillo, idluarijangillo malligomavlugit, omamut tamânut, tarnemullo tamânut, angerkattigêngnerub oma okausertangit aglaksimajut aglangne tamakkonane nellautsititsomavlugit. Innugiartoksuillo illunatik angerkattigêkput.

4 Attanniublo ajochertuijersoak Hilkia, ajochertuijullo nukardlit, upkuallo udsertortingit perkovait, annitsijuksaungmatta Nalekab Tempelinganit, perkutinik tamainik sennajaulauktunik Baalelo Astarotelo, uvloriagaseksuillo pivlugit. Ikkipaillo Jerusalemib sillatâne naksarme Kidroneme; argsangillo âtauvut Bethelemut.

5 Gudingoallo ajochertuijokotingit Judakut attanningita tagvanekolauktangit, isserluanititsikovlugit pôktôjune, Judakullo iglugaseksoangine, Jerusalemiblo avatâne, pejarpait; tapkoalo isserluanititsijut Baalelo, sekkinerlo, takkerlo Planetillo, uvloriagaseksuit tamaita pivlugit.

6 Gudingoallo annitaukovait Nalekab iglunganit Jerusalemib sillatânut, kôngmut Kidronemut, ikkipaillo kôngme Kidroneme, serkalitilaitalo sanningortilugit, sanningillo kângarsorpait innuit erkanangitut illuvingita kânginut.

7 Ajortullijullo iglungit Nalekab iglungata tunganelauktut, itseliorviolauktullo arnanut, Gudingoanut, imitipait.

8 Ajochertuijullo illunaita Judakut iglugaseksoanginit tikkikovait, pôktôjullo, ajochertuijut isserluanititsivigilauktangit ivsornartipait, Gebamit Berseba tikkilugo; poktôjullo itterviksuit sângine asserorpait, tamnalotauk Josuab iglugaseksuit angajokaunerôjungata iglungata sânganelauktok, iglugaseksuit ittervingata saumianêlauktok.

9 Pôktôjulle ajochertuijungit tunnergutinik tunnijilaungilet, Nalekab Altarekotingane Jerusalemêtome, nerrivulle kakkojanik sêrnakangitunik kattangutimik akkorngane.

10 Tofetelo ivsornartipatauk Hinnomib kittorngangita naksanganêtok, kinalonêt ernerminik panniminiglonêt ikkomakorkojikonnago Moloche pirlugo.

11 Horsillo pêjarpait Judakut attanningita tunnergutautilauktangit sekkinermut, pitilauktangit Nalekab iglungata ittervianut, Nethanmelichib iglugaseksuit aiparusinganêtub inningata sânganut; sekkerngublo kammutingit ikkipait.

12 Altarillo Ahasib iglungata kângane Judakut attanningita sennalauktangit, Altarillo Manassib sennalauktangit, Nalekab iglungata innigingne avaluliorsimajungne, attanniub serkomipait, akpangerdlunelo sanningit egipait kôngmut Kidronemut.

13 Poktojuttauk Jerusalemib sivuranêlauktut, kakkab Mashitib attiggub tallerpianêlauktut, Salomob Israelitit attanning- 
ata sennalauktangit, Asthorete Zidonemiut makkojungnartokotingat pivlugo, Kamoselo Moabiterit makkojungnartokotingat pivlugo, Milkomelo Ammonib kittorngangita makkojungnartokotingat pivlugo, attanniub ivsornartipait,

14 Nappajullo serkomipait, nappartullo pêjarpait, inningillo nâmatipait innuit sauninginut.

15 Taimaktauk Altare Betheleme, pôktôjok Jerobeamib Nebatib erningata Israelitinik idluitullititsilauktub sennalauktanga, Altare tamna, pôktojorlo serkomipâk; pôktojorlo ikkipa, sanningortilâlo, nappajullo Gudingoat ikkipait.

16 Josialo keviarpok tækkolaitalo illuvît tagvane kakkamelauktut, tilliklilunelo, saunîllo illuvingnêlauktut aijaukovait, ikkipaillo Altarib kângane, ivsornartipâlo, Nalekab okausingit, Gudib angutekotingata nipliarlauktangit, tamattominga nipliarlauktub, malliklugit.

17 Okarporlo: Illuvek tækkojara kannoêka? Iglugaseksuillo innungita okautivât: Tagva Gudib angutekotingata illuvinga Judamit tikkilauktub nipliadlalauktublo tamattominga pinniarnerijarnik Altaremut Bethelemêtomut.

18 Okarporlo: Taimailingale, sauningit ullapitauniaratik kinamut. Taimak taipsoma sauningit piulijaulaukput, nellautaijub Samariamit tikkilauktub sauningit illagællugit.

19 Poktojullo iglungit illunaita Samariab iglugaseksoangine, Israelitit attanningita sennalauktangit Nalekamik ningaksailutik, pêjarpaittauk; taimaluatsiamiglo pinniarvigivait, sorlo Betheleme pinniarlaungmat.

20 Poktojullo ajochertuijungit, tagvanelauktut tunnergutautipait 1 ltarit kângine, taimaglo innuit sauningit ikkipait kângine. Taimaglo utterpok Jerusalemut.

21 Attanniublo innugiartoksuit perkovait okarlunelo: Passamik attoleritse Nalekamut Gudipsingnut, sorlo aglaksimangmat aglangne, angerkattigêngnelingne.

22 Passa taimaitok attortaulaungimat idluarsaijut nelliuninginit, Israelitinik idluarsailauktut, Israelitillo attanningita Judakullo attanningita nelliuningine.

23 Attanniuble Josiab Jahringita 18 ingane Passa tamanna attortaulaukpok, Nalegak pivlugo Jerusaleme.

24 Taimaktauk Josiab nellautainerluktut illunaita, angakullo, adsingoallo, Gudingoallo, makkojungnartullo illunaita, Judæame Jerusalemelo tækkojaulauktut, pêjarpait, perkojat okausertangit nellautsititsomavlugit aglaksimajut aglangne, Hilkiab ajochertuijub nachvarlauktangine Nalekab iglungane.

25 Attannekalaungilarlo adsinganik tapsoma sivorngane, taimak omamut tamânut, tarnemullo tamânut, pitsartunermullo tamânut sâlauktomik Nalekamut, Mosesib perkojangit tamaita malliklugit; tapsomalo kingorngane adsinga sakkijarlaunginivok. 
26 Sullele Nalekab ningarnime illungertudlarninga, Judakunik ningarutijame, allarlungila, ningaksainerit tamaita pivlugit, Manassib ningaksaijutigijangit tapsominga.

27 Nalegarlo okarpok: Judakut pêjaromagivakka kênama sânganit, sorlo Israelitit pêjarlaurapkit; iglugaseksuillo makkoa annerilauktakka egitsomavakka, tagva Jerusalem, iglolo okautigilauktara: Attera tagvanêtuksauvok.

28 Unipkautsille tamakkoa assingit Josia pivlugo, ahâk, tamanna aglaksimavok Judakut attanningita aglangine.

29 Tapsoma nelliuningane Farao Necho Egiptenemiut attanningat takpaungarpok, koksoarmut Fratemut Assiriamiut attanningat sorsugvigijomavlugo. Attanniuble Josiab aularlune pachrearpa, tokkolunelo Meggidome, tækkolaukârdlugo.

30 Kivgakotingitalo tokkungatillugo aularutivât Meggidomit, âlâllo Jerusalemut, illijartorpâllo illuvinganut. Nunablo innungita Joahas, Josiab erninga tiguvât mingoarlâllo, attanningortipâllo atâtanga innangêrlugo.

31 Joahas Jahrekalaukpok 23 ginik attanningorame, attanniotilunelo takkit pingasut nâlugit Jerusaleme. Anânanga attekalaukpok Hamutalemik, Jeremiab pannia Libnamiovinek.

32 Pinniarporlo Nalekab idluigijanginik, sorlo atâtagilauktangit pinniarlaungmatta.

33 Faraoble Nechob kellaksortaukova Riblateme nuname Hemathemik attelingme, attaniojungnaikovlugo Jerusaleme, nunalo akkilêkova Talentinik 100 dinik Silberemik, Talentemiglo attautsemik Goldemik.

34 Faraubló Nechob Eliakime Josiab erninga attanningortipa atâtanga innangêrlugo, attingalo ablatsangortipa Jojakimemut. Joahasele neksarpa, âlâlo Egiptenemut, tagvane tokkolaukpok.

35 Jojakimiblo Silbere, Goldelo tamakkoa tunnivait Faraomut; nunamiulle tunnijikovait, Silbere tamanna tunnijungnarlugo, Faraub perkojanga malliklugo; attunit perkutingit malliklugit nunab innungit tunnijikovait Silberemik Goldemiglo, tunnijomavlugit Faraomut Nechomut.

36 Jojakime Jahrekalaukpok 25 ginik attanningorame, attanniotilunelo Jahrit 11 it nâlugit Jerusaleme. Anânanga attekalaukpok Sebudamik, Pedajab pannia Rumamiovinek.

37 Pinniarporlo Nalekab idluigijanginik, sorlo atâtagijangit pinniarlaungmatta. 


\section{KAPITEL XXIV.}

\section{Jojakime, Jojachine, Zedekialo attanît Judałunut. Jerusalemib illujaumaninga.}

TAPSOMA nelliuningane majorarlune takpaungarpok Nebukadnezar Babelib attannertanga; Jojakimiblo nalekpa Jahrit pingasut nâvlugit; allârpale nalegungnailugolo.

2 Tagva Nalekab tikkitaukovait sorsuktuksanut Kaldæamit, Siriamillo, Moabemillo, Amoniblo kittornganginit, tapkoa tillivait Judæamut, asseroikovlugit tamattominga, Nalekab okausingit malliklugit, okauserilauktangit kivgamigut nellautaijutigut.

3 Nalekab okausingit malliklugit kissiane Judakut taimak pinniarviovut, kênaminit pêjaromangmagit, Mannassib idluitulliningit pivlugit, pinniarlauktangit.

4 Auk passijaksaungitok kovilauktanga pivlugotauk, Jerusalemelo nâmatilaungmagit aungmut passijaksaungitomut, Nalekab issumagijungnaivigijomalaungilait.

5 Unipkautsille tamakkoa assingit Jojakime pivlugo, tamaitalo pinniarlauktangit, ahâk tamanna aglaksimavok Judakut attanningita aglangine.

6 Jojakimelo sinnisilerpok atâtagijaminut ailune; erningalo Jojachin attanningorpok innangêrlugo.

7 Egiptenemiullo attanningat aularungnairpok nunaminit; Babelemiut attanningata aksarlaungmago tamainik Egiptenemiut attanningata pigijanginik, Egiptenib kônganit, kôk Frate tikkilugo.

8 Jojachin Jahrekalaukpok 18 nik attanningorame, attanniotilunelo takkit pingasut nâlugit Jerusaleme. Anânanga attekalaukpok Nehustamik Elnatanib pannia Jerusalemit.

9 Pinniarporlo Nalekab idluigijanginik, sorlo atâtanga pinniarlaungmat.

10 Nelliutome taipsomane Nebukadnezarib Babelemiut attanningata kivgangit majorarlutik takpaungarput Jerusalemut, tikkilutiglo iglugaseksoarnut, talluliorutiksalijarlutik.

11 Nebukadnezarelo Babelemiut attanningat tikkipok iglugaseksoarnut, kivgangit iglugaseksoarnik illumajitillugit.

12 Jojachinele Judakut attanningat annivok Babelemiut attanninganut, anânane, kivgakotinelo, angajokakotinelo, aulatsijokotinelo illagællugit. Babelemiullo attanningata tiguva attannionime Jahringita 8 ingane;

13 Tiguvaillo tagvangat Nalekab iglunganit, attanniublo 
iglunganit, perkutit pivianartut tamaita, illulerkutillo Goldeôjut Salomob Israelitit attanningata sennajaukolauktangit Nalekab Tempelinganut, serkomipait, sorlo Nalegak okalaungmat.

14 Aularutivaillo Jerusalemiut illuêngarlutik, angajokajat illunaita, sorsuktuksat illunaita, parngnanaigutsaumajut 10000 dit, igloliortut illunaita, sabviortullo illunaita; amiakokarkojilungilarlo nunab innunginik erkananginerpangojunik kissianik.

15 Aularutivâlo Jojachin Babelemut, attanniublo anânanga, attanniublo nulliangit, angajokakotingillo; tapkoalo illaliudlugit sôngojut nuname parngnanaigutsauvlutik aularutikassiutivait Jerusalemit Babelemut.

16 Innuillo erkanarnerpât 7000 dit, igluliortullo, sabviortullo 1000 dit, sorsugungnartut, unatadlarnermut piojut illunaita; tapkoa Babelemiut attanningata parngnanaigutsauvlutik âpait Babelemut.

17 Babelemiullo attanningata Methanja, akkanga, attanningortipa innangêrlugo, attingalo ablatsangortipa Zedekiamut.

18 Zedekia Jahrekalaukpok 21 ginik attanningorame, attanniotilunelo Jahrit 11 it nâvlugit Jerusaleme. Anânanga attekalaukpok Hamitalemik Jeremiab pannia Libnamiovinek.

19 Pinniarporlo Nalekab idluigijanginik, sorlo Jojakime piniarlaungmat.

20 Nalekable ningarninga pitjutigællugo, Jerusalemiut Judakullo taimailiortitaulaukput ajæktuikârtinane tapkoninga kênaminit. Zedekiablo Babelemiut attanningat nalegungnailerpa.

\section{KAPITEL XXV.}

\section{Iglugaselssuit Jerusalemib serkomitaunngit; innugiartoksuillo aularuisauningit Babelemut.}

PiJokalaukPORLO attannioningata Jahringita 9 ingane, takkit 10ingata uvlungita 10 ingane, tagva Nebukadnezar Babelemiut attanningata sorsuktuksaksoakotine tamaita illagællugit tikkipok Jerusalemut; illumavaillo, talluliorlaitalo.

2 Taimak iglugaseksuit illujaumalaukput Zedekiab attannioningata Jahringita 11 ingat tikkidlugo.

3 Takkillo 4 ingata -uvlungita 9 ingane kâksoak angijororpalliarok iglugaseksoarne, nerkiksakalungilarlo nunab innugiartoksoanginut.

4 Iglugaseksuillo pittaktauvut, sorsuktuksallo illunatik unnuarme kemâvut itterviksoakut attanniub perorsevingata avalukita akkorngangnetokut. Kaldæerille iglugaseksuit avatânelaukput. Attannerlo kemmâvok apkosinerme naternamut torartome. 
5 Kaldæerillo sorsuktuksaksoangita attannek udlapât, angulâllo Jerichub naternangane, sorsuktuksaksoakotingillo illunatik siamalerput tapsomangat.

6 Tapkoalo attannek tiguvât, aularutivâllo takpaunga $\mathrm{Ba}-$ belemiut attanninganut Riblatemut; kakkimiojutigivlugo illingajuksauninga okautigivât.

7 Zedekiablo kittorngangit tokkopait ijikita sângangne, ijigiglo tautugungnaitipakit, kellaksorlâllo kellaumgujanut aularutivâllo Babelemut.

8 Takkillo 5ingata uvlungita 7 ingane, tagva Nebukadnezarib Jahringita 19 ingane, Nebusaradan sorsuktuksat angajokaksoangat, Babelemiut attanningata kivganga, Jerusalemut tikkipok,

9 Ikkipaillo Nalekab iglunga, attanniublo iglunga, iglullo illunaita Jerusaleme, iglullo angijut illunaita ikkipait.

10 Kaldæerillo kattimgajoksoangita, sorsuktuksat angajokaksoanganetut Jerusalemib avaluksoangit serkomipait.

11 Innugiartoksuille amiakungit iglugaseksoarne, kemâlauktullo Babelemiut attanninganut, innugiartoksuillo assingita amiakungit, Nebusaradanib sorsuktuksat angajokangata aularutivait.

12 Nunamiullo erkananginerpânginit sorsuktuksat angajokangata illangit nokkarkovait, perorsêvilerijiokovlugit Weineliksanik, kakkojaksaniglo.

13 Kaldæerillo nappajut kannujaujut, Nalekab iglunganêlauktut, ikkovrallo, immarbingoarlo kannujaujok, Nalekab iglungane serkomipait, kannujangillo aularutivait Babelemut.

14 Ukkusillo, poargitillo, sâvillo, allupsautillo, illulerkutillo kannujaujut, kivgartornermut attortaulauktut tiguvait.

15 Taimaktauk sorsuktuksat angajokangata, êlikautit, kallutaujallo, illulerkutillo Goldeôjut, Silbereôjullo tiguvait.

16 Nappajûk, immarbingoarlo, ikkovrallo, Salomob sennalauktangit, Nalekab iglunganut; illulerkutit tamakkoa tamaita kannujangit kiktitarlugit ajornalaukpok.

17 Nappajûk aipa tallitut 18 tut poktôtigijolaukpok, niakojartungalo kânganêtok kannujaulaurivok, tallitullo pingasotut pôktotigijôlaukpok, nulluangoakalaukporlo, paungâlungoakalarmelo, Granatenik attelingnik, niakojartub avatane, illunâllo kannujaulaukpok, nappajûglo aipanga, taimailingalaurivok nulluangoat illaliudlugit.

18 Sorsuktuksallo angajokangata tiguva ajochertuije Seraja, sivurliojit illangat, ajochertuijorlo Zefanja tuglit illangat, udsertortullo upkoanik pingasut,

19 Iglugaseksoarnillo angajokaksuit illangat, angajokangortitaumajok sorsuktuksanut, angutillo tellimat, attanniub najuganetsainarlauktut, naipitaumajut iglugaseksoarne, Soferelo 
sorsuktuksaksoakattigêt angajokangat, innugiartoksoarnik nuname illinniartitsijok sorsungnermik, angutillo 60 git nunab innunginit, iglugaseksoarne naipitaumajut;

20 Tapkoa Nebusaradanib sorsuktuksat angajokaunerojungata tiguvait, âpaillo Babelemiut attanninganut Riblatemut.

21 Babelemiullo attanningata ullimârlugit tokkopait Riblateme, Hematib nunanganetome. Taimak Judakut aularutitaumalaukput nunakotimingnit.

22 Innugiartoksuille amiakunginut nuname, Nebukadnezarib Babelemiut attanningata amiakokojanginut, Gedalja Ahikamib Safanib erningata erninga angajokangortipa.

23 Sorsuktuksallo angajokangit illunatik, angutillo tapkonanêtut tussaramik, Babelemiut attanningata Gedalja angajokangortimago nunamut, tikkiput Gedaljamut Mizpamut, tapkoa: Ismael, Nethanjab erninga, Johananelo Kareab erninga, Serajalo Thanhumetib erninga, Netofatemiovinek, Jaesanjalo, Maechatib erninga, angutekotitik illagilugit.

24 Gedaljablo tapkoa angutekotingillo angermarikpait, okautilaitalo; Sivuratailititse Kaldæamiut naleklugit; nunamêtuaritse, Babelemiullo attanningat naleksiuk, tagva pilloringniarpose.

25 Takkille 7 ingane Ismaele, Nethanjab Elisamab erningata erninga attanniub kinguranginit pijok, tikkipok, angutit 10 illagilugit, Gedaljalo ullimârlugo tokkopât, tappilugillo Judit, Kaldæerillo tapsomanêlauktut Mizpame.

26 Tagva innugiartoksuit illunatik mikkijut angijullo, sorsuktuksallo angajokangit parngnailerput, aularlutiglo Egiptenemut; Kaldæerit illimagigamigit.

27 Jahrillo 37 ingane Jojachin, Judakut attanningat aularudsaulaukârdlune, takkit 12 ingata uvlungita 27 ingane EvilMerodachib Babelemiut attanningata, attannionime Jahringita sivorlingane, Jojachimib Judakut attanningata niakunga kivikpa parngnanairsimavingmit,

28 Illanaksarlunelo okarkattigiva, iksivautangalo sivorliotipa attannît tapsomanêlauktut Babeleme illunatik iksivautanginit;

29 Annorairkovalo parngnanêrsimavingmêsiutinginik; nerrimarkattautsainarlaukporlo tapsoma sângane innôsingane tamât.

30 Nerkiksangillo attoriakartangit, nerkiksat pijariakatsainartangit tunnitsiviôlaukpok attannermut, uvlut tamaita, innôsingane tamât. 


\title{
KRONIKAB AGLANGITA SIVURLINGIT.
}

\author{
$m a x$ \\ KAPITEL I. \\ Kinguvarêt Adamemit Jako tikkilugo.
}

ADAM, Seth, Enos,

2 Kenan, Mahalaleel, Jared,

3 Henoch, Methusala, Lamech,

4 Noa, Sem, Ham, Jafet.

5 Jafetib erningit tapkoangôvut: Gomer, Magog, Medai, Javan, Tubal, Mesech, Tiras.

6 Gomeriblo erningit tapkoangôvut: Askenas, Rifat, Togarma

7 Javanib erningit tapkoangôvut: Elisa, Tarsisa, Kitime, Dodanime.

8 Hamiblo erningit tapkoangôrut: Kus, Mizraime, Put, Kanaan.

9 Kusiblo erningit tapkoangôvut: Seba, Hevila, Sabta, Ragema, Sabtecha. Ragemable erningit tapkoangôvuk: Scheba, Dedanelo.

10 Kusiblo. Nimrod ernetariva; tamna angutaudlalerlaukpok nuname.

11 Mizramiblo ernetarivait: Ludime, Anamime, Lehabime, Naftuhime,

12 Patrusime, Kasluhime, (tapsomangat Filisterit pivut.) Kaftorimelo.

13 Kanaaniblo ernetarivait Zidon, erniangokârtane, Hetelo,

14 Jebuselo, Amorilo, Girgosilo,

15 Hevilo, Arkilo, Sinilo,

16 Arvadilo, Zemarilo, Hematilo.

17 Semib erningit tapkoangôvut: Elame, Assur, Arfasat, Lud, Arame, Uz, Hul, Geter, Masech.

18 Arfasatiblo ernetariva Sala; Salablo ernetariva Eber.

19 Eberelo ernetarpok maggungnik, aipanga attekalaukpok 
Pelegemik, tapsoma nelliuningane nuna aviktaulaungmat, tapsomalo nukanga Jaktanemik attekalaukpok.

20 Jaktaniblo ernetarivait Almodad, Saleflo, Hazarmafetelo, Jaralo,

21 Hadoramelo, Usallo, Diklalo,

22 Ebalelo, Abimaello, Schebalo,

23 Ofirelo, Hevilalo, Jobabelo. Tapkoa illunaita Jaktanib ernerivait.

24 Sem, Arfasat, Sala,

25 Eber, Peleg, Regu,

26 Serug, Nahor, Tara,

27 Abram, tagva Abraham.

28 Abrahamib ernigik tapkoangôvuk: Isaak Ismaelelo.

29 Tamadsa tapkoa kinguvangit: Ismaelib ernerikârtanga Nebajot, Kedarelo, Adbeello, Mibsamelo,

30 Mismalo, Dumalo, Masalo, Hadadelo, Temalo,

31 Jeturelo, Nafiselo, Kedmalo. Tapkoa Ismaelib ernerivait.

32 Keturablo, Abrahamib pannervgangata erningit: erniarivait, Simrame, Jaksanelo, Medanelo, Midianelo, Jesbakelo, Sualo. Jaksaniblo ernigik tapkoangôvuk: Scheba Dedanelo.

33 Midianiblo erningit tapkoangôvut: Efa, Efer, Henoch, Abida, Eldaa. Tapkoa illunaita Keturab ernerivait.

34 Abrahamiblo Isaak ernetariva. Isaakiblo ernigik tapkoangôvuk: Esau, Israelelo.

35 Esaub erningit tapkoangôvut: Elifas, Reguel, Jeus, Jaelamelo, Koralo.

36 Elifasib erningit tapkoangôvut: Teman, Omarelo, Zefillo, Gartamelo, Kenảselo, Timnalo, Amalekelo.

37 Reguelib erningit tapkoangôvut: Nahat, Seralo, Samma, Misalo,

38 Seirib erningit tapkoangôvut: Lotan, Sobalelo, Zibeonelo, Analo, Disonelo, Ezerelo, Disanelo.

39 Lotanib erningit tapkoangôvut: Hori, Homamelo; Lothaniblo Timna najagilaukpa.

40 Sobalib erningit tapkoangôvut: Alian, Manahatelo, Ebalo, Sefilo, Onamelo. Zibeonib ernigik tapkoangôvuk: Aja, Analo. 41 Anab erninga: Dison. Disonib erningit tapkoangôvut: Hamrame, Esbanelo, Jetranelo, Keranelo.

42 Ezerib erningit tapkoangôvut: Bilhan, Saevanelo, Jaekanelo. Disanib ernigik tapkoangôvuk: Uz, Aranelo.

43 Tamadsa attannit, attanniotikârlauktut Edomiterit nunangane, attannît illangat attanniotikârtinago Israelib kittorngangita akkorngane: Bela, Beorib erninga; iglugaseksoakotingillo attekalaukput Dinhabamik.

44 Belalo tokkungmat Jobabe, Serab erninga Bazramiok attanningorpok innangêrdlugo. 
45 Jobabelo tokkungmat, Husame Temaniterit nunanganit pijok attanningorpok innangêrlugo.

46 Husamelo tokkungmat, Hadad Bedadib erninga, Midianiterinik ullimarniktok, Moabiterit naternangane, attanningorpok innangêrlugo; iglugaseksoakotingillo attekalaukput Avitemik.

47 Hadade tokkungmat Samla Masrekemiok innangêrlugo attanningorpok.

48 Samla tokkungmat, Saul Rehobotemit kôngmetomit pijok innangêrlugo attanningorpok.

49 Saulelo tokkungmat, Baal Hanan, Achborib erninga innangêrlugo attanningorpok.

50 Baal Hananelo tokkungmat Hadad attanningorpok innangêrdlugo, iglugaseksoakotingillo attekalaukput Pagimik; nulliangalo, Matredib Mesahab panningata pannia, attekalaukpok Mahetabeelemik.

51 Hadadele tokkungmat, angojokangôrput Edomeme: Angajokâk Timna, angajokâk Alia, angajokâk Jetet,

52 Angajokâk Ahalibama, angajokâk Ela, angajokâk Pinon,

53 Angajokâk Kenas, angajokâk Teman, angajokâk Mibzar,

54 Angajokâk Magdiel, angajokâk Irame. Tamadsa angajokait Edomeme.

\section{KAPITEL II.}

\section{Jakob Judablo erningit.}

TAMADSA Israelib erningit: Ruben, Simeon, Levi, Juda, Isaschar, Sebulon,

2 Dan, Josef, Benjamin, Naftali, Gad, Asser.

3 Judab erningit tapkoangôvut: Ger, Onan, Sela. Tapkoa pingasut ernetarivait Kanaanemiomit Suab panninganit. Gerele Judab erninga ernerikârtanga idluilaukpok Nalekab sângane, tamanna pivlugo tokkopâ.

4 Thamarible ukkoangata erniarivâk tapsomunga, Perez, Seralo; taimak Judab erningit tellimaungmatta.

5 Perezib ernigik tapkoangôvuk: Hezron, Hamullo.

6 Serable erningit tapkoangôvut: Simri, Etan, Heman, Kalkol, Dara. Tapkoa kattilugit tellimauvut.

7 Karmib erninga Achaneôvok, kiksartitsilauktok Israelitinik, nungutaksaujomik tigusilaurame.

8 Etanib erninga, Asarjaôlaukpok.

9 Hezroniblo erningit, erniangomajut tapsomunga, tapkoangôvut: Jerameel, Ramelo, Kalubailo.

10 Ramiblo Aminadabe ernetariva. Aminadab, Nahasson ernetariva, angajokaulauktok Judakunut. 
11. Nahassonib Salma ernetariva. Salmab Boase ernetariva.

12 Boasib Obed ernetariva. Obedib Isai ernetariva.

13 Isaiblo Eliabe ernine ernerikârtane ernetariva, Abinadabelo tuglek, Simealo pingajoat,

14 Nethaneelelo sittamangat, Raddailo tellimangat,

15 Ozemelo pingasojoat, Davidelo 7 ingat.

16 Tapkoalo najakit tapkoangôvuk: Zeruja Abigailelo. Zerujab erningit tapkoangôvut: Abisai, Joabe, Asahelo, tapkoa pingasut.

17 Abigailible Amasa erniariva. Amasable atâtanga Jetereôlaukpok Ismailib kinguvânginit pijok.

18 Kalelo Hezronib erninga ernetârpok Asubamit nulliaminit, Jerigotemillo; tamadsalo tapsoma (Asubab) erningit: Jeser, Sobabelo, Ardonelo.

19 Asubale tokkungmat, Kaleb Efrate nullianikpa; tapsoma Hur erniariva tapsomunga.

20 Hurib Uri ernetariva, Uriblo Bezaleel ernetariva.

21 Kingorngane Hezronib Machirib Gileadib atâtangata pannia innangakattigiva; nullianikpalo Jahrekarlune 60 ginik; tapsomalo Segube erniariva tapsomunga.

22 Segublo Jair ernetariva, tamna iglugaseksoakalaukpok 23 ginik Gileadib nunangane.

23 Tiguvaillo Gesur, Aramelo, Jairiblo iglugasengit, tappilugillo Kenat aiparusingillo, iglugaseksuit 60 git. Tapkoa illunaita Machirib Gileadib atâtangata ernerivait.

24 Hezronelo tokkolaukârmat Kale Efratame, nulliangata Abiab erniariva tapsomunga Ashur, Tekoab atâtanga.

25 Jerameeliblo Hezronib ernerikârtangata erningit, tapkoangôlaukput: Sivurlek Ram, Bunalo, Ozemelo, Ahjalo.

26 Jerameelo nulliakalaukpok sulle assianik, Ataramik attelingmik, tamna anânauvok Onamemut.

27 Ramiblo Jerameelib ernerikârtangata erningit tapkoangôlaukput: Maaz, Jaminelo, Ekerelo.

28 Onamiblo ernigik tapkoangôlaukpuk: Samai, Jadalo. Samaiblo ernigik tapkoangôlaukpuk: Nadabe, Abisurelo.

29 Abisuriblo nullianga attekalaukpok Abihailemik, tapsoma erniarivak tapsomunga Achban, Molidelo.

30 Nadabiblo ernigik tapkoangôvuk: Seled, Appaimelo; Seledelo tokkovok kittorngakarane.

31 Appaimiblo erninga, Jeseiôvok. Jeseib erninga Sesaneôvok; Sesanib erninga Ahelaiôvok.

32 Jadable Samaib nukangata ernigik tapkoangôvuk: Jeter, Jonathanelo. Jeterelo tokkovok kittorngakarane.

33 Jonathaniblo ernigik tapkoangôvuk: Pelet, Sasalo. Tagva Jerameelib erningit. 
34 Sesanele ernekarane pannekalaukpok. Sesanelo kivgakalaukpok Egiptenemiomik, Jarhamik attelingmik.

35 Sesaniblo Jarha kivgane tunnitsivigiva panniminik, nulliangôkovlugo tapsomunga, tapsoma erniariva tapsomunga Atai.

36 Ataiblo ernetariva Nathan. Nathaniblo ernetariva Sabade.

37 Sabadiblo ernetariva Eflal. Eflaliblo ernetariva Obed.

38 Obediblo ernetariva Jehu. Jehublo ernetariva Asarja.

39 Asarjablo ernetariva Halez. Haleziblo ernetariva Elleasa.

40 Elleasablo ernetariva Sissemai. Sisemaiblo ernetariva Sallume.

41 Sallumiblo ernetariva Jekamia. Jekamiablo ernetariva Elisama.

42 Kaleblo Jerameelib nukangata erningit tapkoangôvut: Mesa ernerikârtanga, tamna Sifib atâtagiva, Maresablo Hebronib atâtangata erningit.

43 Hebroniblo erningit tapkoangôvut: Kora, Tapualo, Rekemelo, Samalo.

44 Samablo ernetariva Rahame, Jarkaamib atâtanga. Rekemib ernetariva Samai.

45 Samaib erninga attekalaukpok Maonemik. Maoniblo Betzur erneriva.

46 Efablo Kaleb pannervgangata erniarivait Haran, Mozalo, Gaseselo. Haraniblo Gases ernetariva.

47 Jadaiblo erningit tapkoangôvut: Rekeme, Jothamelo, Gesanelo, Peletelo, Efalo, Saafelo.

48 Maechable Kaleb pannervgangata erniarivak Seber Tirhenalo.

49 Erniarivaglo Saaf, Madmanab atâtanga, Sevalo, Machbenab, Gibeablo atâtangak. Kaleblo pannia Achsaulaukpok.

50 Tamadsa manna Kaleb erningit: Hur, erniangokârtok Efratemit; Sobal, Kiriatjearimib atâtanga;

51 Salma, Bethlehemib atâtanga; Haref, Bethgaderib atâtanga.

52 Sobalelo Kiriatjearimib atâtanga, ernekalaukpok: Haroemik, Hazimiglo, Hamenuhotemiglo.

53 Kinguvarêllo Kiriatjearimemit pijut tapkoangôvut: Jethemiut, Putemiullo, Sumatemiullo, Mizraimiullo. Tapkonangallo pilaukput, Zaregamiut, Esthaholemiullo.

54 Salmablo erningit Bethlehemeôvok, Netofatemiullo,. Atarot, Beth Joab, Manatemiullo ketterkangit Zaraitemiojut.

55 Aglakpaktullo Jabezeme nunakalauktut kinguvarêngit tapkoangôvut, Tireatemiut, Simeatemiullo, Suchatemiullo. Tagva Kiniterit Hamatemit pimmajut, Bethrechab atâtanganit. 


\section{KAPITEL III.}

\section{Davidib erningit. Judakullo attanningit.}

Tamadsa Davidib erningit erniangolauktut tapsomunga Hebroneme: Sivorlek, Amnon, Ahinoamit Jesreelemiovinermit; tuglek Daniel Abigailemit Karmelemiojomit;

2 Pingajoat Absalome Maechab, Talmaib attanniojub Gesureme panningata erninga; sittamangat Adonia, Hagithib erninga;

3 Tellimangat Safatja, Abitalemit; pingasojoat Jetreame, nulliaminit Eglamit.

4 Tapkoa pingasojortut erniangovut tapsomunga Hebroneme; Jahrit 7 it takkillo 6 it nâlugit attanniotigame tagvane, Jerusalemele attanniôtilaukpok Jahrit 33 git nâlugit.

5 Tapkoalo erniangôvut tapsomunga Jerusaleme: Simea, Sobabe, Nathan, Salomo, tapkoa sittamat Bathsuamit Amielib panninganit;

6 Tapkoalo tappilugit, Jebehar, Elisama, Elifalet,

7 Noga, Nefeg, Japia,

8 Elisama, Eliada, Elifalet, tapkoa 9 it.

9 Tagva Davidib erningit, pannervgat erningit illautinagit. Thamarelo najagilaukpât.

10 Salomob erninga Rehabeameôlaukpok, tapsoma erninga Abiaôlaukpok, tapsoma erninga Assaôlaukpok, tapsoma erninga Josafateôlaukpok,

11 Tapsoma erninga Jorameôlaukpok, tapsoma erninga Ahasjaôlaukpok, tapsomalo erninga Joaseôlaukpok,

12 Tapsomalo erninga Amaziaôlaukpok, tapsoma erninga Asarjaôlaukpok, tapsoma erninga Jothameôlaukpok,

13 Tapsoma erninga Ahaseôlaukpok, tapsoma erninga Hiskiaôlaukpok, tapsoma erninga Manasseôlaukpok,

14 Tapsoma erninga Ammoneôlaukpok, tapsoma erninga Josiaôlaukpok.

15 Josiab erningit tapkoangôlaukput: Sivurlek Johanan, tuglek Jojakim, pingajoat Zedekia, sittamangat Sallume.

16 Jojakimiblo erninga Jechanjaôlaukpok, tapsomalo eŕninga Zedekiangôlaukpok.

17 Jechanjablo parngnanêrsortaumalauktub erningit tapkoangôlaukput: Sealthiel,

18 Malchirame, Fadaja, Seneazar, Jekamja, Hosama, Nedabja.

19 Fadajab ernigik tapkoangôvuk: Zerubabel, Simeilo. 
Zerubabelib ernigik tapkoangôlaukpuk: Mesullame, Hananjalo, najangâglo Selomitheolaukpok;

20 Tapkoalo tappilugit, Hasuba, Ohel, Berechja, Hasadja, Josabhesed, tapkoa tellimat.

21 Hananjablo ernigik tapkoangôlaukpuk: Platja, Jesajalo; tapsoma erninga Refajaôlaukpok, tapsomalo erninga Arnanaulaukpok, tapsomalo erninga Obadjaolaukpok, tapsomalo erninga Sachanjaôlaukpok.

22 Sachanjable erninga Semajaulaukpok. Semajablo erningit tapkoangôlaukput: Hattus, Jegeal, Baria, Nearja, Safat, Sesa, tapkoa 6 it.

23 Nearjablo erningit tapkoangôlaukput: Elioenai, Hiskia, Asrikam, tapkoa pingasut.

24 Elioenaiblo erningit tapkoangolaukput: Hodaja, Eliasibe, Plaja, Akube, Johanan, Delaja, Anani, tapkoa 7 it.

\section{KAPITEL IV. \\ Judab Simeoniblo kinguvangit.}

JUDAB erningit tapkoangêlaukput: Perez, Hezron, Karmi, Hur, Sobalelo.

2 Reajablo Sobalib erningata Jahate ernetariva. Jahatib Ahumai Lahadelo ernetarivâk. Tagva kinguvarêt Zaregatemiut.

3 Tapkoalo atâtamit Etamemit pivut: Jesreel, Jesma, Jedbaselo; tapkoalo najangat Hazlelponimik attekalaukpok;

4 Pnuelelo, Gedorib atâtanga; Eserelo Husab atâtanga. Tagva Hurib erningit, erniangokârtub Efratemit, Bethlehemib atâtangata.

5 Ashurelo Tekoab atâtanga nulliakalaukpok maggungnik, Helleamik, Naeramiglo.

6 Naerablo erniarivait tapsomunga Ahusamik, Heferemiglo, Temnimiglo, Ahastaremiglo. Tagva Naerab erningit.

7 Helleablo erningit tapkoangôlaukput: Zerete, Jezoharelo, Etnanelo.

8 Hoziblo ernetarivait, Anube, Hazobealo, Aharheliblo kinguvarêngit, Harumib erningata.

9 Jabezelo opigijaunersaulaukpok kattangutiminit; anânangatalo atserarpa Jabezemik, okarame: Siorniudlarlunga erniarilaukpara.

10 Jabezelo niplialaukpok Israelib Gudinganut, okarlunelo: Pitsartutilaungatôk, kiglilervigalo anginersautilugo, aggakpîllo illaginiarlinga, kannoêtomullo tikkitautailitilunga, ânertaukonnanga tapsomunga. Gudiblo tuksiararijanga tikkerkova. 
11 Kalublo Suhab nukangata Mehir ernetariva, tagva Estonib atâtanga.

12 Estoniblo ernetarivait Betrafa, Passealo, Tehinalo, iglugaseksuit Nahasemik attiggit atâtangat, tagva angutit Rechamit pijut.

13 Kenasib ernigik tapkoangôvuk: Athniel, Serajalo. Athnieliblo erninga Hathateôlaukpok.

14 Meonothaiblo ernetariva Ofra. Serajablo ernetariva Joabe, igluliortut naksangata atâtanga, igluliorteôngmatta.

15 Kaleblo Jefunnib erningata erningit tapkoangôvut: Iru, Elalo, Naamlo. Elab erninga Kenaseôlaukpok.

16 Jehaleeliblo erningit tapkoangôlaukput: Sif, Sifalo, Tirjalo, Asareello.

17 Esrablo erningit tapkoangôlaukput: Jether, Meredelo, Eferelo, Jalonelo, Taharelo, Mirjamlo illaliudlugo, Samailo, Jesbalo, Esthemoab atâtanga.

18 Nulliangatalo Judijab Jared erniariva, Gedorib atâtanga Heberelo, Sochob atâtanga, Jekuthiello, Sanoab atâtanga. Tagva Bitfab Faraub panningata, Maredib nullianilauktangata erningit.

19 Arnablo Hodijab Nahab najangata Kegilab atâtangata erningit tapkoangôlaukput: Garmi Esthemoalo Maechitemiok.

20 Simonib erningit tapkoangôlaukput: Ammon, Rinnalo, Benhananlo, Tilonelo. Jeseib ernigik tapkoangolaukpuk: Sohet, Bensohetelo.

21 Selablo Judab erningata erningit tapkoangôlaukput: Er, Lechab atâtanga, Laedalo, Maresab atâtanga, uvineruksaijaliortillo kinguvarêngit, Asbeakut akkorngane.

22 Taimaktauk Jokime, angutillo Kosbeamit pijut, Joase, Sarafelo attanniotijut Moabemiunut, Jasubi-Lehemiunullo, sorlo unipkautsit nutaungitut okarmatta.

23 Machaliorteôlaukput, tapkoalo nunakalauktut perortut avaluliorsimajut akkorngane; tagvane nunakalaukput attannerme, tapsoma sullijaksangit pinniarlugit.

24 Simeonib kinguvangit tapkoangôlaukput: Nemuel, Jaminelo, Jaribelo, Seralo, Saulelo,

25 Tapsoma erninga Sallumeôlaukpok, tapsoma erninga Mipsameôlaukpok, tapsoma erninga Mismaôlaukpok.

26 Mismablo erninga Hamueleôlaukpok; tapsoma erninga Zahureôlaukpok, tapsoma erninga Simeiôlaukpok,

27 Simeilo ernekalaukpok 16 nik, pannekalarmelo 6 nik, kattangutingillo unnuktunik kittorngakalaungilet; abvakungille illunatik taimak unnuksivallialaungilet Judab kittorngangititut.

28 Nunakalaukpulle Bersebame, Moladamelo, Hazar-Sualemelo,

29 Bilhamelo, Ezememelo, Tolademelo, 
30 Bethuelemelo, Harmamelo, Ziklagemelo,

31 Bethmarchabotemelo, Hazarsusimemelo, Bethbireimelo, Saaraimemelo. Tagva taipkoa iglugaseksoangit, attannek Davide tikkilugo.

32 Iglugasengit tapkoangôlaukput: Ethan, Ainelo, Rimmon, Tochenelo, Asanelo, iglugaseksuit tellimait.

33 Aiparusingillo, iglugasait, iglugaseksuit makkoa avatanelauktut Baal tikkilugit; tagva tapkoa inningit abvakungillo.

34 Mesobabelo, Jamlechelo, Josalo, Amaziab erninga,

35 Joello, Jehulo, Josibjab Serajab erningata, Asielib erningata erninga,

36 Elioenailo, Jaekobalo, Josohalo, Asajalo, Adiello, Ismeello, Benajalo,

37 Sisalo Sifeib erninga, Allonib erningata, Jedajab erningata, Simrib erningata, Semajab erningata.

38 Tapkoa attanniôlaukput illamik akkorṇgane, abvakungillo siamarlaukput unnuksivalliavlutik.

39 Aularpullo Gedoremut nâksab kittâ tikkilugo, nerripkauteliksarsiorlutik saugakotimingnut;

40 Nerripkautiksarsivullo unnuktunik âmanâniglo, nunasilutiglo nerrotôjomik, kassungajomiglo, akluinartomiglo; sivurngane Hamib kinguvângit tagvane nunakalaumgmatta.

41 Tapkoalo manna attingittigut aglaksimajut, tikkiput Hiskiab Judakut attanningata nelliuningane, ullimarpaillo tuppingit innungillo naipitaumajut tagvane, nungupaillo uvlok tamanna tikkilugo, nunakarlutiglo tagvane taipkoa innangêrlugit; nerripkautiksakarmat tagvane saukanut.

42 Tapkonangallo Simonib erninginit 500 dit aularput Seirib kakkanginut, angajokatik Platja, Nearja, Refajalo, Usiello Jeseib erningit illagilugit,

43 Ullimarpaillo Amalekiterit amiakungit kemmâjut; nunakarlutiglo tagvane, uvlok tamanna tikkidlugo.

\section{KAPITEL V.}

\section{Rubenib, Gadiblo, Manassiblo kinguvângit.}

RUBENIB Israelib ernerikârtangata erningit (erniangokârtolaungmat, tamattomungale atâtame innangavia ivsornartilaungmago, erniangokârtoninga tunnijaulaukpok Josefib, Israelib erningata erninginut; tamnalo erniangokârtonasugijaulaungilak;

2 Judale sôngojolauktok kattangutime akkorngane attannionermik tunnitsiviolaukpok, Josefele erniangokârnermik.)

3 Taimak Rubenib Israelib ernerikârtangata erningit tapkoangôvut: Hanoch, Pallulo, Hezronelo, Karmilo. 
4 Joeliblo erninga Semajaulaukpok, tapsoma erninga Gogeôlaukpok, tapsoma erninga Simeiôlaukpok.

5 Tapsoma erninga Michaôlaukpok, tapsoma erninga Reajaulaukpok, tapsoma erninga Baalôlaukpok,

6 Tapsoma erninga Beeraiôlaukpok, Tiglat Pilnesseremut, Assiriamiut attanninganut aularutitaulauktok; angajokaulaukporle Rubenekut akkorngane.

7 Tapsomalo kattangutingit kittitauvlutik kinguvâtik malliklugit, angajokakalaukput, Jejelemik, Sacharjamiglo.

8 Belalo Asanib erninga, Semab erningata, Joelib erningata nunakalaukpok Aroereme, Nebo, Baal-Meonelo tikkilugik.

9 Nunakalaukporlo unane sunakajuitok tikkidlugo, kôb Fratib miksane, nergjutekotingit unnuktolaungmatta Gileadib nunangane.

10 Sauliblo nelliuningane unatarput Hagariterinut, ochotitauvullo tapkoa agganginut, tuppingillo innigivait Grleadib kittâne illunane.

11 Gadible erningit nunakalaukput taipkoa senniane nuname Basaneme, Salcha tikkidlugo.

12 Joel angajokaunerôjok, Safanelo tuglia, Jaenai, Safatelo Basaneme.

13 Kattangutingitalo atâtangit tapkoangôvut: Michael, Mesulamelo, Sebalo, Jorailo, Jaekanelo, Sialo, Eberelo, tapkoa 7 it.

14 Tamadsa Abihailib erningit, Hurib erningata, Jaroab erningata, Gileadib erningata, Michaelib erningata, Jesisaib erningata, Jadob erningata, Busib erningata.

15 Ahi Abdielib Gunib erningata erninga angajokaulaukpok tapkoa atâtangita iglunginut,

16 Nunakalaukpullo Gileademe Basanemêtome, iglogasenginelo, Saroniblo aiparusingine illunaine kiglilervingit tikkidlugit.

17 Tapkoa illunatik kinguvarêkotitik malliklugit kittitaumavut Jothamib Judakut attanningata nelliuningane, Jerobeamiblo Israelitit attanningata nelliuningane.

18 Rubenib erningit, Gadiblo erningit kinguvarêllo Manassekut ketterkangit, angutaujut tapkonangat sorsugungnartut, sappulutalijartut, saviksoalijartullo, pittiksaniglo kellusijungnartut, unatarnermiglo illisimajut, tapkoa 44760 ôlaukput, tapkoa aularput unatadlarnermut. belo.

19 Unatadlarpaillo Hagariterit, Jeturelo, Nafeselo, Noda-

20 Ikajortaulaukpullo tapkoa akkerartorungnarlugit, Hagariterillo illagijangillo illunaita tunnijauvut agganginut. Gudemut nipliarmatta sorsungnerme; tapsomalo tussarpait, tettigingmatsuk.

21 Aularutivaillo taipkoa nergjutekotingit, Kamelit 5000 dit, 
saukello 250000 dit, siutekoktojut 2000 dit, innuillo 100000 dit.

22 Ullimarsimajut unnuktut ochungmatta, unatadlarnek Gudemit pingmat. Nunakarpullo tagvane taipkoa innangêrdlugit, nelliutok parngnanairsortauviat tikkidlugo.

23 Kinguvarêllo Manassekut ketterkangita kittorngangit nunakalaukput nuname, Basanemit, Baal Hermon tikkilugo. Seniremelo kakkamelo Hermoneme, unnuktolaukpullo.

24 Tamadsalo tapkoa atâtangita iglungita angajokangit: Efer, Jesei, Eliel, Asriel, Jeremia, Hodawja, Jadiel, angutaudlartut, angutillo nertornartut, angajokaulutiglo opinartut atâtamik iglungine.

25 Idluitullilaukpulle atâtagijamik Gudinganut, ajortullilutiglo innugiartoksuit Gudib tapkoa sivorngane pêjarlauktangita Gudingoakotingit malliklugit:

26 Tagva Israelib Gudingata Fulib Assiriamiut attanningata annerninga, Tiglat Pilnesseriblo Assiriab attannertangata annerninga kajungersarpâk, aularutivaillo Rubenekut, Gadekullo, kinguvarêllo Manassekut ketterkangit, âpaillo Halamut, Haboremullo, Haramullo, Gosaniblo kônganut, uvlok tamanna tikkilugo.

\section{KAPITEL VI.}

\section{Levikut, Aronekullo; iglogaseksoangillo.}

LEVIB erningit tapkoangôlaukput: Gersom, Kahatelo, Merarilo.

2 Kahatiblo erningit tapkoangôlaukput: Amram, Jezeharelo, Hebronelo, Usielelo.

3 Amramiblo kittorngangit tapkoangôlaukput: Aron, Moselo, Mirjamelo. Aroniblo erningit tapkoangôlaukput: Nadabe, Abihulo, Eliasarelo, Itamarelo.

4 Eliasarib Pinehase ernetariva; Pinnehasib Abisua ernetariva.

5 Abisuab Buki ernetariva; Bukib Usi ernetariva.

6 Usib Seraja ernetariva; Serajab Merajote ernetariva.

7 Merajotib Amarja ernetariva; Amarjab Ahito ernetariva.

8 Ahitob Zadoke ernetariva; Zadokib Ahimaaz ernetariva.

9 Ahimaazib Asarja ernetariva; Asarjab Johannan ernetariva.

10 Johannanib Asarja ernetariva, ajochertuijôlauktok iglome Salomob igloliortangane Jerusaleme.

11 Asarjab Amarja ernetariva; Amarjab Ahito ernetariva.

12 Ahitob Zadoke ernetariva; Zadokib Sallume ernetariva.

13 Sallumib Hilkia ernetariva; Hilkiab Asarja ernetariva.

14 Asarjab Seraja ernetariva; Serajab Jozadake ernetariva. 
15 Jozadakelo aularkassiutitaumalaukpok, Nalekab Judakut, Jerusalemiullo Nebukadnezarekut parngnanairsortauvlutik aulartitaukolaungmagit.

16 Levib erningit tagva tapkoangovut: Gersome, Kahat, Merarilo.

17 Gersomiblo ernigik attekarpuk: Libnimik, Simeimiglo.

18 Kahatible erningit attekarput; Amramemik, Jezeharemiglo, Hebronemiglo, Usielemiglo.

19 Merarib ernigik attekarpuk: Mahelimik, Musimiglo. Tagva Levitit kinguvarêngita atâtagijangit.

20 Gersomib erninga Libniôlaukpok, tapsoma erninga Jahateôlaukpok, tapsoma erninga Simaôlaukpok.

21 Tapsoma erninga Johaôlaukpok, tapsoma erninga Iddoôlaukpok, tapsoma erninga Serahôlaukpok, tapsoma erninga Jeatraiôlaukpok.

22 Kahatib erninga Aminadabeôlaukpok, tapsoma erninga Koraôlaukpok, tapsoma erninga Assirêolaukpok.

23 Tapsoma erninga Elkanaôlaukpok, tapsoma erninga Abiasafeôlaukpok, tapsoma erninga Assireôlaukpok.

24 Tapsoma erninga Tahateôlaukpok, tapsoma erninga Urieleôlaukpok, tapsoma erninga Usijaôlaukpok, tapsoma erninga Sauleôlaukpok.

25 Elkanablo ernigik tapkoangôlaukpuk: Amasai, Ahimotelo.

26 Tapsoma erninga Elkanaôlaukpok, tapsoma erninga Elkanaôlaukpok Zofemiok, tapsoma erninga Nahateôlaukpok.

27 Tapsoma erninga Elijabeôlaukpok, tapsoma erninga Jerohameôlaukpok, tapsoma erninga Elkanaôlaukpok.

28 Tapsoma erninga Samueleôlaukpok, tapsoma ernerikârtanga Vasniôlaukpok, Abijalo.

29 Merarib erninga Maheliôlaukpok, tapsoma erninga Libniôlaukpok, tapsoma erninga Simeiôlaukpok, tapsoma erninga Usaôlaukpok.

30 Tapsoma erninga Simeaôlaukpok, tapsoma erninga Haggijaôlaukpok, tapsoma erninga Asajaôlaukpok.

31 Tamadsale Davidib pitilauktangit imgerkovlugit Nalekab iglungane iklerviub merngoêrservigijangane;

32 Kivgartorlutiglo iglub tuppiub kattimaviojub sângane imgerlutik, Salomo Nalekab iglunganik Jerusaleme igloliorkârtinnago, nangerlutiglo illuserijatik pinniarkojaunermingne malliklugit.

33 Tapkoangovullo nangerlauktut tagvane erningillo: Kahatib erninginit Heman imgerpaktok, Joelib erninga, Samuelib erningata,

34 Elkanab erningata, Jerohamib erningata, Elielib erningata, Toab erningata, 
35 Zufib erningata, Elkanab erningata, Mahatib erningata, Amasaib erningata,

36 Elkanab erningata Joelib erningata, Asarjab erningata, Zefanjab erningata,

37 Tahatib erningata, Asirib erningata, Abiassafib erningata, Korab erningata,

38 J zeharib erningata, Kahatib erningata, Levib erningata, Israelib erningata.

39 Kattangutingalo Assaf nàngerpok tapsoma tallerpiane. Tamnalo Assaf Berechjab erneriva, Simeab erningata,

40 Michaelib erningata, Baesejab erningata, Malchijab erningata,

41 Atnib erningata, Serab erningata, Adajab erningata,

42 Ethanib erningata, Simab erningata, Simeib erningata,

43 Jahatib erningata, Gersomib erningata, Levib erningata.

44 Kattangutingillo Merarib erningit saumiane nangerput: Ethan, Kusib erninga, Abdib erningata, Malluchib erningata,

45 Hasabjab erningata, Amaziab erningata, Hilkiab erningata,

46 Amzib erningata, Banib erningata, Samerib erningata,

47 Mahelib erningata, Musib erningata, Merarib erningata, Levib erningata.

48 Tapkoale kattangutingit Levitit tunnijaumalaukput kivgartornernut sunatuinarnut Nalekab iglungata tuppingane.

49 Aronible erningillo tunnergutinik tunnijivut tunnergutiksaliub ôtaksanik Altaringane, isserluanijutiksaliublo Altaringane, pinniaraksanullo tamainut ivsornainerpangôjome illingalaukput, ullapkoserpaillo Israelitit, taimaluatsiak, sorlo Mosese, Gudib kivganga perkojilaungmat.

50 Tamadsale Aronib erningit: Eleasar, erninga, tapsoma erninga Pinehaseôlaukpok, tapsoma erninga Abisuaôlaukpok,

51 Tapsoma erninga Bukiôlaukpok, tapsoma erninga Usiôlaukpok, tapsoma erninga Serajaôlaukpok.

52 Tapsoma erninga Merajoteôlaukpok, tapsoma erninga Amarjaôlaukpok, tapsoma erninga Ahitobeôlaukpok.

53 Tapsoma erninga Zadokeôlaukpok, tapsoma erninga Ahimaazeôláukpok.

54 Tamadsalo tapkoa iglungit innigijangit kiglilervingine, Aronib erningit, Kahatib kinguvânginit, tapkoa imeraumut nellautaulaungmatta,

55 Tunitsivigivaillo Hebronemik, Judakut nunanganetunik, tapkoalo aiparusingit avatâne.

56 Iglogaseksuille perorsêvinginik iglogasenginiglo tunnivait Kalemut Jefunib erninganut.

57 Aroniblo erninginut tunnivait iglogaseksuit pitlartaujung- 
nairvit Hebron, Libnalo, aiparusingit illaliudlugit, Jater, Estemoalo, aiparusingit illaliudlugit,

58 Hilenelo, Debirelo,

59 Asanelo, Betsemeselo aiparusingit illaliudlugit.

60 Kinguvarênillo Benjaminekunit Geba, Alemetelo, Anatotelo, aiparusingit illaliudlugit. Tapkoalo iglogaseksoangit abvakungine 13 ôlaukput.

61 Kahatible erningita assingit tunnitsiviôvut imeraut attorlugo, kinguvarêt Manassekut ketterkanginit iglogaseksoarnik 10 nik.

62 Gersonib erningit tunitsiviôvut kinguvarênit Isascharekunit, kinguvarênillo Asserekunit, kinguvarênillo Naftalikunit, kinguvarênillo Manassekunit Basanemetunit, iglogaseksoarnik 13 nik.

63 Merarib erningit avakungit malliklugit tunnitsiviovut immeraut attorlugo, kinguvarênit Rubenekunit, kinguvarênillo Gadekunit, kingurarênillo Sebulonekunit, iglogaseksoarnik 12 nik.

64 Taimaglo Israelib kittorngangita Levitit tunnitsivigivait iglogaseksoarnik tapkoninga, aiparusinginiglo;

65 Tunnitsivigivaillo immeraut attorlugo kinguvarênit Judab kittornganginit, kinguvarênillo Simeonib kittornganginit, kinguvarênillo Benjaminib kittornganginit, iglogaseksoarnik taijanginik.

66 Kahatiblo erningita assingita abvakungita iglogaseksoangit kinguvarênit Efraimekunit pivut.

67 Tunnitsiviôvullo iglogaseksoarnik pitlartaujungnaivingnik Sichememik, Efraimib kakkanginêtunik, Gesermiglo,

68 Jakmeamemiglo, Bethoronemiglo,

69 Ajalonemiglo, Gatrimonemiglo, aiparusinginiglo.

70. Kinguvarêllo Manassekut ketterkanginit, Aneremik, Bileamemiglo aiparusinginiglo.

71 Gersoniblo erningit tunnitsivigivait, kinguvarêt Manassekut ketterkanginit Golanemik, Basanemetunik, Astarotemiglo aiparusinginiglo,

72 Kinguvarênillo Isascharekunit Kedesemik, Dabratemiglo,

73 Ramotemiglo, Anememiglo, aiparusinginiglo.

74 Kinguvarênit Asserekunit Masalemik, Abdonemiglo,

75 Hukokemik, Rehobemiglo, aiparusinginiglo.

76 Kinguvarênit Naftalikunit: Kedesemik, Galileametunik, Hamonemik, Kiriataimemiglo, aiparusinginiglo.

77 Merarib kittorngangita assingit tunnitsivigivait kinguvarênit Sebulonekunit: Rimonomik, Taboremiglo, aiparusinginiglo;

78 Jordaniblo akkiane Jerichob nellâne Jordanib kittâne, kinguvarênit Rubenekunit: Bezeremik, sunakajuitometunik, Jazamiglo, aiparusinginiglo. 
79 Kedmotemiglo, Mepatemiglo, aiparusinginiglo.

80 Kinguvarênit Gadekunit: Ramotemik Gileademetunik Mahanaimiglo,

81 Hesbonemiglo, Jaeseremiglo, aiparusinginiglo.

\section{KAPITEL VII.}

Jakob erningita assingita 6 it erningit, tapkoalo kinguvângit. ISASCHARIB erningit tapkoangôlaukput: Tola, Pualo, Jasubelo, Simronelo, tapkoa sittamat.

2 Tolab erningit tapkoangôlaukput: Usi, Refajalo, Jerielo, Jahemailo, Jebsamelo, Samuelelo, niakoôjut atâtagijamik iglungane Tolamit, angutit angutaudlartut illagijamik akkorngane, tapkoa unnurningit Davidib nelliuningane 22600 deôlaukput.

3 Usiblo erninga Jesrajaôlaukpok. Jesrajablo erningit tapkoangôlaukput: Michael, Obadjalo, Joelelo, Jesialo, tapkoa tellimat, niakoôlaukpullo illunatik.

4 Tapkonanelaukpullo kittorngarêngit, atatagijanginit pijut malliklugit, sorsuktukset unatadlarnermut attorakset 36000 dit; unnuktunik nulliakaramik ernekalarmiglo.

5 Kattangutingillo Isascharekut abvakunginit tamainit angutit angutaudlartut kittitaulauktut 87000 diovut.

6 Benjaminih erningit tapkoangôlaukput: Bela, Becherelo, Jediaelelo, tapkoa pingasut.

7 Belablo erningit tapkoangôlaukput: Ezbon, Usilo, Usielelo, Jerimotelo, Irilo, tellimat, niakoôjullo atatagijamik iglungine, angutaudlarlutiglo. Kittitauvullo 22034 illo.

8 Becheriblo erningit tapkoangôlaukput: Semira, Joaselo, Elieserelo, Elioenailo, Amrilo, Jerimotelo, Abialo, Anatotelo, Alametelo; tapkoa illunaita Becherib ernerilaukpait.

9 Unnurningillo abvakungit malliklugit, niakoôjut atâtagijamik iglungine, angutit angutaudlartut 20200 diôlaukput.

10 Jediaeliblo erninga Bilhaneôlaukpok. Bilhaniblo erningit tapkoangôlaukput: Jeus, Benjaminelo, Ehudelo, Knaenalo, Setanelo, Tarsiselo, Ahisaharelo.

11 Tapkoa illunaita Jediaelib ernerivait niakoôjut atâtagijanginut, angutit angutaudlartut 17200 dit, aulartut sorsuklutik unatadlarnermut.

12 Supimelo, Hupimelo erniôlaukpuk Iremut. Husimelo Aherib ernerilaukpa.

13 Naftalib erningit tapkoangôlaukput: Jaziel, Gunilo, Jezerelo, Sallumelo, Bilhab erningit.

14 Manassib erninga: Esrieleôvok, Aramjab panervgangata erniarijanga; tapsoma Machire Gileadib atâtanga ernetariva. 
15 Machiriblo Hupimib, Supimiblo najakit nullianikpa. Najangalo attekalaukpok Maechamik. Erningatalo tugliub attinga, Zelafehadeôlaukpok; Zelafehade pannekalaukpok.

16 Maechablo Machrib nulliangata ernek erniariva, tamna atserarpa Peresemik; nukangalo Sareseôlaukpok, tapsoma ernigik Ulameôlaukpok Rakemeôlarmelo.

17 Ulamib erninga Bedaneôlaukpok. Tagva Gileadib erningit Machirib erningata, Manassib erningata.

18 Tapsomalo najangata Molechetib Ishude, Abieserelo, Mahelalo erniarivait.

19 Semidablo erningit tapkoangôlaukput: Ajan, Sichemelo, Likhilo, Aniamelo.

20 Efraimiblo erningit tapkoangôlaukput: Sutela, tapsoma erninga Beredeôlaukpok, tapsoma erninga Tahateôlaukpok, tapsoma erninga Eleadaôlaukpok, tapsoma erninga Tahateôlaukpok,

21 Tapsoma erninga Sabadeôlaukpok, tapsoma erninga Sutelaôlaukpok, tapsoma ernigik Esereolaukpok, Eleadelo. Angutillo Gatemiut nunab innungita nakterpait, attarlauramik tarkoa nergjutekotingit aksarnigarijomavlugit.

22 Atâtangallo Efraime ogguarlune kiksarlaukpok akkunît, kattangutingitalo tikkipât manigoromavlugo.

23 Nullianelo uvinikattigiva, tamnalo singailerpok, ernilunelo ernermik, tamna atserarpa Briamik, iglungane kanoêtokarmat.

24 Pannialo Seerâ̂laukpok, tapsoma Bethoron iglogaseliorlaukpait, Bethoron atsiktok, kotsiktorlo, Usen-Seeralo.

25 Tapsoma ernigik Refaolaukpok, Resefelo, tapsoma erninga Telaôlaukpok, tapsomale erninga Tahaneôlaukpok.

26 Tapsomalo erninga Laedaneôlaukpok, tapsoma erninga Amihudeôlaukpok, tapsoma erninga Elisamaôlaukpok.

27 Tapsoma erninga Nuneôlaukpok, tapsoma erninga Josuaôlaukpok.

28 Pigijangallo nunakotingallo Beth-Eleôlaukpok aiparusingillo, kittânelo Naeran, pânelo Geser, aiparusingillo, Sechemelo, aiparusingillo. Gasa aiparusingillo tikkidlugit,

29 Manassiblo kittorngangita senniane, Bethsean aiparusingillo, Taenach aiparusingillo, Meggido aiparusingillo, Dor aiparusingillo. Tapkoa Josefib Israelib erningata kittorngangita innigivait.

30 Asserib erningit tapkoangôlaukput: Jemna, Jeswalo, Jeswilo, Brialo, najangallo Sera.

31 Briab ernigik tapkoangôlaukpuk: Heber, Malchielelo, tagva Birsawitib atâtanga.

32 Heberib ernetarivait Jaflet, Somerelo, Hotamelo, Sualo najangat. 
33 Jafletib erningit tapkoangôlaukput: Passa, Bimehalo, Aswatelo; tagva Jafletib erningit.

34 Somerib erningit tapkoangôlaukput: Ahi, Ragalo, Jehubalo, Aramelo.

35 Nukangatalo Helemib erningit tapkoangôlaukput: Zofa, Jemnalo, Seleselo, Amallo.

36 Zofab erningit tapkoangôlaukput: Sua, Harneferelo, Suallo, Berilo, Jemralo,

37 Bezerelo, Hodelo, Samalo, Silsalo, Jetranelo, Béeralo.

38 Jetherib erningit tapkoangôlaukput: Jefune, Fispalo, Aralo,

39 Ullab erningit tapkoangôlaukput: Ara, Hanielelo, Rizjalo.

40 Tapkoa illunaita Asserib ernerilaukpait, niakoôlaukput atâtagijamik iglungine, angutit angutaudlartut annerimajullo, niakoôjullo angajokânut. Kittitaumalaukpullo sorsuktuksanut unatadlarnermut unnurningit malliklugit 26000 dit.

\section{KAPITEL VIII.}

\section{Benjaminio, Sauliblo kinguvângit.}

BenjaminiB Bela ernerikârtane ernetariva, Asbalelo tuglek, Aralo pingajụat,

2 Noha sittamangat, Rafa tellimangat,

3 Belalo ernekalaukpok: Addaremik, Geramiglo, Abihudemiglo.

4 Abisuamiglo, Naemanemiglo, Ahoamiglo,

5 Geramiglo, Sefufanemiglo, Huramemiglo.

6 Tamadsalo Ehudib erningit, niakoôlauktut atâtat akkorngane, Gebamiune, aulârpullo tagvangat Manahatemut.

7 Naemablo, Ahiablo, Gerablo aulârtilaukpait; ernetarivaglo Usa, Ahihudelo.

8 Saharaime ernetarpok Moabiterit nunangane (ipkoa aularkolaukârdlugit) nulliamingnit Husimemit, Baeramillo,

9 Ernetarivaillo nulliaminit Hodesemit: Jobabe, Zibjalo, Mesalo, Malchamelo,

10 Jeuzelo, Sachjalo, Mirmalo. Tagva tapsoma erningit, niakoôjut atâtanut.

11 Husemillo ernetarivuk, Ahitobe, Elpaalo.

12 Elpaaliblo erningit tapkoangôlaukput: Eber, Miseamelo, Samedelo. Tapsoma, Ono, Lodelo, aiparusingillo iglogaselliorpait.

13 Brialo, Samalo niakoôlaukpuk atâtanut Ajalonemiut akkorngane; tapkoa Gathemiut singitipait. 
14 Ahjo, Sasakelo, Jeremotelo,

15 Sebadjalo, Aradelo, Aderelo,

16 Michaelelo, Jespalo, Johalo; tagva Briab erningit.

17 Sebadja, Mesullamelo, Hiskilo, Heberelo,

18 Jesmerailo, Jeslialo, Jobabelo; tagva Epaalib erningit.

19 Jakime, Sichrilo, Sabdilo,

20 Elioenaillo, Ziltailo, Elielelo,

21 Adaja, Brajalo, Simratelo; tagva Simeib erningit.

22 Jespan, Eberelo, Elielelo,

23 Abdonelo, Sichrilo, Hananelo,

24 Hananjalo, Elamelo, Antotjalo,

25 Jefdejalo, Pnuelelo; tagva Sasakib erningit.

26 Samserai, Seharjalo, Athaljalo,

27 Jaeresjalo, Elialo, Sichrilo, tagva Jerohamib erningit.

28 Tagva niakoôjut atâtanut, abvakungine; tapkoa iglokalaukput Jerusaleme.

29 Gibeonemelo, Gibeonib atâtanga nunakalaukpok, nulliangalo attekalaukpok Maechamik.

30 Erningalo ernerikârtanga Abdoneôlaukpok, Zurelo, Kiselo, Baallo, Nadabelo,

31 Gedorelo, Ajolo, Secherelo,

32 Miklotible Simea ernetariva, nunakalaurivuttauk Jerusaleme, kattangutitik illagilugit tapkoa akkiane.

33 Nerib Kise ernetariva, Kisib Saul ernetariva, Saulib, Jonathan, Malchisualo, Abinadabelo, Esbaalo ernetarivait.

34 Jonathanib erninga Meribaaleôlaukpok. Meribaalib Micha ernetariva.

35 Michab erningit tapkoangôlaukput: Piton, Melechelo, Taeralo, Ahaselo;

36 Ahasiblo Joadda ernetariva, Joaddablo ernetarivait Alemet, Asimawetelo, Simrilo. Simriblo Moza ernetariva.

37 Mozab Binea ernetariva, tapsoma erninga Rafaôlaukpok, tapsoma erninga Eleasaôlaukpok, tapsoma erninga Azelôlaukpok.

38 Azelelo ernekalaukpok 6 inik, attingit imailaukput: Esrikame, Bochrulo, Jesmaelelo, Searjale, Obadjalo, Hananelo. Tapkoa illunaita Azelib ernerilaukpait.

39 Nukangata Esekib erningit tapkoangôlaukput: Ulame, ernerikârtanga, Jeuse tuglek, Elifelet pingajuat.

40 Ulamible erningit angutaudlarlaukput, pikarilaukpullo pittikserlutik; ernekalaukpullo erngutekalarmiglo unnuktunik, 150 ginik. Tapkoa illunatik Benjaminib erninginit pivut. 


\section{KAPITEL IX.}

\section{Jerusalemint, Gibeamiullo.}

ISRAELI'IILLO illuêngarlutik kittitaumalaukput, ahâglo aglaksimavut Israelitit, Judakullo attanningita aglangine, aulartitaumavullo mana, idluitullinitik pivlugit Babelemut.

2 Sivorngane nunakalauktut pigijamingne iglugaseksoakotimingnelo Israeliteôlaukput, ajochertuijut, Levitit, Nethinimilo.

3 Jerusalemelo iglokalaukput Judab kittorngangita illangit, Benjaminiblo kittorngangita illangit, Efraimiblo kittorngangita illangit, Manassiblo kittorngangita illangit.

4 Utai, Amihudib erninga, Omrib erningata, Imrib erningata, Banib erningata, Perezib Judab erningata kittornganginit.

5 Silonimillo: Assaja, erniangokârtok, erningillo assingit.

6 Serablo erninginit Jeguel, kattangutingillo 690 it.

7 Benjaminiblo erninginit Sallu, Mesulamib erninga, Hodawjab erningata, Hasnuab erningata;

8 Jebnejalo Jerohamib erninga; Elalo, Usib erninga, Michrib erningata; Mesullamelo, Sefatjab erninga, Reguelib erningata, Jebnejab erningata;

9 Kattangutingillo abvakungit malliklugit 956 it. Angutił tapkoa illunatik niakoôlaukput atâtanut, atâtagijamik iglungine.

10 Ajochertuijunillo: Jedaja, Jojaribelo, Jachinelo;

11 Asarjalo, Hilkiab erninga, Mesullamib erningata, Zadokib erningata, Merajotib erningata, Ahitob erningata, angajokaujok Gudib iglungane;

12 Adajalo, Jerohamib erninga, Pashurib erningata, Malchiab erningata; Maesailo Adielib erninga, Jachserab erningata, Mesullamib erningata, Mesilemitib erningata Immerib erningata;

13 Tapkoalo kattangutingit, niakoôjut atâtamik iglungine, 1760 git erkinaitut sullijeksanut kivgartornerme Nalekab iglungane,

14 Levitinillo, Merarib erninginit: Semaja, Hasub erninga, Asrikamib erningata, Hasabjab erningata.

15 Bahbakarelo, Hereselo, Galalo, Matanjalo, Michab erninga, Sichrib erningata, Assafib erningata.

16 Obadjalo Semajab erninga, Galalib erningata, Jedutunib erningata Berechjalo, Assab erninga, Elkanab erningata, Netofatiterit iglogasengine nunakalauktub.

17 Upkuallo udsertortingit: Sallume Akubelo, Talmonelo, Ahimanelo, kattangutingillo; Sallumelo angajokaulaukpok.

18 Mannalo tikkilugo attanniub upkuangata kittanêtub sânganêput, tapkoa upkuanik udsertortut, Levib erningita tangmarvinganit pijut. 
19 Sallumelo, Koreb erninga, Abiasafib erningata, Korab erningata, kattangutingillo. atâtame iglunganit pijut, Korakut, sullijaksakalaukput tuppiub upkoanga udsertorlugo, atâtangillo Nalekab tangmarviane ittervik udsertorpât.

20 Pinehaselo, Eleaserib erninga angajokagilaukpât; Nalekablo aipariva.

21 Sacharja, Meselemjab erninga udsertorteolaujovok tuppiub kattimaviojub upkoanganik.

22 Tapkoa illunatik annerijaulaukput udsertorkovlugit upkuab manuangane, 212 it. Iglogasakotitik malliklugit aglaktaumalaukput. Davidiblo, Samueliblo, tækkojub, taimaitungortilaukpait nertornarningit pivlugit.

23 Tapkoa, erningillo, Nalekab iglungata tuppiojub ittervinginelaukput udsertorlugit.

24 Annorit sittamat malliklugit upkoat udsertortingit nangerput: kittane, kangianelo, tachânelo, sekkernganelo.

25 Kattangutingille iglogasekotimingnelaukput, kaitsainarkovlugit uvlut 7 ingane, tapkonanekovlugillo.

26 Levitit upkoanik udsertortut angajokaunerôjungit sittamat nertornarlauramik angajokaulaukput inninut perkutinullo pivianartunut Gudib iglunganetunut.

27 Gudiblo iglungata avatâne unnuarmik najivut, pigartuksaulauramik, perkojaulaukpullo uvlâne tamaine ittervît angmarlugit.

28 Tapkoalo illangit pimaklertungortitaumalaukput illulerkutinik, kivgartornernut illingajunik, kittitaumavlutigle ittertipait, kittitaumavlutiglo annipait.

29 Illangillo pimaklertungortitaumalaukput illulerkutinik ivsornaitunik, sennaukamiglo kakkortamik, Weinemiglo, Orksomiglo, Weirauchemiglo, isserluanijutiksaniglo.

30 Ajochertuijullo erningita illangita isserluanijutiksat sennavait.

31 Matitjalo, Levitit illangat, Sallumib ernerikârtanga, Korakut illangat, pimaklertungortitaumalaukpok jarataumajunik kallutaujane.

32 Kahatekunille kattangutinginit illangit, perkojaumalaukput, kakkojanik takkojaksanik sennalutik, Sabbatene tamaine pikovlugit.

33 Tagva imgerpaktut, niakoôjut Levitit atâtangita akkorngane, kivgartoriakalaungitut, uvlotillugo unnuamelo tamattominga sullijaksakalauramik.

34 Tagva niakoôjut Levitit atâtanginut abvakungine. Tapkoa nunakalaukput Jerusaleme.

35 Gibeamelo nunakalaukpok Jejel, Gibeonib atâtanga, nullianga attekalaukpok Maechamik, 
36 Erningalo ernerikârtanga Abdon, Zurelo, Kiselo, Baalelo, Nerelo, Nadabelo,

37 Gedorelo, Ahajolo, Sacharjalo, Miklotelo.

38 Miklotiblo Simeame ernetariva; nunakalaukpullotauk Jerusaleme, kattangutimik akkiane, illagijamik akkorngane.

39 Neriblo Kis ernetariva; Kisib Saul ernetariva. Saulib ernetarivait Jonathan, Malchisualo, Abinadabelo, Esbaalelo.

40 Jonathaniblo erninga Meribaaleôlaukpok, Meribaaliblo Micha ernetariva.

41 Michab erningit tapkoangôlaukput: Piton, Melechlo, Taherealo.

42 Ahasib Jaera ernetariva. Jaerab Alemeth ernetariva, Asmavetelo, Simrilo. Simrib Moza ernetariva.

43 Mozab Binea ernetariva, tapsoma erninga Rafajaôlaukpok, tapsoma erninga Eleasaôlaukpok, tapsoma erninga Azeleĉlaukpok.

44 Azelib ernekalaukpok 6 nik, attekalauktunik: Asrikamemik, Bochrumiglo, Jismaelemiglo, Searjamiglo, Obadjamiglo, Hananemiglo; tagva Azelib erningit.

\section{KAPITEL X.}

Saulib tokikotauninga unatadlarnerme Filisterinut.

FILIsterit Israelitit sorsugvigivait. Israelitillo Filisterit kemâvait, ullimarsimajullo ochovut kakkame Gilboamik attelingme.

2 Filisterille Saul erningillo tunârivlugit unangmivait, ullimarpaillo Jonathan, Abinadabelo, Malchisualo, Saulib erningit.

3 Sorsungnerlo songudlalaukpok Saulemut, pittiksertullo tikkipât, pittiksertunullo ikkilertaulaukpok.

4 Tagva Saul sakkunginik akjarsijomut okarpok: Saviksuit amuleruk kappilungalo tokkolaunga tapsomunga, kippisimangitut tapkoa kaikonnagit, idluitomiglo pinniarvigilunga. Akjarsijokotingale sakkunik pijomalaungilak, angijomik sivuragame. Tagva Saulib saviksoakotine tiguva ochovigilâlo.

5 Akjarsijokotingale sakkunik takkogame Saul tokkungmat, ochotipoktauk saviksoarmut, tokkolunelo.

6 Taimak Saul, erningillo pingasut, iglomiokattingillo tokkovut attautsekut.

7 Israelitillo angutingit, naksamelauktut, takkogamik kemângmatta, Saulelo erningillo takkungmatta, iglugaseksoakotitik kemakpait, kemâlutiglo, Filisterillo tikkilutik innigivait.

8 Kaulermallo Filisterit tikkilerput ullimarsimajut annorai- 
jarlugit; naipipaillo Saule erningillo, nellatillugit kakkame Gilboamik attelingme,

9 Annoraijarpâllo, niakungalo sakkungillo tiguvait, tillikliutilaitalo Filisterit nunanganut sunatuinarnut, okautigijaukojilutiglo tamattominga, Gudingoakotimik innugiartoksuillo illunamassiatik sângine;

10 Sakkungillo illivait Gudimik iglunganut, niakungalo atsungerpât Dagonib iglunganut.

11 Jabesemiullo Gileademe, tussaramik tamainik, Filisterit pinniarlauktanginik Saulemut:

12 Tagva angutit sorsugungnartut illunatik parngnailerput, tigulugillo Saulib erningitalo timingit tokkungajut, âpaillo Jabesemut, illijartorpaillo sauningit nappartub Terebintemik attigub Jabesemetub attâne, nerritaililutiglo uvlut 7 it nâlugit.

13 Taimak Saule tokkovok, idluitullinine pivlugit, Nalekamut idluitullijutigilauktane, Nalekab okausinginut, nalektaulaungitunut tapsomunga; taimaglotauk angakut apperingmagit.

14 Nalegarle apperilaungila: tamanna pivlugo tokkokova, attannionerlo sâtipa Davidemut Isaib erninganut.

\section{KAPITEL XI.}

Davidıb annerijauninganik Israelitinut illunainut attannermut; tapsoma sâlakarninganik Jerusalemiunik; tapsomalo sôngojôkotingit.

ISRAELITILLO illuêngarlutik kattilerput Davidemut Hebronemut, okarlutiglo: Ahâk saunekattigivaptigit, uvinekăttigilutillo.

2 Sivornganelotauk Saule attanniotillugo, igvit Israelitit aulartilugillo ittertilaukpattit; Nalekablo Gudivit okautilaukpâtit: Igvit innukotikka Israelitit pairijaksarivattit, attanniojuksaulutillo innukotimnut Israelitinut.

3 Israelitillo angajokaunerôjungit illunatik tikkilerput attannermut Hebronemut. Davidiblo angerkattigivait Nalekab sângane Hebroneme. Mingoarlugolo Davide attanningortipât Israelitinut, Nalekab okausingit Samuelekut malliklugit.

4 Davidelo, Israelitit illuêngarlutik illagilugit aularpok Jerusalemut, tagva Jebusemut; Jebusiterille nunakalaukput nuname tagvane.

5 Jebusemiullo Davide okautivat: Ovunga ittertuksautsengilatit: Davidible Zionib igloksoanga sôngojok aksarnigariva, tagva Davidib iglugaseksoangit.

6 Davidelo okarpok: Jebusiterinik salakarkârtok tamna 
niakoôjuksauvok angajokangortuksaularmelo. Tagva Joabib, Zerujab erningata, majoarkârpait, angajokangortitaulunelo.

7 Davidible igloksoak sôngojok iglogiva, tamanna pivlugo taijaulaukpok Davidib iglugaseksoanginik.

8 Avatânelo iglogaseliorpait iglogaseksuit, Millomit avatânelo illunane, Joabible iglugaseksuit amiakungit âkiksorpait.

9 Davidelo piganerpok pivallialunelo, Nalekablo Zebaothib aipariva.

10 Tamadsa angajokait Davidib songojokotingita akkorngane, pitsartutitsilauktut tapsominga attannioningane Israelitit illunatik illagællugit, attanningortitaukovlugo Israelitinut, Nalekab okausingit malliklugit.

11 Tamadsalo Davidib sôngojokotingita unnurningit: Jasabeame, Hachmonib erninga, pijariakortonerpâk 30 it akkorngane; kallugiakotine kongmuartipa, ullimârpaillo 300 dit attautsekut.

12 Tapsoma tungâne Eleasareôlaukpok, Dadob erninga, Ahohib erningata, sôngojullo pingasut akkornganelaukpok.

13 Tamna Davidemelaukpok Pasdamime, Filisterit kattimam tilugit tagvane sorsungnermut. Perorsêvikalaukporlo tagvane Gerstelingnik; innugiartoksuillo Filisterit kemâvait.

14 Tagva nangeriartorput perorsêviub kerkânut piulilâlo, Filisterillo ullimârpait; Nalegarlo sâlakarnermik angijomik tunnijivok.

15 Tapkoalo pingasut pijariakôrtojunit 30 ginit attersarput kairtomut, Davidemut kairosungmut Adullamemik attelingmut. Filisterillo tangmarvingat nâksarmelaukpok Refaimemik attelingme.

16 Davidele taipsomane, avaluliorsimajotut îtunelaukpok, Filisterille innugiartoksoangit taipsomane tangmarlaukput Bethleheme.

17 Davidelo ikligosulerpok, okarlunelo: Kia immiksaitoromavânga immektarviub Bethleheme ittertarviub attanêtub iminganit?

18 Tagva pingasut tapkoa Filisterit tangmarvingat ittârpât, Fallulutiglo immermik immektârvingmit Bethlehememêtomit ittertarviub attanêtomit, akjartorpâllo, âlugolo Davidemut. Tapsomale immeromalaungila, kovivâle Nalekamut,

19 Okarlunelo; Nalekab tamanna ungasiktiliuk uvamnit, taimailiorkovlunga, immerlungalo angutit ukkoa aunganik innosingita nangiarnarningane, innosingille nangiarnartomêtillugit tamaungartilaukpât. Tamanna pivlugo immeromalaungilâ. Songojoksuit pingasut tapkoa taimailiorlaukput.

20 Abisailo, Joabib angajua, pijariakortônerpaulaukpok pingasut akkorngane; kallugiakotinelo kongmuartipa ullimârpaillo 300 dit. Okausionginarlaukporlo pingasut akkorngane, 
21 Opigijaunerpângolaukporlo pingasunit, angajokaulunelo tapkonunga, pingasulle taipkoa sivurlit innorpait.

22 Benaja, Jojadab erninga, Ishailib erningata, Kabzeelemiub pinniarnersoakalauktub. Tapsoma angutik Moabittrik Loewetut-îtuk ullimarpâk; attersarporlo Loewelo tokkopâ immektarvingme aputekarviane.

23 Ullimarpâlotauk angut Egiptenemiok tallitut tellimatut takkitigijok, tigumiartorlo kallugiarmik aggangminut umiab napparutangatut itomik. Tapsomale attersarlune aivigiva ajaupialijarlune, kallugiarlo tiguva agganginit, nakterlâlo kallugiakotinganut nangminerijanganut.

24 Taimailiorlaukpok Benaja, Jojadab erninga, okausionginarlaukporlo sôngojoksuit pingasut akkorngane,

25 Opigijaunerpangôlaukporlo 30 git akkorngane, pingasulle taipkoa (sivurlit) innorpait. Davidiblo atannertuijungortipa ingminut.

26 Songojullo sorsuktuksane tapkoangôlaukput: Asahel, Joabib nukkâ. Elhanan, Dodob erninga, Bethlehemiub.

27 Samot, Haroremiok. Helez, Pelonemiok.

28 Ira, Ekeb erninga, Tekoimiok. Abieser, Antotemiok.

29 Sebechai, Husatemiok. Ilai, Ahohitemiok.

30 Maherai, Netofatemiok. Heleḋ, Baenab erninga, Netofatemiub.

31 Itai, Ribaib erninga, Gibeamit, Benjaminib kittorngangita iglogaseksoanginit pijub. Benaja, Pirgatonemiok.

32 Hurai, Gaasib kônginit pijok. Abiel Arbatemiok.

33 Asmavet, Baherumiok. Eliaba, Saalbonemiok.

34 Hasamib Gisonemiub erningit. Jonathan, Sagib erninga, Hararimiok.

35 Ahiame Sacharib erninga, Hararimiok. Elifal, Urib erninga.

36 Hefer, Mecheramiok. Ahia Pelonemiok.

37 Hezro, Karmelemiok. Naerai, Asbaib erninga.

38 Joel, Nathanib kattanguta. Mihebar, Hagrib erninga.

39. Zelek, Amonitereôjok. Naherai, Berotemiok, akjarsijok Joab, Zerujab erningata sakkunginik.

40 Ira Jetrimiok. Gareb, Jetrimiok.

41 Uria, Hetitereôjok. Sabad, Ahelaib erninga.

42 Adina, Sisab erninga, Rubenekunit pijok, angajokaujok Rubenekunut, 30 gillo illagivait.

43 Hanan, Maechab erninga. Josafat, Matonemiok.

44 Usia, Asteratemiok. Sama, Jaelelo, Hotamib ernigik, Aroetemiub.

45 Jediael, Simrib erninga. Joha, kattanguta, Tizemiok. 
46 Eliel, Mahevimiok. Jeribai, Josawjalo, Elmaamib ernigik. Jethma, Moabitereojok.

47 Eliel, Obed, Jaesiel, Mezobajamit.

\section{KAPITEL XII.}

\section{Davidemut tilklitut Ziklagemut aítingit.}

TapKoangovulle Davidemut tikkitut Ziklagemut, allingitariakartillugo sulle Saulemik, Kisib erninganik. Sôngojullo akkornganêlaukput, ikkajorteolutiglo unatadlarnerme,

2 Pittiksertut, sappingitullo ujarkanik illoerearlutik, kargjutiniglo pittiksautjilutik aggangnut tallerpingnut saumingnullo. Saulib kattangutinginit Benjaminekunit pijut;

3 Pijariakortonerpâk Ahieser, Joaselo, Samaab, Gebeamiub ernigik; Jesiel, Peletelo, Asmavetib ernigik; Baracha, Jehulo Antotemiok.

4 Jesmaja Gibeonemiok, angutaudlartok 30 git akkorngane, angajokaujorlo 30 ginut. Jeremia, Jahesiel, Johanan, Josabad. Gederamiok.

5 Eleasai, Jerimot, Bealja, Samarja, Safatja Harochetemiok.

6 Elkana, Jesijalo, Asareelo, Joeserelo, Jasabeamelo, Korakut,

7 Joela, Sabadjalo, Jerohamib ernigik, Gedoremiub.

8 Gadekunit apterput Davidemut kairosungmut sunakajuitometomut, sôngojut sorsugungnartullo sappulutalijartut, kallugialijartullo, tautukalauktut Loewetut, tuktungajutitullo kakkanêtutitut sukkalijutigijut.

9 Sivorlek Eser, tuglek Obadja, pingajuat Eliabe,

10 Sittamangat Masmana, tellimangat Jeremja,

11 Pingasojoat Athai, 7 ingat Eliel,

128 ingat Johanan, 9 ingat Elsabad,

13 Tellimaujoat Jeremja, 11 ingat Machbanai.

14 Tapkoa Gadib erninginit, angajokait sorsuktuksanut, mikkinerpâk 100 dinut, angnek 1000 dinut.

15 Tapkoangôvut ikkârlauktut Jordanemik takkit sivorlerpângane ulliksimatillugo sikjamingne tamangne, kemâtitsijullo naksarmiunik tamainik, taununga paungalo.

16 Tikkitokalaukporletauk Davidemut kairosungmut Benjaminib Judablo kittornganginit.

17 Davidible annivigivait, kiolugillo okautivait: Ullapirsainarluse tikkikupse ikajoromavlunga, tagva omattima illagijaksarivâse; tikkikupsele pingitsertorluse akkerartoromavlunga, 
idluinekangimarigalloartillugo uvamne, tagva atâtagijapta Gudingata takkoliuk, tamanna pitlarlugo.

18 Annerneruble angajokaunerôjok Capitanit kajungersarpa, okarporlo: Igvit pigivaptigut Davide, igvillo Isaib erninga illigivaptigit! Ullapirsautekarit, ullapirsautekarit! ullapirsautekarlit ikkajortitit! Gudivit ikkajormatit. Tagva Davidib illaliorpait, angajokangortipaillo sorsuktuksanut.

19 Manassekunillo, Davidemut tikkiput, tikkitillugo Filisterit illagilugit Saule unatadlarvigijomavlugo, ikkajornagille. Filisterit angajokaksoangita kakkimiolerlaukârlutik aularkolaungmatsuk, okarlutiglo: Angajokane Saule illigingâruniuk tagva niakoêjartaujungnarajarpogut.

20 Ziklagemut ailertillugo manna Manassekunit tikkiput tapsomunga, Arna, Josabad, Jedjael, Michael, Josabad, Elihu, Ziltai, nirkoôjut 1000 dinut Manassekune.

21 Tapkoalo Davide ikkajorpât arktaijut akkerartorlugit; illunatik sôngudlarlaungmatta angutaudlarlutiglo. Capitangortitaulutiglo sorsuktuksanut.

22 Uvlut tamaita tikkitokalaukpok Davidemut ikkajoromavlugo, kattimgajoksoangolerkârtinagit Gudib kattimgajoksoakotingititut.

23 Tamadsale niakoôjut unnurningit oppalungêrsimavlutik sorsungnermut, tikkitut Davidemut Hebronemut Saulib attanniovinga sâtilugo tapsomunga, Nalekab okausingit malliklugit.

24 Judab kittorngangit sappulutalijartut, kallugialijartullo, 6800 ôlaukput, oppalungêrsimavlutik sorsungnermut.

25 Simeonib kittorngangit sôngojut sorsungnermut 7100 ôlaukput.

26 Levib kittorngangit 4600 deôlaukput.

27 Jojadalo angajokaunerôjok Aronekunit illakarlune 3700 dinik.

28 Zadokelo innuosuktok, sôngojok angutaudlartorlo, atâtame iglomiokattingit illagilugit kapitat 22 git.

29 Benjaminib kittorngangit, Saulib kattangutingit 3000. Nelliutok taimna tikkidlugo, unnurnersat tapkonangat Saulekut ungagivait sulle.

30 Efraimib kittorngangit 20800 sôngojut anguteôjullo okausiovaktut atâtagijamik iglungine.

31 Kinguvarêt Manassekut ketterkangit 18000 attermikut taijaulauktut, kaikovlugit Davide attanningortilugo.

32 Isascharib kittorngangit sillatojut, attannertuijullo nelliutune tamaine Israelitit pinniaraksanginik kapitat 200 dit; kattangutingillo illunamassiatik naletsiarlaukput okausinginik.

33 Sebulonekunit, aulartut, kattimgajunut oppalungêrsimavlutik sorsungnermut, sakkulijarlutik sunatuinarnik $50000 \mathrm{dit}$, omattiggit naletsiartunik. 
34 Naftalikunit, kapitat 1000 dit, tapkoalo illagilugit sappulutalijartut, kallugialijartullo 37000 dit.

35 Danekunit oppalungêrsimajut sorsungnermut 28600 dit.

36 Asserekunit aulartut kattimgajunut oppalungêrsimajullo sorsungnermut 40000 dit.

37 Jordaniblo akkianit, Rubenekunit, Gadekunillo, kinguvarêllo Manassekut ketterkanginit sakkolijarlutik sunatuinarnik sorsungnermut 120000 dit.

38 Sorsuktuksat tapkoa illunatik sorsungnermik sungiusimajut, tikkiput omamut tamanut Hebronemut, Davide attanningortitsomavlugo Israelitinut illunamassianginut. Israelitillo, tapkoa assingittauk attautsemik omattekarkattigêlaukput Davide attanningortitaujuksaungmat.

39 Tagvanelo uvlivut Iavideme pingasunik, nerrilutiglo immerlarmiglo, kattangutingita nerkiksalliorlaungmagit.

40 Kanninerpâllo avatingine, Isascharekut, Sebulonekullo, Naftalikullo tikkidlugit, kaitsivut niakojanik, siutekoktojune, Kamelinelo, Horseojartunelo, tuktuvângnelo; sennaukamiglo, Feiginiglo, Weineliksallo paunganginik pannersimajunik, Weinemiglo, orksungmiglo, tuktuvangniglo, saukaniglo unnuktunik; kuviasutiksakarmat Israeleme.

\section{KAPITEL XIII.}

Angersimanerub iklervingata äjauninga Kiriat-Jearimemit; ấauringalo Obed Edomib iglunganut.

DAVIDIBLO angajokait 1000 dinut 100 dinullo, angajokaillo illunaita kachimiokattigivait,

2 Okarlunelo Israelitit illagêngninginut illunainut: Namagijaukpat illipsingnut, Gudemillo Nalegaptingnit pikpat: tagva tilliklerta illunânut, kattangutiptingnut assiptingnut Israelib nunangine illunainetunut, illagilugillo ajochertuijunut Levitinullo iglugaseksoarmingnetunut, kattimalerkovlugit uvaptingnut.

3 Gudiptalo iklervinga ailermilavut uvaptingnut, Saulib nelliuningane kammagilaunginaptigo.

4 Tagva illagêt illuêngarlutik okarput, taimailiortokartuksaungmat; tamanna innugiartoksuit illunatik namagingmatsuk.

5 Taimak Davidib Israelitit illunamassiangit kattikovait, Sihoremit Egiptenib (kônganit) Hemat tikkidlugo, Gudib iklervinga ailugo Kiriat Jearimemit.

6 Davidelo majorarpok Israelitit illunamassiangit illagilugit Kiriat-Jearimemut Judametomut, tagvangat takpaungartitsomavlugo Gudib iklervinga, Nalekab iksivajub Kerubit kângine, attiub tuksiarvioviane.

7 Gudiblo iklervinga kemuksiutitaukorât kamutingnut nu- 
tângnut, Abinadab iglunganit; Usablo Ahiablo kamutik aulatipâkik.

8 Davidele Israelitillo illuêngarlutik Gudib sivorngane pitsartunermingnut tamainut attorput imgerutinik, Harfiniglo, Psalteriniglo, killautiniglo, Cimbeleniglo, tiptulautiniglo,

9 Tikkimattale Kidonib kakkojaksaliorvinganut, Usab aggane issakpait iklervik najumijomavlugo; tuktuvâk sangulermannik.

10 Tagva Nalekab nigarninga illungertulerlaukpok Usa pivlugo, annauvâlo tamana pivlugo aggane issalaungmagit iklervingmut, tokkovorlo tagvane, Gudib sângane.

11 Tagva Davide kiksalerpok Nalegak taimaitomik koppakartitsilaungmat Usame, innelo tamna taiva Perez-Usamik uvlok tamanna tikkilugo.

12 Davidiblo Gude illimagiva uvlorme taipsomane, okarlunelo: Kannok Gudib iklervinga kaititaksarivigo uvamnut?

13 Taimak Davidib Gudib iklervinga ingminut âtaukolaungila Davidib iglugaseksoanginut, pititaukovâle Obed Edomib Gathemiub iglunganut.

14 Taimak Gudib iklervinga takkit pingasut nâlugit Obed Edomib iglunganêtuarpok. Nalekablo Obed Edomib iglomiokattingit, tamaitalo pigijangit pitsartutipait.

\section{KAPITEL XIV.}

Davidib nulliangit kittongangillo. Filisterit salagïjauningit.

H IrRAMiBlo Tirusib attannertangata Davide tilliklervigiva tillijanik, kejungniglo Cederinik, karmaliorteniglo iglulliortiniglo, iglomik iglolliorkovlugit tapsomunga.

2. Davidelo tukkisilerpok Nalekab attannioninga Israelitinut nakkiterlaungmago; attanniovinga angijorortitaungmat innukotingit Israelitit pirlugit.

3 Davidelo arnanik unnurnersanik nullianikpok sulle Jerusaleme, ernetarnersaulunelo pannitarnersaularmelo sulle unnurnersanik.

4 Tamadsalo Jerusaleme erniangolauktut tapsomunga attingit: Samua, Sobabe, Nathan, Salomo,

$\check{5}$ Jebehar, Elisua, Elpalet,

6 Noga, Nefeg, Jafia,

7 Elisamma, Baeljada, Elifalet.

8 Filisterillo tussaramik Davide mingoartauvlune attanningortitaumangmat Israelitit illunamassianginut, tagva illunatik aularlutik majorarput takpaunga Davide kenneromavlugo. Davide tamattominga tussarame aularpok pâromavlugit. 
9 Filisterillo tikkilutik tangmarterput Refaimib naksangane.

10 Davidiblo Gude apperiva, okarlunelo: Paunga majorartuksauvik Filisterit akkerartorlugit, tunnijomavigillo aggamnut? Nalekab okautiva: Majorarit takpaunga, tunnijomavakka aggangnut.

11 Tagva aularput Baalprazimemut, Davidiblo ullimârpait tagvane. Davidelo okarpok: Gudib omigijikka aggaptigut alliktorpait, sorlo immiksuit suppingmatta. Tamanna pivlugo inne tamna atserarpât Baalprazimemik.

12 Gudingoakotitiglo kemmakpait, Davidiblo ikkitaukovait ikkomamut.

13 Filisterille ama tikkilaurivut tangmarterlutiglo naksarme.

14 Davidiblo Gude ama apperiva. Gudiblo okautiva; Majorartuksaungilatit takpaunga kingorngattigut, sangulerille tapkonangat, tikkikovlutit tapkonunga nappartut Maulbeeregit sâdlugit.

15 Tussarniaruvillo nappartut Maulbeeregit pappingita keasungninginik tagva opaksârit sorsungnermut; Gudib aularkârmat illingnit Filisterit kattimgajoksoangit annaulugit.

16 Davidelo taimailiorpok sorlo Gudib perkolaungmago, ullimârpaillo Filisterit kattimgajoksoangit Gibeonemit Gaser tikkidlugo.

17 Davidiblo attinga okausiolaukpok nunane tamaine, Nalekablo innukattigêksuit illunaita tupviotipait sivuranermik tapsominga.

\section{KAPITEL XV.}

Angersimanerub iklervingata âtauninga Jerusalemut.

IGLUNIGLO iglugaselliorpok ingminut Davidib iglugaseksoangine, Gudiblo iklervinga inniksalliorpâ, tuppektorpâlo.

2 Taipsomane Davide okarpok: Gudib iklervinga Levitit assinginut akkigartaujuksaungilak; tapkoa Nalekab annerilaungmagit Nalekab iklervinga akkigarlugo pikovlugit, kivgartorkovlugillo tapsominga soraijuitomik.

3 Davidiblo Israelitit illunamassiangit kattikovait Jerusalemut, Nalekab iklervinga takpaungartilugo pikovlugit, inniksamut âkiksortaukolauktanganut tamna pivlugo.

4 Davidiblo kattikovait Aronib erningit, Levitillo.

5 Kahatib erninginit, Uriel, angajokaunerôjok, kattangutingillo illagilugit 120 git;

6 Merarib erninginit Assaja, angajokaunerôjok, kattangutingillo illagilugit 220 git; 
7 Gersomib erninginit, Joel angajokaunerôjok, kattangutingit illagilugit 130 git.

8 Elizafanib erninginit Semaja angajokaunerôjok, kattangutingit illagilugit 200 dit ;

9 Hebronib erninginit, Eliel angajokaunerôjok, kattangutingit illagilugit 80 git.

10 Usielib kittornganginit Aminadabe angajokaunerôjok, kattangutingit illagilugit 112 it.

11 Davidiblo kaikovait, Zadoke, Abjatharelo, ajochertuijuk; Levitillo, Uriel, Assajalo, Joello, Sémajalo, Elielelo, Aminadabelo;

12 Okautivaillo: Illipse angajokaunerôvose kinguvarênut Levitinut, tagva manna sujungaititse illipsingnik kattangutipsingniglo, Nalekab Israelib Gudingata iklervinga takpaungartilugo pikovluse, tagvunga inniksaliorlauktamnut tapsomunga;

13 Sivurnganele illipse pilaunginase, Nalegak Gudivut kopakartitsilaukpok akkonaptingne, tamana pivlugo kennerlaunginaptigo sorlo pijariakarmat.

14 Tagva ajochertuijut Levitillo sujungaitiput, Nalebab Israelib Gudingata iklervinga takpaungartilugo pikovlugit.

15 Leviblo kittorngangita Gudib Nalekab iklervinga akkigarpât ikkautingnut erksuklugo, sorlo Mosese perkojilaungmat Nalekab okausingit malliklugit.

16 Davidelo Levitit angajokânginut okarpok, kattangutitik, imgerpaktut kaikolugit, nokakseralijarlutik, Psalterilijarlutiglo, Harfelijarlutiglo, Cimbelijarlutiglo pikovlugit, imgerkovlugillo nippekortojomut kuviasuklutik.

17 Tagva Levitit Heman Joelib erninga kaikovât; kattangutinginillo, Assaf, Berechjab erninga; Merariblo kittornganginit kattangutinginit Ethan, Kusajab erninga;

18 Illagællugillo kattangutingit tuglit, Sacharja, Ben, Jaesiel, Semiramot, Jehiel, Unni, Eliab, Benaja, Maeseja, Mathitja, Elifelela, Mikneja, Obed Edom, Jehiel, upkuanik udsertortut.

19 Imgerpaktut Heman, Assaf, Ethanelo perkojauvut Cimbelit, kannujarmit sennamajut nippekortojullo attorlugit,

20 Sacharjalo, Assielo, Semiramotelo, Jehielo, Unilo, Eliabelo, Maesejalo, Benajalo, Psalterit attorlugit ingiorlugit;

21 Matitjale, Elifelejalo, Miknejalo, Obed Edomelo, Jejello, Assasialo Harfesijarlutik imgerkârkovlugit.

22 Kenanjalo Levitit angajokangat aulatsijok imgernermik, illiniartitsikovlugo tapkoninga imgernermik, sillatudlarmat.

23 Berechjalo, Elkanalo, udsertorteolaukpuk iklerviub upkoanganik.

24 Sebanjalo, Josafatelo, Nethaneelelo, Amasailo, Sachar- 
jalo, Benajalo, Elieserelo ajochertuijok tiptulausijarlaukput Gudib iklervingata sivurngane; Obed Edomelo, Jehialo udsertorteolaukput iklerviub upkoanganik.

25 Taimak Davide angajuklîllo Israeleme, angajokaillo 1000 dinut aivut Nalekab angerutingata iklervinga aijomavlugo takpaunga Obed Edomib iglunganit, kuviasuklutik.

26 Gudiblo Levitit Nalekab angerutingata iklervinganik akkigartut ikkajormagit, tunnergumik tunnijivut tuktuvângnik 7 nik saukaniglo angusallungnik 7 nik.

27 Davidelo attisimalaukpok ullingmik sâtomik, taimaktauk Levitit iklervingmik akkigartut, imgertullo, Kenanjalo, imgertunik aulatsijok, imgertut akkorngane, Davidelo attisimalaurivoktauk sillapamik sâtomik.

28 Taimak Israelitit illunamassiangita Nalekab angerutingata iklervinga takpaungartipât, igiakomijarlutik, tiptulausijarlutiglo, Trompetesijarlutiglo, Cimbelisijarlarmiglo nipperkortojunik, Psalteresijarlutiglo, Harfesijarlarmiglo.

29 Nalekab angerutingata iklervinga manna Davidib iglugaseksoanginut tikkilermat, tagva Michal, Saulib panninga nuitavok igalâkut, tækkovâlo attannek Davide kiggertartillugo, okigêsertillugolo Harfesijartillugolo, nachogivâlo omattimigut.

\section{KAPITEL XVI.}

Davidib tuksiarutingit angernerub iklervingata angeraujauninga pivlugo. Kivgariornerub Gudemik âkitssorturninga.

GUDIBLO iklervinga ittertinamitsuk, illivât tuppiub Davidib tuppertorlauktangata tapsomunga illuanut, tunnijilutiglo tunnergutinik ôtaksanik, kujalijutiksaniglo Gudib sângane.

2 Davidiblo tunergutit ôtaksat, tunergutillo kualijutiksat tunijarêramigit, innugiartoksuit saimartipait Nalekab attingane.

3 Aitortuilunelo illunainik Israelitinik, tamangnik angutinik arnaniglo, attunit niakojârsungmik abvakomiglo nerkemik, Weinemiglo ôktut tættælugo.

4 Nalekablo iklervingata sânganut Levitit illangit pitipait kivgartornermut (illingajuksat), Nalegak Israelib Gudinga opigilugo, kujagilugolo, nertorlugolo pikovlugit.

5 Tapkoa Assaf sivurlek, Sacharja tuglek, Jejello, Semiramotelo, Jehiello, Matitjalo, Eliabelo, Benajalo, Obed Edomelo, Jehello, Psalterinik, Harfiniglo attorlutik; Assafible Cimbele nippekortôjok attorlugo.

6 Benajable, Jehasieliblo Trompete attutsainarpâk Gudib angerningata iklervingata sângane. 
7 Taipsomane Davidib Nalegak kujagijaukokârpa Assafekut, kattangutingittigullo :

8 Nalegak kujagisiuk, attinga okalautigisiuk, pinniarningit nellonairtisigik innukattigêksoarnut.

9 Imgervigisiuk, nipliajunik attorutigisiuk, tattamnartulliningillo tamaita tuksiaruselliorutisigik.

10 Attinga heiligeôjok nertorsiuk. Tapkoa Nalekamik kennertut omattingit kuviasulerlit.

11 Nalegak, apperkutigisiuk, pitsartuningalo, kênanga kennitsainarsiuk.

12 Tattamnartulliningit pinniarlauktangit erkarsigik, tattamnartulliningit okausingillo.

13 Illipse, kinguvangôjose Israelemut tapssoma kivganganut, illipse kittorngaujose Jakomut annerimajangit.

14 Tamna Nalegauvok Gudivut, erkartuivok sillaksoarme illunâne.

15 Angerninga erkaumatsainarsiuk soraijuitomik, okautsit perkolauktangit kinguvarênut tausendinut,

16 Angernerilauktanga Abrahamut, okarmaringningalo Isaangmut;

17 Pitipâlo tamanna Jakomut malligeksaukovlugo, Israelemullo angernermut soraijuitomut,

18 Okarlaukporlo: Igvit Kanaanib nunanganik tunnitsivigijomavagit, imerautipse kingormgutijanganut;

19 Ikkitotillugit kêtautilugillo tujormiangotilugillo nuname.

20 Nûlerlaukpullo innukattigêksuit illanganit assianut, attanniovillo illanganit innukattigêksoarnut assinginut.

21 Okumaiksartitaukolaungilait kinamullonêt, attannîllo pitlarpait pivlugit.

22 Mingoartakka aktorniarassigik, nellautaijokotikkalo idluitulliviginiarnagit.

23 Nunaujose illunase Nalegak imgervigisiuk, piuliklerninga akpautigisiuk uvlut tamaita.

24 Unipkaritse ânanaudlarninganik nellojut akkorngane, tattamnartullininginiglo innukattigêksuit akkorngane!

25 Nalegak angijoksôngmat, nertornartoksôlarmelo sivuranaluarporlo Gudinit tamainit.

26 Nellojut illunamassiatik Gudingit Gudingoangôngmatta, Nalekable killak sennalaukpâ.

27 Ananaunekarpok, pinarnarnekarlarmelo tapsoma sângane, tattamnadlartuniglo kuvianadlartuniglo illusekarpok tapsoma inningane.

28 Kaitsileritse Nalekamut innukattigêksoangôjose, kaitsileritse Nalekamut nertortaunermik opigijaunermiglo.

29 Kaitsileritse Nalekab attinganut nertordlernermik; pilli- 
utit kailersigik, sangeriartorlugolo, Nalegarlo tuksiarvigisiuk ivsornainerub ânanaudlarningane.

30 Sillaksoarmiut illunamassiatik sivuragilitsuk; Tapsoma nuna âkiksorlaukpa aulakonnago.

31 Killak kuviasugle, nunalo serridlarle; okautaulelo innukattigêksuit akkorngane Nalegak attanniôtingmat.

32 Immarbiksoak iktullijarle takkamanêtullo; perorsêvit kuviasuglit, tamaitalo tapkonanêtut.

33 Nappartullo illunatik nappartunêtut igiakomijarkogisigik Nalekab sângane, tikkilermat nuna erkartoromavlugo.

34 Nalegak kujagisiuk illanakarmat, saimarsainingalo sorairniatsengimat.

35 Okaritselo: Ikajortigut Gude piulijivut, kattitilutalo piulitigullo nellojunit, attit heiligeôjok kujagilugo, igvillo nertorlutit pikovluta.

36 Nalegak Israelib Gudinga nertortaule issokangitomit issokangitomut, innugiartoksuillo illunatik okarlit: Amen, Nalegarlo nertorlitsuk.

37 Taimak Nalekab angerutingata iklervingata sânganêtsainarkovait Assaf kattangutingillo, kivgartutsainarkovlugit iklerviub sângane uvlut tamaita.

38 Obed Edomele kattangutingillo 68 git, Oded Edomelo, Jedithunib erninga Hossalo udsertortungortipait upkoaksoarnik.

39 Zadokelo ajochertuijok, kattangutingillo ajochertuijut Nalekab iglungata poktojomêtub Gibeame sânganetuarkovait,

40 Uvlut tamaita tunnijikovlugit Nalekamut tunnergutinik ôtaksanik, Altarib tunnergutiksanut ôtaksanut illingajub kângine, uvlarme unnungmelo, sorlo aglaksimangmat Nalekab perkojangine, Israelitinut perkolauktangine.

41 Tapkoalo illagilugit Heman, Jeditunelo, annerijaujullo assingit attermikut taijaumalauktut, Nalegak kujagilugo pitsiarninga sorainiatsengimat.

42 Illagilugillo Heman, Jeditunelo, Trom tit Cimbelilo Gudiblo nokakseralingningit attorlugit. Jeditunible erningit udsertortungortipait upkoaksoarnik.

43 Taimak innugiartoksuit illunatik attunit angerarput iglomingnut; Davidelo sanguvoktauk iglomiokattine saimartitsomavlugit. 


\section{KAPITEL XVII.}

\section{Davidib Tajusimaninga Tempelemik sennajomavlune; Mesiasiblo angerutigïauninga.}

PijokalaukPorlo Davidib iglune innigilauramiuk, okarpok nellautaijomut Nathanemut: Ahâk, uvanga iglo Cederenit sennamajok innigivara, Nalekablo angerningata iklervinga itsat attanêpok.

2 Nathanib Davide okautiva: Tamaita omamigijattit pinniarkit, Gudib aiparingmattit,

3 Unnuarmele taipsomane Gudib okausinga tikkipok Nathanemut, okarlunelo:

4 Ailerit Davidelo kivgara okautiuk: Imâk Nalegak okarpok: Igvit iglolioraksaringilarma inniksamnut illingajomik.

5 Iglome inekalaunginama uvlormit taipsomanemit Israelib kittorngangit aularutilaurapkit, uvlok tamanna tikkilugo; ailaukpungale tuppermit tuppermut, innemillo innemut.

6 Agvertarvigilauktamne Israelitit illunamassiatik akkorngane, idluarsaijut nelliat perkolauktara innukotikka pairilugit, okautilauralloarpigo, okarlungalo: Sôg igloliorlungipissinga Cederelingmik?

7 Tagva mana okalerit kivgamnut Davidemut: Imâk Nalegak Zebaote okarpok: Tigulaukpagit iviksukatalingmit saukat kingornganit, attanniojuksaukovlutit innukotimnut Israelitinut,

8 Aiparilaukpagillo nâmut aivigijarnut, omigijitillo pêjarlaukpakka sivunerne, attekartilaukpagillo sorlo angijoksuit nuname attekarmatta.

9 Innukotikkale Israelitit innekartitsomavakka illijomavakkalo, tagvane innekarkovlugit, aulajungnaititaukovlugillo, innuillo kassêtut nukingêrotititaksarijungnaipait sivornganetut,

10 Nelliutunelo taipsomanetut idluarsaijut perkolaurapkit angajokaukovlugit innukotimnut Israelitinut; omigijitillo illunaita manigotititsomavakka; nellojungnaitipagillo Nalekab igvit iglomik iglolioromangmattit.

11 Uvlokotitille annigoromârpatta, aivlutit atâtagijarnut, tagva kinguvâksattit kingunerne ernivît illangat makkititsomavara, tamna attanniovinganik nakkiterivigijomavara.

12 Tapsoma iglomik iglolioraksarivânga, iksivautangalo nakkiteromavara issokangitomut.

13 Uvanga atâtaujomavunga taipsomunga, taimnalo erniojuksauvok uvamnut. Napkiningningneralo pêjjaromangilara taipsomangat, sorlo pêjarlaurapko taipsomangat sivunernelauktomit. 
14 Pititsomavarale iglumnut attanniovikotimnullo soraijuitomik, iksivautanga sokkosijuikovlugo issokangitomut.

15 Nathaniblo okautsit tamakkoa tamaita malliklugit, tækkotitsug'o tamanna tamât malliklugo Davide okarvigilaukpa.

16 Tagva attannek Davide kaivok nokkangalunelo Nalekab sângane okarlunelo: Kinauvîk Nalegak Gud, iglomiokatikkalo sunauvât, manna tikkidlugo âlauramga?

17 Tamannalo suglugilaukpat sulle Gude, kivgaville iglunga . pivlugo, kanningitut pijomârtullo okautigilaukpattit sulle; tækkonalaukparmalo innub illusinga malliklugo Gudeogalloarlutit, Nalegaujok kotsiktome.

18 Sunamiglo anginersamik Davide okartuksauva illingnut, kivgait ânanausigangne? Kivgait illitarivat.

19 Nalegak kivgait pivlugo, omattit malliklugo tamattominga tamât pijarialiksoarmik pinniarlaukpotit, nellonêrsilutillo pijariakortôjomik tamattominga tamât.

20 Nalegak adsekangilatit, Gudekangilarlo assingnik, taimaimariklutit sorlo siutiptingnut tussarlaurapta.

21 Nanelo innukattigêksoakarka nuname innukotititut Israelititut, Gudib aijutigijangititut, ingminut innugiartoksoarnik piulikleromavlune, attekartitsomavlunelo ingminut, pinniarnernut pijariakortôjunut, kappianadlartunullo, innukattigêksuit pingudlalaurangne innukotivit sivorngane piulilauktavit Egiptenemit?

22 Innukotitillo Israelitit innukotingortilaukpattit illingnut issokangitomut; igvillo Nalegak Gudingorlaukpotit tapkonunga.

23 Manna Nalegak okautsit okauserilauktattit kivgait pivlugo, iglungalo pivlugo, nellautsilerlit soraijuitomik, pınniarlutillo sorlo okalauravit.

24 Illa miksekârlit, attit angijororkovlugo issokangitomut, okartokarkovlugo: Nalegak Zebaoth, Israelib Gudinga Gudeôvok Israelitinut, kivgavillo Davidib iglunga sokkosijuile sângne.

25 Ivgit Nalegak kivgait nellojungnaitilaukpat iglomik iglolioromagangne, tamanna pivlugo kivgait nachvarpok tuksiararijaminik sângne.

26 Manna Nalegak, igvit Gudeôvotit, ajungitorlo taimaitok okautigilaukpat kivgarnut.

27 Manna pigiarit kivgavit iglunga pitsartutilugo sângnetsainarkovlugo soraijuitomik; saimartitattit saimartisimangmatta soraijuitomik. 


\section{KAPITEL XVIII.}

Davidib sâlakarningì sunatuinait; pimaklertokotingillo.

TAMaKKOA kingorngane Davidib Filisterit ullimârpait mannigotitipaillo, aksarnigarivaillo Gathe, aiparusingillo Filisterit agganginit.

2 Moabiterittauk ullimarlaurivait; taimak Moabiterit kivgaulerput Davidemut, âtsilutiglo pilliutinik tapsomunga.

3 Hadad Eseretauk, Zobab Hematemêtub attannertanga, salagigiva ingerarame attanniovine tunganilititsomavlugo kôngme Fratemik attelingme.

4 Davidiblo tiguvait tapsomangat kammutit 1000 dit, horsilło kângine ablangajut 7000 dit, angutillo pissuktut 20000 dit. Davidiblo kammutit illunaita illakungortipait, kammutillo 100 dit amiakokovait.

5 Siriamiullo Damaskusemint tikkilerput Hadadeser Zobab attannertanga ikajoromavlugo. Davidible Siriamiut tapkoa angutit 22000 dit vllimârpait.

6 Sorsuktuksakartitsivorlo Damaskuseme, taimaglo Siriamiut kivgaulerput Davidemut, âtsilutiglo pilliutinik, Nalekab ikajormago aivigijangine tamaine.

7 Davidib sappulutat Goldeôjut Hadadeserib kivgangita pigijangit tiguvait, âlugillo Jerusalemut.

8 Taimaktauk Davide Hadadeserib iglugaseksoakotinginit Tibeatemit, Kunemille tigusivok kannujavaksoarnik. Tamattomangat Salomob immarbingoak kannujaujok, nappajuglo, illulerkutillo kannujaujut sennavait.

9 Togullo Hematib attannertanga tussarame, Davidib $\mathrm{Ha}$ dadeserib Zobab attannertangata sorsuktuksakotingit illunaita ullimarlaungmagit:

10 Ernine Hadoram tilliva attannermut Davidemut, saltutitaukovâlo, saimartitaukolugolo, Hadadeser sorsugvigilaungmago ullimârlaungmagolo. (Togu unatarnekarmat Hadadeseremut,) tamnalo illagællugo illulerkutinik sunatuinarnik Goldeôjunik Silbereôjuniglo, kannujaujuniglo,

11 Tamakkoalotauk attanniub Davidib Silberit Goldillo innukattigêksoarnit, tagva Edomiterinit, Moabiterinillo, Amoniterinillo, Filisterinillo, Amalekiterinillo, tigulauktangit illaliudlugit perkotautipait Nalekamut.

12 Abisaiblo Zerujab erningata Edomiterit ullimârpait, sioraujab nâksangane, 18000 dit;

13 Sorsuktuksakartitsivorlo Edomeme; Edomiterillo illunatik kivgangorput Davidemut. Nalekab Davide ikkajormago aivigijangine tamaine. 
14 Taimak Davide attanniotilaukpok Israelitinut illunamassianginut, attorlunelo idluarsainermik idluarnermiglo innugiartoksoarnut illunamassianginut.

15 Joab Zerujab erninga angajokaulaukpok sorsuktuksaksoarnut. Josafate Ahiludib erninga angajokaulaukpok aglakpaktunut.

16 Zadok, Ahitub erninga, Abimelechelo Abjatarib erninga ajochertuijolaukpuk, Sausa aglakpakteolaukpok.

17 Benaja, Jojadab erninga angajokaulaukpok Kretinut Pletinullo, Davidiblo erningit attanniub najorivait ikkajortigilugit.

\section{KAPITEL XIX.}

Amoniterit idluitulliningit Davidib tillijanginut, Davidibla pitlarningninga tapkoninga.

\section{$\mathrm{M}$}

ATTOMALO kingorngane Nahase Ammonib kittorngangita attannerijangat tokkovok, erningalo attanningorpok innangêrdlugo.

2 Tagva Davide issumalerpok: Napkiningnermik pinniaromavunga Hananemut, Nahasib erninganut, atâtangata napkiningnermik pinniarvigilaungmanga, tillijaniglo tilliklerpok taipsomunga, manigorlugo pikovlugit atâtanga pivlugo. Davidiblo kivgangit Ammonib kittorngangita nunanganut tikkimatta manigoromavlugo;

3 Tagva Ammonib kittorngangita angajokaunerôjungit okarput Hananemut: Isumavît Davidib atâtait opigingmago ijikpît sângangne, manigoijunik tilliklervigilaungmattit? Illa kivgangit tikkisimavut illingnut nausakarlutik, asseroijomavlutiglo, nunalo nautsertoriarlugo.

4 Tagva Hananib Davidib kivgangit tiguvait, kipsarvigivait, anorângillo kipjaumiktorpait ketterarlugit, makkitingit tikkidlugit, aularkolaitalo.

5 Aularpullo, Davidelo okautsaukovât tamattominga angutinut. Tamnale tilliklerpok pachrearlugit, (angutit taipkoa angijomik kangusuktitaulaungmatta.) Attannerlo okarpok: Jerichometuaritse, umise, perorsearilugit; tagvalo uttileritse.

6 Ammonible kittorngangit takkogamik tippiludlaramik Davidib sângane, Hanan Ammoniblo kittorngangit tillikliutjivut Talentinik 1000 dinik Silberemik, tikkikojijomavlutik tapkonunga kamutinik, ablangajuniglo horsit kângine, Mesopotamiamit, Maechamillo, Zobamillo;

7 Tikkikojilutiglo kamutinik 32000 dinik, attannermiglo 
Maechamik innukotine illagilugit; tapkoa tikkilerput tangmarterlutiglo Medbob sivurânut. Ammoniblo kittorngangit kattilerputtauk iglugaseksoakotimingnit, tikkilerlutiglo sorsugomamut.

8 Davidib tamana tussaramiuk Joabe tilliva sorsuktuksaksoakattigêt sôngojut illunamassiatik illagilugit.

9 Ammonible kittorngangit aularsimalaukput, oppalungaijarlutiglo sorsungnermut iglugaseksuit upkoaksoangata sivurâne. Attannille tikkisimajut ingmikorlutik naternamelaukput.

10 Joabele manna tækkogame sorsungnek torartitaungmat tapsomunga sânganit kingornganillo; annerosulerpok Israelib angutinginit innuosuktunit illunainit, parngnailerlunelo Siriamiut akkerartoromavlugit.

11 Innugiartoksuille assingit pitipait angajume Abisaib agganginut, parngnailerkovlugit Ammonib kittorngangit akkerartorlugit.

12 Okarlunelo: Siriamiut sôngoluarniarpatta uvamnut, tagva ikajoriartorlaunga; Ammonible kittorngangit sôngoluarniarpatta illingnut, uvanga ikkajoromavagit.

13 Maksoalerit, maksoavlutalo innukattivut Gudiptalo iglugaseksoakotingit pinniarutigilavut. Nalegak pinniarle namagijaminik.

14 Joabelo innugiartoksuit illagijane illagilugit kaglilerpok Siriamiut sorsugvigivlugit; kemmâvâllo.

15 Ammonible kittorngangit tækkogamik Siriamiut kemângmatta, Abisai angajua kemâgivât, itterlutiglo iglogaseksoarnut. Joabelo tikkipok Jerusalemut.

16 Siriamiulle tækkogamik salagijaunermingnik Israelitinut; tillijanik tilliklerput, tikkikolaitalo Siriamiut kôksub akkianêtut. Sofachiblo Hadadeserib sorsuktuksoakotingita angajokangata. sivorlervigivait.

17 Davide tamattominga okautsaulerame, Israelitit illunamassiatik kattitipait attautsemut, ingergalunelo Jordane ikârlugo; tikkinamigillo parngnailerpok. Davidelo opalungaijarpok Siriamiut sorsugvigijomavlugit, tapkoalo sorsukattigivât.

18 Siriamiulle Israelitit kemâvait. Davidiblo Siriamiunit kamutit 7000 dit innungit, 40000 dillo angutit pissuktut tokkopait; illaliudlugillo Sofache sorsuktuksaksoakattigêt angajokangat tokkopa.

19 Hadadeseriblo kivgangit tækkogamik sâlagijaunermingnik Israelitinut, ullapirsautiksaliorput Davidemut kivganginullo. Siriamiullo Ammonib kittorngangit ikajoromairpait. 


\section{KAPITEL XX.}

Amoniterit iglugaseksoakotingita Rabbamik attigit sâlagïauningat; Filisterillo salagïauningat.

J AHRELO kângermat, nelliutome attannit aularvigivaktangane, Joabib sorsuktuksaksoakattigêt aularutivait, asserorlâlo Amonib kittorngangita nunangat, tikkilunelo Rabba illumavait; Davidele Jerusalemetuarpok. Joabible Rabba sâlagivait, serk milaitalo.

2 Davidiblo attanningata niakotinga tiguva niakunganit, tıkkisilâlo Talentetut okkumaitigijomik Goldekarmat, ujarakarmallo pivianartunik; illijaulaukporlo Davidib niakoanut. Aksarnigaujoksuillo aularutivaittauk iglugaseksoarnit.

3 Innugiartoksuille takkamanetut annitipait avgorlugillo kiblutinut, niksinullo, kuksautinullo kikkiangôjunut, Davidib Amonib kittorngangita iglugaseksoakotingit illunaita taimailiorvigivait. Davidelo innugiartoksuit illagilugit utterpok Jerusalemut.

4 Tamattoma kingorngane sorsungnekalerpok Gasereme: Filisterinut. Taipsomane Sibechaib Husatemiub Sibai innukpait kittorngangita illangat ullimârpa; manigotititauvullo.

5 Amalo sorsungnekalerlaukpok Filisterinut. Tagva Elhananib Jairib erningata Lahemi Goliathib Gathemiub nukanga ullimârpa, kallugiakalauktok ippulingmik umiab naparutangatutitomik.

6 Amalo sorsungnekalaukpok Gatheme. Tagvane angutekarpok innugangoalingmik 6 inik aggangmine ittikaminelo, tagva 24 ôjut, erniangolaurivoktauk innukpanit.

7 Mittautekarporlo Israelitinik. Jonathanible, Simeab, Davidib angajuata, erningata ullimârpa.

8 Tapkoa erniangolaukput innukpanit Gatheme, ochotitaulutiglo Davidib tapsomalo kivgangita agganginut.

\section{KAPITEL XXI.}

Innugiartolssuit kittitauningat, pitlartaunerlo nusilingnartomik.

SATANASIB Israelitit akkerartorpait. Davidelo kajungersarpa Israelitit kittitaukolugit pikovlugo,

2 Davidelo Joabemut innugiartoksuillo angajokanginut okar- 
pok: Israelitit kittijartorsigik Bersebamit Dane tikkilugo; kailugillo uvamnut, kaujikovlunga unnurninginik.

3 Joabe okarpok: Nalegak illautitsilerle innukotiminut sorlo mana illingangmatta 100 deêrtortunik unnurnersanik sulle; kissianele nalegâak attannek, illunaita, nalekama kivgaringilait? Sôgle nalekama tamana apperkutigivauk? Sôg patsitikartuksaulerka Israelitinut?

4 Attanniuble okausingit ajugaulaukput Joabemut, Joabelo aularpok agvertarlunelo Israelitit nunangitigut illunaitigut, tikkiporlo Jerusalemut.

5 Innugiartoksuillo kittisimajut unnurningit tunnivait Davidemut. Innugiartoksuillo illunatik unnurningit 1100000 deolaukput saviksoarmik amusijut, Judakullo 470000 diolaukput saviksoarmik amusijut.

6 Levikullo, Benjaminekullo kittikassiutilaungilait tapkoa akkorngane; Joabib attanniub okausingit makkojugingmagit.

7 Gudible tamana idluigiva; annauvaillo Israelitit.

8 Davidelo okarpok Gudemut: Angijomik idluitullilaukpunga taimailiorlaurama; mannale pêjarlauruk kivgavit idluitullininga, sillainartomik pilaurama.

9 Nalekablo Gade Davidib tækkojokotinga okarvigiva, okarlunelo:

10 Ailerit Davidelo okarkattigiuk: Imâk Nalegak okarpok: Pingasunik adsigêngitunik manijervigivagit annerosulerit tapkoa illanganik, taimailiorkovlunga illingnut.

11 Gadiblo Davide tikkigamiuk okautiva: Imâk Nalegak okarpok: Annerosulerit:

12 Jahrinik pingasunik kângnartuniglonêt; kemânermiglonêt takkit pingasut nâlugit akkerartortitit pivlugit, omigijivillo saviksoangit pivlugit, angukovlugit illingnik; Nalekab saviksoanganiglonêt, nusilingnartomiglo nuname uvlut pingasut nâvlugit, Nalekab Engelinga asseroikovlugo Israelitit kiglilervingine illunaine; tagva issumaksarsiolerit nautsertorlutillo mana sunamik kiojuksaunimnik tapsomunga tillijimnut.

13 Davide okarpok Gademut: Kappiasudlarpunga angijomik; kissianele Nalekab agganginut kattagomavunga, tapsoma napkoarusungninga angijovaksongmat, kattagomangilangalo innuit agganginut.

14 Tagva Nalekab nusilingnartok tikkerkova Israelitinut, ochojokarmat Israeleme angutinik 70000 dinik.

15 Gudiblo Engele tilliva Jerusalemut, asserorlugit pikovlugo. Asserorsaitillugolo Nalekab tækkova tamanna, kakkialerutigivâlo, Engelelo asserorsaijok okautiva: Namaksivok aggaktit erkitikit. Nalekable Engelinga nangerpok Arafnab Jebusitereôjub kakkojaksalliorvingata kiglingane. 
16 Davidelo tækkosarpok, tækkovâlo Nalekab Engelinga nangertillugo killaub nunablo akkorngangne, saviksoarmiglo kenailitakangitomik tigumiartillugo aggangmine, issautjitillugolo tapsominga Jerusalemut. Tagva Davide angajokaunerôjullo pôngnut ulliksimavlutik pamakterput kênatik pûvlugit.

17 Davidiblo Gude okautiva: Uvangaungilanga innugiartoksoarnik kittitaukojijok? Uvangauvunga idluitullilauktok, namalungitomiglo pinniarlauktok; saukalle ukkoa, sunamik pinniarlaukât? Nalegak Gudîk aggaktit torarlit uvamnut atâtamalo iglomiokotinginut akkerartorluta innukotingnulloungitok, tapkoa kappiasuktillugit.

18 Nalekablo Engelingata Gade okautiva: Davide okautilugo pikovlugo, Davide majorartuksaungmat Altarelo sennajaksaringmago Nalekamut, Arafnab Jebusitereôjub kakkojaksalliorvingane.

19 Taimak Davide Gadib okausingit okauserilauktangit Nalekab attingane malliklugit majorarpok.

20 Arnanelo keviarame erningillo sittamat illagilugit Engelemiglo takkogame, ijerput; Arnanible Waizen tullertautipait.

21 Davide mana Arnanemut aingmat, Arnan tækkosarpok, Davidelo tækkova, annilunelo kakkojaksalliorvingınit, kênanelo pûvlugo nunamut Davide opigiva.

22 Davidiblo Arnan okautiva: Inniksamik tunnitsivigilaunga kakkojaksalliorvingme, Altaremik Nalekamut sennajungnarkovlunga tagvunga; namaktomik akkekartillugo tunnijaksarivat uvamnut, pagvisartoâluk soraikovlugo.

23 Arnanele Davidemut okarpok: Tigusilerit Nalegâk attannek, pinniarlutillo sorlo namagijaungmat illingnut, âhâk tuktuvak una tunnivara tunergutiksamut ôtaksamut, tuktuvaublo annungita kejuktangit ikkomaksamut, Weizenelo tunergutiksamut nerkiksamut, tamaita tunnivakka.

24 Attannerle Davide Armanemut okarpok: Taimangungitok, kênaujanulle akkikonartanga malliklugo pissiarijomavara; pigijattit tigujomanginapkit âlugillo Nalekamut, akkekangitomiglo tunnijijomangilanga tunnergutiksamik ikkoalajaksamik.

25 Taimak Davidib Arnan tunnitsivigiva innemut mattomunga Goldemik, Sekeletut 600 ditut okumaitigijomik.

26 Davidiblo tagvane Altare sennava Nalekamut, tunijilunelo tunergutinik ôtaksanik, kujalijutiksaniglo. Nalegarlo nipliarvigingmago tussarpa ikkomakut killangmit tunergutiksaliub ôtaksanik Altaringata kângane.

27 Nalekablo Engele okautiva, saviksoane pôlugo kênailitanganut pikovlugo.

28 Nelliutome taipsomane Davide tækkogame, Nalekab tussarmago Arnanib Jebusitereôjub kakkojaksaliorvigijangane, tunnergutinik tunnijijongovok tagvane. 
29 Nalekable iglunga Mosesib sennalauktanga sunakajuitome, tunnergutiksaliublo ôtaksanik Altaringat nelliutome taipsomane pôktojomêlaukpuk Gibeoneme.

30 Davidele aijungnarlaungilak tapsoma sânganut Gude kennerlugo, koaksarnermut, Nalekab Engelingata saviksoanganik.

\section{KAPITEL XXII.}

Davidib attuinaruterininga sunatuinarnik Tempelemut; lkaiblainingalo ernerminit Salomomik.

$\mathrm{D}_{\mathrm{A}}$ mânelo Altare tunnergutinut ôtaksanut.

2 Davidiblo tujormitjet Israelib nunanganêlauktut katterkovait; ujaralerijokarkojivorlo ujarkat ullimârlugit, Gudib iglunganut illingajuksat.

3 Davidelo âkiksoivok kikkiangnik unnuktunik, kikkitsanut upkoanut itterviksoanganêtuksanut, assinginullo kikkiektortaujariakartunut illingajuksanik, kannujarmiglo taimak angitigijomik kiktitartaujungnangimatta.

4 Kejungniglotauk kittiksaungitunik Zidonemiut Tirusemiullo âtsingmatta kejungnik Zedereôjunik unnuktunik Davidemut.

5 Davide issumagame: Ernera Salomo nukapiangôvok, nikkanarlunelo, iglole igloliortaujuksak Nalekamut angijojuksauvok, attinga nertornautalo angijororkovlugik nunane illunaine, tamana pivlugo attuinaruterivigijomavara sunatuinarnik. Taimak Davide attuinaruterivok unnuktunik tokkokârane.

6 Erninelo Salomo kaikova, perkolugolo Nalekab Israelib Gudingata igluksanga igluliorlugo,

7 Okautivâlo: Ernîk, uvanga kajusimalaukpunga Nalekab Gudima attinga iglomik igloliorlugo.

8 Nalekable okausingata tikkipânga, okarlunelo: Aut unnuktut kovilaukpattit, unatadlarnekalauklutillo angijomik, tamana pivlugo igvit attera iglulioraksaringilat, auksoarmik taimak angijoksoarmik kovisilauravit sâmne nunamut.

9 Ahâk, ernît, erniangojuksak illingnut, tamna angutaujomârpok uimajangitok; uvanga merngoêrserkojomagapko omigijinginit avatinganêtunit tamainit; Salomomik attekartuksaungmat, uvangalo ullapirsaumik, merngoêrsernermiglo tunijijomavunga Israelitinut innôsingane tamât.

10 Tapsoma attera iglomik iglolioraksariva. Ernerijomavara, tapsomalo atâtagijaksarivânga. Uvangalo tapsoma attannioningata iksivautanga Israelitinut nakkiteromavara soraijuitomik. 
11 Mana Nalekab aiparilitit ernîk, sullitsiarkovlutit Nalekab Gudivit igluksanga igluliorlugo pikovlutit, sorlo okautigilaungmattit.

12 Nalekab tunnitsiviginiarpâtittauk sillatunermik illissimanermiglo, Israelitillo perkoniarpait illingnut, mallikovlutit $\mathrm{Na}$. lekab Gudivit perkojanginik.

13 Tagva pilloridlarniarpotit, kamatsiaruvit Gudib perkojangit, idluarijangillo, Nalekab perkolauktangit Mosesekut Israelitinut malliklugit pinniarkovlutit. Maksoalerit mungotaililutillo, sivoraniarnak, koaksanganaglo!

14 Ahâk siorniornimne piksarsiorlaukpunga Nalekab iglunganut illingajuksanik Talentinik 100000 dinik Goldemik, 1000 dillo 1000 deêrtorlutik Talentinik Silberemik, tamakkonungalo kannujarnik kittiktartaujungnangitunik, unnudlarmatta; kejungniglotauk ujarkaniglo piksarsiorlaukpunga, tapkoa illaksakartitsungnarpattit.

15 Sennajullo unnuktut illingnêput, ujaralerijut, igluliortullo, sungiusimajullo sullijaksanik sunatuinarnik.

16 Golde, Silberelo, kannujarlo, kikkierlo, kittiksaungilet. Parngnailerit tagva sennalugolo; Nalekab aipariniarpâtit.

17 Davidiblo Israelitit angajokangit illunaita perkovait ernine Salomo ikajorlugo pikovlugit.

18 Nalekah Gudipse illagingilâse merngoêrsernermiglo tunnitsivigiluse avatipsingne illunane? Tapsoma nunab innungit tunnilaungmagit aggamnut, nunalo nalektitaumavok Nalekamut, innukotinginullo.

19 Taimak mana omattise tarniselo torartisigik Nalegak kennerlugo. Parngnaileritselo igloliorluselo Gudemut Nalekamut irsornaitovingmik, Nalekab angerningata iklervinga, illulerkutillo ivsornaitut pititaukovlugit iglomut Nalekab attinganut igluliortaujuksaujomut.

\section{KAPITEL XXIII.}

\section{Levitit kittitauningit sullïjaksakartitauningillo.}

DAVIDELo innutokaulermat, inôsermiglo namaksigame, ernine Salomo attanningortipa.

2 Davidiblo angajokajet Israeleme illunaita, ajochertuijullo, Levitillo kattikovait,

3 Levitit kittitaukovlugit Jahrekartut 30 inik unnurnersaniglo; unnurningillo niakomit niakomut 38000 diolaukput, anguteôjut sôngojut.

4 Tapkonangat 24000 dit perkojaurut, pinniaraksat Nalekab 
iglungane pinniarlugit; 6000 dillo angajokangốrput, idluarsaijungorlutiglo ;

54000 dillo udsertortungorput upkoanik; 4000 dillo imgerlutik, nertordlertungorput Nalekamik, nokakseralıngnik, sennalauktamnik (Daride okarpok) attorlutik, nertordlerutiksanik imgerlutik pikovlugit.

6 Davidiblo avikpait ingmigolingajunut Levib erningit, tagva Gerson, Kahat, Merarilo, malliklugit.

7 Gersonekunit tapkoangôlaukput: Laedan, Simeilo.

8 Laedamib erningit: Sivorlek Jehiel, Sethamelo, Joello, tapkoa pingasut.

9 Simeiblo erningit tapkoangôlaukput: Salomith, Hasielelo, Haronelo, tapkoa pingasut. Tapkoa angajokaunerôlaukput atâtat Laedanekut akkorngane.

10 Simeiblo erningit: Jahath, Sinalo, Jeuselo, Brialo. Tapkoa sittamat Simeib ernerilaurivait.

11 Jahatelo sivorliôlaukpok, Sinalo tugliôlaukpok. Jeuselo, Brialo ernekalaungilak unnuktunik, tamanna pivlugo atâtab atautsib kinguvângititut kittikassiutitauvut.

12 Kahatib erningit tapkoangôlaukput: Amrame, Jazeharlo, Hebronelo, Usielelo, tapkoa sittamat.

13 Amramib ernigik tapkoangôvuk: Aron, Moselo. Aronele ingmigolingatitaumalaukpok, ivsornaititaukovlugo ivsornainerpangôjomut, tamma erningillo soraijuitomik, iserluanititsilerlutik Nalekab sângane, kivgartorlutiglo saimartitsilutiglo Nalekab attingane soraijuitomik pikovlugit.

14 Mosesiblo Gudib angutingata erningit taikassiutitauvut kinguvarêt Levikut akkorngane. relo.

15 Mosesible ernigik tapkoangolaukpuk: Gersom, Eliese-

16 Gersomib erningit: Sivurlek Sebuel.

17 Elieserib erningit: Rehabja, sivurlek. Elieserelo assianik ernekalaungilak. Rehabjable erningit unnuktovaksolaukput. lek.

18 Jezeharib erningit tapkoangôlaukput: Salomith, sivur-

19 Hebronib erningit tapkoangôlaukput: Jeraja sivurlek, Amarjablo tuglek, Jehasielo pingajoat, Jakmeamelo sittamangat.

20 Usielib erningit tapkoangôlaukput: Micha sivurlek, Jesialo tuglek.

21 Merarib erningit tapkoangôlaukput: Maheli, Musilo. Mahelib erningit tapkoangôlaukput: Eleaser, Kiselo.

22 Eleaserelo tokkovok ernekarane pannekalaukporle, kattangutingitalo Kisib erningita tiguvait.

23 Musib erningit tapkoangôlaukput: Maheli, Ederelo, Jeremothelo, tapkoa pingasut.

24 Tagva Levib erningit atâtagijamik iglomiokattingita atâ- 
tallo angajokaunerôjut akkorngane, sorlo kittitaulaungmatta attingita unnurningit malliklugit, niakomit niakomut, pinniarlauktut pinniaraksanik Nalekab iglungane, Jahrekartunit 20ginik unnurnersaniglo.

25 Davide okarmat: Nalekab Israelib Gudingata innukotine merngoêrsernermik tunnitsivigilaukpait, innekarniarporlo Jerusaleme soraijuitomik.

26 Taimaglo Levitit akjarsijariakarungnaiput iglomik, illulerkutiniglo kivgartornermut pijariakartunik.

27 Davidible okausingita kingorlingit malliklugit Levitit kittitaulaukput, Jahrekartunik 20 ginik unnurnersaniglo.

28 Pititaulaukpullo Aronib erningita agganginut, kivgartornermut Nalekab iglungane, avalut illuane, innenelo aiparutsine sujungaititzikovlugillo ivsornaitunik sunatuinarnik, ikajorkovlugillo sullijaksane Gudib iglungane.

29 Kakkojanullo tækkojaksanut sennaukamullo kalkkortamut, tunnergutiksanut nerkiksalingnut, kakkojanullo sernakangitunut, tapkonungalo jarrajaupaktunut kallutaujame illingajunut; okumailutanullo illunainut, oktutenullo;

30 Nangerkovlugillo uvlane tamaine Nalegak kujagilugo, nertorlugolo, taimaglotauk unnune;

31 Tunnergutiksallo ôtaksat illunaita tunnilugit Nalekamut Sabbatine, takkêlânelo uvloksiorvingnelo, unnurningit sorlo pijariakarmatta malliklugit, taimailiutsainarkovlugit Nalekab sângane ;

32 Pinniarkovlugillo pinniaraksanik tupperme kattimaviôjome, ivsornaitovingmelo, ikajorkovlugillo Aronib erninginik kattangutinginik kivgartornerme Nalekab iglungane.

\section{KAPITEL XXIV.}

\section{Ajochertuijut ingmigolingatitauningit.}

ARONIBLO erningita ingmigolingatitauningit, tapkoangôlaukput. Aronib erningit tapkoangôlaukput: Nadabe, Abihu, Eleasar, Ithamarelo,

2 Nadabele Abihulo tokkokârlaukpuk atâtamingnit, kittorngakalaungilâglo, Eleaserelo, Ithamarelo ajochertuijungorlaukpuk.

3 Davidiblo âkiksorpait imâk: Zadoke, Eleaserib erninginit pijok, Ahimelechelo, Ithamarib erninginit pijok, unnurningit sullijaksangillo malliklugit.

4 Eleaseriblo erninginit unnurnersanik nachvartokalaukpok niakoôjunik angutit akkorngane Ithamarib erninginit; âkiksor- 
paillo imâk: Eleaserib erninginit 16it, angajokangnut atâtamik iglomiokattingita akkorngane, 8 illo Ithamarib erninginit atâtamik iglomiokattingita akkorngane.

5 Imeraumullo âkiksorpait adsigêktomik, angajokaunerôjut ivsornaitome, Gudiblo angajokâkotingit Eleaserib Ithamariblo erninginit pingmatta.

6 Semajablo aglakpâktub Nethaneelib erningata Leviteôjub aglakpait attanniub sângane, angajokaillo Zadokiblo ajochertuijub, A himelechiblo Abjatharib erningata, atâtallo angajokaunerôjut ajochertuijut Levitillo akkorngane; atâtat angajokaunerôjut illangat Eleaserekunit imerautigivât, aipangalo Ithamarekunit.

7 Imeraut sivorlek nellautsilaukpok Jojaribemut, aipanga Jedajamut,

8 Pingajoat Harimemut, sittamangat Seorimemut.

9 Tellimangat Malchijamut, pingasojoat Mejaminemut,

107 ingat Hakozemut, 8 ingat Abiamut

119 ingat Jesuamut, 10 ingat Sechanjamut,

1211 ingat Eliasibemut, 12 ingat Jakimemut,

1313 ingat Hupamut, 14 ingat Jesebeabemut,

1415 ingat Bilgamut, 16 ingat Imeremut,

1517 ingat Hesiremut, 18 ingat Hapizezemut,

1619 ingat Pithajamut, 20 ingat Jeheskelemut,

1721 ingat Jachinemut, 22 ingat Gamulemut.

1823 ingat Delajamut, 24 ingat Maasiamut.

19 Tagva akiksortauningat pinniaraksamingnut itterlutik Gudib iglunganut, illutsitik perkojaumajut atâtamingnut Aronemut malliklugit, sorlo Nalekab Israelib Gudingata perkolaungmago.

20 Leviblo emingita assingit tapkoangôlaukput: Amramib erninginit Subael. Subaelib erninginit Jedea.

21 Rehabjab erninginit Jesia sivorliôlaukpok.

22 Jezeharikunit Slomoth, Slomothib erninginit Jahat.

23 Hebronib erningit tapkoangôlaukput: Jerja sivorlek, Amarja tuglek, Jahesiel pingajoat, Jakmeame sittamangat.

24 Usielib erninga Michaôlaukpok. Michablo erningita illangat Samireôlaukpok.

25 Michab nukanga Jesiaôlaukpok. Jesiab erningita illangat Sacharjaôlaukpok.

26 Merarib erningit tapkoangôlaukput: Maheli, Musilo, tapsoma erninga Jaesiaôlaukpok.

27 Merarib erningit, erninganit Jaesiamit tapkoangôlaukput: Soham, Sakurelo, Ibrilo.

28 Mahelemit Eleasar, tamna ernekalaungilak.

29 Kisemit. Kisib erninga Jerahmeeleôlaukpok.

30 Musib erningit tapkoangôlaukput: Maheli, Ederelo, Je- 
remothelo. Tagva Levitit erningit, atâtagijamik iglungit malliklugit.

31 Tapkoalotauk imerautigijauvut kattangutingita Aronib kittorngangita senniane, attanniub Davidib Zadokiblo, Ahimelechiblo, atâtallo angajokaunerôjut ajochertuijut Levitillo akkorngane sangine; adsigêktomik imerautigijauvut, kattangut mingnerpâk angajokaunerôjotut atâtat akkorngane.

\section{KAPITEL XXV.}

\section{Imgerpaktut aviktauningit ingmigolingajunut 24 inut.}

DAVIDELO sorsuktuksaksoakattigêllo angajokângit aviksivut kivgartornermut Assafib, Hemaniblo, Jedithuniblo erninginit Harfesijartunik, Psalteriniglo, Cimbeliniglo attortunik kittitaulaukpullo sullijaksanut piniarkojaunitik malliklugit.

2 Assafib erninginit: Sakur, Josefelo, Nethanjalo, Asarelalo, Assafib erningit aulatitauvlutik Assafemut nipliajokteôlauktomut attannerme.

3 Jedithunemit. Jedithunib erningit tapkoangôloukput: Gedalja, Zorilo, Jesaijalo, Hasabjalo, Matitjalo, tapkoa 6 it aulatitauvlutik atâtamingnut Jedithunemut, Harfesijarlutik nellautailauktut, Nalegak kujagilugo nertorlugolo.

4 Hemanemit: Hemanib erningit tapkoangôlaukput: Bukja Mathanja, Usiel, Sebuel, Jerimot, Hananja, Hanani, Eliatha, Gidalthi, Romamthi-Eser, Jasbekasa, Malloti, Hothir, Mahesiotelo.

5 Tapkoa illunaita Hemamib ernerivait, attanniub tækkojokotingita, Gudib okausingit attorlugit nertordlertub. Gudib Heman tunnitsivigilaungmago ernernik 14 nik panningniglo pingasunik.

6 Tapkoa illunaita aulatitauvut atâtamingnut Assafemut, Jeditunemut, Hemanemullo, imgerlutik Nalekab iglungane, Cimbelit, Psalterillo, Harfillo attorkassiutilugit, kivgartornerme Gudib iglungane, taimaluatsiak sorlo attanniub Assafe, Jedithunelo Hemanelo perkolaungmagit:

7 Tapkoa kattangutingill, illaliudlugit imgernermik Nalekamut, sungiusimajut illunatik 288 ôlaukput.

8 Pinniarkojaunitiglo imerautigivait adsigêktomik, mingnerpâmut angnerpâmullo, illiniartitsijomut illiniartomullo.

9 Imeraut sivorlek nellautsivok, Assafekut illanganut, Josefemut. Aipanga Gedaljamut, kattangutingit erningillo illaliudlugit, tapkoa 12 ôlaukput.

10 Pingajoat Sakuremut, erningit kattangutingillo illaliudlugit, tapkoa 12 ôlaukput. 
11 Sittamangat Jezrimut, erningit kattangutingillo illaliudlugit, tapkoa 12 ôlaukput.

12 Tellimangat Nethanjamut, erningillo kattangutingillo illaliudlugit, tapkoa 12 ôlaukput.

13 Pingasojoat Bukjamut, erningit, kattangutingillo illaliudlugit, tapkoa 12 ôlaukput.

147 ingat Jesreelamut, erningit kattangutingillo illaliudlugit tapkoa 12 ôlaukput.

158 ingat Jesajamut, erningit kattangutingillo illaliudlugit, tapkoa 12 ôlaukpul.

16. 9 ingat Mathanjamut, erningit kattangutingillo illaliudlugit, tapkoa 12 ôlaukput.

17 Tellimaujoat Simeimut, erningit kattangutingillo illaliudlugit, tapkoa 12 ôlaukput.

1811 ingat Asareelemut, erningit kattangutingillo illaliudlugit, tapkoa 12 ôlaukput.

1912 ingat Hasabjamut, erningit kattangutingillo illaliudlugit, tapkoa 12 ôlaukput.

20.13 ingat Subaelemut, erningit kattangutingillo illaliudlugit, tapkoa 12 ôlaukput.

21 14 ingat Mathitjamut, erningit kattangutingillo illaliudlugit, tapkoa 12 ôlaukput.

2215 ingat Jeremothemut, erningit kattangutingillo illaliudlugit, tapkoa 12 ôlaukput.

2316 ingat Ananjamut, erningit kattangutingillo illaliudlugit, tapkoa 12 ôlaukput.

2417 ingat Jasbekasamut, erningit kattangutingillo illaliudlugit, tapkoa 12 ôlaukput.

2518 ingat Hananimut, erningit kattangutingillo illaliudlugit, tapkoa 12 ôlaukput.

26 19 ingat Mallotimut, erningit kattangutingillo illaliudlugit, tapkoa 12 ôlaukput.

2720 ingat Eliathamut, erningit kattangutingillo illaliudlugit, tapkoa 12 ôlaukput.

2821 ingat Hothiremut, erningit kattangutingillo illaliudlugit, tapkoa 12 ôlaukput.

2922 ingat Gidaltimut, erningit kattangutingillo illaliudlugit, tapkoa 12 ôlaukput.

3023 ingat Mahesiothemut, erningit kattangutingillo illaliudlugit, tapkoa 12 ôlaukput.

31 24 ingat Romamthieseremut, erningit kattangutingillo illaliudlugit, tapkoa 12 ôlaukput. 


\section{KAPITEL XXVI.}

\section{Udsertortut upkoanik; pimaklertit pivianartunik, idluarsä̈ullo.}

UPKOAT udsertortingita ingmigolingatitauningit. Korekunit Meselemjaôlaukpok Korib erninga, Assafib erninginit pijok.

2 Meselemjable erningit tapkoangôlaukput: Sacharja erniangokârtok, Jediael tuglek, Sebadja pingajuat, Jatniel sittamangat,

3 Eliam tellimangat, Johanan pingasojoat, Elioenai 7 ingat,

4 Obed Edomib erningit tapkoangôlaukput: Semaja erniangokârtok, Josabad tuglek, Joa pingajoat, Sachar sittamangat, Nethaneel tellimangat,

5 Amiel pingasojoat, Isaschar 7ingat, Pegulthai 8ingat; Gudib pitsartutilaungmago.

6 Erningalo Semaja erniangoviolaurivok ernernik atâtagijamik iglungane attanniotijunik angutaudlarlauramik sôngolauramiglo.

7 Semajab erningit tapkoangôlaukput: Athni, Refaello, Obedelo, Elsabadelo (kattangutelik erkinaitunik) Elihu, Samachjalo.

8 Tapkoa illunaita Obed-Edomib erninginit pilaukput, tapkoa erningillo kattangutingillo illaliudlugit angutit erkinaitut, pikkariktut sullijaksanut, 62 ôlaukput Obed-Edomemit.

9 Meselemja ernekalaukpok kattangutekalarmelo, angutinik erkinaitunik 18 nik.

10 Hossalo Merarib kittornganginit pijok, ernekalaukpok, Simri erkanarnerpâk (erniangokârtồlaungilak, atâtangatale erkånarnerpângortipâ),

11 Hilkia tuglek, Tebalja pingajoat, Sacharja sittamangat. Hossab erningit kattangutingillo kattilugit 13 ôlaukput.

12 Tapkonunga upkoanik udsertortut ingmigolingajut pilaukput, angutit niakungit malliklugit, pigârtut kattangutimik senniane, tapkoa kivgartortilugit Nalekab iglungane.

13 Imerautillo attortaulaukput pijariakitut, pijariakortojullo pivlugit, upkoat illunatik attunît pivlugit.

14 Imeraut kittâne nellautsivok Meselemjamut; erningale Sacharja attannertuijolauktok sillatôjok, imerautigijaulaurivok, imerautalo nellautsivok tachânut;

15 Obed-Edomemulle erninginullo sekkernganut Esupimib iglungata senniane. 
16 Suppimemullo Hossamullo kangianut upkuame Salackretemik attelingme, apkosiniub takpaungarviane, udsertortut assingita senniane.

17 Kittâne Levitekalaukpok pingasojortunik, tachane uvlut́ tamaita sittamanik, sekkerngane uvlut tamaita sittamanik, Esupimemelo maggungnik, maggungniglo;

18 Parbaremêlo kangiane sittamanik, apkosiniub kiglingane, maggungniglo Parbareme.

19 Tagva upkoat udsertortingita ingmigolinganingit Korib erninginit Merariblo erninginit pijut.

20 Levitinillo Ahia pimaklerteôlankpok Gudib iglungata pivianartokotinginik, pivianartuniglo ivsornaititaumalauktunik Nalekamut.

21 Laedanib erninginit Gersonib erninginit pijomit. Laedanemit angajokakalankpok atâtanut Jehielemik.

22 Jehielib ernigik Setham nukangalo Joel, pimaklerteôlaukpuk Nalekab iglungata pivianartokotinginik.

23 Amramekunit, Jezeharekunit, Hebronekunillo, Usielekunillo.

24 Sebuele, Gersomib erninga, Mosesib erningata angajokaulaukpok pivianartunut.

25 Nukangale Elieser attautsemik ernekalaukpok, Rehabjamik, tapsoma erninga Jesajaôlaukpok, tapsoma erninga Jorameôlaukpok, tapsoma erninga Sichriôlaukpok, tapsoma erninga Salomitheôlaukpok.

26 Salomith tamna kattangutingillo angajokaulaukput perkutenut pivianartunut tamainut, ivsornaititaumalauktunut attanniub Davidib, atâtallo angajokaunerôjut, angajokait 1000 dinut, 100 dinullo, akkorngane, sorsuktuksallo angajokangita ivsornaitilauktanginut.

27 Unatadlarnermit aksarnigaujunillo ivsornaitilaukpait, Gudib iglungața âkiksortaujutiksanganut illingakovlugit.

28 Samueliblo tækkojub ivsornaitilauktangit, Sauliblo, Kisib erningata, Abneriblo, Nerib erningata, Joablo, Zerujab erningata ivsornaitilauktangit, (perkutit) ivsornaititaumajut illunaita Selomithib kattangutingitalo agganginut aulataulaukput.

29 Jezeharikunit Kenanja, erningillo illagællugit sullijaksanut sakkijartunut Israeleme, aulatsijôlaukput, idluarsaijolutiglo.

30 Hebronekunit Hasabja kattangutingillo, innuit erkinaitut 1700 dit, angajokangortitaulaukput Israelitinut Jordanib miksâne, kangiane, Nalekab illusiokojangit tamaita pitjutigællugit, kivgartornerlo attannermik pivlugo.

31 Hebronekunit Jera niakoôlaukpok Hebronekut atâtagijangita kinguvângita akkorngane. Davidib attannioningata Jahringita 40 gingane nautsertortaulaukput, nachvartokalauk- 
porlo akkorngane Jaesereme Gileademêtome angutinik erkinaitunik.

32 Kattangutingillo angutit erkinaitut 2700, illunatik niakoôjut. Davidiblo angajokângôrtipait Rubenekunut, Gadekunullo, kinguvarêllo Manassekut ketterkanginut, Gudib attanniublo illusiokojangit tamaita pitjutigællugit.

\section{KAPITEL XXVII.}

Sôrsuktuksat âkiksortauningit ; kinguvarêt angajokaksoangit, pimaklertit, aulatsïullo.

I'AMADSA Israelib erningit unnurningit malliklugit niakoôjut kinguvarênut, angajokaillo 1000 dinut, 100 dinullo, aulatsijullo tapkoninga, naipertorlauktut attannermik, ingmigolinganimik illusingit tamaita malliklugit, tikkitut aulartullo takkernit takkernut Jahrib takkingine tamaine. Ingmigolingajullo attunit angutekalaukp ut 24000 dinik.

2 Ingmigolingajunut sivorlernut takkit sivorlinganut, Jasabeame, Sabdielib erninga angajokaulaukpok, ingmigolingajokotinganullo angutit 24000 dit, illingalaukput.

3 Perezib erninginit pilaukpok, niakoôlunelo, takkib sivorliub sorsuktuksangita angajokanginut illunainut:

4 Ingmigolingajunullo takkib aipangane Dodai Ahohimiok angajokaulaukpok, Miklothelo (tamna angajokarilugo) aulatsijolaukpok tapkonunga ingmigolingajokotinginullo angutit 24000 dit illingalaukput.

5 Sorsuktuksat angajokangat, takkit pingajuane angajokaunerôjok Benajâ̂laukpok Jojadab ajochertuijub erninga; ingmigolingajokotinginullo 24000 illingavut.

6 Tagva Benaja tamna, sôngojok 30 git akkorngane, angajokaujorlo 30 ginut. Ingmigolingajokotingillo aulataumalaukput erninganut Ammi-Sabademut.

7 Sittamangat, takkit sittamangane Asaheleôlaukpok, Joab nukanga, kingornganelo erninga Sabadja; ingmigolingajokotinginullo 24000 dit illingaleukput.

8 Tellimangat, takkit tellimangane Samehuteôlaukpok, Jesramiok; ingmigolingajokotinginullo 24000 dit illingalaukput.

9 Pingasojoat, takkit 6 ingane Israôlaukpok, Ikkesib erninga Thekoamiok; ingmigolingajokotinginullo 24000 dit illingalaukput.

107 ingat takkit 7 ingane Helezeôlaukpok, Pelonemiok, Efraimib kittornganginit; ingmigolingajokotınginullo 24000 dit illingalaukput. 
11 Sittamaujoat takkit 8 ingane Sibechaiôlaukpok, Husamiok Sarehiterinit; ingmigolingajokotinginullo 24000 dit illingalaukput.

129 ingat, takkit 9 ingane Abieserôlaukpok, Anthomiok Jeminib kittornganginit; ingmigolingajokotinginullo $24000 \mathrm{dit}$ illingalaukput.

13 Tellimaujoat, takkit 10 ingane Maheraiôlaukpok, Netofatemiok, Serahiterinit; ingmigolingajokotinginullo $24000 \mathrm{dit}$ illingavut.

1411 ingat, takkit 11 ingane Benajaôlaukpok, Pirgatomiok, Efraimib kittornganginit; ingmigolingajokotinginullo $24000 \mathrm{dit}$ illingalaukput.

1512 ingat, takkit 12 ingane Heldaiôlaukpok, Netofatemiok Athnielemit; ingmigolingajokotinginullo 24000 dit illingalaukput.

16 Israeliblo kinguvarênginut, tapkoangôlaukput: Rubenekunut Elieser, Sichrib erninga, angajokaulaukpok, Simeonekunut, Sefata Maechab erninga.

17 Levikunut, Hasabja, Kemuelib erninga. Aronekunut $\mathrm{Za}$ dok.

18 Judakunut Elihu Davidib kattangutinginit. Isascharekunut Amri, Michaelib erninga.

19 Sebulonekunut Jesmaja, Obadjab erninga. Naftalikunut Jeremoth, Asrielib erninga.

20 Efraimekunut Hosea, Asasjab erninga. Kinguvarêt Manassekut ketterkanginut Joel, Pedajab erninga.

21 Kinguvarêt Manassekut ketterkanginut Gileademêtunut Iddo, Sacharjab erninga. Benjaminekunut Jaesiel, Abnerib erninga.

22 Danekunut, Asareel, Jerohamib erninga. Tagva Israelib kinguvarêngita angajoksoangit.

23 Davidiblo tapkoa Jahrekartut 20 inik ikkinersaniglo kittikassiutitaukolaungilait, Nalegak okalaungmat, Israelitit unnuksivalliatitsomavlugit uvloritsatitut killangmêtutitut.

24 Joab, Zerujab erninga, pigiarlaukpok kittitsilune, nâlaungilaille, Israelitille tamanna pivlugo ominautemut tikkitaulaukput; tamana pivlugo unnurningit aglaktaulaungilet attanniub Davidib aglakotinginut (Kronikakotinganut).

25 Attanniub pivianartokotinginut Asmaveth, Adielib erninga pimaklerteôlaukpok; pivianartunullo nunametunut iglogaseksoarnetunut, iglugasengnêtunullo, igloksoarnetunullo Jonathan Usiab erninga pimaklerteôlaukpok.

26 Perorsaijunut nuna poargejarlugo perorsarlugolo Eseri Kelubib erninga angajokaulaukpok.

27 Perorsevingnut Weineliksanut Simei Ramatemiok angajokaulaukpok. Kematullivingnullo Weinetalingnut, Weinemullo kematulliosimajunut tamainut Sabdi Sifimiok angajokaulaukpok. 
28 Perorsêvingnut orksutalingnut, nappartutalingnullo Maulbeerelingnik naksarnêtunut Baal Hanan, Gadekut illangat angajokaulaukpok. Orksomut kematulliosimajomut Joase angajokaulaukpok.

29 Tuktuvângnut Saroneme iviksukatalingnêlauktunut Sitra Saronemiok angajokaulaukpok. Tuktuvângnulle naksarnêlauktunut Safat Adlaib erninga angajokaulaukpok.

30 Kamêlinut Obil Ismaelitereôjok angajokaulaukpok. Siutekoktojunut Jehadja Meronomiok angajokaulaukpok.

31 Saukanut Jasise Hagaritereôjok angajokaulaukpok. Tapkoa illunatik angajokaulaukput attanniub Davidib perkutinginut.

32 Jonathanelo Davidib akkanga attannertuijolaukpok, angut sillatôjok, illissimajorlo. Jehielelo, Hachmonib erninga, attanniub erninginêlaukpok.

33 Ahitofelelo attannertuijolaurivok attannermik. Husai Arachimiok illanângolaukpok attannermut.

34 Ahitofeliblo kingorngane Jojada, Benajab erninga, Abjatharelo (attannertuijolaukpuk). Joabelo angajokaunerôlaukpok sorsuktuksaksoakattigênut.

\section{KAPITEL XXVIII.}

Angajokait kattimaningat, Davidiblo âtiksoiningata kingorlerpângat.

$D_{A}$

AVIDIBLO Israelitit angajokangit illunaita kattikovait Jerusalemut, tagva kinguvaret angajokangit, ingmigolingajullo attannermik naipertortut angajokangit, angajokaillo 1000 dinut 100 dinullo, pimaklertit attanniub perkutinginik, nergjutekotinginiglo, erninginiglo, aulatsijullo attanniub iglunganik, sôngojullo angutillo angutaudlartut illunatik illagilugit.

2 Davidelo, attannek nikkovipok nangerlunelo okarlunelo: Naleksinga kattangutikka innukotikkalo. Issumalaukpunga iglomik iglolioromavlunga Nalekab angerningata iklervingata merngoêrserviksanganik, ikkovraksamiglo Gudipta ittigganginut, oppalungaijarlaukpungalo iglolioromavlunga.

3 Gudible okautsaukolaukpânga: Igvit, attera iglolioraksaringilet; sorsuktuksaugavit, aungmiglo kovisilaukpotit.

4 Taimaigalloarmat, Nalekab Israelib Gudingata annerilaukpânga atâtama iglomiokattinginit tamainit attanniokovlunga Israelitinut soraijuitomik. Judakut annerilaungmagit attanniokovlugit, Judakunillo atâtakukka atâtamalo erningita akkorng- 
ane uvanga kuviagilaukpânga attanningortilunga Israelitit illunamassianginut.

5 Ernimalo illunatik akkornganit (Nalekab ernernik unnuktunik tunnitsivigilaungmanga) ernera Salomo annerilaukpa, iksivajuksaungmat Nalekab attanniovingata iksivautaksoangane Israelitinut attanniovlune.

6 Okautilaukpângalo: Ernerpît Salomob igluga, innekotikkalo avaluliorsimajut iglolioraksarivait; annerilaurapko ernerijomarlugo, uvangalo atâtaujomavunga tapsomunga;

7 Attannioningalo nakkititsomavara soraijuitomik, katsungaidlune pinniarmiarpat perkojakka idluarijakkalo malliklugit, sorlo uvlome illingangmat.

8 Manna Israelitit illunamassiangita Nalekab illagêktokotingita Nalekablo siutekita sângangne, (kaiblarpapse): Mallileritse pinniarluselo Nalekab Gudipse perkojanginik tamainik, nuna ajungitok pigênarlugo pikovluse, kingormgutijaksautilugolo kittorngapsingnut pikovluse kingunapse soraijuitomik.

9 Igvillo ernîk Salomo, atâtavit Gudinga illitarileruk, kivgartorlugolo omamut tamânut, tarnimullo kunujuitomut. Nalekab omattit illunaita nautsertormagit, issumaillo tamaita issumaksarsiorningit tukkisivait. Kennerniarungne nachvarniarpat; kemmaniarungnele, ajektorniarpâtit soraijuitomik.

10 Tækkolerit manna, Nalekab annerilaungmattit, iglomik igluliorlutit pikovlutit ivsornaitomut, maksoalerit sennalugolo.

11 Davidiblo ernine Salomo ôktutiksamik tunnitsivigiva torksomut, iglunganullo, inn nullo pivianartotalingnut, inninullo kollânêtunut, aiparutsinullo, kamanêtunut, iksivautablo saimanartoliub inniksanganut;

12 Oktotiksaniglo tamainut omamigijaminut, tagva innemut avaluliorsimajomut Nalekab iglungane, innenullo illunainut avatanê, Gudib iglungata perkotitalinganut, innemullo ivsornaititaumajunut illingajomut;

13 Ajochertuijut, Levitillo ingmigolingatitaumaninginut kivgartornerublo pinniaraksanginullo tamainut Nalekab iglungane; illulerkutinullo tamainut kivgartornermut Nalekab iglungane illingajunut;

14 Goldiojunut, Goldib okumaininga malliklugo, illulerkutirut illunainut kivgartornerit nellianut illingajunut, illulerkutinullo Silbereôjunut, illunainut, okumainingit malliklugit, illulerkutinut tamainut kivgartornerit nellianut illingajunut.

15 Nenergoabvillo Goldeôjut kollingitalo Goldeôjut okumaininginik, nenergoabvit kollingitalo attunît okkumainingit malliklugit, nenergoabvillo Silbereôjut, kollingitalo attunît okumainingit malliklugit, nenergoabvit attunît attoraksangit malliklugit.

16 Tunnitsivigivâlo Goldemik kakkojat tæklkojaksat sânginut, 
sât nellianut okumaijuksauninganik, taimaktauk Silberemik sânut, Silbereôjuksanut.

17 (Oktutiksaniglo) kappukautinut, pôgutanullo illulerkutinullo Goldemit illakangitomit, ermgusiarsungmullo Goldeôjunut, ermgusiarsuit nelliata okumaininga malliklugo, ermgusiarsung-nullo Silbereôjunut, ermgusiarsuit nelliata okkumaininga malliklugo.

18 Iserluanijutiksallo Altaringanut, Goldeôjomit illakangitomit, okumaininga malliklugo; Kerubiglo Goldeôjuk kamutikita oktutiksanganik, issângajuk ulliktuglo kollâne Nalekab angerningata iklervinganik.

19 Tamakkoa tamaita aglagutigijaumavut Nalekab agganginut ajochertortigilauktama oktutiksab piniarninginik tamainik.

20 Davidelo okarpok ernerminut Salomomut: Maksoalerit mungotaililutillo, sennalugolo, sivuraniarnak koaksanganiarnaglo. Gudib Nalekab Gudima aipariniarpâtit, agganelo erkitìniangilait kemangniangilâtillonêt, pijarêrtitsikârtinak piniaraksanik tamainik Nalekab iglungata kivgartortauninganut illingajunik.

21 Ahâglo, manêput ajochertuijut, Levitillo ingmigolingajingit, piniartaukojaujunut illunainut Nalekab iglungane illingajut, illagivâtit sullijaksane illunaine, kunujuilutiglo sillatunekalarmiglo sullijaksanut tamainut; taimaglo angajokaunerôjut innugiartoksuillo illunaita, piniartaukojarnut illinganiarput.

\section{KAPITEL XXIX.}

Angajoliait tunnergutingit Tempelib igluliortauninginut illingajuksat. Davidib tuksiarninga kujalidlarlune. Salomob mingoartauninga. Davidib tokkunga.

ATtannerlo Davide illagênut illunamassianginut okarpok: Gudib Salomo, ernerma illangat, innuosuktok nikkanartorlo sulle annerilaukpa, pinniaraksarle angijôvok, innungmut igluksaujuksaungimat, Nalekamulle Gudemut.

2 Uvangale ajugaringitamnit piksanik kattersoilaukpunga Nalekab iglunganut, Goldemik perkutinut Goldeôjuksanut, Silbere Silbereôjuksanut, kannujarmik kanujangôjuksanut, kikkiangmik kikkiangôjuksanut, kejungmik kejungôjuksanut, ujarkanik Onichemik attelingnik, ujarkaniglo aglalingnik taksepatânik ivertisimajunik, ânanausijutiksanik, ujarkaniglo piviarnartunik sunatuinarnik, ujarkaniglo Marmoremik attelingnik unuktunik. 
3 Tapkoalo tappilugit alliasungnermut Gudib iglunganik, perkutimnik nangminerjamnik, Goldemik Silberemiglo,

4 Talentinik Goldemik 3000 dinik Ofiremit pijunik, Talentiniglo 7000 dink Silberemik illakangitomik, tunnijijomarunga Gudima iglunganut, iglut sennerangita illupiarutaksanginut.

5 Goldeôlerkovlugit Goldeôjuksat, Silbereôkovlugillo Silbereôjuksat. Pikkariktut sennajanginut tamainut. Kinalo mana kunnujuikâ uvlome tigumiaralijarlune tikkilune Nalegak pivlugo?

6 Tagva kunnutuilaukput atâtat angajokangit, kinguvarêllo angajokangit, angajokait 1000 dinut, angajokaillo 100 dinut, angajokaillo attanniub sennajaukojanginut.

7 Tunnijilutiglo attoraksanik Gudib iglunganut, Talentinik 5000 dinik, 10000 diniglo kênaujanik Goldeôjunik, Talentiniglo 10000 dinik Silberemik, Talentiniglo 18000 dinik kannujanik, Talentiniglo 100000 dinik kikkiengmik.

8 Kinalo nachvarviôjok ujarkanik, tamna tunnijivok tapkoninga Nalekab iglungata perkutitalinganut Jehielib Gersoniteôjub agganginut.

9 Innugiartoksuillo tunnergutitik kunnutuilutik tunnijatik kuviasutigivait, tunnigamigit Nalekamut omamut tamânut, kunnutuilutik. Attannerlo Davidetauk kuviasuvaksoangôlaukpok.

10 Davidiblo Gude nertorpa, okarlunelo illagêt illunamassiatik sângine: Igvit Nalegak Israelib atâtapta Gudinga nertortaulerit soraijuitomik.

11 Igvit, anginek, pitsartunerlo ânanaunerlo, sâlakarnerỉo, ussornarnarnerlo pigivattit. Illunaita killangmêtut nunamêtullo nangminerigangne. Igvit Nalegak nalegaunek pigivat, pôktojovaksôvotit angajokaksoangôlutillo illunainut.

12 Akluinerlo opigijaunerlo kênarnit pivok, igvillo attanniotivotit tamainut; aggangnelo pitsartunekarpok, sôngonekalarmelo; aggangnêporlo angijorortitsinek, sokkosijuititsinerlo.

13 Mannalo Gudivut kujagivaptigit, attillo ânanaudlartok nertorpavut.

14 Uvangale sunauvîk? innukotikkalo sunauvât, sappingituksaugapta tunnergutit taimaitut kunnutuidluta tunnilugit? Illingnille pivut tamaita, aggangnillo tunnilaukpavut illingnut.

15 Pigigaptigut, tujormiangolutalo sângne iglomekarvigivaptigillo, atâtaptitut illunaititut. Uvlovut mâne nuname tachaktut îput, nokkangatsengilello.

16 Nalegak Gudivut, kattititaumajut tamaita tunnimajavut igvit iglomik iglulioromavlutit attit heiligeôjok pivlugo, aggangnit pimavut, tamaitalo pigivattit.

17 Nellungilanga Gudiga omat ôktorangne, nellagôrnerlo kuviagigangne. Tamanna pivlugo tamakkoa tamaita (kunnutuidlunga) omamut nellagôrtomut tunnilaukpakka, kuviasuklungalo 
manna tækkolaukpunga innukotitit manêtut, kunnutuidlutik tunnitsivigilaungmattit.

18 Nalegak, atâtagijapta Abrahab, Isaakiblo, Israeliblo Gudingat, innukotivit omattingine taimaitunik issumakartitsitsainalaurit, erkarsautekartitsitsainarlutillo issokangitomut, omattingillo torartilaukit illingnut!

19 Erneralo Salomo omamik nellagôrtomik tunnitsivigilauruk, perkojatit kigligiudsinitillo idluarijattillo naleklugit pikovlugo, tamainik pikovlugo, igluliorkovlugolo igloksoarmik ominga, attuinarutilauktamnik.

20 Davidelo illagêt illunamassianginut okarpok: Nalegak Gudise nertorsiuk! Illagêllo illunamassiatik Nalegak atâtagijamik Gudingat nertorpât, okkolutiglo opigilakillo Nalegak attannerlo;

21 Tunnijilutiglo Nalekamut tunergutinik; uvlublo aipangata uvlângoningane, tunnijivut tunnergutinik ôtaksanik tukturângnik 1000 dinik, saukanik angusallungnik 1000 dinik, saugârsungniglo 1000 dinik, tunnergutiksat immiksat illautilugit, tunnergutiksallo assingit sulle unnuktovaksuit Israelitit illunamassiatik pivlugit.

22 Nerrivullo immerlutiglo uvlorme taipsomane Nalekab sângane kuviasuvaksoadlarlutik. Salomolo Davidib erninga attanningortipsarpât, mingoarlugolo attanningortipât Nalekamut, Zadokelo ajochertuijersoangortipât.

23 Taimak Salomo ingipok Nalekab iksivautaksoanganut, attanniovlune atâtane Davide innangêrdlugo, pilloridlalaukporlo; Israelitillo illunamassiatik nalekpât.

24 Angajokâksuillo songojullo illunatik attanniublo Davidib erningita illunatik attannek Salomo attannerilerpât.

25 Nalekablo Salomo angijoksotipa Israelitit illunamassiatik sângine, tunnitsivigivâlo attannionerub ânanaudlarninganik, tapsoma sivorngane Israelitit attanningita nelliata pigilaungitanganik.

26 Davidele Isaib erninga attanniôlaukpok Israelitinut illunamassianginut.

27 Attanniôtiningatalo nelliuninga Israelitinut, Jahreôvut 40 git. Hebroneme attanniotilaukpok Jahrinik 7 nik, Jerusalemêlo Jahrinik 33 inik.

28 Tokkolunelo innutokaunerme idluartome, kiglimorlune, akluidlarlune, opigijaulunelo. Erningalo Salomo attanningorpok innangêrdlugo.

29 Attanniuble Davidib illuserivalauktangit, sivorlit kingorlîllo, ahâk, makkoa aglagutigijaumavut Samuelib tækkojub unipkausingine, nellautaijublo Nathanib unipkausingine, Gadiblo tækkojub unipkausingine, 
30 Attanniotininga pitsartunekarningalo, nelliunerillo kângertingit, Israelitillo attanniovillo nunane illunatik kângertingit, illaliudlugit.

\section{KRONIKAB AGLANGITA AIPANGIT.}

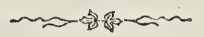

\section{KAPITEL I.}

Salomob tunizïninga tunnergutılssamile Gibeame, sinnektomaningalo, akluiningalo.

SALOMOLO, Davidib erninga pallangêrtaulaukpok nalegauvingmine, Nalekablo Gudingata aipariva, angijovaksôtipalo.

2 Salomoblo Israelitit illunamassiatik okarkattigivait, angajokâksuit 1000 dinut, 100 dinullo, idluarsaijullo, Israelitillo angajokangit illunaita, atâtat anginerôjungit,

3 Aikovlugit, Salomo, illagêllo illunamassiatik illagilugo pôktôjomut Gibeamêtomut; Gudib kattimavinga, tuppek, Mosesib, Nalekab kivgangata sennalauktanga sunakajuitome, tagvanêmat.

4 Davidible Gudib iklervinga takpaungartilaukpa Kiriatjearimemit, tagvunga Davidib âkiksoivigilauktanganut tapsomunga, tuppermik tuppertârlaungmago Jerusaleme.

5 Altarele kannujaujok, Bezaleelib Urib erningata, Hurib erningata sennalauktanga, tagvanêlaukpok Nalekab iglungata sângane; Salomoblo, illagêllo tamna nellipsarpât.

6 Salomolo tunnijivok Altareme kannujaujome, tuppiub kattimaviôjub sânganêtome Nalekab sângane, tunnergutinik ôtaksanik 1000 dinik.

7 Unnuarmele taipsomane Gudib Salomo sakkervigiva okautilâlo: Tuksiarit, sunamik tunnitsivigijaksarigapkit?

8 Salomoblo Gude okautiva: Napkoanersoarmik atâtaga Davide pinniarvigilaukpat, uvangalo taimna innangêrdlugo attanningortilaukparma; 
9 Tagva manna Nalegak Gude okautsitit atâtamnut Davidemut nellautsitikittôk! Uvânga attanningortilaukparma innugiartoksoarnut taimak unnuktigijojunut nunab sanningititut.

10 Tagva manna tunnitsivigilaunga sillatunermik, illitarksinermiglo, innugiartoksuit tapkoa sângine annikovlunga itterkovlungalo; kiale ukkoa innugiartoksoakotitit idluarsarungnarkagit?

11 Tagva Gudib Salomo okautiva: Taimaitunik issumagavit, akluinermiglo perkutinik, nertortaunermiglonêt, omigijivillo tokkunginik, innôtsemiglo sivitôjomik tuksiarlaunginavit; sillatunermigle, illitarksinermiglo, innukotikka attanningortitsijutigijakka illingnik idluarsarungnarlugit :

12 Tagva sillatunermik illitarksinermiglo tunnitsiviomavotit; tamakkoalo tappilugit akluinermik, perkutiniglo, nertortaunermiglo tunnitsivigijomavagit, attannît akkorngane adsekalaukonak sivunerne, kingunernelo, pittakarkonaglonêt.

13 Taimaglo Salomo poktôjomit Gibeonemêtomit tikkipok Jerusalemut, Nalekab tuppingata sânganit; attanniotilunelo Israelitinut.

14 Salomolo kattersoivok kamutingnik aksalloalingnik, ablangajuniglo horsit kângine, kamutekutekalaukporlo 1400 dinik, ablangajokutelaukporlo horsit kângine 12000 dinik, innekarkovaillo iglugaseksoarne kamutingnut illingajune, Jerusalemelo attanniub najugâne.

15 Attanniublo Silberit Goldillo Jerusaleme taimak unnuktigijotipait ujarkatitut, Cederillo nappartutitut Sikomorinik attekartutitut naksarne perortutitut.

16 Salomolo âtsiviolaukpok horsenik Egiptenemit, perkutiniglo niuviaksanik sunatuinarnik. Attanniublo niuvertekotingita niuviaksat tamakkoa pissiarivait.

17 Kaitsiyullo Egiptenemit kammutingnik, attautsingnik kênaujanut Silberlingenik attelingnik 600 dinut, horsemiglo attautsemik Silberlingnut 150 ginut. Taimak âtsivuttauk Hetiterit attanninginut illunainut, Siriamiullo attanninginut illunainut

\section{KAPITEL II.}

Salomob angerkattauninga Tirusemiut attanninganut, Tempelib igloliortauninga pivlugo.

Salomolo issumarok Nalekab attinga iglomik iglolioromavlugo, iglomiglo attannionime innigijaksanganik.

2 Kittitsivorlo angutinik akjarsijunik 70000 dinik, nakkart- 
sijuniglo nappartune 80000 dinik, aulatsijuniglo tapkoninga 3600 dinik.

3 Salomolo tilliklerpok Hiramemut, Tirusib attannertanganut, okautsaukovâlo: Sorlo atâtaga Davide pinniarvigilaurangne, tillikliutjivigilugolo Cederinik igloliorungnarkovlugo ingminut, taimailiorvigilaungatauk.

4 Ahâk, Nalekab Gudima attinga iglomik iglolioromavara, ivsornaititaujutiksamik tapsomunga, isserluanijutiksanik tippitsiariktunik ikkitsiviksamik sângane, kakkojaniglo tækkojaksanik âkiksoiviksamik, tunnergutiksaniglo ôtaksanik tunnijiviksamik uvlâne unnunelo, Sabbatinelo, takkêlanelo, Nalekablo Gudipta uvloksiorvingine. Soraijuitomik Israelitit taimailioriakarput.

5 Iglolo iglolioromajara angijojuksaurok; Gudivut anginersaungmat Gudinit tamainit.

6 Kinale ajoksangilâk tamna igloliorlugo? Killaub killaillo killangita illunamassiatik illumajungnangilât. Uvangalo kinaujuksauvîk igloliorungnarlugo? kissiane, isserluanititsivigilugo.

7 Tagva manna tilliklervigilaunga angumik sillatôjomik, sungiusimajomik Goldelerilune, Silberelerilunelo, kannujalerilunelo, kikkitjerilunelo, Purpurelerilunelo aupaluktamik, Karmesinelerilunelo, kaujimajomiglo ittersaliorlune kepperuklune illissimajut Judamêtut Jerusalemêtullo atâtama kaikolauktangit illagilugit.

8 Tillikliutjivigilaungalo nappartunik Cederinik, Cipressiniglo Sandeliniglo Libanonemit; nellunginama kivgakotitit sungiusimangmatta nappartut nakkarlugit Libanoneme; a.lâglo, kivgakotima kivgakotitit illagijaksarivait,

9 Attuinaruiviokovlunga kejungnik unnuktunik; iglo iglolioraksarijara angijojuksaungmat pijariakortôjuksaungmallo.

10 Ahâglo, kivgakotingnut, nappartunik nakkarsijunut tunnijijomavunga Korinik 20000 dinik Weizenik tugaksimajunik, Koriniglo 20000 dinik Gerstinik, Bathiniglo 20000 dinik Weinemik, Batheniglo 20000 dinik orksumik.

11 Tagva Hirame Tirusemiut attanningat aglaktigut okarpok, tilliklerlunelo Salomomut: Nalekab innukotine nagligingmagit, igvit attanningortilaukpâtit tapkonunga.

12 Hiramelo okaranerpok: Nertortaule Nalegak Israelib Gudinga, killaub nunablo pingortingak, attannek Davide tunnitsivigilaungmago ernermik sillatôjomik pokitomiglo illissimajomiglo, igloliorniartomik igluksamik Nalekamut, attannionimelo igluksanganik.

13 Mannalo tilliklerpunga angumik illissimajomik, sillatunelingmik, Hurame-Abifemik,

14 Erniojomik arnamut Danib panninginit pijomut, atâtangale Tirusemiôlaukpok, tamna Goldelerijungnarpok, silbere- 
lerijungnarlarmelo, kannujalerijungnarlunelo, kikkitjerijungnarlunelo, ujaralerijungnarporlo, kejulerijungnarlunelo, Purpurelerijungnarlarmelo aupaluktamik, Karmesinelerijungnarlunelo, ittersaliorungnarporlo kepperuklune sunatuinarnik, sunatuinarniglo ajornartunik, sennajaukojaujunik tapsomunga sennajungnarpok, sillatojokotitit, nalekamalo Davidib sillatojokotingit illagilugit.

15 Mannalo nalegara tillikliutjile Weizenik, Gersteniglo, orksumiglo, Weinemiglo kivgaminut, sorlo okalaurame.

16 Tagva kejuit nakkaromavavut Libanoneme, pijariakarningititut unnuktigijut, kallingortitautilugillo immarbikut âtsomavavut Joppemut; tagvangallo âtaukojungnarpattit takpaunga Jerusalemut.

17 Salomoblo tujormitjet, Israelitit nunanganetut kittipait, atâtame Davidib kittitsiningata tapkoninga unnurningit malliklugit, nachvartauvullo 153600 dit.

18 Tapkonangallo 70000 dit akjarsijungortipait, 80000 dillo nakkarsijungortipait kakkame nappartune, 3600 dillo aulatsijungortilaita, aulujititsijut innugiartoksoarnik sullijaksane.

\section{KAPITEL III.}

\section{Tempelib igloliortauninga.}

SALOMOLO pigiarpok Nalekab iglunga igloliorlugo Jerusaleme, kakkame Morijamik attelingme, atâtaminut Davidemut unertutigijaulauktome, inneme Davidib âkiksorlauktangane, Arnanib Jebusitereôjub kakkojaksaliorvingane.

2 Pigiarporlo igloliorlune attanniotime Jahringita sittamangata takkingita aipangata uvlungita aipangane.

3 Tungaviglo Salomob illilauktanga Nalekab iglungata igloliortauninganut imailaukpok: tukkimutoninga tallitut 60 gitut, sennimutoninga tallitut 20 gitut.

4 Torksuksoarlo iglub sennimutoningata sânganêlauktok, tallitut 20 gitut takkitigijolaukpok, pôktoningale tallitut 120 gitut îlaukpok; illuanelo illupiarutserpa Goldemik illakangitomik.

כ Iglolo angijok illupiarutserpa sâtujanik Cipressiojunik, pekartipâlo Palmingoanik, kellaumgujangoaniglo.

6 Iglolo sillapârpa ujarkanik pivianartunik ânanausijutiksanganut; Goldele attortaujok Parwaimemit pilaukpok.

7 Iglolo, ikkaitjallo, kattallo, iglublo sennerangit, upkoallo illupiarutserpait Goldemik, itterselliorkojilunelo sennerkane Kerubingoanik.

8 Iglolo ivsornainerpângojok sennagiva, tapsoma tukkimuto- 
ninga, iglub sennimutoninga malliklugo tallitut 20 gitut îlaukpok, sennimutoningalo tallitut 20 gitut îlaurivok; illupiarutserpâlo Goldemik pivianarnerpangôjomik Talentinik 600 dinik.

9 Kikkitsello okumainingit, Sekkelititut Goldeojutitut 50 gitut îlaukput. Innillo kollanêtut illupiarutserlaurivaittauk Goldemik.

10 Iglomelo ivsornainerpangôjome pittakârtitsivok Kerubingoangnik, sennangoarnik kepperuktut illusingit malliklugit sennajaumajungnik, sillapârpâglo Goldemik.

11 Kerubingoaglo esarungita takkingat tallitut 20 gitut îlaukpok, taimak esarok attausek tallitut tellimatut takkitigijolaungmat, aktoalune iglub senneranganut; esarkublo igloa tallitut tellimatut îlaurivok, aktoalunelo Kerubingoab aipata esarunganut.

12 Taimak Kerubingoab aipata esarukita aipa tallitut tellimatut takkitigijolaurivok, aktoalunelo iglub senneranganut, esarkublo iglua tallitut tellimatut îlaurivok, aktoalunelo Kerubingoab aipata esarunganut;

13 Kerubingoak esarungit issangatillugit tallitut 20 gitut takkitigijolaukput; nangerpuglo ittikamingne, kênatiglo sângavuk iglomut.

14 Upkomiglo sennagivok Purpuremit aupaluktomit, tungojoktamillo, Karmesinemillo, Bissusemillc, Kerubingoakartipâlo.

15 Iglublo sângane nappajokartitsivok maggungnik, tallitut 35 gitut pôktutigijungnik, niakojartomiglo kanganut nuvuanut, tallitut tellimatut pôktotigijomik.

16 Kellaumgujaniglo sennavok, taipkotitunak inneme kinguanetomêtutitut, illijilunelo tapkoninga nappajuk kângangnut. Paungâlungoaniglo Granatinik attelingnik 100 dinik sennavok, nivingatipaillo kellaumgujangoanut.

17 Nappajuglo nappartipâk Tempelib sângane, aipa tallerpingmut, aipalo saumingmut; tallerpingmetorlo Jachinemik atserarpa, saumingmêtorlo Boasemik.

\section{KAPITEL IV.}

\section{Tempelib perkutingitu illulerkutingitalo illangit.}

Altaremiglo kannujaujomik sennagivok, tallitut 20 gitut tukkimutotigijomik sennimutotigijomiglo, tallitullo tellimaujortutitut pôktotigijomik.

2 Immarbingoarlo sennava kovilugo, tallitut tellimaujortutitut silliktigijok, sinânit aipanganit, aipa tikkidlugo, angmaloriksarsimajok avatâne, tallitullo tellimatut poktotigijok; nimerlo tallitut 30 gitut takkitigijok namatuinarlaukpok avatane. 
3 Tukturangoakalaukporlo attâne, avatâne illunane; immarbingoablo tallitut tellimaujortutitut silliktigijolauktub avatâne, kuglungangoakalaukpok sennilerêngnik maggoinik, kovikassiutitaumalauktunik.

4 Nappavorle taimak tuktuvangoata kângine, tapkoa pingasut sângmitillugit avunga, pingasut paunga, pingasut aunga, pingasullo taununga; immarbingoarlo kânginelaukpok; okpatingillo illunatik illumut torarput.

5 Ivjoningalo aggaktitut igluînartut sennimutoningititut îlaukpok, sinnâlo ermgusiarsub sinningatut îlaukpok, Lilietut sigisimajotut-îdlune; illulekarungnarlaukporlo Bathenik 3000 dinik.

6 Sennavaillo ukkusit tellimaujortut tapkoa tellimat makkitipait tallerpingmut, tellimallo saumingmut erchortuiviksauvlutik, illulerkutit tamaita tunnergutinut otaksanut attortaulauktut erchortorpait tapkonane; immarbingoarle ubvarviksaulaukpok ajochertuijunut.

7 Sennavaillo nennergoabvit Goldeôjut tellimaujortut, illingajuksauningit malliklugit, pitipaillo Tempelemut, tellimat tallerpingmut, tellimallo saumingmut.

8 Sâlo tellimaujortut-sennavait, pitilaitalo Tempelemut; tellimat tallerpingmut, tellimallo saumingmut. Sennavaillo pogutat Goldeôjut 100 dit.

9 Sennavâlo ajochertuijut inningat avaluliorsimajok, avaluliorsimajorlo angijok, upkoallo avaluliorsimajomut angijomut, upkoallo sillapârpait kannujarmik.

10 Immarbingoarlo nappartipa iglub tallerpiane kittâne sekkerngane.

11 Hiramiblo ukkusit, poargillo, pogutallo sennavait; Taimaglo Hiramib sennajaksat, sennajane attannermut Salomomut, Gudib iglungane innerpait:

12 Tagva nappajuk, niakojartuglo nuvuangnetuk kângangne illagilugik; nulloangoaglo maggoit niakojartuk nappajuk nuvuangnêtuk aksaujakita ulliksangit,

13 Paungâlungoallo Granatit 400 dit nulluangoanut, Granatit sennilerêt maggoit nulluangoanut attautsinut, niakojartuk aksaujakita ulliksanginut.

14 lkkovrallo sennavait, ukkusillo, ikkovrat kânginêtut.

15 Immarbingoak, tuktuvaillo 12 it tapsoma attânêtut.

16 Ukkusillo, poargillo, kappukautillo, illulerkutingillo tamaita illagilugit Huram-Afibib sennavait attannermut Salomomut, Nalekab iglunganut illingakovlugit, kannujarmit illakangitomit.

17 Jordanib erkâne Sochotib Zartaniblo akkorngangne attanniub kovijaukovait macharme. 
18 Salomoblo illulerkutit tapkoa illunaita sennavait unnuktovaksovlutik; kannujangita okumainingit kiktitartaujungnangimatta unnumut.

19 Salomoblo perkutit tamaita Gudib iglungata illuanut illingajut sennavait: tagva Altare Goldeôjok, sâlo, kakkojallo tækkojaksat kânginetuksat;

20 Nennergoabvit, kollingit illaliudlugit, Goldemit illakangitomit, ikkoalakovlugit ivsornainerpângojub sângane, sorlo illusiojuksaungmat;

21 Nuvugolangoangallo kollerne, takkersutillo Goldemit; tapkoa illunatik Goldeolaukput;

22 Sâvillo, pogutallo, allupsautillo, kallutaujallo isserluanijutiksat Goldeôlaukput. Itterviglo upkoangalo illuane ivsornainerpangôjomut, upkoallo iglome Tempeleojome Goldeolaukput.

\section{KAPITEL V. \\ Tempelib attortautainarninga.}

TAIMAGLo sennajaksat Salomob sennajangit Nalekab iglungane innertaumalaukput. Salomoblo ittertipait tamaita atâtame Davidib ivsornaitilauktangit, tagva Golde Silberelo perkutillo sunatuinait, illivaillo perkutitalingmut Gudib iglunganêtomut.

2 Tagva Salomob Israelitit angajokangit illunaita, kinguvarêt angajokaunerôjungit, atâtallo attanningit, kattikovait Jerusalemut, Nalekab angerningata iklervinga âtaukovlugo takpaunga Davidib iglugaseksoanginit, tagva Zionemit.

3 Kattimalerpullo attannermut Israelib angutingit illunamassiatik uvloksiorvingme, tagva takkit 7 ingane.

4 Israelitillo angajokaunerôjungit illunatik tikkiput. Levitillo iklervik erksukpât,

5 Apâllo takpaunga, tuppek kattimaviojok, perkutillo ivsornaitut tuppermetut illaliudlugit, tapkoa ajochertuijut Levitillo takpaungartipait.

6 Attannerlo Salomo, illagêllo Israelitit kattimalauktut tapsomunga, iklerviub sânganelaukput tunnijilutiglo tunergutiksanut saukanik, tuktuvangniglo, taimak unnuktigijunik, kittitaujungnarlaungimatta unnumut.

7 Ajochertuijullo Nalekab angerningata iklervinga ittertipât takkamunga inniksanganut, innemut iglub kinguanêtomut, ivsornainerpangôjomut, Kerubingoak esarungita attânut.

8 Kerubingoaglo esarkotik issangavait iklerviub inningata kollâne, ullikpaillo iklervik akkerautigiglo kollânit.

9 Akkerautiglo taimak takkitigijolaukpuk, issungit tækkojaulaungmatta iklervingmit ivsornaitovingmit, inniub kinguanê- 
tub sângane; igluble sivuanit tækkojaulaungilet. Tagvanêtuarporlo uvlok tamanna tikkilugo.

10 Pittakalaungilarlo sunamik iklervingme aglagvik ujaraujuk assigingnik, Mosesib illilauktakita takkamunga Horebeme, Nale$\mathrm{kab}$ Israelib kittorngangit angerkattigilaungmagit aulartillugit Egiptenemit.

11 Ajochertuijullo annilertillugit ivsornaitovingmit (ajochertuijut najutijut illunamassiatik sujungaitilauramik, ingmigolingatitauningit kamagijaulaungilet),

12 Levitillo imgerpaktut, tapkoa illunatik, Assafe, Hemanelo, Jedithunelo, erningillo, kattangutingillo, annorarsimavlutik sâtunik, Cimbelijarlutik, Psalterilijarlutiglo, Harfelijarlutiglo nangerput Altarib kittâne, najortanginelo ajochertuijut 120 git Trompetesijartut;

13 Imailaukporlo attausênak Trompetesijarajarune imgerajarunelo, nippe attausênak tussartaunajarune Nalegak nertorlugo kujagilugolo. Trompetillo, Cimbelillo nokakseraggillo sunatuinait, nertordlertullo Nalekamik, pitsiarmat napkiningningalo sorairniatsengimat issokangitomut, nippingit pigialermatta: tagra Nalekab iglunga illumertaulerpok nuvujarmik,

14 Ajochertuijut nangerungnarlaungimatta kivgartorlutik nuvujak pivlugo; Nalekab ânanauningata Gudib iglunga illunât nâmangmago.

\section{KAPITEL VI.}

\section{Salomob tuksiarninga Tempele atiortautainartillugo.}

TaIPSOMane Salomo okarpok: Nalegak okalaukpok innekaromagame tâktome.

2 Uvanga iglo igloliolauralloarpara igloksarnut, innelo innigijaksarijat soraijuitomik.

3 Attanniublo kênane allarpa, illagêllo Israelitit illunamassiangit saimartipait, illagêllo Israelitit illunatik nangerput tagvane.

4 Okarporlo: Nertortaule Nalegak Israelib Gudinga, kannerminut okalauktok atâtamnut Davidemut, aggangminullo nellautsititsilauktok, okalaurame:

5 Nelliutomit taipsomangamit aulartitsivigilauktamnit innukotimnik nunamit Egiptenemit, iglugaseksuit nellianik annerosulaungilanga Israelitit kinguvarêngine illunaine, iglo igloliortaukovlugo, attera tagvanêkovlugo, angullo annerilaunginivara attanniokovlugo innukotimnut Israelitinut.

6 Jerusalemele annerilaukpakka attera tagvanekovlugo; Davidelo annerilaukpara angajokaukovlugo innukotimnut Israelitinut. 
7 Davidelo issumalaungmat Nalegak Israelib Gudingata attinga iglomik igloliorlugo,

8 Nalegak atâtamnut Davidemut okarpok: Namaktullilaukpotit issumagavit attera iglomik iglolioromavlugo.

9 Igville iglo tamna iglolioraksaringilat, erniville illingnit piniartub, tapsoma attera iglolioraksariva.

10 Taimak Nalekab manna okautsine okauserilauktane nellautsitimavait, uvangalo atâtaga Davide innangêrlaukpara, iksivalungalo Israelib iksivautaksoangane, sorlo Nalekab okalaungmat, iglolo igloliorlaukpara Nalekab Israelib Gudingata attinganut;

11 Iklerviglo takkamunga pitilaukpara, Nalekab angerninganik pittalik, angerlauktanganik Israelib kittornganginut.

12 Salomoblo Nalekab Altarekotinga sangariartorpa, illagêt Israelitit illunamassiatik najutitillugit, agganelo issangmivait.

13 Salomoble ikkovrak kannujaujok sennalaukpa, nappartilaukpâlo inniub avaluliorsimajub kerkânut, tallitut tellimatut tukkimutotigijok, sennimutotigijorlo, tallitullo pingasotut pôktotigijok; tapsoma kânganut nangeriartorpok, serkortorlunelo illagêt Israelitit illunamassiatik sângine, agganelo issangmivait killangmut,

14 Okarlunelo: Nalegak Israelib Gudinga, Gudekangilak . illiktut killangmêlonêt, nunamêlonêt; sokkosijuititsijotit angernermik, napkiningnermiglo kivgakotingnut, agvertartunut sângne omamut tamânut.

15 Nellautsititsikauvotit kivgakotingnut Davidemut, atâtamnut okauserilauktarnik tapsomunga, kannernut okauserilaukpat, aggangnullo nellautsitilaukpat, sorlo uvlorme tamattomane-îmat.

16 Manna Nalegak Israelib Gudinga, kivgait Davide, atâtaga, sokkosijuititsivigilauruk okauserilauktarnik tapsomunga, okarlutit: Ajoksartuksaungilatit angumik, sâmne iksivajomik Israelib iksivautangane, ernitit kammatsiartuinarpatta apkotigijamingnik, agvertarkovlugit perkojamne, sorlo igvit sâmne agvertarlauravit.

17 Manna Nalegak Israelib Gudinga, okautsitit miksekâxtotikit, okauserilauktatit kivgakotingnut Davidemut.

18 Gude nellâgut innekaromagalloarka innungne nuname? Ahâk killaub killaillo killangita illunamassiatik illumajungnangilatit, kannok tagva iglo una igloliorlauktara pijungnarajarka tamattominga?

19 Sâlaurilletôk Nalegak Gudiga, kivgakotivit tuksiarninganut, kennuninganullo, tussarkovlutit kennunernik tuksiarnerniglo kivgavit attortanginik sângne,

20 Ijikik uitatsainarkovlugik uvlotillugo unuarmelo iglomut omunga, innemut okautigilauktarnut, attit tagvungatitsomagang- 
ne, tussarkovlutit tuksiarnermik, kivgavit tuksiarnerijanganik, inneme tamattomane.

21 Taimak tussalaurit manna kivgavit innukotivillo Israelitit kennuninginik, kennuneriniartanginik inneme omane: tussalaurugle innemit innigijarnit, killangmit, tussarungnelo issumagijungnainilaurit.

22. Kina idluitullirangat innukattiminut, angermaringnermiglo illitsiviokpat, attoraksanganik, angermaringnerlo âtaulerpat Altarekotivit sânganut, iglome omanêtub,

23 Tagva igvit tussalaurit killangmit, pinniarlutillo, kivgatillo erkartorkit, passijaksak akkiniarlugo pikovlutit, agvertarningalo pitilugo niakoanut, idluartorlo idluarnerarlugo pikovlutit, tunnitsivigilugolo idluarninga malliklugo.

24 Innukotitillo Israelitit sâlagijaulerangatta omigijimingnut, idluitullilauramik illingnut, sâgiarpattalo, attillo kigligiutigilugo pikpatta, tuksiarpattalo kennulutiglo illingnut iglome omane;

25 Tagva igvit tussalaurit killangmit, issumagijungnailugillo innukotivit Israelitit ajorningit, uttertilugillo nunamut, tapkonunga, atâtagilauktanginullo tunnilauktarnut.

26 Killak parnanairsimalerangat sillaluluganelo, idluitullilaungmattalo illingnut, tuksiarniarpattalo inneme omane, attillo kigligiutigilugo pikpatta, sâpattalo ajornermingnit manigotitigangne,

27 Tagva igvit tussalaurit killangme, issumagijungnailugillo kivgakotivit innukotivillo Israelitit ajorningit, ajochertorlaukârdlugit apkosinermik ajungitomik, agvertarvigijaksarijanganik; tunnijilutillo sillalungnermik nunakotingnut, tunnilauktarnut innukotingnut, innigijaukovlugo tapkonunga.

28 Kâvikalerangat nuname, nûsilingnartokarangallonêt, perorsijat kakkojaksat kernersilerangattalonêt, piglertaujartokadlarangallonêt nerrijoksoarnik, omigijingitalonêt nuname itter. tarvingit illumajarangagit, pagvinartokarangallonêt, kannimasekarangallonêt nellianik;

29 Tuksiarnerit tamaita, kennunerillo tamaita, tagva pinniartut, innuit nellianut, innukotingnullo Israelitinut illunainut, missigilerpatta illunatik attunît pagvisartaunermingnik, ânianermingniglo, aggatiglo issangminiarpagit iglomut omunga ;

30 Tamanna igvit tussalauruk killangmit, innemit innigijarnit, issumagijungnainilutillo, illunaitalo attunît pinniarvigikit apkosinerilauktangit tamaita malliklugit, sorlo omattingit illitarigangne; igvit kissivit innuit kittorngangita omattingit illitarigangne.

31 Sivoralerkovlugit illingnik, agvertarkovlugillo apkosinekotingne uvlut tamaita, innotillugit nuname tunnilauktarne atâtagijaptingnut.

32 Tækkorngartattauk nelliat, innukotingnut Israelitinut 
illingalungitok, tikkilerangat nunamit kanningitomit, attit angijok, aggaktillo pitsartojut, tallillo issangmijok pivlugit, tikkipallo tuksiarlunelo iglomut omunga;

33 Tagva igvit tussalaurit killangmit, innemit innigijarnit, pinniarniarlutillo tamainik nipliarutigijanganik illingnut, innukattigêksuit nunamêtut illunatik atternik illitarksilerkovlugit, sivuragilutillo pikovlugit, innukotititut Israelitititut, tukkisilerkovlugillo iglo una, igloliolauktara, attit malliklugo atserartaungmat.

34 Innukotitit aularniarangatta unatadlarnermut, omigijitik sorsugvigilugit, apkome tilliklerutigijarne tapkoninga, tuksiarpattalo illingnut, iglugaseksuit ukkoa annerilauktatit sâdlugit, iglolo igloliolauktara atternut sâdlugo:

35 Tagva igvit tuksiarningit, kennuningillo tussarlaukit killangmit, idluartomiglo sakkêvigikit.

36 Idluitulliniaraikpatta illingnut, (innukalungimat idluitullilungitomik) illulugvigigungnelo, tunnilugillo omigijinginut, sâlagijingitalo parngnanairsortauvlutik aularutikpagit nunamut kanningitomut kannitomullonêt;

37 Omamiutarilerpatsuglo nuname parngnanairsimavigijamingne, sâlerpattalo, kemnulerpattalo illingnut, nuname parngnanairsimavigijamingne, okarlutiglo: Idluitullilaukpogut; tammarlauklutalo, innorlulaūkpogut;

38 Taimaglo sâlerpatta illingnut omamut tamânut, nuname parngnanairsimavigijamingne, parngnanairsortauvlutik aulartitauvigijamingne, tuksiarpattalo sâlutik nunagijamingnut atâtagijanginut tunnilauktarnut, iglugaseksoarnullo annerilauktarnut, iglomullo igloliorlauktamnut atternut;

39 Tagva igvit tuksiarningit, kennuningillo tussarlaukit killangmit, innemit innigijarnit, idluartomiglo sakkêvigikit, innukotitillo issumagijungnaivigikit idluitullininginik illingnut.

40 Taimak manna Gudiga ijikik uitalaulik, siutikiglo kammatsiarlaulik, tuksiarnermik inneme omane.

41 Mannalo Nalegak Gude kângatarit innemut merngoêrservingnut, igvit, pitsartunivillo iklervinga. Ajochertuijokotitit Nalegak Gude attititaulit piulijaunermik, idluarijatillo kuviasulerlit ajunginek pivlugo.

42 Igvit Nalegak Gude mingoartaukojavit kênanga allârtiniarnago; erkailaurit saimanermik angerutigijaumajomik kivgakotingnut Davidemut. 


\section{KAPITEL VII.}

Tempelib attortautainarningata nâninga. Gudiblo sakkerninga Salomomut, angerningillo sillungningillo.

SALOMOBLO tuksiarnek nâgamiuk, ikkoma kattakpok killangmit, nerrilunelo tunnergntinik otaksanik, tunnergutiniglo tokkotaksanik; Nalekablo ânanauningata iglo nâmalerpa;

2 Ajochertuijut itterungnarlungimatta Nalekab iglunganut; Nalekab ânanauningata Nalekab iglunga nâmangmago.

3 Israeliblo kittorngangita illunatik ikkoma tækkovât kattaktillugo, Nalekablo ânanauninga iglıb kollâne, okkolerlutiglo kênatik pûvlugit nunamut, opigosulutiglo. Nalegarlo kujagivât pitsiarmat, napkiningningalo sorairniatsengimat.

4 Attannerle innugiartoksuillo illunatik tunnergutinik tunnijivut Nalekab sângane.

5 Attannerlo Salomo tunnijivok tuktuvangnik 22000 dinik, saukaniglo 120000 dinik, taimaglo Salomob innugiartoksuillo Gudib iglunga attortainarlugo ivsornaitipât.

6 Ajochertuijullo nangerput pinniaraksamingne, Levitillo Nalekab nokakseralikotinginik, attanniub Davidib sennajaukolauktanginik attorlugik, Nalegak kujalivigilugo, napkiningninga soraijuimat, Davide imgerlune nertordlerlaurangat tapkutiguna. Ajochertuijullo akkingerlugit Trompetesijarput, Israelitilio illunamassiatik nangerput.

7 Salomoblo avaluliorsimajok ketterdlek, Nalekab iglungata sivurânctok ivsornaitipa, tunigamigit tagvane tunnergutit otaksat, tunnergutillo kujalijutiksat, Altare kannujaujok Salomob sennajaukolauktanga mikkiluarmat tunnergutinut otaksanut, tunnergutinullo nerkiksanut, tunnergutilıo kujalijutiksat orksunginut.

8 Taimaglo Salomob nelliutome taipsomane uvloksiorvik attorpa uvlut 7 it nâlugit, illagællugolo Israelitit illunamassiangit, illagêt unnuktovaksuit Hemathemit Egiptenib kônga tikkidlugo.

9 Uvlullo 8 ingane kattimaniksakarput; Altarible attortautainarninga uvloksiorpât uvlut 7 it nâlugit, uvloksiorviglotauk uvlut 7 it nâlugit.

10 'Takkillo 7 ingata uvlungita 23 gingane innugiartoksuit angerarkovait iglunginut kuviasuktillugit maksoatillugillo, ajungitut tamaita pivlugit Nalekab sakkertilauktangit Davidemut Salomomullo, innukotiminullo Israelitinut.

11 Taimak Salomob Nalekab iglunga, attanniublo iglunga innerpâk, tamaitalo omattime illumigijangit sennajomavlugit Nalekab iglungane, iglominelo sullivait. 
12 Nalekablo Salomo sakkervigiva unnuarme, okautilâlo: Tuksiarnît tussarlaukpara, innelo tamanna annerimavara uvamnut tunniorkaiviksaukovlugo tunnergutiksanik.

13 Ahâk, killak pallangairupko, sillalukonnago, piglertaujartullonêt nerrikogupkit nunamik, nussilingnartorlonêt tikkikogupko innukotima akkornganut,

14 Innukotikkalo attera malliklugo atserarsimajut nikkanarsarpatta, tuksiarpattalo, kênaralo kennerlugo pikpatta, sagiarlutiglo apkotigijamingnit idluitomit; tagva killangmit tussaromavunga, issumagijungnailugillo ajorningit, nunangallo innulilugo.

15 Manna ijikka uitatsainartuksauvuk, siutikkalo kammatsiartuksauvuk tuksiarnernik attortaujunik inneme tamattomane.

16 Taimaglo manna iglo tamanna annerimavara ivsornaitilugolo, attera tagvanêkovlugo issokangitomut, ijikkalo, omattigalo tagvanêtuksauvut nelliutune tamaine.

17 Sâmnelo agvertarniaruvit sorlo atâtait Davide agvertarlaungmat, pıniaruvit taimaluatsiamik sorlo perkogapkit, perkojakkalo idluarijakkalo malligungne;

18 Tagva attanniovikpît iksivautanga sokkosijuititsomavara, sorlo Davide atâtait angervigilaurapko, okalaurama: Ajoksartuksaungilatit angumik, nalegaujomik Israelitinut.

19 Allarniarupsele, idluarijakkalo perkojakkalo tunnilauktakka illipsingnut, kemmagupsigik, Gudiblo assingit kivgartoriartorupsigik, tuksiarvigilugillo pigupse;

20 Tagva nutsungniarpakka nunakotimnit, tunnilauktamnit tapkonunga, iglolo tamna ivsornaiviotilauktara attimnut, eginiarpara kênama sânganit, okausiovaktungortiniarparalo okangoarutingortiniarlugolo innukattigêksuit illunatik akkorngane.

21 Iglolo tamna pijariakortônerpângôlauktok, tapsominga sennerkotsijut kakkiladlarutigijomârpât, okoromârlutiglo: Suna pivlugo Nalekab nuna una, iglolo una taimaiiiorvigilaukpagik?

22 Okartokaromârporlo: Tamanna pivlugo, Nalegak, atâtagijamik Gudingat kemmalaungmatsuk, aulartitsilauktok tapkoninga Egiptenemit, Gudiblo assingit ungagilugit, tuksiarvigilugillo, kivgartorlugillo pilaungmatta; tamanna pivlugo tikkitaukolaukpait kannoêtomut mattomunga tamât. 


\section{KAPITEL VIII.}

\section{Salomob iglogaseliorninga; illusinga kivgartornerme Gudemik; umialctorningalo; tunnergutingillo.}

JAHRILLO 20 git, Salomob attorlauktangita Nalekab iglunga, iglunelo igloliorlugik, kingorngane,

2 Iglugaseksuittauk iglugaseliorpait Huramib tunnijangit Salomomut, Israeliblo kittorngangit nunakarkovait tapkonane.

3 Salomolo aivok Hemath-Zobamut, songotipaillo;

4 Tadmorelo sunakajuitome iglugaseliorpait, iglugaseksuillo kemmatulliviojut illunaita iglugaseliorlauktane Hematheme.

5 Iglugaseliorpaillotauk Beth-Horon kagvasiktok, atsiktorlo, iglugaseksuit sôngojut avaluksoaggit, upkoaksoaggillo, senneroaggillo.

6 Baelathetauk, iglugaseksuillo kemmatulliviojut Salomob pigijane illunaita, iglugaseksuillo illunaita kamutingnut, ablangajunullo horsit kângine illingajut, tamaitalo Salomob tussugijane sennajomavlugit, Jerusalemelo, Libanonemelo, attanniovimelo nunangane illunane.

7 Innugiartoksuit amiakovinît Hetiterinit Amoriterinillo, Feresiterinillo, Heviterinillo, Jebusiterinillo, Israelib kittornganginit pilaungitut,

8 Taipkoa amiakojut, Israelib kittorngangita mungulaungitangita kittorngangit Salomob sennajuksangortipait, uvlok tamanna tikkidlugo,

9 Israelible kittornganginit Salomo kivgangortitsilungilak kikunik sullijaksaminut illingajunik, sorsuktuksaulaukpulle, angajokaulutiglo Kapitanut, aulatsijolutiglo kammutekotinginik, ablangajokotinginiglo horsit kângine.

10 Aulatsijullo angajokaunerồjungit attanniub Salomob sullijaksanginik 250 giôlaukput, aulatsilauktut innugiartoksoarnik.

11 Faraublo pannia Salomob aijaukova takpaunga Davidib iglugaseksoanginit iglomut, (Salomob) igloliorlauktanganut tapsomunga. Okarame: Nulliara iglokartuksaungilak Davidib Israelib attanningata iglungane, ivsornaititaulaungmat, Gudib iklervinga takkamungartaulaungmat.

12 Taimangat Salomo tunnergutinik ôtaksanik tunnijivok Nalekamut, Nalekab Altaringata kângane, sennalauktamine, 1orksub sângane;

13 Tunnergutiksallo uvlunut tamainut illingajut, tunnivait Mosesib perkojangit malliklugit, Sabbatine, takkêlanelo, uvloksiorvingnelo, pingasuêrtorlune Jahreme, tagva uvlokssiorvingme 
kakkojanik sêrnakangitunik, Wochillo uvloksiorvinganelo, uvloksiorvingmelo iglokarvingmik akkerkunik millukattalingnik.

14 Tapsomalo ajochertuijut pitipait ingmigolinganingit malliklugit, pinniaraksanginut, sorlo Davidib oma atâtangata âkiksorlaungmagit; Levitillo sullijaksanginut, nertordlerlutik kivgartorlutiglo ajochertuijut sângine, uvlut tamaita illusingititut; upkoaksoaniglo udsertortut ingmigolinganingit malliklugit, attunît upkoaksoanganut. Taimak Davidib Gudib angutekotingata perkolaungmago.

15 Attanniublo perkojanga ajochertuijut Levitillo, sunatuinaillo pivianartullo pivlugit sennerkotaulaungilak.

16 Taimaglo Salomob pinniaraksangit tamaita pijarêrtitaumavut, Nalekab iglungata tunganilititauningata uvlunga tikkilugo, tapsomalo innertaumaninga tikkilugo, Nalekab iglunga innersimamarikârtinago.

17 Tagva Salomo aularpok Ezeon-Geberemut, Elotemullo immarbiksub siksanganêtunut, Edomemiut nunanganut.

18 Huramiblo tillikliutjivigiva umiaksoarnik kivgakotimigut, immarbiksoarmik illitarksijutigut; tapkoalo Salomob kivgangit illagilugit umiaktorput Ofiremut, aiklerlutiglo tagvangat Goldemik Talentinik 450 ginik, âtsilutiglo tamakkoninga Salomomut.

\section{KAPITEL IX.}

Salomob nellipsartauninga arnamut Arabiamiut attanninganut. Akluininga, tokkungalo.

A ARABIAMIULLO attanningata arnab Salomob tussaumajanga tussaramiuk, kaivok Salomo ôktoromavlugo nellaupsarutiksanut, tikkiporlo Jerusalemut, najortekarlune unnuktunik Kamelinik, mingoarutiksanik pivianartunik, Goldeniglo unnuktunik, ujarkaniglo pivianartunik nangmartunik. Salomomullo tikkiname okarvigiva tamainik, omamigijaminik.

2 Salomoblo tamainik okautiva apperkutigijanginik; nellonartokalaungilarlo sunamik Salomomut, okauserilaungitanganik tapsomunga.

3 Arabiamiullo attanningata arnab Salomob illissimaninga, iglolo igloliorlauktanga tækkogamigit,

4 Nerkiksallo tapsoma nerrimavianut illingajut, kivgakotingitalo iksivaningit, nangerningillo, naipertutsiarningillo, anorângillo, immiksaliorkotingillo, tapkoalo annorângit, majutingillo majoarutigijangit Nalekab iglunganut, tagva uimâmajotut-1̂lerpols. 
5 Attannerlo okautiva: Miksekârtôvok tussarlauktara nunakotimne illinganît illissimanîllo pivlugik.

6 Okausingille okperilaungilakka, tikkikârtinanga, tækkolugolo ijimnut. Ahâglo, illissimanersoarpît koppanganiglonêt okautsaumalaungilanga. Tussaumajak tussarlauktara pitikipet.

7 Pilloridlarput angutekotitit, pilloridlarpullo kivgakotitit nangertsainartut sângne, illissimanerniglo tussarlutik.

8 Nalegak Gudit nertortaule nagligijit, iksivautakotiminut ingitilaungmattit attannertut, Nalegak Gudit pivlugo. Gudivit Israelitit nagligingmagit, sokkosijuititsomavlugit soraijuitomik, igvit attanningortilaukpâtit tapkonunga, namanermik idluarnermiglo attorkovlutit.

9 Tapsomalo arnab attannek tunnitsivigiva Goldinik Talentinik 120 ginik mingoarutiksaniglo tippitsiariktunik unnuktunik, ujarkaniglo pivianartunik. Mingoarutiksakalaungilak tapkotitunak, Arabiamiut attanningata tunnijangititut attannermut Salomomut.

10 Huramiblo kivgangit, Salomoblo kivgangit Goldenik kaitsilauktut Ofiremit, kaitsilaurivuttauk kejungnik Sandelemik attelingnik, ujarkaniglo pivianartunik.

11 Salomolo sennajaukojivok kejungmit Sandelemik attelingmit majutinik Nalekab iglungane, attanniublo iglungane, Harfeniglo nokakseralingniglo imgerpaktunut. Sivurngane kejuit taimaitut tækkojaulaungilet Judakut nunangane.

12 Attanniublo Salomob Arabiamiut attanningat tunnitsivigiva tamainik tussujanginik, tuksiararijanganiglo, tapsoma arnab kailauktangita attannermut assinginik. Allarporlo, aulârlunelo nunakotiminut, kivgakotine illagællugit.

13 Goldele tikkiutjaulauktut Salomomut Jahreme attautseme Talenteôlaukput 666 git,

14 Niuvertit niuverniartullo kaitangit kittikassiutinagit. Arabiamiullo attanningit illunatik, angajokaillo nuname, kaitsivut Goldemik Silberemiglo Salomomut.

15 Attanniublo Salomob sappulutaksat 200 dit sennavait Goldemit namanerpaujomit, Sekkeletut 600 ditut okumaitigijut Golde attorpait sappulutaksamut attautsemut.

16 Tartschillo 300 dit Goldemit namanerpâmit, Sekkeletullo 300 ditut okumaitigijut Golde, attorpait Tartschemut attautsemut.

17 Attanniublo pitipait igloksoarmut, nappartut Libanonemit igloksoanganik attelingmut. Attanniublo iksivautaksoak sennava Elefantit tôganginit, sillaparpâlo Goldemik illakangitomik.

18 Iksivautaksoarlo tungmerakalaukpok pingasojortunik, ikkovrakalaukporlo attajomik Goldeôjomik, ajakpavikalaukporlo maggungnik iglugêngnik tallingnut iksivavingme, Lœwingoaklo nangerpuk ajækpavikita tungangne. 
19 Lœwingoallo 12 it nangerlaukput tagvane tumerangne pingasojortune ittivigêngine. Taimaitomik sennamajokalautsengilak attanniovit nelliane.

20 Salomoblo illulerkutingit immiksakautit Goldeolaukput; iglublo nappartut Libanonemit iglungata illulerkutingit illunatik Goldeolaukput, Silbere sunaunasugijautsengimat Salomob nelliuningane.

21 Attaniub umiaksoakotingit umiaktormatta immarbingme, Huramib kivgangit illagilugit, utterkattarlutiglo Jahrine pingasune attausêarlutik, tikkiutjilutiglo Goldemik Silberemiglo, Eletantillo tôganginik, pissuktuniglo itjuarsijunik, tingmianiglo Fauinik attelingnik.

22 Taimak attannek Salomo anginersaulaukpok attannernit nunamêtunit tamainit, akluinerme illissimanermelo.

23 Attannillo nunamêtut illunatik Salomob kênanga kennerasuarpât sillatuninga tussaromavlugo, Gudib tunnilauktanga tapsoma omattinganut.

24 Illunatiglo attunît atsivigikattarpât pilliutimingnik, illulerkutinik Silbereojunik Goldeojuniglo, annorângniglo, sakkuniglo, mingoarutiksaniglo, horsiniglo, horseojartuniglo Jahrine tamaine.

25 Salomolo horsekutekalaukpok kemmuktunik 4000 dinik, sorsuktuksakutekalarmelo ablangajunik horsit kângine 12000 dinik, innekarkojaulaukpullo iglugaseksoarne, kammutingnut aksalloalingnut illingajune, attanniublo najugâne Jerusaleme.

26 Attanniotilaukporlo attannernut illunainut, kôb miksânit Filisterit nunangat tikkidlugo, Egipteniblo kiglilervinga tikkidlugo.

27 Attanniublo Jerusaleme Silbere taimak unnuktigijotipait ujarkatitut, Cederillo nappartutitut Sikomorinik attekartutitut, naksarne perortutitut.

28 Atsiviomavorlo horsinik Egiptenemit nunanillo illunainit.

29 Unipkautsit assingit sulle Salomo pivlugo, sivurlit kingorlillo, ithâk, tapkoa aglaksimavut nellautaijub Nathanib aglangine, Ahiablo Silomiub nellautaijutigijangine, Jeddiblo tækkojub tækkotitauningine, Jerobeame Nebadib erninga pivlugo.

30 Salomolo attanniotilaukpok Jerusaleme Israelitit illunamassianginut Jahrit 40 git nâlugit.

31 Salomolo sinnisilerpok, atâtagijaminut ailune, illijartortauvorlo Davidib atâtame iglugaseksoangine. Rehabeamelo erninga attanningorpok innangêrdlugo. 


\section{KAPITEL X.}

\section{Kinguvarêt 10 it apterningit Rehabeamemit.}

REHABEAMELO Sichememut aivok, Israelitit illunamassiangit Sichememut tikkisimagamik attanningortitsomavlugo.

2 Jerobeamelo Nebatib erninga, Egiptenemêlauktok, kemâvigijamine Salomo pivlugo, tussarame tamattominga utterpok Egiptenemit.

3 Tilliklerpullo taikunga kaikojaukovâllo. Jerobeamelo Israelitit illunamassiangit illagilugit, tikkilerpok okarkattigivâllo Rehabeame, okarlutiglo:

4 Atâtavit nangmariakartavut okumailuartilaukpa, tagva igvit manna atâtavit kivgartorkojininga okumaitok, nangmagarlo siorniornartok illilauktanga uvaptingnut okinersautitik; tagva nalegomavaptigit.

5 Tapsoma okautivait: Uvlut pingasut nâkpatta kaipsarniaritse uvamnut. Innugiartoksuillo aularput.

6 Attanniublo Rehabeamib angajokaunerôjut atâtame Salomob sângane innôtillugo nangerlauktut kachimiolerkattigivait, okarlunelo: Kannok attannertuivise innugiartoksuit taipkoa kiolugit?

7 Okarkattigivât okarlutiglo: Innugiartoksuit taipkoa pitsiarvigigungne, naletsiarungnelo, illanârnartuniglo okausekarvigigungne, tagva naletsainarniarpâtit.

8 Tapsomale angajuklit attannertuiningat, tunnilauktangat tapsomunga kemakpâ, innuosuktullo ikkingutine nangertullo sângane kachimiolerkattigivait,

9 Okautivaillo: Sunamik illipse attannertuivise, innugiartoksuit taipkoa kiolugit pijuksaugapta okautjigilauktakka, okarlutiglo: Nangmagak atâtavit illilauktanga uvaptingnut okinersautiuk?

10 Innuosuktulle ikkingutingita okarkattigivât okarlutiglo: Imâk okartuksauvotit innugiartoksoarnut, okarkattaulauktunut illingnik, okarlauktunullo: Atâtavit nangmagaksavut okumailuartilaukpa, igville okinersautiuk, okautikillo: Erkekotiga sillinersaujuksauvok atâtama koktoragingnit.

11 Manna atâtama nangmiulaukpasse okumailuartomik; tagva uvanga nangmagaksase illajomavara. Atâtama ipperautinut ipperartorlaukpâse; uvangale Skorpionenut.

12 Jerobeamelo innugiartoksuillo illunatik uvlut pingajuane Rehabeamut tikkimatta, sorlo attannek okalaungmat: Uvlut pingajuane kaipsarniaritse uvamnut; 
13 Attanniub kiovait okumaitomik. Attanniublo Rehabeamib angajuklit attannertuiningat kemmakpa,

14 Okarkattigivaillo innuosuktut attannertuiningat malliklugo, okarlunelo: Atâtama nangmagaksase okumailuartilaukpagit, tagva uvanga okumainersautitsomavakka; atâtama ipperautinut ipperartorlaukpâse, uvangale Skorpionenut sugiaromavapse.

15 Taimaglo attanniub innugiartoksuit nalelaungilait. Gutdemut taimak illingatitaungmat, Nalegak okautsiminik nellàutsititsikovlugo okauserilauktaminik Ahiakut, Silomiokut Jerobeamemut Nebatib erninganut.

16 Israelitille illunamassiangit tækkogamik attanniub nalengimagit, innugiartoksuit attannek kiovât okarlutiglo: Sunamik illangiutivikarkita Davidemik? kingormgutijaksakangilagullo Isaib erningane! Israelitiôjose, aileritse tuppekotipsingnut! Taimak manna igvit iglokotit tækkonâruk Davide. Israelitillo illunamassiatik tupperkotimingnut aivut.

17 Israelib kittornganginut Judakut iglugaseksoangine iglokartunut Rehabeame attanniotivok.

18 Rehabeamible Hadorame angajokaujok sennajuksanut tilliva; Israelible kittorngangita millorlugo tokkopât. Attannerlo Rehabeame tuavidlune ikkivok kamutekotiminut, kemmâlunelo Jerusalemut.

19 Taimak Israelitit aptertut Davidib iglunganit uvlok tamanna tikkilugo.

\section{KAPITEL XI.}

Rehabeamib parngnailerninga unatadlarnermut, innertertauningalo Gudemut. Iglugaseksoakotingit, nulliangillo, kittorngangillo.

Rehabeamelo Jerusalememut tikkiname, Judakut Benjaminekullo katterkovait angutit innuosuktut 120000 dit, Israelitit sorsugvigijomavlugit, attanniovik sattortautitsomavlugo ama Rehabeamut.

2 Nalekable okausinga tikkipok Semajamut Gudib angutekotinganut, okarlunelo:

3 Rehabeame Salomob erninga Judakut attanningat okautiuk, Israelitillo illunaita, Judakunut Benjaminekunullo illingajut, okarlutillo:

4 Imâk Nalegak okarpok: Majorarluse takpaungartuksaangilase, kattangutiselonêt sorsugvigijaksaringilasse, illunatik 
attunît angerarmilit, tamanna uvamnit pingmat. Nalekpaillo Nalekab okausingit nokkarlutiglo aularnermit Jerobeamut.

5 Rehabeamelo iglokarpok Jerusaleme, iglugaseksuillo Judame sôngotipait:

6 Tagva: Bethleheme, Etame, Tekoalo.

7 Betzur, Socho, Adullamelo.

8 Gath, Maresa, Sif,

9 Adoraime, Lacnis, Aseka,

10 Zarega, Ajalon, Hebronelo, tapkoa iglugaseksoangolaukput songotitaksat Judame Benjaminemelo.

11 Tapsomalo sôngotidlarpait, angajokakartilaitalo, piksakartilugillo nerkiksanik, orksumiglo Weinemiglo.

12 Iglugaseksoarnelo illunaine sappulutaksakartitsivok, kallugiakartitsilunelo, sôngotidlarpaillo. Judakullo Benjaminekullo tapsomunga illingavut.

13 Ajochertuijullo Levitillo Israelitit illunamassiangine kiglilervinginelo illunainêtut illagilerpât.

14 Kemmakpaillo iglugaseksoagijatik, perkutitiglo, tikkilutiglo Judakunut Jerusalemut. Jerobeamib erningitalo ajæktormagit, ajochertuijuêrutilugit Nalekamut.

15 Ajochertuijungortitsivorle pôktôjunut, Satanasenullo, nochangoanullo, sennajaukojaminut.

16 Tapkoalo kingorngane Israelib kinguvarênginit illunainit tikkitokalerpok Jerusalemut, omattemingnik tunnijijunik, Nalegak Israelib Gudinga apperkutigilugo, tunnergutinik tunnijijomavlutik Nalekamut atâtagijamik Gudinganut.

17 Pitsartutipâllo Judakut attanniovingat, nakkiterpâllo Rehabeame Salomob erninga Jahrit pingasut nâlugit ; agvertaramik Davidib Salomoblo apkosiningine Jahrit pingasut nâvlugit.

18 Rehabeamiblo Mahelate Jerimotib Davidib erningata pannia tiguva nulliarivlugo, Abihaillo Eliab Isaib erningata pannia.

19 Tapsomalo erniarivait tapsomunga ernerit: Jeus, Semarjalo, Sahamalo.

20 Tapsomalo kingorngane Maecha Absalomib pannia nulliarilerpa; tapsoma erniarivait tapsomunga: Abia, Atailo, Sisalo, Selomitelo.

21 Rehabeamiblo Maecha Absalomib pannia nagliginerpariva nulliaminit illunainit, panervgaminillo illunainit; nulliakarame 18 nik, pannervgakalarmelo 60 ginik; ernertarlunelo 28 ginik, pannetarlarmelo 60 ginik.

22 Rehabeamiblo Abia Maechab erninga niakungortipa angajokangortilâlo kattangutingita akkorngane; issumavorlo attanningortitsomavlugo.

23 Pinniarporlo sillatôlune, siamartipaillo ernine Judakut Benjaminekullo iglugaseksoanginut songojunut illunainut, ner- 
kiksakartipaillo unnuktunik, tigusilunelo tapkonunga illingajunik arnanik unnuktunik.

\section{KAPITEL XII.}

Rehabeamib salagïjauninga Sisakemut, Egiptenemiut attaninganut, tokkungalo.

REHABEAMIBLE attaniôvinga pallangêrsimangmat songongmallo, Nalekab perkojangit kemmakpait, Israelitillo illunamassiangita tamna illagilugo.

2 Rehabeamible attannioningata Jahringita tellimangane aulariune takpaungarpok Sisak Egiptenemiut attanningat Jerusaleme sorsugvigijomavlugit, (idluitullilaungmatța Nalekamut),

3 Najortekarlune kammutingnik aksalloalingnik 1200 dinik, ablangajuniglo horsit kângine 60000 dinik; kittiksaulaungilello innugiartoksuit illaulauktut tapsomunga Egiptenemit Libienemillo, Suchimemillo, Aetheopiamillo.

4 Iglugaseksuillo sôngojut sâlagivait Judamêtut, tikkilunelo Jerusaleme tikkidlugit.

5 Tagva Semajab nellautaijub Rehabeame Judakullo angajokaunerôjungit Jerusaleme kattimalertut sivuramut Sisakemik, okautivaillo: Imâk Nalegak okarpok: Illipse kemmalaukpapsinga, tamanna pivlugo tunnilaukpapse Sisakib agganginut.

6 Tagva angajokaunerôjut Israeleme, attannerlo mannigotitilerput, okarlutiglo: Nalegak idiuarpok.

7 Nalegarle tækkogame manigotitingmatta, Nalekab okausinga Semajamut tikkipok, okarlunelo: Manigotitikauvut, tamanna pivlugo asseroromalungilakka; piulijaunermigle tunnitsivigijomavakka, illungertorneralo kovijaujuksaungilak Jerusalemut Sisakekut.

8 Taimaigalloarmalle kivgartoraksarivât, tukkisilerkovlugit sunaumangat kivgartorlunga, nunallo attanniovingit kivgartorlugit.

9 Taimak Sisak Egiptenemiut attanningat takpaunga majorarpok Jerusalemut, tigusilunelo perkutinik pivianartunik Nalekab iglunganêtunik, attanniublo iglunganêtunik, illunaitalo aksarnigarivait, tiguvaillotauk sappulutaksat Goldeôjut Salomob sennajaukolauktangit.

10 Attanniublo Rehabeamib taipkoa inninginut sappulutaksat kannujaujut sennajaukovait, unertutigivaillo sorsuktuksat, attanniub iglungata upkoanginik udsertortut angajokânginut. 
11 Attannerlo Nalekab iglunganut itterlaurangat, udsertortut akjarkattariartorpait, uttertikattarlaitalo udsertortut iglunganut.

12 Manigotitingmallo Nalekab ningarningata allarpa, asserortaumarikonago. Judakune sulle idluarnekarmat.

13 Taimak attannek Rehabeame pallangilak Jerusaleme, attanniotilunelo. Rehabeame Jahrekalaukpok 41 ginik attanningorame, attanniotilunelo Jahrit 17 it nâvlugit Jerusaleme, iglugaseksoarne Nalekab annerilauktangine Israelib kinguvarênginit tamainit, atterminik pititsikovlugo tagvunga. Anânanga attekalaukpok Naemamik, Amoriteriôjok.

14 Idluitullilaukporlo, omattinelo torartilaungila Nalegak kennerlugo.

15 Rehabeamible pijokalaungningit sivurlit kingorlillo, aglaksimavut Semajab nellautaijub, Iddoblo tækkojub unnipkausing ine; Rehabeamiblo Jerobeamiblo akkorngangne unnatadlartokatsainarpok.

16 Rehabeamelo sinnisilerpok atâtagijaminut ailune, illijartortaulunelo Davidib iglugaseksoangine. Erningalo Abia attanningorpok innangêrdlugo.

\section{KAPITEL XIII.}

\section{Abia Judakut attanningat; tapsoma sâlakarninga Jerobeamik Israelitit attanninganik.}

ATtanniUB Jerobeamib Jahringita 18 ingane, Abia attanningorpok Judame.

2 Attanniotilunelo Jahrit pingasut nâlugit Jerusaleme. Anânanga attekalaukpok Michajamik, Urielib Gibeamiub panninga. Unatadlartokalerporlo Abiablo Jerobeamiblo akkorngangne.

3 Abialo oppalungaijarpok unatadlarnermut angutit innuosuktut 400000 dit sorsugungnartut illagilugit. Jerobeamele oppalungaijarpok sorsukattigijomavlugo, angutit innuosuktut 800000 dit sorsugungnartut illagilugit.

4 Abialo majorarpok kakkamut Zemaraimik attelingmut, Efraimib kakkanginêtomut, okarlunelo: Naleksinga, Jerobeame, Israelitillo illunamassiatik!

5 Nellungilase Nalekab Israelib Gudingata Davide tunnitsivigilaungmago Israelitit attanniovinganik soraijuitomik, tamna, erningillo, angernermut sioraujalingmut?

6 Jerobeamele Nebatib erninga, Salomob Davidib erningata 
kivganga pimariuterlaukpok nalegüngnailunelo nalekaminik. 7 Illaulaukpullo tapsomunga innuit kuksalaitut Belialib kittorngangit, tettitosarvigivâllo Rehabeame, Salomob erninga, Rehabeamele innuosuktolaukpok sulle, omatekalunelo nangiarsaraitomik, sorsuklune agviarlaunginamigit.

8 Manna illipse issumavose Nalekab attanniovikotinga Davidib erninginut illingajok kippalidlaromavlugo, illipse unnuktôgapse, nochangoakarapselo Gold̉eôjungnik Jerobeamib sennalauktangangnik, Gudeôkovlugik illipsingnut.

9 Nalekab ajochertuijokotingit, Aronib erningit Levitillo, pingudlalaungipissigik, ajochertuijungortitsiluselo nangminipsingnut, innukattigêksoatitut nunane? Tikkitok tigumiaralijarlune tuktuvârsungmik, saukaniglo angusallungnik 7 nik, tamna ajochertuijungortitauvok, tapkoa Gudiolungitut pivlugit.

10 Uvagulle Nalegapta Gudipta illagivâtigut, allarlungitapta; ajochertuijullo Nalekamik kivgartortut, Aronib erningit Levitillo sullijaksamingne,

11 Ikkipaillo uvlâne tamaine, umunelo tamaine tunnergutit otaksat, isserluanijutiksallo tippitsiariktut Nalegak pivlugo; illijilutiglo kakkojanik tækkojaksanik sâmut Goldeôjomut illakangitomik; nennergoabviglo Goldeôjok, kollingillo unnune tamaine ikkipait. Uvagut kammagijaksat Nalegak Gudivut pivlugo kammagigaptigik, illipsele kemmalaukpasse.

12 Ahâglo, uvagut illagivâtigut sivuniptingne, Gudib ajochertuijokotingitalo, Trompetillo trompetesijautigijaukovlugit akkingerluse. Kittorngaujose Israelemut, akkerartorniarase Nalekamut atâtagijapse Gudinganut, sullinianginapse.

13 Jerobeamele terliarsivikartitsivok avatâne, tunnuanit tikkitsomavlugit, taimaglo nangerput Judakut sirurâne, terliarsivingmiullo tunnungine.

14 Judakullo manna keviarmatta, ahâk tagva sângine tunnunginelo sorsugvikalaukpok. Tagva niplialerput Nalekamut, ajochertuijullo Trompetit trompetisijautigivait.

15 Angutillo Judakut illunamassiatik nipliarput, angutillo Judakut nipliartillugit, Gudib Jerobeame Israelitillo illunaita annauvait Abiab Judakullo sângine.

16 Israeliblo kittorngangita Judakut kemmâvait, Gudiblo tunnivait tapkoa agganginut.

17 Abialo innukotingillo sâlakarnekartitsivut angijomik tapkonañe, ochovullo Israelitinit ullimarsimajut angutit innuosuktut 500000 dit.

18 Taimak Israelib kittorngangit mannigotititaulaukput taipsomane; Judable kittorngangit maksoalerput, Nalegak at̂̂tagijamik Gudingat tettigigamitsuk.

19 Abiablo Jerøbeame udlava, aksarpâlo iglugaseksoarnik, 
Bethelemik aiparusinginiglo, Jesanamik, aiparusinginiglo, Efronemiglo aiparusinginiglo;

20 Jerobeamelo nukkiktarlungilak Abia innôtillugo, Nalekablo annauva tolkkolugo.

21 Abialo pallangairame arnat 14 it nulliarilerpait, ernertarlunelo 22 ginik, pannetarlarmelo 16 nik.

22 Unipkautsit assingit Abia pirlugo, apkotingillo pinniarningillo, tamakkoa aglaktaumavut Iddob nellautaijub aglangine.

\section{KAPITEL XIV.}

Assa attanningorpok; kivgartornek Gudeungitunik pêjarpa; sâlukarninga Aethiopiamiunik.

ABIALO sinnisilerpok, atâtagijaminut ailune, illijartorpâllo Davidib iglugaseksoangine; Assalo erninga attanningorpok innangêrảlugo. Tapsoma nelliuningane nuna innilangalaukpok Jahrinik 14 nik.

2 Assalo pinniarlaukpok idluartomik, Nalekablo Gudingata namagijanganik.

3 Altarillo tækkorngartat pêjarpait, pôktojullo, nappajoksuillo serkomipait, nappartullo Gudingoaggit nakkarpait.

4 Judakullo okautsaukorait Nalegak, atâtagijamik Gudingat kenneraksaringmatsuk, pinniarkovlugillo malligaksat perkojallo malliklugit.

5 Judakullo iglugaseksoanginit illunainit pôktojut Gudingoallo pêjarpait. Attannioviglo innilangalaukpok sângane.

6 Iglugaseksuillo sôngojut iglugaseliorpait Judame, nuna innilangalaungmat, unatadlatokalaungimallo tapsomunga, Jahrine taipkonane; Nalekab merngoêrserviksakartimago.

7 Judakullo okautivait: Iglugaseksuit ukkoa iglugaseliorlavut, avaluliorlavullo karmaksoarnik, nuvugusekartilugillo, upkoakartilugolo, nuna sulle saptingnêmat, Nalegak Gudivut kennerlauraptigo; kennerlaukpavut, tapsomalo merngoêrservikartilaukpâtigut avatiptingne. Taimaglo igloliorsavut, sullidlarlutiglo.

8 Assalo sorsuktuksakalaukpok sappulutalijartunik, kallugialijartuniglo, Judakunit 300000 dinik, Benjaminekunillo sappulutalijartunik, pittikserungnartuniglo 280000 dinik, tapkoalo illunatik songovlutik angutaudlalaukput.

9 Serable Aethiopiamiub sorsugiartorpait najortekarlune 1000000 nik sorsuktuksanik, kamutingniglo aksaloalingnik 300 dinik, tikkipullo Maresa tikkidlugo.

10 Assalo aularpok pâromavlugit, oppalungaijarpullo sor- 
sungnermut naksarme Zefatamik attelingme Maresab kannitanganêtome.

11 Assablo Nalegak Gudine nipliarvigiva, okarlunelo: $\mathrm{Na}$ legak adsigêngitokangilak illingne, ikajorlutit unnuktut akkorngane, pitsakitorlonêt ikajorlugo. Ikajortigut Nalegak Gudivut; sungertutigigaptigit, atternelo tikkisimavogut, unnuktovaksuit tapkoa pârlugit sorsugomavlugit. Nalegak igvit Gudigivaptigit salagijauniarnak innungnut.

12 Tagva Nalekab Aethiopiamiut annaulerpait Assab Judakullo sivurâne, kemmâkovlugit.

13 Assablo innugiartoksuillo najortane illagilugit udlapait Gerar tikkidlugo. Aethiopiamiullo ochovut taimak unnuktigijovlutik innojokarungnailaungmat tapkonangat, serkomitaulaungmatta Nalekab tapsomalo sorsuktuksaksoakotingita sângine. Aksarnigaujullo unnuktovaksuit neksarpait.

14 Iglugaseksuillo tamaita Gerarib avatâne sâlagivait, Nalekab sivuranarningata tupvigilaungmagit. Iglugaseksuillo illunaita aksarpait, aksarnigaksakarmat tapkonane unnuktovaksoarnik.

15 Nergjutillo tuppingit sâlagivaittauk, saukallo unnuktovaksuit Kamelillo tikkiutivait, utterpullo Jerusalemut.

\section{KAPITEL XV.}

Assafib pigiarninga kivgartornek Gudemik âkiksorlugo.

GUDIBLO annerningata Asarja Odedib erninga tupvigilerpa.

2 Tamna annivok Assa pachreartorlugo, okautilâlo: Naleksinga, Assa Judamiôjoselo, Benjaminemiôjoselo illunase! Nalekab illagivâse, illipse illagigapsiuk; kennerniarupsiuglo tagva nachvartaukoniarpok illipsingnut; allarniarupsiugle, tagva allarniarivâse.

3 Uvlokaromârporle unnuktunik Israeleme, Gudekaromângitunik idluartomik, ajochertuijokaromângituniglo ajochertuijunik, perkojakaromângituniglo.

4 Sagiaromârpattalo kappianartonermingne Nalekamut Israelib Gudinganut, kenneromârpatsuglo, tagva nachvartaukoniarpok.

5 Nelliutome taipsomane kannoêngitsiarnekaromângilak tapsomunga annijomut ittertomullo, aulajadlarnekaromârmat illunainut nunamêtunut.

6 Innukattigêksuit attautsit innukattigêksuit assingit serkomigomârmagit, iglugaseksuillo attautsit iglugaseksuit assingit; Gudib koaksartitsomârmagit kappianartunut tamainut. 
7 Illipsele maksoaleritse, aggasselo kattaktailitilugit pititse, pinniarnise akkiksakarniarmatta.

8 Assable okautsit tamakkoa tussaramigit, nellautaijublo Odedib nellautaijutigijangit, maksoalerpok, péjarpaillo makkojungnartut Judakut Benjaminekullo nunangangnêtut, iglugaseksoarnêtullo aksarnigarilauktamine Efraimib kakkangine. $\mathrm{Na}$ lekablo Altaringa Nalekab torksungata sânganêtok nutângortipa.

9 Kattikovaillo Judakut Benjaminekullo illuêngarlutik, tujormitjallo tapkonanêtut Efraimekunit, Manassekunillo, Simeonekunillo. Israelitinit unnuktut apteramik, tikkipâllo, tækkogamik Nalekab Gudingata aiparingmago.

10 Kattimalerpullo Jerusalemut Assab attannioviata Jahringita 15 ingata takkingita pingajuane,

11 Tunnijilutiglo urlorme taipsomane tunnergutinik Nalekamut aksarnigavinernit tikkiutilauktamingnit, tuktuvait angusallunginik 700 dinik, saukaniglo 7000 dinik.

12 Angerkattigêkpullo, Nalegak atâtagijamik Gudingat kenneromavlugo omamut tamânut, tarnemullo tamânut;

13 Kinalo Nalekamik Israelib Gudinganik kennerniarajangitok, tamna tokkotaujuksaungmat, mikkijômangât angijômangâllonêt, angutaumangallo arnaumangâllonêt.

14 Angervigimarikpâllo Naltgak nippekôrtôjomut, igiakomijarlutiglo, trompetesijarlutiglo, tiptulausijarlarmiglo.

15 Judakullo illunamassiatik angermaringnek tamna kuviasutigivât; angermarilauramik ômamut tamânut, kennerpâllo pijomanermingnut tamânut, nachvartaukolaukporlo tapkonunga ; Nalekablo merngoêrsernekartipait avatingine illunane.

16 Assab attanniojub Maecha anânane attanniojungnaitipâtauk, Gudingoamik sennalaungmat nappartune. Assablo Gudingoakotinga pêjarpa, tugarlugolo serkalitipa, ikkilâlo kôngme Kidronemik attelingme.

17 Pôktojulle Israeleme pêjartaulaungilet; Assable omattinga nellagorlaukpok innosermine tamât.

18 Ittertipaillo Nalekab iglunganut atâtame ivsornaitilauktangit, ivsornaitilauktanelo, Silbere Goldelo illulerkutillo.

19 Unatadlartokalaungilarlo, Assab attannioningata Jahringita 35 ingat tikkidlugo. 


\section{KAPITEL XVI.}

Assab idluitullininga Gudemut, nellautaïokotinganullo; tokkungalo.

AssaB attanniovingata Jahringita 36 ingane Baesa, Israelitit attanningat majorarlune takpaungarpok Judakut akkerartorlugit; iglugaseliorpaillo Rama, Assa Judakut attanningat agviaromavlugo aularnermik itternermiglo.

2 Assale Nalekab iglungata attanniublo iglungata perkotitalingangnit tigusivok Silberemik Goldemiglo, tillikliutigivaillo Benhadademut Siriamiut attanninganut Damaskuseme nunakartomut okautsaukovâlo:

3 Angerkattigêngnekarpok uvamne illingnelo, atâtamnelo atâtangnelo; tamanna pivlugo tillikliutjivigilaukpagit Silberemik Goldemiglo, aøgerkattigêngnek illingnêtok Baesamêtorlo Israelitit attanningane serkomilugo pikovlutit, tamna aularkovlugo uvamnit.

4 Benhadadib attannek Assa nalekpa, sorsuktuksakotimelo angajokangit tillivait Israelitit iglugaseksoanginut; tapkoa ullimarpait Ejon, Danelo, Abelmaimelo, iglugaseksuillo kemmatulliviktaggit Naftalikunêtut illunaita.

5 Baesab tamanna tussaramiuk nokarpok Ramamik iglugaseliorlune, nippotivâlo pinniarnine.

6 Attanniuble Assab Judakut illunamassiangit illasearivait, akjartorpaillo ujarkat kêjuillo Baesab igloliorutigilauktangit Ramamit, tamakkonangallo Geba Mizpalo iglugaseliorpait.

7 Nelliutome taipsomane Hananib tækkojub Assa Judakut attanningat tikkipa, okautilâlo: Siriamiut attanningat sungertutigilaurangne, Nalegarlo Gudit tettigilaunginangne; tamanna pivlugo Siriamiut attanningata sorsuktuksakotingit aggakpit kemâjivait.

8 Aethiopiamiut, Libienemiullo unnuktovaksolaungilet, kammutekarlutik ablangajokarlutiglo horsit kângine unnuktunik? Sullelo Nalekab tunnilaukpait aggangnut sungertutigilaurangne.

9 Nalekab ijikita nunat illunaita tautungmattigik, pitsartutitsikovlugo tapkoninga, omamut tamânut ungajunik tapsomunga. Sillaitomik pinniarlaukpotit; tamanna pivlugo mannamit unatadlartokarniarpotit.

10 Assable tækkojok ningarvigiva, pitilâlo parngnanêrsimavingmut; okalokivigivalo pijokartok tamanna pivlugo. Assablo. nelliutome taipsomane innugiartoksuit illangit nênerpait. 
11 Assable pijokalaungningit, sivurlit kingurlillo, ahâk, aglaksimavut Judakut, Israelitillo attanningita aglangine.

12 Assalo ittigalerilerpok attannionime Jahringita 39 ingane, kannimasingalo sugalukpalliadlarpok kannimavlunele Nalegak kennerlaungila, aniasiortille.

13 Taimak sinnisilerpok atâtagijaminut ailune, tokkovorlo attannionime Jahringita 41 ingane.

14 Illijartortauvorlo illuvikotiminut, sennajaukolauktaminut Davidib iglugaseksoangine. Illivâllo innangavianut tættætaulauktomut isserluanijutiksanik tippitsiariktunik, mingoarutiksaniglo sunatuinarnik, âniasiusiortit illusingit malliklugit sennamajunik; ikkoalanekartitsivullo angijomik.

\section{KAPITEL XVII.}

\section{Josafatıb attanniôtininga.}

ERningalo Josafat attanningorpok innangêrdlugo, makkutjilunelo Israelitinik.

2 Judakullo iglugaseksoangit sôngojut illunaita, sorsuktuksakartipait, aulatsijokartitsivorlo Judakut nunangane, Efraimekullo iglugaseksoangine illunaine, atâtame Assab aksarnigarilauktangine.

3 Nalekablo Josafate aipariva; agvertarmat atâtame Davidib apkosiningine, nutaungitune, kennertaililugillo Baalit,

4 Atâtagijamele Gudinga, agvertarlunelo tapsoma perkojangine, Israelitillo pinniarningit malliklugiungitok.

5 Tamanna pivlugo Nalekab attanniôvik pallangairpa aggangine. Jułakullo illunamassiatik pilliutinik tunnitsivigivât, akluinekalaukporlo, opigijauneliolunelo arklingnartomik.

6 Omattingalo maksoapalliavok Nalekab apkosiningane, piganerporlo pôktojut, nappartullo Gudingoaggit pêjarlugit Judakunit.

7 Attannionime Jahringita pingajuane, angajokakotine Benhail, Obadjalo, Sacharjalo, Nethaneello, Michajalo tillivait, ajochertuijartorkovlugit Judakut iglugaseksoangine;

8 Tapkoalo illagilugit Levitit, Semaja, Nethanjalo, Sebadjalo, Asaello, Semiramotelo, Jonathanelo, Adonialo, Tobialo, Tobadonialo; illagilugillo ajochertuijut Elisamalo, Joramlo.

9 Ajochertuivullo Judame, najortekalutiglo Nalekab aglakotinginik perkotitalingnik, ingergavullo Judakut iglugaseksoangittigut illunaitigut, ajochertuilutiglo innugiartoksoarnik.

10 Nalekablo sivuranarningata nunat attanniovingit Judakut avatinginêtut illunaita tupvigilerpait sorsukonagit Josafatemut. 
11 Filisterillo illangit âtsivut Josafatemut pilliutinik, Silberemiglo nangmakamik attautsemik. Arabiamiullo kaitsivigivât saukanik angusallungnik 7700 dinik, tuktungajuniglo angusallungnik 7700 dinik.

12 Taimaglo Josafate pivalliavok, angijororpallialunelo; igloliorsalunelo Judakut nunangane igloksoarnik avaluliorsimajunik, kemmatullivingniglo.

13 Kemmatullisimajokutekalaukporlo unnuktunik Judakut iglugaseksoangine, angutekutekalarmelo sorsugungnartunik angutaudlartuniglo Jerusaleme.

14 Tamadsalo tapkoa ingmigolinganingit atâtagijamik iglungine, Judakune angajokaunerolauktut tausendinut: Adna angajokaunerôjok, angutillo sorsugungnartut 300000 dit illagivât.

1 J Tungânelo Johananeôlaukpok angajokâk, 280000 dillo illagivât.

16 Tungânelo Amasjaôlaukpok Sichrib erninga, pijarællune kivgautitok Nalekamut, angutillo sorsugungnartut 200000 dit illagivât.

17 Benjaminib kittornganginit Eliadaôlaukpok, angutaudlartok, 200000 dillo illagivât, oppallungêrsimajut pittiksalijarlutik sappulutalijarlutiglo.

18 Tungâne Josabadeôlaukpok; 180000 dillo illagivât oppalungêrsimavlutik sorsungnermut.

19 Tapkoa illunatik attannek naipertorpat, attanniub pitilauktangit iglugaseksoarnut sôngojunut Judame illunâne kittikassiutinagit.

\section{KAPITEL XVIII.}

Josafatib illanâtealerninga Ahabemit. Ahabelo aipariva matadlarnermut Siriamiunut. Ahabib tolkunga.

JoSAFATELO akluinekadlalaukpok, opigijauneliolunelo, Ahabelo illanârilerpa.

2 Jahriglo magguk nângmanik, attersarpok Ahabemut Samariamêtomut. Ahabiblo tamna pivlugo, innugiartoksuillo najortingit pivlugit, saukat tuktuvaillo unnuktut tokkotaukovait. Tilliorpâlo takpaungarkattaukovlugo Ramotemut Gileademêtomut.

3 Ahabiblo Israelitit attanningata Josafate Judakut attanningat okautiva: Aularkattigilaunga Ramotemut Gileademêtomut. Tapsoma okautiva: Uvanga illiktun-îpunga innukotikkalo innukotititut, illagijomavaptigit unnatadlarnermut.

4 Josafatible Israelitit attanningat okautiva: Nagliktara uvlome Nalekab okausinga apperilauruk. 
5 Tagva Israelitit attanningata nellautaijut angutit 400 dit kattikovait, okautilugillo: Sorsugiariartortuksauvita Ramotemut Gileademêtomut nippotitaksarivigolonêt? Tapkoa okarput majorarlutit takpaungarit, Gudib tunniniarpait attanniub agganginut.

6 Josafatele okarpok: Pittakangilâk sulle Nalekab nellautaijokotingita illanganik, tapsomuna appertsorajarluta?

7 Israelitit attanningat Josafatemut okarpok: Angutekârpok sulle attautsemik, appertsorungnarajarutigijaptingnik Nalekamik, kissiane omigivara, ajungitomik nellautaivigingimanga, idluitomigle kissiane; tagva Micha Jemlab erninga. Josafate okarpok: Attannek taimak okarniarane.

8 Israelitillo attanningata angajokakotime illangat kaikova, okarlunelo: Micha Jemlab erninga tamaungartisaruk!

9 Israelitillo attanningat, Josafatelo Judakut attanningat iksivavuk attunic iksivautaksoakotimingne annorarsimavlutik attanneksiutinik, iksivavugle inneme Samariab itterviksoangata sillatanêtome; nellautaijullo illunatik nellautaivut tapkoa sângangne.

10 Zedekialo Knaenab erninga sennavok ingminut naksungnik kikkiangojungnik, okarlunelo: Imâk Nalegak okarpok: Ulkkungnunga Siriamiut nakjumigarniarpattit nungutitsikârtinnak taipkoninga.

11 Nellautaijullo illunatik taimak nellautailaurivut, okarlutiglo: Majorarlutit takpaungarit, sullidlarniarpotit; Nalekab tunniniarpait attanniub agganginut.

12 Tillijablo aimalauktub Micha kaikolugo, okarkattigiva, okarlunelo: Ahâk nellautaijut okalâningit illunatik adsigêktomik attanniub idluarkutinganut illingavut; nagliktara, okautsitit taimailingalaulittauk taipkoa illangata okausingititut, okarlutillo ajungitomik.

13 Michale okarpok: Sorlo Nalegak miksekârtomik innôngmat, Gudima okauseriniartanganik okaromavunga.

14 Attannermullo tikkimat, attanniub okautiva: Micha, aulartuksauvita Ramotemut Gileademêtomut sorsungniarluta, nippotijaksarivigolonêt? Tamna okarpok: Ahaila majorarluse takpaungaritse, sullidlarniarpose, tunnijauniarput aggapsingnut.

15 Attanniublo okautiva: Kapseartorlunga okautimarijariakarpagit, assianik okausekarkonak uvamnut, miksekârnermik kissiane Nalekab attingane?

16 Tagva okarpok: Israelitit illunamassiatik tækkolaukpakka siamarsimatillugit kakkat kângine saukatitut mianerijekangitutitut. Nalegarlo okarpok: Ukkoa angajokakangilet, illunatik attunît angerarlit iglomingnut ullapirsautekarlutik. 
17 Tagva Israelitit attanningata Josafate okautiva: Okautilaungilagit: Nellautaivigilungimanga ajungitomik, idluitomigle?

18. Tamnale okarpok: Tamanna pivlugo Nalekab okausingit tussarsigik: Nalegak tækkolaukpara iksivatillugo iksivautaksoarmine, killangmiugaseksuillo illunamassiatik nangertillugit tallerpiane saumianelo.

19 Nalegarlo okarpok: Kia Ahabe Israelitit attanningat tillioromavaûk, majorarlune takpaungarkovlugo, ochokovlugo Ramoteme Gileademêtome? Tamnalo imâk okarmat, assialo imâk;

20 Annernerit illangat kaivok, Nalegarlo sângeriarpa, okarlunelo: Uvanga tillioromavara. Nalekable okautiva: Sunamut?

21 Tamna okarpok: Aularomavunga, annerniôjomalungalo nellagongitok, tapsoma nellautaijokotingita illunatik kanningine. Tamnalo okarpok: Tilliorniarpet, sullidlarniarlutillo, aularit taimailiorlutillo.

22 Mannalo ahâk, Nalekab annernek nellagôngitok tunnimava nellautaijokotivit tapkoa kanninginut, Nalekablo idluitomik okarutigilaukpâtit.

23 Tagva Zedekia Knaenab erninga tikkilerpok tagvunga, ulloarokpâlo Micha, okarlunelo: Apkosinit nelliagut Nalekab annerninga nûsimava uvamnit, okarkovlugo illingnut?

24 Micha okarpok: Ahâk tækkojomârpet, iglorutsit kamanenerpânganut tikkitsomâruvit ijerkovlutit.

25 Israelitille attanningat okarpok: Tigusiuk Micha, nokarkolugolo Amoneme iglugaseksuit angajokangane, Joasemelo attanniub erningane;

26 Okarluselo: Imâk attannek okarpok: Una parngnanairsimavingmut pitisiuk, nerritilugolo kakkojamik, immertilugolo immermik kiksarnartomik, utterkârtinanga ullapirsautekarlunga.

27 Micha okarpok: Ullapirsautekarlutit utterniaruvit, tagva Nalegak uvapkut okalaungilak. Okarporlo: Tussaritse innugiartoksôjose illunase!

28 Taimak lsraelitit attanningat, Josafatelo Judakut attanningat majorarput takpaunga Ramotemut Gileademêtomut.

29 Israelitillo attanningata Josafate okautiva: Pissertorlunga sorsungnermut aijomavunga; igville annorâkotitit attikit. Israelitillo attanningat pissertorpok ingminik annorâminut, tikkipullo unatadlarnermut.

30 Siriamiulle attanningata kamutekotime angajokangit perkolaukpait, okarlunelo: Sorsuktuksaungilasse mikkijunullonêt, angijunullonêt, Israelitille attanninganut kissiane.

31 Pijokalaukporlo kamutit angajokangita Josafate tækkoga- 
mitsuk aulajitigivât attannionasugivlugo Israelitinut, sangulutiglo tunarilugo sorsugomavlugo. Josafatele nipliadlarpok; Nalekablo ikajorpa, Gudiblo sangutipait tapsomangat.

32 Kamutille angajokângit tækkogamik attanniolungimat Israelitinut, allarpât.

33 Angutille illangata maungatuinak pittiksine kelluva, pittikpâlo Israelitit attanningat sagvingmiutab pêrniublo akkorngagut. Tagva kemuksertokotiminut okarpok: Sangulerit nûtilungalo sorsungnermit, ikkilersimagama.

34 Sorsungnerlo sugalulerpok uvlorme taipsomane. Israelitillo attanningat nangerpok kamutikotimine, Siriamiut akkiane, unnuk tikkilugo, tokkolunelo sekkinek nippilertillugo.

\section{KAPITEL XIX.}

Josafatib suartauninga illanakarninga Ahabemik pivlugo; âkiksoiningalo kivgartornermik Gudemil.

JoSAFATELE Judakut attanningat ullapirsautekarlune utterpok Jerusalemut.

2 Jehublo Hananib erningata tækkojub pachreartorpa, okautilâlo attannek Josafate: Igvit Gudekangitok ikajoraksariviuk, nagligilugillo Nalekamik omisuktut? Tamannalo pivlugo Nalekab ningausingata kollangiarpâtit.

3 Ajungitomigle nachvartokalauralloarpok illingne, nappartut Gudingoaggit pêjarlaurangne nunamit, omattillo torartilaurangne Gude kennerlugo.

4 Taimaglo Josafate Jerusalemêtuarpok. Amalo aularpok innugiartoksuit akkornganut, Bersebamit Efraimib kakkangit tikkidlugit, sattortautipaillo Nalekamut atâtagijangita Gudinganut.

5 Idluarsaijokartitsivorlo nuname Judakut iglugaseksoangine sôngojune illinaine.

6 Okautivaillo idluarsaijut: Kammatsiaritse pinniarnipsingnik innuit pivlugit idluarsailunginapse, Nalekamulle; tapsomalo illagivâse idluarsainerme.

7 Tamanna pivlugo sivuranek Nalekamik illipsingnêle, udsertutsiaritse pinniarluselo, Nalekame Gudiptingne idluinekalungilak, kammanginekangilarlonêt innub kinauninganik, illalliornekangilarlo pilliutinik.

8 Jerusalemelotauk Josafate pittakartitsivok Levitinit ajochertuijunillo, atâtanillo angajokaunerôjunit Israeleme, aulatsijunik Nalekab idluarsaininganik, akkerartortigêngnerniglo, nunakarkolugillo Jerusaleme.

9 Perkovailla, okarlunelo: Taimailioritse sivuranerme Nalekamik, nertornarluse, omamullo nellagortomut. 
10 Illinganerne tamaine âtaujune illipsingnut kattangutipsingnit, iglugaseksoarmingnêtunit, auk pivlugolonêt, malligaksat pivlugillonêt, perkojat pivlugillonêt, illutsit pivlugillonêt, idluarijaunerit pivlugillonêt, tagva ajochertorsigik, idluitullikonagit Nalekamut, ningautsemiglo tikkitokarkonago illipsingnut kattangutipsingnullo. Taimailioritse, tagva idluitullitailiniarpose.

11 Ahâk, Amarja ajochcrtuijersoak angajokaunerôvok illipsingnut Nalekab illingakojiningit tamaita pivlugit. Taimaktauk Sabadja Ismaelib erninga, angajokaksoak Judakunut, attanniub illingakojiningit tamaita pivlugit. Aulatsijullo Levitit sapsingnệput. Maksoaleritse pinniarluselô; Nalekablo ajunginek aipariniarpa.

\section{KAPITEL XX.}

\section{Josafatib sâlałarninga Amoniterinil Moabiteriniglo.}

TAMAKKOA kingorngane, Moab kittorngangit, Amoniblo kittorngangit, illagilugillo Amunitit illangit tikkilerput Josafate sorsugomavlugo.

2 Tikkitokarporlo nellojungnaititsijomik Josafatemik, okarlunelo: Tikkitokarpok opaluktunik illingnik unnuktovaksoarnik, immarbiksub akkianit Siriamit; ahâglo Zazezon Tamaremêput, tagva Engeddime.

3 Tagva Josafate sivuravok, kênanelo torartipa Nalegak kenneromavlugo, nerritailinekarkojivorlo Judakune illunaine.

4. Judakullo kattimalerput Nalegak kenneromavlugo, Judakullo iglugaseksoanginit illunainit tikkiput, Nalegak kennerlugo.

5 Josafatelo, Judakut Jerusalemiullo kattimaniksangane, inneme avaluliorsimajome nutâmetome, Nalekab iglungata sângane, nangerpok,

6 Okarlunelo: Nalegak atâtagijapta Gudingat, igvit Gudeungilatît killangme, aulatsijolungilatîllo innukattigêksuit illunamassiatik attanniovingine? Aggangnelo pitsartunekarpok sôngonekarlunelo, kialonêllo akkerartorungnangilâtit.

7 Igvit Gudivut nunab tamattoma innungit pêjarlaungipigît innukotivit Israelitit sivorngane, tunnilaungipiuglo Abrahab nagligijavit kinguvânginut issokangitomut?

8 Nunagilaukpâllo ivsornaitovingmiglo atternut igloliorlaukput tapsomane, okarlutiglo:

9 Tikkitauleraikupta kannoêtomut, savilksoarmut, pitlartaunermut, nusilingnartomut, kângnartomullonet, tagva nangeriartoromavogut iglub oma sânganut, sângnullo, (attit iglub oma illuanêmat,) nipliarvigijomavaptigillo kappiagijavut pitjutigællugo, igvillo tussarniarpotit, ikajorniarlutillo. 
10 Mannalo ahâk, Amonib Moablo kittorngangit, taipkoalo Seirib kakkanginit, opaktaukolaungitatiit Israclib kittornganginut, Egiptenemit aular'illugit, sennerkojariakalaukpaille, péjarlaungilaillo ;

11 Ahaglo, manna akkiniarlukpâtigut, kailutiglo singitsomavluta perkutigijarnit, tumnilauktarnit uvaptingnut pigitsainarlugo.

12 Gudivut, erkartoromangipigit? Uvaptingne pitsartunekangimat unnuktoksuit taipkoa opaluktivut akkerartorungnarlugit. Nellorogut kannok pinniaraksaptingnik, ijiptale torarvigivâtit.

13 Judakullo illuêngarlutik nangerput Nalekab sângane, kittorngatik, nulliatiglo, ernitiglo illagilugit.

14 Jehasiele Zachariab erninga Benajab erningata, Jehielib erningata, Mathanjab erningata, Leviteôjok Assafib kittornganginit, tupviolerpok Nalekab annerninganik, illagêt kattimajut akkorngane.

15 Okarporlo: Okokse, Judamiôjose illunase, Jerusalemiôjoselo, attanniojotillo Josafate! Imâk Nalegak okarpok illipsingnut: Kattimgajoksuit taipkoa sivuragijaksaringilasse, maksujarutioinagillonêt: illipse sorsuglunginapse, Gudele.

16 Kaupat attersarvigijaksarivasse; ahâglo, majokame Zizemik attelingme majorarniarput, naipiniarpasselo naksab pângane, sunakajuitub Jeruelemik attiggub sângane.

17 Illipse sorsugiariakarniangilase pijokartome tamattomane. Nangeriartortuinaritse, nangerluselo tækkolerluselo Nalekab illagijipse piuliklerninganik, Judamiojose, Jerusalemiojoselo. Sivuraniarase maksujarniaraselo; kaupat aularitse, pâriartorlugit, Nalekab illagivâse.

18 Tagva Josafate okkolerpok kênane pûvlugo nunamut; Judakullo, Jerusalemiullo illunamassiatik pamakterput Nalekab sânganut, Nalegarlo opigivât.

19 Levitillo, Kahatib kittornganginit, Korablo kittornganginit, makkiput Nalegak Israelib Gudinga nertorlugo imgerlutik nippersoarmut killangmut.

20 Uvlarârsukullo parngnailerput, aularlutiglo Teknab sunakajuitunganut. Aulartillugillo Josafate nangerpok okarlunelo: Naleksinga Judamiôjose Jerusalemiôjoselo: Nalegak Gudise okperisiuk, tagva ussornarniarpose; nellautaijokotingillo okperisigik, tagva sullidlarniarpose.

21 Innugiartoksuillo okarkattigivait, imgertokartitsivorlo $\mathrm{Na}$ lekamut, nertordlerkovlugit pinarnarsinerme heiligeôjome, sivurlerkovlugillo oppalungêrsimajunik unatadlarnermut, okarkovlugillo: Nalegak kujagisiuk napkiningninga sorainiatsengimat.

22 Pigiartillugillo kujalilutik nertordlerlutiglo, Nalegak terliarsijokartitsivok Amonib Moablo kittornganginik, tapkoning- 
alo Seirib kakkanginit pijunik, tikkitunik Judamut; ullimârtaulaukpullo.

23 Amoniblo Moabiblo kittorngangita taipkoa Seirib kakkanginit pijut nangerdlutik akkingarpait pêjarlugit nungutsomavlugillo, Seiremiullo nungulerêramigit, tagva tapkoa illangata illane ikajorpa asserortigêkovlugittauk.

24 Judakulle kakkab kânganut tikkimatta sunakajuitub sakkânut, tagva sanguvut kattimgajoksoarnut taipkonunga, ahâglo, timingit tokkungavlutik nellavut nuname, kemâjokalaungilarlo attautsemiglonêt.

25 Tagva Josafate innugiartoksoakotine illagilugit tikkipok, perkutingit aviklugit, nachvarpullo tapkonane unnuktovaksoarnik, perkutinik annorangniglo, illulerkutiniglo pivianartunik, aksarpaillo tamakkoninga taimak unnuktigijunik, akjartortaujungnangimatta; aksarnigaujallo avikpait uvlut pingasut nâlagit, unnuktolaungmatta.

26 Uvlulle sittamangane kattimalerput nertordlernerub naksangane, tagvane Nalegak nertormatsuk. Tamanna pivlugo inne tamna atserartaurok nertordlernerub naksanganik uvlok tamanna tikkilugo.

27 Taimaglo Judamiut Jerusalemiullo angutingit utterput, Josafatelo sivurliovlune, Jerusalemeliarkovlugit kuviasuklutik. Nalekab kuviasungnermik tunnitsivigilaungmagit omigijingit pivlugit.

28 Jerusalemullo itterput Psalterijarlutik, Harfesijarlutiglo Trompetesijarlutiglo Nalekab iglunganut.

29 Gudiblo sivuranarningata attanniovit illunaita nunane tupvigilerpait, tussarmatta Nalekab Israelitit omigijingit sorsugvigilaungmagit.

30 Taimaglo Josafatib attanniovinga innilangalaukpok, Gudiblo merngoêrsernermik tunnitsivigiva avatâne illunane.

31 Josafatelo attanniotipok Judakunut; Jahrekalaukporlo 35 ginik attanningorame, attanniotilunelo Jahrit 25 git nâlugit Jerusaleme. Anânanga attekalaukpok Asubamik, Silhib panninga.

32 Agvertarporlo atâtame Assab apkosiningine, katsungalaungilarlo pinniarlune Nalekab namagijanganik.

33 Pôktôjulle pêjartaulaungilet, sullelo innugiartoksuit omattitik torartilaungilait atâtagijamik Gudinganut.

34 Unipkautsit assingit Josafate pivlugo, sivurlit kingurlillo, ahâk tamakkoa aglaksimavut Jehub Hananib erningata unipkausingine, aglalauktangine, Israelitit attanningita aglanginut. 35 Kingornganelo Josafatib Judakut attanningata Ahasja Israelitit attanningat piungitunik pinniarnelik angerkattigiva.

36 Angerkattigivâlo umijoromavlutik, immarbiksoarmut umiaktortuksanik; umijorpullo Ezeon Gebereme. 
37 Tagva Elieser Dodavab erninga Maresamiok nellautailerpok Josafate kiglornilugo, okarlunelo: Tamanna pivlugo Ahasja angerkattigilaurangne Nalekab pinniarnît asserorpa. Umiaksuillo serkomitauvut, umiaktorungnarlaungilello immarbiksoarmut.

\section{KAPITEL XXI.}

Joramib tokkotsininga nukkaminı, kivgartorningalo Gudingoarik, tokkungalo.

JOSAFATELO sinnisilerpok, atâtagijaminut ailune, illijartortauvorlo atâtagijamine Davidib iglugaseksoangine; erningalo Jorame attanningorpok innangêrdlugo.

2 Nukkakalaukporlo Josafatib erninginik, Assarjamik, Jehielemiglo, Sachariamiglo, Azarjamiglo Michaelemiglo, Sefatjamiglo, tapkoa illunaita Josafatib Judakut attanningata ernerilaukpait.

3 Atâtangatalo tunnitsivigivait tumnergutinik unnuktunik Silberemik Goldemiglo, pivianartuniglo, iglugaseksoarniglo sôngojunik Judamêtunik illaliudjilune, attanniovingmigle Jorame tunnitsivigiva, erniangokârtongmat.

4 Joramele attanningortitaujarêrame, atâtame inningane, pallangaijarêramelo, nukkane illunaita tokkopait saviksoarmut, illagilugillo angajokaunerôjut Israeleme illangit.

5 Jorame Jahrekalaukpok 32 ginik attanningorame, attanniotilunelo Jahrit 8 it nâvlugit Jerusaleme.

6 Agvertarlunelo Israelitit attanningita apkosiningine, sorlo Ahabekut pinniarmatta, Ahabib pannia nulliaringmago; pinniarlunelo Nalekab idluigijanginik.

7 Nalekable Davidib iglunga (kinguvangit) asseroromalaungila, angerkattigêngnek pivlugo Davidemut angernerilauktanga, sorlolo okalaungmat, tamnalo kittorngangillo kaumautekartitsomavlugit nelliutune tamaine.

8 Tapsoma nelliuningane Edomiterit apterput Judakunit attannekartitsivullo ingmingnut.

9 Tagva Jorame angajokâkotine kamutekotinelo aksalloaggit illagælluit, aularpok taipkonunga, unnuamelo parngnailerpok, ullimarpaillo Edomiterit illumajine, kamutillo angajokangit.

10 Taimaglo Edomiterit apterput Judamit uvlok tamanna tikkidlugo. Nelliutome taipsomane Libna (iglugaseksuit) apterivut, Nalegak atâtagijame Gudingat kemangmago.

11 Poktôjuniglo sennavok kakkane Judamêtune, Jerusalemiullo ajortullitipait, Judamiullo uivêrivait.

12 Aglangniglo tikkitokarpok tapsomunga, nellautaijomit Eliamit pijunik, imâk okartunik: Imâk Nalegak, atâtagijavit 
Davidib Gudinga okarpok: Tamanna pivlugo agvertarlaunginavit atâtavit Josafatib apkosiningine, Assablo Judakut attanningata apkosiningine;

13 Agvertaraville Israelitit attanningita apkosiningane, Judamiullo Jerusalemiullo ajortullitigangne, Ahabekut ajortulliningit malliklugit; nukkatillo, atâtavit iglomiokotingit idluarkijattit nakterlaukpattit;

14 Ahak, tagva Nalekab pagvisarnartoksoarmik tikkikojiniarpok, innukotingnut, kittorngakotingnullo, nulliakotingnullo, perkutingnullo illunainut.

15 Igville kannimasekarniarpotit unnuktunik; kannimasermik erchavingne, erchavitit kannimasermut annilerkârtinagit uvlormit uvlormut.

16 Taimaglo Nalekab Filisterit annerningit, Arabiamiullo Aethiopiamiut sennianetut annerningit akkerartortipait Joramemut.

17 Majorarpullo takpaunga Judamut ittârpâllo, aksarnigarivaillo perkutit tamaita attanniub iglunganêlauktut, taimaglotauk erningit, nulliangillo, amiakokalungimat ernermik, Joahasib nukardlerpângojub assianik.

18 Tamakkoalo tamaita kingorngane pagvisarpa kannimasernermut erchavingine âkiktaujungnangitomut.

19 Taimailingalaukporlo uvlormit uvlormut; Jahriglo magguk nângmanik erchavingit annilerput tapsomangat kannimasermut, tokkovorlo serlekidlarlune. Ikkoalanekartitsilungilello kângane, sorlo atâtagijangine pinniarlaungmatta.

20 Jahrekalaukpok 32 ginik attanningorame, attanniotilunelo Jahrit sittamaujortut nâvlugit Jerusaleme, agvertarnekalunelo ânanaulungitomik, annernêngerporlo oggurijaunane. Illijartorpâllo Davidib iglugaseksoangine, attannille illuvinginêungitok.

\section{KAPITEL XXII.}

Ahasjab attanniotininga, tokkungalo. Anânangata Athaljab pinniarlungningit.

JERUSALEMIULLO Ahasja, erningita nukârdlerpângat attanningortipat innangêrdlugo. Sorsuktuksalle Arabiamiunit tikkitut tanginarvingmut, angajuklit illunaita nakterlaukpait. Taimaglo Ahasja Joramib Judakut attanningata erningata attanningorpok.

2 Ahasja Jahrekalaukpok 42 ginik attanningorame, attan- 
niotilunelo Jahremik attautsemik Jerusaleme. Anânanga attekalaukpok Athaljamik, Amrib panninga.

3 Agvertarporlotauk Ahabekut apkosiningane. Anânangata attannertormago idluitullikovlugo.

4 Tamanna pivlugo pinniarpok Nalekab idluigijanginik Ahabekutitut. Taipkoa atâtangata tokkungata kingorngane attanneringmagit asserortaujutiksanganut.

5 Taipkoalo attannertuiningat malliklugo agvertarpok. Joramelo Ahabib Israelitit attanningata erninga aularkattigiva unatadlarnermut Ramotemut Gileademêtomut, Hasaele Siriamiut attanningat sorsugvigijomavlugo. Siriamiulle Jorame ikkilerpât;

6 Tagva utterpok mammititaujomavlune Jesreeleme, ikkinit pilauktaminit Ramoteme Gileademêtome, Hasaele Siriamiut attanningat sorsugvigingmago. Ahasjalo Joramib erninga Judakut attanningat attersarpok Jorame Ahabib erninga Jesreeleme kannimavlune nellajok tækkojartoromavlugo.

7 Ahasjablo asserortauninga Gudemit pilaukpok, tikkininganut Joramemut, tikkisimagamelo Jorame aularkattigiva Jehu, Nimsib erninga, Nalekab mingoarlauktanga Ahabekut pêjarlugit, pachreartorlugo.

8 Pijokalaukporlo Jehub Ahabekut pitlarmagit, Judakut angajokangit Ahasjablo angajungita erningit, Ahasjamik kivgartortut naipipait, tokkopaillo.

9 Ahasjalo kennerpa, tiguvâlo, ijersimatillugo Samariame, âpâllo Jehumut, tokkopâllo, illijartorlâllo. Okarmatta: Josafatib kennerlauktub Nalekamik omamut tamânut erneriva. Ahasjablo iglungane kinakalaungilak attanniolerungnarajartomik.

10 Athaljalo Ahasjab anânanga tækkogame ernine tokkungmat, parngnailerpok tokkotsilunelo Judakut attanningata kinguvânginik illunainik.

11 Josabeatible attanninb najangata Joase Ahasjab erninga tiguva, tiglilalo attanniub kittorngangita tokkotaujut akkornganit, pitipâlo pairijinga aiparællugo innemut sinnigvingmut. Taimak Josabeatib attanniub Joramib panniata ajochertujjub Jojadab nulliangata ijerpa Athaljamit, (Ahasjab najagingmago) tokkotaukonago.

12 Illagilugillo Gudib iglungane ijersimalaukpok Jahrit pingasojortut nâvlugit, Athalja attanniotillugo nuname. 


\section{KAPITEL XXIII.}

Joasib mingoartauvlune attanningortitauninga Jojadamut. Athaljab tokkotauninga; kivgartornerub Baalemik asserortauninga.

JAHRILLE 7 ingane Jojada maksoalerpok, angajokajallo 100 dinut, tagva Asarja Jerohamib erninga, Ismaelelo Johannanib erninga, Asarjalo Obedib erninga, Maesejalo Adajab erninga, Elisafatelo Sichrib erninga, tiguvait, angerkattigilaitalo.

2 Tapkoa ingergavut Judakut nunangatigut, kattikovaillo Levitit Judakut iglugaseksoanginit illunainit, atâtallo Israeleme angajokaunerôjungit, kaikovlugit Jerusalemut.

3 Illagêflo illunamassiangita attannek angerkattigivât Gudib iglungane. Jojadablo okautivait: Attanniub erninga attanniojuksauvok, sorlo Nalekab Davidib erningit okautigilaungmagit.

4 Tagva manna imailiortuksauvose: Illipse pingasoliluse pingajuise attautsit, ajochertuijunit Levitinillo nangeriartortuksauvut Sabbatib iglungane udsertorlutik upkoanik;

5 Pingajuiselo attautsit attanniub iglunganêtuksauvut; pingajuiselo attautsit upkoaksoarme angijomêtuksauvut, innugiartoksuillo illunamassiatik innemêtuksauvut avaluliorsimajome Nalekab iglunganêtome.

6 Kinalo Nalekab iglunganut itterkonnago, ajochertuijut Levitillo kivgartortut assingat, tapkoa ittèrtuksauvut ivsornainamik; innugiartoksuille assingit illunatik udsertortuksauvut Nalekamik.

7 Levitillo attanniub avatânetuksauvut, attunît sakkomingnik tigumiarlutik aggangmingnut. Kinalo iglomut ittertok tokkotaujuksauvok. Tapkoalo attanermêtuksauvut annileraikpat itteraikpallo.

8 Levitillo Judamiullo illunatik taimailiorlaukput, sorlo Jojada ajochertuijok perkojilaungmat, attunillo innukotitik ingmingnut pitipait, tikkitut Sabbateme nûlertuksat illagilugit, Jojadab ajochertuijub ingmigolingajut nûkolaungimagit.

9 Jojadablo ajochertuijub angajokajat 100 dinut tunnitsivigivait kallugiarnik, sappulutaniglo, sakkuniglo, Davidib pigilauktanginik, Gudib iglunganêlauktunik;

10 Innugiartoksuillo illunaita pitipait attunît sakkumingnik tigumiarlutik aggangmingnut, iglub sennerangata tallerpianit iglub sennerangata saumianut tikkidlugo, Altareme, iglomelo attanniub avatâne.

11 Attanniublo erninga annitipât, niakoserpâllo, tunnitsi- 
vigivâllo perkojanik, attanningortilâllo. Jojadablo erningitalo mingoârpât, okarlutiglo: Attannek innôle!

12 Athaljable innugiartoksuit, opaktut, attannermiglo nertordlertut nipliadlarningit tussaramigit, aivok innugiartoksoarnut Nalekab iglunganut.

13 Tækkosarlunelo, ahâglo, attannek nangerpok nangervigijamine ittervingme, angajokajallo, trompetesijartullo attannek nangerkattigivat, nunablo innugiartoksoangit illunatik kuviasuklutik tiptulausijarlutiglo, imgerpaktullo nokakseralingnik sunatuinarnik attorlutik, nertordlerlutiglo. Tagva annorâne allikpait, okarlunelo: Pikkablajoksonek! pikkablajoksonek!

14 Jojadable ajochertuijub angajokajat 100 dinut, angajokaujut sorsuktuksanut tikkerkovait okautilugillo: Annitisiuk, siaktut akkornganut tikkidlugo; malliktorlo tapsominga tokkotaujuksauvok saviksoarmut; ajochertuijok okarmat: Tokkoniarassiuk Nalekab iglungane.

15 Aggatiglo illivait tapsomunga, tikkimallo horsit ittervianut attanniub iglungane, tokkopât tagvane.

16 Jojadalo angerkattigêngnekartitsivok, tapsomane innugiartoksoarnelo illunaine, attannermelo innukutaujuksaugamik Nalekamut.

17 Tagva innugiartoksuit illunatik itterput Baalib iglunganut, immitipâllo, Altaringillo, innungoangillo serkomipait. Mathanelo Baalib ajochertuijokotinga tokkopât Altarit sângine.

18 Jojadablo sullijaksat Nalekab iglungane perkovait ajochertuijunut, Levitinullo Davidib ingmigolingatilauktanginut, Nalekab iglungane tunnergutinik ôtaksanik tunnilutik Nalekamut pikovlugit, sorlo aglaksimangmat Mosesib perkojangine, kuviasuklutik, nertordlerutiksakarlutiglo Davidib illusinga malliklugo.

19 Udsertortokartitsivorlo upkoanik Nalekab iglungata upkoaksoangine, ivsornartomik nellianiglonêt ittertokarkonago.

20 Tiguvaillo angajokajat 100 dinut; erkinarnerpâllo nalegaujullo innugiartoksoarne, nunablo innungit illunaita, attersartipâlllo attannek Nalekab iglunganit, âlâllo upkoaksoakut pôktojokut attanniub iglunganut; ingitipâllo attannek attanniub iksivautaksoanganut.

21 Nunablo innungit kuviasudlalaukput; iglugaseksuillo innilangalaukput; Athaljale saviksoarmut tokkolaukpat. 


\section{KAPITEL XXIV.}

Tempelib akiktauninga. Joasib nakkilersininga tokkotsiningalo nellautaïomik; tokkotauningalo.

J OASE Jahrekalaukpok 7 nik attanningorame, attanniotilunelo Jahrit 40 git nâlugit Jerusaleme. Anânanga attekalaukpok Zibjamik, Bersebamiok.

2 Joaselo pinniarlaukpok Nalekab namagijanganik, ajochertuijok Jojada innotillugo.

3 Jojadablo nulliakartipa maggungnik; ernetarlunelo pannetarlaukpok.

4 Kingornganelo Joase kajusimalerpok Nalekab iglunga nutangortitsomavlugo,

5 Ajochertuijullo Levitillo kattikovait, okautilaitalo: Aularitse Judakut iglugaseksoanginut illunainut, kattersoiluselo kênaujanik Israelitinit illunainit Nalekab Gudipse iglungata âkiktaujutiksanganut illingajuksanik Jahremit Jahremut, errinesukluselo taimailioritse. Levitille tuavilaungilet.

6 Tagva attanniub Jojada ajochertuijersoak kaikova, okautilâlo: Sôg Levitit kammagingipigit, kattersoikovlugit Judamiunit Jerusalemiunillo, tunnergutinik, Mosesib Nalekab kivgangata kattersortaukojanginik Israelitit akkorngane, perkojaktaliub tuppinganut?

7 Athaljable Gudekangitub, erningitalo Gudib iglunga serkomilaukpât, tamaitalotauk Nalekab iglunganut ivsornaititaumalauktut, perkutautilaukpait Baalemut.

8 Tagva attannek perkojivok iklervingmik sennakovlugit, illivâllo Nalekab iglungata itterviksoangata sillatânut.

9 Akpartaukojilunelo Judame Jerusalemelo, kaitsijuksaungmatta Nalekamut Mosesib Gudib kivgangata tunnijaukojanginik Israelitinut sunakajuitome.

10 Tagva angajokajat illunatik innngiartoksuillo illunatik kuviasulerput, kaipaillo, egilugillo iklervingmut, tættækârtinago.

11 Nelliutileraikpallo iklervik âtaujuksaulaungmat Levitinut, attanniub perkojanga malliklugo, tækkopatta kênaujakarmat unnuktunik kammane, tagva attanniub aglakpaktokotinga, ajochertuijersoarmullo perkojaujut kaikattarput navilâllo iklervik, tiguvâllo âpsarlugolo inninganut. Taimailiorput uvlut tamaita, kattersoilutiglo kênaujanik unnuktunik.

12 Attanniublo Jojadabio tunnivait sennajunut sullijaksanik Nalekab iglungane, sullijaksakartitsijunullo, ujaralerijunik, ig- 
loliorteniglo Nalekab iglunga nutângortilugo, sabviorteniglo kikkiangmik, kannujarmiglo Nalekab iglunga âkiksorlugo.

13 Sennajullo sennavut, âkiktaunerlo pivalliavok tapkoa aggangittigut, innermarikpâllo Nalekab iglunga sattortautimilugo, sôngotipâllo.

14 Pijarêramiglo kênaujat amiakojut sulle, âpait attanniub Jojadablo sângangnut; tapkonangallo illulerkutit Nalekab iglunganut illingajuksat sennajauvut, illulerkutit kivgartornermut illingajuksat, tunnergutinullo ôtaksanut, allupsautit, illulerkutit Goldeôjut, Silbereôjullo. Tunnergutiniglo ôtaksanik tunnijitsainarlaukput Jojada innôtillugo.

15 Jojadale innutokaulaukpok, namaksilunelo innoserminik, tokkolarmelo; Jahrekalaukporlo 130 ginik tokkogame.

16 Illijartorpâllo Davidib iglugaseksoangine, attannit akkornganut, ajungitullilaungmat Israelemut, Gudemullo, iglunganullo.

17 Jojadablo tokkungata kingorngane, Judakut angajokaunerôjungit tikkiput, tuksiarvigilugolo attannek; attanniublo nalekpait tagva.

18 Nalekablo atâtagijamik Gudingata iglunga kemmakpât, kivgartorlugillo nappartut Gudingoaggit, Gudingoallo assingit. Tagva Judakut Jerusalemiullo ningautsimut tikkitauvut, idluitullinek tamanna pitjutigællugo.

19 Tapsomalo nellautaijut tillivait tapkonunga, uttertitsomavlugit ama Nalekamut, tennersarpaillo; kissianele kammagingilât.

20 Gudible annerningata Zacharja Jojadab ajochertuijub erninga tupvigilerpa, nangeriartorporlo innugiartoksuit kollânut, okautilaitalo: Gude imâk okarpok: Sôg Nalekab perkojangit tappagivissigîk? Sulliniatsengilase. Nalegak kemagapsiuk, kemaniarivâse.

21 Angerkattigêngnekartitsivulle akkerartorlugo, millorpâllo inneme avaluliorsimajome Nalekab iglunganêtome, attanniub perkojanga malliklugo,

22 Attanniublo Joasib napkoarnek erkarlaungila, Jojadab Zachariasib atâtangata pinniarlauktanga tapsomunga; innuarpâle erninga. Tokkovlunelo okarpok: Nalekab tækkova, akkiniarutigivâlo.

23 Pijokalaukporlo Jahre kângermat Siriamiut sorsuktuksangit majorarlutik takpaungarput Judamut Jerusalemullo, tokkopaillo innugiartoksuit angajokangit illunaita; aksarnigarijatiglo tamaita tillikliutivait Damaskusemiut attanninganut.

24 Siriamiut sorsuktuksangit ikkitotuinarlutik tikkilauralloarput, Nalekable tapkoa agganginut tunnivait sorsuktuksat kattimgajoksuit unnuktut; tamanna pivlugo Nalegak atâtagijamik Gudingat kemalaungmatsuk. Joaselo pitlarpât. 
25 Aularmattalo tapsomangat, (kemakpât kannimadlartillugo) kivgakotingit angerkattigêngnekartitsivut akkerartorlugo, Jojadab ajochertuijub erningita aungit pivlugit, nakterpâllo innangavingane, tokkovorlo. Illijartorpâllo Davidib iglugaseksoangine, attannille illuvingita akkornganeungitok.

26 Tapkoangôvugle angerkattigêngnekartitsilauktuk tapsomunga: Sabade Simeatib erninga, arnab Amonitereôjub, Josabadelo Simritib erninga, arnab Moabitereôjub.

27 Erningille, tunnijariakarningitalo unnurningit, Gudiblo iglungata igluliortauninga, ahâk, tamakkoa aglaksimavut, unipkautsine attannit aglangine. Erningalo Amazia attanningorpok innangêrdlugo.

\section{KAPITEL XXV.}

Amaziab Judakut attanningata attanniotininga.

AMAZIA Jahrekalaukpok 25 ginik attanningorame; attanniotilunelo Jahrit 29 it nâlugit Jerusaleme. Anânanga attekalaukpok Joadamik, Jerusalemiok.

2 Pinniarporlo Nalekab namagijanganik, omamulle tamânuungitok.

3 Attanniovinga manna pallangersimangmat, kivgakotine nakterpak, attannermik atâtanganik ullimarnilauktuk.

4 Kittorngangille tokkolaungilait. Taimak aglaksimangmat perkojane, Mosesib aglangine, Nalegak perkojingmat, okarlunelo: Atâtat tokkotaujuksaungilet kittorngat pivlugit, kittorngallonêt atâtat pivlugit; nellialle ajornine pivlugit tokkotaujuksauvok.

5 Amaziablo Judakut kattikovait, pitipaillo atâtat kinguvângit (iglungit) malliklugit, angajokait 1000 dinut 100 dinullo malliklugit, Judakut illuêngarlutik Benjaminekullo; kittipaillo Jahrelingnit 20 ginik unnurnersaniglo, naipipaillo 300000 diojut annerimajut, aularungnartut unatadlarnermut, kallugialijarungnartut, sappulutalijarungnartullo.

6 Tapkoa tappilugit Israelitinit ikajortiksarsivok angutinik sôngojunik unatadlarnermut 100000 dinik, Talentinut 100 dinut Silberemik.

7 Gudible angutekotingita illangata tikkipa, okarlunelo: Attannek Israelitit sorsuktuksaksoangit illaukoniarnagit illingnut, Nalekab Israelitit Efraimib kittorngangillonêt illagingimagit.

8 Igville aularit, sôngoniarlutillo sorsungnerme, Gudib sapkoniarajarmattit omigijivit sângine; Gudeme pitsartunekarmat ikajorlune, sapkutsilunelo.

9 Amaziab Gudib angutekotinga tamna okautiva: Kan- 
norlo Talentit 100 dit tunnimalauktakka sorsuktuksanut Israelitinit pinniarvigijaujuksauvât? Gudib angutekotinga okarpok: Nalegak unnurnersakarpok sulle makkonangat, tunnijungnartaminik illingnut.

10 Tagva Amaziab sorsuktuksat tapkoa Efraimekunit tikkilauktut tapsomunga apterpait, aulirkovlugit nunamingnut. Tagva ningarningita Judakut illungertutigilerpait, aularlutiglo nunamingnut, ningarnermut illungertorlutik.

11 Amaziale maksoalerpok, innukotinelo aularutivait naksarmut sioraujalingmut, Seiriblo kittorngangit 10000 dit ullimarpait.

12 Israeliblo kittorngangita anguvait tapkonangat 10000 dit innôtillugit; tapkoa tessiorpait kairtub sinâkjuata kânganut, ijukartipaillo kairtub sinâkjuanit, illunatik kârkovlugit.

13 Sorsuktuksalle Amaziab utterkolauktangit, innukotine illagilugit sorsungnermut aikonagit, ittârput Judakut iglugaseksoanginut Samariamit Beth-Horon tikkidlugo, ullimarpaillo tapkonangat 3000 dit, aksarnigaujokartitsivullo unnuktunik.

14 Amazialo utterame Edomiterit sâlagijauninganit, Seirib kittorngangita Gudingoangit neksarpait Gudiotilugillo ingminut, tuksiarvigilugillo isserluanititsivigilaitalo.

15 Tagva Nalekab ningarningata Amazia illungertutigiva, nellautaijullo illangat tilliva tapsomunga, okautilalo: Sôg innugiartoksuit Gudingoangit kennerpigit, innukotimingnik piuliklerungnarlaungitut aggangnit?

16 Okarkattigingmagolo (Amaziab) okautiva: Igvit attannertuijungortitaumavit attannermik? Nippangerit. Sunna pivlugo annaulertaujomavit? Tagva nellautaijok nokkarpok, okarlunelo: Mallugosukpunga, Gude kajusimangmat asseroromavlutit, taimailioravit attannertuineralo nalenginangne.

17 Amazialo Judakut attanningat issumalerpok, tilliklerlunelo Joasemut Joahasib erninganut Jehub erningata, Israelitit attanninganut, okautsaukovâlo: Kait tækkonautilauluk!

18 Joasele Israelitit attanningat tilliklerpok Amaziamut Judakut attanninganut okautsaukovâlo: Orpik Dornemik attelik, Libanonemêtok, tilliklerpok Cederemut Libanonemetomut, okautsaukovâlo: Pannît tunnileruk ernimnut, nulliangokovlugo tapsomunga; omajulle nujuartut Libanonemêtut, orpik Dorneôjok tullerajakpât tullerlugolo serkomipât.

19 Issumavotit: Ahâk Edomiterit salagilaukpakka; tamanna omattit piojorijutigiva, nertornautiksarsiorlutillo; manna nunangnêtualerit. Sôg kannoêtoksiorkit, ochokovlutit, Judakullo illagællutit?

20 Amaziale nalelaungilak; tamannale Gudemit pilaukpok, tunnilugit pikovlugo, Edomiterit Gudingit kennerlaungmagit.

21 Tagva Joase Israelitit attanningat majorarlune tak- 
paungarpok, tækkonautivuglo, tamna Amazialo Judakut attanningat, Bethsemeseme Judamêtome.

22 Judakulle salagijauvut Israelitinut, kemâlutiglo attunît iglomingnut.

23 Joasiblo Joahasib erningata Israelitit attanningata Amazia Judakut attanningat Joasib erninga tiguva Bethsemeseme, tikkiutilâlo Jerusalemut, Jerusalemiblo karmangit (avaluksoangit) serkomipait, itterviksoarmit Efraimemik attelingmit, terrekub itterviksoanga tikkilugo, tallitut 400 ditut takkitigijomik.

24 Goldillo Silberillo tamaita, illulerkutillo tamaita Nalekab iglunganêlauktut Obed-Edomeme, attanniublo iglungata perkutitalingane, sorrutsillo sattoraksauvlutik tiguvait, neksarlugillo Samariamut.

25 Amazialo Joasib erninga Judakut attanningat innôlaukpok sulle Joasib Joahasib erningata Israelitit attanningata tokkungata kingorngane Jahrinik 15 nik.

26 Unipkautsit assingit Amazia pivlugo, sivurlit kingorlillo, ahâk, tamakkoa aglaksimavut Judakut Israelitillo attanningita aglangine.

27 Nelliutomillo taimangat Amaziab allaivianit Nalekamik, angerkattigêngnekartitsivut Jerusaleme akkerartorlugo; kemâvorlo Lachisemut. Tagva malliklugo tilliklerput Lachisemut, tokkopâllo tagvane.

28 Horsekullo âpât, illijartortauvorlo atâtagijamine Judakut iglugaseksoangine.

\section{KAPITEL XXVI.}

\section{Usiab atianniotininga.}

TAGVA innugiartoksuit Judakut illunamassiatik Usia Jahrekalauktok 16 nik tiguvât attanningortilâllo atâtangata Amaziab inningane.

2 Tapsoma Elothe iglugaseliorpait, sattortautilaitalo Judamut, attannek sinnisilaukârdlune atâtagijane illagællugit.

3 Usia Jahrekalaukpok 16 nik attanningorame, attanniotilunelo Jahrinik 52 ginik Jerusaleme. Anânanga attekalaukpok Jechaljamik, Jerusalemiok.

4 Pinniarporlo Nalekab namagijanganik, sorlo atâtanga Amazia pinniarlaungmat.

5 Gudelo kennerpa taimak akkunêrtigijomik Sacharja, tukkisijok Gudib tækkotitanginik, innôtillugo; taimaglo akkunêrtigijomik Nalegak kennerlaungmago, Gudib sullidlarkolaukpa.

6 Aularporlo, Filisterillo sorsukpait, Gatiblo karmangit 
(avalungit) Janiblo karmangit, Asdodiblo karmangit serkomipait; iglugaseliorporlo Asdodib avatâne, Filisterillo akkorngane.

7 Gudiblo ikajorpa Filisterit akkerartorlugit, Arabiamiut Gurbaaleme nunakartut akkerartorlugit, Meuniterillo akkerartorlugit.

8 Amoniterillo Usia tunnitsivigivât pilliutinik, attingalo okausiolaukpok Egipten tikkidlugo; sôngopalliatsainarmat.

9 Usiablo nuvugutsit igloliorpait Jerusaleme, terrekub itterviksoangane, naksablo itterviksoangane, terrekullo assingine, sôngotipaillo.

10 Igloksuillo avaluliorsimajut igloliorsavait sunakajuitome, aggarlunelo immektarvingnik unnuktunik, nergjutekalaurame unnuktunik naksarne, naternanelo; perorsaijokutekalaurivok perorsêvingne, kakkanelo Weineliksalingne, Karmelemelo; perorsainek kuviasutigigamiuk.

11 Usialo sorsuktúksakutekalaukpok sorsugungnartunik, aulartunik unnatadlarnermut, kattimgajovlutik ingmigolingatitauvlutik, sorlo kittitaumangmatta Jejelib aglakpaktub agganginut, Masejamullo aulatsijomut, Hananjab attanniub angajokakotingita illangata aulatsininganut.

12 Atâtallo angajokaunerôjut, sorsuktuksat sôngojut akkorngane unnurningit, 2600 diolaukput.

13 Tapkoalo aulatsiningine sorsuktuksanik 307500 dinik sorsugungnartunik, sôngudlarlutik attannek ikajorlugo omigijingit akkerartorlugit.

14 Usablo sorsuktuksat illunaita pittakartipait sappulutanik, kallugiarniglo, nessâlungniglo, sagvingmiutaniglo, pittikseniglo, ujarkaniglo illoereumut.

15 Tallutiniglo sennavok Jerusaleme tattamnadlartunik, nuvugutsit kânginêtuksanik, terrekullo kânginêtuksanik, pittikserviksaniglo kârgjunik, illoerearviksaniglo ujarkanik. Tussaumajauvorle kanningitomut, tattamnadlartomik ikajortaulaungmat, sôngokârtinago.

16 Sôngoleramele omattinga piojorilerpok asserortaujutiksaminut. Idluitullivorlo Nalekamut Gudiminut, ailunelo Nale$\mathrm{kab}$ Tempelinganut, isserluanititsijomavlune isserluanititsiviub Altaringane.

17 Asarjablo ajochertuijub illagællugo ajochertuijut 80 git, angutit erkomatsiartut, mallikpât,

18 Kiglornilâllo Usia attannek, okautilugolo: Igvit Usia isserluanititsijuksaungilatit Nalekamut; ajochertuijulle, Aronib erningit, ivsornaititaumajut isserluanititsikovlugit. Annit ivsornaitovingmit, tammaravit; nertortaujutiksarnullo Gudib Nalekab sângane illinganiangilak.

19 Usiale ningalerpok illulerkullo isserluanititsivik tigumiarpa aggangminut, ajochertuijunullo okalokitillugo, kallak 
annilerpok kaungane ajochertuijut tækkoæne, Nalekab iglungane, isserluanititsiviub Altaringata sângane.

20 Asarjablo ajochertuinerôjub, ajochertuijullo illunatik tækkosarmatsuk, ahâk, tagva kallaksimalaukpok kaungane, annitipâllo. Nangminerlo tuavilukarpok annileromavlune, Nalekab annaulaungmago.

21 Taimak Usia attannek kallaliôlaukpok tokkone tikkilugo, iglolo ingmigolingajok innigiva kallaliôvlune, avisimagame Nalekab iglunganit. Jothamible attanniub erningata attanniub iglunga aulapa, idluarsarlaitalo innugiartoksuit nuname.

22 Unipkautsille tamakkoa assingit Usia pivlugo, sivurlit kingurlillo nellautaijub Jesaiasib Amozib erningata aglagutigilaukpait.

23 Usialo sinnisilerpok atâtagijaminut ailune, illijartorpâllo atâtagijangine, natername attannit illijartortauvingane. Okarmatta: Kallaliovok. Erningalo Jothame attanningorpok innangêrdlugo.

\section{KAPITEL XXVII.}

\section{Jothamib attanniotininga.}

JOTHAME Jahrekalaukpok 25 ginik attanningorame, attanniotilunelo Jahrit 16 it nâlugit Jerusaleme. Anânanga attekalaukpok Jerusamik, Zadokib pannia.

2 Pinniarporlo Nalekab namagijanganik, taimaluatsiak sorlo atâtanga Usia pinniarlaungmat; kissiane Nalekab Tempelinganut itterlungimat; innugiartoksuille idluitullivut sulle.

3 Tapsoma Nalekab iglungane itterviksoak kogvasiktok igloliorpa, avalungnelo Ofelemik attelingne sennavok unnuktunik.

4 Iglugaseksoarniglo iglugaseliorpok Judab kakkangine, nappartunelo igloliorsavok igloksoarnik avaluliorsimajunik, nuvugusingniglo.

5 Sorsugvigivâlo Amonib kittorngangita attanningat, salagilaitalo; Amonib kittorngangita Jahreme taipsomane tunnitsivigingmatsuk Talentinik 100 dinik Silberemik, Koriniglo 10000 dinik Weizenik, Koriniglo 10000 dinik Gerstenik. Taimak unnuktigijunik Amonib kittorngangita tunnitsivigivattauk Jahrib aipangane, pingajuanelo.

6 Taimak Jothame sôngudlalaukpok; apkotigijane âkigasuarmagit Nalekab Gudime sângane.

7 Unipkautsille tamakkoa assingit Jothame pivlugo, unatadlarningit, pinniarningillo, ahâk, tamakkoa aglaksimavut Israelitit Judakullo attanningita aglangine. 
8 Jahrekalaukpok 25 ginik attanningorame, attanniotilunelo Jahrit 16 it nâlugit Jerusaleme.

9 Jothamelo sinnisilerpok atâtagijaminut ailune, illijartorpâllo Davidib iglugaseksoangine. Erningalo Ahas attanningorpok innangêrdlugo.

\section{KAPITEL XXVIII.}

\section{Ahasib attanniotininga pinniarningillo.}

AHAS Jahrekalaukpok 20 ginik attanningorame, attanniotilunelo Jahrit 16 it nâlugit Jerusaleme, pinniarlungilarlo Nalekab namagijanganik atâtagijamitut Davidetut.

2 Agvertarporle Israelitit attanningita apkosiningine, Gudingoaniglo kovimajunik sennavoktauk Baalemut;

3 Isserluanititsivorlo Hinomib erningita naksangane, erninelo otautipait nellojut Nalekab Israelib kittorngangita sivurngane pêjarlauktangita makkojungnartulliningit malliklugit;

4 Tunnergutiniglo tunnijilaukpok isserluanititsilunelo poktôjune, kakkarulângnelo, nappartullo tungojortut illunatik attâne.

5 Tagva Nalekab Gudingata tunniva Siriamut attanningata agganginut; tapkoalo ullimarpât, anguvaillo innukotinginit unnuktut, parngnanairsortauvlutiglo aularutivait Damaskusemut. Israelitillo attanningata agganginut tunnijaulaurivok, tapsomalo - salagijaunekartipa angijomik.

6 Pekajablo Remaljab erningata Judakune 120000 dit ullimarpait uvlorme attautseme, illunatik angutit sorsugungnartut, Nalegak atâtagijamik Gudingat kemmangmatsuk.

7 Sichriblo, angutaudlartub Efraimekunit, tokkopait Maeseja attanniub erninga, Asrikamelo, aulatsijok attanniub iglunganik, Elkanalo attanniub tuglia.

8 Israeliblo kittorngangita kattangutimingnit aularutivait parngnanairsortaumajut $200000 \mathrm{dit}$, nullit, ernillo pannillo, aksarpaillotauk perkutinik unnuktunik, aksarnigaujullo âpait Samariamut.

9 Tagvanelaukporle Nalekab nellautaijokotingita illangat, Odedemik attelik, tamna annivok sorsuktuksat Samariamut tikkitut pachreartorlugit, okautilugillo: Ahâk, Nalekab atâtagijapse Gudingata Judakut ningaringmagit tunnilaukpait aggapsingnut; illipsele tokkolaukpasse kanningaumajarnermut, killangmut tôngmat.

10 Mannalo kajusimavose Judamiut Jerusalemiullo kittorngangit attanniotivigijomavlugit, angutillo arnallo kippalungortit- 
somavlugillo illipsingnut. Idluinekangilak illipsingne, illa illipsingne, akkerartortomik Nalekamut Gudipsingnut?

11 Tagva naleksinga manna, parnanairsortallo aularutimajase kattangutipsingnit, uttertisigik; Nalekab ningarningata illungertorutigingmasse.

12 Tagva Efraimib kittorngangita angajokaunerôjungita illangit: Asarja, Johananib erninga, Berechjalo, Mesillemotib erninga, Jehiskialo Sallumib erninga, Amasalo, Haldaib erninga, nikkoviput, tapkoa unatadlarnermit tikkitut kiglornilugit.

13 Okautilugillo: Parnanairsortat, tamanngatitaksaringilasse; idluitullijarêrapta Nalekamut, illipse issumavose idluinivut, tappanivullo illatuinaromavlugit, tappanivut angijovaksongmatta, ningautsiblo Israelitit illungertorutigivait.

14 Tagva sakkulijartut, parngnanairsortat, aksarnigaujullo kemmakpait angajokaunerôjut illagêllo illunatik sânginut.

15 Tagva angutit manna attimikut taijaumajut nikkoviput, tiguvaillo parngnanairsortat, illunaitalo annorairsimajut akkorngane, annorarpaillo aksarnigaujunit, annorartipaillo kamiktorlaitalo, tunnitsivigivaillo nerkiksanik, immiksaniglo, mingoarpaillo, sangêtullo ikkitipait siutekoktôjunut, âlugillo Jerichomut iglugaseksoarnut Palmitalingnut, kattangutinginut; utterlutiglo Samariamut.

16 Nelliutome taipsomane attannek Ahase tilliklerpok Assiriamiut attanninginut, ikajorlugo pikovlugit.

17 Edomiterillotauk tikkiput sâlagilugillo Judakut, aularutivaillo illangit.

18 Filisterillo iglugaseksuit naksarnetut Judablo sekkernganêtut ittarpait, aksarnigarivaillo Bethsemese, Ajalonelo, Gederotelo, Socholo, aiparusingit illagilugit, Timnalo, aiparusingit illagilugit, Gimsolo, aiparusingit illagilugit, innigivaillo.

19 Nalekab Judakut mannigotitingmagit, Ahase Judakut attanningat pivlugo, Judakut ussingatimagit, Nalegarlo idluitullivigingmago.

20 Tiglatpilneseriblo Assiriamiut attanningata opaglukpa, tettivâlo, ikajorlungilâlo.

21 Ahasib Nalekab iglunga, attanniublo iglunga, angajokaunerôjullo iglungit aksaralloarpait, tunnilaitalo Assiriamiut attanninganut; sullilaungilarle.

22 Tettijaunimelo nelliuningane, Ahase attannek idluitulliganerpok sulle Nalekamut;

23 Tunnergutiniglo tunnijivok Damaskusemiut, salakarlauktut tapsominga Gudinginut, okarlunelo: Siriamiut attanningita Gudingita ikajorpait tapkoa; tamanna pivlugo tunnergutinik tunnijijomavunga tapkonunga ikajorkovlugittauk uvamnik. Ochojutiksanganulle Israelitillo illunatik ochojutiksanginut illingalaukput. 
24 Ahasiblo Gudib iglungata illulerkutingit kattitipait, avgorpaillo Nalekab iglungata illulerkutingit. Nalekablo iglungata upkoangit pallangairpait; Altarekartitsivorlo ingminut Jerusaleme terrekune illunaine.

25 Judakullo iglugaseksoangine illunaine pôktojokartitsivok, Gudib assingit isserluanititsivigivlugit, Nalegarlo atâtagijame Gudingat ningaksarpa.

26 Unipkautsit assingit tamna pivlugo, apkotigijangillo tamaita, sivorlit kingorlillo, ahâk, tamakkoa aglaksimavut Judakut Israelitillo attanningita aglangine.

27 Ahaselo sinnisilerpok, atâtagijaminut ailune, illijartorpâllo iglugaseksoarne Jerusaleme; âtaulungilarlo Israelitit attanningita illuvinginut. Erningalo Jehiskia, attanningorpok innangêrdlugo.

\section{KAPITEL XXIX.}

\section{Hiskiab kivgartornek Gudemik sattortautipa.}

HISKIA Jahrekalaukpok 25̌ ginik attanningorame, attanniotilunelo Jahrit 29 it nâlugit Jerusaleme. Anânanga attekalaukpok Abjamik Sacharjab pannia.

2 Pinniarporlo Nalekab namagijanganik atâtamitut Davidetut.

3 Attannionimelo Jahringita sivurlerpângata takkingita sivurlerpângane Nalekab iglungata upkoangit pallangairudjarpait, âkilaitalo;

4 Ittertipaillo ajochertuijut Levitillo, kattikolugillo apkosinerme nerrotôjome kittanêtome,

5 Okautivaillo: Naleksinga Leviteôjose: Manna ivsornaitileritse, Nalekablo atâtagijapse Gudingata iglunga ivsornaitilugo pititse, ivsornartut annitisigik ivsornaitovingmit.

6 Atâtagijavut tammarlaungmatta, pinniarlaukpullo Nalekab Gudipta idluigijanginik, kemmalaukpâllo. Kênatik sangulaungmagit Nalekab iglunganit, tunnulaukpâllo;

7 Upkoarlo torksungme pallangairlaukpât, kollillo kammilaukpait, isserluanijutiksaniglo isserluanititsilaungilet, tunnijilaungilello tunnergutinik ôtaksanik ivsornaitovingme Israelib Gudinganut.

8 Tamanna pivlugo Gudib ningausingata Judakut Jerusalemiullo tikkipait, tunnilaukpaillo siorniornartunut kakkilarnartunullo, mittautigijaunermullo, sorlo ijipsingnut tækkogapse.

9 Ahâgle, tamanna pivlugo atâtagijarut ochosimavut saviksoarmut, ernivullo pannivullo nulliavullo aulartitaumavut. 
10 Manna kajusimavunga Nalegak Israelib Gudinga angerkattigijomavlugo ningarninga, kaningaumajarningalo allaikovlugik uvaptingnik.

11 Manna ernikka katsunganiarasse; Nalekab illipse annerilaungmasse nangertuksaugapse sângane, kivgaujuksauluselo isserluanititsijôjuksaugapse tapsomunga.

12 Tagva parngnailerput Levitit tapkoa: Mahat, Amasaib erninga, Joello, Asarjab erninga, Kahatib erninginit; Merariblo erninginit: Kis, Abdib erninga, Asarjalo, Jehaleelib erninga; Gersoniblo erninginit: Joabe, Simmab erninga, Edenelo, Joab erninga;

13 Elizafaniblo erninginit: Simri, Jejelelo; Assafiblo erninginit: Sacharja, Matanjalo.

14 Hemaniblo erninginit: Jehiel, Simeilo; Jedutuniblo erninginit: Semaja, Usielelo.

15 Tapkoalo kattangutitik kattitipait, ivsornaitilutiglo, tikkilerpullo attanniub perkojanga, Nalekab okausinginit pijok malliklugo, Nalekab iglunga sujungaitilugo.

16 Ajochertuijullo itterput Nalekab iglunganut sujungaititsomavlugo, ivsornartullo tamaita, nachvartaujut Nalekab iglungane, annipait innemut avaluliorsimajomut Nalekab iglunganêtomut; Levitillo tiguvait akjartorlaitalo kôngmut Kidronemut.

17 Pigiarpullo ivsornaitilutik takkit sivurlerpângata uvlungita sivurlerpângane; takkiblo tapsoma uvlungita 8 ingane itterput Nalekab torksukotinganut, ivsornaitipâllo Nalekab iglunga uvlut 8 it nâlugit; takkiblo sivurlerpângata uvlungita 16 ingane innersimavât.

18 Tagva itterput attannermut Hiskiamut okarlutiglo: Nalekab iglunga illunât sujungaitimavavut, tunnergutiksat ôtaksat Altaringat, illulerkutingillo tamaita, kakkojallo tækkojaksat sângat, illulerkutingillo tamaita.

19 Illulerkutillo illunaita, attanniub Ahasib attanniôvlune egilauktangit idluitullilune, tapkoa sattortautimavavut ivsornaitilugillo; ahâglo Nalekab Altaringata sânganêput.

20 Tagva attannek Hiskia uvlârorpok, iglugaseksuillo angajokaunerôjungit kattitipait, takpaungarlunelo Nalekab iglunganut.

21 Atsivullo tagvunga tuktuvângnik angusallungnik 7 nik, saukaniglo angusallungnik 7 nik, saugârsungniglo 7 nik, tuktungajuniglo angusallungnik 7 nik, tunnergutiksamut ajortoêrutiksamut illingajuksanik, attanniôvik pivlugo, ivsornaitovik pivlugo, Judakullo pivlugit. Tapsomalo ajochertuijut, Aronib erningit perkovait tunnijikovlugit tunnergutiksanik Nalekab Altaringata kângane.

22 Tagva tuktuvait tokkopait, ajochertuijullo auk tigurât serkerartipâllo Altarib kânganut; 
23 Apaillo tuktungajut angusalluit tunnergutiksamut ajortoêrutiksamut illingajut attanniub illagêllo sânginut, pattikpaillo.

24 Ajochertuijullo tokkopait, aungillo ajortoêrutiksauvlune serkerartipât Altarib kânganut, Israelitit illuêngarlutik ullapkoserlugit. Attannek perkojilaungmat tunnergutit ôtaksat, ajortoêrutiksallo tunnilugit, Israelitit illunamassiangit pivlugit.

25 Pitipaillo Levitit Nalekab iglungane, Cimbelijarlutik, Psalterijarlutiglo, Harfesijarlutiglo, sorlo Davide, Gadelo attanniub tækkojokotinga, Nathanelo nellautaijok perkojilaungmatta, Nalekamille perkojak tamna pivok, nellaitaijokotingittigut.

26 Levitillo nangerput, Davidib nok̇akseralikotinginik najortekarlutik, ajochertuijullo Trompetinik.

27 Hiskialo perkojivok tunnergutit ôtaksat tunnilugit Altareme. Pigiarmattalo tunnergutiksak ôtaksak tunnilugo, tagva imgernek Nalekamut, trompetesijarnerlo, attornerlo Davidib Israelib attanningata nokakseralikotinginik sunatuinarnik pigiarivut.

28 Illagêllo illunatik pamakterput; imgernerlo tussaksaulaukpok, Trompetillo nipliadlarput tunnergut ôtaksak tunnijaujarêrkârtinnago.

29 Tumnergullo ôtaksak tunnijaujarêrmat, attannek okkovok, illunatiglo najuganêlauktut, opigosulutiglo.

30 Attanniublo Hiskiab angajokaunerôjullo, Levitit perkovait Nalegak nertorlugo Davidib Assafiblo tækkojub imgerutingit attorlugit pikovlugit. Kuviasuklutiglo nertordlerput okkolutiglo, opigosularmiglo.

31 Hiskialo kiovlune okarpok: Manna tigumiaralijarluse ikkipose Nalekamut, tamaungariartoritse, kaitsiluselo tamaunga tunnergutiksanik, tunnergutiksaniglo nertordlerutiksanik Nalekab iglunganut. Illagêllo âtsivut tunnergutinik, tunnergutiniglo nertordlerutinik, illunatiglo kunnutuitut tunnergutinik ôtaksanik.

32 Tunnergutillo ôtaksat illagêt kaitangita unnurningit tuktuvangôlaukput 70 git, saukat angusalluit 100 dit, saugârsuillo 200 dit, tapkoa illunatik tunnergutiksaulaukput ôtaksat Nalekamut.

33 Ivsornaitipaillo tuktuvait 600 dit, saukallo 3000 dit.

34 Ajochertuijulle ikkitoluarlaukput tunnergutiksat ôtaksat illunaita aktorungnarlaungilait tamanna pivlugo kattangutinginut Levitinut ikajortaulaukput pinniarnek tamanna pijarêrkârtinago, ajochertuijullo ivsornaitikârtinagit. Levitit illungertornersaulaungmatta ivsornaitilutik ingminik ajochertuijunit.

35 Tunnergutille ôtaksat, tunnergutit kujalijutiksat orksungit illaliudlugit, tunnergutiksallo immiksat tunnergutinut 
ôtaksanut illingajut unnuktolaukputtauk. Taimaglo kivgartornek Nalekab iglungane âkiktaumalaukpok.

36 Hiskialo kuviasulaukpok innugiartoksuit illunatik illagilugit, Gudib innugiartoksuit oppalungaijarlaungmagit, tagvainak pinniartaulaungmat.

\section{KAPITEL XXX.}

Hiskia Passamik uvloksiorkojivok innugiartoksuit kuviasutiksanginut.

HISKIaLo tilliklerpok Israelitinut illunainut, Judamiunullo, aglangniglo aglakpok Efraimekunut Manassekunullo, kaikovlugit Nalekab iglunganut Jerusalemut, Passamik uvlosiorlutik Nalekamut Israelib Gudinganut.

2 Attanniublo angajokâkotine illagêllo illunaita kachimiolerkattigivait Passa attoromavlugo takkib aipangane,

3 Attorungnarlaungimatsuk nelliutome taipsomane, ajochertuijut ivsornaitisimajut ikkitoluarlaungmatta, innugiartoksuillo tikkilungimatta Jerusalemut.

4 Attanniublo illingatitaunek tamanna idluariva, illagêllo illunamassiatik,

5 Perkojivullo tamanna akpautigijaukovlugo Israelitine illunaine Bersebamit Dane tikkidlugo, kaikovlugit Passamik uvloksiorlutik Nalekamut Israelib Gudinganut Jerusaleme; akkunît attorlaungimatsuk sorlo aglaksimangmat.

6 Tagva akpangertut aivut, aglait attanniub angajokakotingitalo agganginit pijut, neksarlugit, Israelitit, Judakullo illunatik akkorngattigut, attanniublo perkojanga malliklugo okarput: Kittorngaujose Israelemut, sâleritse Nalekamut, Abrahab, Isaakiblo Israeliblo Gudinganut; tagva tamna sâlerniarivok amiakojunut illipsingnit, kemâlauktunut Assiriamiut attanningata agganginit;

7 Atâtagijapsetullo kattangutigijapsetullo idluitullilauktutitut Nalekamut atâtagijamik Gudinganut, tunnilauktangititullo tamanna pivlugo asserornartomut, sorlo nangminek tækkogapse, îniarase.

8 Tagva tappatôniarase manna atâtagijapsetut, aggassele tunnissigik Nalekamut, kaititselo ivsornaitovikotinganut ivsornaitilauktanganut issokangitomut, Nalegarlo Gudise kivgartorsiuk, ningarninga illungertortok sangulerkovlugo illipsingnit.

9 Illipsele sâlerniarupse Nalekamut, tagva kattangutise kittorngaselo napkigijaunekarniarput parnanairsortimingne, nunamut tamattomunga utterkovlugit, Nalegak Gudise saimarsai- 
gosungmat, napkigosuklunelo, kênanelo sânguniangilâ illipsingnit, illipse sâvigigupsiuk.

10 Akpangertullo aivut iglugaseksoarnit iglugaseksoarnut Efraimekut, Manassekullo nunangane, Sebulonekullo tikkidlugit, ijorutigivaille mittautigilaitalo.

11 Illangille Asserekunit, Manassekunillo, Sebulonekunillo manigotitiput ingminik tikkigalloarlutiglo Jerusalemut.

12 Nalekab aggangita Judakuttauk tikkipait adsigêktunik issumakartimagit, pinniarkovlugit attanniub angajokaunerôjullo perkojangat, Gudib okausinginit pijok, malliklugo.

13 Innugiartoksuillo unnuktut kattimalerput Jerusaleme uvloksiorvik kakkojanik sêrnakangitunik uvloksioromavlugo, takkib aipangane, illagêt unnuktovaksuit.

14 Parngnailerpullo Altarillo Jerusalemelauktut pêjarpait, Altarillo isserluanititsivit illunaita pêjarpait, êgilugillo kôngmut Kidronemut;

15 Tokkotsivullo Passasiorutiksamik takkib aipangata uvlungita 14 ingane. Ajochertuijullo Levitillo kangusulaukput ivsornaitilutiglo, atsilutiglo tunnergutiksanik ôtaksanik Nalekab iglunganut;

16 Nangerpullo innimingne, sorlo illusiolaungmat Mosesib Gudib angutekotingata perkojangit malliklugit. Ajochertuijullo auk serkerartipât Levitit agganginit.

17 Unnuktolaungmatta illagêktune ivsornaitilaungitut ingminik, tamanna pivlugo Levitit saugarsuit passasiorutiksat tokkopait, illunaita sujungaitilungitut akkisartorlugit, ivsornaitilaitalo Nalekamut.

18 Innugiartoksoarnillo unnuktut, Efraimekunillo unnuktut, Manassekunillo, Isascharekunillo, Sebulonekunillo sujungaitilaungitut ingminik, nerrilutiglo Passamik aglait malliklugiungitok. Hiskiable tuksiupait okarlunelo: Nalekab ajungitub illunaita issumagijungnaivigiligit,

19 Omattimingnik torartitsilauktut Gude Nalegak, atâtagijamik Gudingat kennerlugo, unêt ivsornaimarilungigalloarlutik.

20 Nalekablo Hiskia tussarpa nekkoksitilaitalo innugiartoksuit.

21 Taimak Israelib kittorngangita Jerusaleme najutijut uvloksiorvik kakkojanik sêrnakangitunik uvloksiorpat uvlut 7 it nalugit kuviasuvaksoadlarlutik. Levitillo ajochertuijullo Nalegak uvlut tamaita nertorpât Nalekab nok̇akseralikotingit nipliadlartut attorlugit.

22 Hiskiablo Levitit illunaita sungiusimajut kivgartornermik ânanâmik Nalekamik, pitsiarlune okarkattigivait. Nerrivaillo uvloksiorviub tunnergutingit uvlut 7 it nâlugit, tunnijilutik tunnergutinik kujalijutiksanik, imgerlutiglo nertordlerutiksanik Nalekamut atâtagijamik Gudinganut. 
23 Kattimajullo illunatik kajusimavut, ama uvlut 7 it uvloksioromavlugit; tapkoalo uvlut 7 it uvloksiorpaittauk kuviasuklutik.

24 Hiskiab Judakut attanningata kattimajut tunnitsivigingmagit tuktuvângnik angusallungnik 1000 dinik, saukaniglo 7000 dinik. Angajokajallo illagat tunnitsivigivait tuktuvangnik 1000 dinik, saukaniglo 12000 dinik. Taimak ajochertuijut unnuktut ivsornaitiput ingminik.

25 Kattimajullo Judakut, illunamassiatik kuviasudlalaukput, ajochertuijut Levitillo, kattimajullo Israelitinit tikkimalauktut, tujormitjello Israelitit nunanganit tikkimalauktut Judamelo nunakartut.

26 Kuviasuvaksoadlarnekarporlo Jerusaleme. Salomonib Davidib erningata, Israelitit attanningata nelliuninganit taimaitokalaungilak Jerusaleme.

27 Ajochertuijullo Levitillo nikkovilerput saimartilaitalo innugiartoksuit, nippingallo tussartaulaukpok, tuksiarningallo tikkilerpok kamunga innigijanganut ivsornaitomut killangmut.

\section{KAPITEL XXXI.}

Hiskiab asseroininga kivgartornermik Gudingoanik; pattangaititsiningalo ajochertuijunit.

TAMAKKOA tamaita pijarêrlaungmatta Israelitit illunamassia-
tik, najutigut tagvane, aularput Judakut iglugaseksoanginut,
serkomipaillo nappajoksuit, nakkarlugillo nappartut Gudingoag-
git, pôktojullo, Altarillo serkomipait, Judakunêtut, Benjamine-
kunêtullo, Efraimekunelo, Manassekunelo, nungutaumarikârtina-
git; tagva Israelib kittorngangit illunatik utterput attunît per-
kutigijamingnut, iglugaseksoakotimingnullo.

2 Hiskiablo ajochertuijut Levitillo ingmigolinganingit pitipait, attunît ingmigolinganingit malliklugit, ajochertuijut Levitillo, tunnijinermut tunnergutiksanik otaksanik, tunnergutiksaniglo kujalijutiksanik, imgernermullo nertordlerutiksanik, nertordlernermullo Nalekab tangmarviata upkoaksoangine.

3 Attanniublo perkutime illangit tunnikattarpait tunnergutiksanut ôtaksanut uvlaksiutinut, unnuksiutinullo, tunnergutiksanullo ôtaksanut, Sabbatesiutinut, takkêlasiutinullo, uvloksiorviksiutinullo; sorlo Nalekab perkojangine aglaksimangmat.

4 Innugiartoksuillo Jerusalemiut perkovait, ajochertuijut Levitillo pijaksangit tunnilugit pikovlugit, tapkoa katsungainersaujungnarkovlugit perkojanut.

5 Perkojarlo tamna tunnijaujarêrmat Israelib kittorngangit 
âtsivut innerkârtunik kakkojaksanik, Weiniblo paungangita iptinginik, orksumiglo, igupsaujartullo orksunginik, perorsêviublo perortunginik sunatuinarnik; tamaitalo tellimaujortungit âpait unnuktut.

6 Israeliblo Judablo kittorngangit, Judakut iglugaseksoangine nunnakartut, âtsivuttauk tuktuvait saukallo tellimaujunginik, ivsornaititaumajullo, ivsornaitilauktamik (tunnilauktamik) Nalekamut tellimaujunginik, kattititakartitsivullo mânelo ikkanelo.

7 Takkit pingajuane pigiarput, kattititat kattitilerlugit, takkillo 7 ingane namaksilerpait.

8 Hiskialo angajokaunerôjut illagilugit ittermat kattititaumajullo tækkogamigit, tagva Nalegak innukotingillo Israelitit nertorpait.

9 Hiskiablo ajochertuijut Levitillo apperivait kattititaumajut pitjutigællugit.

10 Asarjablo ajochertuijub, angajokaunerôjub Zadokekunit, okautiva: Taimangamit pigiarlaungmatta tunnergutit âlugit $\mathrm{Na}$ lekab iglunganut nerrilaukpogut akkeartorlutalo, amiakokartitsivogullo sulle unnuktunik; Nalekab innukotine pitsartutilaungmagit, tamanna pivlugo kattititaumajut ukkoa amiakovut.

11 Tagva attannek perkojivok inniorkovlugit Nalekab iglungane. Sennajauvullo;

12 Ittertipaillo illuanut nertornarlutik, tunnergutit perkutit tellimaujungit, ivsornaititaumajullo. Kananjalo Levitiôjok angajokaulaukpok tapkonunga, Simeilo kattangutine ikajortigiva.

13 Jehiello, Asasjalo, Nagatelo, Asahello, Jerimotelo, Eliello, Jesmachjalo, Mahatelo, Benajalo aulatsijôlaukput, Kananja kattangutalo Simei ikajorlugik, attanniub Hiskiab perkojanga malliklugo. Asarjale attanniolaukpok Gudib iglungane.

14 Korelo Jemnab erninga Leviteôjok, udsertorte upkoamik kittanêtomik angajokaulaukpok Gudib tunnergusianginut, pijarællune tunnijaulauktunut, aulailugit tunnergutit Nalekamut, ivsornainerpângôjunuilo.

15 Aulatsijoninganêlaukpullo: Edene, Minjaminelo, Jesualo, Semjalo, Amarjalo, Sachanjalo, ajochertuijut iglugaseksoangine, tunnijikovlugit kattangutimingnut nertornarlutik, attunît illinganingit malliklugit, mingnerpâk anginerpâktut;

16 Angutit kittitaumajut Jahrelingnit pingasunik, unnurnersaniglo, senniane, illunainut Nalekab iglunganut tikkitunut uvlut tamaita kivgartornermingnut sullijaksamingne, ingmigolinganingit malliklugit.

17 Ajochertuijunullo kittitaumajunut atâtagijangita iglungit (kinguvângit) malliklugit, Levitinullo Jahrelingnit 20 ginik unnurnersaniglo, sullijaksangine, ingmigolinganinginelo;

18 Kittitaumajullo kittornganginut, nullianginut, erninginut, 
panninginullo illunamassianginut; nertornarlutik ingminik ivsornaitingmatta kivgartornermut.

19 Aronib erningit, ajochertuijut pivlugittauk iglugaseksuit illunatik aiparusingita perorsêvingine angutekarkojauvok attermikut taijaumajunik, tunnijikovlugit angutinut ajochertuijojunut, Levitinullo aglaksimajunut illunainut, pijaksanginik.

20 Taimailiorlaukporlo Hiskia Judame illunane, pinniarporlo Nalekab Gudime namagijanginik, idluarijanginiglo, nellagôrtorijanginiglo,

21 Pinniarnermine tamaine, pinniarnerijamine Gudib iglungane, innatsinelo, perkojanelo Gudine kennerlugo; omamut tamânut pinniarpait, sullidlarlunelo.

\section{KAPITEL XXXII.}

Hiskiab piulijauninga tattamnadlartok Sanherib aggangenit, kannimasernermillo.

Pijokartullo tamakkoa, nertornarnerublo kingorngane, kaivok Sanheribe Assiriamiut attanningat, tikkilunelo Judamut, tangmarlunelo iglugaseksuit sôngojut sivurânut, aksarnigarijomanasugivaillo nangmineliutilugit.

2 Hiskialo tækkogame Sanheribe tikkilermat, kênangalo torarmat Jerusaleme sorsugvigijomavlugit;

3 Angajokâkotine, sôngojokotinelo kachimiokattigivait, immektârvit iglugaseksuit sillatânêtut mattujomavlugit; ikajorpâllo.

4 Innugiartoksuillo kattimalerput, mattuvaillo puailavît illunaita, kổllo nunakut kôktut, okarpullo: Assiriamiut attanningit, tikkilutik immiksarsijuksaukonagit unnuktunik.

5 Maksoalaukporlo, karmaillo avaluksuit sujuksimaningit âkiksorpait, kallerêktipaillo nuvugutsit tikkilugit, sillatânelo karmaliorivok sulle assianik, Millolo Davidib iglugaseksoangine sôngotipa, sakkoliorsavorlo, sappulutaliorsalunelo unnuktunik.

6 Angajokâkartitsivorlo innugiartoksoarnik, kattitilugillo ingminut iglugaseksuit apkosiningane silliktome, upkoaksub kannitangane, okausekarlunelo tapkonunga illanârnartunik, okarlunelo:

7 Maksoaleritse pitsartuluselo, Assiriamiullo attanningat, unnuktoksuillo illagijangit sivuraginiarassigik, maksujarutiginassigiglo; illagijavut unnurnersaungmatta illagijanginit.

8 Talliub uviniôjub tamna aipariva, uvagulle Nalekab Gudipta illagivâtigut, ikajorluta, aulalugolo sorsungnivut. Innugiartoksuillo Hiskiab Judakut attanningata okausingit tettigivait. 
9 Tamakkoa kingorngane Sanherib Assiriamiut attanningata kivgane tillivait Jerusalemut (tamna Lachisib sivurâne tangmarsimangmat, nalektine illunaita illagællugit) Hiskiamut Judakut attanninganut, Judakunullo illunainut Jerusaleme najutijunut, okautsaukovâlo:

10 Imâk Sanheribe Assiriamiut attanningat okarpok: Suna sungertutigivissiuk, Jerusaleme illumatjaumajomêtuarapse?

11 Hiskiab tilliorlungipasse tunniluse tokkomut, kângmullo kêlernermullo, okarlune: Nalekab Gudipta piuliniarpâtigut Assiriamiut attanningata agganginit?

12 Hiskiaôlungilâk, poktôjokotinginik, Altarekotinginiglo pêjailauktok, okarlauktorlo Judakunut Jerusalemiunullo: Altaretojub sângane tuksiartuksauvose, kânganelo isserluanititsijuksauvose?

13 Kaujimangilase, sunamik uvanga atâtagijamalo nunat illunatik innugiartoksoangit pinniarvigilauraptigit? Nunat innugiartoksoangita Gudingita nunakotitik piulijungnalaurivattigik aggamnit?

14 Kia innukattigêksuit taipkoa atâtagijama pêjarlauktangita Gudinginit tamainit innukotine piulijungnarlaukpagit aggamnit, Gudipse illipse piulijaksarinajarmasse aggamnit?

15 Taimak mana uivêrijautailititse, tilliortautailitiluselo, okperiniarasiuglo. Innugiartoksuille attanniôvillo Gudingita nelliata innukotine piulijungnarlaungimagit aggamnit, atâtagijamalo agganginit, tagva Gudigijapse piulinianginivâse aggamnit.

16 Kivgangillo sulle unnurnersanik okausekarput Nalegak Gude akkerartorlugo, kivgangalo Hiskia akkerartorlugo.

17 Aglangniktauk aglakpok Nalegak Israelib Gudinga mittautigivlugo, okautigivâlo okarlunelo: Sorlo innugiartoksuit nunane Gudingita innukotitik piulilaungimattigik aggamnit, taimak. Hiskiab Gudingata innukotine piulinianginivait aggamnit.

18 Nippekortôvlutiglo Judit okausingit attorlugit innugiartoksuit Jerusalemiut karmaksuit (avaluksuit) kânganêlauktut nipliarvigivait sivurasaromavlugit koaksartitsomavlugillo, iglugaseksuit salagilugit pikovlugit;

19 Jerusalemiullo Gudingat okautigivat, innugiartoksuit nuname Gudingoangititut, innuit aggangita sennajangititut.

20 Attanerlo Hiskia nellautaijorlo Jesaiase tuksiarpuk tamanna pivlugo, nipliadlarlutiglo killangmut.

21 Tagva Nalekab Engelit illangat tilliva, tapsoma sorsuktuksat sôngojungit illunaita, attannillo angajokaunerôjullo illunaita Assiriamiut attanningata tangmarviane nungupait, kangusuklunelo utterpok nunaminut. Gudigijamelo iglunganut ittermat, ochotipâk tagvane saviksoarmut, timinganit nangminerijanganit pilauktuk.

22 Taimak Nalekab Hiskia Jerusalemiullo ikajorpait San- 
herib Assiriamiut attanningata agganginit, assingitalo illunatik agganginit, serngnigivaillo avatingine illunâne.

23 Unnuktullo Nalegak pilliutinik âtsivigivât Jerusalemut, Hiskialo Judakut attannıngat pivianartunik. Kingornganelo angijolaukpok innukattigêksuit illunatik ijingita sângine.

24 Nelliutome taipsomane Hiskia kannimalaukpok tokkoajarlune, tuksiarporlo Nalekamut, tapsomalo okarvigiva tunnitsivigilugolo nellonaikutamik tattamnadlartomik.

25 Hiskiable ajungitullivionine akkiniarutigilaungila, omattingale kagverpok, tamanna pivlugo ningautsib tamna, Judamiullo, Jerusalemiullo tikkipait.

26 Tagva Hiskia mannigotitipok omattime kagverninga pivlugo; tamna, Jerusalemiullo tamanna pivlugo Nalekab ningausingata tikkilaungilait Hiskia innôtillugo.

27 Hiskialo akluinekadlalaukpok, nertortaunekadlalunelo, perkutetalititsilunelo ingminut, Silbereôjunik Goldeojuniglo, ujarkaniglo pivianartunik mingoarutiksaniglo tippitsiariktunik, sappulutaksaniglo illulerkutiriglo pivianartunik sunatuinamik.

28 Kematullivikartitsivorlo perortunut kaitaujunut kakkojaksanut, Weinemullo, orksumullo; igloliorsalınelo nergjutit sumatuinait igluksanginik, avaluliorsalunelo saukanut.

29 Iglugaseliorlunelo ingminut, nergjutekalaukporlo saukanik, tuktuvângniglo unnuktovaksoarnik, Gudib tunnitsivigingmago perkutevaksoarnik.

30 Hiskiablo oma puailaviub Gihonemik attiggub pânga kogvasiktok simmikpa kôktilugolo unnunga Davidib iglugaseksoangita kangianut. Hiskialo pilloridlalaukpok pinniarnermine tamaine.

31 Babelemiulle attanningita tillijangit tikkinamik tattamnartok pilauktok nuname apperkutigilugo, Gudib taimak kemakpâ ôktoramiuk, illitarileromavlugit tamaita omattingata illumigijangit.

32 Pijokalauktulle assingit Hiskia pivlugo, pinniarningillo idluartut, ahâk tamanna aglaksimavok Jesaiasib Amozib erningata nellautaijub tækkotitauningine, Judakullo Israelitillo attanningita aglangine.

33 Hiskialo sinnisilerpok atâtagijaminut ailune, illijartorpâllo Davidib erningita illuvingita poktojungane. Judakulio illuêngarlutik Jerusalemiullo opigivât tokkungane. Erningalo Manasse attanningorpok innangêrdlugo.

\section{KAPITEL XXXIII.}

\section{Munassib, Amoniblo attanniotinikik.}

MANASSE Jahrekalaukpok 12 nik attanningorame attanniotilunelo Jahrinik 55 ginik Jerusaleme. 
2 Pinniarporlo Nalekab idluigijanginik, innukattigêksuit Nalekab pêjarlauktangita Israelib kittorngangita sivurngane, makkojungnartulliningit malliklugit.

3 Poktojullo atâtame Hiskiab serkomilauktangit sattortautipait, Altarekartitsivorlo Baalemut, sennalunelo nappartunik Gudingoalingnik, tuksiarvigivaillo uvloriagaseksuit illunaita, kivgartorlaitalo.

4 Altariniglo sennavoktauk Nalekab iglungane, Nalekab okautigilauktangane: Attera Jerusalemêtsainartuksauvok issokangitomut.

5 Altareliorsavaillo killaub kattimgajungit sunatuinait, Nalekab iglungata innigingne avaluliorsimajungne tamangne.

6 Erninelo ikkomakorkovait Hinomib erningata naksangane, tingmitjello nipjarningit kamagivait, uvlullo annerivait, angakoktorlunelo, nellautainarluklarmelo, elisênarlunelo, pinniarporlo unnuktunik Nalekab idluigijanginik, ningaksarlugo.

7 Innungoallo, Gudingoallo sennajaukojane pitipaittauk Gudib iglunganut, Nalekab Davidemut erninganullo Salomomut okautigijanganut: Iglomut tamattomunga, Jerusalemullo annerilauktamnut Israelib kinguvarênginit tamainit, attera pititsomavara soraijuitomik;

8 Israelitillo ittiggangit aularkojomairpakka nunamit, tunnilauktamnit atâtagilauktanginut, kamatsiartuinarpatta pinniarlutik perkojat tamaita, innatsillo, idluarijaunerillo, Mosesekut perkolauktakka tapkonunga, malliklugit.

9 Manassible Judakut Jerusalemiullo uivêrivait, sugaluluartomik pinniarkovlugit innukattigêksoarnit Nalekab pêjarlauktanginit Israelib kittorngangita sivorngane.

10 Nalekablo Manasse innukotingillo okarvigivait, kamalungilelle tamattominga.

11 Tamanna pivlugo Nalekab tikkitaukovait Assiriamiut attanningata sorsuktuksangita angajokânginut, tapkoa Manasse parngnanairsortautipât niksinut, -kellaksorlâllo kellaumgujanut, tikkiutipâllo Babelemut.

12 Kappianartomênamelo kennuvok Nalekamut Gudiminut mannigotitilunelo Nalekab atâtagijame Gudingata sângane.

13 Tuksiarporlo kennulunelo tapsomunga. Tagvalo kennuninga tussarpa, uttertilâlo Jerusalemut, attanniovinganut. Tagva Manasse illitarksilerpok Nalegak Gudeôngmat.

14 Kingorngane Davidib iglugaseksoangita karmangit (avaluksoangit) ungardlerpât, sennavait kângianit Gihonemit naksarmêtomit, ittervik upkoaksoarmut mingeritjanik attelingnut tikkilugo, Ofeliblo avatâne, pôktosilaitalo, pittakartitsivorlo angajokaksoarnik sorsuktuksanut Judakut iglugaseksoangine sôngojune illunaine.

15 Pêjarpaillo Gudit tujormitjat, Gudingoallo Nalekab ig- 
lunganit, Altarillo illunaita sennalauktane Nalekab iglungati kakkangane Jerusaleme, egilaitalo iglugaseksuit sillatânut.

16 Sattortautipâlo Nalekab Altaringa, tunnijilunelo kângane tunnergutinik nertordlerutiksanik, kujalijutiksaniglo, perkovaillo Judakut Nalegak Israelib Gudinga kivgartoraksaringmatsuk.

17 Innugiartoksuille tunnergutinik tunnijivut pôktôjune, Gudemut Nalekamingnuvalloak.

18 Unipkautsille assingit Manasse pivlugo, tuksiarninga Gudiminut, tækkojullo Nalekab Israelib Gudingata attingane okautjingita okalâningit, ahâk, tamakkoa Israelib attanningita pijokarningita akkornganêput.

19 Tuksiarningillo, kannorlo tussartauninga, ajorningillo, idluitulliningillo, innillo sennavigilauktangit pôktôjunik, nappartuniglo Gudingoalingnik, Gudingoaniglo, manigotitikârtinago, ahâk, tamakkoa aglaksimavut tækkojut unipkausingine.

20 Manasselo sinnisilerpok atâtagijaminut ailune, illijartorpâllo iglungane. Erningalo Amon attanningorpok innangêrdlugo.

21 Amon Jahrekalaukpok 22 ginik attanningorame, attanniotilunelo Jahrik magguk nâlugik Jerusaleme.

22 Pinniarporlo Nalekab idluigijanginik sorlo atâtanga Manasse pinniarlaungmat. Amonelo tunnergutinik tunijilaukpok Gudingoanut illunainut atâtame sennalauktanginut, kivgartorlaitalo.

23 Manigotitilaungilarlo Nalekab sângane, Manassetut atâtangatut; tamna Amon idluitullingmat unnuktunik.

24 Kivgangillo angerkattigêngnekartitsivut akkerartorlugo, tokkopâllo iglungane.

25 Tagva innugiartoksuit nuname ullimârpait illunaita angerkattigêngnekartitsilauktut, attannek Amon akkerartorlugo. Nunablo innugiartoksoangita Josia erninga attanningortipât innangêrdlugo.

\section{KAPITEL XXXIV.}

\section{Josiab attanniotinanga idluartok.}

JOSIA Jahrekalaukpok sittamaujortunik attanningorame, attanniotilunelo Jahrit 31 git nâlugit Jerusaleme.

2 Pinniarporlo Nalekab namagijanginik, agvertarlunelo atâtagijame Davidib apkosiningine, sangunanelo tallerpingmullonêt, saumingmullonêt.

3 Attannionime Jahringita 8 ingane nukapiangôvlune sulle pigiarpok atâtagijame Davidib Gudinga kennerlugo, Jahring- 
italo 12 ingane, pigiarpok Juda Jerusalemelo pêjaivigilugit pôktôjunik, nappartuniglo Gudingoalingnik, Gudingoaniglo, adsingoaniglo kovimajunik;

4 Najutitillugolo Baalit Altaringit asserorpait, adsingoallo kollanêtut nakkarpait, nappartullo Gudingoaggit, Gudingoallo, adsingoallo serkomipait, sanningortilaitalo, kângasorpaillo taipkoa, tunnergutinik tunnijilauktut tapkonunga, illuvingita kânginut.

5 Ikkipaillo ajochertuijut sauningit Altarit kângine, êpkejarpaillo Juda, Jerusalemelo;

6 Manassekullo, Efraimekullo, Simeonekullo, Naftalikullo tikkilugit iglugaseksoangine, iglungita illungit nautertorpait, avatânê illunane.

7 Altarillo nappartullo Gudingoaggit, asserorêramigit, Gudingoallo serkalimagamigit, adsingoallo illunaita Israelitit nunanganêlauktut nakkarsimagamigit, utterpok Jerusalemut.

8 Attannionime Jahringita 18 ingane, nuna igiolo êpkejarêramigit, tillivait Safan, Azaljab erninga Maesejalo iglugaseksuit angajokangat, Joalo Joahasib erninga, aglakpaktut angajokangat, Nalekab Gudingata iglunga âkigasuarlugo.

9 Tikkipullo ajochertuijersoarmut Hilkiamut. Tunnitsiviôvullo kênaujanik, Gudib iglunganut âtaumajunik, Levitit upkoanik udsertortut kattersorlauktanginik Manassekunit, Etraimekunillo, Israelitillo amiakunginit illunainit, Judakunillo illunainit, Benjaminekunillo, tapkonangallo Jerusaleme iglokartunit.

10 Tunnivaillo sennajut, aulatsikojaujut sullijaksanik Nalekab iglungane, agganginut. Tapkoalo tunnivait tapkonunga, sennajunut Nalekab iglungane, sujuksimanekarvingine, iglo sattortautilugo âkiksorlugolo pikovlugit.

11 Tapkoa tunnivait igloliortunut, sennajúnullo, ujarkat ullimârsimajut, kejuillo ikâksat pissiarilugit, ikaksallo iglunut Judakut attanningita asserorlauktanginut.

12 Angutillo sennavut pinniaraksame tamattomane nertornarlutik. Angajokakartitaulaukpullo Jahatemik, Obadjamiglo, Leviteôjungnik, Merarib erninginit, Sacharjamik Mesullamemiglo Kahatib erninginit, pinniarnek tamanna aulalugo. Levitillo tapkoa illunatik sungiusimalaukput nokakseraggit attorlugit;

13 Angajokaulaurivuttauk akjarsijunut, aulatsijolutiglo sennajunik tamainik, sullijaksat nellianik; Levitinillo illangit aglakpaktôlaukput, aulatsijôlutiglo, udsertorteôlutiglo upkoaksoarnik.

14 Kênaujallo Nalekab iglunganut âtaumalauktut tigungmagit tagvangat, ajochertuijub Hilkiab Nalekab aglakotingit perkojaktaggit, Mosesekut tunnijaumajut, nachvarpait.

15 Hilkialo kiovok okarlunelo Safanemut aglakpaktomut: 
Aglait perkojaktaggit nachvarpakka Nalekab iglungane. Hilkiablo aglait tunnivait Safanemut.

16 Safaniblo aglait âpait attannermut, unipkautilâlo attannek, okarlunelo: Tamaita sennajaukojaujut kivgakotingnut, sennavait.

17 Kênaujallo nachvartaujut Nalekab iglungane navilaukpait, tunnilauklugillo sennakojijut aulatsijullo pinniaraksanik agganginut.

18 Safaniblo aglakpaktub attannek nellojungnaitipa okarlunelo: Hilkiab ajochertuijub aglangnik tunnitsivigilaukpânga. Safanelo attuarsivok tapkonane attanniub sângane.

19 Attanniublo perkojat okausertangit tussaramigit annorane allikpait.

20 Attanniublo Hilkia, Ahikamelo Safanib erninga, Abdonelo, Michab erninga, Safanelo aglakpaktok, Asajalo attanniub kivganga, perkovait okarlunelo:

21 Nalegak apperiartorsiuk pivlunga, Israelitillo amiakungit pivlugit, Judakullo pivlugit, aglait nachvartaumajut pitjutigællugit; Nalekab ningarninga kovijaumajok uvaptingnut angijovaksôngmat, atâtagijapta Nalekab okausingit mallilaungimagit, pinniartaililutik sorlo aglaksimangmat aglangne tapkonane.

22 Tagva Hilkia tapkoa illagilugit, attanniub tillilauktangit, aivok nellautaijomut arnamut Huldamut, Sallumib, Takehatib erningata, Hasrab, udsertortub annorânik erningata nullianganut, iglokartomut Jerusaleme abvakungita aipangine, okarvigivâllo tamattominga.

23 Tapsomalo okautivait: Imâk Nalegak Israelib Gudinga okarpok: Angut tillijise uvamnut, okautisiuk:

24 Imâk Nalegak okarpok: Ahâk kannoêtomik kaisijomavunga iglugaseksoarnut makkonunga, innunginullo, ominautinik tamainik, aglaksimajunik aglangne Judakut attanningata sângane attuartaulauktune;

25 Tamanna pivlugo, kemmalaungmanga, Gudiblo assingit isserluanititsivigilaungmattigik, ningaksarlunga aggangita pinniarninginut sunatuinarnut. Ningarneralo navijaujuksauvok iglugaseksoarnut makkonunga, kamitauniangilarlo.

26 Judakullo attanninganut tillijipsingnut Nalegak apperilugo, okartuksauvose: Imâk Nalegak Israclib Gudinga okarpok, okautsit tussarlauktatit pivlugit:

27 Tamanna pivlugo omattit auksititaumangmat, manigotitilauravillo Gudib sângane, okausingit tussarlaurangne, iglugaseksuit makkoa pivlugit, innungillo pitjutigællugit, manigotitilauravillo sâmne annorakotitit allilaurangne, kugviolauravillo sâmne, tagva uvanga tussarlaurivagittauk, Nalegak okarpok.

28 Ahâk illaliutitsomavagit atâtagilauktarnut, ullapirsautekarlutillo illautitaujuksauvotit illuvingnut, ijikpillo kannoêtut 
tamaita tikkerkojomârtakka iglugaseksoarnut makkonunga innunginullo tækkojaksaringilait. Tapkoalo tamakkoa okautigivait attannermut.

29 Tagva attannek tilliklerpok kattikovaillo Judakut Jerusalemiullo angajuklingit illunaita.

30 Attannerlo majorarpok takpaunga Nalekab iglunganut, Judakullo angutingit Jerusalemiullo illunatik, ajochertuijut Levitillo, innugiartoksuillo illunatik, mikkijut angijullo; tapsomalo attuarpait siutingita sângine, aglait angerkattigêngniggit nachvartaumajut Nalekab iglungane okausertangit tamaita.

31 Attannerlo nangerpok innimine, angerkattigêngnekartitsivorlo Nalekab sângane, Nalegak malliklugo agvertaromagamik, perkojangillo, illusiokojangillo, idluarijangillo malligomavlugit omamut tamânut, tarnemullo tamânut, angernerub okausertangit aglaksimajut aglangne tapkonane tamaita malliklugit pinniaromagamik.

32 Illangutikovaillo (angerkattigêngnermik) Jerusalemiut illunaita, Benjaminekunillo najutijut, Jerusalemiullo pinniarput Gudib atâtagijamik Gudingata angerninga malliklugo.

33 Josiablo makkojungnartut tamaita pêjarpait nunanit Israelib kittornganginut illingajunit; illunaitalo Israeleme naipitaumajut kivgartortipait Nalekamik Gudigijamingnik. Josia innotillugo nûlungilet Nalekamit atâtagijamik Gudinganit.

\section{KAPITEL XXXV.}

\section{Josiab attorninga Passamik, tokkotauningalo unaiadlarnerme.}

JoSIALO Passamik attorpok Jerusaleme Nalegak pivlugo, tokkotsivullo Passamik takkib sivurliub uvlungita 14 ingane.

2 Ajochertuijullo pitipait sullijaksanginut, kajungersarpaillo pinniarninginut Nalekab iglungane.

3 Okarporlo Levitinut, Israelitinik illunainik ajochertuijunut, Nalekamullo ivsornaititaumajunut: Iklervik ittertisiuk iglomut Salomob Davidib erningata Israelitit attanningata igloliorlauktanganut. Erksugaksaringilasse. Tagva manna Nalegak Gudise innukotingillo Israelitit kivgartorsigik.

4 Opalungaijaritselo illipsingnik atâtagijapse iglungita ingmigolinganingit malliklugit, sorlo Davidemut Israelitit attanninganut, erninganullo Salomomut aglaksimangmat;

5 Nangerluselo ivsornaitovingme, atâtagijapse iglungita ingmigolinganingit malliklugit, kattangutipse innugiartoksuit erningita akkorngane, Levitillo atâtagijamik iglungita ingmigolinganingit malliklugit.

6 Tokkotsiluselo Passamik, ivsornaitiluselo, kattangutiselo 
opalungaijarsigik, pinniarkovlugit Nalekab okausingit Mosesekut malliklugit.

7 Josialo innugiartoksoarnut tunnijivok saugârsungnik tuktungajoarkaniglo (tamainik Passamut illingajuksanik illunainut najutijunut) unnurningit 30000 diolaukput, tuktuvângniglo 3000 dinik; illunatiglo attanniub perkutinginit.

8 Angajokakotingille tunnijivut pijarallutik innugiartoksoarnut, ajochertuijunullo, Levitinullo, (tagva Hilka, Sacharjalo, Jehielelo angajokaunerôjut Gudib iglungane ajochertuijut akkorngane,) Passamut 2600 dinik saukanik, tuktungajoarkaniglo, tuktuvângniglo 300 dinik.

9 Kananjale, Semajalo, Nethaneelelo, kattangutingillo Hasabja Jejel, Josabadelo, angajokaunerôjut Levitinut, tunnijivut Levitinut Passab tunnergutiksanginut 5000 dinik saugârsungnik tuktungajoarkaniglo, tuktuvângniglo 500 dinik.

10 Taimaglo kivgartornek Gudemik akiksortaumalaukpok; ajochertuijullo nangerput innimingne, Levitillo ingmigolinganitik malliklugit, attanniub perkojanga malliklugo.

11 Passamiglo tokkotsivut, ajochertuijullo tapkoa agganginit auk serkerartipât, Levitillo aktorpait.

12 Apterpaillo tunnergutiksat ôtaksat, tunnilugit atâtat iglungita abvakunginut, tunnergutautilugit Nalekamut, sorlo aglaksimangmat Mosesib aglangine. Taimailiorlaukpaittauk tuktuvait.

13 Passalo ikkoalavât ikkomamut sorlo pijuksaungmat, ivsornaititaumajulle igavait ukkusingne kattanelo kallutanelo; tuavitomiglo âpait innugiartoksoarnut.

14 Kingornganelo âkiksoivuttauk nangminermingnut, ajochertuijunullo. Ajochertuijut Aronib erningita tunnergutit ôtaksat, orksuglo sulliarivait unnuak tikkidlugo. Tamanna pivlugo Levitit attuinaruteriakalaukput nangmineriningnut ajochertuijunullo Aronib erninginut.

15 Imgerpaktullo Assafib erningit nangerput innimingne Davidib attanniub Assafiblo, Hemaniblo, Jedituniblo tækkojub perkojangat malliklugo, upkoaniglo udsertortut upkoaksoarne (nangerput) nûlungilello sullijaksamingnit, kattangutingit Levitit attuinaruteringmatta pivlugit.

16 Taimak kivgartornek Gudemik Nalekamik âkiksorsimalaukpok uvlorme taipsomane, Passa attorlugo, tunnergutillo ôtaksat tunnilugit Nalekab Altaringata kângane, attanniub Josiab perkojanga malliklugo.

17 Taimak Israelib kittorngangita, najutijut, Passa attorpât, uvloksiorvik kakkojanik sêrnakangitunik nelliutome taipsomane, uvlut 7 it nâlugit.

18 Tapsotunarle Passamik attortokalaungilak Israeleme, Samuelib nellautaijub nelliuninganit, Israelitillo attanningit 
illunatik Passamik attorlaungilet taimaitomik Josiab, ajochertuijullo, Levitillo, Judamiullo illunatik Israelitinillo najutijut, Jerusalemiullo attorlauktangititut.

19 Josiab attanniovingata Jahringita 18 ingane Passa tamanna attortaulaukpok.

20 Tamakkoa Josiab sattortautitsiningata iglomik kingorngane, NechoEgiptenemiut attanningat takpaungarpok, Karchemis Fratemetut sorsugomavlugit. Josialo aularpok pâromavlugo.

21 Taipsomale tillijanik tilliklervigiva okautsaukolâlo: Suniarpigit Judakut attanninga? Opalugvigingilagit manna innugiartoksuille taipkoa unatadlartakka; Gudelo okarpok tuavituksaugama. Gude aiparijiga akkerartorungnairuk, asseroikonago illingnik.

22 Josiable kênane sangulungila tapsomangat, anorâminiglo pissertorpok sorsukattigijomavlugo, Nechublo okausingit, Gudib kaninganit nalengilait; tikkiporlo Megiddob naternanganut sorsukattigijomavlugo.

23 Pittiksertulle attannek Josia pittikpât; attanniublo kivgane okautivait: Nûtisinga, angijomik ikkilersimagama.

24 Kivgangitalo niotipât kamutingnit ikkitilâlo kamutekotingita aipanganut, âpâllo Jerusalemut; tokkovorlo illijartortaulunelo atâtagijame illuvingita akkorngane. Judakullo illunamassiatik Jerusalemiullo Josia oggurivât.

25 Jeremiablo Josia ârusutigiva; imgerpaktullo illunatik angutit arnallo ogguarsiutitik okâlatigivait Josia pivlugo, uvlok tamanna tikkilugo, illusiotitaulaukporlo Israeleme. Ahâk aglaksimavok imgerutine ogguarsiutine.

26 Unipkautsille assingit Josia pivlugo, pinniarningillo idluartut, Nalekab aglangit perkojaktaggit malliklugit pijut,

27 Pijokalauktangillo, sivurlit kingurlillo, ahâk, tamakkoa aglaksimavut Israelitit, Judakullo attanningita aglangine.

\section{KAPITEL XXXVI.}

Josiab malliktingit sittamat; Judakut aulartitauningat Babelemut. Utterkojauningat Koresemut.

\footnotetext{
Nunablo innugiartoksoangita Josiab erninga Joahas tiguvât, attanningortilâllo atâtanga innangêrlugo.

2 Joahase Jahrekalaukpok 23 ginik attanningorame, attanniotilunelo Jerusaleme takkit pingasut nâlugit.

3 Egiptenemiullo attanningata attanniojungnaitipa Jerusaleme, nunalo akkilêkova Talentinik Silberinik 100 dinik, Talentemiglo attautsemik Goldemik.
} 
4 Egiptenemiullo attanningata Eliakime kattanguta attanningortipa Judakunut Jerusalemiunullo, attingalo ablatsangortipa Jojakimemut. Nechoble kattanguta Joahase tiguva tikkiutilâlo Egiptenemut.

5 Jojakime Jahrekalaukpok 2 ǧ ginik attanningorame, attanniotilunelo Jahrit 11 it nâlugit Jerusaleme, pinniarporlo Nalekab Gudime idluigijanginik.

6 Nebukadnezarelo Babelemiut attanningat takpaungardlune opalukpa, kellaksorlâlo kellaumgujanut, aularutijomavlugo Babelemut.

7 Nalekab iglungata illulerkutingita illangit Nebukadnezarib Babelemiut attanningata tikkiutipaittauk Babelemut, pitilaitalo Tempelekotiminut Babelemetomut.

8 Unipkautsit assingit Jojakime pivlugo, makkojungnartulliningillo, nachvartaulertut tapsomane, ahâk, tamakkoa aglaksimavut Israelitit Judakullo attanningita aglangine. Erningalo Jojachin attanningorpok innangêrdlugo.

9 Jojachin Jahrekalaukpok 18 nik attanningorame, attanniotilunelo takkit pingasut, uvlullo tellimaujortut nâlugit Jerusaleme, pinniarporlo Nalekab idluigijanginik.

10 Jahrelo kângermat, Nebukadnezar tilliklerpok aijaukolâlo Babelemut, illulerkutit pivianartut Nalekab iglunganêtut illagilugit, Zedekialo kattanguta attanningortipa Judakunut Jerusalemiunullo.

11 Zedekialo Jahrekalaukpok 21 nik attanningorame, attanniotilunelo Jahrit 11 it nâlugit Jerusaleme.

12 Pinniarporlo Nalekab Gudime idluigijanginik, manigotitilungilarlo nellautaijub Jeremiab okâlalauktub Nalekab kanninganit sângane.

13 Nalegungnaipâlo Nebukadnezar Babelemiut attanningat, angermaringnermik Gudeme tigusilauktok tapsomangat, tappatôsilunelo, omattinelo mangaitipa, sâlerkonago Nalekamut Israelib Gudinganut.

14 Ajochertuijut angajokaunerojungit illunatiktauk innugiartoksuit illagilugit idluinekartitsivut unnuktunik, nellojut makkojungnartulliningit sunatuinait malliklugit, ivsornartipâllo Nalekab iglunga, ivsornaitilauktanga Jerusaleme.

15 Nalegarlo atâtagijangita Gudingat tilliklerpok tapkonunga, tillijamigut uvlamit. Innukotine iglunelo ivlerigamigit.

16 Tapkoale Gudib tillijangit mittautigivait nachogilaitalo okausingit, ijorivaillo nellautaijokotingit, Nalekab kanningajarninga innukotine pivlugit angijororkârtinago, innulijaujungnarungnaimatta.

17 Tagva tikkitaukovait Kaldæerit attanninganut, tapsoma angutingit innuosuktut tokkopait iglome ivsornaitovingane, iv- 
lerilaungilaillo innuosuktut angutillonêt uigârsuillonêt innutokaujullonêt kêrtojullonêt; Gudib illunaita tunnivait tapsoma agganginut.

18 Illulerkutillo Gudib iglunganêtut mikkijut angijullo, perkutillo Nalekab iglunganêtut, attanniublo angajokâkotingitalo perkutingit pivianartut, âtaukovait Babelemut.

19 Gudiblo iglunga ikkipât, Jerusalemiblo avalungita karmangit serkomipait, igloksoakotingillo illunaita ikkipait, perkutingillo pivianartut illunaita asserorpait.

20 Aularutivaillo Babelemut saviksoarmit amiakolauktut, tagvanelo kivgaulaukput tapsomunga erninginullo, Persiamiut attanniovingat attanniotilerkârtinago.

21 Nellautsilerkovlugit Nalekab okausingit Jeremiab kanningagut, nunab Sabbatingit namaksilerkârtinagit. Nelliutok tamna illunât nuna asserorsimatillugo Sabbateôlaungmat, Jahrit 70 it nâlerkârtinagit.

22 Koresible Persiamiut attanningata Jahringita sivurlingane, nellautsilerkovlugit Nalekab okausingit, okauserilauktangit Jeremiab kanningagut, Nalekab Koresib Persiamiut attanningata annerninga tuppaksarpa, akpautigijaukojikovlugo attanniovingmine illunane, aglaktigullotauk, okarkojilunelo:

23 Imâk Korese Persiamiut attanningat okarpok: Nalekab killaub Gudingata tunnitsivigilaukpânga attanniovingnik tamainik nunane, perkolaukpângalo iglomik igloliorlugo Jerusaleme Judame. Kina manna akkonapsingne tapsoma innukotinginut illingajok, tamna Nalekab Gudingata aipariliuk, takpaungarlunelo.

\section{ESRAB AGLANGIT.}

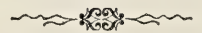

\section{KAPITEL I.}

Koresib Persiamiut attanningata perkojininga Judit kippalutjarsimavlutit utterungnarmatta, T'empeliorungnarmattalo.

Ko ORESIB Persiamiut attanningata Jahringita sivurlingane, nellautsilerkovlugit Nalekab okausingit, okauserilauktangit Jere- 
miab kanningagut, Nalekab Koresib Persiamiut attanningata annerninga tuppaksarpa, akpautigijaukojikovlugo attanniovingmine illunane, aglaktigullotauk, okarkojilunelo:

2 Imâk Korese Persiamiut attanningat okarpok: Nalekab killaub Gudingata tunnitsivigilaukpânga attanniovingnik tamainik nunane, perkolaukpângalo, iglomik. igluliorlugo Jerusaleme Judame.

3 Kina mana akkunapsingne tapsoma innukotinginut illingajok, tamna Nalekab Gudingata aipariliuk, takpaungarlunelo Jerusalemut Judamêtomut, igloliorlunelo Nalekab Israelib Gudingata iglunganik. Gudeôvok tamna Jerusalemêtok.

4 Amiakojorlo sulle innine tamaine tujormiangovingine, tamna inningata innungita ikajorlitsuk, Silberemut, Goldemullo, perkutinullo, nergjutinullo, tunnergutit kunnutuinermut tunnijaulertut Gudib iglunganut Jerusalemêtomut, tappilugit.

$\check{5}$ Tagva angajokaunerôjut atâtanit Judakunit, Benjaminekunillo, ajochertuijullo, Levitillo, illunatik annernermigut tuppaksartaujut Gudemut, parngnailerput takpaungaromavlutik, igloliorlugolo Nalekab igluksanga Jerusaleme.

6 Illunatiglo avatinginêtut ikajorpait, illulerkutinut Silbereôjunut, Goldeôjunullo, perkutinullo, nergjutinullo, pivianartunullo, tunnergutingit pijarællutik tappilugit.

7 Attanniublo Koresib Nalekab iglungata illulerkutingit ' annipait Nebukadnezarib tigulauktangit Jerusalemit, Gudimelo iglunganut pitilauktangit.

8 Koresible Persiamiut attanningata annipait Mitredatekut pimaklerteôjokut perkutinik, kittitautipaillo Sesbazaremut Judakut angajokanganut.

9 Tamadsa unnurningit: Pogutat Goldeôjut 30 git, pogutallo Silbereôjut 1000 dit, sâvillo 20 git.

10 Ermgusiarsuillo 30 git, ermgusiarsuillo assingit Silbereôjut 410 ôlaukput.

11 Illulerkutit illunatik Goldeôjut, Silbereôjullo 5400 diôlaukput. Sesbazarib tapkoa illunaita takpaungautipait tapkoa illagallugit parngnanairsimavingmit Babelemit takpaungartut Jerusalemut.

KAPITEL II.

Judit uttertut Babelemit unnurningit, attingillo; tunnergutingillo Tempeleliornermut.

TAMADSA nunab (Judab) kittorngangit, takpaungartut parngnanairsimavingmit, Nebukadnezarib Balelemiut attanningata 
tikkiutilauktangit Babelemut, uttertullo Jerusalemut Judamullo attunît iglugaseksoarnut iglokarvigilauktamingnut.

2 Tikkipullo Serubabel, Jesualo, Nehemialo, Serajalo, Reeljalo, Mardochailo, Bilsanelo, Misparelo, Bigevaillo, Rehumelo, Baenalo illagilugit. 'lamadsa manna Israelib innugiartoksoangita angutingita unnurningit:

3 Pareosib kittorngangit, 2172 git.

4 Sefatjab kittorngangit, 372 git.

5 Arab kittorngangit, 775 git.

6 Pahat Moabib kittorngangit Jesuab Joabiblo kittorngangita akkorngane, 2812 it.

7 Elamib kittorngangit, 1254 git.

8 Sathub kittorngangit, 945 git.

9 Sakaib kittorngangit, 760 git.

10 Banib kittorngangit, 642 git.

11 Bebaib kittorngangit, 623 git.

12 Asgadib kittorngangit, 1222 git.

13 Adonikamib kittorngangit, 666 git.

14 Bigevaib kittorngangit, 2056 git.

15 Adinib kittorngangit, 454 git.

16 Aterib kittorngangit Hiskiab kinguvânginit, 98 git.

17 Bezaib kittorngangit, 323 git.

18 Jorab kittorngangit, 112 it.

19 Hasumib kittorngangit, 223 git.

20 Gibbarib kittorngangit, 95 git.

21 Bethlehemib kittorngangit, 123 git.

22 Netofamiut, 56 git.

23 Anatotemiut, 128 git

24 Asmavetib kittorngangit, 42 git.

25 Kiriatarimib Kafirablo Beerotiblo kittorngangit 743 git.

26 Ramab Gabablo kittorngangit 621 git.

27 Michmasemiut 122 git.

28 Bethelmiut, Aimiullo 223 git.

29 Nebob kittorngangit, 52 git.

30. Magbisemiut, 156 git,

31 Elamib aipangata kittorngangit 1254 git.

32 Harimib kittorngangit, 320 git.

33 Lodib, Hadidiblo, Onoblo kittorngangit, 725 git.

34 Jerehob kittorngangit, 345 git.

35 Senaab kittorngangit, 3630 git.

36 Ajochertuijut: Jejadab kittorngangit Jesuakunit, 973 git.

37 Immerib kittorngangit, 1052 git.

38 Pashurib kittorngangit, 1247 git.

39 Harimib kittorngangit, 1017 it.

40 Levitit: Jesuab Kadmieliblo kittorngangit, Hodavab kittornganginit pijut 74 git. 
41 Imgerpaktut: Asafib kittorngangit 128 git.

42 Udsertortut upkoanik kittorngangit: Sallumib kittorngangit, Aterib kittorngangit, Thalmonib kittorngangit, Akubib kittorngangit, Hatitab kittorngangit, Sobaiblo kittorngangit illunatik kattilugit, 139 git.

43 Nethinimit: Zihab kittorngangit, Hasufab kittorngangit, Tabaotib kittorngangit,

44 Kerosib kittorngangit, Siehab kittorngangit, Padonib kittorngangit,

45 Lebanab kittorngangit, Hagabab kittorngangit, Akub kittorngangit,

46 Hagab kittorngangit, Samlaib kittorngangit, Hananib kittorngangit,

47 Giddelib kittorngangit, Gaharib kittorngangit, Reajab kittorngangit,

48 Rezimib kittorngangit, Nekodab kittorngangit, Gasamib kittorngangit,

49 Usab kittorngangit, Passeab kittorngangit, Bassaib kittorngangit,

50 Asnab kittorngangit, Meunimib kittorngangit, Nefusimib kittorngangit,

51 Bakbukib kittorngangit, Hakufab kittorngangit, Harhurib kittorngangit,

52 Batzelutib kittorngangit, Mehidab kittorngangit, Harsab kittorngangit,

53 Barkomib kittorngangit, Sisserab kittorngangit, Thamab kittorngangit,

54 Neziab kittorngangit, Natifab kittorngangit.

55 Salomob kivgangita kittorngangit: Sotaib kittorngangit, Soferetib kittorngangit, Prudab kittorngangit,

56 Jaelab kittorngangit, Darkonib kittorngangit, Giddelib kittorngangit,

57 Sefatajab kittorngangit, Hattilib kittorngangit, Pocheretib Zebaimiub kittorngangit, Amib kittorngangit.

58 Nethinimit illunatik Salomoblo kivgangita kittorngangit kattilugit 392 giôlaukput.

59 Tapkoalo takpaungarkattaugivut: Mithel, Mela, Thel, Harsa, Kerube, Addon, Immerelo; atâtagijamigle kinguvângit sivurlitiglo nellonailijungnarlungilait, Israelemit pingmangata.

60 Delajab kittorngangit, Tobiab kittorngangit, Nekodab kittorngangit, 652 git.

61 Ajochertuijullo kittornganginit: Habajab kittorngangit, Hakozib kittorngangit, Barsilaib kittorngangit, Barsilaib Gileademiub panninginit nullianilauktub, taipsotunarlo atserartaulauktub.

62 Tapkoa siyurlimik abvakungita nippisiorutingit kenner- 
pait, nachvarlungilaillo; tamanna pivlugo ajochertuijoêrotilerput.

63 Hathirsatablo okautivait nerrijuksaungimatta ivsornainerpangôjomit, ajochertuijokarkârtinago kaumaniutiksalijartomik idluarsaijutiksalijartomiglo.

64 Illagêt illunatik kattilugit 42360 giôvut.

65 Kivgangit angutit, arnallo kittinagit, tapkoa 7337 ôlaukput. Imgerpaktokalaukpullo angutinik arnaniglo 200 dinik.

66 Horsekotingit 736 giôlaukput, Horseojartokotingillo 245 giôlaukput.

67 Kameelekotingit 435 giôlaukput; siutekoktôjokotingillo 6720 giôlaukput.

68 Atâtallo angajokaunerôjut illangit, tikkinamik Nalekab iglunganut Jerusalemut, kunnutuilutik tunnijivut aitusiamik Gudib iglunganut, igluliortaumikovlugo innigilauktanganut;

69 Tunijivullo ajugaringitamingnik sullijaksab akkiliutiksanganut kênaujanik Goldeôjunik Darikenik attelingnik 61000 dinik, Silberemiglo paundinik 5000 dinik, ullingniglo ajochertuijunut 100 dinik.

70 Taimak ajochertuijut Levitillo innugiartoksuillo illangita, imgerpaktullo, upkoaniglo udsertortut, Nethinimillo iglugaseksoakotitik innigilerpait, Israelitillo illunatik iglugaseksoakotitik.

\section{KAPITEL III.}

Altarib sennajauninga. Uvloksiorviub iglokarnermit akkerkunik millukattalingnik attortauninga. Tempelib tungaviata illïauninga.

TAKKILLO 7 ingat nelliutilermat, Israeliblo kittorngangit mana iglugaseksoakotimingnêmatta, tagva innugiartoksuit kattimalerput Jerusaleme angutitut attautsetut sorlo.

2 Jesualo Jozadakib erninga, kattangutingillo ajochertuijut, Serubabelelo, Sealthib erninga kattangutingillo parngnailerput, sennalutiglo Israelib Gudingata Altaringanik, tunnergutinik ôtaksanik tunijilune kângane, sorlo aglaksimangmat Mosesib Gudib angutekotingata perkojangine.

3 Sennavâllo Altare innigilauktangane, (koaksarnermut tikkitaumagamik innukattigêksuit nunane pivlugit;) tunnijilutiglo kângane Nalekamut tunnergutinik ôtaksanik, uvlâne unnunelo.

4 Uvloksiorlutiglo uvloksiorvingmik iglokarnermik akkerkunik millukatalingnik, sorlo aglaksimangmat; tunnijilutiglo tunnergutinik ôtaksanik uvlut tamaita unnurningit, sorlo pijuksaungmatta, uvlut attunît tunnergutinginik, 
5 Kingurnganelotauk tunninivait tunnergutit ôtaksat pitsainartuksat takkêlanelo, Nalekablo uvloksiorvingine tamaine, ivsornaititaumajune, tunnergutillo pijarijut, tunnijangit pijarællutik Nalekamut.

6 Takkit 7 ingata uvlungita sivurlingane pigiarput tunnergutit ôtaksat tunnilugit Nalekamut. Nalekable Tempelingata tungavia illijaulaungilak sulle.

7 Tunnijivulle kênaujanik ujaralerijunut, igluliortunullo, nerkiksaniglo, immiksaniglo, orksumiglo Zidonemiunut, Tirusemiunullo kejungnik Cedereôjunik âtsikovlugit Libanonemit immarbikut Jafomut, Koresib Persiamiut attaningata perkojanga tapkonunga malliklugo.

8 Tikkiningatalo Nalekab iglunganut Jerusalemut, Jahringita aipangata takkingita aipangane, Serubabel Sealthielib erninga Jesualo, Jozadakib erninga, assingillo kattangutingit, ajochertuijut Levitillo, illunatiglo parngnairsimavingmit tikkisimajut Jerusalemut, pigiarput, pitilaitalo Levitit Jahrekartut 20 ginik unnurnersaniglo, aulatsilerkovlugit sullijaksanik Nalekab iglungane.

9 Jesualo erningillo, kattangutinelo illagilugit, Kadmielelo erningillo, Judablo kittorngangit nangerput angutetut attautsetut, sennajut Gudib iglungane aulujitilugit, taimaktauk Henadadib kittorngangit, kittorngatik, kattangutitiglo, Levitit illagilugit.

10 Iglomiglo sennajut tungavingmik illijitilugit Nalekab Tempelekotinganut, ajochertuijut nangerput tagvane annorasimavlutik (ajochertuisiutinik) Trompetelijarlutik, Levitillo Assafib kittorngangit, Cimbelilijarlutik Nalegak nertorlugo, Israelib attanningata Davidib illusiokojanga malliklugo.

11 Imgerpullo Nalegak nertorlugo kujagilugolo, pitsiarmat, napkoarningalo sorainiangmat Israelemut. Innugiartoksuillo illunatik nipliadlarput Nalegak nertorlugo Nalekab iglungata tungavia illijaumangmat.

12 Ajochertuijulle innutokaujut unnuktut, Levitillo atâtailo angajokaunerôjut, iglub sivorlinganik tækkolauktut, iglolo tamna manna ijingita sângane tunganilititaumangmat, kêavaksoadlarput; unnuktulle nipliadlarput kuviasumut.

13 Innugiartoksuillo nipliadlarnek kuviasumut illitarijungnarlaungilât, kêavaksub innugiartoksuit akkornganêtub nippevaksoanganit, innugiartoksuit kaggudlarmatta, nippersoanga tussaksaungmat kanningitomut. 


\section{KAPITEL IV.}

\section{Tempelib sennajauningata agviartauninga.}

JUDAKULLE Benjaminekullo akkerartortingit tussaramik, parngnanairsortaumalauktut kittorngangit Tempeleliormatta Nalekamut Israelib Gudinganut;

2 Tikkiput Serubabelemut atâtanullo angajokaunerôjunut, okautivaillo: Igluliorkattigijomavapse, Gudise kenneraptigo, illipsetut; tapsomungalo tunnergutinik tunnijivogut, AsserHaddonib Assiriamiut attanningata, tamaungartitipta nelliuninganit.

3 Serubabelible, Jesuablo, atâtallo angajokaunerôjut Israelitit akkorngane, kiovait: Namatsengilak igluliorkattigilugo Nalegapta iglunga, uvagutôlutale iglulioromavogut Nalekamut Israelib Gudinganut, sorlo Koresib Persiamiut attanningata perkolaungmattigut.

4 Tagva nunab innungita innugiartoksuit Judakut aggangit katsungatinasuarpait, koaksartilugillo igluliortailitilugit.

5 Kaikojivullo attannertuijunik, kajusimaninginiglo agviarput Kores Persiamiut attanningat innotillugo, Dariusib Persiamiut attanningata attanniotininga tikkilugo.

6 Ahasverosible attanniotiningane, attanniotiningata pigiarningane, passiklerutiksanik aglakput Judamiut, Jerusalemiullo pivlugit.

7 Artasastablo nelliuningane, Bislame, Mitredatelo, Tabeelo assingillo illangit aglakput Artasastamut Persiamiut attanninganut. Aglait tapkoa aglangit aglaksimalaukput Siriamiut okausingit attorlugit, illijaulutiglo Siriamiut okausinginut.

8 Rehumib attanniotitub, Simsaiblo aglakpaktub aglait tapkoa aglakpait Jerusalemiut akkerartorlugit, Artasastamut attannermut:

9 Uvagut: Rehume, angajokaujunga, Simsailo aglakpaktok, illangitalo assingit, attannertuijut Dinamiut, Afarsachemiullo, Tatplatemiullo, Persiamiullo, Arachemiullo, Babelemiullo, Susanemiullo, Dehamiullo, Elamemiullo.

10 Innugiartoksuillo assingit Asnafarib angijub, okausiovaktublo tamaungartilauktangit, pitilauktangillo Samariab iglugaseksoanginut, assinginullo kôksub miksânêtunut, Kanaanemêtunullo.

11 Tamadsalo aglait tapkoa tillikliutilauktangita attannermut Artasastamut tukkingit: Kivgakotitit, angutit kôksub miksânêtut Kanaanemêtullo :

12 Attannek nellojungnaititaule, Judit, illingnit takpaung- 
arsimajut uvaptingnut Jerusalemut, iglugaseksuit pikkablâjoksôjut idluitullo, iglugaselliormagit, avaluksoangillo (karmangit) sennangmagit, tungavingillo sattortautimagit.

13 Taimak manna attannek nellojungnaititaule, iglugaseksuit taipkoa iglugaseliortaupatta, avaluksoangillo sattortautitaupatta, tagva Schossemik, Zollemiglo, akkiliaksaniglo Jahrine tamaine tunnijiniangilat, kajusimaningallo, attanniub aksartaujutiksanganut illinganiarpok.

14 Uvagulle manna illunata najûtimagapta Tempelemik asseroilauktogut, attanniub nachogijauninga akkunênersamik tækkonâromalaungilavut, tamanna pivlugo tilliklerpogut nellonaijarlugolo tamanna attannermut,

15 Atâtagijavit pijokalaungningita aglangine kennertokarkovlugo, tagva nachvarniarpotit pijokalauktut aglangine tapkonane, tukkisiniarlutillo, iglugaseksuit tapkoa pikkablâjoksôngmatta, idluitoksôngmattalo attannernut, nunanullo, assitiglotauk nalegungnaisarpait itsaksoarmit; tamanna pivlugo iglugaseksuit tapkoa asserortaumalaurivut.

16 Tamanna pivlugo attannek nellojungnaitipavut iglugaseksuit ukkoa iglugaseliortauniarpatta, avaluksoangillo pijarêrtauniarpatta, tagva tapkoa pivlugit kôksub miksane illangiutivikarungnainiaravit.

17 Tagva attannek kigusermik tillikliutjivok Rehumemut attanniotijomut, Simsaimullo aglakpaktomut, illanginullo Samariame nunakartunut, assinginullo kôksub miksânêtunut: Ullapirsaunek salutitaunerlo!

18 Aglait tillikliutilauktase uvaptingnut, sagvartome attuartaulaukput sâmne.

19 Perkojilaukpungalo kennertokarkovlugo. Nachvartaumavorlo, iglugaseksuit taipkoa itsaksoarnit akkerartorpalaungmatta, pikkablatoksonekalaungmallo, nalegungnainekadlalarmelo taipkonane.

20 Taimaktauk attannekalaukpok Jerusaleme pitsartujunik, attanniotilauktunik illunainut kôksub ungatânetunut, Zollemiglo Schossemiglo akkiliaksaniglo Jahrine tamaine tunnitsiviolaukput.

21 Tagva perkojamik tunnijileritse manna innuit taipkoa agviartaukovlugit, iglugaseksuillo ukkua iglugaseliortaukonagit, perkojilerkârtinanga.

22 Udsertutsiaritselo katsungakonase taimailiornerme attannek aksartaukonnago.

23 Attanniub Artasasthab aglangit attuartaujarêrlaungmatta Rehumib, Simsaiblo aglakpaktub, attannertuijokotingitalo sâng ine, tagva tuavilutik aularput takpaunga Jerusalemut Judinut, agviarlugillo tallermut sôngojomut.

24 Tagva sullijaksak Gudib iglungane Jerusalemêtome nok- 
karpok, taimailingalunelo Dariusib Persiamiut attanningata Jahringita aipanga tikkilugo.

\section{KAPITEL V.}

\section{Tempeliornerub pigannerninga nellautaïĥl Hagaib Sachar- jablo nellautainingit malliklugit.}

\section{NeLLaUtAIJUGLE Haggai, Sacharjalo Iddob erninga nellau- tailaukpûk Israelib Gudingata attingane Judinut Judametunut Jerusalemetunullo.}

2 Tagva Serubabel Sealthielib erninga, Jesualo Jozadokib erninga parngnailerpuk Gudib iglunga igluliorlugo Jerusaleme, illagilugiglo Gudib nellautaijokotingit nekkoksijut tapkoninga.

3 Nelliutome taipsomane Thatnaib nunab aulatinga kôb miksâne, Star Bosnailo illangillo tikkiput tapkonunga, okautilugillo imâk: Kia perkolaukpâsse iglo manna igluliorlugo, karmangillo sennalugit?

4 Tagva okautivavut, kannok angutit iglomik ominga igluliortut attekarninginik.

5 Gudingatalo ijingata Judit angajokângit kollangiarpait agviarkonagit tapkoninga, pijokartok tamanna Dariusemut tillijaujutaukârtinago aglangniglo tikkitokarkârtinago tamanna pivlugo.

6 Tamadsale Tatnaib nunab kôksub miksânêtub aulatingata, Star Bosnaiblo attannertuijokotingitalo Afarsachemiut, kôksub miksânetut aglangita tillikliutilauktangita attannermut Dariusemut tukkingit.

7 Okautsillo tillikliutilauktangit tapsomunga imailaukput: Attannek Dariuse ullapirsautekarle illunane!

8 Attannek nellojungnaititaule uvagut tikkisimagapta Judit nunanganut, Gudib angijoksojub iglunganut, igluliortaujomut ujarkanit angijunit iktoriktunit, ikkatjello illijauvut senneranginut sullijaksarlo tamanna katsungaitomik pinnasuartaurok pivalliadlarlunelo taipkoa agganginut.

9 Uvagulle angajokaunerôjut apperilaukpavut okautilugillo : Kia perkolaukpâsse iglo tamna igluliorlugo, karmangillo sennalugit?

10 Apperilaukpavuttauk, kikkômangata, nellonaijaikovluta tapkoninga illingnut, angutillo angajokaunerôjungita attingit aglalaukpavut.

11 Taipkoale okautsinik imaitunik kigusiksakalaukput uvaptingnut, okarlutiglo: Uvagut kivgauvogut killaub nunablo Gudingangnut, iglolo tamna igloliorpavut, Jahrit unnuktut mat- 
toma sivorngane igluliortaulauktok, Israelitit attanningata illangata angijub igluliorlauktanga nappartilauktangalo.

12 Atâtagilauktaptale killaub Gudingat ningaksarlaungmatsuk, tunnilaukpait Nebukadnezarib Babelemiut attanningata, Kaldæereôjub agganginut; tapsoma iglo tamanna serkomilaukpa, innugiartoksuillo aularutilaukpait Babelemut.

13 Koresible Babelemiut attanningata Jahringita sivurlingane, attannek Korese tamna perkojilaukpok, Gudib iglunga tamanna igluliorlugo.

14 Illulerkutittauk Goldeôjut Silbereôjullo Gudib iglungane, Nebukadnezarib tigulauktangit Tempelemit Jerusalemêtomit, tikkiutilauktangillo Tempelemut Babelemêtomut, attanniub Koresib tigulaukpait Tempelemit Babelemetomit, tunnivaillo Sesbazaremik attelingmut, attanningortilauktaminut nunamut;

15 Okarlunelo: Illulerkutit makkoa tigukit, aulârlutillo tikkiutilaitalo Tempelemut Jerusalemêtomut, Gudiblo iglunga igluliortaule innigilauktangane.

16 Tagva Sesbazar tamnautsainak tikkisimavok illijilauklunelo tungavingmik Gudib iglunganut Jerusaleme. Taimangamillo igluliorput innersimangilarlo sulle.

17 Namagijaulerpallo manna attannermut, tagva kennerkojile attanniub pivianartotalingane Babelemêtome, taimaimangât, attannermut Koresemut perkojaulaungmangât Gudib iglunga Jerusaleme . igluliorlugo: tillikliutjivigitigullo attanniub issumanginik tamanna pivlugo.

\section{KAPITEL VI.}

Tempelib innertaulerninga, attortautainarningalo, Passablo attortauninga.

TAGVA attannek Dariuse perkojivok, kennertokakovlugo attanniub agliakotitalingane Babelemêtome.

2 Tagva Ahmetame Medenemêtome igloksoarme aglait nachvartauvut, imâglo pijokalauktomik aglaksimajokalaukpok tapkonane:

3 Attanniub Koresib Jahringita sivurlingane attannek Kores perkojivok, Gudib iglunga Jerusaleme igluliorlugo, tunnergutiksat tunnijauviat, tungavialo illilugo, pôktôninga tallitut 60 gitut, sennimutuningalo tallitut 60 gitut;

4 Sennerangnik pingasunik ujarkanit iktogriktunit, senneraglo attausek kejungmit; akkiliutiksallo tunnijaujuksauvut attanniub iglunganit. 
5 Gudiblo igIungata illulerkutingit Goldeôjut Silbereôjullo Nebukadnezarib tigulauktangit Tempelemit Jerusalemêtomit, Babelemullo tikkiutilauktangit, tunnijaujuksaugivut uttertaukovlugit Tempelemut Jerusalemêtomut, innigilauktanganut Gudib iglungane.

6 Manna taimaimat igvit Tatnai aulateôjotit kôksub ungatâne, Star Bosnailo, illangillo Afarsachemiôjose kôksub ungatânetose ungasilersigik.

7 Sennalit Gudib iglunganik Judit nunangata aulatingata angajokaunerôjungitalo Gudib iglunga igluliorlugo inningane pikovlugit.

8 Perkojaumavorlotauk uvamnut, sunamik pinniartuksaunipsingnik Judakut angajokaunerôjunginut, tapkonunga Gudib iglunga igluliorlugo, imâgle, attanniub perkutinginit, akkiliaksanit kôksub ungatâne, angutit tapkoa akkiliutigijariakartangit, tunnijautsiartuksauvut agviartaukonagit.

9 Pijariakaraikpattalo angusalluârsungnik, saugârsungniglo, tunnergutiksanut ôtaksanut killaub Gudinganut, Weizenmiglo, sioraujamiglo, Weinemiglo orksumiglo, ajochertuijut Jerusalemêtut illusingit malliklugit; tagva tunnitsiviojuksauvut tamakkoninga uvlut tamaita pijariakartanginik, tamannalo kâtuimauvlune piniartaukonnago;

10 Tunnergutinik tunnijikovlugit killaub Gudingata naimatsiarninganut, tuksiutjikovlugillo attanniub innosinganik, kittornganginiglo.

11 Uvamnit perkojak tamanna tunnijaumavok; innuit nelliat okautsinik ukkuninga ablatsangortitsilerpat, tagva tapsoma iglunganit ikâtjet illangat tigujaujuksauvok nappartaujuksaulunelo, tamnalo nivingatilugo tapsomunga; iglungalo aksivingortitaule.

12 Gudelo kujusimalauktok tagvane innekaromavlune, tokkotsilerle attannernik innukattigêksoarniglo tamainik, aggamingnik issaktunik Gudib iglunga Jerusalemêtok ablatsangortitsomavlugo serkomitsomavlugolonêt. Uvanga Dariuseôjunga tamanna perkolaukpara, illungertornermut piniartaukovlugo.

13 Tagva Tatnai nunab aulatinga kôksub ungatâne, Star Bosnailo, illangillo, tilliklerviomajut attannermut Dariusemut, taimailiorput illungertorlutik.

14 Judillo angajokaunerôjungit igluliorput; sullitsiarlaukpullo nellautaijuk Haggaib, Sacharjablo Iddob erningata nellautainingitigut, igluliorput nappartilugolo, Israelib Gudingata perkojanga malliklugo, Koresiblo, Dariusiblo Arthasasthablo Persiamiut attanningita perkojangit malliklugit.

15 Innerpâllo iglo takkib Adaremik attiggub uvlungita pingajuane, tagva attanniub Dariusib attanniotiningata Jah ringita pingasojoat. 
16 Israeliblo kittorngangita, ajochertuijut, Levitit parngnanairsortaumalauktullo kittorngangita assingita Gudib iglungata attortautainarninga uvloksiorpât kuviasuklutik;

17 Tunnijivullo tunnergutinut Gudib iglungata attortautainarninganut, tuktuvângnik angusallungnik 100 dinik, saugârsungniglo 200 dinik, tuktungajuniglo angusalungnik 400 dinik, tunnergutiksamullo ajortoêrutiksamut Israelitit illuêngârlutik pivlugit tuktungajunik angusallungnik 12 nik, Israelib kinguvarêngita unnurningit malliklugit.

18 Pitipaillo ajochertuijut inninginut, Levitillo ingmigolinganinginut, Gude Israelemêtok kivgartorlugo sorlo aglaksimangmat Mosesib aglangine.

19 Kittorngallo parngnanairsortaumalauktut Passamik attorput, takkit sivorliub uvlungita 14 ingane.

20 Ajochertuijut, Levitillo, sujungaitilaukput ingminik, illunatik ivsornaimatta angutut attautsetut sorlo; tokkotsilutiglo Passasiorutiksamik kittorngat parngnanairsortaumajut illunatik pivlugit, kattangutitiglo ajochertuijut pivlugit, pivlutiglo.

21. Israeliblo kittorngangit parngnanairsimavingmit utterlauktut, illunatiglo apterlauktut nellojut ivsornarninginit tapkonunga, Nalegak Israelib Gudingat kenneromavlugo, Passamik nerrivut,

22 Uvloksiorviglo kakkojanik sêrnakangitunik attorpât uvlut 7 it nâvlugit kuviasuklutik; Nalekab kuviasuktilaungmagit, Assiriamiullo attanningata omattinga torartilaungmago tapkonunga, ikajorlugit sullijaksame Gudib Israelib Gudingata iglungane.

\section{KAPITEL VII.}

Esrab perkojauninga attannermut Jerusalemut ingerarungnarlune, kivgartornerlo Gudemik akiksorlugo.

PIJOKalaUKTUT
tanningata attannioving
paungarpok Babelemit
ata, Hilkiab erningata,

2 Sallumib erningata, Zadokib erningata, Ahitub erningata,

3 Amarjab erningata, Asarjab erningata, Merajotib erningata.

4 Serajab erningata, Usib erningata, Bukib erningata,

5 Abisuab erningata, Pinehasib erningata, Eleaserib erningata, Aronib ajochertuinerôjub erningata.

6 Illisimatsiangolauktok aglangnik, Mosesib perkojanginik Nalekab Israelib Gudingata tunnilauktanginik, Attanniublo 
tunnitsivigiva tamainik pijomajanganik, Nalekab Gudingata aggangita kollangiarninginut tapsominga.

7 Takpaungârpullo (illagællugo) Jerusalemut Israelib kittorngangita illangit, kapsit, ajochertuijullo, Levitillo, imgerpâktullo, upkoaniglo udsertortut, Nethinimillo, attanniub Arthasasthab Jahringita 7 ingane.

8 Tikkipullo Jerusalemut takkit tellimangane, tagva attanniub Jahringita 7 ingata.

9 Takkib sivurliub uvlungita sivurlingane kajusimalerlaurame Babelemit takpaungaromavlune, takkillo tellimangata uvlungita sivurlingane tikkipok Jerusalemut, Gudib aggangita pitsiartut kollangiarninginut tapsominga.

10 Esrable omattine torartipa Nalekab perkojangit kenneromavlugit, pinniaromavlunelo ajochertuijomavlunelo Israeleme perkojanik idluarnerniglo.

11 Tamadsalo aglait tukkingit attanniub Arthasasthab tunnilauktangita Esramut, ajochertuijomut, aglangniglo illisimajomut, ajochertuijolauktomut Nalekab okausinginik, perkojanginiglo Israelemut:

12 Artasastha, attanneôjok attannernut illunainut, Esramut, ajochertuijôjomut aglangniglo illissimajôjomut killaub Gudingata perkojanginik, ullapirsaumik salutitaunermiglo.

13 Uvamnit perkojak tunnijaumavok, illunatik nalegauvimnêtut innukattigênit Israelitinit, ajochertuijunginillo Levitinillo, kunnutuilutik aularomajut Jerusalemut, illaukattaujungnarmatta illingnut;

14 Igvit attannermut attannertuijokotinginullo 7 inut tillijaumagavit, Juda Jerusalemelo nautsertoriartorlugit Gudib perkojangit aggangnêtut oktutigivlugit.

15 Neksarlugillo Silbere, Goldelo attanniub attannertuijokotingitalo pijarallutik tunnijangit Israelib Gudinganut, iglokartomut Jerusaleme;

16 Silberelo, Goldelo tamaita nachvarungnartattit nuname Babeleme, tapkoa illaliudlugit innugiartoksuit ajochertuijullo pijarallutik tunnijangit Gudib iglunganut Jerusalemêtomut;

17 Tamakkoa tamaita tigukit, attaruilivlutillo pissiniarit kênaujanut tapkonunga, tuktuvângnik angusallungnik, saukaniglo angusallungnik, saugârsungniglo, tunnergutiksaniglo nerkiksanik, tunnergutiksaniglo immiksanik, tunnergutaukovlugit Altareme Gudipse iglunganêtome Jerusaleme.

18 Sunalo igvit kattangutivillo idluarigapsiuk kênaujat amiakojut pitjutigællugit, tamanna pinniarsiuk Gudipse pijomajanganut;

19 Illulerkutillo tunnijaumajut illingnut, kivgartornermut Gudivit iglungane, tapkoa unertutigikit Gudib sângane Jerusaleme. 
20 Tapkoalo tappilugit pijariakartut Gudivit iglunganut, allaijariakarkattartatit, tamakkoa tunnijaukokit attanniub pivianartotalinganit.

21 Uvanga attanniôjunga Arthasastha, tamanna perkokauvara pimaklertunut illunainut kôksub ungatânetunut, sunamik Esrab, ajochertuijub, aglangniglo illissimajub killaub Gudingata perkojanginik, kaitaukojanginik illipsingnit, tammakkoninga attaruilivluse pinniarkovluse;

22 Silberemik Talentit 100 dit tikkilugit, Weizenemik Korit 100 dit tikkidlugit, Weinemiglo Bathit 100 dit tikkidlugit, sioraujaniglo oktortaulungitunik.

23 Tamaitalo killaub Gudingata perkojiningit tunnijautsiatsainartuksauvut killaub Gudingata iglunganut, ningautsemik tikkitokarkonago attanniovingmut attannermullo, erninginullo.

24 Illipselo nellojungnaileritse pitsartunekanginapse, Zinsenik, Zolleniglo, akkiliaksaniglonêt Jahrine tamaine, ajochertuijut nelliallonêt, Levitillonêt, imgerpâktullonêt, upkoanik udsertortullonêt, Nethinimillonêt kivgartortullonêt Gudib oma iglungane tunnijikolugit.

25 Igville Esra, Gudivit sillatuninga, aggangnêtok malliklugo, idluarsaijungortitsilerit pairksijingortitsilutillo, idluarsaijunik innugiartoksoarnik kôksub ungatanêtunik, illunainik $\mathrm{Gu}$ divit perkojanginik nellolungitunik; kaujimangitullo tapkoninga, tapkoa ajochertorsigik tapkoninga.

26 Nelliallo attaruilivlune pinniarlungitok Gudivit perkojanginik attanniublo perkojanginik, tamna namaktomik pinniarviojuksauvok, tokkolugolonêt, nûkolugolonêt nunanganit, akkilêkolugolonêt perkumik, pitilugolonêt parngnanairsimavingmut.

27 Nertortaule Nalegak atâtagijapta Gudingat tamattominga attannermik illumêlauktok, Gudib iglunga Jerusalemêtok ânanausijomavlugo,

28 Napkiningnermiglo torartitsilauktok uvamnut attanniub sângane, attanniublo attannertuijokotingita, sôngojokotingitalo illunatik sângine. Maksoalaukpungalo Nalekab Gudima aggangita kollangiarninginut uvamnik, kattikovakkalo niakoôjut Israelitinit, takpaungakattigællunga pikovlugit.

\section{KAPITEL VIII.}

\section{Esrab ingerarninga Jerusalemut.}

IAMADSALO niakoôjut atâtanut kittitaulauktut, takpaungarkattigilauktakka Babelemit attanniub Artasasthab attanniotiningata nelliuningane. 
2 Pinehasib kittornganginit: Gersome; Itemarib kittornganginit: Daniel. Davidib kittornganginit: Hattus.

3 Sechanjab kittornganginit, Pareosib kittorngangita: Sacharja, illagilugo kittitaumajut angutit 150 git.

4 Pahat Moabib kittornganginit: Elioenai, Serajab erninga, illagælugolo angutit 200 dit.

5 Sechanjab erninginit: Jehasielib erninga, illagilugolo angutit 300 dit.

6 Adin Ebedib kittornganginit: Jonathanib erninga, illagilugolo angutit 50 git.

7 Elamib kittornganginit: Jesaja, Athaljab erninga, illagællugo angutit 70 git.

8 Sefatjab kittornganginit: Sebadja Michailib erninga, illagællugolo angutit 80 git.

9 Joabib kittornganginil: Obadja, Jehielib erninga, iilagællugolo angutit 218 it.

10 Selomitib kittornganginit: Josifiab erninga, illagællúgolo angutit 160 it.

11 Bebaib kittornganginit: Sacharja, Bebaib erninga, illagællugolo angutit 28 it.

12 Asgadib kittornganginit: Johanan, erningita nukârdlerpângat, illagællugolo angutit 110 it.

13 Adonikamib kittornganginit kingorlernit, tamadsalo attingit: Elifelet, Jehielelo Semajalo, illagilugillo angutit $60 \mathrm{it.}$

14 Bigevaib kittornganginit: Utai, Sabudelo, illagilugillo angutit 70 git.

15 Uvangalo kattitilaukpakka kôngme Ahevamut kôktome; uvlilutalo tagvane pingasunik. Ullernaigigapkillo innugiartoksuit, ajochertuijullo, Levitisilaun gilanga tagvane.

16 Tagva tilliklilaukpunga Elieseremut, Arielemullo, Semajamullo, Elnatanemullo, Jaribemullo, Elnatanemullo, Natane. mullo, Sacharjamullo Mesulamemullo, niakoôjunut, Jojaribemullo, Elnathanemullo, illissimajungnut.

17 Tillivakkalo Iddomut, angajokaunerôjomut Kasfiame, okausekartipakkalo, okauserilugit Iddomut kattangutinginullo Nethiniminut Kasfiametunut, kaitsivigivluta kivgaksanik Gudipta iglunganut, pikovlugit.

18 Kaitsivigivâtigullo Gudipta aggangita pitsiartut kollangiarninginut uvaptingnik, angumik sillatôjomik Mahelib erninginit, Levib erningata, Israelib erningata, Serebjamik, erningit, kattangutingillo illaliudlugit, 18 nik.

19 Hasabjamiglo, illagællugolo Jesajamik, Merarib erninginit, erningit kattangutingillo illaliudlugit 20 ginik.

20 Nethiniminiglo, Davidib angajokakotingitalo tunnilauktanginik Levitit kivgartorlugit, 220 ginik, illunatik attingitigut aglaksimavlutik. 
21 Tagvanelo kôngme Ahevametome nerritailinek akpautigijaukovara, nikkanarsarluta Gudipta sângane pikovluta, kennerluta tapsomangat apkomik tammarnangitomik uvaptingnut kittorngaptingnullo, perkutiptingnullo tamainut.

22 Kangusulaurama attannermit sorsuktuksat, ablangajullo horsine kaitaukolugit, sapputiluta omisuktunit apkome pikovlugit. Attannek okautilauraptigo: Gudipta agganģita illunaita, kennertut tapsominga kollangiarpait idiuarkutiksanginut; pitsartuningatale ningarningatalo illunaita akkerartorpait allaijut tapsominga.

23 Taimaglo nerritaililaukpogut kennuarivavullo tamanna Gudiptingnit; tapsomalo tussarpâtigut.

24 Uvangalo ajochertuijunit anginerôjunit 12.it ingmigolingatipakka: Serebja, Hasabjalo, 10 illo kattangutinginit;

25 Kiktitarivigivakkalo Silberemik, Goldemiglo Gudib iglungata tunnergusianganik, attanniub attannertuijokotingitalo, angajokâkotingitalo, Israelitillo najutilauktut illunatik tunnilauktanginik.

26 Kiktitarpakkalo agganginut tunnilugit Talentit 650 git Silbere, illulerkutillo Silbereôjut, Talentitut 100 ditut; Goldelo Talentit 100 dit;

27 Ermgusiarsuit Goldeôjut 20 git, Darikitut 1000 ditut akkitôtigijut; illulerkutiglo magguk kannujaujuk, Goldetun-îtuk.

28 Okautivakkalo: Illipse ivsornaipose Nalekamut, illulerkutillo tapkoa ivsornainivut, Silbere, Goldelo tapkoa tunnergutauvut pijarijut Nalekamut atâtagijapse Gudinganut.

29 Udsertorsigik pairilugillo, kiktitarikârtinasse makkoninga, ajochertuijut, Levitillo angajokaunerôjut, atâtallo angajokaunerôjut, Israelitit akkorngane, sângine Jerusaleme, Nalekab iglungata inningine.

30 Tagva ajochertuijut Levitillo Silbere, Goldelo, illulerkutillo kiktitarsimajut tiguvait, tikkiutitsomavlugit Jerusalemut Nalekab Gudipta iglunganut.

31 Taimaglo aulârpogut Ahevab kônganit takkit sivorlingata uvlungita 12 ingane, ingerarkovluta Jerusalemut. Gudiptalo aggangita kollangiarpâtigut, piulilutalo omisuktut, terliarinasuartullo uvaptingnik apkome agganginit.

32 Jerusalemullo tikkinapta merngoêrserpogut tagvane uvlut pingasut nâlugit.

33 Uvlulle sittamangane Silbere, Goldelo illulerkutillo kiktitartaulerput Gudib iglungane, Meromotib Uriab erningata ajochertuijub agganginut; illagivalo Eleaser Pinehasib erninga; tapkoalo illagivait Josabad Jesuab erninga, Noadjalo, Benunib erninga Leviteôjuk.

34 Illunatik attunît unnurningit okumainingillo malliklugit; okumainingillo taipsomane illunaita aglaktaulaukput. 
35 Kittorngallo parngnanairsortaumalauktut parngnanairsimavingmit uttersimajut, tunnijivut tunnergutinik ôtaksanik Israelib Gudinganut; tuktuvângnik angusallungnik 12 nik Israelitit illuengârlutik pivlugit, saukanik 96 nik, saugârsungnik 77 nik, tuktungajuniglo angusallungnik 12 inik, tunnergutiksamut ajortoêrutiksamut; illunatik tunnergutiksamut ôtaksamut Nalekamut.

36 Attanniublo perkojanga unertutigivat attanniub pimaklertokotinginut, nunablo aulatinginut kôksub miksâne, tapkoalo innugiartoksuit Gudiblo iglunga kakkitipait.

\section{KAPITEL IX.}

\section{Esrab tuksiarninga.}

TaMaKkoALO tamaita namaksimangmatta angajokaunerôjut tikkipânga okautilungalo: Innugiartoksuit Israelitit, ajochertuijullo, Levitillo aptersimavlutik ingmigolingalungilet, innukattigêksoarnit nunane makkojungnartulliningit pitjutigallugit, tagva Kananiterit, Hetiterit, Feresiterit, Jebusiterit, Amoniterit, Moabiterit, Egipterit, Amoriterillo;

2 Tapkoa panningit tigulaungmagit nulliangokovlugit ingminut ernermingnullo, kinguvaillo ivsornaitut akkumavait nunat innukattigêksoanginut. Angajokaunerôjullo aulatsijullo aggangit idluitullinerme tamattomane sugaluluarlaukput.

3 Tamattominga tussarama annorâka allikpakka, ulligalo, niakungmalo nutjangit, umikkalo nutsukpâkka, iksivalungalo unainalivlunga.

4 Kattimalerpullo uvamnut illunatik Nalekab Israelib Gudingata okausinginik sivurajut, tammartovinersoak pitjutigallugo; iksivalungalo unainalivlunga tunnergut unnuksiut tikkilugo.

5 Tunnergutiblo unnuksiutib tunnijauviane nikkovipunga paksarnimnit annoralijarlunga, ullilijarlungalo alliksimajunik, serkortorpungalo aggakkalo issangmilugit Nalekamut Gudimnut,

6 Okarlungalo: Gudiga, kangusukpunga, illerasuklungalo uilunga illingnut Gudiga; idluitullinivut aglisimangmatta niakupta kollangittigut, passijaksaunivullo angijovaksôvok killangmut tôklune.

7 Atâtagijapta nelliuninganit passijaksaudlalaukpogut uvlok tamanna tikkidlugo; idluitullinivullo pitjutigællugit uvagut, attannivullo, ajochertuijôvullo, tunnijaumavogut nunat attannertangita agganginut, saviksoarmut, parngnanairsimavingmullo, 
aksartaunermullo, kênaptalo kangusuktitauninginut, sorlo uvlorme tamattomane illingangmat.

8 Mannalo kêtamik saimarvionermik pivogut Nalekamit Gudiptingnit, amiakokarapta sulle ikkitunik, annigoimajunik, tunnitsivigilutalo kikkiangmik attautsemik innimine ivsornaitome, Gudivut ijiptingnik kaumaksaimat, innungmaumitilutalo kêtamik kivgauniptingne.

9 Kivgauvogulle, Gudiptale kemmalaungilâtigut kivgauniptingne, napkiningnerlo torartilaukpa uvaptingnut Persiamiut attanningita sângine, innokojikovlugit uvaptingnik, Gudipta iglunga igluliorlugo pikovluta, asserorsimaningillo sattortautilugit pikovluta, tunnitsivigivlutalo avalunik, Judame Jerusalemelo.

10 Mannalo kannok okartuksauvita Gudivut tamakkoa kingorngane? perkojattit kemmalauraptigik,

11 Perkolauktattit kivgakotikut nellautaijutigut okarlauklutillo: Nuna tikkiniartasse kingormgutiniarlugo nunauvok ivsornartok nunat innukattigêksoangita ivsornarninginut makkojungnartullininginut, nâjijutigijanginut tapsominga issuanit aipanit, aipanga tikkidlugo sallumainermik.

12 Tagva manna pannise tunnijaksaringilasse taipkoa erninginut, taipkoalo panningit tigujaksarmngilasse ernipsingnut nulliangokovlugit, ullapirsautiksangallo, idluarkutiksangallo kenneraksaringilasse sorairase, sôngokovluse, nerrikovluselo nunab pinginik, kingormgutijaksautilugolo kittorngapsingnut, issokangitomut.

13 Tamakkoalo tamaita tikkitautigijapta, pinniarniâluvut, passijaksaudlarnivullo pivlugit, kingorngane, igvit Gudivut pitlarnikinersarilaukpaptigut pijuksauniptingnit idluitullinivut pitjutigællugit, piulijaunekartilaukpaptigullo, sorlo mana imat.

14 Uvagulle ama perkojatit serkomijaksarivitigik innukattigêksuillo makkoa, taimaitunik makkojungnartoggit aipanikattigivlugit. Ningariniangipittigut, nungutaukârtinata, amiakokarungnaikârtinago, piulijaunekarungnaikârtinagolonêt?

15 Nalegak, Israelib Gudinga, igvit idluarpotit, uvagut amiakogapta, annigoimavlutalo, sorlo uvlorme tamattomane illingangmat. Ahâk, nangerpogut sângne passijaksauniptingne; tamannale pivlugo nangertokarungnangilak sângne.

\section{KAPITEL X.}

\section{Nullit nellojunit pïut aptertauvut Israelitinit.}

ESRalo taimak tuksiartillugo, kigligiksarlunelo kêavlunelo pamangatillugo Gudib iglungata sângane, kattimalerput tapso- 
munga Israelitinit illagêt unnuktoksuit, angutit arnallo, sorutsillo, innugiartoksuit kêavaksoadlarmatta.

2 Tagva Sachanja Jehielib erninga Elamib erninginit, kiovok okarlunelo Esramut: Unêt idluitulilaukpogut Gudiptingnut, takkorngartanik nullianilaurapta nunab innukattigêksoanginit; Israelitille pivlugit nerriungnekarpok sulle tamanna pivlugo.

3 Tagva manna Gudivut angerkattigilavut, nullit takkorngartat illunaita, erniangomajullo tapkonangat, apterlugit pikovluta nalekama (Esråb) attannertuininga malliklugo, tapkoalo Gudib perkojanginik sivurajut (attannertuiningit) perkojat mallikiugo pinniartaukovlugo.

4 Tagva makkilerit, igville pijokartok tamanna pinniaraksarivet; uvagut illigijomavaptigit. Maksoalerit, pinniarlugolo.

5 Tagva Esra makkipok, tigusilunelo angermaringnermik ajochertuijut, Levitillo angajokaunerôjunginit Israelitinillo illunainit, okausek tamanna malliklugo pinniartuksaungmatta; angerpullo.

6 Esralo Gudib iglungata sânganit nûpok, itterlunelo Johananib Eliasib erningata inninganut; itteramelo tagvunga nerritailivok niakojamik, immertaililunelo immermik, taipkoa parngnanairsortaumalauktut idluitulliningit sanikterijutigigamigit.

7 Akpautigijaukovâllo Judame illunane, Jerusalemelo kittornganut parngnanairsortaumalauktunut illunainut, Jerusalemut kattimalerkovlugit.

8 Uvlullo pingasut kingorngane tikkiniangitub, angajokaunerôjut, angajuklillo kachimioningat malliklugo, tapsoma perkotingit tamaita nungutaujuksaungmatta, tamnalo avitauniarajarmat illagênit parngnanairsortaumalauktunit.

9 Tagva illunatik Judakut Benjaminekullo angutingit kattilerput Jerusalemut uvlune pingasune, tagva takkit 9 ingata uvlungita 20 ingane. Innugiartoksuillo illunatik iksivavut apkosinerme Gudib iglungata sângane, sajuklutiglo pijokartok tamana pivlugo, sillalungnerlo pivlugo.

10 Tagva Esra ajochertuijôjok, nikovipok, okautilugillo: Idluitullilaukpose, takkorngartanik nullianilaurapse, taimaglo Israelitit passijaksauningat angijorortigapsiuk.

11 Tagva manna Nalegak atâtagijapse Gudingat kigligiksarvigisiuk, pinniarluselo namagijanganik, apterluselo nunab innukattigêksoanginit, nullinillo takkorngartanit.

12 Tagva illagêktut illunamassiangit kiovut, okarlutiglo nippekôrtôvlutik: Taimainiarle, sorlo okalauravit.

13 Innugiartoksuille unnuktôvut, sillalukporlo, ajornarlunelo sillametuinarlune, pinniarnerlo tamanna piniartaujungnanginivok uvlune maggungne pingasunelonêt; unnuktogapta tammarlauktogut idluitullinermut taimaitomut. 
14 Angajokavut illagêktune illunamassiangine perkolavut, illunatik takkorngârtunik nullianilauktut iglugaseksoakotiptingne, nelliutune okautigijaujune kaikovlugit, iglugaseksuillo attunit angajokangit, idluarsaijungillo illautilugit, Gudib ningausingat illungertortok pijokartok tamanna pitjutigallugo sangutitaukârtinago uvaptingnit.

15 Tagva Jonathan Asahelib erninga, Jehasjalo Tikwab erninga kaikojauvuk pijokartut tapkoa pivlugit, Mesullamiblo Sabtaiblo Levitiojuk ikajorpâk.

16 Kittorngallo parngnanairsortaumalauktut taimailiorput. Ajochertuijublo Esrab atâtallo angajokaunerôjut atâtagijamik iglungine, illunatiglo taijaumajut manna apterpait; ingipullo takkit 10 ingata uvlungata sivurlingane, pijokartut tapkoa nauttertoromavlugit;

17 Namaksivaillo angutit illunaita takkorngartanik nulliakartut takkit sivorlingata uvlungita sivurlingane.

18 Nachvartaulaukpullo ajochertuijut erningita akkorngane, takkorngartanik nullianilauktut imâk: Jesuab Jozadakib erningata erninginit, kattangutinginillo: Maeseja Elieserelo, Jaribelo, Gedaljalo.

19 Tapkoalo aggatik tunnivait angerlutik nullit takkorngartat ajaktoromagamigit, atsilutiglo saukamik angusalungmik tunnergutiksamut ajortoêrutiksamut tammarnitik pivlugit.

20 Imerib erninginit: Hanani, Sebadjalo.

21 Harimib erninginit: Maeseja, Elialo, Semajalo, Jehielo, Usialo.

22 Pashurib erninginit: Elioenai, Maesejalo, Ismaello, Nethaneelo, Josabadelo, Eleasalo.

23 Levitinillo: Josabad, Simeilo, Kelajalo (Klitaovok), Petajalo, Judalo, Eliezerlo.

24 Imgerpaktunit: Eliasibe; upkoanik udsertortunit: Sallume, Telemelo, Urilo.

25 Israelitinillo, Pareob erninginit: Ramja, Jesialo, Malchajalo, Mejaminelo, Eliasarlo, Malchjalo, Benajalo.

26 Elamib erninginit: Mathanja, Sacharjalo, Jehielo, Abdillo, Jeremotelo, Elialo.

27 Satub erninginit: Elioenai, Eliasibelo, Mathanjalo, Jeremotelo, Sabadelo, Asisalo.

28 Bebaib erninginit: Johanan, Hananjalo, Sabailo, Atlailo.

29 Banib erninginit: Mesullame, Malluchelo, Adajalo, Jasubelo, Seallo, Jaramotelo;

30 Pahat Moabib erninginit: Adna, Kelallo, Benajalo, Maesejalo, Matanjalo, Bezaleelo, Benunillo, Manasselo.

31 Harimib kittornganginit: Elieser, Jesialo, Malchalo, Semajalo, Simeonelo.

32 Benjaminelo, Maluchelo, Semarjalo; 
33 Hasumib erninginit: Matnai, Matata, Sabade, Elifeletelo, Jeremailo, Manasselo, Semailo.

34 Banib erninginit: Maedai, Amramelo, Huello,

35 Benajalo, Bedjalo, Keluilo,

36 Najalo, Meremotelo, Eliasibelo,

37 Matanjalo, Matnaillo, Jaesanelo,

38 Benilo, Bennuilo, Simeilo,

39 Selemjalo, Nathanelo, Adajalo,

40 Machnadbaillo, Sasailo, Sarailo,

41 Asareello, Selemjalo, Samarjalo,

42 Sallumelo, Amarjalo, Josefelo.

43 Nebob erninginit: Jejel, Matitjalo, Sabadelo, Sebinelo, Jaddailo, Joelo, Banajalo.

44 Tapkoa illunatik nullianilaukput takkorngârtanik; nullillo tapkoa illangit, nutakkanik ernilaukput.

\section{NEHEMIAB AGLANGIT.}

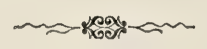

\section{KAPITEL I.}

Nehemiab tussarninga Jerusalemik, tuksiarningalo tapkoa pivlugit.

TamadsA Nehemiab, Hachaljab erningata pijokalaungningit. Pijokalaukpok takkerme Kisleuemik attelingme, Jahrit 20 gingane, igloksoarmêtillunga Susanemêtome.

2 Hanani kattangutima illangat, angutit illangit illagilugit tikkipok Judamit. Appertsorpakkalo Judit kemmâmajut, amiakojullo parngnanairsortaunermit, Jerusalemelo pitjutigivlugit.

3 Tapkoalo okautivânga: Amiakut parngnanairsortaunermit tagvane nuname kannoêtomêput angijome mittautigijauner- 
mêlutiglo; Jerusalemib avaloksoangit serkomisimavut, upkoaksoangillo ikkisimavut.

4 Okautsille taimaitut tussarapkit, iksivalerpunga kêalungalo, ogguarlarmalo uvlut kapsit nâvlugit, nerritaililungalo tuksiarlungalo killaub Gudingata sângane,

5 Okarlungalo: Nalegak killaub Gudinga, Gudeôjoksôjotit, sivuranartoksôlutillo, angersimanermik, napkiningnermiglo torkorsijok tapkonunga naglingniktunat tapsominga, perkojanginiglo nalektunut;

6 Siutikik kamatsiarlîktôk, ijikiglo uitalerlîk, tussarkovlutit kivgakotivit tuksiarninganik, tuksiarutigijamnik manna sângne uvlotillugo unnuamelo, Israelitit kivgakotitit pivlugit; kannorlo Israelib kittorngangita idluitulliningit, pinniarlauktavut illingnut, kigligiutigigapkit; uvangalo atâtamalo iglomiokattangit idluitullilaurivogut.

7 Idluitullivigilaukpaptigit, perkojallo, innatsinerillo, idluarnerillo, perkolauktatit kivgakotingnut Mosemut, mallilaungilavut.

8 Erkarugletôk okausek imna, perkolauktat kivgarnut Mosemut, okalauklutillo: Namangitulliniarupse, tagva siamartitsomavapse innukattigêksuit akkornganut.

9 Sâniarupsele uvamnut perkojakkala kammagigupsigik, pinniarutigigupsigiglo, ajæktortaumagalloarupselo killaub issua tikkidlugo; sulle tagvangat kattersoromavapse, atsomaluselo innemut annerijamnut, attera tagvanekovlugo.

10 Illâle kivgarivattit innukotigilugillo, piulilauktattit pitsartunersoarnut, aggangnullo sôngojunut.

11 Kanoktôk Nalegak siutikik kamatsiarlik, kivgakotivit tuksiarninganik, kivgakotivillo assima tuksiarninganik, tussudlartut attit sivuragijomavlugo; kivgakotillo uvlome sullitilauruk, napkiningneksikolugolo angutib ipsoma kênangata sângane. Immiksaliorteôlaurama attannermut.

\section{KAPITEL II.}

Nehemiab pertojauninga attannermut, Jerusalem iglugaseliorungnarlugit.

PiJokalaukporlo attanniub Artasastab attanniotiningata Jahringita 20 ingane, takkerme Nisanemik attelingme, sâmiktillugo Weinemik, Weine kivikpara, tunnilâlo attannermut; tautukarpungalo kiksartomik.

2 Tagva attanniub okautivânga: Sôg kênait tautorluka? 
Kannimalungigalloartotit? Tamannaungilak, omattille okumaipok. Uvangale sivuravaksoadlalaukpunga,

3 Okarlungalo attannermut: Attannek innôle issokangitomut, uvanga tautorluktuksaungilanga? Iglugaseksuit atâtagijama illijartortaumaningita najugangat asserorsimavut, upkoaksoangit ikkomamut nerrijaumavut.

4 Tagva attanniub okautivânga: Sunamigle tuksiarkît? Tagva killaub Gudinganut tuksiarpunga,

5 Okarlungalo attannermut: Namagijaukpat attannermut, kivgakotingnullo sângnêtunut, tillilunga pikovlutit Judamut, atâtagijama illijartortaumaningita iglugaseksoanginut, iglugaseliorlugit pikovlunga.

6 Attanniublo okautivânga, aipangatalo senniane iksivajub: Ingerganît kannok akkunêrtigijoniarka? Kakkugolo utteromârkît? Attanniublo namagiva tillilunga. Nelliunerlo nellonairpara tapsomunga,

7 Okarlungalo attannermut: Attanniub namagiguniuk, tagva aglanik tunnitsivigilinga aijuksanik, nunat kôb ungatanêtut aulatinginut, kannilarkovlugit uvamnik, tikkikârtinanga Judamut.

8 Aglaniglo Assafemut attanniub pimaklertokotinganut kêjukotinginik, tunnijikovlugo uvamnut kêjungnik ikâksanut illingajunik, igloksub Tempelib kannitanganêtub itterviksoanginut, iglugaseksuillo avaluksoanginut, iglomullo uvanga iglogijomajamnut. Attanniublo tunnitsivigivânga Gudima aggangit ajungitut kollimnêtut malliklugit.

9 Tikkinamalo nunat aulatinginut kôb akkianut tunnitsivigilaukpakka attanniub aglanginik. Attanniublo sorsuktuksat angajokângit, ablangajullo horsit kângine tillivait illaukovlugit uvamnut.

10 Saneballatible Horonitereôjub, Tobiablo kivgab Amonitereôjub, tamanna tussaramikko, sinnaungadlalaukpok tapkungnunga, tikkitokarmat innungmik, Israelib kittorngangita idluarkutiksanginik kennertomik.

11 Jerusalemullo tikkinama, uvlilaukârdlunga pingasunik tagvane,

12 Unnuarme parngnailerpunga, angutillo ikkitut illagivânga; innuille nelliallonêt okautilaungilara sunamik Gudib illumertilaungmanga Jerusaleme pinniarvigilugit; najortekalaungilanga nergjumik ablangavigijama assianik.

13 Unnuarmelo horsekut annilerpunga nâksab itterviksoangagut, puailavingmut Drachemik attelingmut, itterviksoarmullo perorsaijomik attelingmut tikkidlunga; kemergovakkalo Jerusalemib avaluksoangita karmangit serkomisimajut, upkoaksoangillo ikkomamut nerrijaumalauktut.

14 Sivumuarpungalo itterviksoarmut, puailavingmik atteling- 
mut, attanniublo tessinganut; inniksakalaungilarlo tagvane nergjutekotimnut tagvûnârungnarlune attimne.

15 Kôngmelo majorarpunga unnuarme, avaluksuillo kemmergovakka, ingergamilungalo nâksab itterviksoangagut, utterlungalo.

16 Angajokajallo kaujimalaungilet nâmut ailaungmangarma, sunaliormangarmalo; taipsomane tikkidlugo, Judit ajochertuijullo attannertuijullo, angajokajallo, assingillo pinniaraksame tapsomane sennalauktut okautilaungimarikapkit sunamik.

17 Tagva okautivakka: Takkovasse kannoêtok attortavut, Jerusaleme asserorsimangmat, upkoaksoangillo ikkisimangmatta. Kaititse Jerusalemiblo avaluksoangita karmangit sennalerlavut, mittautiksaujungnailerkovluta.

18 Unipkautivakkalo kannok Gudima aggangita pitsiartut kollangiarlaungmanga, taimaglotauk attanniub okausinginik okauserilauktanginik uvamnut. Tapkoalo okarput: Attê parngnailerta avaluliorlutalo! Taimaglo aggatik pitsartutipait sullijaksak ajungitok tamna pinniaromavlugo.

19 Saneballatible Horitereôjub, Tobiablo kivgab Amonitereôjub, Gosemiblo Arabiamiub, tamanna tussaramitsuk, mittautigivâtigut, nachogilutalo, okarlutiglo: Sunauva pinniartasse? Attannek nalegungnairomavissiuk?

20 Tagva uvanga kiovakka, okarlungalo: Killaub Gudingata sullitiniarpâtigut tamattominga; uvagullo kivgaujogut tapsomunga, parngnaileromavogut, sennajomalutalo, illipsele illangiutivikangilase, idluarnekaraselonêt, erkaumajaksakaraselonêt Jerusaleme.

\section{KAPITEL III.}

\section{Unipkautsit kannok Jerusalemib avaluksoangit sennajaulaung-} matta.

AJOCHERTUIJERSOARLO Eliasibe parngnailerpok kattangutine ajochertuijut illagællugit, sennalutiglo saukat itterviksoanganik; tuksiarulugolo ivsornaitipât, upkoagiglo illivakit, ivsornaitipâlle nuvugusek Meamik attelik, tagva Hananeelib nuvugusinga tikkilugo,

2 Tapsoma senniane angutit Jerichomiut sennavut; Sachurelo Imrib erninga sennavoktauk senniane.

3 Mingeriable itterviksoanga Senaab kittorngangita sennavât; kâkartipât, upkoangalo illivât, pallangairuservikartilâllo, senneroaktârlugolo.

4 Tapkoa senniane sennavok Meremote, Uriab erninga, 
Hakozib erningata. Tapkoa senniane sennavok Mesullame Berechiab erninga, Mesesabeelib erningata. Tapkoa senniane. sennavok Zadoke, Baenab erninga,

5 Tapkoa senniane Tekoamiut sennavut, tapkoale angajokaunerojungita kongesitik okkotilaungilait Nalekamik pinniartaukojanganut.

6 Jojadab Passeab erningata, Mesullamiblo, Besodjab erningata ittertarviksoak nutaungitok sennavâk; kâkartipâk, upkuangalo, pallangairuservingillo, senneroangillo illivait.

7 Tapkoa senniane sennavut Melatja Gibeonemiok, Jadonelo Meronemiok, angutillo Gibeonemiut, Mizpamiullo, nunab aulatingata kôksub miksânetub attanniovinganut illingajut.

8 Tapkoalo senniane sennavok Usiel, Harhajab erninga Goldelerijok. Tapsoma senniane sennavok Hananja,-âniasiusiortit illangata erninga; sennavullo Jerusalemib sillatânut karmak silliktok tikkidlugo.

9 Tapsoma senniane sennavok Refaja Hurib erninga, Jerusalemib ketterkangita angajokaunerôjungat.

10 Tapsoma senniane sennavok Jedaja, Harumafib erninga, iglome akkiane. Tapsoma senniane sennavok Hattus Hasabenjab erninga.

11 Malchjable Harimib erningata, Hasubiblo, Pohat-Moabib erningata illangutivigik sennavakik, kiaksautiblo nuvugosinga.

12 Tapsoma senniane sennavok Sallume, Haloheb erninga, Jerusalemib ketterkangita aipaingita angajokaunerôjungat, tamna panningillo.

13 Hanunib Sanoamiullo nâksab itterviksoanga sennavât; sennavât illilugillo upkoangit, pallangairuservingillo, senneroangillo, tallitullo 1000 dit avaluksoarme, perorsaijut itterviksoangat tikkilugo.

14 Malchjable, Rechabib erningata, Beth Hakaremib erkângata abvakungata angajokaunerôjungata perorsaijut itterviksoangat sennava; sennava upkoangalo pallangairuservingillo, senneroangillo illivait.

15 Sallumible, Kalhosib erningata, Nizpab erkângata angajokaunerôjungata, puailaviub itterviksoanga sennava; sennavâ kâkartilâlo, upkoangalo, pallangairuservingillo, senneroangillo illivait; avaluksuillo sennava Siloab tessingane, attanniub perorsêvikotingata kiglingane, tumerkat tikkidlugit, Davidib iglugaseksoanginit unnungartut.

16 Tapsoma tungâne sennavok Nehemia, Asbukib erninga, Bethzurib erkângata koppangata angajokaunerôjungat, Davidib illuvingita akkia tikkidlugo, Asujablo tessinga, sôngojullo ig-lungat tikkidlugik.

17 Tapsoma tungâne Levitit sennavut, Rehume, Banib er- 
ninga. Tapsoma senniane sennavok Hasabja, Kegilab erkângata koppangata angajokaunerôjungat, illangutivigijamine.

18 Tapsoma tungâne kattangutingit sennavut, Bavai, Henadab erninga, Kegilab erkângata ketterkangata aipangata angajokaunerôjungat.

19 Tapsoma senniane sennavok Eser, Jesuab erninga, Mizpamiut angajokaunerôjungat, illangutiviub aipanganik, majokals torartub sakkutalingmut akkiane avaloksuit terrekoane.

20 Tapsoma tungane sennavok illungertorlune Baruch Sabaib erninga illangutiviub assianik, terrekungmit Eliasibib ajochertuijersub iglungata upkoanga tikkidlugo.

21 Tapsoma tungâne sennavok Meremot, Uriab erninga, Hokozib erningata, illangutiviub aipanganik, Eliasibib iglungata upkoanganit, Eliasibib iglungata issua tikkilugo.

22 Tapsoma tungâne ajochertuijut sennavut angutit Jordanib erkânit pijut.

23 Tapkoa tungâne Benjamin sennavok, Hasubelo, iglungmik akkiangne. Tapkoa tungangne sennavok Asarja Maeseab erninga, Ananjab erningata, iglome senniane.

24 Tapsoma tungâne sennavok Benuni Henadadib erninga illangutiviub aipanganik, Asarjab iglunganit, avalut sanguningat tikkidlugo, tagva terrekok tikkidlugo.

25 Palal Usaib erninga, terrekub, nuvugutsiblo poktojub attanniub iglunganit nuitajub akkiane, parngnanairsimaviub inningata avaluliorsimajub tungâne. Tapsoma tungâne Pedaja, Pareosib erninga.

26 Nethinimillo iglokalaukput Ofeleme immiub itterviksoangata kitta tikkilugo, nuvugutsib nuitavia Ofeliblo karmanga tikkidlugo.

27 Tapkoa tungâne Tekoamiut sennavut illangutiviub assianik, nuvugoseksub nuitajub akkianit Ofelib karmanga tikkilugo.

28 Horsille itterviksoanganit ajochertuijut sennavut attunît iglomik akkiane.

29 Tapkoa tungâne sennavok Zadok, Immerib erninga, iglome akkiane. Tapsoma tungâne sennavok Semaja Sechanjab erninga upkoaksoamik udsertorte kittane.

30 Tapsoma tungâne sennavok Hananja Selemjab erninga, Hanunelo Salafib erningita 6 ingat, illangutiviub aipanganik. Tapsoma tungâne sennavok Mesullame Berechjab erninga innime akkiane.

31 Tapsoma tungâne sennavok Malchja Goldelerijub erninga, Nethinimit niuvertillo iglungit tikkidlugit, kachimiojut itterviksoangata akkiane, innelo terrekomêtok tikkilugo.

32 Inniblo terrekometub, saukallo itterviksoangata akkorngangne Goldelerijut niuvertillo sennavut. 


\section{KAPITEL IV.}

\section{Avalut pivallianingit, Nehemia tuksiarmat, omigijinginut ag- viartaugalloarmatta.}

Sanneballatele tussarame, uvagut avaloksuit sennagaptigik, ningalerpok, kaningaumajarlunelo, mittautigivaillo Judit,

2 Okarlunelo kattangutiminut, pitsartunelingnullo Samariamêtunut: Judit nukkekangitut sunaliorkat? Taimailingakojautsainarniarkat? Tunnergutinik tunnijiniarkat? Uvlut illangane innerniaralloarkatsuk? Ujarkat omartiniarpattigik patsalungavlutik kallerêktisimajut ikkitaumajullo?

3 Tobiale Amonitereôjok senniane nangerpok okarlunelo: Sennatuinarlit; terrianiat taikungarajarunik karmangit ujaraggit serkominiarpallukpait.

4 Gudivut tussarit, kannok nachogijaugapta! Mittautekarningit uttertikit niakunginut nangminerijanginut, tunnilugit pikovlutit nachogijaunermut parngnanairsortaumaningata nunangane!

5 Idluitulliningit ulliktaililugit pît, ajorningillo pêjarniarnagit sângne; sennajut ningaksarlaungmattigik.

6 Uvagulle avaluksuit sennavavut, tokkitilugillo pôktojuksauningata koppanga tikkidlugo. Innugiartoksuillo omattekalerlaukput maksoajunik sennanermut.

7 Saneballatele Tobialo, Arabiamullo, Moabiterillo, Asdodemiullo tussaramik, Jerusalemib avaluksoangita karmangit âkiktaujarêrmatta kingingillo tokkititaulermatta, ningavaksoadlalerput,

8 Angerkattigêklutiglo tikkitsomavlutik, Jerusalemelo sorsuklugit agviaromavlugillo.

9 Uvagulle tagva tuksiarpogut Gudiptingnut, udsertortokartitsivogullo uvlotillugo unnuarmelo taipkoa pivlugit.

10 Judakullo okarput: Akjarsijut pitsartuningit amigarput, sannillo unnuluadlarput; avalune sennajungnangilagut.

11 Akkerartortivullo okarput: Kaujijuksaungilet, tækkojuksaunatiglonêt, akkornganêmarikârtinata, nakterikârtinatalo taipkoninga, pinniarnerlo sorairtilugo.

12 Judille taipkoa senniane nunakalauktut, tikkimatta okautilutalo tamattominga, tellimaujortoêrtorlutik aglât, inninit nunagijamingnit tamainit avatiptingne;

13 Tagva pititsivunga innugiartoksoarnik kittorngarêt malliklugit avlaloksuit ungatânut ittersanut, saviksoakotitik, kallugiarkotitiglo, pittiksekotitiglo najortigilugit.

14 Allakausijarpungalo parngnailerlungalo, okarlungalo er- 
kanartunut angajokaunerôjunullo, innugiartoksoarnullo assinginut: Sivuraginiarassigik, Nalegaksoarle erksinartoksoak erkarsiuk, kattangutiselo erniselo, panniselo, nulliaselo, iglokotiselo sorsugutigisigik.

15 Omigijivulle tussarmatta, uvagut nellojungnaititaumagapta tamattominga, tagva Gudib kajusimaningat asserorpa. Uvagullo illunata utterpogut avaluksoarnut, attunît sullijaksaptingnut.

16 Pijokalaukporlo nelliutomit taipsomangamit, innuosuktut ketterkangit sennangmatta, ketterkangitalo aipangita tigumiarpait kallugitsat sappulutallo, pittiksallo, sagvingmiutallo; angajokaunerôjullo Judakut illunamassiatik tunnuane nangerput,

17 Sennajut avaluksoarne, akjarsijullo akjaraksanik tapkonangat illijilauktunit tapkonunga, agganut igluînarnut sennavut sullijaksanik, igluanullo sakkut tigumiarpait.

18 Sennajullo illunatik tapserusersimalaukput saviksoarmingnik makkitimikut, sennalutiglo taimailingavlutik; tiptulausijartorlo sennimnelaukpok.

19 Okarpungalo erkanartunut, angajokajanullo: Sullijaksak angijôvok, kanningitomullo illingavok, uvagullo siamarsimavogut avaloksuit kângane kanningitigêklutalo.

20 Nanelo manna tiptulaut tiptulausijautigijauninga tussarniarangapsiuk, tagvunga kattimaleritse uvaptingnut, Gudipta sorsugutiginiarpâtigut.

21 Taimaglo sennalaukpogut sullijaksame; innugiartoksuillo ketterkangita aipangita kallugitsat tigumiarpait kaub aupalungningata nuilerninganit uvloritset nuilerningat tikkidlugo.

22 'I'aipsomane innugiartoksoarnut okalaukpungatauk: Illunatik kivgatik illagællugit unnuakut sinniktarlit Jerusaleme, unnuane udsertorteôkovlugit uvaptingnut, uvlotillugolo sennakovlugit.

23 Uvangale kattangutikkalonêt, kivgakotikkalonêt, angutillonêt udsertorteôjut, malliktut uvamnik, annorairlungimarikpogut, illunatiglo timitik ubvartailivait.

\section{KAPITEL V.}

Judit ârusungningit akkiliksatik pivlugit; Nehemiablo suadlarninga tapkoninga, pitjutarjunik tamattomunga.

NipliadlarneKsoakalerPorlo innugiartoksoarnit nullianginillo, kattangutitik Judit akkerartorlugit.

2 Illangillo tagvanêtut okarput: Ernivut pannivullo unnuk- 
tôvut, tamanna pirlugo kakkojaksanik pinasuarta, nerrijungnarkovluta innôlutalo.

3 Illangille okarput: Perorsêvikotivut, perorsêvivullo Weineliksat, iglovullo sattoraksautiniarlavut, kakkojaksarsikovluta kângmut.

4 Illangille okarput: Kênaujanik taukteliksarsilaukpogut akkiliaksanut, attannermut illingajunik, perorsêrikotivut, perorsêvikotivullo Weineliksat sattoraksautigivlugit.

5 Taimaigalloarmallo kattangutipta timingit timiptitut îput, kittorngangillo kittorngaptitut îput. "Ahâglo, uvagut ernivut pannivullo kivgangortitaukojariakarparut; panniptalo illangit kivgangortitaumajarêrput, uvagullo perkutekangilagut aggaptingne, perorsêvivullo, perorsêvivullo Weineliksat assiptingnut illingavut.

6 Tagva ningavaksoadlalerlaukpunga, nipliadlarningit okausingit tapkoa tussarapkit.

7 Omattigalo illumne issumaksarsiorpok, suarpakkalo erkanartut, aulatsijullo, okautilugillo: Illipse attunît kattangutipsingnit, taukteliksarsijunit illipsingnit tappiksamik tigusijomavise? Illagêllo unnuktut akkerartortipakka tapkonunga,

8 Okautivakkalo: Uvagut kattangutivut Judit nellojunut niorvgutigijaumalauktut pissiarilugit kippalutjarlaukpavut, ajugaringitaptingnit; illipselo kattangutisse niorvgotigijomavissigik, uvagut pissiarilauktavut? Tagva nippangerput, kiogutiksarsijungnaratiglo.

9 Okalaukpungalo: Namalungilak pinniartase. Agvertartuksaungilasê sivuranerme Nalekamik, nellojut omigijipta mittautekarningit pivlugit?

10 Uvangalo kattangutimalo kivgakotimalo taukteliksakartilaukpavuttauk kênaujanik, kakkojaksaniglo; kennuvigivapsele tappiksakarkojiniarata.

11 Tagva manna uvlorme tamattomane perorsêvingit, perorsêvingillo Weineliksat, perorsêvingillo orksuggit, iglungillo sattortautisigik tapkonunga, kênaujallo kakkojaksallo, Weineliksallo iptingit, orksuglo tigulauktapse tapkonangat tappiksamut 100 dingat.

12 Tagva okarput: Sattortautitsomavavut tapkonunga, kaitsikojomangilavullo sunamik, pinniaromalutalo, sorlo igvit okalauravit. Ajochertuijullo kaikovakka, angermarikolugillo taimailiortuksaungmatta.

13 Ikkiaratauk nâvilugo ipsortorlaukpara, okarlungalo: Taimak Gudib nelliat ipsortorlitsuk iglunganit sullijaksanganillo, okautsemik ominga nellautsititsilungitok, ipsortauvlune navisimajotun, illulêrtotullo îkovlugo. Illagêllo illunamassiatik okarput: Taimaile, Nalegarlo nertorpât. Innugiartoksuillo taimailiorput, okautsit tapkoa malliklugit.

14 Nelliutomit taipsomangamit perkojauvimnit aulatsijuk- 
saugama Judakut nunanganik, tagva attanniub Artasastab Jahringita 20 inganit 32 ingat tikkidlugo, tagva Jahrit 12 it nâlugit, uvanga kattangutimalo, nunab aulatingata nerkigivaktangit nerrilaungilavut.

15 Aulatsijut sivunimnêlauktut innugiartoksuit okumaiksartilaukpait, tigusilauklutiglo tapkonangat niakojamik Weinemiglo, tapkoalo tappilugit Sekelinik 40 ginik Silberemik. Uvangale taimailiorlaungitunga, suvuranek Gudemik pivlugo.

16 Sennalaukpungatauk avaluksoarne, perorsêviksilaungilangalo; kivgakotikkalo illunatik kattimalaukput tagvane sullijaksamut.

17 Judinillo aulatsijunillo 150 git uvlut tamaita nerrimarkattauvut nerrimavimne, tikkilauktut uvamnut nellojut avatiptingnêtut akkornganit.

18 Uvlullo tamaita pivlunga attuinautitauvut, tuktuvak angusalluk attausek, saukallo kuinititaumajut pingasojoritut, tingmitsello, uvlunelo tellimaujortune attausiardlune Weinit sunatuinait arklingnartut. Taimaigalloarmat nunab aulatingata nerkiksangit kaitaukolaungilakka, kivgartornek okumaidlalaungmat innugiartoksoarnut.

19 Gudîk, innugiartoksoarnut tapkonunga pinniarlauktakka tamaita erkarutigikit uvamnik, idluarkutiksamnut!

\section{KAPITEL VI.}

\section{Nehemiab unangmijauninga, uniorgariningalo.}

Saneballatelo, Tobialo, Gosemelo Arabiamiok, omigijiptalo assingit tussarmatta avaloksuit sennajarêrapkit, kingekarungnaimatta, upkoat illilaungigalloarapkit sulle itterviksoarnut;

2 Saneballate Gosemelo tilliklerpûk uvamnut, okautsauko-lungalo: Kait kattimalerlutalo iglugasengne naternamêtune Onomik attelingme. Idluitullivigijomanasugilaukpângale.

3 Uvangale tillijanik tilliklervigilaukpakka, okautsaukolugillo: Sullijaksakarpunga pijariakortôjomik, attersarungnarlungitungalo. Sôg sullijaksak nokkartuksauva, kemagupko attersarajarlungalo illipsingnut?

4 Sittamairtorlutigle taimaitomik illusekarlutik tilliklervigivânga, uvangalo kiotsainarpakka taimaitomik illusekarlunga.

5 Tagva Saneballate tellimangane tilliklerpok uvamnut kivgaminik agganginut tigumiartomik aglanik nippitisimangitunik.

6 Tapkonane aglaksimajokalaukpok: Nellojut akkorngane tussartaumavok, Gosemelo okalaukpok tamattominga, igvit Judillo issumakarapse attannek pikablavigijomavlugo, tamannalo 
pivlugo avaloksuit -sennagangne, igvillo attanniojomagavit tapkonunga, pinniarnerit tamakkoa malliklugit.

7 Igvillo nellautaijokartitsilauravit illingnik akpaujijuksanik Jerusaleme, okarlutik: Tamna attanniovok Judame. Mannalo tamanna tussartautitauniarpok attannermut. Tamannalo pivlugo kailaurit kachimiokattigilaulavut.

8 Uvangale tilliklerpunga tapsomunga okautsaukolugolo: Taimaitomik pinniartaujokalaungilak, okauserijarnik; omattingnut issumertorlaukpat.

9 Taipkoa illunatik sivurasaromalaungmattigut, issumalutiglo: Aggatik ergititaksarivait sullijaksamit, pinniartaukonago. Mannale tamanna pivlugo, Gudik, aggakka pitsartutilaukit!

10 Tikkilaukpungalo Semajab, Delajab erningata Meheletabeelib erningata iglunganut; tamnalo pallangairutivok ingminik, okarlunelo: Kattimalerta Gudib iglungane, Tempelib kerkane, Tempeliblo upkoangit pallangairlavut; tikkiniarmatta nakteromavlutit, unnuarmelo tikkiniarput nakterikovlugit illingnik.

11 Uvangale okarpunga: Angut uvaptun-îtok kemâjuksauva? Angullo taimaitok uvaptut Tempelemut aijuksaunajarka innokojomavlune? Kamungaromalungitunga.

12 Mallugosukama Gudib tillilaungimago. Nellautailune okautigilauralloarpânga, Tobiable Saneballatiblo kênaujanik tunnitsivigilaukpâk.

13 Tamanna pivlugo kênaujanik tigusivok sivurajuksaugama, taimailiortuksaugamalo idluitullijuksaulungalo, okausiksâlukaromangmatta pivlunga, mittautigilungalo pijungnaromavlutik.

14 Gudîk, Tobiase .Saneballatelo issumagikik pinniarningit tapkoa malliklugit; taimaglotauk nellautaijok arnak Noadja, nellautaijullo assingit sivurasaromalauktut uvamnik.

15 Avaloksuillo pijarêrlaukput takkib Elulemik attigub uvlungita 25 ingane, uvlune 52 ine.

16 Omigijiptalo illunatik tamanna tussaramitsuk, nellojut avatiptingnêtut illunatik sivuravut, mungolerlutiglo, mallugosukamik pinniarnek tamanna Gudemit pingmat.

17 Nelliutome taipsomane angajokaunerôjokalaukpoktauk unnuktunik Judame, aglanik tillikliutjijunik Tobiamut, Tobiamillo taimaitut tikkiput tapkonunga.

18 Unnuktôlaungmatta Judame angermarilauktut tapsomunga; tapsomale Sachanja, Arab erninga ningaugingmago, erningatalo Johananab Mesullamib Berachjab erningata pannia nullianilaukpa.

19 Ajungitomiglo okarpaktut pivlugo sâmne, okautsikkala akpautigivait tapsomunga. Tobiablo aglait tillikliutivait sivurasâromavlunga. 


\section{KAPITEL VII. \\ Nehemiab akiksoiningit sunatuinait.}

PiJokalaukPORLO avaloksuit sennamajarêraptigik, upkoat illivakka, upkoaksuillo udsertortingit imgertuksallo, Levitillo nellonairtauvut.

2 Kattangutigalo Hanani, Hananjalo igloksub Jerusaleme angajokanga (anguteôngmat nertornartok, Gudemiglo sivuraluartok assinginit unnuktunit) perkovâka,

3 Okautilugiglo: Upkoaksuit Jerusaleme upkoêrtaujuksaungilet sekkinek kitserkârtinago; sennaviksautillugolo sulle, upkoartaujuksauvut, senneroartaujuksaularmiglo. Udsertortokartitauvorlo Jerusalemiunit, attunit illangutivingmine, iglomiglo avatingine.

4 Iglugaseksuille angijolaukput nerrotôlarmiglo, innuisadlalaukporle, igluillo igloliortaulaungilet sulle.

5 Gudimalo issumakartipânga omattimne, kattimakolugit erkanartut angajokaunerôjullo, innugiartoksuillo kittitilugit. Unnurningitalo nippisiorutingit nachvarpakka.

6 Taipkoa sivurngane takpaungarlauktut parngnanairsimavingmit, Nebukadnezarib Babelib attannertangata aulartilauktangita, Jerusalemêlo nunakalauktut, Judamelo attunît iglugaseksoamingne.

7 Tikkilauktullo' Serubabele, Jesualo, Nehemialo, Asarjalo, Raamjalo, Nahemanilo, Mardochailo, Bilsanelo, Misperetelo, Bigevailo, Nehumelo, Baenalo illagilugit: Tamadsa Israelib innugiartoksoangita angutingita unnurningit.

- 8 Pareosib kittorngangit, 2172 ôlaukput.

9 Safatjab kittorngangit 372 ôlaukput.

10 Arab kittorngangit, 652 ôlaukput.

11 Pahat-Moabib kittorngangit, Jesuab, Joabiblo kittorngangita akkorngane 2818 ôlaukput.

12 Elamib kittorngangit 2254 ôlaukput.

13 Satub kittorngangit, 845 ôlaukput.

14 Sakaib kittorngangit, 760 ôlaukput.

15 Benuib kittorngangit, 648 ôlaukput.

16 Bebaib kittorrıgangit, 628 ôlaukput.

17 Asgadib kittorngangit, 2322 ôlaukput.

18 Adonikamib kittorngangit, 667 ôlaukput.

19 Bigevaib kittorngangit, 2067 ôlaukput.

20 Adinib kittorngangit, 655 olaukput.

21 Aterib kittorngangit Hiskiamit, 98 ôlaukput.

22 Hasumib kittorngangit, 328 ôlaukput. 
23 Bezaib kittorngangit, 324 ôlaukput.

24 Harifib kittorngangit, 112 ôlaukput.

25 Gibeonib kittorngangit 95 ôlaukput.

26 Angutit Bethlehemiut, Netofamiullo 188 ôlaukput.

27 Angutit Anatotemiut 128 ôlaukput.

28 Angutit Beth-Asmavetemiut 42 ôlaukput.

29 Angutit Kiriatjearimemiut, Kafiramiullo, Beerotemiullo 743 ôlaukput.

30 Angutit Ramamiut, Gabamiullo 621 ôlaukput.

31 Angutit Michmasemiut 122 ôlaukput.

32 Angutit Bethelemiut Aimiullo 123 ôlaukput.

33 Angutit Nebomiut 52 ôlaukput.

34 Elamib aipangata kittorngangit 1254 ôlaukput.

35 Harimib kittorngangit, 320 ôlaukput.

36 Jerehob kittorngangit, 345 ôlaukput.

37 Lodib, Hadidiblo, Onoblo kittorngangit 721 ôlaukput.

38 Senaab kittorngangit 3930 ôlaukput.

39 Ajochertuijut: Jedajab kittorngangit, Jesuab kinguvanginit 973.

40 Immerib kittorngangit 1052 ôlaukput.

41 Pashurib kittorngangit 1247 ôlaukput.

42 Harimib kittorngangit, 1017 ôlaukput.

43 Levitit: Jesuab Kadmieliblo kittorngangit, Hodiab kittorngangita akkorngane 74 ôlaukput.

44 Imgerpaktut: Assafib kittorngangit, 148 ôlaukput.

45 Upkoanik udsertortut, tapkoangolaukput: Sallumib kittorngangit, Aterib kittorngangit, Talomonib kittorngangit, Akubib kittorngangit, Hatitab kittorngangit, Sobaib kittorngangit; illunatik kattilugit 138 ôlaukput.

46 Nethinimit: Zihab kittorngangit, Hasufab kittorngangit, Tabaotib kittorngangit,

47 K॰rosib kittorngangit, Siab kittorngangit, Padonib kittorngangit,

48 Libanab kittorngangit, Hagabab kittorngangit, Salmaib kittorngangit,

49 Hananib kittorngangit, Giddelib kittorngangit, Gaharib kittorngangit,

50 Reajab kittorngangit, Rezinib kittorngangit, Nekodab kittorngangit,

51 Gasamib kittorngangit, Usab kittorngangit, Paseab kittorngangit,

52 Bessaib kittorngangit, Megunimib kittorngangit, Nefusimib kittorngangit,

53 Bakbukib kittorngangit, Hakufab kittorngangit, Hahurib kittorngangit, 
54 Bazlitib kittorngangit, Mehidab kittorngangit, Harsab kittorngangit,

55 Barkob kittorngangit, Sisserab kittorngangit, Thamab kittorngangit,

56 Neziab kittorngangit, Hatifab kittorngangit,

57 Salomob kivgangita kittorngangit tapkoangolaukput: Sotaib kittorngangit, Soferetib kittorngangit, Pridab kittorngangit,

58 Jaelab kittorngangit, Darkonib kittorngangit, Giddelib kittorngangit,

59 Sefatjab kittorngangit, Hatilib kittorngangit, Pochretib Zebaimiub kittorngangit, Amonib kittorngangit,

60 Nethinimit kittorngangit, Salomoblo kivgangita kittorngangit kattilugit 392 ôlaukput.

61 Tapkoalotauk takpaungarkattauvut, Mithel, Thel-Harsa, Kerube, Addon, Immerelo; atâtagijamigle kinguvângit, sivurlitiglo nellonailijungnarlaungilait, Israelemit pingmangata.

62 Delajab kittorngangit, Tobiab kittorngangit, Nekodablo kittorngangit, 642 ôlaukput.

63 Ajochertuijunillo, Habajab kittorngangit, Hakoziblo kittorngangit, Barsilaib kittorngangit, Barsilaib Gileademiub paninginit nullianilauktub, taipsotunarlo atserartaulauktub.

64 Tapkoa sivurlimik abvakungita nippisiorutingit kennerpait; nachvarlungilaillo; tamanna pivlugo ajochertuijoêrotilerput.

65 Hatirsastablo (nunab aulatingata) okautivait, nerrijuksaungimatta ivsornainerpângojomit, ajochertuijokarkârtinago kaumaniutiksalijartomik, idluarsaijutiksalijartomiglo.

66 Illagêt illunatik kattilugit 42360 ôlaukput;

67 Kivgangit angutit, arnallo kittinagit; tapkoa 7337 ôlaukput. Imgerpaktokalaukpullo 245 ginik, angutinik arnaniglo,

68 Horsekotingit 736 ôlaukput, Horseojartokotingillo 245 ôlaukput,

69 Kamelekotingit 435 ôlaukput, siutekortojokotingillo 6720 ôlaukput.

70 Atâtallo angajokaunerôjut illangit tunnijivut aitusiamik Gudib iglunganut. Hatirsastalo tunnijivok perkutitalingmut sullijaksab akkiliutiksanganut Goldemik Darikenik 1000 dinik, pôgutaniglo 50 ginik, ullingniglo ajochertuijunut illingajunik 530 ginik.

71 Atâtallo angajokaunerôjut illangit tunnijivut perkutitalingmut sullijaksab akkiliutiksanganut Goldemik Darikenik 20000 dinik, Silberemiglo paundenik 2200 dinik.

72 Innugiartoksuillo assingit tunnijivut Darikenik 20000 dinik, Silberemiglo paundinik 2000 dinik, ullingniglo ajochertuijunut illingajunik 67 ginik. 
73 Taimak ajochertuijut, Levitillo, upkoaniglo udsertortut, imgerpaktullo, innugiartoksuillo illangita, Nethinimillo, Israelitillo illunatik iglugaseksoakotitik innigilerpait.

\section{KAPITEL VIII.}

Perkojat attuartauningat. Uvloksiorviublo iglokarnermik akkerkunik millukattalingnik attortauninga.

$\mathrm{T}$

AKKILLO 7 ingat manna tikkilermat, Israeliblo kittorngangit iglugaseksoakotimingnêmatta, innugiartoksuit illuêngarlutik kattimalerput angutetut attautsetut sorlo, apkosinerme silliktome immiub itterviksoangata sângane, okarlutiglo Esramut, aglanik illissimajomut, aiklerkovlugo Mosesib aglanginik perkojaktalingnik, Nalekab perkolauktanginik Israelitinut.

2 Esrablo ajochertuijub perkojat âpait illagêt, angutit arnallo illunatiglo tussarungnartut tapkoninga sânginut, takkit 7 ingata uvlungita sivurlingane,

3 Attuarpaillo apkosinerme silliktome immiub itterviksoangata sânganêtome, uvlarârsungmit uvlub ketterarninga tikkidlugo. Innugiartoksuillo siutingit illunatik torarput aglanut perkojaktalingnut.

4 Esralo aglanik illissimajok nangerpok ikovrangme kejungôjome, sennalauktangane tamanna pivlugo. Sennianelo nangerput: Matitja, Semalo, Anajalo, Urialo, Hilkialo, Maesealo, tallerpiane; saumianelo, Pedaja, Misaelo, Malchjalo, Hasumelo, Hasbadanalo, Sacharjalo, Mesullamelo.

5 Esrablo aglait kopperpait innugiartoksuit illunamassiatik tækkoane, nuitangmat innugiartoksoarnit tamainit; koppermagillo innugiartoksuit illunamassiatik nikkovilerput.

6 Esrablo Nalegak Gudejoksoak nertorpa. Innugiartoksuillo illunamassiatik kiovut Taimaile! taimaile! aggatik kongmuartilugit pivlutik; okkolutiglo, opigosuglarmiglo Nalekamik kênatik pûvlugit nunamut.

7 Jesuablo, Baniblo, Serebjablo, Jaminiblo, Akubiblo, Sabtaiblo, Hodajablo, Maesejablo, Klitablo, Assarjablo, Josabadiblo, Hananablo, Plajablo, Levitillo perkojat tukkisinartipait innugiartoksoarnut; innugiartoksuillo nangerput innimingne.

8 Attuarpaillo Gudib aglangit perkojaktagit tukkisinartomik, tukkingillo nellonairpait, tukkisinartilugillo attuartautilugit.

9 Nehemiablo Hatirsastaôjub (nunab aulatingata) Esrablo ajochertuijub, aglanik illissimajub, Levitillo, innugiartoksoarnik ajochertuijut, innugiartoksuit illunamassiatik okautivait: Uv- 
lok tamanna ivsornaitôvok Nalekamut Gudipsingnut; kiksarniarase kêaniaraselo. Innugiartoksuit illunamassiatik kêagamik perkojat okausertangit tussaramigit.

10 Tamanna pivlugo okautivait: Aileritse nerriluselo orksulingmik, immerluselo mamaktomik; pajungniluselo aitusiamik akiksoilaungitunut ingmingnut; uvlok tamanna ivsornaitôngmat Nalegaptingnut; tamanna pivlugo issumajarniarase, kuviasungnek Nalekamik pitsartutigigapsiuk.

11 Levitillo innugiartoksuit saimarsarpait, okarlutiglo: Imangeritse, uvlok tamanna ivsornaitongmat, issumajarniarase.

12 Innugiartoksuillo illunatik aivut nerrijomavlutik, immeromavlutiglo, pajungnigomavlutiglo aitusianik, kuviasungnekartitsilarmiglo angijomik; okautsit tukkisilauramigit, okautigijaulauktut tapkonunga.

13 Uvlublo aipangane atâtat innugiartoksuit illunamassiatik angajokaunerôjungit, ajochertuijullo, Levitillo kattimalerput Esramut, aglanik illisimajomut, ajochertortaujomavlutik tapsomunga perkojat okausertanginik.

14 Nachvarpullo perkojane, Nalekab Mosesekut tunnilauktangine, aglaksimangmat, Israelib kittorngangit uvloksiorvingme takkit 7 ingane, iglunêtuksaungmatta akkerkunit millukatalingnit sennamajune.

15 Tamannalo tussartaukovât, akpautigijaukolugolo iglugaseksoamingne illunaine Jerusalemelo, okarkojilutiglo: Aileritse kakkanut, aiklerluselo nappartut, orksuliub, Balsamiblo, Mirteniblo, Palmillo akkerunginik, nappartullo assingita akkulaitunik akkeroggit, iglunik akkerolingnik sennajokarkovlugo, sorlo aglaksimangmat.

16 Innugiartoksuillo aivut aiklerlutiglo, iglokartitsivullo ingmingnut akkerkunik, attunît iglomik kângine, innemingnelo avaluliorsimajune, Gudiblo iglungata inningine avaluliorsimajune, apkosinermelo silliktome immiub itterviksoangata sângane, apkosinermelo silliktome Efraimib itterviksoangata kannitangane.

17 Illagêllo illunamassiatik uttersimajut parngnanairsimavingmit, sennavut iglunik akkerolingnik, innigivaillo. Israelib kittorngangit Josuab Nunib erningata nelliuninganit uvlok tamanna tikkidlugo taimailiorlaungimatta; kuviasungnevaksoakalaukporlo.

18 Gudiblo aglangit perkojaktaggit attuartauvut uvlut tamaita, uvlut sivurlerpânganit kingorlerpâk tikkilugo; uvloksiorviglo attorpât uvlut 7 it nâlugit; uvlullo 8 ingane uvloksiorviub kattimaniksanga, sorlo pijuksaungmat. 


\section{KAPITEL IX.}

\section{Innugiartoksuit kakkialerningat sagvartome.}

TAKKIB tapsoma uvlungita 24 ingane Israelib kittorngangit kattimalerput, nerritaililutik, pôksaijamiglo (silliktunik illerksalingnik) attisimavlutik, ivjolijarlutiglo niakomik kângine.

2 Israeliblo kinguvangit avitipait tækkorngartat kittornganginit tamainit, nangeriartorlutiglo ajornitik, atâtagijamiglo idluitulliningit kigligiutigilugit.

3 Nikkovipullo innimingne, Nalekablo Gudimik aglangit perkojaktagit attuartauvut, uvlorlo sittamaulilugo, sittamangat nâlugo attuarsivut, sittamangallo aipangane kigligiutsivut, tuksiarlutiglo Nalekamut Gudemingnut.

4 Levitillo poktojokotinganut nangeriartorput Jesua, Banillo, Kadmiello, Sebanjalo, Bunilo, Serebjalo, Banilo, Kenanilo, nipliarlutiglo nippekortojomut Nalekamut Gudemingnut.

5 Levitillo Jesua, Kadmiello, Banilo, Hasabenjalo, Serebjalo, Hodjalo, Sebanjalo, Petatjalo okarput: Nikkovititse, Nalegarlo Gudise nertorsiuk issokangitomit issokangitomut! Attillo ânanaudlartok, anginersaujok nertordlerutiksanit, nakosarnernillo tamainit, nertortaule!

6 Nalegak igvitôvotit, igvit killak pingortilaukpat, killaillo tamaita killangit, kattimgajoksoangit illaliudlugit, nuna tamaitalo tapsomanêtut, immarbiksuit, tamaitalo tapkoa illuanêtut. Igvillo tamaita pairivattit; killangmiugaseksuillo tuksiarvigivâtit.

7 Igvit Nalegauvotit Gude, Abramik annerosulauktotit, tessiornilauktotillo taipsominga Uremit Kaldæamêtomit, adserarlugolo Abrahamik.

8 Omattingalo nertornartok nachvarlaukpat sângne, angerkattigilaukpello kinguvângit tunnitsivigijomavlugit, Kanaaniterit, Hetiterillo, Amoriterillo, Feresiterillo, Jebusiterillo, Girgositerillo nunangit; okautsitillo nellautsitilaukpattit, idluartogavit!

9 Igvillo atâtagijapta ajulerningat Egipteneme tækkonârlaukpet nipliarningallo immarbingme iviktalingme suvlulingnik, tussarlaukpet,

10 Nellonaikutekartitsilaukpotit tattamnartullilutillo Faraome, kivgakotinginelo illunaine, nunangatalo innugiartoksoangine illunaine; igvit illitarksilauravit perajarlugvigingmagit; attekartilaukpotillo sorlo uvlome îmat.

11 Immarbiglo avilaukpet sivurngane, immarbik kerkagôr- 
vigilugo ipteriliktokut pikovlugit, unangmijingillo ittijomut egilaukpattit, sorlo ujarak egitauvangmat immaksoarmut;

12. Uvlokarningane tessiorlaukpattit nappajome nuvujaujome, unnuakarninganelo nappajome ikkomaujome, kaumarlugit apkome ingerarvigijangane.

13 Akkarlaukpotillo kakkamut, Sinaimik attelingmut, okarvigilaukpattillo killangmit, tunnitsivigilugillo iduarnermik tammarnangitomik, malligaksaniglo mikselingnik, perkojaniglo illusiksaniglo ajungitunik;

14 Sabbatekotillo ivsornaitok nellonairlaukpet tapkonunga; perkojaniglo, illusiksaniglo, malligaksaniglo perkolaukpattit Mosekut kivgakotikut.

15 Kakkojamiglo killangmit tunnitsivigilaukpattit kâktillugit, immermiglo annikojilaukpotit tapkonunga kairtomit immerulauktillugit; okarvigivattillo aijuksaungmatta nuna nangmineliutilugo, aggangnik issajutigilauktat kôngmut, tunnijomavlugo tapkonunga.

16 Atâtagijavulle piojorilerlaukput, tappatolerlutiglo, nalelugatiglo perkojarnik.

17 Kunnulaukpullo tussarnermik, tattamnartullinitillo pinniarlauktattit erkarlaungilait, tettitosarlaukput angajokâkartitsivullo ingmingnut utteromamut kivgaunermingnut keppeserlutik. Igville Gudiga issumagijungnainilaukpotit, saimarvikalaukpotit, napkigosuklutillo, kennuêdlalarpillo, napkoartoksôlaukpotit, kemmalaungilaillo.

18 Nochangoarmiglo kovimajomik sennalauralloaramik, okarlutiglo: Tagva (Israele) Gudit Egiptenemit aulartilauktit! mittautiksakarlutiglo taimak angijomik;

19 Sullele sunakajuitome kemmalaungilattit napkoatoksônit pitjutigilugo, nappajorlo nuvujaujok nulaungilak taipkonangat uvlotillugo, tessiorlugit apkome, nappajorlonêt ikkomaujok unnuame, kaumarlugit apkome ingergavigijangane.

20 Annernerniglo ajunginermik tunnitsivigilaukpattit, ajochertorlugit, Manakotitillo sangutilaungilattit kanninginit; immermiglo tunnitsivigilaukpattit immeruktillugit.

21 Jahrit 40 git nâlugit pattangaitilaukpattit sunakajuitome, ajoksalaukonagit sunamiglonêt. Anorângit samgusengorlaungilet, ittiggangillo puvilerlaungilet;

22 Tunnitsivigilaukpattillo attanniovingnik, innukattigêksoarniglo, avilaukpattillo maunga ikungalo, aksarnigarivâllo Sihonib nunanga, Hesbonib attannertangata, Ogiblo nunanga, Basanib attannertangata;

23 Kittorngangillo unnuksivalliatilaukpattit uvloritsetitut killangmêtutitut, âlaukpattillo nunamut atâtagijanginut okarutigilauktarnut, ittervigilugo nangmineliutilugolo pijuksaungmatta.

24 Kittorngangitalo ittervigivât nangmineliutilâlo; igvillo 
nunab innungit taipkoa sivurngane mannigotitilaukpattit, Kanaaniterit, tunnilaukpattillo agganginut, attanningillo innukattigêksoangillo, pinniarvigilugit pijomajamingnut.

25 Iglugaseksuillo sôngojut aksarnigarivait, nunalo pitsornartok, iglullo perkutitaggit sunatuinarnik nangmineliutilerpait, immektarvillo ullimarsimajut, perorsêvillo Weineliksat, perorsêvillo orksugit, nappartullo nerkiksagit unnuktut, nerrilaukpullo akkeartorlutiglo, kuinilerlarmiglo, innosekalutiglo allianartomik, pitsiarnersoarnut.

26 Kissianele tappatolerlaukput, akkerartorpâtillo, malligaksaukojattit egipait tunnumingnut, nellautaijokotitillo nakterpait, kigligiutsijut tapkonunga, salertuksaungmatta illingnut, okarnerlutjilutiglo angijomik.

27 Tamanna pivlugo tunnilaukpattit omigijingita agganginut, kappiasuktitsikovlugit tapkoninga. Kappiasungnimiglo nelliuningane nipliarlaukput illingnut; igvillo tussarlaukpattit killangmit, napkoarosuktoksônernullo piuliklertunik tunnitsivigilaukpattit, piuliklertunik tapkoninga omigijingita agganginit.

28 Merngoêrserviksakalauruniglo mumiktilerput idluitullilutik sângne. Tagva kemmalaukpattit omigijingita agganginut, attanniotikovlugit tapkonunga. Tagva sâlerput nipliarlutiglo illingnut; igvillo tussarlaukpattit killangmit piulikattarlugillo napkoarosuktoksônernut,

29 Kigligiudsiviokolaukpattillo sâlertuksaungmatta malligaksaukojarnut. Piojorilerlaukpulle, nalelaungilaillo perkojattit, idluitullilutiglo idluarijattit akkerartorlugit, (tapkoale innuit nelliata pinniarpagit innôgutigivlugit pingmagit) tuitiglo sangutipait, tappatolerlutiglo, nalengnatiglo.

30 Igvillo Jahrit unnuktut nâvlugit kennuêrsarvigilaukpattit, kigligiutsiviokolaukpattillo annernermut, nellautaijokotingnêtomut; illaliorlaungilâlle siutimingnut. Tamana pivlugo tunnilaukpattit innukattigêksuit nunanetut agganginut.

31 Napkoarosuktoksônît malliklugo nungumaritaililaukpattit, kemmalaungilattillonêt; Gudeôgavit saimarvikarosuktok, napkoarosuktorlo.

32 Mannalo Gudivut, Gudeojoksôjotit, sôngojotit kappianadlartotillo, nellautsititsijotit angerkattigêngnermik, napkiningnermiglo, siorniornartut tamaita tikkitautigijavut, attannivullo, angajokaunerôjovullo, ajochertortivullo, nellautaijovullo, atâtavullo, innukotitillo tamaita, Assiriamiut attanningita nelliuninginit uvlok tamanna tikkidlugo, mikkijonasugitailikittôk.

33 Igville idluarpotit, tamaine tikkitaukolauktarne uvaptingnut, igvit namaktullilauravit, uvagulle namangitoâlungmik pinniarlaukpogut.

34 Attanniptalo, angajokaunerôjuptalo ajochertortiptalo, atâtagijaptalo malligaksaukojattit pinniarlaungilait, perkojattillo, 
kigligiutsinitillo kigligiutigijaukolauktattit tapkonunga, kammagilaungilait.

35 Kivgartorlaungilâtillo attanniovingmingne, perkutevaksoarnerne, tunnilauktarne tapkonunga, nunamelo nerrotôjome, pitsornartomelo unnertutigijarne tapkonunga, sâlerlaungilello pinniarnermingnit idluitunit.

36 Ahâk, manna kivgauvogut; nunamêpogullo atâtagijaptingnut tunnilauktarne, perortungit perkutingillo nerrilugit pikovluta; ahâk, tapsomane kivgauvogut;

37 Kaitangillo unnuksivalliavut attannernut, illilauktarnut uvaptingnut, ajornivut pivlugit; attanniotivullo timiptingnut, nergjutekotiptingnullo pijomajamingnut, uvagullo siorniornadlartomêpogut.

38 Taimailingagalloarlutalo angerkattigêngnekartitsivogut sokkosijuitomik, aglaklutalo, angajokaunerôjuptingnullo, Levitinullo, ajochertortiptingnullo nakkitertaukovavut.

\section{KAPITEL X.}

Innugiartoksuit angermaringningit malligalesat malligomavlugit.

$\mathrm{N}$ (nunab aulatinga) Hachaljab erninga, Zedekialo,

2 Serajalo, Asarjalo, Jeremjalo,

3 Pashurelo, Amarjalo, Malchjalo,

4 Hattuselo, Sebanjalo, Malluchelo,

5 Harimelo, Meremotelo, Obadjalo,

6 Danielelo, Gintonelo, Baruchelo,

7 Mesullamelo, Abjalo, Mejaminelo,

8 Mâsjalo Bigailo, Semajalo, tagva ajochertuijut.

9 Levitillo tapkoangôlaukput: Jesua, Asanjab erninga, Benuniblo, Henadadib, Kadmieliblo kittorngangita akkorngane, 10 Kattangutingillo: Sechanja, Hodjalo, Klitalo, Plajalo, Hananjalo,

11 Michalo, Rehobelo, Hasabjalo,

12 Sachurlo, Serebjalo, Sebanjalo,

13 Hodjalo, Banilo, Beninulo.

14 Niakoôjullo innugiartoksoarne tapkoangôlaukput: Pareos, Pahat Moab, Elame, Satu, Banilo,

15 Buni, Asgad, Bebai,

16 Adonja, Bigvai, Adine,

17 Ater, Hiskia, Asur,

18 Hodja, Hasume, Bezai.

19 Harife, Anatote, Neubai,

20 Magpiase, Mesullame, Hesire, 
21 Masesabeel, Zadok, Jadua,

22 Platja, Hanan, Anaja,

23 Hosea, Hananja, Hasube,

24 Halohes, Pilha, Sobek,

25 Rehume, Hasabna, Maeseja,

26 Ahia, Hanan, Anan,

27 Malluch, Harim, Baenalo.

28 Innugiartoksuillo assingit, ajochertuijut, Levitillo, upkoaniglo udsertortut, imgerpaktullo, Nethinimillo, illunatiglo, apterlauktut innukattigêksoarnit nunane Gudib malligaksaukojanginut, nulliatik, ernitiglo, pannitiglo illagilugit, illunatik tukkisijungnartut,

29 Illauvut kattangutimingnut erkanartunut, tikkipullo angerlutiglo angermaringnermut, agvertaromagamik Gudib malligaksaukojangit malliklugit, tunnilauktangit Mosekut, Gudib kivgakotingagut, nellautsititsijomagamik, pinniaromagamiglo Nalekab attanniotivigijipta perkojangit, idluarijangillo, illusiksaukojangillo tamaita malliklugit;

30 Pannivullo tunnijomanginaptigik innukattigêksoarnut nunane, pənningillonêt tigujomanagit erniptingnut;

31 Nunablo innukattigêksoangita Sabbatib uvlungane, kaitsileraikpatta niorvgutiksanik, nerkiksaniglo sunatuinarnik niorvgosijomavlutik, tigujomanginaptigik Sabbatine, uvlunelo ivsornaitune; Jahrillo 7 ingane akkiliaksat sunatuinait akkilertaujungnairkojomagaptigik.

32 Perkojakartipogullo, Jahrine tamaine kênaujak Sekele pingasuilimajub pingajuak, tunnijomagaptigo, kivgartornermut Gudipta iglungane;

33 Tagva kakkojaksanut tækkojaksanut, tunnergutiksanullo nerkiksanut uvlunut tamainut illingajunut, tunnergutiksanullo otaksanut uvlunut tamainut, Sabbatinullo, takkêlanullo uvloksiorvingnullo illingajunut, ivsornaititaumajunut, tunnergutiksanullo ajortoêrutiksanut, Israelitit ullapkosertaukovlugit, pinniaraksanullo tamainut Gudipta iglungine.

34 Imeraut attorpavuttauk ajochertuijunut, Levitinullo, innugiartoksoarnullo, kejuit pivlugit tunnergutiksanut attoriakartut, Nalekab Gudipta iglunganut âtaksaulauktut, atâtagijapta iglungit malliklugit, nelliutune nellonairsimajune, Jahrine tamaine, ikkoalakovlugit Nalekab Gudipta Altarekotingata kângane, sorlo malligaksane aglaksimangmat.

35 Jahrinelo tamaine nunapta perortungita innerkârtungit, nappartullo tamaita paungâlungita innerkârtungit, âtsomagaptigik Nalekab iglunganut;

36 Ernivullo ernerikârtavut, nergjutekotiptalo erniarikârtangit, sorlo aglaksimangmat malligaksane, tuktuvakotiptalo, 
saugakotiptalo erniarikârtangit, tamakkoa tamaita âtaksarigaptigik ajochertuijunut, Gudib iglungane kivgartortunut.

37 Kakkojaksaptalo innerkârtungit, tunnergutiksaptalo sivurlingit, nappartullo tamaita paungâlungit, Weineliksallo iptingit, orksuglo innerkârtut âtsomagaptigik ajochertuijunut inninut Gudib iglunganêtunut; nunaptalo tellimaujoanik Levitinut, Levitit tellimaujoanik pekarkovlugit iglugaseksoaptingne perorsêviktalingne tamaine.

38 Ajochertuijorlo Aronib erninga, Levitinut illaujuksauvok Levitit tellimaujoanik tiguleraikpatta, Levitit tellimaujoakotimik tellimaujoanik âtsikovlugit takpaunga Gudipta iglunganut innenut perkotitalingmêtunut.

39 Israelib kittorngangita, Leviblo kittorngangita tunnergutiksat kakkojaksat, Weineliksallo iptingit, orksuglo âtaksaringmagit takpaunga inninut. Tagvanêput ivsornaitoviub illulerkutingit, ajochertuijullo kivgartortut, upkuaniglo udsertortut, imgerpaktullo; uvagullo Gudipta iglunga kemmagomangilavut.

\section{KAPITEL XI.}

\section{Iglugaseksuit Jerusalemib innungit.}

INNUGIARTOKSUILLO angajokaunerôjungit Jerusaleme iglokalaukput. Innugiartoksuille assingita immeraut attorpât, tellimaujortunit attausek Jerusalemut iglugaseksoarnut ivsornaitunut tikkikovlugo nunagilugit pikovlugo, 9 illo iglugaseksoarne assingine.

2 Innugiartoksuillo angutit illunaita saimartipait pijarællutik Jerusaleme nunakaromalauktut.

3 Tamadsa niakoôjut nunab innunginit Jerusaleme nunakartut. Judable iglugaseksoangine, illunatik attunît nangminerijamingne nunakalaukput iglugaseksoarmingne: tagva Israelitit, ajochertuijullo, Levitillo, Nethinimillo, Salomoblo kivgangita kittorngangit.

4 Jerusalemelo nunakalaukput Judab Benjaminiblo kittorngangita illangit. Judab kittornganginit: Athaja, Usiab erninga, Sacharjab erningata, Amarjab erningata, Sefatjab erningata, Mahelaleelib erningata, Perezib kittornganginit.

5 Maesalo, Baruchib erninga, Kal-Hosib erningata, Adajab erningata, Jojarib erningata, Sacharjab erningata.

6 Perezib erningit Jerusaleme nunakalauktut kattilugit 468 ôlaukput, illunatik angutit erkinaitut.

7 Tamadsa Benjaminib erningit: Sallu, Mesullamib erninga, 
Joedib erningata, Pedajab erningata, Kolajab erningata, Jesajab erningata;

8 Tapsomalo tungâne Gabai, Sallailo, 928 ôjut;

9 Joello Sichrib erninga, aulatsijolaukpok tapkoninga, Judalo Hasnuab erninga angajokaulaukpok iglugaseksuit abvakungita aipanginut.

10 Ajochertuijunit nunakalaukput: Jejada, Jojarib erninga, Jachinelo,

11 Serajalo, Hilkiab erninga Mesullamib erningata, Zadokib erningata, Merajotib erningata, Ahitob erningata, attanniolaukpok Gudib iglungane.

12 Kattangutingillo iglome kivgartortut, tapkoa 822 ôlaukput. Adajalo Jerohamib erninga, Plajab erningata, Amzib erningata, Sacharjab erningata, Pashurib erningata, Malchab erningata,

13 Kattangutingillo angajokaunerôjut atâtat akkorngane 242 ôlaukput. Amasailo, Asreelib erningata, Ahusaib erningata, Mesillemotib erningata, Immerib erningata,

14 Kattangutingillo 128 ôlaukput, angutit erkinartut. Aulatingallo Sabdieleôlaukpok, Gedolimib erninga.

15 Levitinit: Semaja, Hasubib erninga, Asrikamib erningata, Hasabjab erningata, Bunib erningata,

16 Sabtailo, Josabadelo, Levitit angajokaunerôjunginit sullijaksanut pijariakinersanut Gudib iglungane angajokaujunit.

17 Mathanjalo, Michab erninga, Sabdib erningata, Assafib erningata, niakoôlauktuk, nertordlerutiksamik pigiarkârtok tuksiarnerme; Bakbukjalo tugliolauktok kattangutime akkorngane, Abda, Samuab erninga, Galab erningata, Jeditunib erningata.

18 Levitit illunatik iglugaseksoarne ivsornaitunelauktut 284 ôlaukput.

19 Upkoaniglo udsertorteôjut: Akube, Thalmonelo, kattangutingillo, upkoaksoarne udsertortut 172 ôlaukput.

20 Israelitillo assingit, ajochertuijut, Levitillo Judab iglugaseksoangine illunainêlaukput, attunît nangminerijamingne.

21 Nethinimillo Ofeleme iglokalaukput. Zihalo, Gispalo Nethiniminut angajokaulaukpûk.

22 Levitillo aulatingat Jerusaleme Usiôlaukpok, Banib erninga, Hasabjab erningata, Mathanjab erningata, Michab erningata. Assafib erninginit imgerpaktunit aulatsijokalaukpok sullijaksanik Gudib iglungane.

23 Perkojakalaungmat attannermit tapkoa pivlugit, imgerpaktut uvlut tamaita aitusiamik nellonairsimajomik pijuksaungmatta.

24 Pethajalo Mesesabeelib erninga', Serab Judab erningata erninginit aulatsijolaukpok attanniub pinniartaukojanginik tamainik innugiartoksoarnut. 
25 Judablo kittornganginit iglugasengne numamingne nunakartunit illangit nunakalaukput Kiriat-Arbame, aiparusinginelo, Dibonemelo, aiparusinginelo, Kabzeelemelo, aiparusinginelo.

26 Jesuamelo, Moladamelo, Bethpalatemelo,

27 Hazarsualemelo, Bersebamelo, aiparusinginelo,

28 Ziklagemelo, Mochonamelo aiparusinginelo,

29 Enrimmonemelo, Zaregamelo, Jeremuthemelo,

30 Sanoame, Adullamelo, aiparusinganelo, Lachisemelo, perorsêvinginelo, Asekamêlo, aiparusinginelo. Nunakarpullo Bersebamit Hinomib naksanga tikkilugo.

31 Benjaminible kittorngangit nunakalaukput Gabamit, Michmaseme, Ajamelo, Bethelemelo, aiparusinginelo.

32 Anatotemelo, Nobemelo, Ananjamelo,

33 Hazoremelo, Ramamelo, Gitaimelo,

34 Hadidemelo, Ziboimelo, Neballatemelo,

35 Lodemelo, Onomelo, igloliortullo naksangane.

36 Levitillo illangit illangiutivikalauktut Judame, nunakarput Benjaminekut akkorngane.

\section{KAPITEL XII.}

Ajochertü̈ut Levitillo attingit. Iglugaselsuit avaluksoangita innersimaningita uvloksiortauningat. Aulatsïut tunnergutinik,

\section{Tempelemut âtaumajunik.}

TAMADSA ajochertuijut Levitillo, Serubabele, Sealtib erninga, Jesualo illagilugit takpaungarlauktut: Seraja, Jeremia, Esra,

2 Amarja, Malluch, Hattus,

3 Sechanja, Rehum, Meremot,

4 Iddo, Gintoi, Abja,

5 Mejamin, Maadja, Bilga,

6 Semaja, Jojaribe, Jedaja,

7 Sallu, Amok, Hilkia, Jedaja. Tapkoa niakoôlaukput ajochertuijut kattangutingitalo akkorngane, Jesuab nelliuningane.

8 Levitillo tapkoangôlaukput: Jesua, Benuni, Kadmiel, Serebja, Juda, Mathanja, nertordlerutiksanut illingajut, tamna kattangutingillo.

9 Bakbukja, Unnilo, kattangutigik, akkinginêlaukpuk udsertorteôlutik.

10 Jesuab Jojakime ernetariva, Jojakimib Eliasibe ernetariva, Eliasibib Jojada ernetariva,

11 Jojadab Jonathan ernetariva, Jonathanib Jaddua ernetariva. 
12 Jojakimiblo nelliuningane tapkoa atâtauvlutik angajokaunerôlaukput, ajochertuijut akkorngane: tagva Serajamit, Merajaôlaukpok; Jeremjamit, Hananjaôlaukpok;

13 Esramit Mesullameôlaukpok; Amarjamit Johananôlaukpok ;

14 Malluchemit Jonathaneôlaukpok; Sebanjamit Josefeôlaukpok;

15 Harimemit Adnaulaukpok; Merajotemit Helkaiôlaukpok;

16 Iddomit Sacharjaôlaukpok; Gintonemit Mesullameôlaukpok;

17 Abjamit Sichriôlaukpok; Mejamin-Moadjamit Piltaiôlaukpok ;

18 Bilgamit Samuaôlaukpok; Semajamit Jonathaneôlaukpok;

19 Jojaribemit Mathnaiôlaukpok; Jedajamit Usiôlaukpok;

20 Sallaimit Kallaiôlaukpok; Amokemit Ebereôlaukpok;

21 Hilkiamit Hasabjaôlaukpok; Jedajamit Nethaneeleôlaukpok.

22 Eliasibiblo, Jojadablo, Johananiblo, Jadduablo nelliuningine, atâtat angajokaunerôjut Levitinit aglaktaulaukput, ajochertuijunillo Dariusib, Persiab attannertangata attanniotiningane.

23 Levible erningita angajokaunerôjungit aglaktaumavut nelliunerit pijokarningita aglanginut, Johananib Eliasibib erningata nelliuninga tikkilugo.

24 Tapkoalo angajokaunerôlaukput Levitit akkorngane: Hasabja, Serabja, Jesualo Kadmielib erninga, kattangutingillo senniane, nertordlerlutik kujalilutiglo; sorlo Davide Gudib angutekotinga perkojilaungmat, udsertortut, udsertortut sennianêdlutik.

25 Mathanja Bakbukjalo, Obadjalo, Mesullamelo, Talmonelo, Akubelo udsertorteôlaukput upkuanik, udsertorpullo manuangine.

26 Tapkoa Jojakimib Jesuab erningata, Jozadakib erningata, nelliuninganêlaukput, Nehemiablo nunab aulatingata, Esrablo aglanik illissimajub nelliuningine.

27 Jerusalemiblo avaluksoangita ivsornaititauninganut Levitit illunaita nunangine kennertaulaukput, Jerusalemut tikkikovlugit, avaluksuit ivsornaititsomavlugit, kuviasuklutik, kujalilutiglo, imgerlutiglo, Cimbelillo, Psalterillo, Harfillo attorlugit.

28 Imgerpaktullo erningit, Jerusalemib erkânit avatânit kattilerput, Netofatiterillo iglugasenginit,

29 Gilgaliblo iglunganit, Gibeablo, Asmavetiblo perorsêvinginit: imgerpaktut Jerusalemib avatâne illunane iglugasengnik igloliorsalaungmatta ingmingnut. 
30 Ajochertuijullo Levitillo, sujungaitilerput ingmingnik, sujungaititsilutiglo innugiartoksoarnik, itterviksoarniglo, avaluksoarniglo.

31 Judakullo angajokaunerôjungit majoarkovakka avaluksuit kânganut, kattimgajokartitsivungalo maggoinik angijunik, kujalidlartunik; tapkoa aipangit pissukput avaluksuit kângine tallerpianut perorsaijut itterviksoanganut torarlutik;

32 Tapkoalo kingorngane mallikput Hosaja, Judakullo angajokaunerôjungita ketterkangit.

33 Asarjalo, Esralo, Mesullamelo,

34 Judalo, Benjaminelo, Semajalo, Jeremialo,

35 Ajochertuijullo erningita illangit Trompetelijarlutik: Tagva Sacharja, Jonathanab erninga, Semajab erningata, Mathanjab erningata, Michajab erningata, Sachurib erningata Assafib erningata;

36 Kattangutingillo, Semaja, Asraeello, Milalai, Maai, Nethaneel, Judalo, Hananilo Davidib Gudib angutekotingata nokakseralikotingit attorlugit; Esrale aglanik illissimajok sivurnganêdlune,

37 Puailaviublo itterviksoangane, akkinginetome Davidib iglugaseksoangita majutingit majoarpait, avaluksuit pôktoninginut Davidib igloksoanganut, immiub itterviksoanga kittanêtok tikkidlugo.

38 Kattimgajullo kujalidlartut aipangit pissulaukput tapkoa igluanut, uvangalo mallikpakka, innugiartoksuillo ketterkangit illagilugit, avaluksuit kângagorvigivlugit, kiæksautiblo nuvugosinga kângagorvigivlugo, karmak silliktok tikkilugo.

39 Efraimiblo itterviksoanga kângagorvigilugo, itterviksublo nutaungitub kângagut, Hananeelib nuvugosinganut, saukat itterviksoangat tikkidlugo; nokkarpullo parngnanairsortat itterviksoangane.

40 Nangerpullo taimak kattimgajuk maggoit nertordlertut Gudib iglungane, uvangalo angajokaunerôjullo ketterkangita illagilunga;

41 Ajochertuijullo: Eljakime, Maeseja, Minjamin, Michaja, Elioenai, Sacharja, Hananjalo trompetelijarlutik;

42 Maesejalo, Semajalo, Eleasarlo, Usilo; Johananelo, Malchjalo, Eleamelo, Asarlo. Imgerpaktullo nipperkortovlutik imgerput, Jesrajalo aulatsijôlaukpok tapkoninga.

43 Uvlormelo taipsomane tunnergutit unnuktut tunnijauvut, kuviasulaukpullo; Gudib kuviasungnekartilaungmagit angijomik, arnallo sorrutsillo kuviasulaukputtauk, Jerusalemiullo kuviasungningat tussartaulaukpok kanningitomit.

44 Nelliutomelo taipsomane angutit nellonairtaulaukput angajokaukovlugit perkutitalingnut tunnergutiksanik, perortullo sivurlinginik, tellimaujuaniglo pitalingnut, kattersoikovlugit 
perorsêvingnit iglugaseksuillo avatânit, aitortuijutigilugit malligaksat malliklugit ajochertuijunut, Levitinullo, Judakut kuviasutekarmatta ajochertuijunik Levitiniglo, nangermatta,

45 Kamagilugit kamagijaksat Gude pivlugo, kamagijaksallo sujungaititaunek pivlugo. Imgerpaktullo upkoaniglo udsertortut nangerput Davidib, erningatalo Salomob perkojagik malliklugik.

46 Davidib Assafiblo nelliuningangne, itsaksoarme aulatsijokalaungmat imgerpaktunik, nertordlerutiksakalarmelo, kujalijutiksakalunelo Gudemut illingajunik.

47 Israelitillo illunamassiatik Serubabelib nelliuningane Nehemiablo nelliuningane tunnijilaukput aitusianik imgerpaktunut upkoaniglo udsertortunut, uvlut tamaita aitusianginik; ivsornaititaumajuniglo tunnijivut Levitinut, Levitillo ivsornaititaumajunit aitortuivut Aronib kittornganginut.

\section{KAPITEL XIII.}

\section{Nehemiab aliksoininga illinganernit sunatuinarnit.}

Nelliutomelo taipsomane Mosesib aglangit attuartaulaukput innugiartoksuit siutingita sângine; nachvartauvorlo tapkonane aglaksimangmat, Amoniterit Moabiterillo Gudib illagêktokotinginut pijuksautsengimatta;

2 Tamanna pivlugo Israelib kittorngangit pachrearlaungimagit âtsivigilugit kakkojamik immermiglo, pissiarilugolo Bileame tikkerkolaungmatsuk akkerartorlugit, ominarsijaukojikovlugo tapkoninga; Gudiptale ominarsinek ablatsangortipa saimartitaunermut.

3 Malligaksallo manna tussaramigit, tækkorngartat illunaita avitipait Israelitinit.

4 Tamattomalo sivurngane Eliasibe ajochertuijok, angajokaulauktok inninut Gudipta iglunganêtunut, illijilaukpok tapkonunga Tobiab tunnergutinganik.

5 Akiksoilaungmat taipsomunga innemik angijomik takkamungalo illijilaukpok sivurngane tunnergutiksanik nerkiksanik, Weihrauchemiglo, illulerkutiniglo, kakkojaksallo tellimaujoanik, Weineliksallo iptinginik orksumiglo, aitusiaksanik Levitinut, imgerpaktunullo, upkoaniglo udsertortunut, tunnergutiksallo ajochertuijunut.

6 Nelliutomele taipsomane tamât uvanga Jerusalemelaungilanga. Artasastab Babelib attannertangata Jahringita 32 ingane tikkinama attannermut, uvlullo kapsit kingorngane kennuarivara attannermit.

7 Taimaglo Jerusalemut tikkipunga, mallugivaralo namang- 
itullinek Eliasibib pinniarlauktanga Tobia pivlugo, inniliorlaungmago Gudib iglungata inningane avaluliorsimajome;

8 Illulugutigilaukparalo angijomik, egipakkalo inniub illuanit Tobiab iglungata perkutingit tamaita.

9 Perkojivungalo inne sujungaititaukovlugo; Nalekablo iglungata perkutingit ittertinivakka takkamunga, tunnergutiksat nerkiksat, Weihrauchelo.

10 Tussarpungalo Levitit aitusiaksangit tunnijaulaungimatta tapkonunga; tamana pivlugo Levitit imgerpaktullo, pinniaraksanik pinniartuksat, kemmâlaukput attunît perorsêvingmingnut.

11 Tagva angajokaunerôjut suarpakka, okarlungalo: Sôg Gudib iglunga kemaktaumava? Kattikovakkale pitilugillo innigijaksanginut.

12 Tagva Judakut illunamassiatik kakkojaksat! Weineliksallo iptingita orksullo tellimaujoat âpait perkutitalingmut.

13 Angajokangortipakkalo perkutinut Selemja ajochertuijok, Zadokelo aglanik illisimajok, Pedajalo Levitinit, Hananlo Sachurib erninga, Mathanjab erningata ikajortingortilugo tapkonunga; nertornartonasugijaungmatta, perkojauvullo aitortuikovlugit kattangutimingnut.

14 Erkarlaunga tamana pivlugo Gudîk, puigortautailitikillo pinniarnikka ajungitut, pinniarlauktakka Gudima iglunga pivlugo, illusiokojangillo pivlugit!

15 Nelliutome taipsomane Judame tækkovunga Weineliksat iptêjaivinganik tullertunik Sabbatib uvlungane keppitaniglo kakkojaksanik ittertitsingmatta, siutekoktôjuniglo nangmalingnik Weinemik, Weineliksallo paunganginik, Feigeniglo, nangmaniglo pittalingnik sunatuinarnik ittertitsingmatta Jerusalemut Sabbatib uvlungane. Tennersarpakkalo uvlorme taipsomane nerkiksanik niorvgusingmatta.

16 Tiriamiokalaurivok nunakartunik tagvane, mingeritjanik âtsijunik, niorvgutiksaniglo sunatuinarnik, niorvgosilutiglo Judab kittornganginut, Jerusalemiunullo Sabbateme.

17 Tagva Judakut angajokaunerôjungit suarpakka, okautilugillo: Sunauva idluitok pinniartase Sabbatib uvlunga serkomigapsiuk?

18 Atâtagijavut taimailiorlaungilât, Gudiptalo kannoêtut tapkoa tamaita tikkerkovait uvaptingnut iglugaseksoarnullo ukkununga? Illipselo anginersamik sulle ningarnekartitsivose Israelitinut, Sabbate serkomigapsiuk?

19 Pijokalaukporlo Jerusalemib itterviksoangine tâksilermat, Sabbatib uvlungata sivurngane, perkojivunga upkoat pallangairtaukovlugit, perkojivungalo pallangairutjartaukonagit, Sabbatib kingorngane kissiane. Kivgakotimalo illangit tillivakka itterviksoarnut, akjarsijunik ittertokarkonago Sabbateme. 
20 Tagva niuvertit niorvgosijullo niorvgutiksanik sunatuinarnik najortekarlutik unnuivut attausêarlutik, maggoêrtorlutiglo Jerusalemib sillatâne.

21 Tagva kigligiutsivigivakka okautilugillo: Sôg unnuivise avaluksuit sillatâne? Taimailiormigupse, tagva aggakka illiniarpakka illipsingnut. Nelliutomit taipsomanemit tikkitsungnailaukput Sabbatib uvlungane.

22 Levitillo perkovakka sujungaitikovlugit ingmingnik, tikkikovlugillo upkoat udsertorlugit, Sabbatib uvlunga ivlerilugo. Gudîk, tamanna pivlugotauk erkarlaunga, ivlerilungalo napkoartoksonit pivlugo!

23 Nelliutome taipsomane tækkolaukpungatauk Judinik, nullianiktunik Asdodemiunit, Amoniterinillo, Moabiterinillo.

24 Tapkoalo kittorngangit okausekalaukput akkusimajunik, ketterarlutik Asdodemiut okausinginik; okarungnaratiglo Judit okausinginik, innukattigêksuille nellit okausingit malliklugit.

25 Suarpakkalo, okarnerlugvigilugillo, angutillo illangit annaulerpakka nussuilugillo, angermaringnermiglo Gudib sângane tigusivunga tapkonangat: Pannise tunnijaksaringilasse taipkoa erninginut panningillonêt tigunagit ernipsingnut, nangminipsingnullonêt.

26 Salomob Israelitit attanningata tamanna idluitullijutigilaungikauk? Innukattigêksuillo unnuktut akkorngane, attannekalaungilak tapsotunak, Gudingatalo nagligilaukpa, Gudiblo attanningortipa Israelitinut; taimailingagalloarlune arnat tækkorngârtat idluitullitipât.

27 Tamanna tussarlaungipissiuk, taimak idluitoksoarmik pinniarapse, Gudivut idluitullivigilugo, nullianingnermut tækkorngartanik?

28 Jojadablo Eliasibib ajochertuijersub erningata erningita illangata Saneballate Horonitereojok illanârilerpa; uvangale kemâtilaukpara uvamnit.

29 Issumagikit Gudîk ajochertuinerôniub ivsornartitauninga pivlugo, ajochertuijullo Levitillo angerkattigêngningata sujuktauninga pivlugo.

30 Taimaglo illakarungnaitilaukpakka takkorngartanit tamainit; nellonairpakkalo ajochertuijut Levitillo pinniaraksangit, attunît sullijaksanginut,

31 Tunnergutillo kejuit nelliutunut nellonairsimajunut, perortullo nergjutillo innerkârtungit erniarikârtangillo. Gudîk, erkarlaunga idluarkutiksamnut! 


\section{ESTERIB AGLANGIT.}

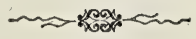

KAPITEL I.

Persiamiut attanningata nulliangata ajoktortauninga uiminut Ahasverosemut.

Ahasverosib attanniolauktub Indiamit Aetiopia tikkidlugo, nunanut 127 ginut, nelliuningane pijokalaukpok,

2 Iksivavlune attannionime iksivautaksoangane, igloksoarme sôngojome iglugaseksoarnetome Susanemik attelingne,

3 Attanniotinime Jahringita pingajuane nerrimartitsivok angajokakotiminut kivgaminullo illunainut, tagva pitsartunelingnut Persiame, Medenemelo, erkanarnerpânullo aulatsijunullo nunamine,

4 Tækkojautitsijomavlune attanniônime akluininganik ânanaudlartomik, pijariakôrtonimelo pinniarnarninganik pivianartoksoarmik, uvlut unnuktut nâvlugit, tagva uvlut 180 git nâvlugit.

5 Uvlullo tapkoa nâlaungmatta, attannek nerrimârtitsivok innugiartoksoarnut, tammangnut angijunut mikkijunullo Susanemêtunut, iglugaseksoarne attanniub igloksoakarvigijangine; uvlut 7 it nâlugit, avaluliorsimajome, perorsêvingmêtome, attanniub igloksoangata sennianêtome.

6 Tagvane nivingajokalaukpok kongeselitanik kakkortanik, aupalluktaniglo, korgsutaniglo, aklunane ivaluksaijamit sennamajune, (aklunaneio) aupalloriktune, suvlolingne silbereojunik, siakititaumajune nappajune ujaraujune Marmoremik attelingne. Iksivautat Goldeôlaukput, Silbereôlutiglo tungavikartut Marmoremik kakkortamik, korgsutamiglo kennertamiglo.

7 Immiksallo akjartaulaukput illulerkutine Goldeôjune, assetartaukattartune, Weinekalaukporlo attannersiumik arklingnartomik, attanniojub illusia maliiklugo. 
8 Kinalo perkojaulaungilak sunamik immertuksaunerminik: attanniuble aulatsijut iglunganik perkolaukpait, illunatik attunît namagijamingnik pinniarungnartuksaungmatta.

9 Attanniublo nullianga, Vasthimik attelik nerrimartitsivoktauk arnanut, attanniub Ahasverosib attannioningata iglungane.

10 Uvlullo 7 ingane, attanniub omattinga kuviasudlalauktillugo Weinemut, perkovait Mehumane, Bistalo, Harbonalo, Bigtalo, Abagtalo, Setarlo, Karkaselo, aulatsiôjut 7 it, kivgartorlauktut attanniub sângane,

11 Attanniub nullianga Vasthi ailugo attanniub sânganut pikovlugit, niakotelijartillugo attanniojub niakotinganik; taipsoma ânanauninga tækkojautitsomangmago innukattigêksoarnut, angajokaksoarnullo; tækkoranidlalaungmat.

12 Attannerle Vasthi kunnulaukpok tikkilune attanniub okausingit aulatsijokotimigut malliklugit. Tagva attannek ningadlalerpok, kanningajarningalo illungertudlalerlaukpok illumine.

13 Attanniublo illissimajut nunab illusinginik kaujimajut okarvigivait (attanniub illinganingit illunaita pinniartaujariakalaungmatta, illisimajut idluarnernik illutsiniglo sângine ;)

14 Kanninerpâllo tapsomunga tapkoangolaukput: Karsena, Setar, Admata, Tarsis, Meres, Marsena, Memuchanelo, Persiamiut Medenmiullo angajokaunerôjungit 7 it, attanniub kênanganik tækkonarlauktut, issukliovlutiglo iksivalauktut attaniovingme:

15 Sunamik idluarnek malliklugo, attannek Vasthi pinniarviojuksauva, pinniarlaungimat attanniub Ahasverosib perkojininganik aulatsijokotimigut?

16 Tagva Memuchane okarpok attanniub angajokâvaksuillo illunatik sângine: Attanniub nulliangata Vasthib attannek kissiat idluitullivigilaungila, angajokaksuille illunaitatauk, innukattigêksuillo illunaita attanniub Ahasverosib nunanginêtut.

17 Attanniub nulliangata pinniarninga tamanna tussartauniarmat nullianut illunainut, nachokovlugit uimingnik ijimik sângine, okarniarlutiglo: Attanniub Ahasverosib aipane Vasthi kaikojaukolauralloarpa sâminut, kaijomalaungilarle.

18 Taimaglo manna angajokaksuit Persiamêtut Medenemêtullo nulliangit okarniarputtauk, attanniub angajokakotinginut illunainut, attanniub aipangata pinniarninganik taimaitomik tussarniarunik; nachonekadlarniarporlo ningarnekadlalarmelo.

19 Attanniub namagikpago, tagva perkojamik attannersiumik annijokarle tapsomangat, aglaktaujokarlelo Persiamiut Medenmiullo malligaksangit tappagijaujuksaulungitut, malliklugit, Vasthi attanniub Ahasverosib sânganut tikkitsungnaikovlugo, attanniublo taipsoma attannioningata illinganinga tunniliuk, taipsoma innukattinganut idluarkejanganut; 
20 Attanniub aglangit tapkoa, aglaktauniartut, tussartitaukovlugit attanniovingane angijoksojome illunâne, nullit illunatik uimingnik naletsiarkovlugit, pijariakôrtune pijariakitunelo.

21 Attanniublo angajokaksuillo tamanna namagivât; attannerlo pinniarlaukpok Memuchanib okausingit mallikıugit.

22 Tagva aglait tillikliutijauvut attanniub nunakotinginut illunainut, nunailo illunatik attunît aglangit malliklugit, innukattigêksuillo illunatik attunît okausingit malliklugit, uit illunatik attunît angajokaujuksaungmatta iglomingne; okautigijaujuksaulermallo innukattigêksuit attunît okausingit malliklugit.

\section{KAPITEL II.}

Esterib annerïauninga attanniub nullianganut. Mardachaib, tapsoma innugoijingata angerkattigêngnek attannek akkera'm torlugo mallugiva, sagvarlâlo.

Pijokalauktut tamakkoa kingorngane attanniub Ahasverosib kaningajarninga sorairlaukârtillugo, Vasthi erkarpa, pinniarlauktanga, kajusimavioningalo.

2 Tagva attanniub kivgangit naipertortut tapsominga, okarput: Kennertokarle attannek pivlugo, innuosuktunik uigarsungnik pinnarnartunik;

3 Attannerlo kemmergojokartitsilerle attanniovime nunangine illunaine, uigârsungnik innuosuktunik pinniarnartuniglo sunatuinarnik kattitsilutik âtsikovlugit attanniub igloksoanganut Susanemêtomut, arnat iglunganut, Hegaib, attanniub pimaklertokotingata, arnanik aulatsijub agganginut, pinarnarninginik tunnijikovlugo tapkonunga;

4 Uigasuillo nelliat attannermut ânanaugijaujok, tamna annerijaule Vasthi innangêrlugo aipaukovlugo attannermut. Attanniublo tamanna namagiva, taimailiorlunelo.

5 Angutekalaukporle Judeôjomik iglugaseksoarne Susaneme attanniub iglokarvigijangine, Mardachaimik attelingmik, Jairib erninganik, Simeib erningata, Kisib erningata, Benjaminekut illanganik,

6 Aularkassiutitaumalauktomik Jerusalemit, Jechanja, Judakut attanningat Nebukadnezarib aulartilauktanga aulartitautillugo.

7 Innugoijolaukporlo Hadassamik, tagva Esteremik, akkame panninganik, atâtakalungimat, anânakangimallonêt. Uigârsôlaukporlo ânanaudlartok, pinnarnadlartorlo. Atâtangalo anânangalo tokkungmanik Mardachaib illaliorpa pannimetut.

8 Attanniublo perkojanga malligaksaukojangalo tussartauler- 
mat, uigâsuillo unnuktut âtaungmatta attanniub igloksoanganut Susanemêtomut, Hegaib agganginut, Esteretauk âtaulaukpok attanniub iglunganut, Hegaib, arnanik udsertortib agganginut.

9 Tapsomalo uigâsuk tamna ânanaugiva, Esterelo napkiningneksivok sângane. Tuavilaukporlo pinnarnarsilugo, tunnitsivigilugolo nerkiksanginik, uigârsungniglo 7 nik ânanânik, attanniub iglunganit pijunik. Pitipalo uigasukotingit illagilugit innemut namanerpâmut, arnat iglunganêtomut.

10 Esteriblo okautilaungila innukotiminik illaminiglo; Mardachaib perkolaungmago, tamattominga okartuksaungimat.

11 Mardachailo agvertarlaukpok uvlut tamaita arnat iglungata avluliorsimaningata sângane, tussarasuaromavlune Estere kanoêngitsiarmangat, kannorlo pinniarvioninganik.

12 Uigâsuille nelliata nelliuninga nellonairsimajok ittertuksauvia attannermut Ahasverosemut nelliutilaukpat, arnat pinarnarsijauninganêlaukârdlune takkit 12 it nâlugit (pinarnarsijauningat taimak akkunêrtigijôjariakalaungmat, takkit pingasujortut nâlugit Mirrit orksunginut, takkillo pingasujortut nâlugit mingoarutiksanut tippitsiariktunut, assinginullo, arnat pinarnarsijaujutiksanginut illingajunut);

13 Tagva uigâsuk tamna itterpok attannermut, pijomajangalo aipaukojaujariakalaukpok tapsomunga, arnat iglunganit attanniub iglunganut.

14 Nelliallo unnungme itterlaurune, tamna uvlâme annilerpok tapsomangat, ailunelo arnat iglungata aipanganut, Saasgasemut attanniub pimaklertokotinganut, udsertortemut pannervganik, utterungnaituksaulaukporlo attannermut, kissiane attanniub tussugilauruniuk, attingagullo kaikojaukoguniuk.

15 Esterib, Abihailib Mardachaib akkangata panningata (Mardachaib illaliorlauktangata pannimetut) nelliuninga ittertuksauvia attannermut nelliusimangmat, tussulungilak sunamik, Hegaib attanniub pimaklertingata, arnanik udsertortib, okarningata assianik. Esterelo saimaneksivok illunatik tækkotime ijingita sângine.

16 Esterelo âtaulaukpok attannermut Ahasverosemut attanniub iglunganut, takkit tellimaujoane, Tebetemik attelingme, attanniotiningata Jahringita 7 ingane.

17 Attanniublo Estere nagliginerpârilerpa arnanit tamainit, saimaneksilunelo, napkiningneksilualerpok tapsoma sângane uigasungnit illunainit. Tapsomalo niakote attannersiut illiva niakoanut, attanningortilâlo, aipangortipa ingminut Vasthi innangêrdlugo.

18. Attannerlo nerrimârtitsivok angijoksoarmik angajokakotiminut, kivgaminullo illunainut, (nerrimaraksak tamna Estere pivlugo pilaukpok) nunallo (innuit) akkiliaksanik akkilêtailikovait, pilliutiniglo attannersiutinik tunnijivok. 
19 Aipanganiglo uigârsuit kattertautilugit, Mardachai iksivalaukpok attanniub itterviksoangane.

20 Esterelo nellonairsilaungilak illaminik, innukotiminiglonêt; sorlo Mardachai perkolaungmago. Estere Mardachaib okausingit taimaluatsiak malliklugit pinniarame, sulle sivurnganetut pairijaunermine tapsomunga.

21 Nelliutome taipsomane Mardachai iksivatillugo attanniub itterviksoangane, attanniub pimaklertokotigik magguk, Bigtanelo Thereslo ningalerpûk, pinnasuarpûglo aggatik illijomavlugit attannermut Ahasverosemut.

22 Tamanna kaujijaulerpok Mardachaimut, tapsomalo okautigiva attannermut Esteremut (attanniub aipanganut;) Esteriblo okautigiva attannermut Mardachaib attingane.

23 Tamannalo nautsertortaulermat nachvartaulaukpok taimak. Tammarmiglo kimiktaulaukpûk nappartome, tamannalo aglaktaulaukpok attanniub pijokarningita aglanginut.

\section{KAPITEL III.}

\section{Hamanib piojorininga illusiâlungillo -Judinut.}

Pijokalauktut tamakkoa kingorngane, attanniub Ahasverosib Haman Meedatab erninga, Agagitereôjok angijorortipa, kagvertilâlo, iksivautangalo issakliotipa angajokaksoaminit tapsomanêtunit illunainit.

2 Attanniublo kivgangita attanniub itterviksoanganêlauktut illunatik pamaktervigivât, opigilâlo; attannek taimak perkojilaungmat. Mardachaile pamakterlungilak, opiginagolo.

3 Tagva attanniub kivgangita, attanniub itterviksoanganêlauktut Mardachai okautivât: Sôg attanniub perkojanga tappagiviuk?

4 Uvlullo tamaita taimak okautipsarkattaramitsuk, tapsomalo nalengimagit okautigivât Hamanemut, tækkojomavlutik Mardachaib pinniarninga sokkosijuijungnaralloarmangat; okautilauramigit Judeôgame.

5 Hamanelo tækkogame Mardachaib okkungavigingimago pamaktervigingimagolo, illumilerpok kaningaumajarnermik.

6 Nachogivâlo aggane illilugit Mardachaimut kissiane, Mardachaib innukattingit okautigilaungmagit tapsomunga; pinnasuarporle Mardachaib innugaseksoakotingit Judit illunaita Ahasverosib attanniovingane illunanêtut nungutitsomavlugit.

7 Takkit sivurlerpângane, tagva takkerme Nisanemik attelingme, attanniub Ahasverosib attannioningata Jahringita 12 ingane, immeraut attortaulaukpok Hamanib sângane, uvlormit 
uvlormut, takkermit takkermut, takkit 12 ingat tikkidlugo, tagva takkek Adaremik attelik.

8 Hamanelo attannermut Ahasverosemut okarpok: Innukattigêksoakarpok siamarsimajunik, avilerpullo innukattigêksuit attanniovît nunanginetut illunatik akkornganut, malligaksangillo innukattigêksuit assingita malligaksangititut-ilungilet, attanniublo malligaksaukojangit malliklugit pinniarlungilet; attannermullo namalungilak taipkoa taimailingatsainarkolugit.

9 Attannermut namagijaukpat, tagva aglarle, tokkotaukovlugit, tagva Talentinik 10000 dinik Silberemik kittiktarijomavunga pimaklertut agganginut, âtaukovlugit attanniub pivianartokutalinganut.

10 Tagva attanniub ketterdlermêkotine pêrpa aggangminit tunnilâlo Hamanemut, Medatab erninganut, Agagitereôjomut Judit omigijinganut.

11 Attanniublo Haman okautiva: Silberit taipkoa tunnijaumavut illingnut, tappilugillotauk innukattigêksuit taipkoa, namagijarnik pinniarvigilugit pikovlutit.

12 Tagva attanniub aglakpaktokotingit kaikojauvut takkit sivurlingata uvlungita 13 ingane; aglaktaulerporlo taimaluatsiak sorlo Haman perkojingmat, attanniub angajokaksoakotinginut, aulatsijunullo nunanik, nunane sunatuinarne, innukattigêksuillo attunît nunane angajokaunerôjunginut, innukattigêksuit attunît aglaningit okausingillo malliklugit, att:nniub Ahasverosib attingane, attanniublo ketterdlermêkotinganut nakkitertauvlutik.

13 Aglaillo tapkoa tillikliutijauvut akpangertutigut attanniub nunakotinginut illunainut, Judit illunaita nungutilugit, nakterlugillo, innuarlugillo, tammarmik innuosuktut innutokaujullo, sorrutsillo, arnallo, illunaita uvlorme attautseme, tagva takkit 12 ingata uvglungita 13 ingane, tagva takkib Adaremik attiggub perkutingillo arktautigilugit.

14 Aglait tapkoa tukkingit taimailingalaukput, perkojak tunnijaumangmat nunane illunaine, innukattigêksoarnut illunainut, nellonairtaujuksak, uvlorme taipsomane oppalungairsimajuksaungmatta.

15 Akpangertullo aularput tuavilukarlutik attanniub perkojanga malliklugo. Perkojarlo tamna tunnijaumalaukpok attanniub igloksoangane Susaneme. Attannerlo Hamanelo iksivavûk, immerlutiglo; iglugaseksoarmiullo kakkiladlalaukput. 
KAPITEL IV.

Mardachaib kajungersaininga Esteremik, aikovlugo attannermut, tuksiarvigilugo pikovlune, perkojab tokkotsikojæ̋ub Judinik attortaujungnaininganik.

MARDACHAIB tamaita pijokalauktut tukkisigamigit, annorâne allikpait, attitilunelo pôksaijamik arkisamiglo, annilunelo iglugaseksuit kerkânut, nipliakortôvlunelo ârusuklune.

2 Tikkiporlo attanniub upkoaksoangata sânganut. Kinalonêt annoralijarlune pôksaijamik attanniub itterviksoanganut ittertuksaungimat.

3 Nunanelo tamaine attanniub okausingita perkojangitalo tikkivingine ârusutiksakadlalerpok nerritailinekalarmelo, kiksadlarnekalunelo Judit akkorngane; unnuktullo nellâvut pôksaijame arksamelo.

4 Tagva Esterib innuosuktokotingit arnat, kivgangitalo angutit illangat, tikkiput okautivâlo tamattominga. Tagva attannek Ester koaksadlarpok. Annoraniglo tillikliutjivigiva Mardachai, attitikovlugo tapkoninga, pôksaijamiglo pêrsikovlugo; illaliorlungilaille.

5 Tagva Esterib Hatache attanniub pimaklertokotingita illangat, tillilauktanga naipertorkovlugo tapsominga, kaikova, perkojakartipalo Mardachaimut illingajomik, nellojungnaititaujomavlune sunaumangat tamanna, sunalo pivlugo taimailiormangat?

6 Tagva Hatachib Mardachai annivigiva iglugaseksuit apkosininganut, attanniub itterviksoangata sânganêtomut.

7 Mardachaiblo okautiva tamainik tikkitautigijaminik, Silberillo unnurninginik, Hamanib okautigilauktanginik, kittiktaromajanginik attanniub kênaujakotitalinganut, Judit pivlugit nungutitsomavlugit.

8 Perkojablo tunnijaumalauktub Susaneme Judit nungutilugit adsinginik tunnitsivigiva, tækkojautitsikovlugo tamattominga Esteremut, okautjikovlugo tapsominga, perkolugolo pikovlugo, attannermut itterkovlugo, tuksiarvigilugo, innukotiminiglo tuksiarutjikovlugo.

9 Taimaglo Hatach itterpok akpautigivaillo Mardachaib okausingit Esteremut.

10 Esteriblo Hatach okautiva, okarkolâlo Mardachaimut:

11 Attanniub kivgangit illunatik, innugiartoksuillo attanniub nunangine kaujimavut, attannermut ittertokarangat avaluliorsimajub illuanut, angullonêt, arnarlonêt, kaikojaulungitomik, 
tamna perkojak malliklugo tokkotaksaungmat; kissianeúngitok attanniub ajaupiane opigijautiksak Goldeôjok issautiguniuk tapsomunga, innôkovlugo. Uvangale mana uvlune 30 gine kaikojaulaungilanga takkamunga attannermut.

12 Esteriblo okausingit okautigijaulermatta Mardachaimut,

13 Mardachaib Ester okautsaukopsarpa: Issumaniarnak innôtsitit piulijatuariniarangne attanniub iglunganênavit, Judinit tamainit;

14 Mannale nelliutome tamattomane niplitailiniaruvit, tagva Judit ikajortauniksarsiniarput, piulijauniksarsiniarlutiglo nakit assingnit, igville atâtavillo illangit nungutauniarpose. Imakkâlo nelliutok tamanna pitjutigællugo attanningortitaulaungimangarpit?

15 Esteriblo Mardachai kiojaukova:

16 Ailerit Judillo Susanemêtut illunaita katterkokit nerritailijutigissingalo, nerrikonase immerkonaselo uvlune pingasune, uvlotillugolonêt unnuarmelonêt; uvanga uigasukotikkalo, nerritailijomavoguttauk taimak. Taimaglo attannermut itteromavunga, perkojak akkerartorlugo; tokkotauniaruma, tagva tokkotauvunga.

17. Mardachailo aularpok, pinniarlunelo tamainik Esterib pinniarkolauktanginik tapsomunga.

\section{KAPITEL V。}

Esterib aininga atiannermut. Hamanib pijugaklerninga Mardachaimit.

UVLULLO pingajuane Estere attitipok attannersiutinik, nangeriartorlunelo avaluliorsimajub attanniub iglunganêtub illuanut, attanniub iglungata akkianut. Attannerlo iksivavok iksivautaksoarmine attannersiume attanniub iglungane, iglub upkoangata akkiane.

2 Attanniublo Estere attannek tækkungmago nangertillugo avalut illuane, saimaneksilerpok tapsoma ijikita sângangne. Attanniublo ajaupiane opigijautiksak Goldeôjok tigumiartane issautiva Esteremut. Tagva Estere ablorpok tagrunga, aktorlâlo opigijautiksab nuvua.

3 Tagva attanniub okautiva: Suvît attannek Estere? Sunamiglo tussuvit? Attannioviub koppanganiglonêt aglat tunnijaujuksauvok illingnut.

4 Estere okarpok: Attannermut namagijaupat, tagva attannek Hamanelo tikkilerlik uvlome nerrimaraksamut âkiksorsimajamnut. 
5 Attannek okarpok: Tuavititse Haman pinniarkovlugo sorlo Estere okalaungmat. Attannerlo Hamanelo nerrimaraksamut, Esterib âkiksorlauktanganut tikkingmanik,

6 Attanniub Estere okautivâ Weinetorlaukârdlune: Suna mik kennuvît Estere? Tunnijaujuksauvok illingnut. Sunalo tussugiviuk? Attanniôviub koppanga pinajarpat aglât, taimaituksaulerpok.

7 Tagva Estere kiovok okarlunelo: Tuksiarnera tussuneralo imaipok:

8 Saimaneksiguma attanniub sângane, attanniublo namagikpago tuksiaramnik tunnitsivigilunga, tussuneralo pinniarlugo, tagva attannek Hamanelo tikkilerlîk nerrimaraksamut âkiksoromajamnut pivlugik: tagva kaupat pinniaromavunga attanniub okausingit malliklugit.

9 Tagva Haman uvlorme taipsomane annilerpok kuviasuklune maksoavlunelo. Mardachaile trkkogamiuk attanniub itterviksoangane nikkovilungimat, aulalungimallonêt sângane, tættælerpok ningarnermik Mardachai pivlugo.

10 Nunolaukporle. Angeraramelo tilliklerpok aijaukolugillo illanârijane; nullianelo Serese;

11 Unipkautivaillo akluinime ânanaudlarninganik, kittorngamelo unnurninginik, tamainiglo kannok attanniub angijorortilaungmago, attanniublo angajokaksoakotingita, kivgakotingita kollânut kagversimagame.

12 Hamanelo okarpok: Attanniublo Esterib kinalonêt kaikolaungila attannek aiparællugo nerrimaraksamut âkiksorlauktaminut, uvanga kissimaulunga; kaupamullotauk kaikojaumavunga taipsomunga, attannek aiparællugo.

13 Tamannale tamât kuviasutigingilara Mardachai Judiôjok tækkojautillugo uvamnut, iksivatillugo attanniub itterviksoangane.

14 Tagva nulliangata Seresib okautiva illanârijangatalo illunatik: Nappartomik sennajokarle tallitut 50 gitut pôktotigijomik, attannerlo kaupat okautsaulerle, Mardachai kimiktaukovlugo tapsomane, tagva kuviasuklutit attannek aiparællugo nerrimarviliarniarpotit. Hamanib tamanna namagiva, âkiksortaukolugolo nappartok. 


\section{KAPITEL VI.}

\section{Hamanib Mardachai sagvartome opigïariakarpa attanniut. attingane.} UNNUARME taipsomane attannek sinnigungnarlaungilak, kai-
taukovaillo aglait, unipkautsit pijokalauktunik, attuartaulaukpullo attanniub sângane;

2 Tagvanelo naipitauvok aglaksimangmat, kannok Mardachai okarlaungmat, attanniub pimaklertokotigik magguk Bigtan Thereslo upkoab manuane udsertortûk, aggatik illijomanasuarlaungmagit attannermut Ahasverosemut.

3 Attannerlo okarpok: Sunamik nertortaunermik, ajungitullivionermiglo Mardachai pinniarvigilaukpittigo tamanna pivlugo? Tagva kivgat kivgartortut tapsominga okarput: Pinniarviolaungilak sunamik.

4 Attannerlo okarpok: Kina avaluliorsimajub illuaneka? (Haman avaluliorsimajub illuanut, attanniub iglungata sânganêtub ittersimalaurame, attannek okautitsomavlugo, Mardachai kimiktaukolugo nappartome, tapsoma akiksorlauktangane taimna pivlugo.)

5 Attanniublo kivgangita okautivât: Ahâk Haman avaluliorsimajub illuane nangerpok. Attannek okarpok: Itterle.

6 Hamanelo itterpok. Attanniublo okautiva: Angut attanniub opigijomajanga sunaliorviojuksauva? Hamanele omattimigut issumalerpok: Attanniub kina assimnit opigijomanajaralloarkauk?

7 Hamaniblo attannek okautiva: Angut taimna attanniub opigijomajanga,

8 Tamaungartaujuksauvok attititaukovlugo annorânik attannersiutinik, attanniub attorpaktanginik, horselo attanniub ablangavigivaktanga tamaungartaujuksauvoktauk; illijiviokovlugolo (angut taimna) niakunganut attanniub niakutânik;

9 Annorâglo tamna, horselo tunnijaujuksauvûk, attanniub angajokâksoangita illangata agganginut, tapsoma angut taimna attanniub opigijomajanga attitilugo pikovlugo, tessiorlugolo, ablangatillugo horsib kầngane, iglugaseksuit apkosiningittigut, nipliarkojilunelo sivurngane: Taimailiorvioniarpok angut attanniub opigijomajanga.

10 Attannek Hamanemut okarpok: Tuavitit, annorâglo, horselo tigukik, sorlo okalauravit, Mardachailo, Judeôjok attanniub itterviksoangata sillatane iksivajok taimailiorvigiuk; innoksartokarniarane sumik okautigilauktarnik. 
11 Tagva Hamanib annorâk horselo tiguvâk, attitipâlo Mardachai, tessiorlâlo iglugaseksuit apkosiningittigut, niplialunelo sivurngane: Imailiorviojuksauvok angut tamna, attanniub opigijomajanga.

12 Mardachailo tikkinivok attanniub itterviksoanganut. Hamanele aisadlarpok iglominut, kiksarlune kênanelo ulliklugo,

13 Unipkautivâlo nulliane Seres, illanârijanelo, tamainik tikkitautigijaminik. Tagva illisimajokotingita nulliangatalo Seresib okautivat: Mardachai ocholerutigijat Judit kinguvânginit pikpat, tagva sulliniatsengilatit tamna pivlugo, ochojutiginiarpalle.

14 Okartillugille sulle tapsomunga, attanniub pimaklertokotingit tikkiput, errinesarpâllo Haman nerrimaraksamut kaikovlugo, Esterib âkiksorlauktanganut.

\section{KAPITEL VII.}

Hamanib kimiktauninga nappartome Mardachai pivlugo nappalauktamine.

TAIMAGLO attannek Hamanelo tikkipuk nerrimâraksamut attanniub Esterib âkiksorlauktanganut;

2 Attanniublo ama uvlub aipangane Weinetorlaukârdlune Estere okautipsarpa: Sunamik tuksiarkît, attannek Ester? tunnitsiviojuksauvotit tamattominga. Sunalo tussugiviuk? Attannioviub koppanga pinajarpat aglât, taimaituksaulerpok.

3 Attannek Ester kiovok, okarlunelo: Saimavioneksiguma sângne attannîk! attanniublo namagikpago, tagva tunnitsivigilaunga innôsimnik tuksiarnera pitjutigællugo, innukotimniglo tussunera pitjutigællugo.

4 Niorvgutigijaumagapta, uvanga innukotikkalo, nungutaukovluta, naktertaukovlutalo, innuartaukovlutalo. Niorvgutigijaunajarupta arnaujogut angutaujogullo kivgaksaukovluta, tagva nippekarajangilanga; omisuktele sappidlarpok attanniub assiojijangit sattortautilugit.

5 Tagva attannek Ahasverose okausekarpok, okarlunelo attannermut Esteremut: Kinauva taimna? Kinauvalonêt taimaitomik issumalerungnartok taimailioromavlune?

6 Estere okarpok: Omisukte tamna, akkerartortorlo tamna, Hamaneôvok idluitok una. Hamanible attannek, aipangalo kakkiladlarutigivak.

7 Attannerlo kanningaumajarlune nikkovipok nerrimarnermit, Weinetornermillo, annilunelo perorsêvingmut iglub tunganêtomut. Hamanelo nikkovipok tuksiarvigivâlo attannek Ester 
innotsiminik, tækkogame kannoêtomik kajusimaviojarêrame attannermut.

8 Attannerlo uttertillugo perorsêvingmit iglub tunganêtomit nerrimarvingmut, Haman pamangavok iksivautab, Esterib iksivavigijangata tungâne. Tagva attannek okarpok: Attanniub aipanga aksorotijomavauk aglât najugamne iglome? Okausek tamanna attanniub kanninganit annilermat, Hamanib kênanga ullikpât.

9 Harbonalo pimaklertut illangat attanniub sângane, okarpok: Ahâk nappartomik nappasimajokarpok Hamanib iglungane, tallitut 50 tut pôktotigijomik, tapsoma sennajaukolauktanganik Mardachai, attanniub idluarkutiksanganik okalauktok, pivlugo. Attannek okarpok: Kimiktaule tapsomane.

10 Taimak Haman kimiktaulaukpok nappartome sennalauktamine Mardachai pivlugo. Tagva attanniub ningarninga sorailaukpok.

\section{KAPITEL VIII.}

Mardachaib angïorortitauninga. Attanniub perkojanga nutâk, Judit idluarkutiksanganut illingajok.

Uy VLORME taipsomane attanniub Ahasverosib attannek Estere tunnitsivigiva Hamanib Judit omigijingata iglunganik. Mardachailo tikkipok attanniub sânganut; Esterib okautilaungmago sugijaminik tapsominga.

2 Attanniublo ketterdlermêkotine Hamanemit tigulauktane pêrpa tunnilâlo Mardachaimut. Esteriblo Mardachai pimaklertingortipa Hamanib iglunganik.

3 Esterelo ama okarivok attanniub sângane, pamakterlunelo ittiggangita sânganut kennuvigivâlo pêjaikovlugo Hamanib Agagitereôjub kassêninganik, kajusimaninganiglo. Judit akkerartorlugit issumagilauktanginik.

4 Attanniublo ajaupiak opigijautiksak Goldeojok issautiva Esteremut. Tagva Estere makkipok, sângerlâlo attannek,

5 Okarlunelo: Attanniub namagikpago, saimavioneksigumalo sângne, idluarijaukpâllo attannermut, ânanaugijautgumalo tapsomunga, tagva aglaktokarle, aglait Hamanib Medatab erningata Agagitereôjub kajusimaningit aglalauktangit Judit tokkolugit attanniub nunangine, pitsartuêrotitaukovlugit.

6 Kannorle kannoêtok innukotima tikkitautiginajartangat tækkonarungnarajarpigo? Kanorlo tækkonârungnarkîk illama nungutauninginik?

7 Tagva attanniub Ahasverosib attannek Estere Mardachailo 
Judeôjok okautivak: Ahâk Hamanib iglunga tunnilaukpara Esteremut, taimnalo kimiktaumavok nappartome, aggane illilauramigit Judinut;

8 Tagva manna illiptik aglaleritik attanniub attingane Judit pivlugit namagijaptingnik, nakkiterlugillo attanniub ketterdlermêkotinganut. Aglait attanniub attingane aglaksimajut, attanniublo ketterdlermêkotinganut nalkikitersimajut pitsartuêrotitaujungnangimatta.

9 Tagva attanniub aglapaktokotingit nelliutome taipsomane kaikojauvut takkit pingajuane, tagva takkerme Sivanemik attelingme, uvlungita 23 ingane kaikojauvut, aglaktaulaukporlo, sorlo Mardachai perkojingmat, Judinut angajokaksoarnullo, nunallo aulatinginut, kappitanullo nunane, Indiamit Aethiopia tikkilugo; tagva nunane 127 gine, nunat illunatik aglagviôvut attunît aglatik malliklugit, innukattigêksuillo attunît okausitik malliklugit, Judillo, aglatik okautsitiglo malliklugit.

10 Aglakporlo (Mardachai) attanniub Ahasverosib attingane, nakkiterpaillo attanniub ketterdlermêkotinganut. Aglaillo tillikliutivait tillijattigut horseojartut innutsût kângine ablangajatigut.

11 Tapkonane attanniub Judit perkovait, nâne iglugaseksoarnekunik kattimalerungnaramik, innôtsitiglo sorsugutigilugit, nungutilugillo nakterlugillo tokkolugillo innugiartoksuit illunatik, nunablo kappiasuktijimik pitsartuningit, sorrutsit arnallo illaliudlugit perkutingillo arktautigilugit.

12 Uvlorme attautseme, attanniub Ahasverosib nunakotingine illunaine, tagva takkit 12 ingata, tagva takkib Adaremik attigub uvlungita 13 ingane.

13 Aglait tapkoa tukkingit imailaukput, perkojamik tunnijaumajokarmat nunane illunaine innukattigêksuit illunaita nellojungnairtilugit, Judit uvlorme taipsomane oppalungairsimajuksaugamik omigijitik akkiorlugit.

14 Tillijallo ablangajut horseojartut kângine, sukkalidlarlutik tuavilutiglo aularput attanniub okausingit malliklugit. Perkojarlo tamna tunnijaulaukpok attanniub igloksoangane Susaneme.

15 Mardachaile annivok attannermit annorarsimavlune attannersiutinik, korksutanik, kakkortaniglo, niakotelijarlunelo Goldeôjomik angijomik, ullilijarlarmelo Bissuseôjomik, Purpureôjomiglo. Iglugaseksuillo Susan (innuit) igiakomijarput, kuviasudlarlutiglo.

16 Judit kaumautekalaukput, kuviasutekalarmiglo, erkiertornekalutiglo, nertortaunekalaukpullo.

17 Nunanelo illunaine, iglugaseksoarnelo tikkitaujune attanniub okausinginut, perkojanganullo, kuviasungnekalaukpok, erkiêrtornekalarmelo Judit akkorngane, nerrimartitsivut uvlok- 
siorlutiglo. Unnuktullo nunat innunginit Judingorlaukput; Judit sivuranarningita tupvigilaungmagit.

\section{KAPITEL IX.}

\section{Judit akkiorningit omigïimingnik, uvloksiorvikartitsiningallo piulijaunimik erkaumajauvianik.}

TAKKIT 12 ingane, tagva takkib Adarib uvlungita 13 ingane, attanniub okausingita perkojangatalo nellautsititauviane, uvlorme taipsomane Judit omigijingit nerriulauramik sâlagilugit, ablatsangolerpok Judit omigijitik sâlagijaksaringmagit.

2 Tagva Judit kattimalerput iglugaseksoarmingne, attanniub Ahasverosib nunangine illunaine, aggatik illijomavlugit tapkonunga idluitullivigijomajinginut. Kialo agviarungnarlaungilait; sivuranarningat tikkisimalaungmat innukattigêksoarnut illunainut.

3 Angajokaunerôjullo nunane, angajokaksuillo, aulatsijullo nunanik, attanniublo pimaklertokotingita Judit kagvertipait, Mardachaib sivuranarningata tikkigamigit.

4 Mardachai angijolaungmat attanniub iglungane, tussaumajauningalo tussaksauiaukpok nunanut illunainut, kannok aglivalliangmat angijorormallo.

5 Taimaglo Judit omigijitik illunaita ullimârpait saviksoarmut, nakterlugillo, tokkolaitalo, pinniarvigivaillo issumamingnik omisuktut tapkoninga.

6 Susanemelo iglugaseksoarne, attanniub igloksoakarvigijangine, Judit nakterpait tokkolaitalo angutit 500 dit.

7 Parsandatalo, Dalfonelo, Aspatalo,

8 Poratalo, Adaljalo, Aridatalo,

9 Parmhastalo, Arisailo, Aridailo, Vajesatalo,

10 Hamanib Medatab erningata, Judit omigijingata erningit tellimaujortut; perkutinginulle aggatik illilaungilait.

11 Uvlorme taipsomane tokkotaumajut iglugaseksoarne Susaneme unnurningit pivut attanniub sânganut.

12 Attannerlo aipaminut Esteremut okarpok: Judit iglugaseksoarne Susaneme angutit 500 dit Hamaniblo erningit tellimaujortut nakterlugillo tokkolaukpait; kannok pinniaralloarkat attanniub nunangine assingine? Sunamiglo tuksiarkit? tunnitsiviojuksauvotit tamattominga. Sunalo tussugiviuk sulle? taimailertuksauvok.

13 Estere okarpok: Attannermut namagijaukpat, tagva Judit kaupattauk Susaneme pinniarlit perkojak uvlomênitak 
malliklugo, nivingaikovlugit Hamanib erninginik tellimaujortunik nappartomut.

14 Attannerlo perkojivok taimailiorkovlugit. Perkojarlo tamna tunnijaulaukpok Susaneme, Hamaniblo erningit tellimaujortut nivingartaulaukput.

15 Judillo kattimalerput Susaneme takkib Adarib uvlungita 14 ingane, nakterpaillo Susaneme angutit 300dit; perkutinginulle aggatik illilaungilait.

16 Judille tapkoa assingit attanniub nunangine, kattimalerlaukputtauk, innôtsitiglo sorsugutigivait, ullapirsautekartilerpullo omigijimingnit, tokkotsilutiglo omigijimingnit 75000 dinik, perkutinginulle aggatik illilaungilait.

17 Tamanna pilaukpok takkib Adarib uvlungita 13 ingane, merngoêrserlutiglo takkib tapsoma 14 ingane, tamna uvlungortipât nerrimârtitsinermut kuviasungnermullo illingajomut.

18 Judille Susaneme kattimalaukput uvlûngne tamangne, 13 me 14 melo, merngoêrserlutiglo uvlut 15 ingane, uvlorlo tamna uvlungortipât nerrimârtitsinermut kuviasungnermullo illingajomut.

19 Tamanna pivlugo Judit iglugasengne iglugaseksoarnelo avaluksoakangitune nunakartut, takkib Adarib uvlungita 14 ingat uvlungortipât nerrimartitsinermut kuviasungnermullo uvloksiorvingmullo, pajuktigêngnermullo aitusianik illingajomut.

20 Mardachaiblo pijokartut tamakkoa aglagutigivait, aglaillo tillikliutivait Judinut illunainut, kannitomêtunut kanningitomêtunullo.

21 Tamattominga aulajuititsivigijomavlugit, uvloksiorkattarlugik pikovlugit Jahrine tamaine, takkib Adarib uvlungita 14 ingat 15 ingallo,

22 Uvluktitut Judit ullapirsautiksarsivingititut omigijimingnit, takkertullo kappiasungningita ablatsangortitauvingatut kuviasungnermut, kiksarningitalo ablatsangortitauvingatut uvlunut ajungitunut; uvlûk tapkoa uvloksioraksaringmattikik uvlûktitut nerrimartitsinermut kuviasungnermullo pajuktigêngnermullo aitusianik pajungningnermullo aklujunut illingajûktut.

23 Judillo illaliorpait pigiarlauktatik pinniarlugit, Mardachaiblo aglalauktangit tapkonunga :

24 Kannok Haman Medatab erninga Agagitereôjok Judit illunatik omigijingat issumalaungmat Judit illunaita tokkotsomavlugit, kannorlo immeraut attortaukolaungmago, tapkoa koaksartitsomavlugit, innuaromavlugillo;

25 Kannorlo Estere attannermut ailaungmat, okalaungmallo, aglangnut tapsoma kajusimaningit idluitut, Judit pivlugit issumagilauktangit, sangutitaukovlugit tapsoma niakoanut; kanorlo tamna erningillo nivingartaulaungmatta nappartomut.

26 Tamanna pivlugo uvlûk tapkoa atserarpâk Purimik, 
okausek Pur, (tagva imeraut) malliklugo. Tamanna pivlugo aglait tapkoa okausertangit illunaita malliklugit, nangminerlo tækkolauktatik, tikkitautigilauktatiglo malliklugit,

27 Judit sokkosijuititsivut, illaliorlutiglo, pivlutik, kinguvaksatiglo pivlugit, illunaitalo pivlugit tapkonunga illingajomanajartut, attortautailikonago uvlûk tapkoa uvloksioromavlugik, oktutiksak malliklugo, urlungne nellonairsimajungne Jahrine tamaine ;

28 Uvlûglo tapkoa erkaumajautsainartuksaungmanik, uvloksiortaujuksaulutiglo kinguvarêngne illunaine, kittorngarêngnelo illunaine; nunane illunaine, iglugaseksoarnelo illunaine; uvlûglo tapkoa Purimik attilik pijungnaituksaungimanik Judit akkorngane, erkaumajauningallo sorairtuksaungimat kinguvângine.

29 Attannerlo Estèr Abihalib pannia, Mardachailo Judiôjok aglalaukpûk illungertorlutik, aglait aipangit tapkoa Purimemik okartut nakkiterlugit;

30 Aglaillo tapkoa tillikliutivait Judinut illunainut, Ahasverosib attanniovingata nunangine, 127 ginêtunut, okautsit illanarnartut nertornartullo.

31 Uvlûk Purimemik attelîk nakkiterlugik pikovlugit nelliutome nellonairtaumajome, sorlo Mardachaib Judeôjub Esteriblo attanniub nellonairsivigilaungmattigik tamattominga, sorlolo nangminek pivlutik, kingurakotitiglo pivlugit sokkosijuititsilaungmatta, nerritailinerub kêanerublo pijokarningangnik.

32 Esteriblo perkojiningata pijokartok Purime tamanna nakkiterpa; aglaktaulaukporlo aglangnut.

\section{KAPITEL X.}

Mardachaib pitsartunetiub innukotime idluarkutiksangat pinnasuarpa.

ATTANNIUBLO Ahasverosib nuna, kikkertallo immarbingme (innuit) tunnijikovait akkiliaksanik.

2 Sôngoningatale, pitsartuningatalo pinniarningit illunaita, Mardachaiblo ânanaudlarninga, attanniub tunnilauktanga tapsomunga; ahâk tamakkoa aglaksimavut Medenemiut Persiamiullo attanningita aglakotingine. 
3 Mardachai Judiôjok tugliolaungmat attannermut Ahasverosemut, angijôlunelo Judit akkorngane, kuviagijaulunelo kattangutime unnurninginut, kennerlauktok innukotime idluarkutiksanganik, okalauktorlo namanerpângôjomik kinguvakotine illunaita pivlugit. 








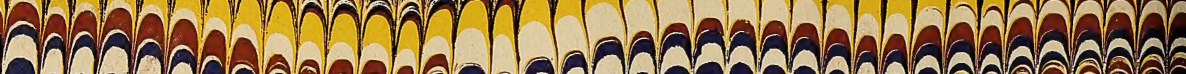
A

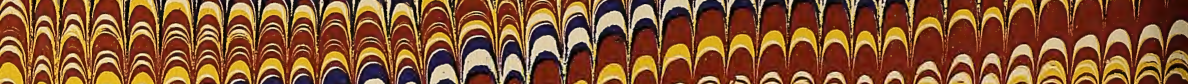

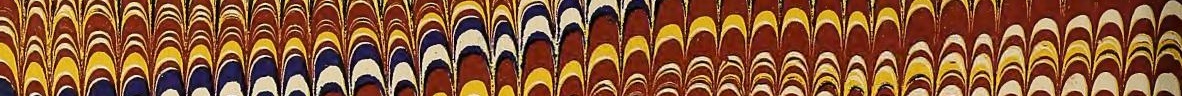
ลด

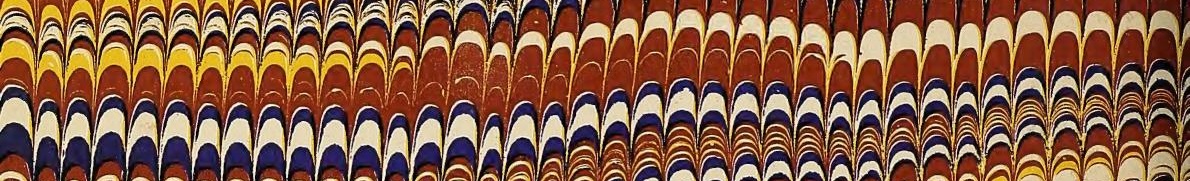
以ล

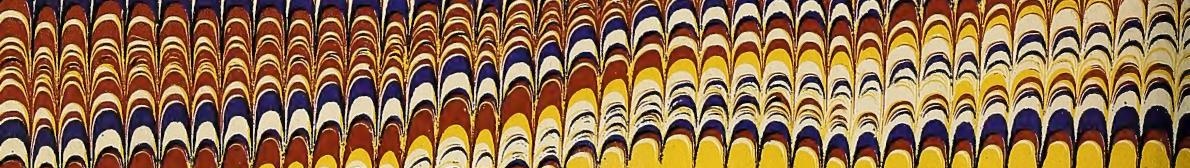

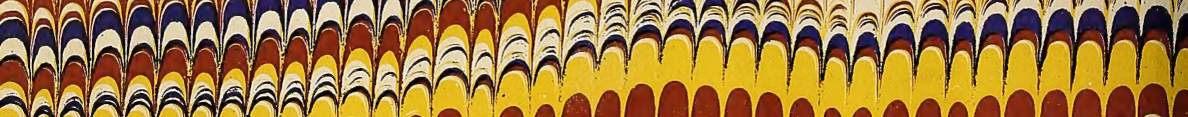

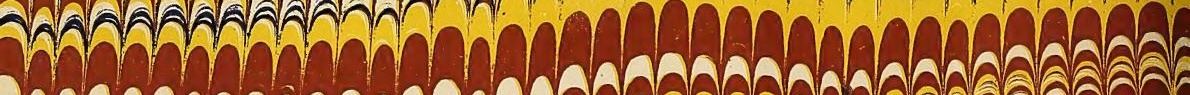
*

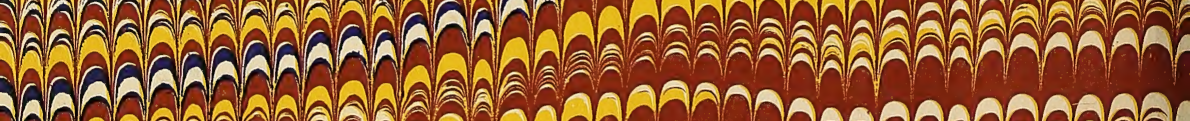
Whanh

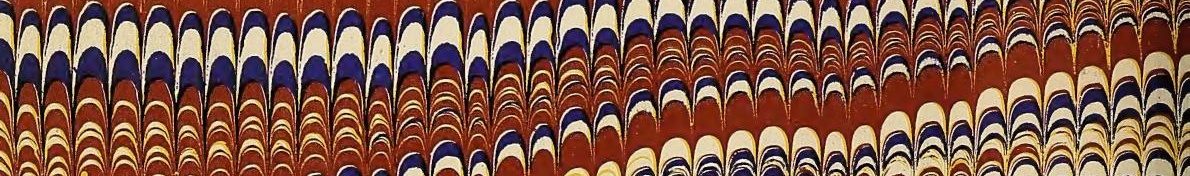
ตร

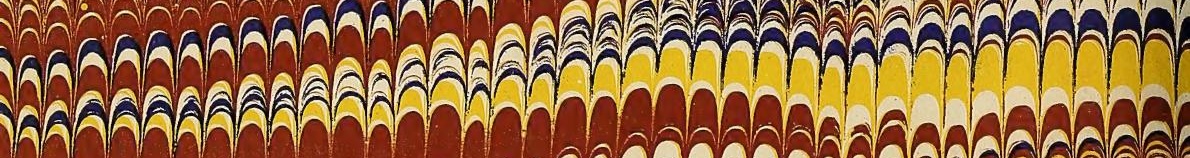
WhanamanaAna

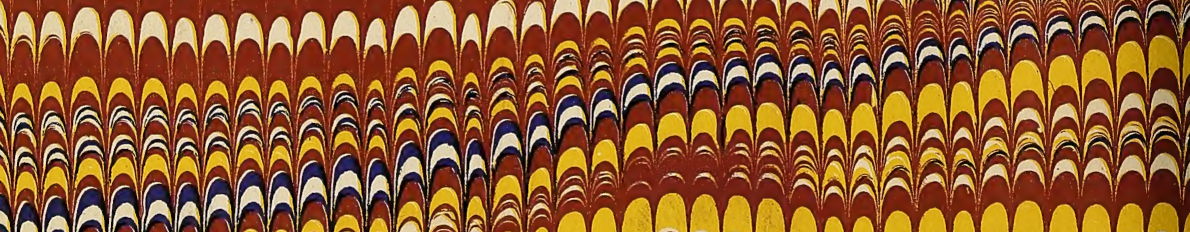
a 


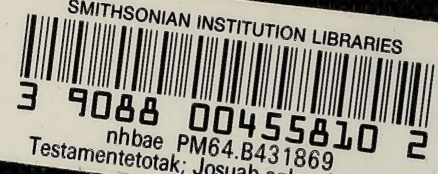
osuab agla 